\title{
Charakterisierung minimaler und maximaler Hemmkonzentrationen verschiedener Spurenelemente in Biogasprozessen und Entwicklung eines vereinfachten Bestimmungsverfahrens
}

\author{
Dissertation \\ zur Erlangung des mathematisch-naturwissenschaftlichen Doktorgrades \\ "Doctor rerum naturalium" \\ der Georg-August-Universität Göttingen \\ im Promotionsprogramm Geowissenschaften / Geographie \\ der Georg-August University School of Science (GAUSS) \\ vorgelegt von \\ Tino Pasold \\ aus Karl-Marx-Stadt \\ (Geburtsort)
}

Göttingen 2020 
Betreuungsausschuss:

Prof. Dr. Hans Ruppert, Abteilung Sedimentologie/Umweltgeologie,

Geowissenschaftliches Zentrum (GZG), Interdisziplinäres Zentrum für Nachhaltige Entwicklung (IZNE), Georg-August-Universität Göttingen

Dr. Benedikt Sauer, Abteilung Sedimentologie/Umweltgeologie, Geowissenschaftliches Zentrum (GZG), Interdisziplinäres Zentrum für Nachhaltige Entwicklung (IZNE), Georg-August-Universität Göttingen

\section{Mitglieder der Prüfungskommission:}

Referent: Prof. Dr. Hans Ruppert, Abteilung Sedimentologie/Umweltgeologie, Geowissenschaftliches Zentrum (GZG), Interdisziplinäres Zentrum für Nachhaltige Entwicklung (IZNE), Georg-August-Universität Göttingen

Korreferent: Prof. Dr. Volker Thiel, Abteilung Geobiologie, Geowissenschaftliches Zentrum (GZG), Georg-August-Universität Göttingen

weitere Mitglieder der Prüfungskommission:

Prof. Dr. Hilmar von Eynatten, Abteilung Sedimentologie/Umweltgeologie, Geowissenschaftliches Zentrum (GZG), Georg-August-Universität Göttingen

Prof. Dr. Matthias Willbold, Abteilung Isotopengeologie,

Geowissenschaftliches Zentrum (GZG), Georg-August-Universität Göttingen

Dr. Volker Karius, Abteilung Sedimentologie/Umweltgeologie, Geowissenschaftliches Zentrum (GZG), Georg-August-Universität Göttingen

Dr. Bernd Leiss, Abteilung Strukturgeologie und Geodynamik, Geowissenschaftliches Zentrum (GZG), Georg-August-Universität Göttingen

Tag der mündlichen Prüfung: $\quad$ 08.07.2020 
Wir wissen jetzt, daß die Ideen der Menschen nach bestimmten Gesetzen der Natur und des menschlichen Geistes organisch sich entwickeln, und sehen den Baum menschlicher Erkenntnis, den die Griechen gepflanzt, auf dem Boden der Zivilisation und mit dessen Pflege, wachsen und sich entwickeln ohne Unterbrechung, und im Sonnenschein der Freiheit blühen und Früchte tragen zur richtigen Zeit.

JUSTUS FREIHERR VON LIEBIG (1866) 


\section{Kurzfassung}

Charakterisierung minimaler und maximaler Hemmkonzentrationen verschiedener Spurenelemente in Biogasprozessen und Entwicklung eines vereinfachten Bestimmungsverfahrens

Für optimale Biogasbildungsprozesse in Biogasanlagen müssen zahlreiche Betriebsparameter innerhalb gewisser Grenzen liegen. Eine ausreichende Versorgung mit essenziellen Makro- und Mikronährstoffen muss für die Umsetzungs- und Abbauprozesse der gasbildenden Mikroorganismen gewährleistet sein. Insbesondere die Elemente Cobalt und Nickel spielen für die Gasausbeute landwirtschaftlicher Biogasanlagen eine herausragende Rolle. Allerdings reichen bei alleinigem Mais-Silage-Input die Spurenelemente für eine optimale Methanbildung nicht aus, so dass in Deutschland in geschätzt etwa der Hälfte aller Biogasanlagen synthetische Spurenelementadditive zugefüttert werden, um dem Mangel zu begegnen. Das birgt verschiedene Umweltrisiken, wie die Belastung von Böden, verursacht zusätzliche Kosten und steht im Konflikt mit der Düngemittelverordnung. Alternative Energiepflanzen kumulieren im Vergleich zu Mais erheblich mehr an essenziellen Spurenelementen. Gegenstand dieser Arbeit war, in Nassfermentationsversuchen im Labormaßstab zunächst für Cobalt und Nickel die minimalen Schwellenwerte im Biogasprozess zu erarbeiten und dann herauszufinden, ob durch eine Zumischung der alternativen Energiepflanzen-Silagen Winterackerbohne, Amarant, Deutsches Weidelgras bzw. Durchwachsene Silphie zu Mais auf die Zugabe von synthetischen Spurenelementadditiven weitgehend verzichtet werden kann. In geringer Konzentration positiv wirkende Elemente können jedoch bei höheren Gehalten inhibierend oder sogar toxisch auf die Mikroorganismen und damit die Gasbildungsprozesse im Fermenter wirken. So wurden zudem in dieser Arbeit in diskontinuierlichen Gärversuchen auf Basis von ORGA-Tests für Cobalt, Nickel, Molybdän, Kupfer sowie Cadmium und Blei die oberen Schwellenwerte erarbeitet, die beginnende Hemm- bzw. Toxizitätswirkungen anzeigen. Zur besseren Charakterisierung des reaktiven Spurenelementanteils im Fermenter wurden außerdem Trennungs- und Filtrationsversuche mit verschiedenen Fraktionen des Fermentermaterials durchgeführt. Ein vereinfachtes Bestimmungsverfahren wird vorgeschlagen, um auf Spurenelementmangelsituationen schneller reagieren und gegensteuern zu können. 


\begin{abstract}
Characterization of minimum and maximum inhibitory concentrations of various trace elements in biogas processes and development of a simplified determination method
\end{abstract}

For optimal biogas formation processes in biogas plants numerous operating parameters must be within certain limits. A sufficient supply of essential macro- and micronutrients must be guaranteed for the conversion and degradation processes of biomass by the gas producing microorganisms. The trace elements cobalt and nickel in particular play an outstanding role in the gas yield of agricultural biogas plants. However, with maize silage input only, the trace elements are not sufficient for optimal methane formation, so that in Germany in about half of all biogas plants synthetic trace element additives are added to counteract the deficiency. This involves various environmental risks, such as soil contamination, causes additional costs and conflicts with the German fertilizer regulation. Alternative energy crops accumulate considerably more essential trace elements than maize. The subject of this work was first to work out the minimum threshold values for cobalt and nickel in the biogas process in laboratory-scale wet fermentation tests. In a second step it should be found out if the addition of alternative energy crop silages such as winter faba bean, amaranth, perennial ryegrass and cup plant to maize can largely avoid the addition of synthetic trace element cocktails. However, elements with a positive effect in low concentrations can have at higher concentrations an inhibiting or even toxic effect on the microorganisms and the gas formation processes in the biogas digester. In this work, the upper threshold values indicating the beginning inhibitory and toxicity effects were worked out for cobalt, nickel, molybdenum, copper as well as cadmium and lead in discontinuous fermentation assays based on ORGAtests. For better characterization of the reactive trace element content in the biogas digester, separation and filtration assays were also carried out with different fractions of the digester material. A simplified determination method is proposed in order to be able to react more quickly to counteract deficiencies in trace elements in the biogas digester. 


\section{Danksagung}

Ich möchte mich herzlich bei allen bedanken, die maßgeblich zum Gelingen dieser Arbeit beigetragen haben. Mein ganz besonderer Dank gilt hierbei meinen beiden Betreuern, meinem Doktorvater Herrn Prof. Dr. Hans Ruppert für die inspirierenden wissenschaftlichen und auch nicht-wissenschaftlichen Diskussionen sowie Herrn Dr. Benedikt Sauer, der immer ein offenes Ohr für meine Anliegen hatte und mir oft gute Impulse gab. Die vielen schönen und ideenreichen Mensagespräche und die angenehme Arbeitsatmosphäre mit euch werden mir immer in Erinnerung bleiben.

Diese Dissertation wurde im Rahmen des NiCo-Verbundprojektes mit Mitteln des Bundesministeriums für Ernährung und Landwirtschaft (BMEL) über die Fachagentur Nachwachsende Rohstoffe e.V. (FNR) als Projektträger des BMEL gefördert, wofür ich mich nochmals bedanken möchte. Mein Dank für die zielführende Zusammenarbeit gilt außerdem den Projektpartnern in Leipzig am Deutschen Biomasseforschungszentrum gGmbH (DBFZ), Herrn Michael Zechendorf und Herrn Dr. Jürgen Pröter, sowie am Helmholtz-Zentrum für Umweltforschung (UFZ), Frau Dr. Heike Sträuber. Auch bei Dietmar Grimm, Rene Schieritz, Tom Heyner und Robert Kießling, die im Rahmen ihrer Master-, Bachelor- und Praxisarbeiten am DBFZ mit ins NiCo-Verbundprojekt involviert waren, möchte ich mich bedanken. Ferner bedanke ich mich für die sehr gute Zusammenarbeit bei Herrn Prof. Dr. Rolf Rauber und Doktorandin Frau Katharina Hey von der Abteilung Pflanzenbau an der Georg-August-Universität Göttingen.

Weiter gilt mein Dank Herrn Prof. Dr. Volker Thiel für die Übernahme des Korreferats sowie Herrn Prof. Dr. Hilmar von Eynatten, Herrn Prof. Dr. Matthias Willbold, Herrn Dr. Volker Karius und Herrn Dr. Bernd Leiss für die Mitwirkung in der Prüfungskommission. Außerdem bedanke ich mich bei Frau Irina Ottenbacher, Frau Cornelia Friedrich und Frau Judit Dunklne-Nagy für die Hilfe bei allen Laborgerätefragen und die netten Laborgespräche, bei Herrn Dr. Klaus Simon und Herrn Jakob Rauscher für die ICP-MS-Messungen, bei Herrn Lothar Laake und Herrn Bernd Thiele für den schnellen Bau von „Zubehörteilen“ für die Laborgeräte und bei Herrn Dr. Adam Feher für die wertvollen Tipps für meine ORGA-Tests.

Die Laborunterstützung durch meine studentischen Hilfskräfte Frau Janina Sandoval Saldias, Frau Fraya Annisa und Herrn Jakob Popp war Gold wert. Habt vielen Dank! 
Frau Dr. Wiebke Fahlbusch, Frau Dr. Solveig Pospiech und Frau Dr. Thùy Phương Nguyễn möchte ich danken für die vielen schönen Bürostunden und Diskussionsrunden in unserer Arbeitsgruppe Umweltgeochemie. Den beiden Herren Clemens und Heinrich Ropeter von der Praxisbiogasanlage in Verliehausen danke ich für die sehr angenehme und unkomplizierte Zusammenarbeit über all die Jahre hinweg. Zudem danke ich Frau Dr. Marianne Karpenstein-Machan, Herrn Mathias Hartel und Herrn Dr. Michael Lebuhn für den fachlichen Austausch. Auch den Studierenden Henrike Munier, Josefine Neuhäuser, Ruth Rosenbauer, Lukas Zwosta, André Petering, Anton Pfeiffer, Tobias Stürzebecher, Max Caesar und Clemens Jakob, die ich bei ihren Bachelorarbeiten als Zweitgutachter und/oder bei den Laborarbeiten betreute, danke ich für die gute und problemlose Zusammenarbeit.

Abschließend möchte ich mich bei meiner Familie, insbesondere Katharina und meiner Mutter, für die unglaubliche Unterstützung und Geduld bedanken, die mir in all den Jahren zuteil wurde und stets Rückhalt gab. 


\section{Inhalt}

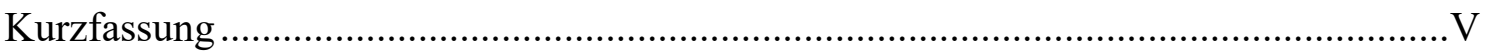

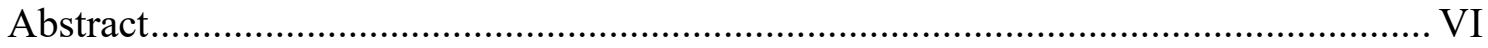

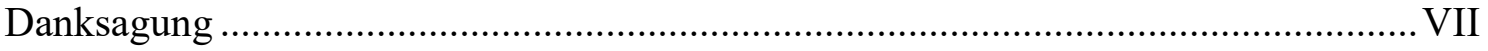

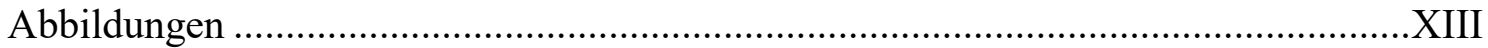

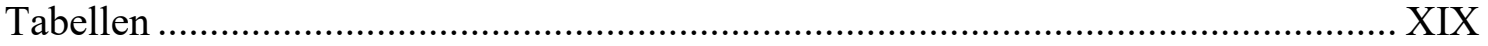

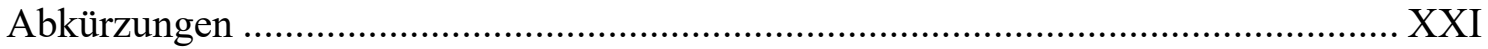

Kapitel 1: Einleitung und Zielstellung............................................................................ 1

Kapitel 2: Grundlagen ..................................................................................................................... 5

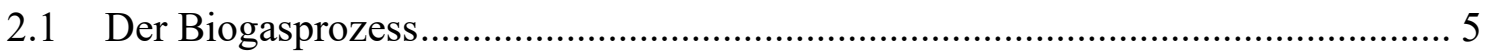

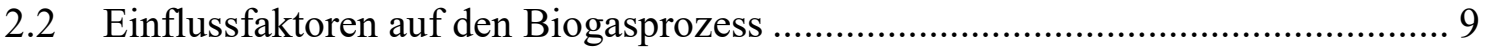

2.2.1 Raumbelastung, Verweilzeit und Durchmischung...................................... 10

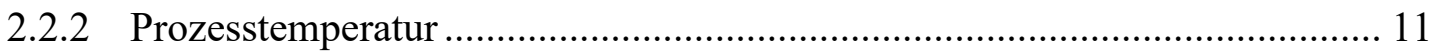

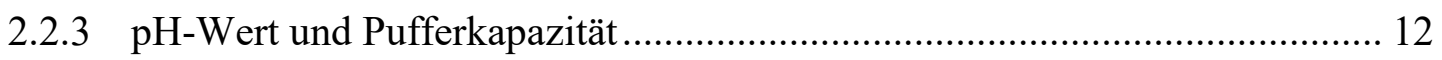

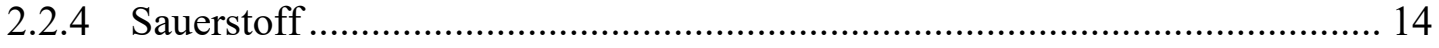

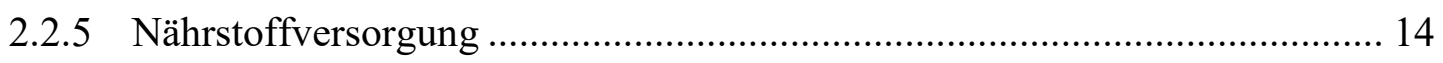

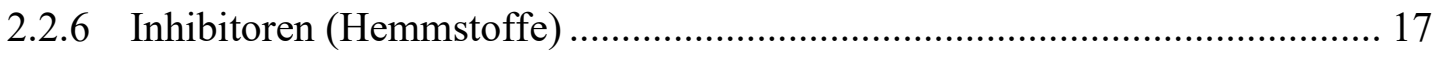

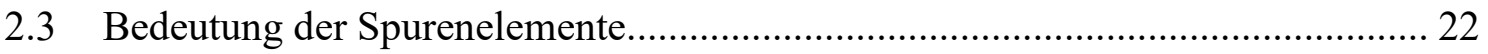

Kapitel 3: Allgemeine Methodik ............................................................................................... 29

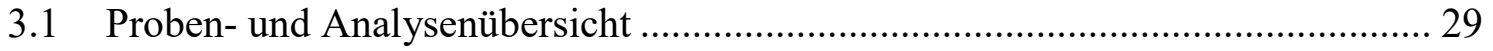

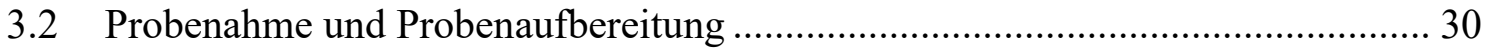

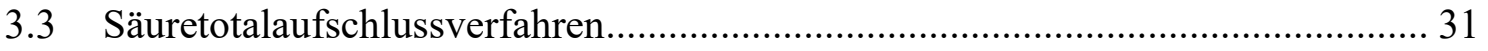

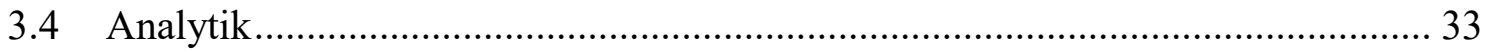

3.4.1 Trockensubstanzgehalte der Gärgemisch- und Silageproben ....................... 33

3.4.2 Organische Trockensubstanzgehalte der Gärgemisch- und Silageproben ..... 33

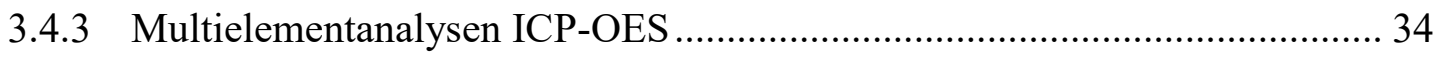

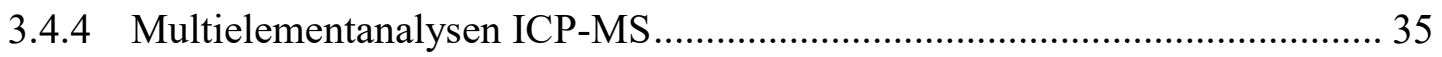

3.5 Fehlerbetrachtung und Qualitätssicherung................................................... 36

3.5.1 Kurzbetrachtung systematischer und methodischer Fehlerquellen................ 36

3.5.2 Qualität der Messergebnisse und Elementauswahl ..................................... 37

3.5.3 Nickel-Chrom-Problematik bei den Laborfermenterversuchen..................... 42

Kapitel 4: (Quasi)kontinuierliche Laborfermenterversuche..................................... 43

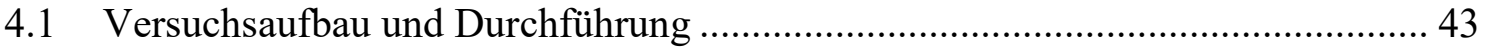

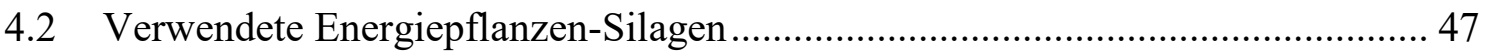


4.3 Verarmungsversuche für Cobalt und Nickel ..................................................... 51

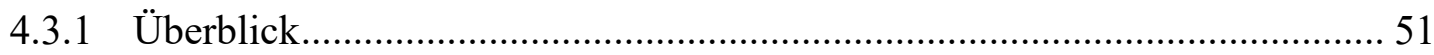

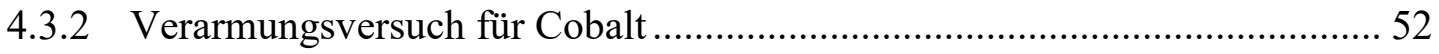

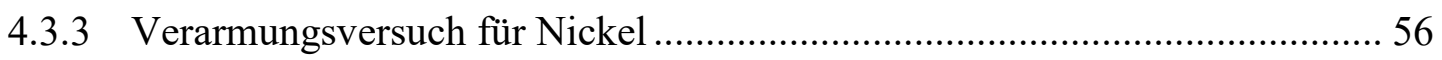

4.3.4 Mögliche Zinkhemmung im Verarmungsversuch für Nickel ....................... 61

4.3.5 Bewertung weiterer Elemente in den Verarmungsversuchen ......................... 64

4.3.6 Eisen/Schwefel-Verhältnisse in den Verarmungsversuchen.......................... 70

4.3.7 Mikrobiologischer Überblick zur Zusammensetzung, Dynamik und Aktivität der mikrobiellen Gemeinschaften in den Verarmungsversuchen ... 71

4.4 Substitutionsversuche von Mais-Silage mit Silagen von Winterackerbohne und

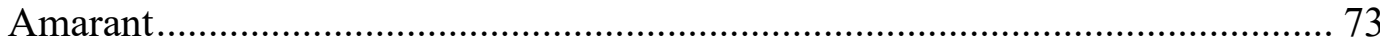

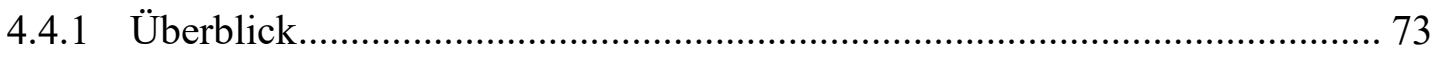

4.4.2 Substitutionsversuch 1: Vergärung von $30 \%$ Silage aus Winterackerbohne zu 70 \% Mais-Silage (oTS-bezogen)............................ 75

4.4.3 Substitutionsversuch 2: Vergärung von $30 \%$ Silage aus Amarant zu 70 \% Mais-Silage (oTS-bezogen) ......................................... 82

4.4.4 Bewertung weiterer Elemente in den Substitutionsversuchen 1 und 2 .......... 87

4.4.5 Eisen/Schwefel-Verhältnisse in den Substitutionsversuchen 1 und 2 ........... 96

4.4.6 Mikrobiologischer Überblick zur Zusammensetzung und Dynamik der mikrobiellen Gemeinschaften in den Substitutionsversuchen 1 und 2 ......... 97

4.5 Substitutionsversuche von Mais-Silage mit Silagen von Deutsches Weidelgras

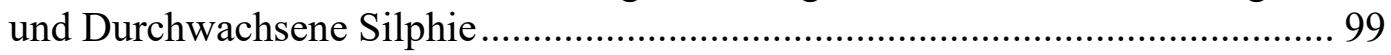

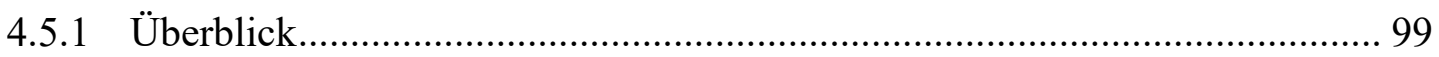

4.5.2 Substitutionsversuch 3: Vergärung von $30 \%$ Silage aus Deutsches Weidelgras zu 70 \% Mais-Silage (oTS-bezogen)...................... 101

4.5.3 Substitutionsversuch 4: Vergärung von $30 \%$ Silage aus Durchwachsene Silphie zu 70 \% Mais-Silage (oTS-bezogen) ................... 110

4.5.4 Nickel-Chrom-Problematik in den Substitutionsversuchen 3 und 4............ 116

4.5.5 Bewertung weiterer Elemente in den Substitutionsversuchen 3 und 4 ........ 118

4.5.6 Eisen/Schwefel-Verhältnisse in den Substitutionsversuchen 3 und 4 ......... 126

4.5.7 Mikrobiologischer Überblick zur Zusammensetzung und Dynamik der mikrobiellen Gemeinschaften in den Substitutionsversuchen 3 und 4 ....... 128

4.6 Zusatzversuch mit Mais-Silage-Monovergärung unter reduzierter

Spurenelementzugabe

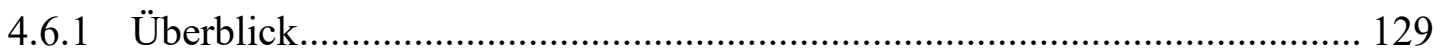

4.6.2 Charakterisierung der Prozessparameter und Cobalt- und Nickelgehalte ... 130

4.6.3 Bewertung weiterer Elemente im Zusatzversuch ...................................... 134

4.6.4 Eisen/Schwefel-Verhältnisse im Zusatzversuch ...................................... 139

Kapitel 5: Diskontinuierliche Gärversuche (ORGA-Tests) ................................. 141

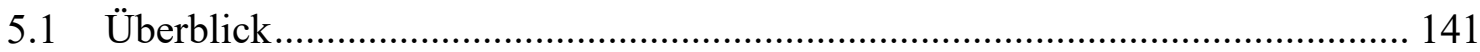

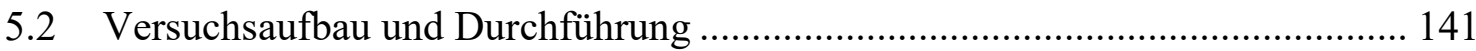


5.3 Obere Hemm- und Toxizitätswirkung von Nickel........................................... 146

5.4 Obere Hemm- und Toxizitätswirkung von Cobalt............................................ 152

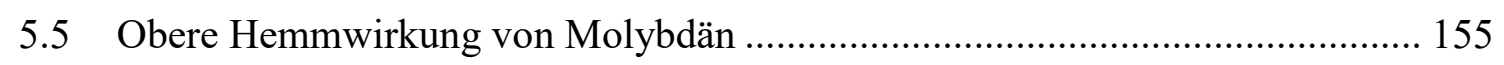

5.6 Obere Hemm- und Toxizitätswirkung von Kupfer ........................................... 156

5.7 Obere Hemm- und Toxizitätswirkung von Cadmium....................................... 159

5.8 Obere Hemmwirkung von Blei .................................................................... 162

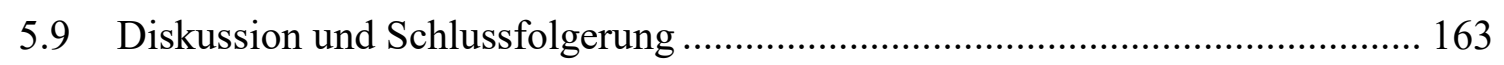

Kapitel 6: Vereinfachtes Bestimmungsverfahren................................................. 167

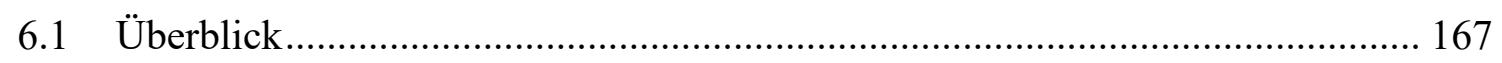

6.2 Durchführung von Versuchen mit verschiedenen Fraktionen von

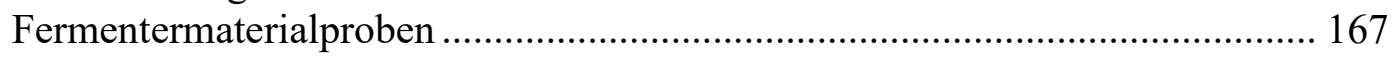

6.3 Ergebnisse und Interpretation der Trennungs- und Filtrationsversuche ............. 171

6.3.1 Elementmengenvergleiche zur Abschätzung der Aufschlusseffizienz ........ 171

6.3.2 Elementkonzentrationen der Filtrate $(<0,45 \mu \mathrm{m})$................................... 173

6.3.3 Elementkonzentrationen der Presssäfte $(<63 \mu \mathrm{m})$.................................... 175

6.3.4 Elementkonzentrationen der Pressrückstände $(>63 \mu \mathrm{m})$............................ 177

6.3.5 Vergleich der Elementkonzentrationen sowie der Elementanteile in der Festsubstanz und flüssigen Phase der verschiedenen Fraktionen.......... 179

6.3.6 Überlegungen zu Löslichkeiten von Schwermetallen im Fermenter ............ 183

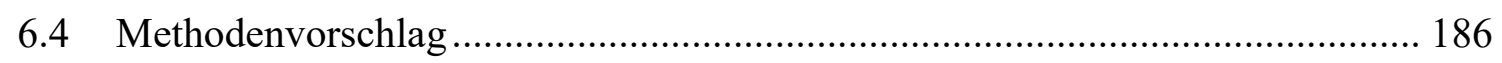

Zusammenfassung ....................................................................................................................... 187

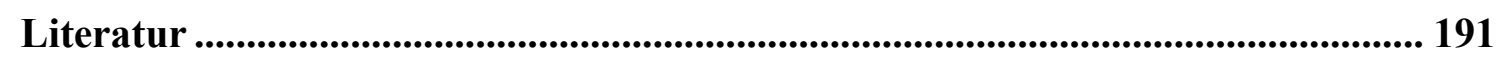

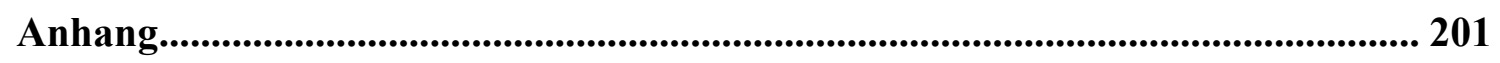

Wissenschaftlicher Werdegang ........................................................................ 217 


\section{Abbildungen}

Abbildung 1: Vereinfachte schematische Darstellung des anaeroben Abbauprozesses bei der einphasigen Biogasproduktion (modifiziert nach LEBUHN \& GRONAUER, 2009; SCHIEDER ET AL., 2010).

Abbildung 2: Klassifizierung der elementaren Grundelemente und Nährstoffe für anaerobe Mikroorganismen im Periodensystem der Elemente (modifiziert nach WACKETT ET AL., 2004 und WEINRICH, 2017).

Abbildung 3: Vereinfachte schematische Darstellung der Versorgung und Verfügbarkeit von Spurenelementen im Fermenter (modifiziert nach BAUER ET AL., 2009).

Abbildung 4: Vereinfachtes Schema wesentlicher Prozesse, die die Bioverfügbarkeit von Spurenelementen im Fermenter beeinflussen (modifiziert nach NRC, 2003 und VAN HULLEBUSCH ET AL., 2016).

Abbildung 5: Fotoaufnahmen eines mehrstufigen Säuretotalaufschlussverfahrens mit dem Druckaufschluss-System DAS 30 der Firma PicoTrace.

Abbildung 6: Schematischer Querschnitt und Aufbau eines im Verbundprojekt betriebenen Versuchsbiogasreaktors am DBFZ (modifiziert nach SCHIERITZ, 2016).

Abbildung 7: Fotoaufnahmen der fünf am DBFZ (quasi)kontinuierlich betriebenen Versuchsbiogasreaktoren (R 4.5 bis R 4.9), die im Verbundprojekt benutzt wurden.

Abbildung 8: Verlauf der gemittelten Gassummenkurven der ORGA-Testreihe zur Ermittlung der spezifischen Biogaserträge der bei den (quasi)kontinuierlichen Laborfermenterversuchen verwendeten Energiepflanzen-Silagen.

Abbildung 9: $\quad$ FOS/TAC (FAL)-Verläufe des Verarmungsversuchs für Cobalt in den Versuchsbiogasreaktoren (mit Referenzreaktor).

Abbildung 10: $\quad$ pH-Wert-Verläufe des Verarmungsversuchs für Cobalt in den Versuchsbiogasreaktoren (mit Referenzreaktor).

Abbildung 11: Verläufe der normierten Gasproduktion des Verarmungsversuchs für Cobalt in den Versuchsbiogasreaktoren (mit Referenzreaktor)

Abbildung 12: Verläufe des normierten Methangehalts des Verarmungsversuchs für Cobalt in den Versuchsbiogasreaktoren (mit Referenzreaktor).

Abbildung 13: Gegenüberstellung der Verläufe der FOS/TAC (FAL)-Werte und der normierten Gasproduktion des Verarmungsversuchs für Cobalt in den Versuchsbiogasreaktoren (mit Referenzreaktor).

Abbildung 14: Verläufe der Cobaltkonzentrationen des Verarmungsversuchs für Cobalt in den Versuchsbiogasreaktoren (mit Referenzreaktor).

Abbildung 15: FOS/TAC (FAL)-Verläufe des Verarmungsversuchs für Nickel in den Versuchsbiogasreaktoren (mit Referenzreaktor)

Abbildung 16: pH-Wert-Verläufe des Verarmungsversuchs für Nickel in den Versuchsbiogasreaktoren (mit Referenzreaktor)

Abbildung 17: Verläufe der normierten Gasproduktion des Verarmungsversuchs für Nickel

in den Versuchsbiogasreaktoren (mit Referenzreaktor).

Abbildung 18: Verläufe des normierten Methangehalts des Verarmungsversuchs für Nickel in den Versuchsbiogasreaktoren (mit Referenzreaktor).

Abbildung 19: Gegenüberstellung der Verläufe der FOS/TAC (FAL)-Werte und der normierten Gasproduktion des Verarmungsversuchs für Nickel in den Versuchsbiogasreaktoren (mit Referenzreaktor).

Abbildung 20: Verläufe der Nickelkonzentrationen des Verarmungsversuchs für Nickel in den Versuchsbiogasreaktoren (mit Referenzreaktor). 
Abbildung 21: Verläufe der Zinkkonzentrationen des Verarmungsversuchs für Nickel in den Versuchsbiogasreaktoren (mit Referenzreaktor).

Abbildung 22: Verläufe der Zinkkonzentrationen des Verarmungsversuchs für Cobalt in den Versuchsbiogasreaktoren (mit Referenzreaktor).

Abbildung 23: Verläufe der Calcium-, Kalium-, Magnesium- und Natriumgehalte der Verarmungsversuche für Cobalt und Nickel in den Versuchsbiogasreaktoren (mit Referenzreaktor).

Abbildung 24: Verläufe der Phosphor- und Schwefelgehalte der Verarmungsversuche für Cobalt und Nickel in den Versuchsbiogasreaktoren (mit Referenzreaktor).

Abbildung 25: Verläufe der Eisen-, Kupfer-, Mangan- und Molybdängehalte der Verarmungsversuche für Cobalt und Nickel in den Versuchsbiogasreaktoren (mit Referenzreaktor).

Abbildung 26: Verläufe der Cadmium-, Chrom- und Bleigehalte der Verarmungsversuche für Cobalt und Nickel in den Versuchsbiogasreaktoren (mit Referenzreaktor).

Abbildung 27: Verläufe der Aluminium-, Lanthan-, Titan- und Strontiumgehalte der Verarmungsversuche für Cobalt und Nickel in den Versuchsbiogasreaktoren (mit Referenzreaktor).

Abbildung 28: Verläufe der Eisen/Schwefel-Verhältnisse der Verarmungsversuche für Cobalt und Nickel in den Versuchsbiogasreaktoren (mit Referenzreaktor).

Abbildung 29: FOS/TAC (FAL)-Verläufe des Substitutionsversuchs 1 mit $30 \%$ Winterackerbohne- zu $70 \%$ Mais-Silage (oTS-bezogen) in den Versuchsbiogasreaktoren (mit Referenzreaktor).

Abbildung 30: $\quad$ pH-Wert-Verläufe des Substitutionsversuchs 1 mit $30 \%$ Winterackerbohne- zu $70 \%$ Mais-Silage (oTS-bezogen) in den Versuchsbiogasreaktoren (mit Referenzreaktor).

Abbildung 31: Verläufe der normierten Gasproduktion des Substitutionsversuchs 1 mit $30 \%$ Winterackerbohne- zu $70 \%$ Mais-Silage (oTS-bezogen) in den Versuchsbiogasreaktoren (mit Referenzreaktor).

Abbildung 32: Verläufe des normierten Methangehalts des Substitutionsversuchs 1 mit $30 \%$ Winterackerbohne- zu $70 \%$ Mais-Silage (oTS-bezogen) in den Versuchsbiogasreaktoren (mit Referenzreaktor).

Abbildung 33: Gegenüberstellung der Verläufe der FOS/TAC (FAL)-Werte und der normierten Gasproduktion des Substitutionsversuchs 1 mit $30 \%$ Winterackerbohne- zu $70 \%$ Mais-Silage (oTS-bezogen) in den Versuchsbiogasreaktoren (mit Referenzreaktor).

Abbildung 34: Verläufe der Cobaltkonzentrationen des Substitutionsversuchs 1 mit $30 \%$ Winterackerbohne- zu 70 \% Mais-Silage (oTS-bezogen) in den Versuchsbiogasreaktoren (mit Referenzreaktor).

Abbildung 35: Verläufe der Nickelkonzentrationen des Substitutionsversuchs 1 mit $30 \%$ Winterackerbohne- zu $70 \%$ Mais-Silage (oTS-bezogen) in den Versuchsbiogasreaktoren (mit Referenzreaktor).

Abbildung 36: FOS/TAC (FAL)-Verläufe des Substitutionsversuchs 2 mit $30 \%$ Amarant- zu $70 \%$ Mais-Silage (oTS-bezogen)

in den Versuchsbiogasreaktoren (mit Referenzreaktor).

Abbildung 37: pH-Wert-Verläufe des Substitutionsversuchs 2 mit $30 \%$ Amarant- zu $70 \%$ Mais-Silage (oTS-bezogen) in den Versuchsbiogasreaktoren (mit Referenzreaktor).

Abbildung 38: Verläufe der normierten Gasproduktion des Substitutionsversuchs 2 mit $30 \%$ Amarant- zu $70 \%$ Mais-Silage (oTS-bezogen) in den Versuchsbiogasreaktoren (mit Referenzreaktor). 
Abbildung 39: Verläufe des normierten Methangehalts des Substitutionsversuchs 2 mit

$30 \%$ Amarant- zu 70 \% Mais-Silage (oTS-bezogen)

in den Versuchsbiogasreaktoren (mit Referenzreaktor).

Abbildung 40: Gegenüberstellung der Verläufe der FOS/TAC (FAL)-Werte und der

normierten Gasproduktion des Substitutionsversuchs 2 mit

$30 \%$ Amarant- zu $70 \%$ Mais-Silage (oTS-bezogen)

in den Versuchsbiogasreaktoren (mit Referenzreaktor).

Abbildung 41: Verläufe der Cobaltkonzentrationen des Substitutionsversuchs 2 mit

$30 \%$ Amarant- zu 70 \% Mais-Silage (oTS-bezogen)

in den Versuchsbiogasreaktoren (mit Referenzreaktor).

Abbildung 42: Verläufe der Nickelkonzentrationen des Substitutionsversuchs 2 mit $30 \%$ Amarant- zu $70 \%$ Mais-Silage (oTS-bezogen)

in den Versuchsbiogasreaktoren (mit Referenzreaktor).

Abbildung 43: Verläufe der Calcium-, Kalium-, Magnesium- und Natriumgehalte der Substitutionsversuche 1 und 2 in den Versuchsbiogasreaktoren (mit Referenzreaktor).

Abbildung 44: Verläufe der Phosphor- und Schwefelgehalte der Substitutionsversuche 1 und 2 in den Versuchsbiogasreaktoren (mit Referenzreaktor).

Abbildung 45: Verläufe der Eisen-, Kupfer-, Mangan- und Molybdängehalte der

Substitutionsversuche 1 und 2 in den Versuchsbiogasreaktoren

(mit Referenzreaktor).

Abbildung 46: Verläufe der Zink-, Selen- und Vanadiumgehalte der

Substitutionsversuche 1 und 2 in den Versuchsbiogasreaktoren

(mit Referenzreaktor).

Abbildung 47: Verläufe der Arsen-, Cadmium-, Chrom- und Bleigehalte der

Substitutionsversuche 1 und 2 in den Versuchsbiogasreaktoren

(mit Referenzreaktor).

Abbildung 48: Verläufe der Aluminium-, Lanthan-, Titan- und Strontiumgehalte der

Substitutionsversuche 1 und 2 in den Versuchsbiogasreaktoren

(mit Referenzreaktor).

Abbildung 49: Verläufe der Eisen/Schwefel-Verhältnisse der Substitutionsversuche 1 und 2 in den Versuchsbiogasreaktoren (mit Referenzreaktor).

Abbildung 50: $\quad$ FOS/TAC (FAL)-Verläufe des Substitutionsversuchs 3 mit

$30 \%$ Deutsches Weidelgras- zu $70 \%$ Mais-Silage (oTS-bezogen)

in den Versuchsbiogasreaktoren (mit Referenzreaktor).

Abbildung 51: $\quad$ pH-Wert-Verläufe des Substitutionsversuchs 3 mit

$30 \%$ Deutsches Weidelgras- zu 70 \% Mais-Silage (oTS-bezogen)

in den Versuchsbiogasreaktoren (mit Referenzreaktor).

Abbildung 52: Verläufe der normierten Gasproduktion des Substitutionsversuchs 3 mit

$30 \%$ Deutsches Weidelgras- zu 70 \% Mais-Silage (oTS-bezogen)

in den Versuchsbiogasreaktoren (mit Referenzreaktor).

Abbildung 53: Verläufe des normierten Methangehalts des Substitutionsversuchs 3 mit $30 \%$ Deutsches Weidelgras- zu $70 \%$ Mais-Silage (oTS-bezogen)

in den Versuchsbiogasreaktoren (mit Referenzreaktor).

Abbildung 54: Gegenüberstellung der Verläufe der FOS/TAC (FAL)-Werte und der normierten Gasproduktion des Substitutionsversuchs 3 mit $30 \%$ Deutsches Weidelgras- zu 70 \% Mais-Silage (oTS-bezogen) in den Versuchsbiogasreaktoren (mit Referenzreaktor).

Abbildung 55: Verläufe der Cobaltkonzentrationen des Substitutionsversuchs 3 mit $30 \%$ Deutsches Weidelgras- zu 70 \% Mais-Silage (oTS-bezogen) in den Versuchsbiogasreaktoren (mit Referenzreaktor). 
Abbildung 56: Verläufe der Nickelkonzentrationen des Substitutionsversuchs 3 mit 30 \% Deutsches Weidelgras- zu 70 \% Mais-Silage (oTS-bezogen) in den Versuchsbiogasreaktoren (mit Referenzreaktor).

Abbildung 57: $\quad$ FOS/TAC (FAL)-Verläufe des Substitutionsversuchs 4 mit $30 \%$ Durchwachsene Silphie- zu 70 \% Mais-Silage (oTS-bezogen) in den Versuchsbiogasreaktoren (mit Referenzreaktor).

Abbildung 58: $\quad$ pH-Wert-Verläufe des Substitutionsversuchs 4 mit $30 \%$ Durchwachsene Silphie- zu 70 \% Mais-Silage (oTS-bezogen) in den Versuchsbiogasreaktoren (mit Referenzreaktor).

Abbildung 59: Verläufe der normierten Gasproduktion des Substitutionsversuchs 4 mit $30 \%$ Durchwachsene Silphie- zu 70 \% Mais-Silage (oTS-bezogen) in den Versuchsbiogasreaktoren (mit Referenzreaktor)

Abbildung 60: Verläufe des normierten Methangehalts des Substitutionsversuchs 4 mit 30 \% Durchwachsene Silphie- zu 70 \% Mais-Silage (oTS-bezogen) in den Versuchsbiogasreaktoren (mit Referenzreaktor).

Abbildung 61: Gegenüberstellung der Verläufe der FOS/TAC (FAL)-Werte und der normierten Gasproduktion des Substitutionsversuchs 4 mit $30 \%$ Durchwachsene Silphie- zu $70 \%$ Mais-Silage (oTS-bezogen) in den Versuchsbiogasreaktoren (mit Referenzreaktor).

Abbildung 62: Verläufe der Cobaltkonzentrationen des Substitutionsversuchs 4 mit $30 \%$ Durchwachsene Silphie- zu $70 \%$ Mais-Silage (oTS-bezogen) in den Versuchsbiogasreaktoren (mit Referenzreaktor).

Abbildung 63: Verläufe der Nickelkonzentrationen des Substitutionsversuchs 4 mit $30 \%$ Durchwachsene Silphie- zu 70 \% Mais-Silage (oTS-bezogen) in den Versuchsbiogasreaktoren (mit Referenzreaktor).

Abbildung 64: Verläufe der Nickel- und Chromkonzentrationen des Substitutionsversuchs 3 mit 30 \% Deutsches Weidelgras- zu 70 \% Mais-Silage (oTS-bezogen) in den Versuchsbiogasreaktoren.

Abbildung 65: Verläufe der Nickel- und Chromkonzentrationen des Substitutionsversuchs 4 mit $30 \%$ Durchwachsene Silphie- zu 70 \% Mais-Silage (oTS-bezogen) in den Versuchsbiogasreaktoren.

Abbildung 66: Verläufe der Nickel- und Chromkonzentrationen im Referenzreaktor während der Substitutionsversuche 3 und 4.

Abbildung 67: Verläufe der Calcium-, Kalium-, Magnesium- und Natriumgehalte der Substitutionsversuche 3 und 4 in den Versuchsbiogasreaktoren (mit Referenzreaktor).

Abbildung 68: Verläufe der Phosphor- und Schwefelgehalte der Substitutionsversuche 3 und 4 in den Versuchsbiogasreaktoren (mit Referenzreaktor).

Abbildung 69: Verläufe der Eisen-, Kupfer-, Mangan- und Molybdängehalte der Substitutionsversuche 3 und 4 in den Versuchsbiogasreaktoren (mit Referenzreaktor).

Abbildung 70: Verläufe der Zink-, Selen- und Vanadiumgehalte der Substitutionsversuche 3 und 4 in den Versuchsbiogasreaktoren (mit Referenzreaktor).

Abbildung 71: Verläufe der Arsen-, Cadmium- und Bleigehalte der Substitutionsversuche 3 und 4 in den Versuchsbiogasreaktoren (mit Referenzreaktor).

Abbildung 72: Verläufe der Aluminium-, Lanthan-, Titan- und Strontiumgehalte der Substitutionsversuche 3 und 4 in den Versuchsbiogasreaktoren (mit Referenzreaktor). 
Abbildung 73: Verläufe der Eisen/Schwefel-Verhältnisse des Substitutionsversuchs 3 mit $30 \%$ Deutsches Weidelgras- zu 70 \% Mais-Silage (oTS-bezogen) in den Versuchsbiogasreaktoren (mit Referenzreaktor).

Abbildung 74: Verläufe der Eisen/Schwefel-Verhältnisse des Substitutionsversuchs 4 mit $30 \%$ Durchwachsene Silphie- zu 70 \% Mais-Silage (oTS-bezogen) in den Versuchsbiogasreaktoren (mit Referenzreaktor).

Abbildung 75: $\quad$ FOS/TAC (FAL)-Verläufe des Zusatzversuchs mit Mais-Silage-Monovergärung unter reduzierter Spurenelementzugabe in den Versuchsbiogasreaktoren.

Abbildung 76: pH-Wert-Verläufe des Zusatzversuchs mit Mais-Silage-Monovergärung unter reduzierter Spurenelementzugabe in den Versuchsbiogasreaktoren.

Abbildung 77: Verläufe der normierten Gasproduktion des Zusatzversuchs mit Mais-Silage-Monovergärung unter reduzierter Spurenelementzugabe in den Versuchsbiogasreaktoren.

Abbildung 78: Gegenüberstellung der pH-Wert- und FOS/TAC (FAL)-Verläufe sowie der normierten Gasproduktion des Zusatzversuchs mit Mais-Silage-Monovergärung unter reduzierter Spurenelementzugabe in den Versuchsbiogasreaktoren.

Abbildung 79: Verläufe der Cobaltkonzentrationen des Zusatzversuchs mit Mais-Silage-Monovergärung unter reduzierter Spurenelementzugabe in den Versuchsbiogasreaktoren.

Abbildung 80: Verläufe der Nickelkonzentrationen des Zusatzversuchs mit Mais-Silage-Monovergärung unter reduzierter Spurenelementzugabe in den Versuchsbiogasreaktoren

Abbildung 81: Verläufe der Calcium-, Kalium-, Magnesium-, Natrium-, Phosphor- und Schwefelgehalte des Zusatzversuchs mit Mais-Silage-Monovergärung unter reduzierter Spurenelementzugabe in den Versuchsbiogasreaktoren.

Abbildung 82: Verläufe der Eisen-, Kupfer-, Mangan-, Molybdän-, Zink-, Selen- und Vanadiumgehalte des Zusatzversuchs mit Mais-Silage-Monovergärung unter reduzierter Spurenelementzugabe in den Versuchsbiogasreaktoren.

Abbildung 83: Verläufe der Arsen-, Cadmium-, Chrom-, Blei-, Aluminium-, Lanthan-, Titanund Strontiumgehalte des Zusatzversuchs mit Mais-Silage-Monovergärung unter reduzierter Spurenelementzugabe in den Versuchsbiogasreaktoren.

Abbildung 84: Verläufe der Eisen/Schwefel-Verhältnisse des Zusatzversuchs mit Mais-Silage-Monovergärung unter reduzierter Spurenelementzugabe in den Versuchsbiogasreaktoren.

Abbildung 85: Fotoaufnahmen eines diskontinuierlichen Gärversuchs auf Basis des Oberhausen-Rostock-Göttinger Aktivitätstests (ORGA-Test).

Abbildung 86: Verlauf der gemittelten Gassummenkurven der ORGA-Versuchsreihe „Nickel 1“ zur Überprüfung der oberen Hemm- und Toxizitätswirkung von Nickel.

Abbildung 87: Verlauf der gemittelten Gassummenkurven der ORGA-Versuchsreihe „Nickel 2“ zur Überprüfung der oberen Hemm- und Toxizitätswirkung von Nickel

Abbildung 88: Verlauf der gemittelten Gassummenkurven der ORGA-Testreihe „Nickel 3“ zur Überprüfung der oberen Hemm- und Toxizitätswirkung von Nickel.

Abbildung 89: Verlauf der gemittelten Gassummenkurven der ORGA-Testreihe „Nickel 4“ zur Überprüfung der oberen Hemm- und Toxizitätswirkung von Nickel.

Abbildung 90: Verlauf der gemittelten Gassummenkurven der ORGA-Testreihen „Nickel 2“ bis „Nickel 4“ zur Überprüfung der oberen Hemm- und Toxizitätswirkung von Nickel.

Abbildung 91: Verlauf der gemittelten Gassummenkurven der ORGA-Testreihe „Cobalt 1“ zur Überprüfung der oberen Hemm- und Toxizitätswirkung von Cobalt. 
Abbildung 92: Verlauf der gemittelten Gassummenkurven der ORGA-Testreihe „Cobalt 2“ zur Überprüfung der oberen Hemm- und Toxizitätswirkung von Cobalt.

Abbildung 93: Verlauf der gemittelten Gassummenkurven der ORGA-Testreihe zur Überprüfung der oberen Hemmwirkung von Molybdän.

Abbildung 94: Verlauf der gemittelten Gassummenkurven der ORGA-Testreihe „Kupfer 1“ zur Überprüfung der oberen Hemm- und Toxizitätswirkung von Kupfer.

Abbildung 95: Verlauf der gemittelten Gassummenkurven der ORGA-Testreihe „Kupfer 2“ zur Überprüfung der oberen Hemm- und Toxizitätswirkung von Kupfer.

Abbildung 96: Verlauf der gemittelten Gassummenkurven der ORGA-Testreihe „Cadmium 1“ zur Überprüfung der Hemm- und Toxizitätswirkung von Cadmium.

Abbildung 97: Verlauf der gemittelten Gassummenkurven der ORGA-Testreihe „Cadmium 2“ zur Überprüfung der Hemm- und Toxizitätswirkung von Cadmium.

Abbildung 98: Verlauf der gemittelten Gassummenkurven der ORGA-Testreihe zur Überprüfung der Hemm- und Toxizitätswirkung von Blei.

Abbildung 99: Fotoaufnahme von zwei Teflon-Filtrationsapparaturen mit Filtraten $(<0,45 \mu \mathrm{m})$ einer Fermenterprobe in den beiden Verdünnungsstufen 1:5 (links) und 1:10 (rechts).

Abbildung 100: Elementkonzentrationsverhältnisse der Filtratproben $(<0,45 \mu \mathrm{m}$, 1:20-Verdünnungen, Aufschlussvariante 1) verschiedener Gärmaterialien aus der Praxisbiogasanlage in Verliehausen

Abbildung 101: Elementkonzentrationsverhältnisse der Filtratproben $(<0,45 \mu \mathrm{m}$, 1:20-Verdünnungen, Aufschlussvariante 2) verschiedener Gärmaterialien aus der Praxisbiogasanlage in Verliehausen

Abbildung 102: Elementkonzentrationsverhältnisse der Presssaftproben $(<63 \mu \mathrm{m})$ ausgewählter Referenzreaktorproben der dritten Projektphase am DBFZ und einer Fermentermaterialprobe aus der Praxisbiogasanlage (BGA) in Verliehausen.

Abbildung 103: Elementkonzentrationsverhältnisse der Pressrückstände ( $>63 \mu \mathrm{m})$ ausgewählter Referenzreaktorproben der dritten Projektphase am DBFZ und einer Fermentermaterialprobe aus der Praxisbiogasanlage (BGA) in Verliehausen......

Abbildung 104: Vergleich der Elementkonzentrationsverhältnisse der Fraktionen Filtrat $(<0,45 \mu \mathrm{m})$, Presssaft $(<63 \mu \mathrm{m})$ und Pressrückstand $(>63 \mu \mathrm{m})$ einer Fermentermaterialprobe (Fermenter BGA 07/17) aus der Praxisbiogasanlage in Verliehausen.

Abbildung 105: Vergleich der Elementkonzentrationsverhältnisse der Fraktionen Presssäfte $(<63 \mu \mathrm{m})$ und Pressrückstände $(>63 \mu \mathrm{m})$ ausgewählter Referenzreaktorproben der dritten Projektphase am DBFZ und einer Fermentermaterialprobe (Fermenter BGA 07/17) aus der Praxisbiogasanlage in Verliehausen.

Abbildung 106: Verteilung der Elemente in der Festsubstanz und der flüssigen Phase in der Gesamtprobe (oben), im Pressrückstand ( $>63 \mu \mathrm{m}$, Mitte) sowie im Presssaft $(<63 \mu \mathrm{m}$, unten) einer Fermentermaterialprobe (Fermenter BGA 07/17) aus der Praxisbiogasanlage in Verliehausen.

Abbildung 107: Schematische Darstellung des Einflusses von Spurenelementen auf den Biogasprozess bei zu geringen, optimalen, hemmenden und toxischen Konzentrationen mit Angabe von jeweiligen Schwellenwerten, stark modifiziert nach VAN HULLEBUSCH ET AL. (2016). 


\section{Tabellen}

Tabelle 1: Zusammenstellung minimaler Bedarfswerte (Minimalgehalte, $\mathrm{mg} / \mathrm{L}_{\mathrm{FM}}$ ) wichtiger essenzieller Mikronährstoffe für optimale Biogasprozesse.

Tabelle 2: Übersicht der erhobenen Proben, mit Anzahl der Multielementmessungen mittels ICP-OES bzw. ICP-MS.

Tabelle 3: Verwendete Referenzmaterialien (Blatt I) mit Angabe der gemittelten Mess- und Bezugswerte $[\mathrm{mg} / \mathrm{kg}]$ sowie der prozentualen Richtigkeit (R).

Tabelle 4: Verwendete Referenzmaterialien (Blatt II) mit Angabe der gemittelten Mess- und Bezugswerte $[\mathrm{mg} / \mathrm{kg}]$ sowie der prozentualen Richtigkeit (R).

Tabelle 5: $\quad$ Auswahl der bevorzugten Spektrallinien (ICP-OES) und Isotopenmassen (ICP-MS)

für die Bestimmung der Elementkonzentrationen in den Gärgemisch- und

Silage-Proben.

Tabelle 6: Zusammensetzung der im Verbundprojekt am DBFZ hergestellten und verwendeten Spurenelementlösung bei Vollversorgung.

Tabelle 7: Zusammenstellung wichtiger pflanzenbaulicher und gasanalytischer Parameter der verwendeten Energiepflanzen-Silagen für die (quasi)kontinuierlichen Nassfermentationsversuche am DBFZ.

Tabelle 8: Durchschnittsgehalte $\left[\mathrm{mg} / \mathrm{kg}_{\mathrm{FM}}\right]$ der verwendeten Energiepflanzen-Silagen

für die (quasi)kontinuierlichen Nassfermentationsversuche.

Tabelle 9: $\quad$ Überblick über die erste Projektphase der (quasi)kontinuierlichen Nassfermentationsversuche am DBFZ.

Tabelle 10: Überblick über die zweite Projektphase der (quasi)kontinuierlichen Nassfermentationsversuche am DBFZ.

Tabelle 11: Überblick über die dritte Projektphase der (quasi)kontinuierlichen

Nassfermentationsversuche am DBFZ.

Tabelle 12: Überblick über den Zusatzversuch der (quasi)kontinuierlichen Nassfermentationsversuche am DBFZ

Tabelle 13: Überblick über die diskontinuierlichen Gärversuche (ORGA-Tests) zur Erarbeitung von Hemm- und Toxizitätskonzentrationen verschiedener Spurenelemente

Tabelle 14: Überblick über die Parameter der diskontinuierlichen Gärversuche (ORGA-Tests). ......... 145

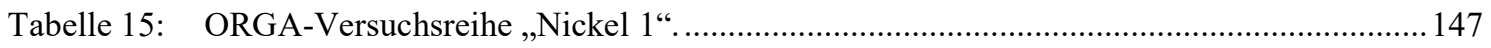

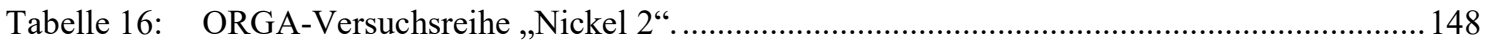

Tabelle 17: ORGA-Versuchsreihe „Nickel 3“................................................................................ 149

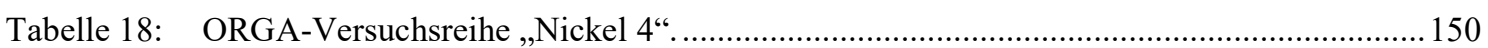

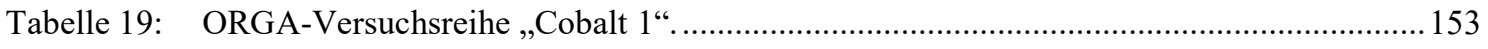

Tabelle 20: ORGA-Versuchsreihe „Cobalt 2“...................................................................................... 154

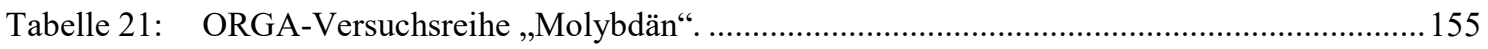

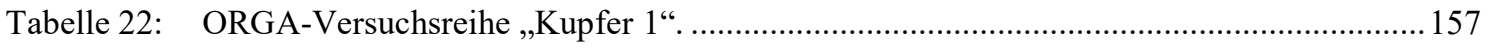

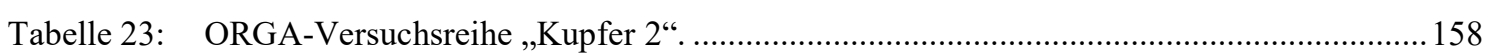

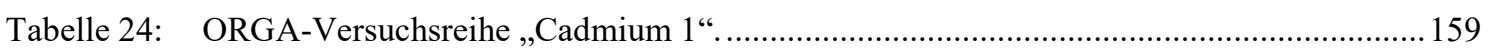

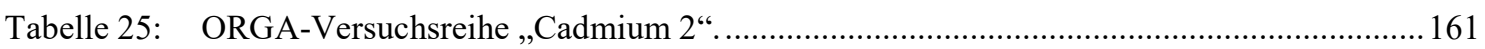

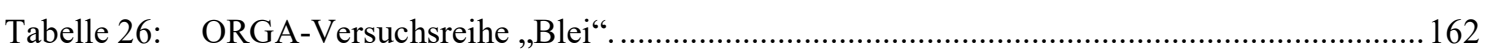

Tabelle 27: Obere Hemm- und Toxizitätskonzentrationen $[\mathrm{mg} / \mathrm{L}]$ der mit Hilfe von ORGA-Tests untersuchten Spuren- und potenziellen Schadelemente. .................................163 
Tabelle 28: Zusammenstellung oberer Hemm- und Toxizitätskonzentrationen [mg/L] ausgewählter Schwermetalle in anaeroben Prozessen.

Tabelle 29: Berechnungen zu den verwendeten Energiepflanzen-Silagen

für die (quasi)kontinuierlichen Nassfermentationsversuche am DBFZ

(Konzentrationsangaben in $\mathrm{mg} / \mathrm{kg}_{\mathrm{FM}}$ ).

Tabelle 30: Vergleich der Summe der Elementmengen im Pressrückstand ( $>63 \mu \mathrm{m})$ plus Presssaft $(<63 \mu \mathrm{m})$ gegenüber der Elementmenge in der Gesamtprobe ausgewählter Referenzreaktorproben der dritten Projektphase am DBFZ und einer Fermentermaterialprobe (Fermenter BGA 07/17) aus der Praxisbiogasanlage in Verliehausen

Tabelle 31: Übersicht der Elementkonzentrationen $\left[\mathrm{mg} / \mathrm{kg}_{\mathrm{FM}}\right]$ der Filtratproben $(<0,45 \mu \mathrm{m}$, 1:20-Verdünnungen, Aufschlussvarianten V1 und V2) und der Gesamtproben verschiedener Gärmaterialien aus der Praxisbiogasanlage (BGA) in Verliehausen.

Tabelle 32: Übersicht der Elementkonzentrationen $\left[\mathrm{mg} / \mathrm{kg}_{\mathrm{FM}}\right]$ der Presssäfte $(<63 \mu \mathrm{m}, \mathrm{PS})$ und der Gesamtproben $(\mathrm{G})$ ausgewählter Referenzreaktorproben der dritten Projektphase am DBFZ und einer Fermentermaterialprobe aus der Praxisbiogasanlage (BGA) in Verliehausen.

Tabelle 33: Übersicht der Elementkonzentrationen $\left[\mathrm{mg} / \mathrm{kg}_{\mathrm{FM}}\right]$ der Pressrückstände ( $\left.>63 \mu \mathrm{m}, \mathrm{PR}\right)$ und der Gesamtproben $(\mathrm{G})$ ausgewählter Referenzreaktorproben der dritten Projektphase am DBFZ und einer Fermentermaterialprobe aus der Praxisbiogasanlage (BGA) in Verliehausen.

Tabelle 34: Empfohlener Wertebereich sowie Minimal- und Optimalwerte $\left[\mathrm{mg} / \mathrm{kg}_{\text {FM }}\right.$ ] wichtiger essenzieller Mikronährstoffe und Natrium für optimale Biogasbildungsprozesse. 


\section{Abkürzungen}

\section{Begriffe / Formelzeichen / Symbole}

\begin{tabular}{|c|c|c|c|}
\hline BGA & (Praxis)Biogasanlage & $\mathrm{Me}$ & Metall \\
\hline BMEL & $\begin{array}{l}\text { Bundesministerium für Ernährung } \\
\text { und Landwirtschaft }\end{array}$ & $\mathrm{mg}$ & Milligramm \\
\hline $\mathrm{B}_{\mathrm{R}}$ & Raumbelastung & $\min$ & Minute \\
\hline BRENDA & $\begin{array}{l}\text { BRaunschweig ENzyme DAtabase } \\
\text { (Online-Enzym-Datenbank) }\end{array}$ & $\mathrm{mL}$ & Milliliter \\
\hline$d$ & Tag & $\mathrm{n}$ & Anzahl \\
\hline DBFZ & $\begin{array}{l}\text { Deutsches Biomasseforschungs- } \\
\text { zentrum gGmbH }\end{array}$ & na & nicht analysiert / nicht angegeben \\
\hline FAL & $\begin{array}{l}\text { Bundesforschungsanstalt für } \\
\text { Landwirtschaft }\end{array}$ & NawaRo & nachwachsende Rohstoffe \\
\hline FM & Frischmasse & ORGA-Test & $\begin{array}{l}\text { Oberhausen-Rostock-Göttinger } \\
\text { Aktivitätstest }\end{array}$ \\
\hline FNR & $\begin{array}{l}\text { Fachagentur Nachwachsende } \\
\text { Rohstoffe e.V. }\end{array}$ & oTS & $\begin{array}{l}\text { organische Trockensubstanz, } \\
\text { organischer Trockensubstanzgehalt }\end{array}$ \\
\hline G & Gesamtprobe & ppm & parts per million (Anteile pro Million \\
\hline $\mathrm{g}$ & Gramm & ppq & $\begin{array}{l}\text { part per quadrillion (Anteile pro } \\
\text { Billiarde) }\end{array}$ \\
\hline Gew.-\% & Gewichtsprozent & PR & Pressrückstand \\
\hline GPS & (Getreide-)Ganzpflanzen-Silage & PS & Presssaft \\
\hline GZG & $\begin{array}{l}\text { Geowissenschaftliches Zentrum } \\
\text { der Universität Göttingen }\end{array}$ & qPCR & $\begin{array}{l}\text { quantitative Echtzeit-Polymerase- } \\
\text { Kettenreaktion }\end{array}$ \\
\hline $\mathrm{h}$ & Stunde & $\mathrm{R}$ & Richtigkeit \\
\hline HRT & $\begin{array}{l}\text { hydraulic retention time } \\
\text { (hydraulische Verweilzeit) }\end{array}$ & $\mathrm{RF}$ & Radiofrequenz \\
\hline ICP-MS & $\begin{array}{l}\text { Inductively Coupled Plasma - } \\
\text { Mass Spectrometry }\end{array}$ & $\mathrm{rpm}$ & $\begin{array}{l}\text { revolutions per minute } \\
\text { (Umdrehungen pro Minute) }\end{array}$ \\
\hline ICP-OES & $\begin{array}{l}\text { Inductively Coupled Plasma - } \\
\text { Optical Emission Spectrometry }\end{array}$ & $\mathrm{s}$ & Sekunde \\
\hline $\operatorname{IrA}$ & Index der relativen Anbauwürdigkeit & $\mathrm{t}$ & Tonne \\
\hline IZNE & $\begin{array}{l}\text { Interdisziplinäres Zentrum für } \\
\text { Nachhaltige Entwicklung }\end{array}$ & $t_{250}$ & Zeitparameter (Aktivitätskriterium) \\
\hline $\mathrm{K}$ & Kelvin & T-RFLP & $\begin{array}{l}\text { Terminaler Restriktionsfragment- } \\
\text { Längenpolymorphismus }\end{array}$ \\
\hline $\mathrm{K}_{\mathrm{sp}}$ & Löslichkeitsprodukt & TS & $\begin{array}{l}\text { Trockensubstanz, } \\
\text { Trockensubstanzgehalt }\end{array}$ \\
\hline KED & $\begin{array}{l}\text { Kinetic Energy Discrimination } \\
\text { (kinetische Energiediskriminierung) }\end{array}$ & UFZ & $\begin{array}{l}\text { Helmholtz-Zentrum für } \\
\text { Umweltforschung }\end{array}$ \\
\hline $\mathrm{kg}$ & Kilogramm & ${ }^{\circ} \mathrm{C}$ & Grad Celcius \\
\hline $\mathrm{kPa}$ & Kilopascal & $\%$ & Prozent \\
\hline $\mathrm{L}$ & Liter & $\mu \mathrm{L}$ & Mikroliter \\
\hline $\mathrm{m}^{3}$ & Kubikmeter & $\mu \mathrm{m}$ & Mikrometer \\
\hline
\end{tabular}




\section{Chemische Symbole / Formeln / Kurzformen}

\begin{tabular}{|c|c|c|c|}
\hline $\mathrm{Ag}$ & Silber & $\mathrm{La}$ & Lanthan \\
\hline $\mathrm{Al}$ & Aluminium & $\mathrm{Mg}$ & Magnesium \\
\hline $\mathrm{Ar}$ & Argon & $\mathrm{Mn}$ & Mangan \\
\hline As & Arsen & Mo & Molybdän \\
\hline ATP & Adenosintriphosphat & $\mathrm{N}_{2}(\mathrm{~N})$ & Stickstoff \\
\hline $\mathrm{Au}$ & Gold & $\mathrm{Na}$ & Natrium \\
\hline B & Bor & NADP & $\begin{array}{l}\text { Nicotinsäureamid-Adenin-Dinukleotid- } \\
\text { Phosphat }\end{array}$ \\
\hline $\mathrm{Be}$ & Beryllium & $\mathrm{NaHCO}_{3}$ & Natriumhydrogencarbonat \\
\hline $\mathrm{C}$ & Kohlenstoff & $\mathrm{NaOH}$ & Natriumhydroxid (Natronlauge) \\
\hline $\mathrm{Ca}$ & Calcium & $\mathrm{Nb}$ & Niob \\
\hline $\mathrm{CaCO}_{3}$ & Calciumcarbonat & $\mathrm{NH}_{3}$ & Ammoniak \\
\hline $\mathrm{Cd}$ & Cadmium & $\mathrm{NH}_{4}^{+}$ & Ammonium-Ion \\
\hline $\mathrm{CH}_{4}$ & Methan & $\mathrm{NH}_{4}-\mathrm{N}$ & "Ammoniumstickstoff" \\
\hline $\mathrm{Cl}_{2}(\mathrm{Cl})$ & Chlor & $\mathrm{Ni}$ & Nickel \\
\hline Co & Cobalt & $\mathrm{O}_{2}(\mathrm{O})$ & Sauerstoff \\
\hline $\mathrm{CO}$ & Kohlenstoffmonoxid & $\mathrm{OH}^{-}$ & Hydroxid-Ion \\
\hline $\mathrm{CO}_{2}$ & Kohlenstoffdioxid & Os & Osmium \\
\hline $\mathrm{CO}_{3}{ }^{2-}$ & Carbonat-Ion & $\mathrm{P}$ & Phosphor \\
\hline $\mathrm{Cr}$ & Chrom & $\mathrm{Pb}$ & Blei \\
\hline $\mathrm{Cu}$ & Kupfer & $\mathrm{Pd}$ & Palladium \\
\hline DNA & Desoxyribonukleinsäure & PE & Polyethylen \\
\hline FDH & Formiat-Dehydrogenase & $\mathrm{PO}_{4}{ }^{3-}$ & Phosphat-Ion \\
\hline $\mathrm{Fe}$ & Eisen & $\mathrm{Pt}$ & Platin \\
\hline FMDH & Formylmethanofurandehydrogenase & PTFE & Polytetrafluorethylen (Teflon) \\
\hline FOS & Flüchtige organische Säuren & $\mathrm{Re}$ & Rhenium \\
\hline FOS (Kapp) & $\begin{array}{l}\text { Titrationsmethode zur Bestimmung } \\
\text { der Säurenkonzentration (FOS) }\end{array}$ & $\mathrm{Rh}$ & Rhodium \\
\hline FOS/TAC (FAL) & $\begin{array}{l}\text { Titrationsmethode, Verhältnis FOS zur } \\
\text { Carbonat-Pufferkapazität (TAC) }\end{array}$ & RNA & Ribonukleinsäure \\
\hline $\mathrm{Ga}$ & Gallium & $\mathrm{Ru}$ & Ruthenium \\
\hline $\mathrm{Ge}$ & Germanium & $\mathrm{S}$ & Schwefel \\
\hline $\mathrm{H}_{2}(\mathrm{H})$ & Wasserstoff & $\mathrm{S}^{2-}$ & Sulfid-Ion \\
\hline $\mathrm{H}_{2} \mathrm{O}$ & Wasser & $\mathrm{Se}$ & Selen \\
\hline $\mathrm{H}_{2} \mathrm{~S}$ & Schwefelwasserstoff & $\mathrm{Si}$ & Silicium \\
\hline $\mathrm{HCl}$ & Salzsäure & Sn & Zinn \\
\hline $\mathrm{HClO}_{4}$ & Perchlorsäure & $\mathrm{SO}_{4}{ }^{2-}$ & Sulfat-Ion \\
\hline $\mathrm{HCO}_{3}^{-}$ & Hydrogencarbonat-Ion & $\mathrm{Sr}$ & Strontium \\
\hline $\mathrm{He}$ & Helium & TAC & $\begin{array}{l}\text { Totales anorganisches Carbonat } \\
\text { (alkalische Pufferkapazität) }\end{array}$ \\
\hline $\mathrm{HF}$ & Flusssäure & $\mathrm{Te}$ & Tellur \\
\hline $\mathrm{Hg}$ & Quecksilber & $\mathrm{Ti}$ & Titan \\
\hline $\mathrm{HNO}_{3}$ & Salpetersäure & $\mathrm{V}$ & Vanadium \\
\hline $\mathrm{HS}^{-}$ & Hydrogensulfid-Ion & $\mathrm{W}$ & Wolfram \\
\hline In & Indium & $\mathrm{Zn}$ & Zink \\
\hline Ir & Iridium & $\mathrm{Zr}$ & Zirconium \\
\hline K & Kalium & & \\
\hline
\end{tabular}




\section{Kapitel 1: Einleitung und Zielstellung}

Für optimale Vergärungs- und Biogasbildungsprozesse muss in Biogasanlagen eine Vielzahl an Betriebsparametern innerhalb bestimmter Toleranzbereiche liegen. Um eine optimale Umwandlung des in den Inputsubstraten organisch gebundenen Kohlenstoffs in Methan erreichen zu können, sollten alle Stufen im Biogasprozess stabil und ideal ablaufen. Insbesondere muss eine ausreichende Versorgung mit essenziellen Makround Mikronährstoffen für die Umsetzungs- und Stoffwechselprozesse der gasbildenden Mikroorganismen gewährleistet sein. Eine wichtige Rolle für die optimale Gasausbeute landwirtschaftlicher Biogasanlagen scheinen dabei unter anderem die Elemente Cobalt und Nickel zu spielen.

Bei alleiniger Verwendung von Mais-Silage als am häufigsten eingesetztes Inputsubstrat in landwirtschaftlichen Biogasanlagen reichen die Spurenelementgehalte im Mais für eine optimale Biogasbildung generell nicht aus. Die Ursache liegt im geringen Spurenelemente-Aufnahmevermögen von Mais. Dies führt dazu, dass in Deutschland in geschätzt circa der Hälfte aller Biogasanlagen synthetische Spurenelementadditive als Prozesshilfsstoffe zugefüttert werden, um einem potenziellen Mangel entgegenzuwirken (FAHLBUSCH ET AL., 2018).

Der Einsatz synthetischer Spurenelementadditive kann jedoch mit diversen Umweltund Gesundheitsrisiken verbunden sein, wie der potenziellen Gefahr einer Belastung des Bodens durch die Aufbringung von mit Schwermetallen angereicherten Gärresten oder beim Arbeiten mit den schwermetallreichen Additivpulvern oder -lösungen. Zudem verursachen Spurenelementadditive zusätzlich hohe Kosten und geltende Grenzwerte der Düngemittelverordnung (DüMV, 2012) können durch die Elementzugaben überschritten werden, sodass die Gärreste als Abfallstoffe zu handhaben wären. Deshalb sollte bzw. muss bei der Verwendung synthetischer Spurenelementadditive immer ein Minimierungsgebot gelten, d.h. minimaler Einsatz der schwermetallreichen Pulver oder Lösungen bei optimaler Biogasausbeute.

Im Vergleich zu Mais weisen alternative Energiepflanzen hingegen deutlich höhere essenzielle Spurenelementkonzentrationen auf. So schien es nach Berechnungen von SAUER ET AL. (2014) möglich, durch die Zumischung von anbauwürdigen spurenelementakkumulierenden Energiepflanzen zum Mais, eine ausreichende Versorgung mit essenziellen Makro- und Mikronährstoffen für den Biogasprozess zu 
gewährleisten, wodurch auf synthetische Spurenelementadditive verzichtet werden könnte. Diese theoretischen Erkenntnisse für ausgewählte Energiepflanzenarten bzw. -mischungen im Labormaßstab praktisch zu prüfen war u.a. Bestandteil dieser Forschungsarbeit.

Die Dissertationsschrift war eingebettet im Forschungsprojekt „Spurenelemente durch Energiepflanzen - Stoffströme und Handlungsempfehlungen für eine optimierte Prozessbiologie in Biogasanlagen (NiCo)“ (SAUER ET AL., 2018), gefördert mit Mitteln des Bundesministeriums für Ernährung und Landwirtschaft (BMEL) über die Fachagentur Nachwachsende Rohstoffe e.V. (FNR) als Projektträger des BMEL. Beteiligt im Forschungsverbund waren:

- Arbeitsgruppe Umweltgeochemie in der Abteilung Sedimentologie/Umweltgeologie am Geowissenschaftlichen Zentrum (GZG) und dem Interdisziplinären Zentrum für Nachhaltige Entwicklung (IZNE), Georg-August-Universität Göttingen,

- Abteilung Pflanzenbau im Department für Nutzpflanzenwissenschaften an der Fakultät für Agrarwissenschaften, Georg-August-Universität Göttingen,

- Arbeitsgruppe Charakterisierung und Entwicklung anaerober Prozesse im Department Biochemische Konversion am Deutschen Biomasseforschungszentrum gGmbH (DBFZ) in Leipzig,

- Arbeitsgruppe Mikrobiologie anaerober Systeme im Department Umweltmikrobiologie am Helmholtz-Zentrum für Umweltforschung (UFZ) in Leipzig.

Im NiCo-Forschungsverbund wurden auf zwei Freilandversuchsfeldern in Schoningen, einem Ortsteil der Stadt Uslar, und am Versuchsgut Reinshof der Universität Göttingen verschiedene alternative Energiepflanzenarten angebaut (Bearbeitung: Doktorandin K. Hey, Abteilung Pflanzenbau, Dissertation geplant 2020). Sie wurden u.a. im Hinblick auf ihre Spurenelementaufnahme untersucht (Bearbeitung: Dr. W. Fahlbusch, Arbeitsgruppe Umweltgeochemie, Dissertation 2018) sowie praxisorientierte Anbausysteme erarbeitet, überprüft und weitere pflanzenbauliche, soziale und ökonomische Parameter erhoben. Mit dem daraus entwickelten Index der relativen Anbauwürdigkeit (IrA) erfolgte eine pflanzenbauliche Bewertung der alternativen Energiepflanzen für die Biogaserzeugung (Bearbeitung: Doktorandin K. Hey). Nähere Erläuterungen hierzu und die wichtigsten Ergebnisse dieser Projektteile fasst u.a. der Schlussbericht zum Vorhaben unter SAUER ET AL. (2018) zusammen. 
Gegenstand dieser Forschungsarbeit ist, auf Basis von Fermenterversuchen im Labormaßstab zunächst für die beiden essenziellen Spurenelemente Cobalt und Nickel deren Minimalkonzentrationen im Biogasprozess zu erarbeiten und dann herauszufinden, ob durch eine Zumischung von spurenelementreichen anbauwürdigen Energiepflanzen bei der Vergärung von Mais-Silage auf die Anwendung bzw. Zugabe von synthetischen Spurenelementadditiven in Biogasanlagen verzichtet werden kann. Hierfür wurden die am Versuchsgut Reinshof angebauten Energiepflanzenvarianten Winterackerbohne, Amarant, Deutsches Weidelgras sowie Durchwachsene Silphie als Substitutionssilagen ausgewählt und getestet. Die Versuche wurden im Biogaslabor des Fachbereichs Biochemische Konversion am DBFZ in drei Projektphasen mit Nassfermentation in fünf baugleichen, (quasi)kontinuierlich betriebenen Laborreaktoren durchgeführt und prozessanalytisch verfolgt (Koordination: M. Zechendorf):

- 1. Projektphase: Verarmungsversuche für Cobalt und Nickel (Kapitel 4.3),

- 2. Projektphase: Substitutionsversuche 1 und 2: Mais-Silage mit Silagen von Winterackerbohne und Amarant (Kapitel 4.4),

- 3. Projektphase: Substitutionsversuche 3 und 4: Mais-Silage mit Silagen von Deutsches Weidelgras und Durchwachsene Silphie (Kapitel 4.5).

Gegen Ende der zweiten Projektphase wurde parallel ein zusätzlicher Versuch mit Mais-Silage-Monovergärung unter reduzierter Spurenelementzugabe in zwei weiteren Laborreaktoren durchgeführt, um $\mathrm{zu}$ testen, ob eine stark reduzierte Additivzugabe genügt, stabile Biogasprozesse in den Fermentern aufrechtzuerhalten (Kapitel 4.6). Um die Zielfragestellungen zu beantworten bzw. grundlegende (spuren)elementanalytische Zusammenhänge in Biogasreaktoren aufzuzeigen, wurden die physikochemischen und gasanalytischen Prozessparameter der (quasi)kontinuierlichen Laborfermenterversuche in der vorliegenden Arbeit zusammengeführt, ausgewertet und mit gemessenen Elementkonzentrationen der Reaktormaterialproben aus diesen Versuchen verglichen.

Parallel $\mathrm{zu}$ den Erhebungen der physikochemischen und gasanalytischen Prozessparameter am DBFZ sowie den elementanalytischen Untersuchungen wurden in der Arbeitsgruppe Mikrobiologie anaerober Systeme am UFZ am Reaktormaterial der genannten Laborfermenterversuche mikrobiologische Untersuchungen durchgeführt. Es wurden sowohl die methanogenen als auch die bakteriellen Gemeinschaften hinsichtlich ihrer Zusammensetzung und Dynamik (DNA-basierte T-RFLP-Analysen) sowie ihrer 
transkriptionalen Aktivität (nur für die Methanbildner, qPCR und RNA-basierte T-RFLP-Analysen) charakterisiert (Bearbeitung: Dr. Heike Sträuber). Bei einer Cobaltund Nickelverarmung sollten Effekte auf die mikrobiellen Gemeinschaften erkannt und mikrobielle Indikatororganismen für eine Cobalt- bzw. Nickel-Unterversorgung identifiziert werden. In der vorliegenden Arbeit sind die wichtigsten mikrobiologischen Erkenntnisse jeweils im letzten Unterkapitel der drei Projektphasen zusammengestellt (Kapitel 4.3.7, 4.4.6 und 4.5.7). Ausführlichere Beschreibungen zur Mikrobiologie finden sich im Schlussbericht zum Forschungsvorhaben unter SAUER ET AL. (2018).

In geringen Mengen positiv wirkende (Spuren)Elemente können hingegen bei höheren Konzentrationen hemmend oder sogar toxisch auf die Mikroorganismen bzw. die Gasbildung im Fermenter wirken. Systematische wissenschaftliche Untersuchungen hierzu fehlen bisher weitestgehend in der Literatur. So wurden als weitere Zielstellungen in dieser Arbeit in diskontinuierlichen Batchversuchen mit Hilfe des Oberhausen-RostockGöttinger Aktivitätstest (ORGA-Test) obere hemmende und toxische Konzentrationsbereiche für verschiedene Spuren- und Schadelemente erarbeitet, ab denen der Biogasbildungsprozess stark gehemmt wird bzw. bei weiterer Konzentrationserhöhung sogar zusammenbricht (Kapitel 5).

Ein weiterer Bestandteil dieser Arbeit war die Durchführung von Press- und Filtrationsversuchen mit verschiedenen Fraktionen des Fermentermaterials, um den reaktiven Spurenelementanteil im Fermenter besser charakterisieren bzw. eingrenzen zu können (Kapitel 6). Vergleiche von Elementanalysen nach einfachen Salpetersäureaufschlüssen gegenüber dem etablierten mehrstufigen Säuretotalaufschlussverfahren sollten ferner Hinweise über die Aufschlusseffizienz v.a. der Spurenelemente liefern. Ziel war es letztlich, ein vereinfachtes Verfahren zur Bestimmung der für den Biogasprozess wichtigen Spurenelemente vorzuschlagen, um den Zeitraum zwischen Probenahme, Probenaufbereitung, Analyse und Interpretation stark zu verkürzen, was den Anlagenbetreibern und Analyselaboren Vorteile brächte. So kann neben einer Kostenersparnis v.a. auf Spurenelementmangelsituationen im Biogasreaktor schneller reagiert und gegengesteuert werden. 


\section{Kapitel 2: Grundlagen}

\subsection{Der Biogasprozess}

Unter anaeroben Bedingungen wird organisches Material von einer Vielzahl von Mikroorganismen zersetzt. Bei den Abbau- bzw. Vergärungsprozessen wird Biogas gebildet, ein Gasgemisch, das typischerweise etwa aus $45-75$ Vol.-\% aus Methan $\left(\mathrm{CH}_{4}\right)$ und $25-55$ Vol.-\% aus Kohlenstoffdioxid $\left(\mathrm{CO}_{2}\right)$ besteht und $2-7$ Vol.-\% Wasser $\left(\mathrm{H}_{2} \mathrm{O}\right.$, bei $\left.20-40{ }^{\circ} \mathrm{C}\right),<5$ Vol.- $\%$ Stickstoff $\left(\mathrm{N}_{2}\right),<3$ Vol.- $\%$ Sauerstoff $\left(\mathrm{O}_{2}\right),<2$ Vol.- $\%$ Schwefelwasserstoff $\left(\mathrm{H}_{2} \mathrm{~S}\right)$ sowie $<1$ Vol.-\% Wasserstoff $\left(\mathrm{H}_{2}\right)$ enthält (SCHOLWIN ET AL., 2009). Eine natürliche Vergärung organischer Substanzen findet in Sümpfen und Mooren, im Sediment sauerstoffarmer Gewässer, auf Reisfeldern, aber auch in Güllelagern, Mülldeponien oder im Verdauungstrakt von Menschen und Tieren, insbesondere im Pansen von Wiederkäuern statt. Bei der industriellen Nutzung von Gärprozessen hat sich der Begriff Fermentation etabliert (LEMMER ET AL., 2011). In Biogasanlagen werden diese natürlichen Prozesse technisch genutzt, um aus biologisch abbaubaren Substraten wie organischen Reststoffen, Energiepflanzen und Gülle Biogas zu erzeugen.

Der anaerobe Abbau- bzw. Vergärungsprozess wird grundsätzlich in vier wesentliche Reaktionsschritte unterteilt: Hydrolyse (Verflüssigung), Acidogenese (Versäuerung), Acetogenese (Essigsäurebildung) und Methanogenese (Methanbildung). Entsprechend ihrer Leistung in den einzelnen Schritten werden die beteiligten Mikroorganismen (Bakterien und Archaeen) als hydrolytisch-acidogene, acetogene und methanogene Mikroorganismen bezeichnet. Ihre Vielfalt und Aktivität sowie das Wachstum werden vor allem durch die Gärtemperatur, den pH-Wert, das Nährstoffangebot, die Verweilzeit und Raumbelastung sowie weiteren Faktoren beeinflusst (SCHIEDER ET AL., 2010).

Abbildung 1 zeigt eine schematische Darstellung des anaeroben Abbauprozesses bei der einphasigen Biogasproduktion. Dies ist der häufigste Fall in der Biogaspraxis. Alle vier Phasen laufen räumlich und zeitlich simultan in der Biogasanlage ab. Durch den gemeinsamen und syntrophen Abbau der komplexen Inputsubstrate ergeben sich hohe Anforderungen an die im Reaktor vorherrschenden chemischen und physikalischen Bedingungen, um eine optimale Biogasbildung zu gewährleisten. Bei einer räumlichen Trennung von Hydrolyse/Acidogenese und Acetogenese/Methanogenese wird die Biogasproduktion als zweiphasig bezeichnet. 


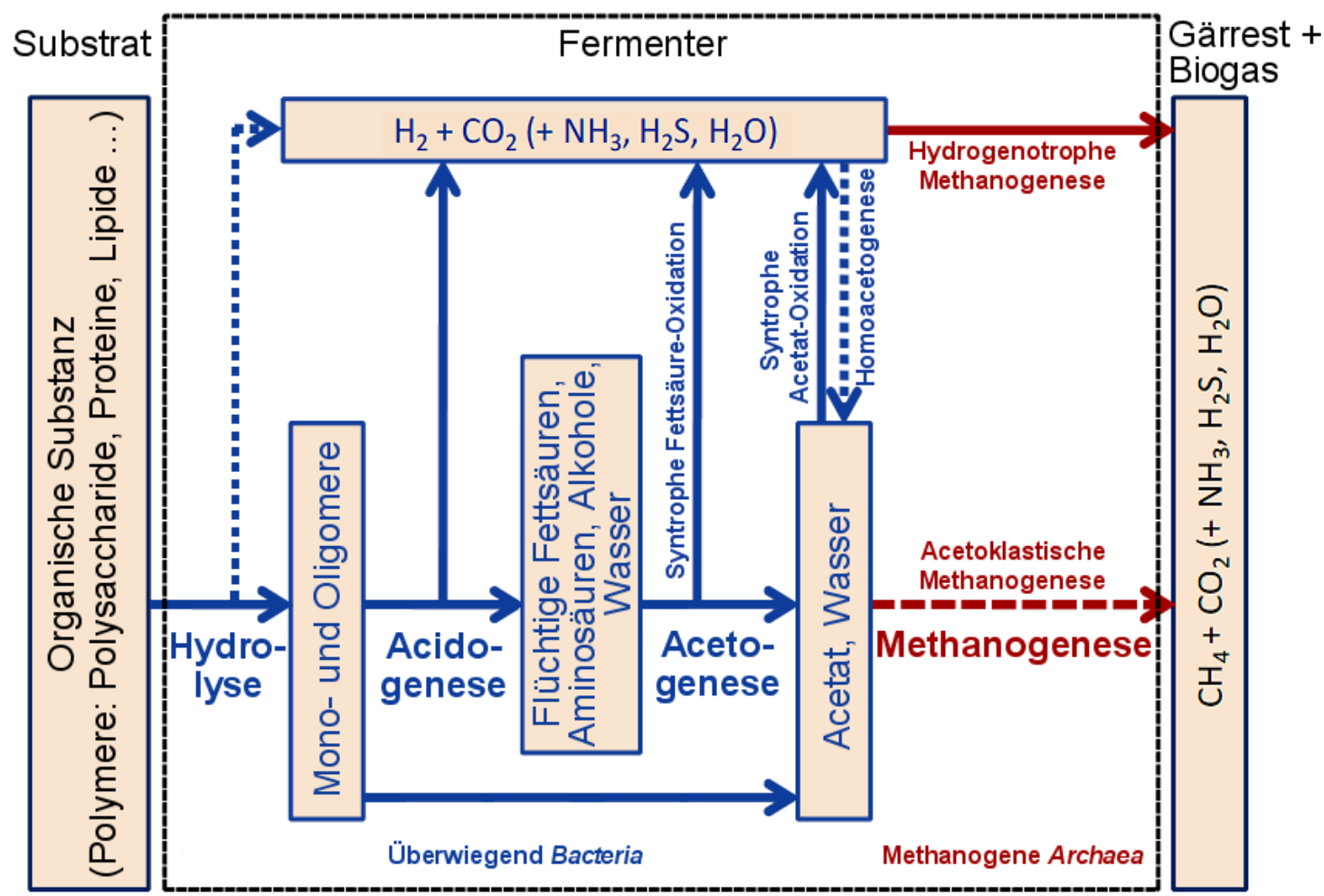

Abbildung 1: $\quad$ Vereinfachte schematische Darstellung des anaeroben Abbauprozesses bei der einphasigen Biogasproduktion (modifiziert nach LEBUHN \& GRONAUER, 2009; SCHIEDER ET AL., 2010).

In der ersten Stufe des Vergärungsprozesses, der Hydrolyse (Verflüssigung), werden größtenteils ungelöste, komplexe organische Makromoleküle wie z.B. Polysaccharide, Proteine und Lipide in den Inputsubstraten in kürzere niedermolekulare Spaltprodukte (Mono- und Oligomere) wie z.B. Oligosaccharide, Oligopeptide, Triglyceride und Monosaccharide, Amino- und Fettsäuren oder Glycerin zerlegt. Dabei werden in den Makromolekülen die Wasserstoffbrückenbindungen aufgetrennt und die Polymere durch gleichzeitige Einbindung von Wasser aufgelöst (LEMMER ET AL., 2011). In der Regel wird die hydrolytische Spaltung der komplexen Polymere in bakterienverfügbare, wasserlösliche Mono- und Oligomere durch zellwandgebundene extrazelluläre Enzyme (Hydrolasen) katalysiert (SAHM, 1981; KOCH ET AL., 2011). Die Geschwindigkeit und Effektivität der Hydrolyse ist dabei von den einzelnen Ausgangsstoffen in den verwendeten Inputsubstraten abhängig. Die meisten organischen Polymere lassen sich vergleichsweise leicht und schnell spalten. Komplexe lignocellulosehaltige Materialien können jedoch nur sehr langsam und unvollständig abgebaut werden, da Lignine anaerob nicht gespaltet werden können und stark hemmend auf die enzymatische Hydrolyse der Polysaccharide Cellulose und Hemicellulose wirken (SAHM, 1981). So sind stärkehaltige Inputsubstrate wie Mais-Silage schneller abbaubar und liefern höhere 
Biogasausbeuten als stark cellulosehaltige Substrate. Die Hydrolysierbarkeit der komplexen Ausgangsstoffe ist damit der die Abbaugeschwindigkeit bestimmende Schritt während des Biogasbildungsprozesses. Schon in dieser ersten Prozessstufe werden bei einigen Reaktionen Wasserstoff und Kohlenstoffdioxid sowie neben Wasser gegebenenfalls auch Ammoniak und Schwefelwasserstoff gebildet. Die beiden letztgenannten Gase können bereits während der Hydrolyse entstehen, wenn die Inputsubstrate höhere Konzentrationen an Schwefel (S) und Stickstoff enthalten (SCHIEDER ET AL., 2010).

Die durch die Hydrolyse entstandenen Mono- und Oligomere werden in der zweiten Prozessstufe, der Acidogenese (Versäuerung), von diversen hydrolytisch-acidogenen (fermentativen) Bakterien zu kurzkettigen Fettsäuren (flüchtige Fettsäuren) wie z.B. Essig-, Propion-, Butter-, Valerian- oder Capronsäure und weiteren organischen Säuren wie z.B. Milch-, Bernsteinsäure oder Aminosäuren sowie zu Alkoholen und Wasser gespalten bzw. umgebaut. Vor allem bei dieser Prozessstufe werden Wasserstoff und Kohlenstoffdioxid sowie gegebenenfalls Ammoniak und Schwefelwasserstoff erzeugt (SCHIEDER ET AL., 2010). Der Abbau durch die fermentativen Bakterien kann in der Acidogenese über unterschiedliche Stoffwechselwege erfolgen und wird stark vom Wasserstoffpartialdruck beeinflusst (SAHM, 1981). So werden bei höheren Wasserstoffkonzentrationen mehr Propion-, Butter-, Milchsäure oder Ethanol und weniger Essigsäure und Kohlenstoffdioxid gebildet als bei niedrigen Wasserstoffkonzentrationen (Scholwin ET AL., 2009). Flüchtige Fettsäuren wie Propion-, Butter-, Valerian-, Capron- oder Önanthsäure sind für die Mikroorganismen der nächsten beiden Prozessstufen schlechter abbaubar. Bei höheren Säuregehalten kommt es zu einem Abfallen des pH-Wertes bei gleichzeitiger Hemmung der Acetogenese und Methanogenese, welche im ungünstigsten Falle ganz zum Erliegen kommen. Ein verstärktes Auftreten dieser Säuren ist somit ein Indikator für einen instabilen Biogasprozess, in dem die Acetogenese und die Methanogenese nicht effizient ablaufen (BAUER ET AL., 2009). Neben dem Wasserstoffpartialdruck hängt das Spektrum der gebildeten organischen Säuren außerdem stark von der Gärtemperatur, dem pH-Wert und den vorliegenden Gehalten an längerkettigen Kohlenstoffverbindungen (v.a. Fettsäuren) ab. Die ersten beiden Prozessstufen Hydrolyse und Acidogenese sind verfahrenstechnisch meist nicht klar voneinander $\mathrm{zu}$ trennen, da sie typischerweise gemeinsam ablaufen (BAUER ET AL., 2009). 
In der dritten Stufe des Biogasprozesses, der Acetogenese (Essigsäurebildung), werden die Stoffwechselprodukte der vorherigen Prozessstufen von acetogenen Bakterien durch Oxidations- und Decarboxylierungsreaktionen v.a. zu Essigsäure (Acetat-Anionen), Wasser, Wasserstoff und Kohlenstoffdioxid (Hydrogencarbonat-Anionen) abgebaut. Wie bei der Acidogenese bereits aufgeführt, spielt hierbei der Wasserstoffpartialdruck eine entscheidende Rolle. Aus energetischen Gründen verhindern zu hohe Wasserstoffkonzentrationen den Abbau der Stoffwechselprodukte der Acidogenese (FRIEHE ET AL., 2016). Viele der säurebildenden Reaktionen sind thermodynamisch ungünstig (endergon) und laufen nicht spontan ab. Um thermodynamisch günstige (exergone) Bedingungen $\mathrm{zu}$ erreichen, muss der gebildete Wasserstoff kontinuierlich aufgebraucht werden (SCHINK, 1997). Die wasserstoffbildenden acetogenen Bakterien stehen daher in einer engen Wechselbeziehung (Syntrophie) mit den methanogenen Archaeen, die den Wasserstoff zusammen mit Kohlenstoffdioxid bei der Bildung von Methan (Methanogenese) verbrauchen. Dieser Wasserstoff-Transfer (interspecies hydrogen transfer) setzt eine enge räumliche Nachbarschaft der beteiligten acetogenen Bakterien und methanogenen Archaeen voraus. Bei ungenügender Populationsdichte, z.B. durch zu starkes Rühren, kann es zu Störungen dieses synergistischen Effekts kommen (WANDREY \& AIVASIDIS, 1983). Das bedeutet, dass die anaerobe Umsetzung der flüchtigen Fettsäuren, Aminosäuren und Alkohole energetisch gesehen auf Kosten der methanogenen Archaeen geht, diese aber umgekehrt von den acetogenen bzw. syntrophen Bakterien ihre Substrate erhalten (SAHM, 1981). Aber auch bei der Homoacetogenese kann gebildeter Wasserstoff für die Reaktion mit Kohlenstoffdioxid zu Acetat-Anionen verbraucht werden. Die Homoacetogenese spielt gegenüber der hydrogenotrophen Methanbildung jedoch nur eine wesentliche Rolle unter besonderen Bedingungen, z.B. bei niedrigen Temperaturen (CONRAD \& WETTER, 1990). Die syntrophe Acetat-Oxidation, also die Umsetzung von Acetat-Anionen zu Wasserstoff und Kohlenstoffdioxid, stellt die Edukte für die hydrogenotrophe Methanogenese bereit (GALlERT \& WinTER, 2015). Die einzelnen syntrophen Abbaureaktionen sind teilweise komplizierte Umsetzungsprozesse über mehrere Zwischenstufen und noch nicht im Detail geklärt (BAUER ET AL., 2009).

In der letzten Prozessstufe der Biogasbildung, der Methanogenese (Methanbildung), werden Methan und Kohlenstoffdioxid vorwiegend über zwei verschiedene Reaktionswege von methanogenen Archaeen gebildet, dem hydrogenotrophen (wasserstoff- 
verwertenden) und acetoklastischen (essigsäurespaltenden). Nur bestimmte Archaeen des Stamms Euryarchaeota sind zu einer nennenswerten Bildung von Methan fähig. Sie sind damit einzigartig in der Natur (BAUER ET AL., 2009). Die methanogenen Archaeen weisen ein eng begrenztes Substratspektrum auf und sind strikt auf sauerstofffreie Milieubedingungen angewiesen. Von den Stoffwechselprodukten der fermentativen und acetogenen Bakterien können nur Wasserstoff, Kohlenstoffdioxid, Kohlenstoffmonoxid (CO) und die flüchtigen Fettsäure-Anionen Acetat und Formiat als Hauptsubstrate sowie untergeordnet einige Methylverbindungen verwendet werden. Liegen SulfatIonen in der Prozessstufe vor, konkurrieren Sulfatreduzierer meist erfolgreich mit den Methanbildnern um diese Substrate (GALLERT \& WiNTER, 2015). Da alle Reaktionswege der Methanogenese energieliefernd sind, können sie die energiezehrenden Abbaureaktionen der Acetogenese bzw. den Wasserstoff-Transfer (siehe Acetogenese) ermöglichen (SCHINK, 1997; BAUER ET AL., 2009). In landwirtschaftlichen Biogasanlagen verläuft die Methanogenese bei höherer Raumbelastung bzw. geringerer Verweilzeit und höherer Temperatur vorwiegend über den hydrogenotrophen Reaktionsweg. Die Acetat-Ionen werden weniger acetoklastisch gespalten, sondern über die syntrophe Acetat-Oxidation verstärkt zu den Stoffwechselprodukten Kohlenstoffdioxid (bzw. Hydrogencarbonat) und Wasserstoff abgebaut und dann über die hydrogenotrophe Methanogenese in Methan umgesetzt (SCHNÜRER ET AL., 1999). Nur bei geringer Raumbelastung bzw. längerer Verweilzeit sowie niedrigeren Acetatgehalten ist der acetoklastische Reaktionsweg von größerer Bedeutung (BAUER ET AL., 2008, 2009).

\subsection{Einflussfaktoren auf den Biogasprozess}

Für eine große Vielfalt, ein optimales Wachstum und eine hohe Aktivität der Mikroorganismen in den vier Prozessstufen müssen verschiedene Anforderungen erfüllt und die vorherrschenden Umgebungsbedingungen im Fermenter optimal aufeinander abgestimmt und angepasst sein. Am anfälligsten gegenüber Prozessstörungen sind die methanogenen Archaeen, die strikt auf ein anaerobes Milieu angewiesen sind. So werden in der Biogaspraxis die Prozessbedingungen in erster Linie an diese Mikroorganismengruppe angepasst (KAISER ET AL., 2008). Da die häufigsten Biogasfermenter einphasig und semikontinuierlich betrieben werden, d.h. dem Fermenter werden in bestimmten Zeitabständen, z.B. einmal täglich, Inputsubstrate zugegeben und die entsprechende Menge an Gärrest entnommen, werden dem System gleichzeitig Mikro- 
organismen entzogen. Damit der Biogasbildungsprozess stabil und im Gleichgewicht bleibt, muss gewährleistet sein, dass die Verluste durch den Entzug durch die Wachstumsrate der Mikroorganismengruppen kompensiert werden. Für die Biogasbildung sind verschiedene weitere Prozessgrößen wichtig, die die Abbauprozesse und damit die Biogasproduktion beeinflussen, die im Folgenden kurz beschrieben werden.

\subsubsection{Raumbelastung, Verweilzeit und Durchmischung}

Wichtige ökonomische und prozesskinetische Kenngrößen beim Fermenterbetrieb sind die Raumbelastung, die Verweilzeit und die Durchmischung. Die Raumbelastung $\left(\mathbf{B}_{\mathbf{R}}\right)$ wird häufig als Kennwert für die Anlagenauslegung verwendet und stellt ein Maß für die Belastung des Fermenters mit organischem Material dar (SCHOLWIN ET AL., 2009). Sie ist definiert als der Quotient aus der dem Fermenter zugeführten Masse organischer Trockenubstanz (oTS) und dem verfügbaren Reaktionsraum (nutzbares Fermentervolumen) pro Zeiteinheit und wird in $\mathrm{kg}_{\mathrm{oTS}} /\left(\mathrm{m}^{3} \cdot \mathrm{d}\right)$ oder $\mathrm{g}_{\mathrm{oTS}} /(\mathrm{L} \cdot \mathrm{d})$ angegeben. Die Verweilzeit bzw. hydraulische Aufenthaltszeit (HRT) beschreibt die theoretische (rechnerisch ermittelte) durchschnittliche Aufenthaltszeit des Inputsubstrates im Fermenter zwischen der Einbringung und dem Austrag (in Tagen). Die reale Verweilzeit weicht davon ab, da z.B. durch Kurzschlussströmungen einzelne Komponenten den Fermenter unterschiedlich schnell verlassen (FRIEHE ET AL., 2016). Die hydraulische Verweilzeit muss so gewählt werden, dass nicht mehr Mikroorganismen aus dem Fermenter ausgewaschen werden, als in ihm nachwachsen können bzw. festgehalten werden (SCHOLWIN ET AL., 2009). So ist es notwendig, sie an die spezifische Abbaugeschwindigkeit der eingesetzten Inputsubstrate anzupassen (KAISER ET AL., 2008).

Zwischen den beiden Belastungsparametern besteht ein direkter Zusammenhang: Da mit steigender Raumbelastung dem Fermenter mehr Inputsubstrat zugeführt wird und somit bei konstantem Fermentervolumen die Ablaufmenge erhöht werden muss, sinkt die Verweilzeit. Eine längere Verweilzeit bewirkt jedoch eine höhere Abbauleistung des Substrates und somit eine in der Summe gesteigerte Biogasproduktion und Methanfreisetzung (KAISER ET AL., 2008). Nachdem die optimale Biogasausbeute pro Zeiteinheit erreicht ist, sinkt sie wieder. Deshalb wird in der Biogaspraxis nicht der maximal mögliche Gasertrag bzw. der vollständige Abbau der im Substrat enthaltenen organischen Stoffe angestrebt, sondern eine mit einem ökonomisch vertretbaren Aufwand erreichbare optimale Ausbeute pro Zeiteinheit (SCHOLWIN ET AL., 2009). 
Eine weitere Kenngröße ist der Grad der Durchmischung des Fermenterinhaltes. Eine moderate Durchmischung intensiviert den Kontakt zwischen den Mikroorganismen und den Inputsubstraten und sorgt außerdem für einen Ausgleich von Temperatur- und Konzentrationsunterschieden im Gärmaterial. Entfällt die Durchmischung tritt durch die Dichteunterschiede im Gärmaterial Schichtenbildung auf, die nur schwer zu beseitigen ist. Zudem erschweren Schwimmschichten u.a. den Gasaustritt (KAISER ET AL., 2008).

\subsubsection{Prozesstemperatur}

Die Prozesstemperatur ist einer der wichtigsten Parameter für die Stabilität des Biogasprozesses bzw. das Wachstum und die Aktivität der Mikroorganismen. In der Natur steigen die Umsetzungsgeschwindigkeiten chemischer Reaktionen grundsätzlich mit einer Erhöhung der Temperatur. Für Mikroorganismen bzw. anaerobe Abbau- und Umsetzungsprozesse gilt das nur bedingt, da sich die beteiligten mikrobiellen Gruppen nur in fixen Temperaturbereichen optimal entwickeln können (KAISER ET AL., 2008). Sie lassen sich grob in drei Gruppen einteilen: psychrophile Mikroorganismen etablieren sich optimal in einem Temperaturbereich bis $25^{\circ} \mathrm{C}$, mesophile bei etwa 35 bis $43{ }^{\circ} \mathrm{C}$ und thermophile bei 50 bis $57^{\circ} \mathrm{C}$ (SCHOLWIN ET AL., 2009). Weicht die Gärtemperatur vom optimalen Bereich ab, v.a. bei schnellen Temperaturänderungen, können Hemmwirkungen im Biogasprozess auftreten bis hin zu irreversiblen Schäden der Populationsdichte der Mikroorganismen (FRIEHE ET AL., 2016).

Der Betrieb eines Fermenters im psychrophilen Temperaturbereich hätte zwar den Vorteil, dass eine Beheizung des Reaktors nicht nötig ist; allerdings wäre aufgrund der geringen Abbaugeschwindigkeit der Substrate und geringerer Biogaserträge der Betrieb wirtschaftlich ungünstig. Ein Betrieb im thermophilen Temperaturbereich bei eventuell hohen Substratumsatzraten (aufgrund der hohen Temperatur) hätte den Vorteil, dass eine Hygienisierung der Gärreste erreicht wird. Das könnte vor allem bei der Vergärung von Klärschlämmen, Biomüll oder industriellen Reststoffen relevant sein (GALLERT \& WINTER, 2015). Jedoch besteht durch das Aufheizen auch ein höherer Energiebedarf und der Gärprozess reagiert empfindlicher auf Prozessstörungen, Unregelmäßigkeiten in der Substratzufuhr oder der Betriebsweise des Fermenters (FRIEHE ET AL., 2016). Die meisten Biogasanlagen werden in einem Temperaturbereich zwischen 38 und $42{ }^{\circ} \mathrm{C}$ (WEILAND, 2010) betrieben, da der größte Anteil der bekannten Methanbildner ein Wachstumsoptimum im mesophilen Bereich aufweist (KAISER ET AL., 2008) und damit 
relativ hohe Biogaserträge bei guter Prozessstabilität erreicht werden können (FRIEHE ET AL., 2016). Der mesophile Temperaturbereich gewährleistet im Gegensatz zum psychrophilen moderate bis gute Abbaugeschwindigkeiten der Substrate, ohne dass ein hoher Energiebedarf durch das Beheizen des Reaktors wie im thermophilen Temperaturbereich notwendig ist.

Aufgrund der breiteren Übergänge zwischen den einzelnen Temperaturbereichen wirken sich die Temperaturen jedoch nicht so gravierend auf den Biogasbildungsprozess aus als rasche Temperaturänderungen. Die methanogenen Archaeen können sich nur bei langsamer Temperaturänderung an unterschiedliche Temperaturen anpassen. Daher ist für die Prozessstabilität weniger die absolute Temperatur, sondern vielmehr die Einhaltung einer Konstanz in einem Temperaturniveau wichtig (FRIEHE ET AL., 2016).

\subsection{3 pH-Wert und Pufferkapazität}

Durch eine Vielzahl chemischer bzw. mikrobiologischer Reaktionen werden beim Biogasprozess $\mathrm{pH}$-Wert-Veränderungen hervorgerufen, die sich unter optimalen und stabilen Prozessbedingungen jedoch in engen Bereichen bewegen. Durch das gebildete Kohlenstoffdioxid bzw. die Carbonatpuffer- $\left(\mathrm{HCO}_{3}{ }^{-} / \mathrm{CO}_{3}{ }^{2-}\right)$ und Ammoniumpufferkapazität $\left(\mathrm{NH}_{4}{ }^{+} / \mathrm{NH}_{3}\right)$ sind die Milieubedingungen im Gärprozess insgesamt neutral bis leicht alkalisch bei einem $\mathrm{pH}-$ Wert zwischen 6,8 und 7,8. Dieser $\mathrm{pH}$-Bereich stellt nach SCHIEDER ET AL. (2010) für die methanogenen Archaeen optimale Lebensbedingungen dar, sodass die Methanogenese ideal ablaufen kann. Andere Autoren beschreiben für die Biogasbildung optimale pH-Wert-Bereiche von 6,7 bis 7,5 (WEILAND, 2001), 6,8 bis 7,4 (BRAUn, 1982), 6,8 bis 7,5 (KAISER ET AL., 2008) oder 7,0 bis 7,5 (SCHOlWIN ET AL., 2009). Die hydrolytisch-acidogenen Bakterien bevorzugen eher eine schwach saure bis saure Umgebung bei einem pH-Wert von 5,0 bis 6,5 (SCHOLWIN ET AL., 2009). Andere Autoren geben pH-Wert-Bereiche von 4,5 bis 6,3 (KAISER ET AL., 2008), 4,7 bis 7,0 (SCHIEDER ET AL. 2010) oder 5,2 bis 6,3 (WeILAND, 2001) an. Bei einem pH-Wert unter 4,5 hemmen die hydrolytisch-acidogenen Bakterien ihr Wachstum durch ihre eigenen Ausscheidungsprodukte (SCHOLWIN ET AL., 2009). Die säurebildenden Bakterien sind allerdings in der Lage, sich auch in größeren $\mathrm{pH}-$ Wert-Bereichen $\mathrm{zu}$ entwickeln und anzupassen. Die methanogenen Archaeen werden dagegen durch eine zu stark saure oder zu stark alkalische Umgebung schnell gehemmt. 
So stellt sich bei einphasiger Biogasproduktion in der Regel ein $\mathrm{pH}-$ Wert im optimalen Bereich $(7,0$ bis 7,5$)$ ein, da die Bakterien und methanogenen Archaeen, wie in Kapitel 2.1 bereits aufgeführt, ein selbstregulierendes syntrophes System bilden (SCHOLWIN ET AL., 2009). Bei einer zweiphasigen Biogasproduktion sind die ersten beiden Prozessstufen der Hydrolyse und Acidogenese wie beschrieben gewöhnlich deutlich saurer (pH-Wert 5,0 bis 6,5; SCHOLWIN ET AL., 2009) als die beiden Stufen der Acetogenese und Methanogenese ( $\mathrm{pH}-$ Wert 7,5 bis 8,5; BAUER ET AL., 2009). Denn die Methanbildner selbst erzeugen im Gegensatz zu den hydrolytisch-acidogenen Bakterien ein eher alkalisches Milieu: einerseits durch die Verwertung der Acetat-Anionen (das gebildete Kohlenstoffdioxid löst sich teilweise und schafft Carbonatpufferkapazität), andererseits durch den Abbau des Wasserstoffs durch die Syntrophie mit den acetogenen Bakterien beim Säurenabbau (BAUER ET AL., 2009).

Durch das bei den Abbauprozessen freigesetzte Kohlenstoffdioxid wird der pH-Wert im Normalfall im neutralen bis schwach sauren $\mathrm{pH}$-Bereich gepuffert. Das $\mathrm{CO}_{2}$ liegt im Gleichgewicht mit dem Hydrogencarbonat (SCHOLWIN ET AL., 2009) und ist im sauren Milieu weniger gut löslich als im basischen, sodass die Freisetzung von Kohlenstoffdioxid ins Biogas mit niedrigerem pH-Wert günstiger wird (SCHIEDER ET AL., 2010). Die Ammoniumpufferung $\left(\mathrm{NH}_{4}^{+} / \mathrm{NH}_{3}\right)$ bewirkt eine Stabilisierung im schwach basischen pH-Wert-Bereich. Hemmwirkungen durch höhere Konzentrationen von Ammoniak $\left(\mathrm{NH}_{3}\right)$ im Biogas sind verstärkt nur bei basischen $\mathrm{pH}-$ Werten zu erwarten. Allerdings ist Ammoniak bei dem in Fermentern üblichen optimalen pH-Wert-Bereich sehr gut wasserlöslich, sodass es bevorzugt im Fermentermaterial verbleibt. So gelangen im Normalfall nur sehr geringe Mengen $\mathrm{NH}_{3}$ ins Biogas, selbst wenn die Inputsubstrate höhere Konzentrationen an Stickstoff enthalten (SCHIEDER ET AL., 2010).

Das Gleichgewicht v.a. durch die Carbonatpuffer- und Ammoniumpufferkapazität bzw. durch die syntrophe Wechselbeziehung der Mikroorganismen ist im Fermenter jedoch empfindlich. Wird der $\mathrm{pH}-$ Wert durch das freigesetzte Kohlenstoffdioxid nicht mehr ausreichend gepuffert z.B. durch zu hohe Inputsubstratmengen, beginnt er schnell abzusinken. Das führt zu einem Aufschaukelungsprozess: Weil die Methanogenen in ihren Stoffwechselprozessen stark gehemmt sind, werden die Säuren aus der Acidogenese zusätzlich aufkonzentriert. Infolgedessen sinkt der pH-Wert immer weiter ab, der Biogasprozess versäuert (SCHOLWIN ET AL., 2009). 
Der Versäuerung kann durch eine Reduzierung oder eines Stopps der Substratzufuhr entgegengewirkt werden, wodurch die methanogenen Archaeen wieder Zeit bekommen, die vorhandenen überschüssigen Säuren abzubauen. Gegebenenfalls kann durch die Zugabe von basischen Zuschlagstoffen wie z.B. Natriumhydrogencarbonat $\left(\mathrm{NaHCO}_{3}\right)$ der pH-Wert reguliert werden (KAISER ET AL., 2008).

\subsubsection{Sauerstoff}

Methanogene Archaeen gehören zu den ältesten Lebensformen der Erde. Sie entstanden vor etwa 3,5 Milliarden Jahren und waren bzw. sind auf eine sauerstofffreie Lebensumgebung angewiesen, um dauerhaft überleben zu können (BRAUN, 1982). Da sie keine effektiven Schutzmechanismen gegen die hemmende bzw. toxische Wirkung von Sauerstoff aufweisen, werden die meisten Arten schon durch geringe Sauerstoffmengen abgetötet (FrIEHE ET AL., 2016). Bei jeder Substratzugabe in den Fermenter kommt es zu geringen Sauerstoffeinträgen, da es sich bei den meisten Anlagen um Durchflussfermenter handelt. Einige am anaeroben Abbau- bzw. Vergärungsprozess beteiligte fakultativ anaerob lebende Bakterien besitzen jedoch die Fähigkeit, den in den Biogasprozess eingetragenen Sauerstoff zu verbrauchen. Sie gehören vor allem $\mathrm{zu}$ den acidogenen Bakterien und können sowohl unter Sauerstoffeinfluss als auch ohne Sauerstoff leben (KAISER ET AL., 2008). Das bedeutet, solange der Sauerstoffeintrag nicht zu groß ist, $<\mathbf{0 , 1} \mathbf{~ m g / L ~} \mathbf{O}_{2}$ (WEILAND, 2001), wird er von diesen Bakterien verstoffwechselt, bevor er die methanogenen Archaeen schädigen kann. Auch der in den Fermenter eingetragene Luftsauerstoff bei biologischer Entschwefelung hat daher im Normalfall keinen negativen Einfluss auf die Methanbildner (WEILAND, 2001).

\subsubsection{Nährstoffversorgung}

Die komplexen organischen Ausgangsstoffe liefern für die Vergärung wichtige Makround Mikronährstoffe. Der Bedarf ist für die Vielzahl an Mikroorganismen im Biogasprozess artspezifisch, die Konzentrationen und die Verfügbarkeit beeinflussen die Wachstumsrate und Aktivität der verschiedenen Arten (FRIEHE ET AL., 2016). So kann ein Mangel an Nährstoffen zu einem gehemmten Wachstum und einer geringeren Aktivität bzw. zu höheren Säurekonzentrationen im Fermenter und niedrigeren Biogaserträgen führen und die Stabilität und Effizienz des Biogasbildungsprozesses gefährden (Lebuhn et Al., 2008; Demirel \& Scherer, 2011; Schattauer et Al., 2011). Für 
einen stabilen Prozess ist ein ausgeglichenes Verhältnis von essenziellen Makro- und Mikronährstoffe von enormer Bedeutung. Die elementaren Nährstoffe für anaerobe Mikroorganismen lassen sich in Anlehnung an WACKETT ET AL. (2004) aufgrund ihrer benötigten Konzentration bzw. ihrer Bedeutung und Funktion allgemein in verschiedene Gruppen einteilen (Abbildung 2).

\begin{tabular}{|c|c|c|c|c|c|c|c|c|c|c|c|c|c|c|c|c|c|}
\hline $\mathrm{H}$ & ${ }_{\| 1}^{2}$ & $\begin{array}{l}\square \\
\square\end{array}$ & $\begin{array}{l}\text { Esse } \\
\text { Esse }\end{array}$ & zielle $M$ & kronähr & $\begin{array}{l}\text { nte } \\
\text { toffe }\end{array}$ & $\square$ & Essenziel & e Mikro & ährstoff & & ${ }_{\mathrm{IIA}}^{13}$ & $\begin{array}{l}14 \\
\text { IVA }\end{array}$ & $\begin{array}{l}15 \\
\text { VA }\end{array}$ & $\begin{array}{l}16 \\
\text { VIA }\end{array}$ & $\begin{array}{c}17 \\
\text { VIIA }\end{array}$ & $\mathrm{He}$ \\
\hline${ }^{3} \mathrm{Li}$ & ${ }^{4} \mathrm{Be}$ & & & $\begin{array}{l}27 \\
\text { Co }\end{array}$ & $\begin{array}{l}\leftarrow \text { Ord } \\
\leftarrow \text { Elen }\end{array}$ & $\begin{array}{l}\text { Ingszahl } \\
\text { ntsyymo }\end{array}$ & & 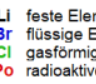 & $\begin{array}{l}\text { lente } \\
\text { emente (b } \\
\text { eElement } \\
\text { Elemente }\end{array}$ & $\left.20^{\circ} \mathrm{C}\right)$ & & $\begin{array}{c}{ }^{5} \\
{ }^{B}\end{array}$ & $\begin{array}{c}{ }^{6} c^{c} \\
C\end{array}$ & ${ }^{7} \mathrm{~N}$ & ${ }^{8} 0$ & ${ }^{9} \mathrm{~F}$ & ${ }^{10} \mathrm{Ne}$ \\
\hline $\begin{array}{l}11 \\
\mathrm{Na}\end{array}$ & $\begin{array}{l}12 \\
\mathrm{Mg}\end{array}$ & ${ }_{\text {III }}^{3}$ & $\begin{array}{l}4 \\
\text { IVB }\end{array}$ & $\begin{array}{l}5 \\
\text { VB }\end{array}$ & $\begin{array}{c}6 \\
\text { viB }\end{array}$ & $\begin{array}{l}7 \\
\text { VIIB }\end{array}$ & $\begin{array}{c}8 \\
\text { vimB }\end{array}$ & $\begin{array}{c}99 \\
\text { viाв }\end{array}$ & $\begin{array}{l}10 \\
\text { VIIIB }\end{array}$ & ${ }_{18}^{11}$ & $\begin{array}{ll}12 \\
\text { IIB }\end{array}$ & $\begin{array}{l}13 \\
\mathrm{Al}\end{array}$ & ${ }^{14} \mathbf{S i}$ & ${ }^{15} \mathbf{P}$ & ${ }^{16} \mathrm{~S}$ & $\begin{array}{l}{ }^{17} \\
\mathrm{Cl}\end{array}$ & ${ }^{18} \mathrm{Ar}$ \\
\hline${ }^{19} \mathbf{K}$ & $\begin{array}{l}20 \\
\mathrm{Ca}\end{array}$ & ${ }^{21} \mathrm{Sc}$ & ${ }^{22} \mathrm{Ti}$ & ${ }^{23} \mathrm{~V}$ & ${ }^{24} \mathrm{Cr}$ & $\begin{array}{l}25 \\
\text { Mn }\end{array}$ & Fe & ${ }^{27} \mathrm{Co}$ & $\begin{array}{l}28 \\
\mathrm{Ni}\end{array}$ & ${ }^{29} \mathrm{Cu}$ & ${ }^{30} \mathrm{Zn}$ & ${ }^{31} \mathrm{Ga}$ & Ge & As & Se & ${ }^{35} \mathrm{Br}$ & ${ }^{36} \mathrm{Kr}$ \\
\hline $\begin{array}{l}37 \\
\mathrm{Rb}\end{array}$ & ${ }^{38} \mathrm{Sr}$ & ${ }^{39} \mathbf{Y}$ & $\begin{array}{l}40 \\
\mathrm{Zr}\end{array}$ & $\begin{array}{l}41 \\
\mathrm{Nb}\end{array}$ & $\begin{array}{l}42 \\
\text { Mo }\end{array}$ & $\begin{array}{l}43 \\
\text { Tc }\end{array}$ & $\begin{array}{l}44 \\
\mathrm{Ru}\end{array}$ & $\begin{array}{l}45 \\
\text { Rh }\end{array}$ & $\begin{array}{l}46 \\
P d\end{array}$ & $\begin{array}{l}47 \\
\mathrm{Ag}\end{array}$ & ${ }^{48} \mathrm{Cd}$ & $\begin{array}{l}49 \\
\text { In }\end{array}$ & Sn & Sb & $\begin{array}{l}52 \\
\mathrm{Te}\end{array}$ & $\begin{array}{l}53 \\
\quad\end{array}$ & $\begin{array}{l}54 \\
\mathrm{Xe}\end{array}$ \\
\hline${ }^{55} \mathrm{Cs}$ & $\begin{array}{l}56 \\
\mathrm{Ba}\end{array}$ & $\begin{array}{c}57-71 \\
\text { La }- \text { Lu }\end{array}$ & $\begin{array}{l}72 \\
\mathrm{Hf}\end{array}$ & $\begin{array}{l}73 \\
\mathrm{Ta}\end{array}$ & ${ }^{74}$ W & $\begin{array}{l}75 \\
\mathrm{Re}\end{array}$ & $\begin{array}{l}76 \\
\text { Os }\end{array}$ & $\begin{array}{l}77 \\
\text { Ir }\end{array}$ & $\begin{array}{l}{ }^{78} \\
\mathrm{Pt}\end{array}$ & $\begin{array}{l}79 \\
\mathrm{Au}\end{array}$ & $\begin{array}{l}80 \\
\mathrm{Hg}\end{array}$ & $\begin{array}{l}81 \\
\mathrm{TI}\end{array}$ & ${ }^{82} \mathrm{~Pb}$ & ${ }^{83} \mathrm{Bi}$ & $\begin{array}{l}84 \\
\text { Po }\end{array}$ & ${ }^{85}$ At & $\begin{array}{l}86 \\
\mathrm{Rn}\end{array}$ \\
\hline
\end{tabular}

Abbildung 2: $\quad$ Klassifizierung der elementaren Grundelemente und Nährstoffe für anaerobe Mikroorganismen im Periodensystem der Elemente (modifiziert nach WACKETT ET AL., 2004 und WEINRICH, 2017).

Aufgrund der Vielzahl an Funktionen erfolgt der Übersicht halber in dieser Arbeit eine Zuordnung der wichtigsten Elemente für die anaeroben Mikroorganismen im Biogasprozess in drei wesentliche Gruppen:

I: Essenzielle Grundelemente und Makronährstoffe: $\quad$ C, N, P, S, Ca, Cl, K, Mg, Na

II: Essenzielle Mikronährstoffe: Co, Ni, Mo, Fe, Cu, Mn, Zn, Se, V, W

III: Weitere Elemente: As, $\mathrm{Cd}, \mathrm{Cr}, \mathrm{Pb}, \mathrm{Al}, \mathrm{La}, \mathrm{Ti}, \mathrm{Sr}, \ldots$

In Gruppe I sind die Makronährstoffe aufgeführt, also alle essenziellen Elemente, die von den anaeroben Mikroben in größerer Menge benötigt werden. Mikronährstoffe bzw. Spurenelemente (Gruppe II) werden in geringeren Konzentrationen benötigt. Alle weiteren Elemente sind in Gruppe III zusammengefasst. Die genannten Elemente werden in dieser Arbeit näher betrachtet.

Nach den Grundelementen Wasserstoff und Sauerstoff ist Kohlenstoff Hauptbestandteil der Mikroben. Für den Aufbau der Zellsubstanz und ihren Stoffwechsel benötigen die Mikroorganismen neben Kohlenstoff vor allem die essenziellen Grundelemente 
Stickstoff, Phosphor und Schwefel (LEMMER ET AL., 2011). Den Kohlenstoff beziehen die Mikroorganismen im Wesentlichen aus den Substratzugaben und nutzen ihn zum Aufbau ihrer Zellstruktur und als Hauptenergiequelle. Stickstoff benötigen die Mikroorganismen vor allem für die Proteinbiosynthese, d.h. zur Bildung verschiedener Enzyme, die die Stoffwechselreaktionen durchführen. Stark erhöhte Stickstoffgehalte im Inputsubstrat können jedoch zu einer Hemmung der mikrobiellen Aktivität im Fermenter führen (BAUER ET AL., 2009). Für die Synthese zellulärer Energieträger wie ATP (Adenosintriphosphat) und NADP (Nicotinsäureamid-Adenin-DinukleotidPhosphat) sind die Phosphatkonzentrationen im Fermentermaterial wichtig. Ein Phosphatmangel kann die Stoffwechselprozesse im Fermenter vermindern. Häufiger Bestandteil bedeutsamer Aminosäuren wie Cystein oder Methionin ist Schwefel. Er ist damit essenziell für die Bildung wichtiger Stoffwechselenzyme (BAUER ET AL., 2009). Zudem dienen verschiedene Schwefelverbindungen teilweise auch als Redoxpartner beim Elektronentransfer (LEMMER ET AL., 2011). Zu hohe Schwefelgehalte reduzieren andererseits aber die Bioverfügbarkeit von Elementen durch die Bildung unlöslicher Metallsulfide (ZANDVOORT ET AL., 2005, 2006). Einige Archaeen benötigen neben Phosphat noch Natrium für die ATP-Bildung (LEMMER ET AL., 2011). Natrium spielt zudem eine wichtige Rolle beim Nährstofftransport (TAKASHIMA ET AL., 1990). Weitere essenzielle Makronährstoffe und wichtige Strukturelemente für die Enzyme bzw. zum Zellsubstanzaufbau sind neben Wasserstoff, Kohlenstoff, Stickstoff, Sauerstoff, Phosphor, Schwefel und Natrium noch Calcium, Chlor, Kalium und Magnesium.

Um optimale Prozessbedingungen aufrechtzuerhalten, ist generell nicht nur die Menge eines Nährstoffs, sondern auch ein optimales Verhältnis aller Nährstoffe zueinander wichtig (BAUER ET AL., 2009). Zur hinreichenden Versorgung der Methanogenen mit Nährstoffen sollte das C:N:P:S-Verhältnis bei ca. 600:15:5:1 liegen (WEILAND, 2010). Die Verhältnisangaben sind abhängig von den jeweiligen Betriebsparametern im Fermenter und somit nicht generell übertragbar auf alle Biogasprozesse. Sie stellen aber gute Richtwerte dar. Auch das C:N-Verhältnis der eingesetzten Substrate ist von Bedeutung. Ist das Verhältnis zu hoch durch zu hohe Kohlenstoff- und zu niedrige Stickstoffgehalte, kann der im Prozess vorhandene Kohlenstoff durch einen unzureichenden Stoffwechsel nicht vollständig umgesetzt werden, sodass die maximal möglichen Methanausbeuten im Fermenter nicht erreicht werden (FRIEHE ET AL., 2016). Im umgekehrten Fall kann es durch einen hohen Stickstoffüberschuss zu einer 
übermäßigen Bildung von Ammoniak bzw. von Ammonium-Ionen kommen, die sich inhibierend auf das Wachstum der Mikroorganismen bzw. die Substratabbaurate auswirken können, v.a. bei (steigenden) basischen pH-Werten (BRAUN, 1982; WeILAND, 2008). Für einen ungestörten Prozessablauf sollte das C:N-Verhältnis deshalb im Bereich von etwa 15 bis 30 (KOCH ET AL., 2017) bzw. 20 bis 40 (SCHOLWIN ET AL., 2009) liegen.

Für einen stabilen Biogasprozess sind neben den Makronährstoffen die Mikronährstoffe bzw. Spurenelemente von großer Bedeutung. In Fermentern, die ausschließlich mit nachwachsenden Rohstoffen, in erster Linie Mais-Silage oder sonstiger pflanzlicher Biomasse und nur einem sehr geringen Anteil an Gülle oder Mist gefüttert werden, liegt oftmals ein Spurenelementmangel vor (HINKEN ET AL., 2008; LEBUHN ET AL., 2008; Munk et AL., 2010; Pobeheim et AL., 2010, 2011; Demirel \& SCHERER, 2011; Koch ET AL., 2011; LEMMER ET AL., 2011; LINDORFER ET AL., 2012; FAHLBUSCH ET AL., 2018). Größtenteils wird diese Unterversorgung durch eine zusätzliche Spurenelementzugabe von synthetischen Additiven kompensiert. Aufgrund der Bedeutsamkeit im Biogasprozess wird in Kapitel 2.3 näher auf die Rolle der Spurenelemente eingegangen.

\subsubsection{Inhibitoren (Hemmstoffe)}

Generell sind für den Biogasprozess alle biogenen Roh- und Reststoffe geeignet, die anaerob weitestgehend abgebaut werden können und Hemm- bzw. Schadstoffe in so geringen Konzentrationen enthalten, dass weder der Gasbildungsprozess noch die nachfolgende landwirtschaftliche Verwertung der Gärreste nachteilig beeinflusst wird (WEILAND, 2000). Einige Verbindungen und Elemente können jedoch bei zu hohen Konzentrationen einerseits durch die zugegebenen organischen Inputsubstrate, potenziellen Prozesshilfsstoffe oder durch Geräte- und Materialabrieb bzw. andererseits durch die entstehenden Abbauprodukte hemmende oder toxische Wirkungen auf die Mikroorganismen ausüben und den anaeroben Vergärungsprozess behindern. Die inhibierende Wirkung hängt dabei im Wesentlichen neben den Konzentrationen auch von der Gärtemperatur, dem pH-Wert und weiteren Betriebsparametern (z.B. Art der Substratzugabe, Fermentertyp, Raumbelastung, Verweilzeit und Durchmischung) ab. In der Literatur existiert eine Vielzahl an Untersuchungen zu einzelnen Hemmstoffen, die sich aber z.B. aufgrund von vielschichtigen Wechselwirkungen, unterschiedlich langen Adaptionszeiten oder Komplexbildungen in Abhängigkeit der zugrunde liegenden 
jeweiligen Prozess- und Milieubedingungen stark voneinander unterscheiden (Chen et AL., 2008; Scholwin ET AL., 2009). Eine umfassende Zusammenstellung über die Inhibitoren im anaeroben Gärprozess geben CHEN ET AL. (2008).

So können über die Inputsubstrate beispielsweise Antibiotika-, Desinfektions- und Lösungsmittel- oder Herbizidrückstände und Schwermetalle vor allem durch die Zugabe von Wirtschaftsdüngern (Gülle oder Festmist), tierische Fette oder Prozesshilfsstoffe in den Gärprozess gelangen (FRIEHE ET AL., 2016). Abbau- und Stoffwechselprodukte mit potenzieller Hemmwirkung bei zu hohen Konzentrationen in den einzelnen Stufen im Biogasprozess sind v.a. Ammoniak, Schwefelwasserstoff, Wasserstoff und ein weites Spektrum der gebildeten organischen Säuren (vgl. Kapitel 2.1).

Wie bereits in Kapitel 2.2.2 und 2.2.4 aufgeführt, können zudem durch schnelle Temperaturänderungen oder zu hohe Sauerstoffeinträge Hemmwirkungen im Biogasprozess auftreten. Auch Änderungen des pH-Wertes durch eine nicht mehr ausreichende Pufferwirkung können sich inhibierend oder toxisch auf das Wachstum und die Aktivität sowohl der hydrolytisch-acidogenen Bakterien (bei sauren $\mathrm{pH}-$ Werten) als auch der methanogenen Archaeen (bei zu stark saurer oder zu stark alkalischer Umgebung) auswirken (vgl. Kapitel 2.2.3).

Während die Ammonium-Ionen den meisten Bakterien als Stickstoffquelle dienen, kann Ammoniak in undissoziierter Form schon in geringen Mengen hemmend auf die Mikroorganismen wirken (KAISER ET AL., 2008). Freies Ammoniak kann besonders in der acetoklastischen Methanogenese in die Zellen der sensiblen Methanogenen diffundieren, was zu einer Änderung des intrazellulären $\mathrm{pH}-$ Werts führt. Verschiedene Mechanismen verhindern dann, dass bestimmte Enzymreaktionen optimal ablaufen können bzw. der zelluläre Energieträger ATP gebildet wird (BRAUN, 1982; WITTMANN ET AL., 1995; BAUER ET AL., 2009). Jedoch sind Hemmwirkungen durch höhere Konzentrationen von Ammoniak bei dem in Biogasanlagen optimalen pH-Wert-Bereich zwischen 7,0 und 7,5 als eher gering einzustufen (vgl. Kapitel 2.2.3). So kommt es im Fermenter nur bei hohen Stickstoffüberschüssen, bei basischen $\mathrm{pH}-$ Werten oder höheren (v.a. thermophilen) Temperaturen durch Verschiebung des Dissoziationsgleichgewichts $\mathrm{zu}$ einer übermäßigen Ammoniakbildung (BRAUN, 1982). Eine Abnahme des $\mathrm{pH}-$ Wertes infolge höherer Anteile an organischen Säuren im Biogasprozess durch die Hemmung der Methanbildner durch Ammoniak führt dann wiederum zu einer 
Verringerung des undissoziierten Ammoniaks. Das Dissoziationsgleichgewicht verschiebt sich erneut in Richtung der Ammonium-Ionen und schwächt damit die Hemmung ab. Sie ist also in einem gewissen Maße reversibel. So gehen die Angaben von Hemmkonzentrationen für Ammoniak/Ammonium in der Literatur aufgrund der beschriebenen pH-Wert- und Temperaturabhängigkeit bzw. einer hohen Adaptionsfähigkeit der Mikroorganismen weit auseinander (BAUER ET AL., 2009). Bei einem $\mathrm{pH}$-Wert von 7,0 werden beispielsweise als Richtwerte für Hemmungwirkungen Konzentrationen von Ammoniumstickstoff von > 3,5 g/L $\mathbf{~ N H}_{4}{ }^{+}$(WEILAND, 2001) oder $>\mathbf{2 , 0} \mathbf{~ g / L ~ N H} \mathbf{~ N H}^{+}$(KAISER ET AL., 2008) bzw. bei Ammoniak von $>\mathbf{1 5 0} \mathbf{~ m g / L ~ N H}$ (SCHOLWIN ET AL., 2009) genannt. WEINRICH (2017) gibt nach einer Zusammenstellung von Literaturwerten hemmende Ammoniakkonzentrationen im mesophilen Betrieb in einem Bereich zwischen 20 und $150 \mathrm{mg} / \mathrm{L} \mathrm{NH}_{3}$ an.

Obwohl Schwefel für die Mikroorganismen ein Makronährstoff ist (vgl. Kapitel 2.2.5) kann gelöster, nicht dissoziierter Schwefelwasserstoff $\left(\mathrm{H}_{2} \mathrm{~S}\right)$ analog zur Ammoniakhemmung als weiterer Inhibitor des Biogasbildungsprozesses auftreten. Er wirkt in höheren Konzentrationen v.a. auf die methanogenen Archaeen toxisch, da er leicht in die Zellen diffundieren kann (KOSTER ET AL., 1986). Schwefelwasserstoff entsteht im Wesentlichen durch den Abbau schwefelhaltiger Proteine und die Reduktion von Sulfaten durch Desulfurikation. Die sulfatreduzierenden Bakterien stehen jedoch in Konkurrenz mit den Methanogenen, da beide die gleichen Zwischenprodukte (Wasserstoff und Acetat-Ionen) umsetzen. Die Desulfurizierer sind gegenüber den Methanbildnern bei den Abbauprozessen energetisch begünstigt, sodass die Zwischenprodukte bevorzugt für die Sulfatreduktion genutzt werden und nur der verbleibende Rest für die Methanbildung zur Verfügung steht (KroISS \& SVARDAL, 2005). Eine Hemmung der Methanogenen führt meist zu einer Anreicherung der organischen Säuren im Fermenter und damit zu einem sinkenden $\mathrm{pH}-$ Wert und einem Rückgang der Gasproduktion. Mit fallendem $\mathrm{pH}-$ Wert steigt aber auch der Anteil an undissoziiertem Schwefelwasserstoff gegenüber den gelösten Sulfid- und Hydrogensulfid (HS-)-Ionen aufgrund der Verschiebung des Dissoziationsgleichgewichts, was die Hemmung der Methanproduktion verstärkt (BRAUN, 1982; KROISS \& SVARDAL, 2005). Jedoch ist anzunehmen, dass eine toxische Wirkung nicht zu erwarten ist, da vorher die Sulfatreduktion selbst durch hohe Schwefelwasserstoffgehalte gehemmt wird. Solange aber die Konzentration an gelöstem, nicht dissoziiertem Schwefelwasserstoff keine 
Hemmwirkung hervorruft, kann die Desulfurikation sich auch positiv auf die Abbauprozesse auswirken: Einerseits werden Hydroxid $\left(\mathrm{OH}^{-}\right)$-Ionen freigesetzt, was zu einer pH-Wert-Anhebung führt, andererseits bewirken die Desulfurizierer einen besonders niedrigen Wasserstoffpartialdruck im Fermenter, was die Methanbildung und v.a. den acetogenen Propionsäureabbau begünstigt (KROISS \& SVARDAL, 2005). Im gebildeten Biogas verringern erhöhte Schwefelwasserstoffgehalte einerseits die Gasqualität, andererseits wirkt Schwefelwasserstoff korrosionsfördernd und kann zu Schäden im Blockheizkraftwerk führen (BAUER ET AL., 2009). So muss ein kontinuierliches Durchmischen des Fermenters gewährleistet sein, um erhöhte Schwefelwasserstoffkonzentrationen zu vermeiden und das Ausgasen zu fördern. Durch die Zufuhr von geringen Mengen Luftsauerstoff ist eine einfache Entschwefelung (Oxidation des Schwefelwasserstoffs zu elementarem Schwefel durch diverse Arten von Schwefelbakterien) möglich (vgl. Kapitel 2.2.4). Aber auch durch die Zugabe von Schwermetallsalzen wie Eisen(II)- oder Eisen(III)-chlorid kann durch Eisensulfidbildung der Schwefelwasserstoff gebunden werden (vgl. nächster Absatz). Als grobe Richtwerte für Hemmungwirkungen werden $>\mathbf{3 0} \mathbf{~ m g} / \mathbf{L ~ \mathbf { H } _ { 2 }} \mathbf{S}$ (KroISS \& SvardaL, 2005) bzw.

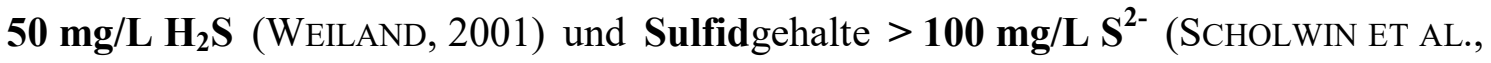
2009) genannt.

In geringeren Konzentrationen wirken einige Schwermetalle stimulierend auf die Aktivität der Mikroorganismen, erfüllen wichtige Funktionen im anaeroben Gärprozess und dienen als essenzielle Mikronährstoffe (vgl. Kapitel 2.2.5 und 2.3). Die Grenzen zwischen Stimulation, beginnenden Hemmwirkungen bis hin zu toxischen Einflüssen sind fließend und stark differierend je nach Schwermetallart und -gehalt sowie den chemischen und physikalischen Milieubedingungen (KroISS \& SVARDAL, 2005). Den Biogasprozess inhibierende Schadelemente können z.B. Arsen, Blei, Cadmium, Chrom, Cobalt, Kupfer, Nickel, Quecksilber und Zink sein. Diese Elemente stören dann den Gärprozess, wenn sie in gelöster Form vorliegen (OLESZKIEWICZ \& SHARMA, 1990; KAISER ET AL., 2008; FrieHE ET AL., 2016). Die Hemmwirkung zeigt sich durch einen Rückgang der Biogasproduktion. Durch die Inaktivierung bzw. Intoxikation der Methanbildner steigen die Gehalte der flüchtigen organischen Säuren im Fermenter an, was zu einem Absinken des pH-Wertes führt. Dies wiederum verstärkt dann zusätzlich die Verfügbarkeit und Mobilität der Schwermetalle (KroISS \& SVARDAL, 2005). 
Durch die Anwesenheit anderer Elemente/Verbindungen und hieraus resultierenden antagonistischen oder synergistischen Effekten kann sich die gegenseitige Wirkung verschiedener Inhibitoren beeinflussen (SCHOLWIN ET AL., 2009). Beispielsweise hängt die Hemmwirkung von Schwermetallen davon ab, welche Anionen sich gleichzeitig im Gesamtprozess befinden, da unter bestimmten Reaktionsbedingungen Schwermetalle in Anwesenheit von z.B. Schwefel (Schwefelwasserstoff) oder Phosphor (Phosphat) und einigen organischen Liganden ausgefällt bzw. in Komplexen gebunden werden (vgl. auch Kapitel 2.3). Jedoch können z.B. andererseits zu hohe Sulfidionenkonzentrationen, abhängig von der Temperatur bzw. dem $\mathrm{pH}-$ Wert, selbst wieder toxisch wirken. So kann auch die Reduktion eines potenziellen Schwermetalls durch Zugabe eines antagonistisch wirkenden Stoffes aufgrund verschiedener Wechselwirkungen problematisch sein. Meist jedoch ist die mikrobielle Aktivität im Fermenter gegenüber Hemmwirkungen von Schwermetallen bis in höhere Konzentrationsbereiche relativ unempfindlich, da in der Regel immer ausreichend Schwefelwasserstoff zur Fällung oder Komplexbildner im Prozess vorhanden sind (BRAun, 1982). Zum Teil sind außerdem auch Anpassungen an ursprünglich hemmende Schwermetallgehalte möglich (SCHOLWIN ET AL., 2009).

Freie Kupfer-Ionen wirken in hoher Konzentration (vgl. Kapitel 5.9) hemmend bzw. toxisch auf die Mikroorganismen. Kupfer- und auch Zink-Verbindungen finden aufgrund ihrer antibakteriellen Wirkung in landwirtschaftlichen Betrieben Anwendung, besonders als Desinfektionsmittel bei der Behandlung von Klauen und Hufen (Klauenbäder) oder zur Wundheilung in der Veterinärmedizin (Zink). So können in Biogasanlagen mit Güllezusatz (v.a. Schweinegülle) erhöhte Kupfer- und Zinkgehalte auftreten (BAUER ET AL., 2009). Auch sind erhöhte Schwermetalleinträge in den Fermenter über die Wirtschaftsdünger durch mit Schwermetallen belastete Futtermittel oder Futterzusatzstoffe in der Viehhaltung, durch alte Leitungsrohre oder durch Geräte- und Materialabrieb möglich.

Aufgrund der hohen Vielfalt an Bakterien und Archaeen im Biogasprozess und deren unterschiedlich stark ausgeprägten Adaptionsfähigkeiten an höhere Schwermetallkonzentrationen sowie der Abhängigkeit von den jeweiligen Prozessparametern ist es schwierig, allgemeingültige Maximalkonzentrationen festzulegen. In der Fachliteratur lassen sich nur wenige Angaben finden. Eine Auflistung von oberen Hemm- und Toxizitätskonzentrationen für ausgewählte Schwermetalle ist in Kapitel 5.9 zu finden. 


\subsection{Bedeutung der Spurenelemente}

Die methanogenen Archaeen benötigen für ihr Wachstum und ihre Aktivität bestimmte Spurenelemente. Nach bisherigem Kenntnisstand spielen Cobalt, Nickel, Molybdän, Eisen, Kupfer, Mangan, Zink, Selen, Vanadium und Wolfram eine wichtige Rolle (SCHÖNHeit ET AL., 1979; Scherer \& SAHM, 1981; FATHePure, 1987; OlesZKIEWICZ \& Sharma, 1990; Jarvis et AL., 1997; GonZAleZ-Gil et AL., 1999; KIDA ET AL., 2001; ZAndvoort et AL., 2006; Lebuhn et AL., 2008; Feng Et AL., 2010; MunK ET AL., 2010; Thauer et AL., 2010; Demirel \& Scherer, 2011; Pobeheim et AL., 2011; LindORFER ET AL., 2012). Ein Mangel an Spurenelementen führt zu einer Limitierung des Wachstums und einer eingeschränkten Aktivität der Mikroorganismen und damit zu geminderten Biogaserträgen mit der Folge von finanziellen Einbußen durch eine suboptimale Biogasstrom- und -wärmegewinnung sowie geringeren Umweltleistungen. Liegen die Ausfuhrraten der Mikroorganismen deutlich über den Wachstumsraten ist ein stabiler Fermenterbetrieb nicht mehr möglich. Wie oben erwähnt, können jedoch auch bei zu hohen Konzentrationen einige Spurenelemente, v.a. Schwermetalle, hemmend oder toxisch auf die Mikroben wirken (KROISS \& SVARDAL, 2005; KAISER ET AL., 2008; PoBeHeIM ET AL., 2010).

Die Funktionen vieler Spurenelemente im Biogasprozess bzw. für die spezifischen Archaeen und Bakterien werden seit über 40 Jahren erforscht und wesentliche Aspekte konnten dazu herausgefunden und das Wissen stark erweitert werden. Dennoch ist bis heute die komplette chemische und mikrobiologische Dynamik der Prozesse im Fermenter noch nicht bis ins Detail bekannt. So können oft nur allgemeingültigere Aussagen über diverse (Teil)Prozesse getroffen werden. Das vielfältige Wechselspiel bei der anaeroben Vergärung ist so immer noch ein attraktiver Forschungsgegenstand, da sich Mangelzustände meist erst in der Methanogenese zeigen. Tabellen mit Zusammenstellungen wichtiger Funktionen einzelner Spurenelementen finden sich z.B. bei OleszKiewicz \& Sharma (1990), TAKASHima et AL. (1990), KayHanian \& Rich (1995), Zandvoort et Al. (2006), Schattauer et AL. (2011), BAnKs \& Heaven (2013) oder ChOONG ET AL. (2016). Aber nur wenige Autoren geben minimale Bedarfswerte für Spurenelementgehalte an, $a b$ denen optimale Biogaserträge im Fermenter möglich sind. Tabelle 1 gibt einen Überblick über zusammengestellte Literaturwerte mit Angabe des Untersuchungsgegenstands bzw. Substrates. 
Tabelle 1: $\quad$ Zusammenstellung minimaler Bedarfswerte (Minimalgehalte, $m g / L_{F M}$ ) wichtiger essenzieller Mikronährstoffe für optimale Biogasprozesse.

\begin{tabular}{|c|c|c|c|c|c|c|c|c|c|}
\hline $\begin{array}{l}\left.\text { [mg/ } / \mathrm{L}_{\mathrm{FM}}\right] \\
\text { Quelle }\end{array}$ & $\begin{array}{r}\text { Cobalt } \\
\text { Co }\end{array}$ & $\begin{array}{r}\text { Nickel } \\
\mathrm{Ni}\end{array}$ & $\begin{array}{r}\text { Molybdän } \\
\text { Mo }\end{array}$ & $\begin{array}{r}\text { Eisen } \\
\mathrm{Fe} \\
\end{array}$ & $\begin{array}{r}\text { Kupfer } \\
\mathrm{Cu} \\
\end{array}$ & $\begin{array}{r}\text { Mangan } \\
\text { Mn }\end{array}$ & $\begin{array}{r}\text { Zink } \\
\text { Zn }\end{array}$ & $\begin{array}{r}\text { Selen } \\
\text { Se }\end{array}$ & $\begin{array}{r}\text { Wolfram } \\
\text { W }\end{array}$ \\
\hline$a$ & 0,06 & 0,25 & 0,16 & 60 & 1,1 & 6,0 & 6,0 & 0,02 & 0,05 \\
\hline$b$ & 0,05 & 0,6 & & & & & & & \\
\hline$c$ & 0,03 & 0,33 & 0,10 & 20 & 2,2 & 2,4 & 4,5 & 0,002 & 0,005 \\
\hline d & 0,07 & 0,33 & 0,18 & & 1,5 & & 6,2 & & \\
\hline e & 0,026 & 0,031 & & & & & & & \\
\hline$f$ & 0,12 & 0,12 & 0,19 & 5,6 & & & & & \\
\hline$g$ & 0,06 & 0,006 & 0,05 & 5,6 & 0,006 & 0,005 & & 0,008 & \\
\hline $\mathrm{h}$ & 0,0006 & 0,006 & 0,001 & 0,28 & & & & & \\
\hline
\end{tabular}

a: Lindorfer, 2014 (Praxisdaten, 600 Nawaro-Fermenter); b: Pobeheim et al., 2011 (Laborversuche, synthetisches Substrat); c: Munk et al., 2010 (Laborversuche, Mais-Silage); d: Sauer, 2010 (Praxisdaten, zwei Praxisbiogasanlagen); e: Kida et al., 2001 (Laborversuche, synthetisches Abwassersubstrat); f: Fathepure, 1987 (Laborversuche, synthetische Medien); g: Scherer \& Sahm, 1981 (Laborversuche, synthetische Medien); h: Schönheit et al., 1979 (Laborversuche, synthetische Medien).

Es wird deutlich, dass die aufgeführten Bedarfswerte einiger Elemente teilweise um mehrere Größenordnungen auseinanderliegen. Einige Autoren ermittelten die Bedarfskonzentrationen zudem auf Basis von synthetischen Medien bzw. Substraten, was im Allgemeinen nicht den realen Verhältnissen in landwirtschaftlichen Biogasanlagen entspricht. Auch die in den Laborversuchen zugrundeliegenden Prozessparameter sind oft kaum anwendbar auf Praxisfermenter. Die Bedarfswerte (Minimalgehalte) sind jedoch von großer Bedeutung: Sie sollten bei Additivzusätzen unbedingt eingehalten und nicht überschritten werden (Minimierungsgebot), um mögliche Hemmwirkungen zu vermeiden bzw. durch das aufgebrachte Gärsubstrat die landwirtschaftlichen Flächen möglichst wenig zu belasten und Kosten einzusparen.

Entscheidend für optimale Konzentrationen von Spurenelementen im Biogasprozess sind jedoch nicht allein die Gesamtgehalte, sondern auch deren Bioverfügbarkeit im Fermenter für die Bakterien und Archaeen (ZANDVOORT ET AL., 2006; AQUINO \& Stuckey, 2007; Lebuhn et Al., 2008; BAuer et Al., 2009; Demirel \& Scherer, 2011; BAnKs \& HEAVEn, 2013; FeHER ET AL., 2013; GuStaVSSON ET AL., 2013; Thanh et Al., 2016; VAn Hullebusch et Al., 2016). In Abbildung 3 ist eine modifizierte, vereinfachte Übersicht der Versorgung und Verfügbarkeit von Spurenelementen im Biogasprozess dargestellt. 


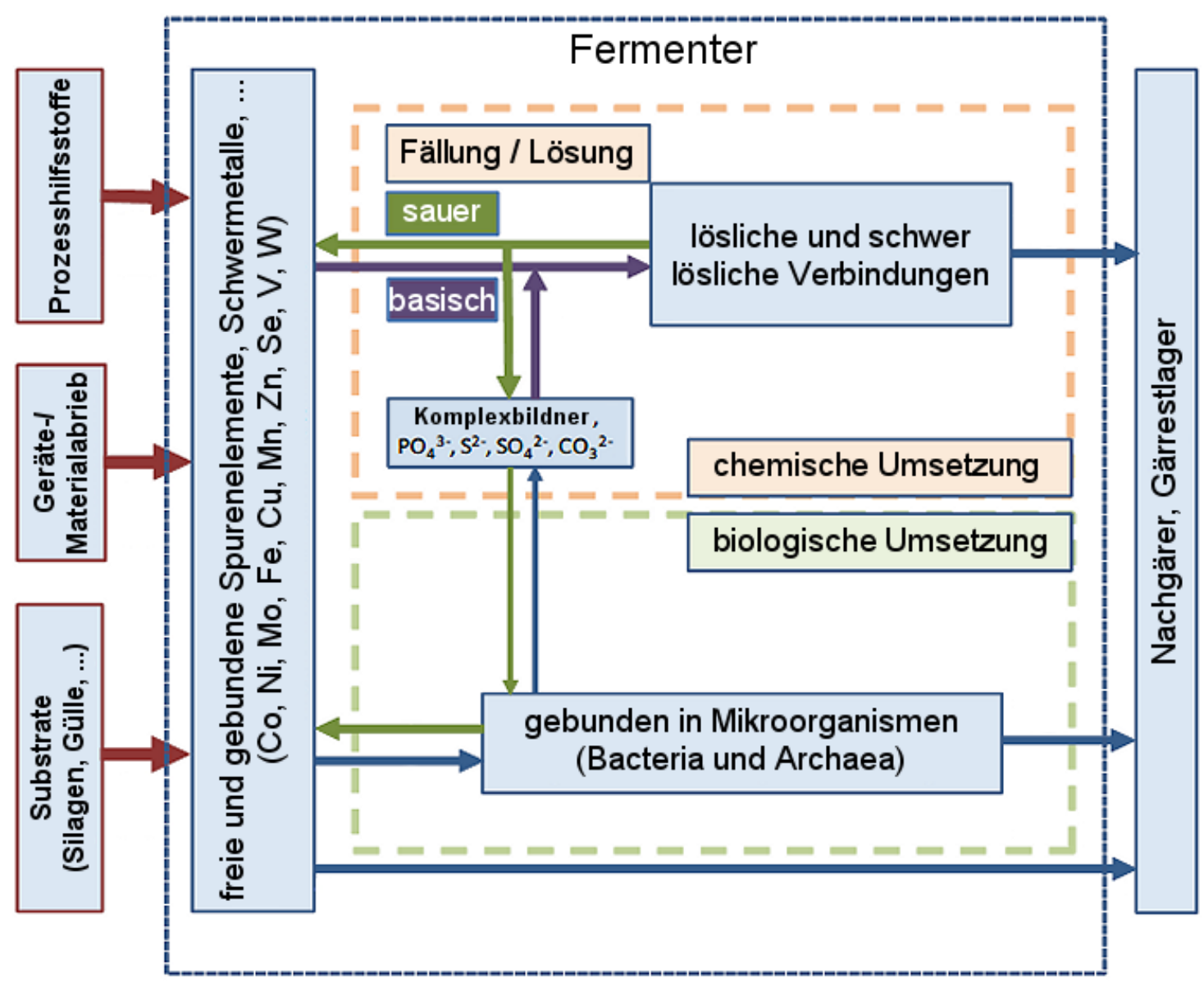

Abbildung 3: Vereinfachte schematische Darstellung der Versorgung und Verfügbarkeit von Spurenelementen im Fermenter (modifiziert nach BAUER ET AL., 2009).

Die Spurenelemente (und Schadelemente) gelangen durch die Inputsubstrate, durch Hilfsstoffe wie insbesondere Prozessadditive, aber auch über Geräte- und Materialabrieb und anhaftendes (Boden)Material an den Pflanzensilagen in unterschiedlich biologisch verfügbarer Form in den Fermenter. Sie können jedoch durch eine Vielzahl von biochemischen Umsetzungsprozessen aus ihren Bindungsformen gelöst und mikrobiell verfügbar werden. Mit Sulfiden, Carbonaten und Phosphaten im Fermenter können sie zwar unlösliche Präzipitate bilden (BANKS \& HEAVEN, 2013), aber v.a. durch Komplexierung mit organischen Liganden auch löslich gehalten werden.

Bei den anaeroben Abbauprozessen spielen die Spurenelemente v.a. in enzymatischen Komplexen eine wesentliche Rolle, in denen sie beispielsweise als Zentralionen für katalytische Funktionen genutzt werden. Die beteiligten Mikroorganismen haben hierbei spezifische Bedarfe an den verschiedenen Spurenelementen (WINTSCHE ET AL., 2016). 
Das Verhalten der Spurenelemente ist abhängig von den jeweiligen Umgebungsbedingungen wie z.B. dem pH-Wert, der Art und dem Angebot an organischen (und anorganischen) Liganden und Chelatbildnern oder dem Redoxpotential (CALLANDER \& BARFORD, 1983). Es entsteht eine Art pseudochemisches Gleichgewicht, durch das die einzelnen Spurenelementfraktionen definiert sind - auch die bioverfügbare Fraktion (OleszKiewicz \& Sharma, 1990; VAn Hullebusch et Al., 2016). Abbildung 4 zeigt ein weiteres stark vereinfachtes Schema über die wesentlichen Prozesse, die die Bioverfügbarkeit von Spurenelementen in anaeroben Prozessen bestimmen.

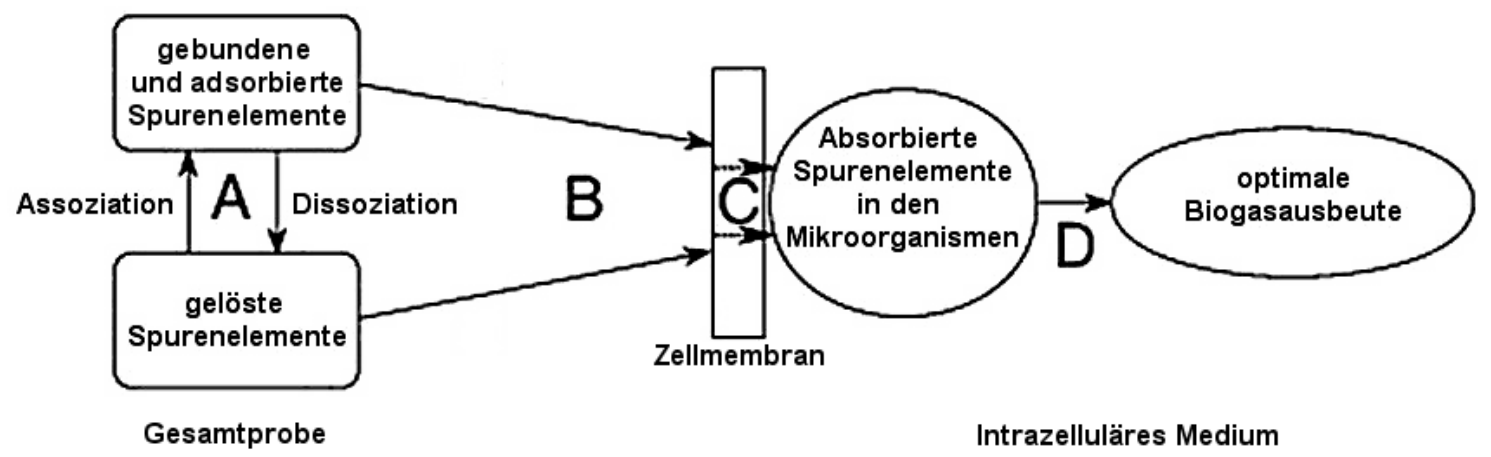

Abbildung 4: $\quad$ Vereinfachtes Schema wesentlicher Prozesse, die die Bioverfügbarkeit von Spurenelementen im Fermenter beeinflussen (modifiziert nach NRC, 2003 und VAN HULLEBUSCH ET AL., 2016).

A: Interaktionen (Assoziation und Dissoziation); B: Transfer zu den Mikroorganismen; $C$ : Aufnahme durch die Zellmembran; D: positive biologische Folgereaktion.

Nach VAN Hullebusch ET AL. (2016) können die bioverfügbaren Spurenelemente entsprechend ihren Aufnahmemechanismen in zwei Kategorien eingeteilt werden: (1) diejenigen mit aktiver Aufnahme, d.h. die für die Internalisierungsprozesse eine direkte metabolische Aktivität der Mikroorganismen erfordern, um die Spurenelemente durch die Zellmembran zu übertragen; (2) diejenigen mit passiver Aufnahme, d.h. deren Aufnahme durch die Zellmembran auf Basis von Konzentrationsgradienten geschieht. Zusätzlich zu diesen Prozessen wird die Bioverfügbarkeit durch die Spurenelementverteilung zwischen den flüssigen und festen Phasen und der Diffussion durch die mikrobiellen Membranoberflächen beeinflusst. Einmal internalisiert können die intrazellulär wirksamen Spurenelemente z.B. zu deutlich gesteigerten Biogasausbeuten führen. Die bioverfügbare Fraktion, hier definiert als die für die Interaktionen mit der mikrobiellen Gemeinschaft nutzbaren Spurenelementgehalte, ist jedoch generell sehr schwierig zu bestimmen bzw. zu quantifizieren (VAN HuLLEBUSCH ET AL., 2016). 
Die essenziellen Spurenelemente sind maßgeblich am Aufbau der Enzyme, Coenzyme und Cofaktoren beteiligt, die im methanogenen Stoffwechsel eine zentrale Rolle spielen (BANKS \& HEAVEN, 2013; BAUER ET AL., 2009). Sie können in gelöster Form in bestimmten Konzentrationsbereichen auf das Wachstum der methanogenen Archaeen stimulierend wirken bzw. die Reproduktion fördern (OLESZKIEWICZ \& SHARMA, 1990). Enzyme, Coenzyme und Cofaktoren dienen im methanogenen Stoffwechsel u.a. als wichtige Biokatalysatoren und Elektronenakzeptoren. Um die Funktionsfähigkeit der Enzyme und Coenzyme bzw. optimale Stoffwechselprozesse zu gewährleisten, ist eine hinreichende Versorgung mit Spurenelementen über die Substratzufuhr erforderlich.

Neben den hinreichenden Spurenelementkonzentrationen in den Inputsubstraten und/oder Prozesshilfsstoffen hängt die Enzymaktivität aber auch z.B. von der Gärtemperatur, dem pH-Wert, der Raumbelastung, der Verweilzeit und Durchmischung sowie dem Vorhandensein von potenziell hemmenden Inhibitoren ab. So nimmt mit ansteigender Temperatur zunächst die Reaktionsgeschwindigkeit zu (vgl. Kapitel 2.2.2). Bei zu hohen Temperaturänderungen können jedoch Strukturveränderungen in den Enzymen und Coenzymen auftreten und diese unwirksam werden. Gleiches gilt für schnelle oder größere pH-Wert-Veränderungen außerhalb der spezifischen Toleranzbereiche (Koch ET AL., 2011; KROISS \& SvARDAL, 2005; vgl. Kapitel 2.2.3).

Sehr umfassende, detaillierte Informationen für biochemische und molekularbiologische Daten über einzelne Enzyme, katalytische Reaktionen und Stoffwechselwege sind auf der frei zugänglichen Online-Enzym-Datenbank BRENDA (BRaunschweig ENzyme DAtabase) der Technischen Universität Braunschweig unter www.brenda-enzymes.org zusammengestellt. Im Folgenden wird ein kurzer Überblick über wichtige Funktionen der essenziellen Spurenelemente im Biogasprozess gegeben.

Cobalt fungiert v.a. in Corrinoiden und Vitamin B12-Enzymen und -Coenzymen als essenzielles Spurenelement und Zentralatom. Sowohl bei den methanogenen Archaeen als auch acetogenen Bakterien treten cobalthaltige Enzyme und Coenzyme auf. Bei den Methanbildnern sind diese Enzyme und Coenzyme an der Methylübertragung als Cofaktor von Methyltransferasen beteiligt, bei den acetogenen Bakterien beeinflussen sie die Bildung des Acetyl-Coenzyms A (LEMMER ET AL., 2011). 
Der relativ hohe Bedarf der Methanbildner an Nickel stellt eine Besonderheit dar, der für das Wachstum anderer Bakterien generell nicht erforderlich ist. Nickel ist essenziell für alle Methanogenen, da es für die Synthese der Zellkomponente Cofaktor $\mathrm{F}_{430}$ als Zentralatom und der Enzyme Hydrogenase und Kohlenstoffmonoxid-Dehydrogenase einen erforderlichen Baustein darstellt (KUNST, 2005). Der Cofaktor $\mathrm{F}_{430}$ ist wichtiger Bestandteil des Enzyms Methyl-Coenzym M-Reduktase. Dieses katalysiert den letzten Schritt bei der acetoklastischen und hydrogenotrophen Methanogenese, die Bildung von Methan (BAuer ET AL., 2009; SChATTAUER ET AL., 2011).

Molybdän und Wolfram werden für die Bildung verschiedener Enzyme gebraucht und spielen katalytisch z.B. bei den Reduktionsreaktionen von Kohlenstoffdioxid zu Methan eine Rolle (KLetzin \&AdAMS, 1996). Beide Elemente weisen ähnliche chemische Eigenschaften auf und übernehmen während der anaeroben Abbauprozesse auch vergleichbare Aufgaben. In Untersuchungen wurde nachgewiesen, dass Molybdän in einigen Fällen durch Wolfram ersetzt werden kann. Es ist damit der einzig bekannte Fall, bei dem in einem Enzym ohne Verlust der enzymspezifischen Wirkung ein Zentralatom durch ein anderes ausgetauscht werden konnte (LEMMER ET AL., 2011). Molybdän ist v.a. an der Bildung der Enzyme Formiat-Dehydrogenase (FDH) und Formylmethanofurandehydrogenase (FMDH) beteiligt (LEMMER ET AL., 2011) und bildet mit Molybdopterin einen wichtigen Cofaktor der Methanogenese (BAUER ET AL., 2009).

Das Element Eisen zählt zu den wichtigsten Mikronährstoffen. Es erfüllt wichtige Funktionen im Biogasprozess. Eisen ist in vielen Enzymen enthalten, beispielsweise in Ferrotoxin oder als Bestandteil in Eisen-Nickel-Schwefel- oder Eisen-Clustern, die als Cofaktoren von Hydrogenasen oder Kohlenstoffmonoxid-Dehydrogenasen auftreten (LEMMER ET AL., 2011). Auch das extrem hohe Reduktionsvermögen ist von Bedeutung. Eisen kann durch Sulfidfällung mit Schwefel im Fermenter die Sulfidtoxizität herabsetzen, aber auch die Ausscheidung von extrazellulären Polymeren fördern, die eine chelatisierende Wirkung haben können (BANKS \& HEAVEN, 2013).

Zink und Kupfer werden v.a. bei der Bildung des Enzyms Hydrogenase verwendet; Zink zudem beim Enzym Formiat-Dehydrogenase (KAYHANIAN \& RiCH, 1995). Bei der Wasserstoffaufnahme spielt Zink neben Nickel und Selen, bei der Kohlenstoffdioxidaufnahme neben Eisen, Molybdän, Wolfram und Selen eine wichtige Rolle. Bei der Acetatspaltung und der Methylübertragung ist Zink unverzichtbar und wird zusätzlich 
für die Synthese des zellulären Energieträgers ATP gebraucht (BAUER ET AL., 2009). Vanadium wirkt ebenfalls als Cofaktor für Nitrogenasen (LEE ET AL., 2010; REHDER, 2000).

Mangan ist Bestandteil verschiedener Enzyme und weist im Biogasprozess ähnliche chemische Eigenschaften und Funktionen wie Eisen auf. Es kann Methyltransferasen in den Methanbildnern aktivieren bzw. stabilisieren, ist bei Kinasen-Reaktionen häufig mit Magnesium austauschbar. Außerdem ist Mangan wichtiger Cofaktor verschiedener Enzyme (Banks \& Heaven, 2013; Oleszkiewicz \& Sharma, 1990; Schattauer ET AL., 2011).

Selen ist ebenfalls ein Baustein für viele Enzyme, die bei neutralem pH-Wert eine hohe Aktivität zeigen, ein geringes Redoxpotential besitzen und bei der Umsetzung der Fettsäuren wirken (KAYHANIAN \& RICH, 1995). Zum Beispiel ist Selen, wie zuvor erwähnt, neben Eisen, Molybdän und Wolfram an der Bildung des Enzyms FormiatDehydrogenase (FDH) beteiligt, das den Abbau von Formiat zu Kohlenstoffdioxid katalysiert. Selen spielt zusätzlich im Stoffwechsel von sulfatreduzierenden Bakterien eine wichtige Rolle und ist außerdem v.a. für den Aufbau von Proteinen wie beispielsweise Selenocystein oder Selenomethionin von Bedeutung. Selenhaltige Proteine werden von einigen Methanogenen zur Oxidation von Wasserstoff benötigt (LEMMER ET AL., 2011). 


\section{Kapitel 3: Allgemeine Methodik}

\subsection{Proben- und Analysenübersicht}

Für die vorliegende Arbeit wurden im Rahmen des Forschungsvorhabens insgesamt 1066 Proben genommen und aufbereitet. Anschließend wurden sie mittels der Multielementanalysemethoden ICP-OES und ICP-MS untersucht. Tabelle 2 gibt einen Überblick aller Proben und Messungen, unterteilt nach verschiedenen Kategorien. Die Kategorie „Reaktormaterialien“ weist die Proben und Messungen der Versuchsbiogasreaktoren am DBFZ sowie der Praxisbiogasanlage in Verliehausen (Fermenter-, Nachgärer- und Gärrestmaterialien) auf, in der Kategorie „Inputsubstrate“ ist die Anzahl der Proben und Messungen diverser Pflanzensilagen (Mais-, Winterackerbohne-, Amarant-, Deutsches Weidelgras-, Durchwachsene Silphie-, Grünroggen- und GrasSilagen) sowie weiterer Substrate (Mist, Gülle, Hühnertrockenkot) aufgeführt. Die Proben und Messungen für die „Aktivitätstests (ORGA)“ zur Ermittlung von Hemmund Toxizitätskonzentrationen verschiedener Spurenelemente (vgl. Kapitel 5) sowie für die Tests zur Erarbeitung eines vereinfachten Bestimmungsverfahrens (vgl. Kapitel 6) stellen die weiteren Kategorien dar. Die für die Datenauswertung wichtigen Referenzmaterialien und Blindproben bilden die letzte Kategorie der „Begleitproben“. Alle Elementdaten wurden im Rahmen des Vorhabens in eine Datenbank aufgenommen.

Tabelle 2: $\quad$ Übersicht der erhobenen Proben, mit Anzahl der Multielementmessungen mittels ICP-OES bzw. ICP-MS.

\begin{tabular}{|c|c|c|c|c|}
\hline Kategorie & Proben & $\begin{array}{r}\text { Messungen }^{*} \\
\text { ICP-OES } \\
\end{array}$ & $\begin{array}{r}\text { Messungen* }^{*} \\
\text { ICP-MS }\end{array}$ & $\begin{array}{r}\text { Messungen } \\
\text { gesamt }\end{array}$ \\
\hline \multicolumn{5}{|l|}{ Reaktormaterialien } \\
\hline Laborfermenter DBFZ & 614 & 636 & 549 & 1185 \\
\hline Praxisbiogasanlage Verliehausen & 49 & 52 & 42 & 94 \\
\hline \multicolumn{5}{|l|}{ Inputsubstrate } \\
\hline Diverse Pflanzensilagen & 36 & 36 & 33 & 69 \\
\hline Sonstige Substrate & 14 & 16 & 14 & 30 \\
\hline Proben für Aktivitätstests (ORGA) & 35 & 37 & 27 & 64 \\
\hline Proben für Bestimmungsverfahren & 133 & 133 & 12 & 145 \\
\hline Summe & 881 & 910 & 677 & 1587 \\
\hline \multicolumn{5}{|l|}{ Begleitproben } \\
\hline Referenzmaterialien & 93 & 162 & 85 & 247 \\
\hline Blindproben & 91 & 148 & 84 & 232 \\
\hline Summe & 184 & 310 & 169 & 479 \\
\hline Gesamt & 1065 & 1220 & 846 & 2066 \\
\hline
\end{tabular}

*: Inklusive Mehrfachmessungen 


\subsection{Probenahme und Probenaufbereitung}

Mit dem Start des Forschungsvorhabens begann die Aufnahme verschiedener Betriebsparameter und die Probenahme in der Praxisbiogasanlage in Verliehausen. Für die Aktivitätstests und Multielementanalysen wurden weitestgehend ein- bis zweimonatlich Proben des Fermenters bzw. ggf. Nachgärers in 1-Liter-Weithals-Kunststoff-Flaschen entnommen, vor Ort tiefgefroren, regelmäßig abgeholt und später in den Laboren am GZG aufbereitet. Vom Biogasanlagenbetreiber wurden für die vergleichenden Analysen und Auswertungen die von ihm bzw. vom beauftragten Analyselabor ermittelten Parameter wie der $\mathrm{pH}-\mathrm{Wert}(\mathrm{pH})$, die flüchtigen organischen Säuren(FOS), die alkalische Pufferkapazität (TAC $=$ Totales anorganisches Carbonat), der Quotient FOS/TAC und verschiedene Säurespektren zur Verfügung gestellt. Für die Multielementanalysen wurden außerdem Gärrest-Proben von der Gärrestaufbringung auf die beiden Freilandversuchsfelder am Versuchsgut Reinshof der Universität Göttingen (Schlag Garte Nord) und in Schoningen bei Verliehausen (Schlag Sömmerling) genommen, ebenfalls tiefgefroren und später aufbereitet. Probenmaterial der Versuchsbiogasreaktoren in Leipzig und von eingesetzten Silagen wurde am DBFZ ab Mai 2015 nach festgelegten Probenplänen gesammelt, tiefgefroren, in regelmäßigen Abständen abgeholt oder verschickt und dann in den Laboren am GZG ebenfalls bearbeitet. Im Anhang (1) bis (3) sind in Übersichten die Versuchs- und Probenahmetage der drei Projektphasen der (quasi)kontinuierlichen Laborfermenterversuche inklusive des Zusatzversuchs aufgeführt.

Nach dem Auftauen der genommenen Proben erfolgte im Trockenschrank (Memmert UF 1060) die Trocknung repräsentativer Probenaliquote in 1-Liter-Messbechern bei $105{ }^{\circ} \mathrm{C}$ bis zur Gewichtskonstanz. Der Trockensubstanzgehalt wurde in Anlehnung an DIN EN 12880:2001 gravimetrisch bestimmt (vgl. Kapitel 3.4.1).

Die nächsten Schritte der Aufbereitung waren die Vorzerkleinerung und Feinmahlung der getrockneten Probenaliquote, wodurch die Proben stärker homogenisiert werden sowie leichter und zügiger mit den eingesetzten Säuren aufschließbar sind. Hierfür wurden die Proben mit einer Planeten-Kugelmühle (Fritsch pulverisette Typ 05.102) analysenfein gemahlen. Die gewählte Mahldauer für die Fermenterproben betrug, abhängig vom Feinheitsgrad des Materials und der Füllmenge in den Mahlbechern, etwa 5 bis 10 Minuten, für die Silageproben ca. 15 bis 25 Minuten. Die gemahlenen Probenpulver wurden in Schnappdeckelgläser zur Aufbewahrung abgefüllt. 


\subsection{Säuretotalaufschlussverfahren}

Alle Proben (mit Ausnahme der Filtrationsproben in Kapitel 6) wurden in einem mehrstufigen Säuretotalaufschlussverfahren nach RUPPERT (2007) und POSPIECH ET AL. (2017a) unter Druck und Einsatz eines Gemisches aus 65 \%iger Salpetersäure $\left(\mathrm{HNO}_{3}\right)$, 40 \%iger Flusssäure (HF) und 70 \%iger Perchlorsäure $\left(\mathrm{HClO}_{4}\right)$ mit dem Druckaufschluss-System (PicoTrace DAS 30) vollständig in Lösung gebracht (Abbildung 5). Zusätzlich wurde bei den Gärgemischproben in der Auflösephase 30 \%ige Salzsäure ( $\mathrm{HCl}$ zugegeben. Je Aufschlussserie, die 32 Polytetrafluorethylen (PTFE)-Becher umfasste, wurden die Probenpulver zusammen mit mindestens zwei Blind- sowie mindestens zwei internationalen oder hausinternen Pflanzen- und Gesteinsreferenzproben vollständig gelöst (vgl. Kapitel 3.5.2). Insgesamt wurden 34 Säuretotalaufschlussserien für alle Feststoffproben sowie drei Säureaufschlussserien mit 65 \%iger Salpetersäure $\left(\mathrm{HNO}_{3}\right)$ für die Filtrationsproben (vgl. Kapitel 6) durchgeführt.

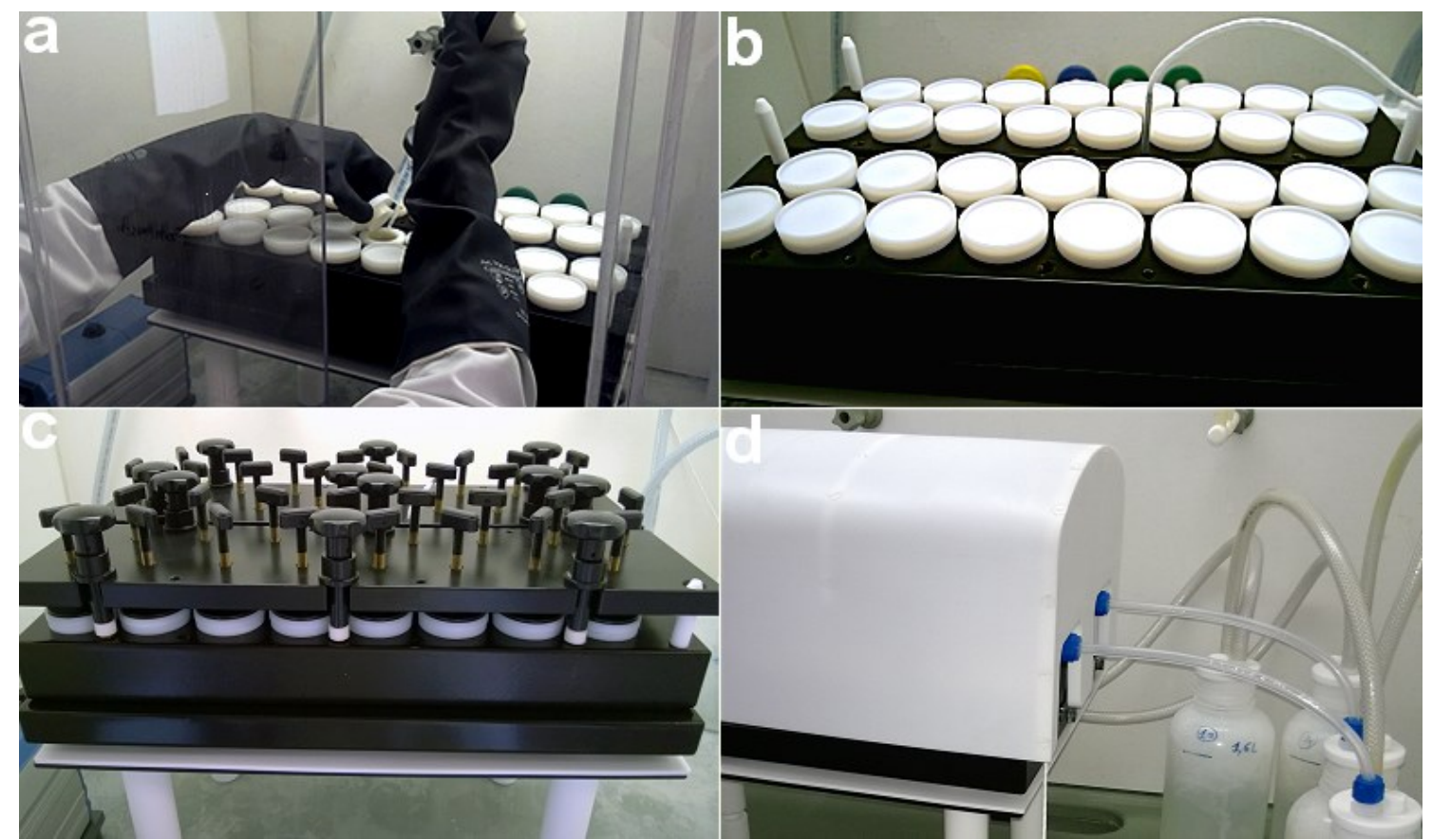

Abbildung 5: $\quad$ Fotoaufnahmen eines mehrstufigen Säuretotalaufschlussverfahrens mit dem Druckaufschluss-System DAS 30 der Firma PicoTrace. a) Säurezugabe; b) Vorreaktionsphase; c) Erste Druckphase bzw. Auflösephase (zweite Druckphase); d) Abrauchphase.

Einwaage: Die Einwaage der feingemahlenen Pulver der Silage- und Pflanzenreferenzproben in die Aufschlussbecher lag bei circa $700 \mathrm{mg}$. Die Einwaage der Gärgemischproben betrug ca. $400 \mathrm{mg}$, die Einwaage der Gesteinsreferenzproben ca. $100 \mathrm{mg}$, jeweils auf $0,1 \mathrm{mg}$ genau bestimmt. 
Vorreaktionsphase: Zum Oxidieren der organischen Substanz wurden in der Vorreaktionsphase bei Raumtemperatur je $4 \mathrm{~mL} \mathrm{HNO}_{3}$ in die Aufschlussbecher zu den eingewogenen Probenpulvern gegeben und die Becherdeckel aufgelegt. Es schloss sich folgendes Heizprogramm an: $10 \mathrm{~h}$ hochheizen auf $30{ }^{\circ} \mathrm{C}$ und $12 \mathrm{~h}$ halten dieser Temperatur; hochheizen in $10 \mathrm{~h}$ von $30^{\circ} \mathrm{C}$ auf $50{ }^{\circ} \mathrm{C}$ und $12 \mathrm{~h}$ halten dieser Temperatur; dann auf Raumtemperatur abkühlen lassen. Danach wurden bei Raumtemperatur $2 \mathrm{~mL} \mathrm{HF}$ sowie $3 \mathrm{~mL} \mathrm{HClO}_{4}$ in die Becher gegeben, die Becherdeckel aufgelegt und folgendes Programm als Vorbereitung der Druckphase gestartet: $2 \mathrm{~h}$ hochheizen auf $100{ }^{\circ} \mathrm{C}$ und $5 \mathrm{~h}$ halten dieser Temperatur.

Erste Druckphase: Hiernach wurden für die erste Druckphase die Druckscheiben auf die Becherdeckel gelegt, die Druckplatten montiert, fest verschraubt und folgendes Programm ausgeführt: Ausgehend von $100{ }^{\circ} \mathrm{C}$ in $3 \mathrm{~h}$ hochheizen auf $150{ }^{\circ} \mathrm{C}$ und $15 \mathrm{~h}$ halten dieser Temperatur; dann auf Raumtemperatur abkühlen lassen. Danach wurden die Druckplatten wieder abgeschraubt und die Becherdeckel abgenommen.

Abrauchphase: Die Abrauch- und Andruckplatten wurden für die folgende Phase aufgeschraubt, der Abrauchtunnel aufgesetzt, die Neutralisationsflaschen mit $4 \%$ iger Natronlauge $(\mathrm{NaOH})$ angeschlossen und die Membranpumpe zum Absaugen der Säuredämpfe eingeschaltet. Folgendes Programm wurde gestartet: 4 h hochheizen auf $100{ }^{\circ} \mathrm{C}$; $8 \mathrm{~h}$ hochheizen auf $180^{\circ} \mathrm{C}$ und $10 \mathrm{~h}$ halten bei dieser Temperatur; auf Raumtemperatur abkühlen lassen.

Auflösephase: Für eine zweite Druckphase wurde zum Auflösen des Abrauchrückstandes der Abrauchtunnel abgenommen sowie die Abrauch- und Andruckplatten abgeschraubt. In die Aufschlussbecher wurden je $1 \mathrm{~mL} \mathrm{HNO}_{3}, 0,25 \mathrm{~mL} \mathrm{HCl}$ (nur bei den Gärgemischproben), $10 \mathrm{~mL}$ Reinstwasser (Milli-Q) sowie $100 \mu \mathrm{L}$ eines internen Standards mit 50 ppm Beryllium (Be), 5 ppm Indium (In), 5 ppm Rhenium (Re) und 10 ppm Rhodium (Rh) für die ICP-MS-Messungen zugegeben. Nach Auflage der Becherdeckel und der Druckscheiben wurden wieder die Druckplatten montiert und folgendes Programm ausgeführt: $2 \mathrm{~h}$ hochheizen auf $150{ }^{\circ} \mathrm{C}$ und $2 \mathrm{~h}$ halten bei dieser Temperatur; dann auf Raumtemperatur abkühlen lassen.

Überführen: Die nunmehr klaren Lösungen wurden abschließend aus den Aufschlussbechern quantitativ jeweils in einen $50 \mathrm{~mL}$-Messkolben überführt, dieser bis zur Ringmarke mit Milli-Q-Wasser aufgefüllt, gut homogenisiert und dann ein Polyethylen (PE)- 
Fläschchen für die Aufbewahrung der Aufschlusslösung mit wenigen mL Probenlösung aus dem Kolben konditioniert. Hiernach erfolgte jeweils das endgültige Überführen der Probenlösung in das PE-Fläschchen für die Multielementanalysen mittels ICP-Massenspektrometrie bzw. ICP-Optische Spektrometrie (vgl. Kapitel 3.4.3 und 3.4.4).

\subsection{Analytik}

\subsubsection{Trockensubstanzgehalte der Gärgemisch- und Silageproben}

Die prozentualen Trockensubstanzgehalte (TS; manchmal auch unzureichend Trockenmasse genannt) der Gärgemisch- und Silage-Proben wurden in Anlehnung an DIN EN 12880:2001 als Quotient der bei $105^{\circ} \mathrm{C}$ getrockneten Probenmasse $m_{\text {trocken }}$ zur jeweiligen frischen Probenmasse $m_{\text {feucht }}$ multipliziert mit 100 bestimmt:

$$
T S=\frac{m_{\text {trocken }}}{m_{\text {feucht }}} \cdot 100 \quad[\text { Gew. }-\%]
$$

Die Ergebnisse sind in den Elementdatentabellen im Anhang (4) bis (15) sowie der Daten-Datei aufgeführt.

\subsubsection{Organische Trockensubstanzgehalte der Gärgemisch- und Silageproben}

Als weiterer Betriebsparameter wurde für die getrockneten, feingemahlenen Pulver der Gärgemisch- und Silage-Proben gravimetrisch die organische Trockensubstanz (oTS) in Anlehnung an DIN EN 12879:2001 ermittelt. Hierfür wurden in Doppelwiederholung jeweils ca. $500 \mathrm{mg}$ des Pulvers auf 0,1 mg genau in vorher ausgeglühte Keramiktiegel eingewogen $\left(m_{\text {Einwaage }}\right)$, die Tiegel direkt nach der Einwaage in den Exsikkator gegeben und dann im Muffelofen (Nabertherm N20/HR) in zweistufigen Rampen (1. Rampe: hochheizen auf $220^{\circ} \mathrm{C}$, 2. Rampe: hochheizen auf $550{ }^{\circ} \mathrm{C}$ ) 2 Stunden bei $550{ }^{\circ} \mathrm{C}$ verascht. Nach Abkühlung auf $80^{\circ} \mathrm{C}$ wurden die Tiegel mit den veraschten Proben in den Exsikkator zurückgestellt, um auf Raumtemperatur abzukühlen und ausgewogen zu werden $\left(m_{\text {Auswaage }}\right)$. Der beim Glühen erlittene Massenverlust multipliziert mit 100 entspricht im Wesentlichen dem prozentualen organischen Trockensubstanzgehalt:

$$
\text { oTS }=\frac{m_{\text {Einwaage }}-m_{\text {Auswaage }}}{m_{\text {Einwaage }}} \cdot 100 \quad[\text { Gew. }-\%]
$$

Die Ergebnisse sind in den Elementdatentabellen im Anhang (4) bis (15) sowie der Daten-Datei aufgeführt. 


\subsubsection{Multielementanalysen ICP-OES}

Für die Multielementanalysen mittels ICP-OES (Inductively Coupled Plasma - Optical Emission Spectrometry) fand ein optisches Emissionsspektrometer (Agilent $5100 \mathrm{VDV}$ ) mit einem induktiv gekoppelten Argon-Plasma als Quelle Anwendung. Das ArgonPlasma in der Fackel erzeugt bei extrem hoher Temperatur (5000 bis $10000 \mathrm{~K})$ ausreichend Energie zur Anregung nahezu aller Haupt- und Spurenelemente und ermöglicht in Kombination von hochauflösenden Gittern und Prismen auf Basis der elementspezifisch emittierten Atom- oder Ionenstrahlung Multielementanalysen mit hoher Präzision und Richtigkeit. Für die Berechnung der Elementkonzentrationen werden die Emissionsintensitäten der elementspezifischen Spektrallinien der angeregten Probe gemessen und mit Intensitäten von Multielementkalibrationslösungen verglichen. Ausführliche Beschreibungen der Messmethode und des Messprinzips sind z.B. bei HEINRICHS \& HERRMANN (1990) und SKOOG ET AL. (2017) aufgeführt.

Die Multielementkalibrationslösungen, die in unserer Arbeitsgruppe Umweltgeochemie für verschiedene Probensets (Boden-/Gesteins- bzw. Pflanzen-/organische Proben) erstellt wurden, umfassten insgesamt 32 Elemente (POSPIECH ET AL., 2017a). Für die Bestimmung der Gärgemisch- und Silage-Proben wurde das Kalibrationslösungsset „Pflanzen-/organische Proben“ gewählt, das bis Sommer 2016 aus 22 Elementen, ab Herbst 2016 dann aus 32 Elemente bestand, von denen insgesamt 26 Elemente sehr gut messbar waren (vgl. Kapitel 3.5.2).

Für die spätere Korrektur der Gerätedrift bzw. für die Blankwertkontrolle wurde in jeder Messserie jeweils nach etwa 12 bis 15 Analysen wiederholt eine Kalibrationslösung (mittlere Kalibrationsstufe, 25 \%-Lösung) und eine Blanklösung gemessen. Hintergrund ist, dass die Gerätedrift bei den Datenauswertungen für die Qualitätssicherung der Messungen einbezogen werden muss. Mögliche Verunreinigungen durch die Reagenzien bei den Multielementanalysen (Spüllösung und Matrix der Kalibrationslösungen) werden durch die mitgemessenen Blanklösungen berücksichtigt; mögliche Verunreinigungen durch die eingesetzten Reagenzien (Säuren) bei den Säuretotalaufschlussverfahren und bei der Handhabung (Laborkontaminationen) durch die Aufschluss-Blindproben beschrieben. Über die dreifache Standardabweichung der mitgemessenen Aufschluss-Blindproben pro Messserie wurden die jeweiligen Nachweisgrenzen der chemischen Elemente berechnet. 
Die Genauigkeit und die Richtigkeit der Analysen wurde mit Hilfe der relativen Abweichungen der Messwerte zum jeweiligen Bezugswert der in Kapitel 3.5.2 aufgeführten Referenzmaterialien überprüft. Die Elementgesamtgehalte der Gärgemisch- und Silageproben sind nach Driftkorrektur, Blindwertabzug, Umrechnung auf die Festphase und unter Berücksichtigung der Nachweisgrenze in den Elementdatentabellen im Anhang (4) bis (15) sowie der Daten-Datei zusammengestellt.

\subsubsection{Multielementanalysen ICP-MS}

Die Multielementanalysen mittels ICP-MS (Inductively Coupled Plasma - Mass Spectrometry) wurden an zwei Standard-Massenspektrometern durchgeführt (Perkin Elmer Elan DRC II bzw. ab Sommer 2016 Thermo Fisher Scientific Thermo Scientific $i C A P Q)$. Hierbei ermöglichen ein hoch effizient ionisierendes Argon-Plasma, ein schnell scannender Quadrupol-Massenanalysator und der einen weiten dynamischen Intensitätsbereich überdeckende Detektor Analysen der Ionenhäufigkeit sehr vieler Elemente im Plasma innerhalb weniger Minuten bis in den Ultraspuren-(ppq)-Elementbereich (SimON, 2009; SkoOg ET AL., 2017). Analog zu den Multielementanalysen mittels ICP-OES wird das mit einem Zerstäuber erzeugte Aerosol der Probenlösung nach Durchgang durch eine Zerstäuberkammer in den zentralen Argonstrom einer Fackel geführt, in der die Atome durch induktiv gekoppelte, hochfrequente Energie im extrem heißen Argon-Plasma ionisiert werden, mit Ausnahme einiger weniger Elemente mit sehr hoher erster Ionisierungssenergie. Nach Eintritt der Ionen in das Massenspektrometer und ihrer Fokussierung auf das Quadrupolfeld werden die Ionen dort nach ihrem Masse/Ladungs-Verhältnis getrennt und im Detektor nachgewiesen. Interferenzen durch Molekülionen (z.B. $\mathrm{Ar}^{40} \mathrm{Cl}^{35}$ auf $\mathrm{As}^{75}$ ) konnten ab Sommer 2016 mit dem iCAP Q-Gerät im Helium (He)-Modus mittels der KED-Methode (Kinetic Energy Discrimination) weitgehend beseitigt werden.

Die Multielementkalibrationslösungen für die ICP-MS-Messungen, die in der Abteilung Geochemie am GZG erstellt wurden, umfassten 62 Elemente (POSPIECH ET AL., 2017a). Davon waren 40 Elemente sehr gut messbar bzw. für die Dateninterpretation geeignet (vgl. Kapitel 3.5.2).

Für die spätere Bestimmung der driftkorrigierten Konzentrationen der analysierten Proben und zur Referenzwertkontrolle wurden, ähnlich der Multielementanalysen mittels ICP-OES, ebenfalls in regelmäßigen Abständen eine Kalibrations- sowie eine 
Referenzmaterial-Lösung (GSJ JA-2) wiederholt mitgemessen. Der bei den Säuretotalaufschlüssen in die Proben zugegebene interne Standard mit definierten Elementkonzentrationen (50 ppm Beryllium (Be), $10 \mathrm{ppm}$ Rhodium (Rh), $5 \mathrm{ppm}$ Indium (In) und 5 ppm Rhenium (Re), vgl. Kapitel 3.3) diente dazu, Schwankungen während der Messungen auszugleichen, indem im jeweiligen Massenbereich Intensitätsverhältnisse zu diesen internen Standardelementen gemessen wurden (POSPIECH ET AL., 2017a).

Die jeweiligen Nachweisgrenzen der Elemente wurden über die dreifache Standardabweichung der mitgemessenen Aufschluss-Blindproben pro Messserie ermittelt. Die Genauigkeit und Richtigkeit der Analysen wurde mit Hilfe der relativen Abweichungen der Messwerte zum jeweiligen Bezugswert der in Kapitel 3.5.2 aufgeführten Referenzmaterialien überprüft. Die Elementgesamtgehalte der Gärgemisch- und Silageproben sind nach Driftkorrektur, Blindwertabzug, Umrechnung auf die Festphase und unter Berücksichtigung der Nachweisgrenze in den Elementdatentabellen im Anhang zusammengestellt.

\subsection{Fehlerbetrachtung und Qualitätssicherung}

\subsubsection{Kurzbetrachtung systematischer und methodischer Fehlerquellen}

Die genommenen Gärgemisch- oder Silageproben stellen immer nur zeitliche und stoffliche Teilausschnitte des zu beprobenden Systems dar und sind somit lediglich oft nicht repräsentative Stichproben aus einer Gesamtmenge. Bei den Probenahmen der (quasi)kontinuierlichen Laborfermenterversuche am DBFZ (vgl. Kapitel 3.2 und 4) wurde darauf geachtet, eine repräsentative Menge an Reaktormaterial zu nehmen. Eine Mehrfachbeprobung an einem Versuchstag war bei den Versuchen aufgrund des geringen Nennvolumens der Reaktoren nicht möglich. Es wurde allerdings in kritischen Versuchszeiträumen das Probenahmeintervall verkürzt.

Bei der Gärgemisch- und Silageproben-Aufbereitung wurde darauf geachtet, möglichst repräsentative, homogene und analysenfeine Probenpulver bei der Feinmahlung mit der Planeten-Kugelmühle zu gewährleisten (vgl. Kapitel 3.2). Die geringen Einwaagemengen des Mahlguts von etwa $400 \mathrm{mg}$ (auf $50 \mathrm{~mL}$ Volumen) für die Gärgemischproben und circa $700 \mathrm{mg}$ (auf $50 \mathrm{~mL}$ Volumen) für die Silageproben bei den Säuretotalaufschlüssen (vgl. Kapitel 3.3) bergen zwar eine potenzielle Gefahr von Inhomogenitäten in sich, jedoch sind die Fehler der Probenhomogenisierung im Rahmen der Aufbereitung als sehr gering einzuschätzen. 


\subsubsection{Qualität der Messergebnisse und Elementauswahl}

Zur Überprüfung der internationalen Vergleichbarkeit (Richtigkeit) und Präzision der Säuretotalaufschlussverfahren (vgl. Kapitel 3.3) bzw. der Messwerte der Multielementanalysen (vgl. Kapitel 3.4.3 und 3.4.4) wurden folgende Pflanzenreferenzmaterialien, in denen Gesamtgehalte zahlreicher Elemente international zertifiziert sind, mit aufgeschlossen und analysiert:

- NCS DC 73349 (Buschzweige und -blätter, China, $\mathrm{n}=37$ ),

- WEPAL-IPE-126 (Mais (Pflanze), Niederlande, $\mathrm{n}=22$ ),

- WEPAl-IPE-168 (Sonnenblume, Niederlande, $\mathrm{n}=20$ ) sowie

- GBW10052 (Grüner Tee, China, $\mathrm{n}=7$ ).

Zusätzlich kamen folgende Gesteinsreferenzmaterialien in geringerem Umfang hinzu:

- GSJ JA-2 (Andesit, Japan, $\mathrm{n}=3$ ),

- TW-45 (12) (Wissenbacher Tonschiefer, hausinterne Referenz, $\mathrm{n}=4$ ).

Tabelle 3 und Tabelle 4 enthalten Zusammenstellungen aller verwendeten Referenzmaterialien mit den gemittelten Messwerten und den Bezugswerten der Elementgesamtgehalte (zertifiziert, als Richtwert oder informativ bzw. hausintern). Für einige Elemente konnten anhand vieler Messungen über mehrere Jahre hinweg bzw. durch Vergleich mit den anderen Referenzmessungen eigene genauere bzw. neue Bezugswerte (vor allem für Richt- oder Informationswerte) etabliert werden, die ebenfalls für die Auswertungen herangezogen wurden.

Anhand der relativen Abweichung (relativer Fehler) zwischen dem gemessenen Wert und dem Bezugswert wurde die prozentuale Richtigkeit des Messergebnisses [(gemessener Wert - Bezugswert) / Bezugswert - 100] ermittelt und so systematische Fehler der Säuretotalaufschlüsse und der Multielementmessungen erfasst. Die relativen Abweichungen zu den Bezugswerten lagen für die meisten Elemente der Pflanzenreferenzmaterialien $<5 \%$ (in Ausnahmen $<10 \%$ ). Nur sehr wenige Elemente (Selen und Zinn) wiesen aufgrund von Interferenzen oder größeren Streuwerten zum Teil Abweichungen $>10 \%$ auf. In Tabelle 3 und Tabelle 4 sind die Abweichungen des jeweils gemittelten Messwertes zum Bezugswert mit aufgeführt. 
Tabelle 3: $\quad$ Verwendete Referenzmaterialien (Blatt I) mit Angabe der gemittelten Mess- und Bezugswerte [mg/kg] sowie der prozentualen Richtigkeit (R).

\begin{tabular}{|c|c|c|c|c|c|c|c|c|c|}
\hline \multirow{2}{*}{$\begin{array}{l}\text { [mg/kg] } \\
\text { Element }\end{array}$} & \multicolumn{3}{|c|}{$\begin{array}{c}\text { NCS DC } 73349 \\
\text { Buschzweige und -blätter }\end{array}$} & \multicolumn{3}{|c|}{$\begin{array}{l}\text { WEPAL-IPE-126 } \\
\text { Mais (Pflanze) }\end{array}$} & \multicolumn{3}{|c|}{$\begin{array}{l}\text { WEPAL-IPE-168 } \\
\text { Sonnenblume }\end{array}$} \\
\hline & $\begin{array}{c}\text { Messwert } \\
(\mathrm{n}=37)\end{array}$ & Bezugswert & $\begin{array}{l}\mathrm{R} \\
{[\%]}\end{array}$ & $\begin{array}{c}\text { Messwert } \\
(n=22)\end{array}$ & Bezugswert & $\begin{array}{l}\mathrm{R} \\
{[\%]}\end{array}$ & $\begin{array}{c}\text { Messwert } \\
(n=20)\end{array}$ & Bezugswert & $\begin{array}{r}R \\
{[\%]}\end{array}$ \\
\hline Al & $2010 \pm 40$ & $2000 \pm 300$ & $+0,4$ & $\underline{810} \pm 50$ & 700 & & $67 \pm 2$ & $68 \pm 10$ & $-2,4$ \\
\hline As & $1,29 \pm 0,11$ & $1,25 \pm 0,15$ & $+3,1$ & $\mathbf{0 , 3 6} \pm 0,04$ & $\mathbf{0 , 3 5} \pm 0,06$ & $+2,9$ & $\mathbf{0 , 0 6 7} \pm 0,004$ & $\mathbf{0 , 0 6 9} \pm 0,008$ & $-3,1$ \\
\hline B & & $38 \pm 6$ & & & $7,1 \pm 1,5$ & & & $30,4 \pm 2,7$ & \\
\hline $\mathrm{Ba}$ & $17 \pm 0,8$ & $18 \pm 2$ & $-2,8$ & $\underline{5,1} \pm 0,4$ & $\mathbf{2 , 4} \pm 0,3$ & & $9,3 \pm 0,4$ & $9,2 \pm 0,9$ & $+1,6$ \\
\hline $\mathrm{Be}$ & & $\mathbf{0 , 0 5 1} \pm 0,004$ & & & $0,022 \pm 0,003$ & & & 0,0051 & \\
\hline $\mathbf{B i}$ & $\underline{0,016} \pm 0,001$ & $\mathbf{0 , 0 2 3} \pm 0,005$ & & $0,0060 \pm 0,004$ & 0,0058 & $+2,6$ & $\underline{0,0025} \pm 0,0004$ & 0,0040 & \\
\hline $\mathrm{Ca}$ & $\overline{16300} \pm 430$ & $16800 \pm 1100$ & $-2,7$ & $3500 \pm 130$ & $3640 \pm 240$ & $-3,9$ & $10700 \pm 330$ & $11000 \pm 580$ & $-2,9$ \\
\hline Cd & $\underline{0,72} \pm 0,03$ & 0,38 & & $\mathbf{0 , 1 3 4} \pm 0,007$ & $\mathbf{0 , 1 3 2} \pm 0,021$ & $+1,8$ & $\mathbf{0 , 4 5} \pm 0,02$ & $\mathbf{0 , 4 5} \pm 0,03$ & $+0,3$ \\
\hline $\mathrm{Ce}$ & $\overline{\mathbf{2 , 2}} \pm 0,2$ & $2,2 \pm 0,1$ & $+0,9$ & $\underline{0,96} \pm 0,09$ & & & $\underline{0,12} \pm 0,02$ & & \\
\hline Co & $\mathbf{0 , 4 1} \pm 0,02$ & $\mathbf{0 , 4 1} \pm 0,05$ & $-0,7$ & $\mathbf{0 , 1 6 3} \pm 0,013$ & $\mathbf{0 , 1 6 6} \pm 0,022$ & $-2,0$ & $\mathbf{0 , 1 1 4} \pm 0,01$ & $\mathbf{0 , 1 2 0} \pm 0,016$ & $-4,7$ \\
\hline $\mathrm{Cr}$ & $\underline{\mathbf{2}, \mathbf{0}} \pm 0,3$ & $\mathbf{2 , 6} \pm 0,2$ & & $\underline{\mathbf{1 , 5 3}} \pm 0,11$ & $1,11 \pm 0,19$ & & $0,58 \pm 0,13$ & $0,56 \pm 0,24$ & $+3,4$ \\
\hline Cs & $\mathbf{0 , 2 6} \pm 0,01$ & $\mathbf{0 , 2 7} \pm 0,02$ & $-2,7$ & $0,177 \pm 0,009$ & 0,185 & $-4,3$ & $0,0138 \pm 0,0006$ & 0,0130 & $+5,8$ \\
\hline $\mathrm{Cu}$ & $6,4 \pm 0,4$ & $\mathbf{6 , 6} \pm 0,8$ & $-2,3$ & $\mathbf{4 , 4} \pm 0,3$ & $\mathbf{4 , 4} \pm 0,7$ & $-0,1$ & $\mathbf{8 , 0} \pm 0,2$ & $\mathbf{8 , 1} \pm 0,8$ & $-1,8$ \\
\hline Dy & $\underline{0,15} \pm 0,01$ & 0,13 & & $\underline{0,069} \pm 0,007$ & & & $\underline{0,0054} \pm 0,0011$ & & \\
\hline $\mathrm{Er}$ & $\underline{0,081} \pm 0,005$ & & & $\underline{0,039} \pm 0,004$ & & & $\underline{0,0028} \pm 0,0007$ & & \\
\hline Eu & $\overline{\mathbf{0 , 0 3 7}} \pm 0,003$ & $\mathbf{0 , 0 3 9} \pm 0,003$ & $-4,4$ & $\underline{\overline{0,018}} \pm 0,002$ & & & & & \\
\hline $\mathrm{Fe}$ & $1030 \pm 30$ & $1070 \pm 57$ & $-3,4$ & $\underline{624} \pm 34$ & $530 \pm 64$ & & $95 \pm 4$ & $88 \pm 11$ & $+8,0$ \\
\hline $\mathbf{G a}$ & $\underline{0,49} \pm 0,07$ & & & $\underline{\underline{0,23}} \pm 0,04$ & & & & 0,058 & \\
\hline $\begin{array}{l}\text { Gd } \\
\text { Ge }\end{array}$ & $0,19 \pm 0,01$ & 0,19 & $-2,1$ & $\underline{0,082} \pm 0,006$ & & & $\underline{0,0079} \pm 0,001$ & & \\
\hline $\begin{array}{l}\mathrm{Hf} \\
\mathrm{Hg}\end{array}$ & $\underline{0,10} \pm 0,01$ & 0,15 & & $\underline{0,067} \pm 0,01$ & $0,0054 \pm 0,0014$ & & $\underline{0,0058} \pm 0,0014$ & $\mathbf{0 , 0 0 5 8} \pm 0,0009$ & \\
\hline $\begin{array}{l}\text { Ho } \\
\text { Ir }\end{array}$ & $0,032 \pm 0,002$ & 0,033 & $-3,7$ & $\underline{0,015} \pm 0,001$ & & & $\underline{0,0011} \pm 0,0002$ & & \\
\hline $\mathbf{K}$ & $9600 \pm 290$ & $9200 \pm 1000$ & $+4,3$ & $18400 \pm 580$ & $18800 \pm 1050$ & $-2,2$ & $26800 \pm 560$ & $27500 \pm 1600$ & $-2,6$ \\
\hline La & $1,16 \pm 0,13$ & $1,25 \pm 0,06$ & $-6,9$ & $\underline{0,49} \pm 0,05$ & & & $\underline{0,095} \pm 0,012$ & & \\
\hline $\mathbf{L i}$ & $\mathbf{2 , 6} \pm 0,05$ & $\mathbf{2 , 6} \pm 0,4$ & $-0,6$ & $\underline{\overline{0,75}} \pm 0,03$ & $0,54 \pm 0,13$ & & $\overline{0,34} \pm 0,03$ & $0,33 \pm 0,04$ & $+2,1$ \\
\hline Lu & $0,011 \pm 0,001$ & 0,011 & $-1,1$ & $\underline{0,0055} \pm 0,0006$ & & & $\underline{0,0003} \pm 0,0001$ & & \\
\hline Mg & $4500 \pm 140$ & $4800 \pm 400$ & $-6,0$ & $1760 \pm 50$ & $1770 \pm 110$ & $-0,5$ & $1920 \pm 50$ & $2000 \pm 110$ & $-4,2$ \\
\hline$M n$ & $62 \pm 3$ & $61 \pm 5$ & $+0,8$ & $29,1 \pm 1,6$ & $27,9 \pm 3$ & $+4,3$ & $126 \pm 4$ & $124 \pm 7$ & $+1,4$ \\
\hline Mo & $\mathbf{0 , 2 7} \pm 0,03$ & $\mathbf{0 , 2 8} \pm 0,05$ & $-4,1$ & $\mathbf{0 , 5 4} \pm 0,03$ & $\mathbf{0 , 5 2} \pm 0,06$ & $+3,2$ & $1,17 \pm 0,08$ & $1,13 \pm 0,11$ & $+3,8$ \\
\hline $\mathrm{Na}$ & $19100 \pm 650$ & $19600 \pm 1800$ & $-2,8$ & $\underline{253} \pm 15$ & $179 \pm 48$ & & $384 \pm 11$ & $388 \pm 39$ & $-1,0$ \\
\hline $\mathrm{Nb}$ & $\underline{0,40} \pm 0,03$ & & & $\underline{0,23} \pm 0,02$ & & & $\underline{0,016} \pm 0,001$ & & \\
\hline Nd & $\overline{1,0} \pm 0,07$ & $1,0 \pm 0,1$ & $+0,1$ & $\underline{0,45} \pm 0,04$ & & & $\underline{0,044} \pm 0,008$ & & \\
\hline $\mathbf{N i}$ & $\underline{1,38} \pm 0,07$ & $1,70 \pm 0,3$ & & $\overline{\mathbf{0 , 7 2}} \pm 0,07$ & $\mathbf{0 , 7 2} \pm 0,13$ & $+0,4$ & $\mathbf{1 , 1 1} \pm 0,05$ & $1,07 \pm 0,12$ & $+4,0$ \\
\hline $\mathbf{P}$ & $\overline{930} \pm 20$ & $1000 \pm 40$ & $-7,3$ & $1800 \pm 70$ & $1800 \pm 104$ & $-0,7$ & $3550 \pm 80$ & $3640 \pm 180$ & $-2,6$ \\
\hline $\mathrm{Pb}$ & $47 \pm 3$ & $47 \pm 3$ & $-0,5$ & $1,01 \pm 0,07$ & $\mathbf{0 , 9 6} \pm 0,22$ & $+4,7$ & $\mathbf{0 , 6 1} \pm 0,06$ & $\mathbf{0 , 6 7} \pm 0,11$ & $-8,4$ \\
\hline $\begin{array}{l}\mathrm{Pr} \\
\mathrm{Pt}\end{array}$ & $0,25 \pm 0,02$ & 0,24 & $+3,1$ & $\underline{0,11} \pm 0,01$ & & & $\underline{0,012} \pm 0,002$ & & \\
\hline $\mathbf{R b}$ & $4,5 \pm 0,4$ & $4,5 \pm 0,6$ & $-0,8$ & $21,9 \pm 1$ & 21,4 & $+2,4$ & $21,3 \pm 1,4$ & $20,5 \pm 1,8$ & $+3,7$ \\
\hline $\mathbf{S}$ & $7100 \pm 180$ & $7300 \pm 600$ & $-2,6$ & $970 \pm 30$ & $1030 \pm 75$ & $-6,2$ & $1770 \pm 40$ & $1850 \pm 150$ & $-4,3$ \\
\hline Sb & $\mathbf{0 , 0 9 6} \pm 0,007$ & $\mathbf{0 , 0 9 5} \pm 0,014$ & $+1,6$ & $\underline{0,036} \pm 0,004$ & $0,015 \pm 0,005$ & & $\underline{0,035} \pm 0,003$ & $0,030 \pm 0,006$ & \\
\hline Sc & $\mathbf{0 , 3 0} \pm 0,01$ & $\mathbf{0 , 3 2} \pm 0,04$ & $-6,8$ & $\underline{0,130} \pm 0,008$ & & & & & \\
\hline $\mathrm{Se}$ & $\mathbf{0 , 0 9 0} \pm 0,005$ & $\mathbf{0 , 1 2 0} \pm 0,02$ & -25 & $\overline{0,038} \pm 0,005$ & $0,035 \pm 0,012$ & $+9,4$ & $\underline{0,039} \pm 0,004$ & $\mathbf{0 , 2 3} \pm 0,05$ & \\
\hline Sm & $\mathbf{0 , 1 9 0} \pm 0,013$ & $0,190 \pm 0,02$ & $+0,2$ & $\underline{0,087} \pm 0,006$ & & & $\underline{\underline{0,0072}} \pm 0,0012$ & & \\
\hline Sn & $\underline{0,48} \pm 0,12$ & 0,27 & & $0,093 \pm 0,013$ & 0,080 & +16 & $0,052 \pm 0,015$ & 0,068 & -23 \\
\hline $\mathrm{Sr}$ & $\overline{\mathbf{2 4 8}} \pm 16$ & $246 \pm 16$ & $+0,7$ & $13,5 \pm 0,5$ & $13,2 \pm 0,5$ & $+2,4$ & $\mathbf{2 8 , 8} \pm 1,6$ & $28,4 \pm 2,5$ & $+1,3$ \\
\hline $\mathrm{Ta}$ & $\underline{0,027} \pm 0,002$ & & & $\underline{0,015} \pm 0,001$ & & & $\underline{0,0011} \pm 0,0003$ & & \\
\hline $\begin{array}{l}\text { Tb } \\
\text { Te }\end{array}$ & $\overline{\mathbf{0 , 0 2 6}} \pm 0,001$ & $\mathbf{0 , 0 2 5} \pm 0,003$ & $+2,6$ & $\underline{\underline{0,012}} \pm 0,001$ & & & $\underline{0,0010} \pm 0,0001$ & & \\
\hline Th & $\mathbf{0 , 3 7} \pm 0,03$ & $\mathbf{0 , 3 6} \pm 0,04$ & $+2,1$ & $\underline{0,14} \pm 0,01$ & & & $\underline{0,009} \pm 0,002$ & & \\
\hline $\mathrm{Ti}$ & $103 \pm 5$ & $95 \pm 20$ & $+8,2$ & $\underline{58} \pm 4$ & 24,9 & & $\underline{4,6} \pm 0,4$ & 3,3 & \\
\hline TI & $\underline{0,020} \pm 0,001$ & & & $\underline{0,014} \pm 0,001$ & & & $\underline{0,018} \pm 0,001$ & & \\
\hline $\mathrm{Tm}$ & $\underline{0,011} \pm 0,001$ & & & $\underline{\underline{0,0055}} \pm 0,0006$ & & & $\underline{\underline{0,0004}} \pm 0,0001$ & & \\
\hline $\mathbf{U}$ & $0,13 \pm 0,01$ & 0,12 & $+5,0$ & $\underline{0,037} \pm 0,002$ & & & $\underline{\underline{0,0029}} \pm 0,0004$ & & \\
\hline $\mathbf{V}$ & $2,3 \pm 0,07$ & $2,4 \pm 0,4$ & $-4,1$ & $1,19 \pm 0,06$ & $1,02 \pm 0,24$ & +16 & $\underline{0,123} \pm 0,012$ & $0,093 \pm 0,027$ & \\
\hline $\mathbf{W}$ & & 0,060 & & & & & & & \\
\hline $\mathbf{Y}$ & $\underline{0,83} \pm 0,07$ & $\mathbf{0 , 6 8} \pm 0,02$ & & $\underline{0,37} \pm 0,04$ & & & $\underline{0,037} \pm 0,0003$ & & \\
\hline Yb & $\underline{0,076} \pm 0,007$ & $\mathbf{0 , 0 6 3} \pm 0,009$ & & $\underline{0,039} \pm 0,004$ & & & $\underline{0,0024} \pm 0,0003$ & & \\
\hline $\mathrm{Zn}$ & $54 \pm 2$ & $55 \pm 4$ & $-2,2$ & $\mathbf{3 1 , 2} \pm 1,4$ & $30,4 \pm 2,7$ & $+2,7$ & $153 \pm 6$ & $148 \pm 11$ & $+3,5$ \\
\hline $\mathrm{Zr}$ & $\underline{3,9} \pm 0,5$ & & & $\underline{2,6} \pm 0,4$ & & & $\underline{0,23} \pm 0,06$ & & \\
\hline Fett: & Zertifizierter W & $\mathrm{t}$ (mit mittleren & n Fehle & & & & & & \\
\hline $\begin{array}{l}\text { Normal: } \\
\text { Unterstrichen: }\end{array}$ & Richtwert (mit & $\begin{array}{l}\text { ttlerem Fehler } \\
\text { er Richtwert a }\end{array}$ & ) oder & $\begin{array}{l}\text { rt zur Informatio } \\
\text { er Messungen }\end{array}$ & & & & & \\
\hline
\end{tabular}


Tabelle 4: $\quad$ Verwendete Referenzmaterialien (Blatt II) mit Angabe der gemittelten Mess- und Bezugswerte [mg/kg] sowie der prozentualen Richtigkeit (R).

\begin{tabular}{|c|c|c|c|c|c|c|c|c|c|}
\hline \multirow{2}{*}{$\begin{array}{l}\text { [mg/kg] } \\
\text { Element }\end{array}$} & \multicolumn{3}{|c|}{$\begin{array}{l}\text { GBW10052 } \\
\text { Grüner Tee }\end{array}$} & \multicolumn{3}{|c|}{$\begin{array}{l}\text { GSJ JA-2 } \\
\text { Andesit }\end{array}$} & \multicolumn{3}{|c|}{$\begin{array}{c}\text { TW45* }^{*} \\
\text { Wissenbacher Tonschiefer }\end{array}$} \\
\hline & $\begin{array}{l}\text { Messwert } \\
\quad(n=7)\end{array}$ & Bezugswert & $\begin{array}{l}\mathrm{R} \\
{[\%]}\end{array}$ & $\begin{array}{l}\text { Messwert } \\
\quad(n=3)\end{array}$ & Bezugswert & $\begin{array}{l}\mathrm{R} \\
{[\%]}\end{array}$ & $\begin{array}{l}\text { Messwert } \\
\quad(n=4)\end{array}$ & Bezugswert & $\begin{array}{l}\mathrm{R} \\
{[\%]}\end{array}$ \\
\hline Al & $\underline{1670} \pm 50$ & & & $86500 \pm 3600$ & $82100 \pm 600$ & $+5,4$ & $82700 \pm 2300$ & 81500 & $+1,4$ \\
\hline As & $\overline{\mathbf{0 , 2 7}} \pm 0,04$ & $\mathbf{0 , 2 7} \pm 0,05$ & $-1,1$ & & $0,71 \pm 0,28$ & & 8,5 & 8,5 & $+0,5$ \\
\hline B & & $14,1 \pm 1,2$ & & & $21,1 \pm 1,9$ & & & & \\
\hline $\mathrm{Ba}$ & $40 \pm 1,4$ & $41 \pm 4$ & $-3,5$ & $306 \pm 15$ & $308 \pm 5$ & $-0,7$ & $366 \pm 13$ & 380 & $-3,7$ \\
\hline $\mathrm{Be}$ & & $\mathbf{0 , 0 2 5} \pm 0,003$ & & & $\mathbf{2 , 3} \pm 0,19$ & & & 3,0 & \\
\hline $\mathbf{B i}$ & $\mathbf{0 , 0 3 7} \pm 0,002$ & $\mathbf{0 , 0 4 0} \pm 0,011$ & $-7,7$ & $\mathbf{0 , 1 0 8} \pm 0,004$ & $0,092 \pm 0,0073$ & +18 & $0,29 \pm 0,008$ & 0,27 & $+5,9$ \\
\hline $\mathrm{Ca}$ & $11400 \pm 310$ & $12100 \pm 300$ & $-5,9$ & $45800 \pm 2100$ & $44700 \pm 400$ & $+2,5$ & $25900 \pm 1400$ & 25400 & $+1,9$ \\
\hline Cd & $\mathbf{0 , 0 8 7} \pm 0,006$ & $\mathbf{0 , 0 7 6} \pm 0,004$ & +14 & $0,058 \pm 0,01$ & $0,069 \pm 0,019$ & -16 & $0,20 \pm 0,005$ & 0,23 & -12 \\
\hline $\mathrm{Ce}$ & $\mathbf{0 , 8 1} \pm 0,04$ & $\mathbf{0 , 8 1} \pm 0,03$ & $-0,5$ & $\mathbf{3 1 , 4} \pm 1,5$ & $32,9 \pm 0,85$ & $-4,4$ & $81 \pm 2,5$ & 81 & $+0,2$ \\
\hline Co & $\mathbf{0 , 2 8} \pm 0,02$ & $\mathbf{0 , 3 0} \pm 0,02$ & $-7,1$ & $30,6 \pm 1,6$ & $28,3 \pm 0,97$ & $+8,1$ & $18,00 \pm 0,6$ & 18 & $-0,9$ \\
\hline $\mathrm{Cr}$ & $\mathbf{0 , 9 1} \pm 0,09$ & $\mathbf{0 , 9 2} \pm 0,2$ & $-1,2$ & $414 \pm 30$ & $425 \pm 9,3$ & $-2,6$ & $108 \pm 18$ & 105 & $+3,1$ \\
\hline Cs & $\mathbf{0 , 5 9} \pm 0,04$ & $\mathbf{0 , 5 8} \pm 0,03$ & $+1,4$ & $\mathbf{5 , 2} \pm 0,4$ & $4,8 \pm 0,087$ & $+9,4$ & $7,7 \pm 0,4$ & 7,8 & $-1,8$ \\
\hline $\mathrm{Cu}$ & $24 \pm 0,7$ & $24 \pm 1$ & $+1,3$ & $\mathbf{2 9 , 2} \pm 1,6$ & $29,0 \pm 1,5$ & $+0,8$ & $36 \pm 0,9$ & 35 & $+1,5$ \\
\hline Dy & $\mathbf{0 , 0 6 4} \pm 0,005$ & $\mathbf{0 , 0 6 5} \pm 0,007$ & $-1,7$ & $3,2 \pm 0,04$ & $2,9 \pm 0,071$ & +12 & $5,8 \pm 0,3$ & 5,5 & $+4,6$ \\
\hline $\mathrm{Er}$ & $\mathbf{0 , 0 3 8} \pm 0,003$ & $\mathbf{0 , 0 3 7} \pm 0,006$ & $+3,1$ & $1,85 \pm 0,05$ & $1,68 \pm 0,031$ & +10 & $3,3 \pm 0,2$ & 3,1 & $+5,8$ \\
\hline Eu & $\underline{0,015} \pm 0,002$ & $\mathbf{0 , 0 2 2} \pm 0,006$ & & $1,02 \pm 0,08$ & $\mathbf{0 , 8 9} \pm 0,018$ & +14 & $1,22 \pm 0,06$ & 1,20 & $+1,6$ \\
\hline $\mathrm{Fe}$ & $347 \pm 17$ & $322 \pm 23$ & $+7,6$ & $47700 \pm 3000$ & $44000 \pm 300$ & $+8,4$ & $47500 \pm 2100$ & 45000 & $+5,5$ \\
\hline $\mathbf{G a}$ & $\underline{0,23} \pm 0,03$ & & & $17,9 \pm 0,67$ & $16,9 \pm 0,77$ & $+6,1$ & $22 \pm 0,9$ & 21 & $+5,5$ \\
\hline Gd & $\mathbf{0 , 0 7 3} \pm 0,006$ & $\mathbf{0 , 0 7 6} \pm 0,011$ & $-3,5$ & $3,6 \pm 0,3$ & $3,0 \pm 0,09$ & +20 & $6,4 \pm 0,2$ & 5,9 & $+8,2$ \\
\hline Ge & & $\mathbf{0 , 0 1 5} \pm 0,005$ & & & 1,1 & & & & \\
\hline Hf & $\underline{0,19} \pm 0,03$ & & & $3,5 \pm 0,4$ & $\mathbf{2 , 8} \pm 0,062$ & +25 & $4,9 \pm 0,1$ & 4,7 & $+3,2$ \\
\hline $\mathrm{Hg}$ & & $\mathbf{0 , 0 0 8 1} \pm 0,0015$ & & & 0,0018 & & & 0,08 & \\
\hline Ho & $\mathbf{0 , 0 1 4} \pm 0,001$ & $\mathbf{0 , 0 1 3} \pm 0,002$ & $+7,7$ & $\mathbf{0 , 6 8} \pm 0,03$ & $\mathbf{0 , 5 9} \pm 0,015$ & +15 & $1,19 \pm 0,02$ & 1,10 & $+8,0$ \\
\hline Ir & & & & & 0,000016 & & & & \\
\hline $\mathbf{K}$ & $15300 \pm 430$ & $15500 \pm 700$ & $-1,4$ & $15400 \pm 650$ & $14800 \pm 100$ & $+3,9$ & $30800 \pm 970$ & 30300 & $+1,6$ \\
\hline La & $\mathbf{0 , 5 5} \pm 0,02$ & $\mathbf{0 , 5 4} \pm 0,04$ & $+2,2$ & $15,8 \pm 0,8$ & $15,5 \pm 0,4$ & $+2,4$ & $41 \pm 1,3$ & 40 & $+3,2$ \\
\hline Li & $\mathbf{0 , 5 3} \pm 0,02$ & $\mathbf{0 , 5 2} \pm 0,04$ & $+1,6$ & $32,0 \pm 1,5$ & $29,2 \pm 0,56$ & $+9,7$ & $51 \pm 1,8$ & 48 & $+5,6$ \\
\hline Lu & $\mathbf{0 , 0 0 6 1} \pm 0,0005$ & $\mathbf{0 , 0 0 6 2} \pm 0,001$ & $-1,4$ & $\mathbf{0 , 2 9} \pm 0,03$ & $\mathbf{0 , 2 5} \pm 0,009$ & +14 & $0,47 \pm 0,02$ & 0,44 & $+7,7$ \\
\hline Mg & $2000 \pm 70$ & $2200 \pm 80$ & $-8,0$ & $49300 \pm 2600$ & $47300 \pm 500$ & $+4,1$ & $23300 \pm 550$ & 23500 & $-0,7$ \\
\hline$M n$ & $1210 \pm 40$ & $1170 \pm 60$ & $+3,1$ & $840 \pm 30$ & $850 \pm 16$ & $-0,9$ & $554 \pm 20$ & 550 & $+0,7$ \\
\hline Mo & $\underline{0,065} \pm 0,014$ & $0,110 \pm 0,02$ & & $\mathbf{0 , 4 8} \pm 0,18$ & $\mathbf{0 , 5 8} \pm 0,035$ & -18 & & 0,48 & \\
\hline $\mathrm{Na}$ & $105 \pm 6$ & $100 \pm 10$ & $+4,9$ & $24400 \pm 1300$ & $22800 \pm 300$ & $+7,0$ & $6500 \pm 200$ & 6600 & $-1,8$ \\
\hline $\mathrm{Nb}$ & $\underline{0,100} \pm 0,014$ & 0,050 & & $10,2 \pm 0,7$ & $\mathbf{9 , 3} \pm 0,24$ & $+9,2$ & $18 \pm 1,1$ & 16 & +13 \\
\hline Nd & $\mathbf{0 , 3 5} \pm 0,02$ & $\mathbf{0 , 3 5} \pm 0,04$ & $+0,8$ & $16,1 \pm 1,1$ & $14,0 \pm 0,24$ & +15 & $38 \pm 1,3$ & 38 & $+0,7$ \\
\hline $\mathrm{Ni}$ & $\mathbf{5 , 2} \pm 0,4$ & $\mathbf{5 , 4} \pm 0,4$ & $-3,2$ & $153 \pm 10$ & $136 \pm 2,2$ & +12 & $73 \pm 4$ & 72 & $+0,8$ \\
\hline $\mathbf{P}$ & $2700 \pm 80$ & $2800 \pm 100$ & $-4,5$ & $690 \pm 40$ & $660 \pm 14$ & $+4,9$ & $378 \pm 23$ & 393 & $-3,9$ \\
\hline $\mathrm{Pb}$ & $1,62 \pm 0,12$ & $1,60 \pm 0,2$ & $+1,1$ & $19,9 \pm 0,8$ & $18,9 \pm 0,29$ & $+5,6$ & $37 \pm 1,4$ & 38 & $-1,5$ \\
\hline Pr & $\mathbf{0 , 0 9 0} \pm 0,004$ & $\mathbf{0 , 0 9 3} \pm 0,008$ & $-2,8$ & $3,9 \pm 0,2$ & $3,7 \pm 0,079$ & $+6,5$ & $9,8 \pm 0,4$ & 9,3 & $+5,1$ \\
\hline $\mathbf{P t}$ & & & & & 0,00017 & & & & \\
\hline $\mathbf{R b}$ & $87 \pm 7$ & $89 \pm 9$ & $-2,7$ & $79 \pm 4$ & $70 \pm 1,3$ & +13 & $161 \pm 3$ & 155 & $+3,7$ \\
\hline S & $4200 \pm 140$ & 4200 & $+0,3$ & & 6,8 & & $4300 \pm 220$ & 4000 & $+6,9$ \\
\hline Sb & $0,053 \pm 0,005$ & 0,052 & $+2,7$ & $\mathbf{0 , 1 6} \pm 0,01$ & $\mathbf{0 , 1 5} \pm 0,03$ & $+7,2$ & $1,04 \pm 0,03$ & 1,0 & $+4,0$ \\
\hline Sc & $\underline{0,080} \pm 0,004$ & 0,070 & & $18,7 \pm 0,6$ & $18,9 \pm 0,34$ & $-1,2$ & $14 \pm 0,3$ & 14 & $+3,2$ \\
\hline $\mathrm{Se}$ & & $\mathbf{0 , 1 0 0} \pm 0,03$ & & & $0,0062 \pm 0,0041$ & & & 0,70 & \\
\hline Sm & $\mathbf{0 , 0 6 7} \pm 0,006$ & $\mathbf{0 , 0 6 6} \pm 0,01$ & $+1,7$ & $3,2 \pm 0,1$ & $\mathbf{3 , 0} \pm 0,043$ & $+7,1$ & $7,1 \pm 0,2$ & 6,9 & $+2,4$ \\
\hline Sn & $0,17 \pm 0,03$ & 0,17 & $+1,0$ & $1,56 \pm 0,14$ & $1,69 \pm 0,15$ & $-7,5$ & $3,5 \pm 0,2$ & 3,3 & $+6,7$ \\
\hline $\mathrm{Sr}$ & $34 \pm 3$ & $36 \pm 2$ & $-5,5$ & $242 \pm 10$ & $246 \pm 3$ & $-1,6$ & $89 \pm 6$ & 90 & $-1,4$ \\
\hline $\mathrm{Ta}$ & & & & $\mathbf{0 , 7 4} \pm 0,04$ & $\mathbf{0 , 6 5} \pm 0,017$ & +14 & $\underline{1,2} \pm 0,1$ & 2,0 & \\
\hline $\mathrm{Tb}$ & $\mathbf{0 , 0 1 0 2} \pm 0,0009$ & $\mathbf{0 , 0 1 1 4} \pm 0,002$ & -10 & $\mathbf{0 , 5 2} \pm 0,03$ & $\mathbf{0 , 4 8} \pm 0,008$ & $+9,6$ & $0,93 \pm 0,04$ & 0,85 & $+9,7$ \\
\hline Te & & & & & 0,0011 & & & & \\
\hline Th & $\mathbf{0 , 0 7 8} \pm 0,003$ & $\mathbf{0 , 0 7 9} \pm 0,012$ & $-1,5$ & $\mathbf{5 , 3} \pm 0,5$ & $4,8 \pm 0,11$ & +11 & $13 \pm 0,6$ & 13 & $+1,3$ \\
\hline $\mathrm{Ti}$ & $\underline{29} \pm 2$ & 21 & & $4100 \pm 170$ & $4010 \pm 40$ & $+2,1$ & $4400 \pm 170$ & 4300 & $+1,0$ \\
\hline TI & $\underline{0,047} \pm 0,003$ & $\mathbf{0 , 0 5 7} \pm 0,011$ & & $\mathbf{0 , 3 4} \pm 0,03$ & $\mathbf{0 , 3 3} \pm 0,013$ & $+3,3$ & $0,75 \pm 0,003$ & 0,77 & $-2,2$ \\
\hline $\mathrm{Tm}$ & $\mathbf{0 , 0 0 5 8} \pm 0,0005$ & $\mathbf{0 , 0 0 5 9} \pm 0,001$ & $-2,5$ & $\mathbf{0 , 2 6} \pm 0,01$ & $\mathbf{0 , 2 5} \pm 0,0065$ & $+3,9$ & $0,48 \pm 0,02$ & 0,50 & $-4,3$ \\
\hline $\mathbf{U}$ & $\mathbf{0 , 0 4 9} \pm 0,003$ & $\mathbf{0 , 0 4 7} \pm 0,007$ & $+5,0$ & $\mathbf{2 , 3} \pm 0,3$ & $2,2 \pm 0,061$ & $+7,3$ & $3,2 \pm 0,2$ & 3,3 & $-2,8$ \\
\hline V & $\mathbf{0 , 6 1} \pm 0,03$ & $\mathbf{0 , 6 0} \pm 0,15$ & $+1,9$ & $128 \pm 5$ & $120 \pm 2,4$ & $+7,0$ & $109 \pm 3$ & 105 & $+3,7$ \\
\hline $\mathbf{W}$ & & & & & $1,15 \pm 0,03$ & & & 2,0 & \\
\hline $\mathbf{Y}$ & $\underline{0,58} \pm 0,02$ & $\mathbf{0 , 5 2} \pm 0,03$ & & $17,5 \pm 0,6$ & $16,9 \pm 0,58$ & $+3,5$ & $31 \pm 0,9$ & 31 & $+0,1$ \\
\hline $\mathrm{Yb}$ & $\mathbf{0 , 0 3 8} \pm 0,002$ & $\mathbf{0 , 0 3 8} \pm 0,005$ & $+1,1$ & $1,82 \pm 0,04$ & $1,65 \pm 0,036$ & +11 & $3,3 \pm 0,2$ & 2,9 & +14 \\
\hline $\mathrm{Zn}$ & $35 \pm 1,3$ & $35 \pm 2$ & $-0,2$ & $67 \pm 3$ & $65 \pm 2,3$ & $+4,1$ & $124 \pm 4$ & 115 & $+8,1$ \\
\hline $\mathrm{Zr}$ & & & & $115 \pm 8$ & $109 \pm 2,6$ & $+5,9$ & $166 \pm 8$ & 180 & $-8,0$ \\
\hline Fett: & Zertifizierter Wer & rt (mit mittlerem $\mathrm{F}$ & Fehler) & & & & & & \\
\hline Normal: & Richtwert (mit mi & ittlerem Fehler) o & der We & ur Information & & & & & \\
\hline Unterstrichen: & Selbst festgelegt & ter Richtwert anh & land vie & Messungen & & & & & \\
\hline *: & Hausinterne Ref & erenz & & & & & & & \\
\hline
\end{tabular}


Die beiden Multielementanalysemethoden ICP-OES (vgl. Kapitel 3.4.3) und ICP-MS (vgl. Kapitel 3.4.4) lieferten eine weite Bandbreite an Gehalten bis in den Spuren- und Ultraspurenelementbereich. Die folgenden Elemente konnten nicht bestimmt werden: Bor (B), Kohlenstoff (C), Quecksilber (Hg), Sauerstoff (O), Silicium (Si), Stickstoff (N), Wasserstoff $(\mathrm{H})$ sowie die Halogene und Edelgase, die sich beim Säuretotalaufschluss verflüchtigen bzw. in Wasser, den Säuren und dem Messgas vorhanden sind; weitere Elemente, die mit Interferenzen behaftet sind oder nicht stabil in Lösung gehalten werden können bzw. immer unter der Nachweisgrenze liegen: beispielsweise Gold ( $\mathrm{Au}$ ), Iridium (Ir), Osmium (Os), Palladium (Pd), Platin (Pt), Ruthenium (Ru), Silber (Ag), Tellur (Te) und Wolfram (W).

Aufgrund von spektralen Interferenzen oder höheren Nachweisgrenzen konnten bei den ICP-OES-Messungen die Elemente Gallium (Ga), Niob (Nb), Selen (Se), Zinn (Sn) sowie Zirconium $(\mathrm{Zr})$, die im Kalibrationslösungsset enthalten waren, nach Vergleich mit den Bezugswerten der Pflanzenreferenzproben nicht für die Dateninterpretation verwendet werden. Beryllium (Be) war Element des internen Standards für die ICP-MSMessungen (vgl. Kapitel 3.4.4), wodurch die originären Probenkonzentrationen nicht quantifizierbar waren. Die Exaktheit der Zugabe des internen Standards beim Säuretotalaufschlussverfahren (vgl. Kapitel 3.3) konnte aber mittels der ICP-OES-Messungen überprüft werden und war generell ausgezeichnet.

Wegen der hohen Sensitivität der ICP-MS- gegenüber den ICP-OES-Messungen bei den Makronährelementen Calcium (Ca), Kalium (K), Magnesium (Mg), Natrium (Na) und Phosphor (P) bzw. Interferenzen oder höheren Nachweisgrenzen bei Chrom (Cr), Vanadium (V) und Zinn (Sn) sowie zu wenigen Bezugswerten bei den Pflanzenreferenzproben bei Gallium $(\mathrm{Ga})$ und Germanium $(\mathrm{Ge})$ konnten diese Elemente für die Dateninterpretation der ICP-MS-Messungen nicht herangezogen werden. Auch Indium (In), Rhenium (Re) und Rhodium (Rh) dienten neben Beryllium (Be) bei den ICP-MSMessungen als interne Standardelemente (vgl. Kapitel 3.4.4) und wurden nicht in der Elementauswahl berücksichtigt.

Für die Elementauswahl wurden bevorzugt die Konzentrationen von 23 Elementen aus den ICP-OES-Messungen und von 28 Elementen aus den ICP-MS-Messungen verwendet, die bezüglich ihrer Übereinstimmung mit den Referenzproben (Richtigkeit, Präzision) bzw. entsprechend günstigen Nachweisgrenzen verlässlich sind (Tabelle 5). 
Tabelle 5: $\quad$ Auswahl der bevorzugten Spektrallinien (ICP-OES) und Isotopenmassen (ICP-MS) für die Bestimmung der Elementkonzentrationen in den Gärgemisch- und Silage-Proben.

(ICP-OES: axialer Modus, wenn nicht rad = radialer Modus; ICP-MS:

$K E D=$ Kinetic Energy Discrimination, seit Sommer 2016 möglich).

\begin{tabular}{|c|c|c|c|}
\hline Symbol & Element & $\begin{array}{l}\text { ICP-OES } \\
\text { Spektrallinie [nm] }\end{array}$ & $\begin{array}{l}\text { ICP-MS } \\
\text { Isotopenmasse }\end{array}$ \\
\hline $\mathbf{A l}$ & Aluminium & 396,152 & \\
\hline As & Arsen & 188,980 & 75 KED \\
\hline $\mathbf{B a}$ & Barium & $455,403 \mathrm{rad}$ & 137 \\
\hline $\mathbf{B i}$ & Bismut & & 209 \\
\hline $\mathrm{Ca}$ & Calcium & $317,933 \mathrm{rad}$ & \\
\hline Cd & Cadmium & 228,802 & 114 KED \\
\hline $\mathrm{Ce}$ & Cer & 418,659 & 140 \\
\hline Co & Cobalt & 230,786 & 59 KED \\
\hline $\mathrm{Cr}$ & Chrom & 267,716 & \\
\hline Cs & Caesium & & 133 \\
\hline $\mathrm{Cu}$ & Kupfer & 327,393 & 65 \\
\hline Dy & Dysprosium & & 163 \\
\hline $\mathrm{Er}$ & Erbium & & 166 \\
\hline Eu & Europium & & 151 \\
\hline $\mathrm{Fe}$ & Eisen & 234,350 & \\
\hline Gd & Gadolinium & & 157 \\
\hline Hf & Hafnium & & 178 \\
\hline Ho & Holmium & & 165 \\
\hline $\mathbf{K}$ & Kalium & $766,491 \mathrm{rad}$ & \\
\hline La & Lanthan & 408,671 & 139 \\
\hline Li & Lithium & 670,783 & 7 \\
\hline Lu & Lutetium & & 175 \\
\hline Mg & Magnesium & $279,078 \mathrm{rad}$ & \\
\hline Mn & Mangan & 257,610 & 55 KED \\
\hline Mo & Molybdän & 202,032 & 98 \\
\hline $\mathrm{Na}$ & Natrium & $588,995 \mathrm{rad}$ & \\
\hline Nb & Niob & & 93 \\
\hline Nd & Neodym & & 146 \\
\hline $\mathrm{Ni}$ & Nickel & 231,604 & 62 KED \\
\hline $\mathbf{P}$ & Phosphor & 178,222 & \\
\hline $\mathrm{Pb}$ & Blei & 220,353 & 206 \\
\hline $\mathrm{Pr}$ & Praseodym & & 141 \\
\hline Rb & Rubidium & & 85 \\
\hline $\mathbf{S}$ & Schwefel & 181,972 & \\
\hline $\mathrm{Sb}$ & Antimon & & 121 \\
\hline Sc & Scandium & 335,372 & 45 KED \\
\hline Se & Selen & & 77 KED \\
\hline Sm & Samarium & & 147 \\
\hline Sr & Strontium & 421,552 rad & 88 \\
\hline $\mathrm{Ta}$ & Tantal & & 181 \\
\hline Tb & Terbium & & 159 \\
\hline Th & Thorium & & 232 \\
\hline $\mathrm{Ti}$ & Titan & 368,520 & \\
\hline TI & Thallium & & 205 \\
\hline Tm & Thulium & & 169 \\
\hline $\mathbf{U}$ & Uran & & 238 \\
\hline V & Vanadium & 292,401 & \\
\hline $\mathbf{Y}$ & Yttrium & & 89 \\
\hline Yb & Ytterbium & & 174 \\
\hline $\mathrm{Zn}$ & Zink & 206,200 & 66 KED \\
\hline $\mathrm{Zr}$ & Zirconium & & 90 \\
\hline
\end{tabular}

Fett: Bevorzugte Spektrallinie oder Isotopenmasse für die Elementauswahl 


\subsubsection{Nickel-Chrom-Problematik bei den Laborfermenterversuchen}

Bei den (quasi)kontinuierlichen Nassfermentationsversuchen am DBFZ fielen im Zuge der Auswertungen der Elementdaten auffällige Anstiege der Chromkonzentrationen in allen Projektphasen auf. In der ersten Projektphase war der Referenzreaktor gegen Ende der Versuchsreihe betroffen (vgl. Kapitel 4.3.5), in der zweiten und dritten Projektphase vier der fünf Versuchsbiogasreaktoren (vgl. Kapitel 4.4.4 und 4.5.4) sowie im Zusatzversuch beide Reaktoren (vgl. Kapitel 4.6.3).

Nach Überprüfung aller mittels ICP-OES bzw. ICP-MS gemessenen Elementgehalte in den Reaktorproben kann festgestellt werden, dass die Konzentrationsanstiege von Chrom genau mit denen der Nickelgehalte korrelieren (Diagramme hierzu sind in Kapitel 4.5.4 aufgeführt). Es muss sich demnach um eine systematische Fehlerquelle handeln. Die zugeführten Mengen der Spurenelementlösung (welche kein Chrom enthielt) können nicht verantwortlich sein. Die in Betracht zu ziehenden Mais-Silagen sowie die bei den Substitutionsversuchen eingesetzten weiteren EnergiepflanzenSilagen können auch ausgeschlossen werden, da solche hohen Konzentrationen in den Silagen selbst nicht nachgewiesen wurden (vgl. Kapitel 4.2). Zudem müssten in diesem Fall alle Reaktoren gleichermaßen von dieser Problematik betroffen sein. Auch andere systematische Fehler wie z.B. Probenverschleppungen oder Probenverunreinigungen können seitens der sorgfältigen und kontaminationsfreien Probenaufbereitung und Messanalytik ebenfalls ausgeschlossen werden (vgl. Kapitel 3.2, 3.3, 3.4.3 und 3.4.4). Eine Kontamination durch die Rührkesselreaktoren scheint auch nicht möglich, da sie aus Kunststoff bestehen.

In Rücksprache mit dem Verbundpartner am DBFZ können als Quelle dieser NickelChrom-Problematik höchstwahrscheinlich nur die mittig in den Reaktoren fixierten Ankerrührer mit den Rührblättern in Frage kommen. Sie bestehen aus rostfreiem Edelstahl, der laut DIN EN 10088-1:2005 Chrom und Nickel als Hauptlegierungselemente enthält, mit mindestens einem Chrom-Anteil von 10,5\%. Die genaue Zusammensetzung des Edelstahls konnte nicht bestimmt werden aufgrund nicht vorliegender Herstellerangaben bzw. nicht mehr durchführbaren Elementanalysen. So traten vermutlich über die langen Versuchszeiträume Abrasions- oder Ablösungsvorgänge an den metallischen Oberflächen durch organische Liganden aus der Biomasse oder durch mikrobielle Interaktionen auf. Die genaue Ursache dieser Nickel-Chrom-Problematik kann letztlich jedoch nicht abschließend geklärt werden. 


\section{Kapitel 4: (Quasi)kontinuierliche Laborfermenterversuche}

\subsection{Versuchsaufbau und Durchführung}

Für die drei Projektphasen der (quasi)kontinuierlichen Laborfermenterversuche am DBFZ wurde in den fünf zylinderförmigen, doppelwandigen Rührkesselreaktoren aus Kunststoff (Reaktor 4.5 bis 4.9, Nennvolumen: 15 Liter) ein Arbeitsvolumen von 8 Litern gewählt. In Abbildung 6 ist schematisch der Aufbau eines Versuchsbiogasreaktors im Querschnitt dargestellt (modifiziert nach SCHIERITZ, 2016).
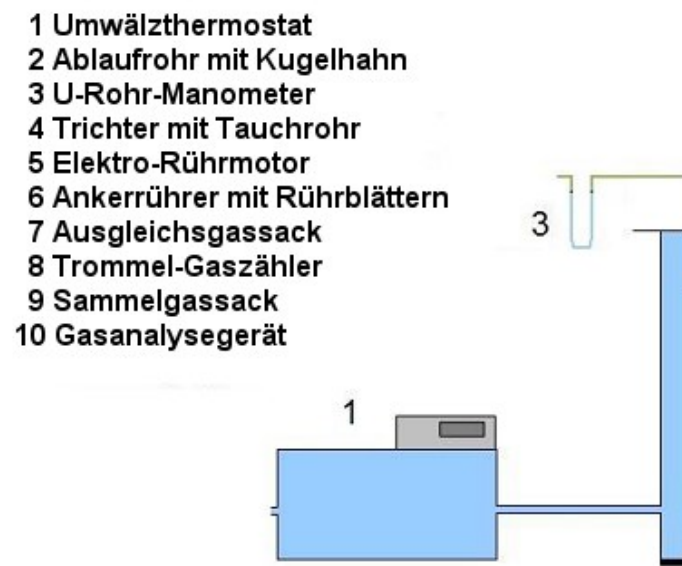

3

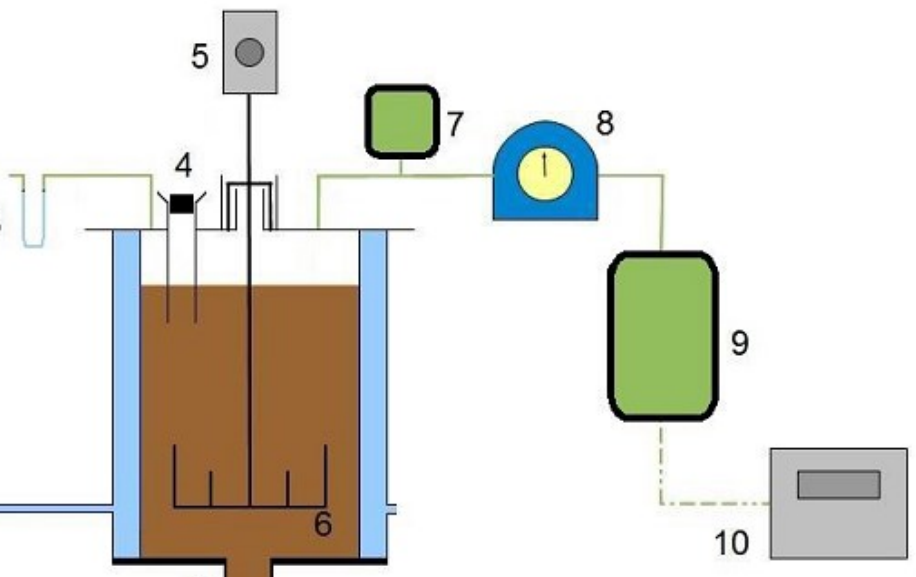

$2+$

Abbildung 6: $\quad$ Schematischer Querschnitt und Aufbau eines im Verbundprojekt betriebenen Versuchsbiogasreaktors am DBFZ (modifiziert nach SCHIERITZ, 2016).

Die Beschickung mit den Silagen, Spurenelementadditiven und destilliertem Wasser wurde quasikontinuierlich einmal täglich durchgeführt, jeweils über einen Trichter mit Tauchrohr aus Edelstahl, dessen unteres Ende zur Vermeidung von Biogasverlusten bzw. eines zu starken Lufteintrages in den oberen Bereich des Fermenterinhaltes eintauchte. Der mit einem Gummistopfen luftdicht verschlossene Trichter wurde nur zur Substratzugabe geöffnet.

Die Durchmischung des Fermentermaterials erfolgte kontinuierlich mittels eines Elektro-Rührmotors (Heidolph RZR 2102 control) und eines jeweils mittig im Reaktor fixierten Ankerrührers aus Edelstahl mit vier vertikal orientierten Rührblättern. Die Rührgeschwindigkeit lag bei allen Versuchen konstant bei $20 \mathrm{rpm}$ (20 Umdrehungen pro Minute). 
Neben dem Trichter mit Tauchrohr und der Rührwelle mit Ankerrührer waren im transparenten Reaktordeckel jeweils zwei gasdichte Schlauchsysteme angebracht. Ein Schlauchsystem fungierte dabei als U-Rohr-Manometer (mit destilliertem Wasser gefüllt) zur Kontrolle des Reaktorinnendrucks, das andere führte zu einem TrommelGaszähler (Ritter TG05) zur Messung der Gasvolumenströme. Von dort aus wurde das Biogas in einen Sammelgassack geführt und gespeichert. Die Gaszusammensetzung wurde zweimal täglich automatisch mit dem Gasanalysegerät (Awite AwiFLEX Serie 7) gemessen. Vor dem Trommel-Gaszähler befand sich zusätzlich ein weiterer gasdichter Schlauch, der aus einem Ausgleichsgassack einen im Reaktor entstehenden Unterdruck im Bedarfsfall ausgleichen (z.B. bei der Gärrestentnahme) oder in den Biogas strömen konnte (z.B. beim Abklemmen der Zufuhr zum Gaszähler bei der Substratzugabe).

In den ebenen Böden der Reaktoren waren senkrechte Ablaufrohre mit Kugelhähnen zur Entnahme der Reaktormaterialien eingelassen. Um die Füllstände bzw. Arbeitsvolumina konstant bei 8 Litern zu halten, wurden aus den Reaktoren an Werktagen (überwiegend täglich) Gärreste entsprechend den Beschickungsmengen entnommen. An den Wochenenden oder Tagen ohne Gärrestentnahme stiegen die Reaktorvolumina leicht an. Die Beschickungsmengen wurden in diesen Fällen dann auf die leicht höheren Volumina angepasst, um die Raumbelastung konstant zu halten.

Die Versuche wurden unter mesophilen Bedingungen bei einer Kerntemperatur von $38^{\circ} \mathrm{C}$ bzw. einer Vorlauftemperatur von $40^{\circ} \mathrm{C}$ durchgeführt. Die Temperierung der Reaktoren wurde mittels eines beheizten Wasserkreislauf- bzw. -pumpsystems in den Zwischenräumen der Doppelwände der Reaktoren über ein Umwälzthermostat (Julabo EC-13) gewährleistet. Die Außenwände der Reaktoren waren nahezu vollständig mit Schaumstoff isoliert, um einen Wärmetransport bzw. Wärmeverluste nach außen zu verringern. Lediglich ein schmales Sichtfenster zur Füllstandskontrolle blieb offen.

In allen Projektphasen wurden die Biogasreaktoren bei einer Raumbelastung von 5 $\left[\mathrm{B}_{\mathrm{R}}=5 \mathrm{~g}_{\mathrm{oTS}} /(\mathrm{L} \cdot \mathrm{d})\right]$ und einer mittleren hydraulischen Verweilzeit von 40 Tagen $(\mathrm{HRT}=40 \mathrm{~d})$ betrieben. Diese Raumbelastung lag im oberen Hochlastbereich der für Biogasanlagen empfohlenen täglichen Fracht an organischer Trockensubstanz und wurde in Kombination mit der dadurch bedingten, kürzeren Verweilzeit gewählt, um frühzeitig Mangelzustände bzw. positive oder negative Effekte zu erkennen. Parallel zu den fünf Rührkesselreaktoren wurde für das Impfmaterial ein 100-Liter-Inokulumsreaktor (Reaktor 3.2) mit den gleichen Belastungsparametern unterhalten. 
Die täglichen Beschickungsmengen bzw. Substratvolumenströme wurden über die Arbeitsvolumina der Reaktoren ( $8 \mathrm{~L}$ ) und die mittlere hydraulische Verweilzeit (40 d) berechnet und lagen bei $200 \mathrm{~mL}$ pro Tag. Sie setzten sich zusammen aus a) den entsprechenden Hauptsubstraten der Pflanzensilagen, b) destilliertem Wasser, das vor allem zur Einstellung der mittleren hydraulischen Verweilzeit und zum Niedrighalten der Viskositäten der Reaktorinhalte gebraucht wurde, sowie c) ggf. Spurenelementadditiven, die als Lösungen nach den Optimalwerten von OECHSNER ET AL. (2008) im Labor aus den entsprechenden Salzverbindungen hergestellt wurden (Tabelle 6). Die Zugabe in die Versuchsbiogasreaktoren erfolgte quasikontinuierlich einmal täglich. Sobald in einem der fünf Versuchsbiogasreaktoren die Ammoniumstickstoff $\left(\mathrm{NH}_{4}-\mathrm{N}\right)$ Konzentration von $2 \mathrm{~g} / \mathrm{L}$ unterschritten wurde, erfolgte die Zugabe von einer auf diesen Wert berechneten Menge an Harnstoff $\left(\mathrm{CH}_{4} \mathrm{~N}_{2} \mathrm{O}\right)$, um eine kontinuierlich konstante, gesicherte Versorgung mit Stickstoff für die Mikroorganismen zu gewährleisten bzw. einer Unterversorgung entgegenzuwirken.

Tabelle 6: $\quad$ Zusammensetzung der im Verbundprojekt am DBFZ hergestellten und verwendeten Spurenelementlösung bei Vollversorgung.

\begin{tabular}{|c|c|c|c|c|c|c|}
\hline \multirow[t]{2}{*}{ Symbol } & \multirow[t]{2}{*}{ Element } & \multirow[t]{2}{*}{$\begin{array}{l}\text { Chemische } \\
\text { Verbindung }\end{array}$} & $\begin{array}{r}\text { Optimalwert } \\
\text { Oechsner et al. } \\
(2008)\end{array}$ & $\begin{array}{r}\text { Lösungs- } \\
\text { konzentration }\end{array}$ & $\begin{array}{r}\text { Tägliche } \\
\text { Zugabemenge } \\
\text { der Verbindung }\end{array}$ & \multirow{2}{*}{$\begin{array}{r}\text { Tägliche } \\
\text { Zugabemenge* } \\
\text { Spurenelement } \\
{[\mathrm{mg} / \mathrm{d}]}\end{array}$} \\
\hline & & & {$\left[\mathrm{mg} / \mathrm{kg}_{\mathrm{TS}}\right]$} & {$[\mathrm{g} / \mathrm{L}]$} & {$[\mathrm{mg} / \mathrm{d}]$} & \\
\hline Co & Cobalt & $\mathrm{Co}(\mathrm{II}) \mathrm{Cl}_{2} \cdot 6 \mathrm{H}_{2} \mathrm{O}$ & 1,8 & 0,38 & 0,31 & 0,076 \\
\hline $\mathbf{N i}$ & Nickel & $\mathrm{Ni}(\mathrm{II}) \mathrm{Cl}_{2} \cdot 6 \mathrm{H}_{2} \mathrm{O}$ & 16 & 3,4 & 2,7 & 0,67 \\
\hline Mo & \multicolumn{2}{|c|}{ Molybdän $\left(\mathrm{NH}_{4}\right)_{2} \mathrm{MoO}_{4}$} & 4 & 0,51 & 0,34 & 0,168 \\
\hline $\mathrm{Fe}$ & Eisen & $\mathrm{Fe}(\mathrm{III}) \mathrm{Cl}_{3} \cdot 6 \mathrm{H}_{2} \mathrm{O}$ & 2400 & 610 & 489 & 101 \\
\hline Mn & Mangan & $\mathrm{Mn}(\mathrm{II}) \mathrm{Cl}_{2} \cdot 4 \mathrm{H}_{2} \mathrm{O}$ & 300 & 57 & 45,5 & 12,6 \\
\hline $\mathbf{Z n}$ & Zink & $\mathrm{ZnCl}_{2}$ & 200 & 22,3 & 17,5 & 8,4 \\
\hline Se & Selen & CaSe & 0,5 & 0,040 & 0,032 & 0,021 \\
\hline W & Wolfram & $\left(\mathrm{NH}_{4}\right)_{6} \mathrm{H}_{2} \mathrm{~W}_{12} \mathrm{O}_{40} \times \mathrm{XH}_{2} \mathrm{O}$ & 0,6 & 0,042 & 0,034 & 0,0021 \\
\hline
\end{tabular}

*: pro 8 Liter Reaktorvolumen 0,8 mL Spurenelementlösung pro Tag

Weitere Details zum Versuchsaufbau und der Durchführung der Nassfermentationsversuche sind in den im Rahmen des Forschungsprojektes am DBFZ angefertigten Masterarbeiten von GRIMM (2016), SCHIERITZ (2016) und HEYNER (2017) sowie in den im Anhang dieser Arbeit befindlichen Reaktorprotokollen aufgeführt. Abbildung 7 zeigt Fotoaufnahmen der fünf (quasi)kontinuierlich betriebenen Versuchsbiogasreaktoren in den drei Projektphasen am DBFZ. 


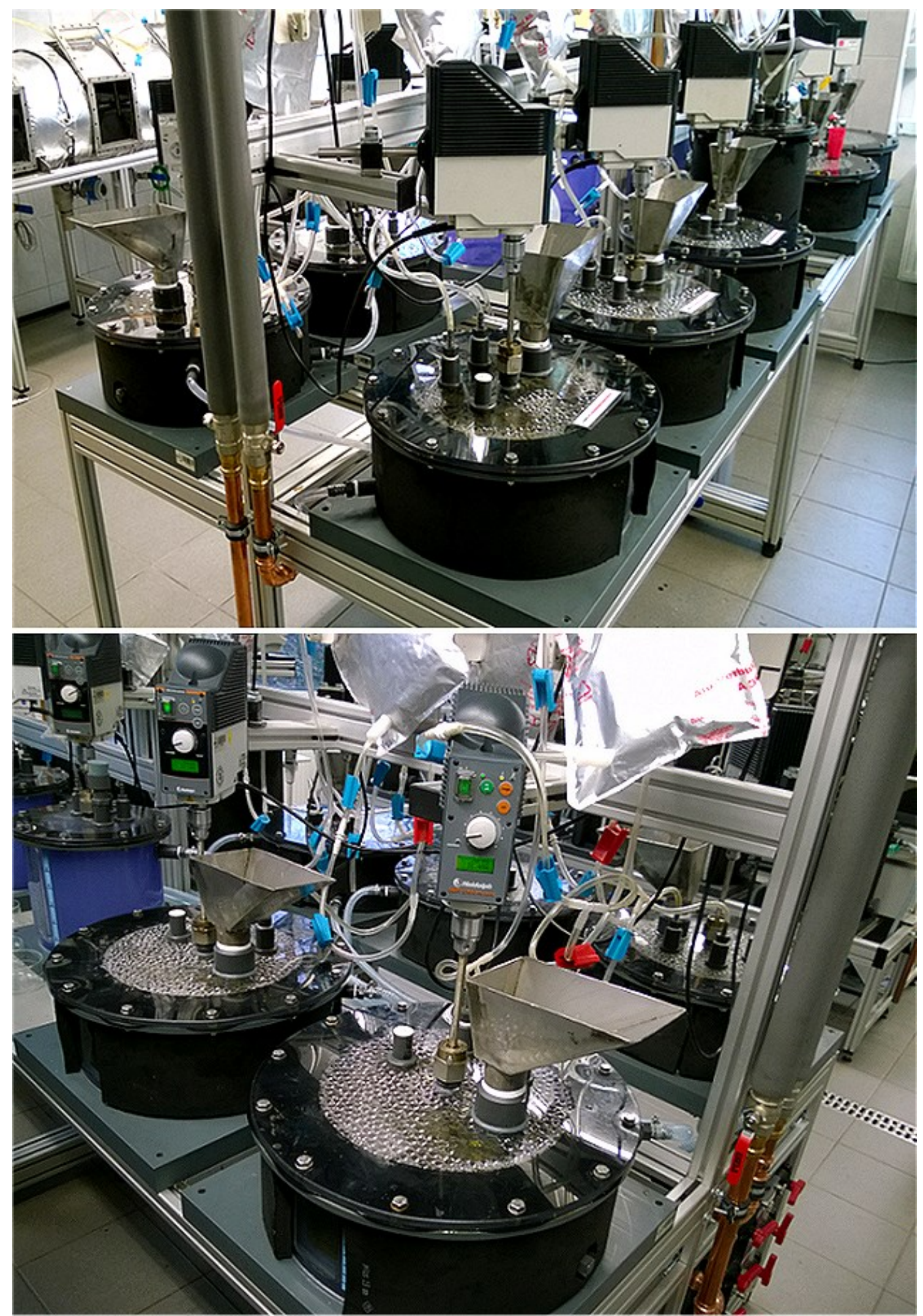

Abbildung 7: $\quad$ Fotoaufnahmen der fünf am DBFZ (quasi)kontinuierlich betriebenen Versuchsbiogasreaktoren ( $R$ 4.5 bis $R$ 4.9), die im Verbundprojekt benutzt wurden. 


\subsection{Verwendete Energiepflanzen-Silagen}

Der Anbau, die Ernten und die Silierung der für die (quasi)kontinuierlichen Nassfermentationsversuche vorgesehenen Energiepflanzenvarianten Winterackerbohne, Amarant, Deutsches Weidelgras und Durchwachsene Silphie wurden, wie in Kapitel 1 erwähnt, am Versuchsgut Reinshof (Schlag Garte Nord) von der Abteilung Pflanzenbau der Universität Göttingen vorgenommen. Nach der Zerkleinerung mit einem Laborhäcksler (Wintersteiger) wurde das jeweilige Erntegut mit einem Silierhilfsmittel (Kofasil ${ }^{\circledR}$ Liquid, 3 L/t Siliergut) versetzt, in 60 L-Kunststofffässer gefüllt und für den Silierprozess luftdicht verschlossen. Das Silierhilfsmittel wurde aufgrund von z.T. niedrigen Trockensubstanzgehalten der Energiepflanzenvarianten zur Vermeidung von Fehlgärungen gewählt. Außerdem wurden die Varianten vor der Silierung z.T. bis zu mehrere Tage anwelken gelassen, um die Trockensubstanzgehalte zu erhöhen. Das in den Laborfermenterversuchen eingesetzte Inputsubstrat Mais-Silage stammte von einer landwirtschaftlichen Biogasanlage aus der Nähe von Leipzig (Trebsen/Mulde, Ortsteil Neichen) und wurde als abgedeckte Schüttung auf dem Gelände des DBFZ gelagert. Jede neue Mais-Silage-Charge wurde am DBFZ einer Futtermittelanalyse unterzogen.

Repräsentative Probenaliquote der Energiepflanzen-Silagen wurden, wie in Kapitel 3 ausführlich beschrieben, in den Laboren am GZG aufbereitet, aufgeschlossen, die prozentualen Trockensubstanzgehalte (TS) und organischen Trockensubstanzgehalte (oTS) ermittelt sowie mittels ICP-OES und ICP-MS elementanalytisch untersucht. Am DBFZ erfolgte die Bestimmung gasanalytischer Parameter wie z.B. der spezifischen Biogas- und Methanerträge sowie der Volumenanteile an Methan und Kohlenstoffdioxid mittels diskontinuierlicher Batchversuche (Eudiometer-Versuche).

Die wichtigsten pflanzenbaulichen und gasanalytischen Parameter der verwendeten Energiepflanzen-Silagen fasst Tabelle 7 zusammen. Zum Vergleich der spezifischen Biogaserträge wurde für die vorliegende Arbeit ferner eine ORGA-Testreihe mit den in den Laborfermenterversuchen verwendeten Pflanzensilagen in Doppelwiederholung durchgeführt (Abbildung 8), analog zu den diskontinuierlichen ORGA-Testreihen zur Bestimmung von oberen Hemm- und Toxizitätskonzentrationen für verschiedene Spurenelemente (vgl. Kapitel 5). Eine detaillierte Beschreibung dieser ORGA-Testmethode ist in Kapitel $5.2 \mathrm{zu}$ finden. 
Kapitel 4: (Quasi)kontinuierliche Laborfermenterversuche

Tabelle 7: $\quad$ Zusammenstellung wichtiger pflanzenbaulicher und gasanalytischer Parameter der verwendeten Energiepflanzen-Silagen für die (quasi)kontinuierlichen Nassfermentationsversuche am DBFZ.

\begin{tabular}{|c|c|c|c|c|c|c|}
\hline \multicolumn{2}{|l|}{ Parameter } & Mais & $\begin{array}{l}\text { Winter- } \\
\text { ackerbohne }\end{array}$ & Amarant & $\begin{array}{l}\text { Durchwachsene } \\
\text { Silphie }\end{array}$ & $\begin{array}{l}\text { Deutsches } \\
\text { Weidelgras }^{1} \\
\end{array}$ \\
\hline \multicolumn{2}{|c|}{ Botanische Bezeichnung } & Zea mays L. & Vicia faba $\mathrm{L}$. & $\begin{array}{l}\text { Amaranthus } \\
\text { cruentus L. }\end{array}$ & $\begin{array}{l}\text { Silphium } \\
\text { perfoliatum L. }\end{array}$ & $\begin{array}{l}\text { Lolium } \\
\text { perenne L. }\end{array}$ \\
\hline \multicolumn{2}{|c|}{ Anbausystem (Variante) } & & $\begin{array}{l}\text { Winter- } \\
\text { zwischenfrucht }^{*}\end{array}$ & $\begin{array}{l}\text { Sommer- } \\
\text { hauptfrucht }\end{array}$ & Mehrjährige Art & $\begin{array}{l}\text { Mehrjährige Art } \\
\text { ( }{ }^{1} \text { : Erster Schnitt) }\end{array}$ \\
\hline \multicolumn{2}{|l|}{ Sorte } & & Nordica & Bärnkrafft & N.L. Chrestensen & Alligator \\
\hline TS & [Gew.-\%] & 32,6 & 19,8 & 21,2 & 38,6 & 41,3 \\
\hline oTS & [Gew.-\%] & 96,2 & 90,4 & 84,9 & 89,3 & 90,4 \\
\hline $\begin{array}{l}\text { Spezifischer } \\
\text { Biogasertrag } \\
\text { (DBFZ) }\end{array}$ & {$\left[\mathrm{mL}_{\mathrm{N}} / \mathrm{g}_{\circ \mathrm{TS}}\right]$} & $583 \pm 15$ & $361 \pm 19$ & $463 \pm 13$ & $379 \pm 17$ & $508 \pm 13$ \\
\hline $\begin{array}{l}\text { Spezifischer } \\
\text { Biogasertrag } \\
\text { (ORGA) }\end{array}$ & {$\left[\mathrm{mL}_{N} / \mathrm{g}_{\circ \mathrm{TS}}\right]$} & $564 \pm 10$ & $363 \pm 1$ & $460 \pm 2$ & $410 \pm 14$ & $501 \pm 6$ \\
\hline $\begin{array}{l}\text { Spezifischer } \\
\text { Methanertrag } \\
\text { (DBFZ) }\end{array}$ & {$\left[\mathrm{mL}_{\mathrm{N}} \mathrm{CH}_{4} / \mathrm{g}_{\mathrm{oTS}}\right]$} & 327 & 220 & 284 & 217 & 282 \\
\hline $\mathrm{CH}_{4}$ & [Vol.-\%] & 56 & 61 & 61 & 57 & 55 \\
\hline $\mathrm{CO}_{2}$ & [Vol.-\%] & 44 & 39 & 39 & 43 & 45 \\
\hline \multicolumn{2}{|c|}{ Fugatfaktor (berechnet) } & 0,77 & 0,92 & 0,90 & 0,84 & 0,76 \\
\hline \multicolumn{2}{|c|}{$\begin{array}{l}\text { Aufkonzentrationsfaktor } \\
\text { (TS-bezogen, berechnet) }\end{array}$} & 3,4 & 1,68 & 1,89 & 1,71 & 2,4 \\
\hline \multicolumn{2}{|c|}{$\begin{array}{l}\text { Aufkonzentrationsfaktor } \\
\text { (FM-bezogen, berechnet) }\end{array}$} & 1,30 & 1,09 & 1,11 & 1,19 & 1,32 \\
\hline
\end{tabular}

*: Im Zweikulturnutzungssystem mit Mais

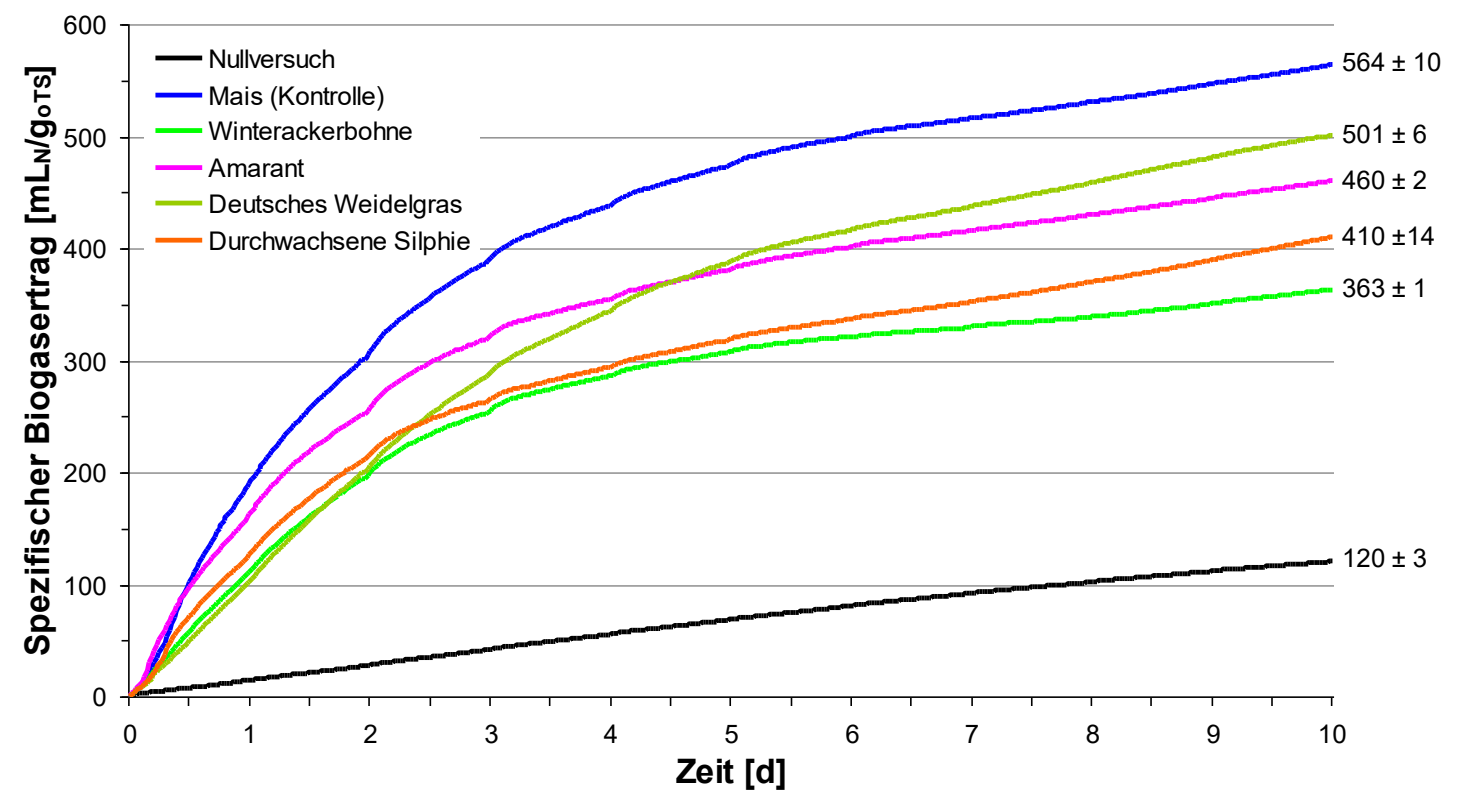

Abbildung 8: $\quad$ Verlauf der gemittelten Gassummenkurven der ORGA-Testreihe zur Ermittlung der spezifischen Biogaserträge der bei den (quasi)kontinuierlichen Laborfermenterversuchen verwendeten Energiepflanzen-Silagen 
Der Vergleich der spezifischen Biogaserträge der Silagen am Ende der ORGA-Testreihe (Abbildung 8) zeigt eine sehr gute Übereinstimmung mit den Eudiometer-Ergebnissen am DBFZ (Tabelle 7). Damit erweist sich der ORGA-Test als ein gut geeignetes, alternatives Verfahren zur Bestimmung der Gaserträge von Substraten im Biogasprozess. Die gemittelten, auf trockenes Gas und Normbedingungen $(273,15 \mathrm{~K}, 101,325 \mathrm{kPa})$ umgerechneten Methan $\left(\mathrm{CH}_{4}\right)$ - und Kohlenstoffdioxid $\left(\mathrm{CO}_{2}\right)$-Anteile im Biogas der Silagen von Durchwachsene Silphie (57 und 43 Vol.-\%) und Deutsches Weidelgras (55 und 45 Vol.-\%) unterscheiden sich gegenüber Mais-Silage (56 und 44 Vol.-\%) nur geringfügig. Die Silagen von Winterackerbohne und Amarant (beide 61 und 39 Vol.-\%) zeigen gegenüber Mais-Silage dagegen um fünf Volumenprozent höhere Methan- bzw. um fünf Volumenprozent niedrigere Kohlenstoffdioxidgehalte. Allerdings reichen die spezifischen Biogas- und Methanerträge der alternativen Energiepflanzen-Silagen nicht an die von Mais-Silage heran (Tabelle 7 und Abbildung 8). Zudem sind in Tabelle 7 berechnete Fugatfaktoren und Aufkonzentrationsfaktoren für die Silagen aufgeführt. Der Fugatfaktor beschreibt die Substratmassenverringerung bei der Konvertierung zu Biogas. Beispielsweise bedeutet ein Fugatfaktor von 0,77 bei der Mais-Silage, dass nach der Vergärung noch $77 \%$ der ursprünglichen Substratmasse vorhanden sind, also $23 \%$ in Biogas umgesetzt wurden. Der Aufkonzentrationsfaktor gibt in Anlehnung an SAUER (2010) einen durchschnittlichen theoretischen Wert für die Elementanreicherung von den gefütterten Substraten (Input) bis zum Gärrest (Output) an. Seine Berechnung basiert auf dem Trockensubstanzgehalt des jeweils eingesetzten Substrates unter Einbeziehung des zugehörigen Fugatfaktors. Er ist bei der Abschätzung von Outputkonzentrationen aller Elemente, die im anaeroben Vergärungsprozess nicht über das Biogas abgeführt werden, ein wichtiger Parameter. Durch Wasserzugabe verringert sich der Aufkonzentrationsfaktor aufgrund der Verdünnungswirkung entsprechend.

Tabelle 8 zeigt eine Zusammenstellung der Durchschnittsgehalte der EnergiepflanzenSilagen aller in der Multielementauswahl berücksichtigten gemessenen Elemente (vgl. Kapitel 3.5.2). Die vier Alternativsilagen weisen für einige der essenziellen Makro- und Mikronährstoffe deutlich höhere Konzentrationen gegenüber der spurenelementarmen Mais-Silage auf. Zur Verdeutlichung sind alle um Faktor 3 höheren Gehalte gegenüber Mais-Silage fett gedruckt, alle um Faktor 3 verarmten Gehalte unterstrichen. Nähere Erläuterungen $\mathrm{zu}$ einzelnen Elementgehalten und deren Einflüsse auf die Biogasprozesse in den Versuchen sind in den zugehörigen Kapiteln 4.3 bis 4.6 beschrieben. 
Tabelle 8: $\quad$ Durchschnittsgehalte [ $\left.\mathrm{mg} / \mathrm{kg}_{F M}\right]$ der verwendeten EnergiepflanzenSilagen für die (quasi)kontinuierlichen Nassfermentationsversuche.

\begin{tabular}{|c|c|c|c|c|c|}
\hline $\begin{array}{l}\left.\text { [mg/kg } \mathbf{g}_{\mathrm{FM}}\right] \\
\text { Element }\end{array}$ & $\begin{array}{l}\text { Mais } \\
(n=11)\end{array}$ & $\begin{array}{c}\text { Winter- } \\
\text { ackerbohne } \\
(n=3)\end{array}$ & $\begin{array}{l}\text { Amarant } \\
(n=4)\end{array}$ & $\begin{array}{c}\text { Durchwachsene } \\
\text { Silphie } \\
(n=3)\end{array}$ & $\begin{array}{c}\text { Deutsches } \\
\text { Weidelgras* } \\
(n=3)\end{array}$ \\
\hline Al & 30 & 66 & 86 & 71 & 120 \\
\hline As & 0,021 & 0,018 & 0,019 & 0,021 & 0,026 \\
\hline $\mathrm{Ba}$ & 1,4 & 1,4 & 4,3 & 5,8 & 6,8 \\
\hline $\mathbf{B i}$ & 0,0009 & 0,0034 & 0,0020 & 0,0014 & 0,0026 \\
\hline $\mathrm{Ca}$ & 670 & 1600 & 4900 & 7400 & 1600 \\
\hline Cd & 0,019 & $\underline{0,005}$ & 0,026 & 0,009 & 0,008 \\
\hline $\mathrm{Ce}$ & 0,04 & $\overline{0,10}$ & 0,14 & 0,14 & 0,20 \\
\hline Co & 0,010 & 0,032 & 0,020 & 0,025 & 0,023 \\
\hline $\mathrm{Cr}$ & 0,10 & 0,24 & 0,18 & 0,19 & 0,32 \\
\hline Cs & 0,004 & 0,008 & 0,016 & 0,008 & 0,011 \\
\hline $\mathrm{Cu}$ & 1,4 & 1,7 & 1,1 & 1,6 & 1,5 \\
\hline Dy & 0,003 & 0,006 & 0,008 & 0,007 & 0,011 \\
\hline $\mathrm{Er}$ & 0,0017 & 0,004 & 0,005 & 0,005 & 0,007 \\
\hline Eu & 0,0006 & 0,0015 & 0,0015 & 0,0017 & 0,0023 \\
\hline $\mathrm{Fe}$ & 28 & 55 & 46 & 45 & 70 \\
\hline Gd & 0,004 & 0,008 & 0,010 & 0,010 & 0,013 \\
\hline Hf & 0,005 & 0,013 & 0,018 & 0,016 & 0,025 \\
\hline Ho & 0,0007 & 0,0014 & 0,0018 & 0,0017 & 0,0026 \\
\hline $\mathbf{K}$ & 3400 & 6000 & 8300 & 8300 & 10500 \\
\hline La & 0,02 & 0,06 & 0,08 & 0,10 & 0,10 \\
\hline Li & 0,019 & 0,06 & 0,06 & 0,10 & 0,11 \\
\hline Lu & 0,0002 & 0,0006 & 0,0007 & 0,0007 & 0,0011 \\
\hline Mg & 520 & 360 & 850 & 1300 & 500 \\
\hline Mn & 7,5 & 7,4 & 9,0 & 14 & 30 \\
\hline Mo & 0,20 & 0,20 & 0,26 & $\underline{0,05}$ & 1,0 \\
\hline $\mathbf{N a}$ & 7,8 & 630 & 270 & 290 & 740 \\
\hline $\mathrm{Nb}$ & 0,011 & 0,04 & 0,06 & 0,05 & 0,08 \\
\hline Nd & 0,018 & 0,04 & 0,06 & 0,06 & 0,08 \\
\hline $\mathbf{N i}$ & 0,06 & 0,19 & 0,08 & 0,18 & 0,31 \\
\hline $\mathbf{P}$ & 710 & 660 & 920 & 630 & 1100 \\
\hline $\mathbf{P b}$ & 0,06 & 0,05 & 0,09 & 0,11 & 0,12 \\
\hline Pr & 0,005 & 0,011 & 0,015 & 0,013 & 0,017 \\
\hline $\mathbf{R b}$ & 1,2 & 2,3 & 8,3 & 2,9 & 3,5 \\
\hline $\mathbf{S}$ & 300 & 200 & 460 & 300 & 640 \\
\hline Sb & 0,003 & 0,005 & 0,005 & 0,008 & 0,010 \\
\hline Sc & 0,005 & 0,008 & 0,011 & 0,008 & 0,016 \\
\hline Se & 0,002 & 0,004 & 0,007 & 0,006 & 0,008 \\
\hline $\mathrm{Sm}$ & 0,003 & 0,008 & 0,011 & 0,010 & 0,014 \\
\hline $\mathrm{Sr}$ & 3,9 & 4,2 & 11 & 18 & 5,1 \\
\hline $\mathrm{Ta}$ & 0,0008 & 0,0019 & 0,002 & 0,002 & 0,004 \\
\hline Tb & 0,0015 & 0,0010 & 0,0013 & 0,0013 & 0,0019 \\
\hline Th & 0,005 & 0,018 & 0,02 & 0,017 & 0,03 \\
\hline $\mathrm{Ti}$ & 2,8 & 6,2 & 8,0 & 7,2 & 11 \\
\hline $\mathrm{TI}$ & 0,0007 & 0,0010 & 0,004 & 0,0015 & 0,0014 \\
\hline $\mathrm{Tm}$ & 0,0002 & 0,0005 & 0,0007 & 0,0007 & 0,0011 \\
\hline $\mathbf{U}$ & 0,0018 & 0,003 & 0,006 & 0,005 & 0,010 \\
\hline $\mathbf{V}$ & 0,06 & 0,11 & 0,12 & 0,11 & 0,17 \\
\hline $\mathbf{Y}$ & 0,016 & 0,04 & 0,05 & 0,05 & 0,07 \\
\hline $\mathrm{Yb}$ & 0,0017 & 0,004 & 0,005 & 0,005 & 0,008 \\
\hline $\mathrm{Zn}$ & 8,5 & 4,9 & 4,0 & 4,1 & 8,2 \\
\hline $\mathrm{Zr}$ & 0,18 & 0,58 & 0,84 & 0,74 & 1,1 \\
\hline & Erster Schnitt & & & & \\
\hline Fett: & Gegenüber Ma & $m$ mindecten & ktor 3 ange & & \\
\hline Unterstrichen: & Gegenüber Ma & $\mathrm{m}$ mindesten & ttor 3 verarn & & \\
\hline
\end{tabular}




\subsection{Verarmungsversuche für Cobalt und Nickel}

\subsection{1 Überblick}

In der ersten Projektphase der Laborfermenterversuche wurde systematisch untersucht, wie sich Cobalt- und Nickelmangelzustände auf (quasi)kontinuierliche Biogasprozesse auswirken und die mikrobiellen Gemeinschaften in ihrer Zusammensetzung und Aktivität beeinflussen. Ziel war es, minimale Schwellenwerte für die beiden Elemente Cobalt und Nickel einzugrenzen bzw. festzulegen. Dafür wurden u.a. wichtige prozessbeschreibende Parameter wie FOS (Kapp), FOS/TAC (FAL), pH-Wert, Ammoniumstickstoff $\left(\mathrm{NH}_{4}-\mathrm{N}\right)$, das organische Säurespektrum der Gärreste und Substrate sowie die normierte und spezifische Biogas- und Methanproduktion mit den Spurenelementkonzentrationen aus den Multielementmessungen verglichen. Eine ausführliche Methodenbeschreibung zur Bestimmung der physikochemischen und gasanalytischen Prozessparameter am DBFZ ist in LIEBETRAU ET AL. (2013) zusammengestellt. Tabelle 9 gibt einen Überblick über die einzelnen Prozessphasen der Verarmungsversuche für Cobalt und Nickel.

Tabelle 9: $\quad$ Überblick über die erste Projektphase der (quasi)kontinuierlichen Nassfermentationsversuche am DBFZ.

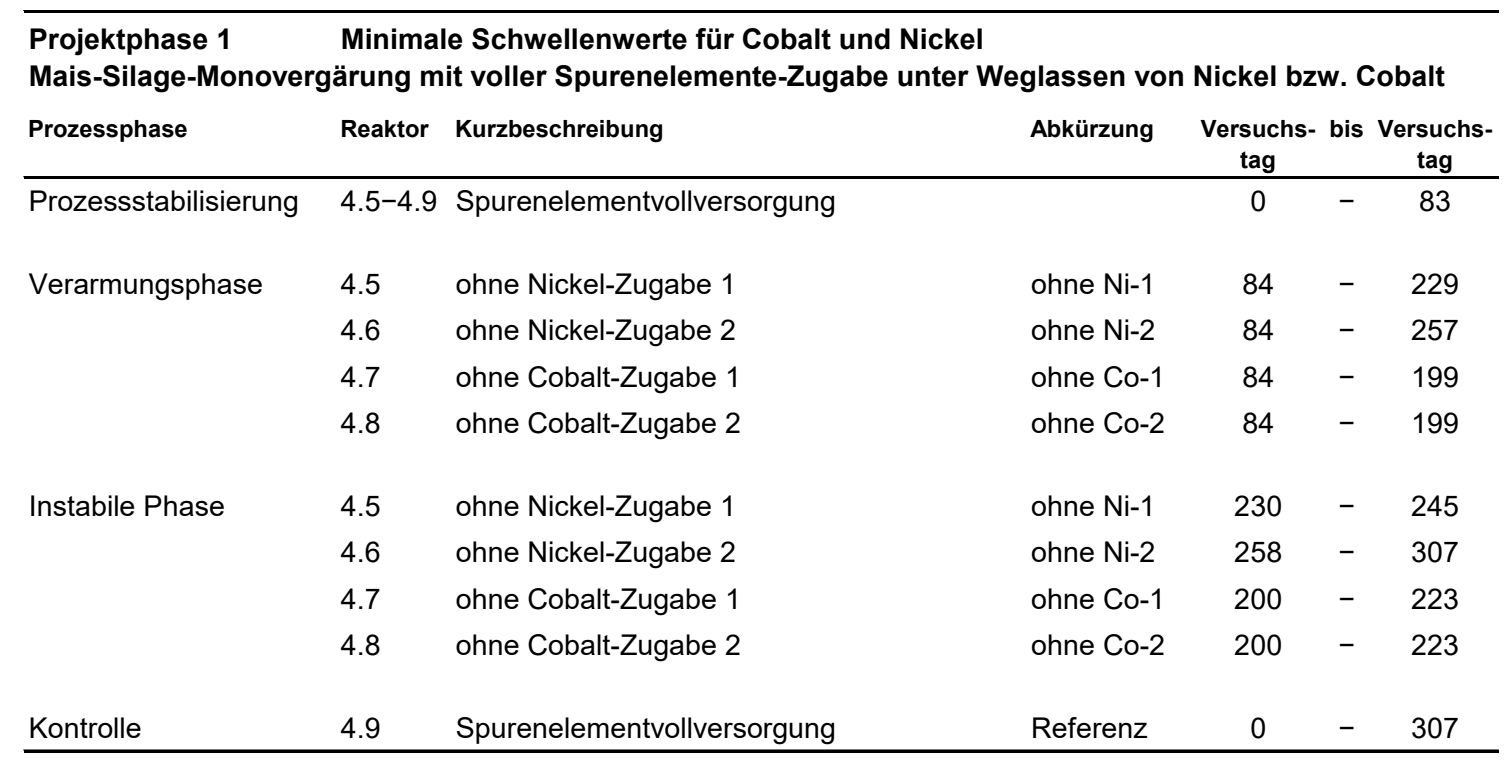

Zunächst musste mit Inbetriebnahme der fünf Versuchsbiogasreaktoren ein stabiles Prozessniveau zur Adaption der Mikroorganismen geschaffen werden (Phase der Prozessstabilisierung). Hierfür wurden alle fünf Reaktoren mit Gärmaterial aus dem Inokulumsreaktor (Reaktor 3.2) angeimpft. Das verwendete Inokulum war bereits seit 
ca. einem halben Jahr vor Versuchsstart gezielt auf die geplanten Versuche gezüchtet worden. Als Standard-Inputsubstrat wurde spurenelementarme Mais-Silage gewählt mit einer zusätzlichen synthetischen Spurenelementadditiv-Vollversorgung. Nach einer anfänglichen „Anfahrphase“ begann ab Versuchstag 19 die „Stabilisierungsphase“, die circa anderthalb Verweilzeiten bis Versuchstag 83 andauerte.

Nach der Etablierung stabiler Biogasprozesse in allen fünf Versuchsbiogasreaktoren wurden in der Verarmungsphase ab Versuchstag 84 jeweils zwei Reaktoren ohne zusätzliche Cobalt-Zugabe (Reaktor 4.7 und Reaktor 4.8) bzw. zwei Reaktoren ohne zusätzliche Nickel-Zugabe (Reaktor 4.5 und Reaktor 4.6) betrieben, wobei die Zusätze der anderen Spurenelemente weiterhin unverändert erfolgten. Als Kontrolle wurde parallel dazu der Referenzreaktor (Reaktor 4.9) mit synthetischer Spurenelementvollversorgung weiterbetrieben.

Im dritten Versuchsabschnitt der Verarmungsversuche, der instabilen Phase, wiesen die vier Mangelreaktoren Instabilitäten bei den physikochemischen und gasanalytischen Prozessparametern auf und die Biogasprozesse brachen erwartungsgemäß allmählich bzw. gegen Ende der Versuchsreihen komplett zusammen. Der Referenzreaktor lief über die gesamte Versuchsdauer stabil.

\subsubsection{Verarmungsversuch für Cobalt}

Nach Abgleich der physikochemischen und gasanalytischen Prozessparameter konnte der Beginn der instabilen Phase in den beiden Cobaltmangel-Reaktoren (Reaktor $4.7=$ ohne Co-1, Reaktor $4.8=$ ohne Co-2) auf Versuchstag 200 festgelegt werden, demnach drei Verweilzeiten nach Start der Verarmungsphase ab Versuchstag 84. Für diese zeitliche Festlegung wurden v.a. die steigenden Verläufe der FOS/TAC-Verhältnisse (nach FAL, Abbildung 9) und die auf Normbedingungen $(273,15 \mathrm{~K}, 101,325 \mathrm{kPa}$ ) umgerechneten sinkenden Volumenanteile an Methan (Abbildung 12) herangezogen.

Die Verläufe der pH-Werte (Abbildung 10) und der auf Normbedingungen umgerechneten Gasproduktion (Abbildung 11) in den beiden Cobaltmangel-Reaktoren begannen wenige Tage später ab Versuchstag 207 rapide zu fallen. Am Versuchstag 223, also nach dreieinhalb Verweilzeiten ohne zusätzliche Cobalt-Applikation, waren die Gasbildungsprozesse in den beiden Reaktoren zusammengebrochen und diese Versuchsreihe wurde beendet. Zum direkten Vergleich sind in den Abbildungen die Verläufe des Kontrollreaktors (Reaktor 4.9= Referenz) mit aufgeführt. 


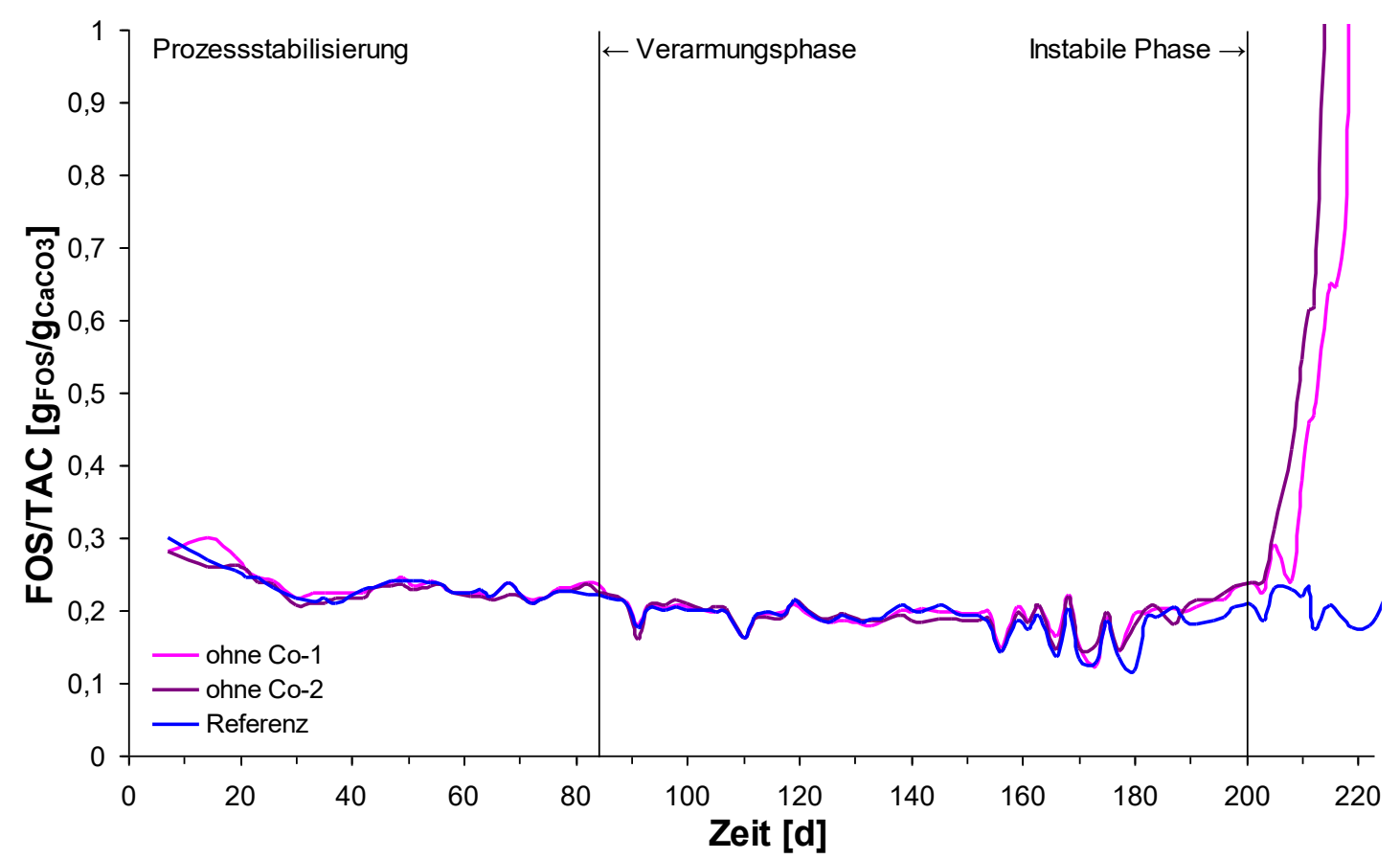

Abbildung 9: $\quad$ FOS/TAC (FAL)-Verläufe des Verarmungsversuchs für Cobalt in den Versuchsbiogasreaktoren (mit Referenzreaktor).

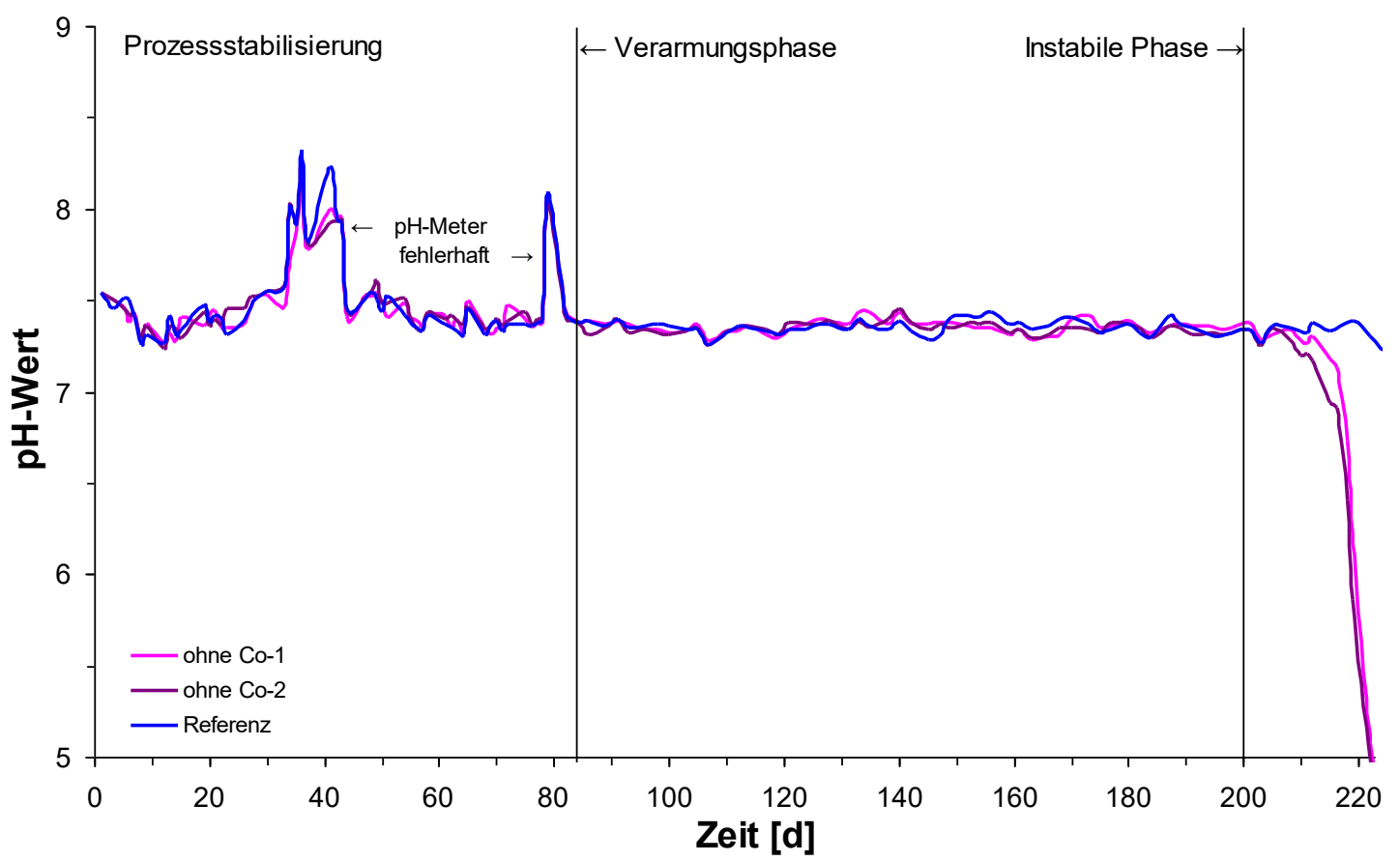

Abbildung 10: $\quad$ pH-Wert-Verläufe des Verarmungsversuchs für Cobalt in den Versuchsbiogasreaktoren (mit Referenzreaktor). 


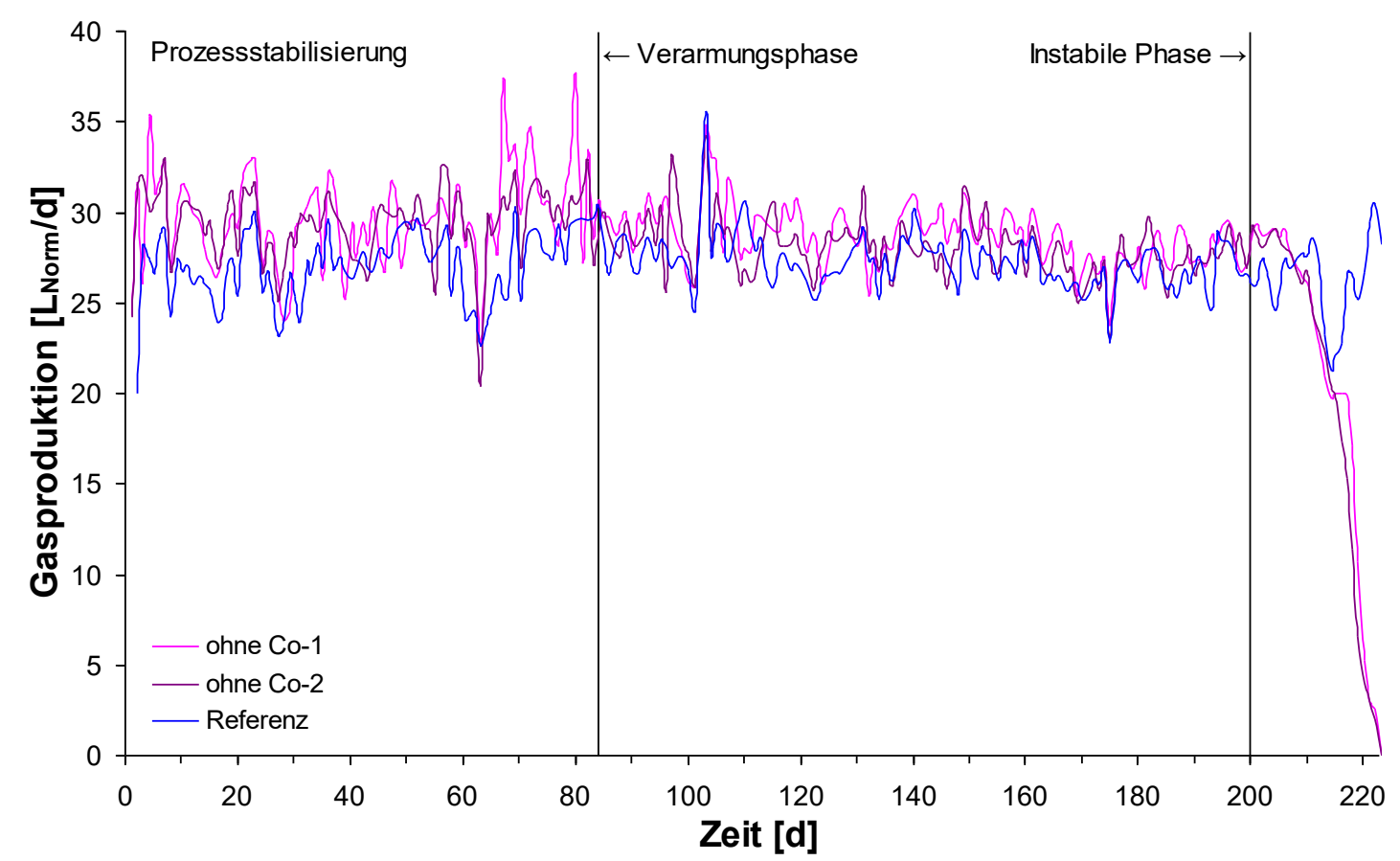

Abbildung 11: $\quad$ Verläufe der normierten Gasproduktion des Verarmungsversuchs für Cobalt in den Versuchsbiogasreaktoren (mit Referenzreaktor).

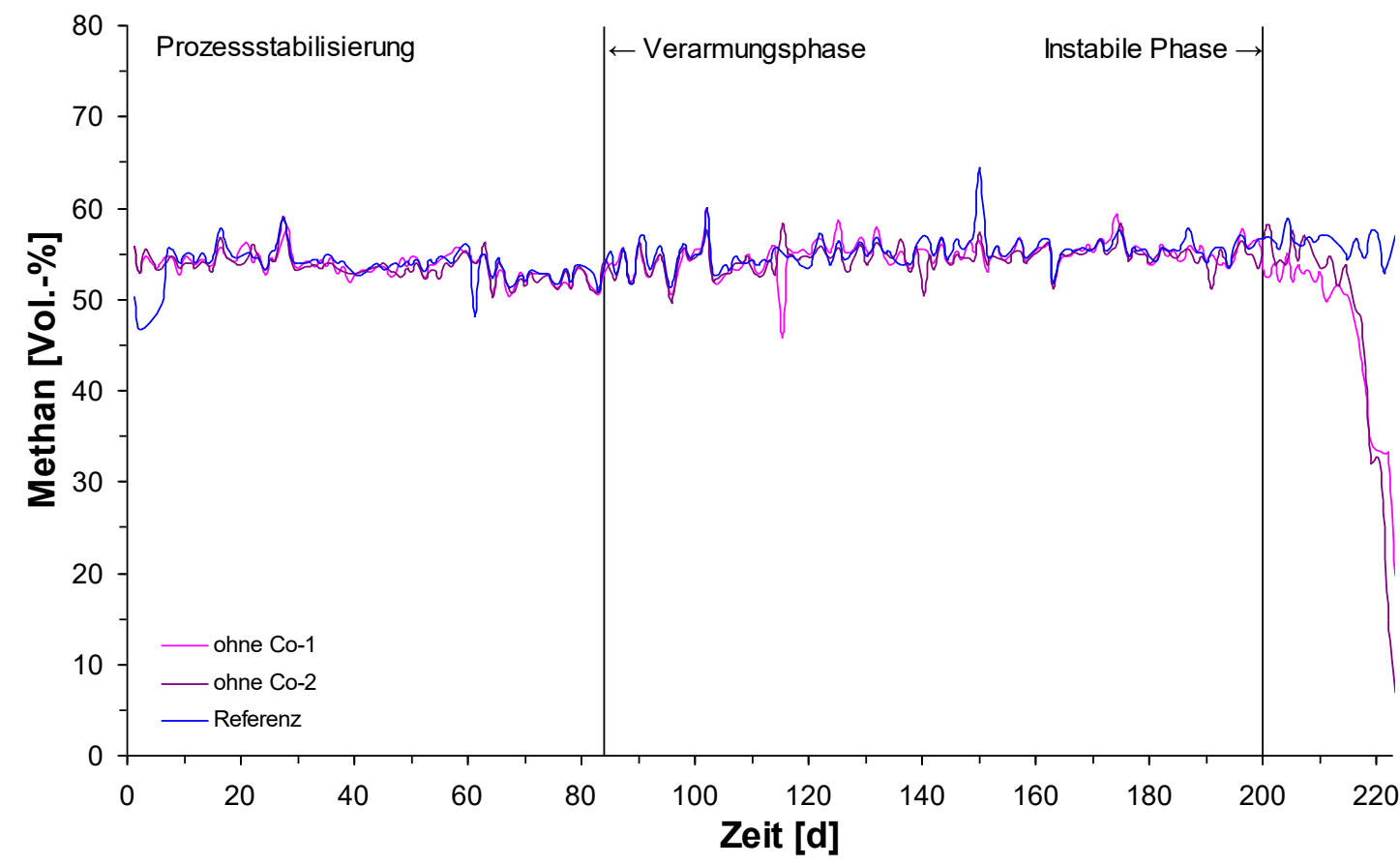

Abbildung 12: Verläufe des normierten Methangehalts des Verarmungsversuchs für Cobalt in den Versuchsbiogasreaktoren (mit Referenzreaktor).

Ferner war bei der Versuchsreihe mit Cobaltmangel in Abbildung 10 bis Abbildung 12 deutlich zu erkennen, dass sich in der Verarmungsphase die Verläufe der $\mathrm{pH}$-Werte, der normierten Gasproduktion und der normierten Methangehalte den Schwankungen der 
FOS/TAC (FAL)-Werte im Bereich der Versuchstage 154 bis 182 (Abbildung 9) nicht anschlossen, sondern bis zum steilen Anstieg der FOS/TAC (FAL)-Werte ab Versuchstag 203 in der instabilen Phase nahezu durchweg stabil verliefen und dann mit wenigen Tagen Verzögerung ab Versuchstag 207 schnell in negativer Korrelation mit den stark steigenden FOS/TAC (FAL)-Werten abfielen. Zum besseren Überblick sind in Abbildung 13 die Verläufe der FOS/TAC (FAL)-Werte und der normierten Gasproduktion direkt gegenübergestellt. Ab Versuchstag 207 in der instabilen Phase verschlechterten sich die Bedingungen für die Methanogenese derart, dass die entsprechenden Mikroorganismen ihren Stoffwechsel einstellten.

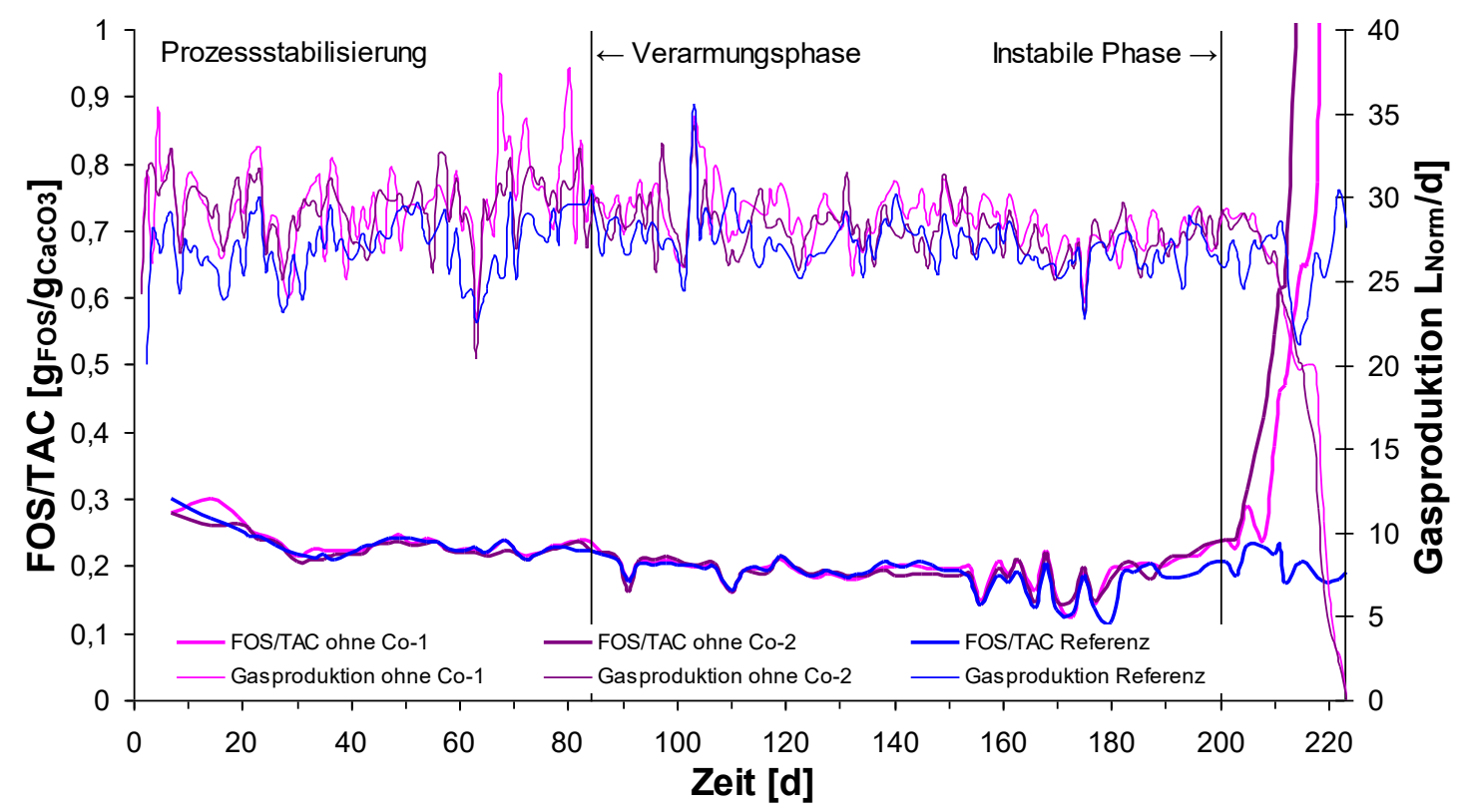

Abbildung 13: Gegenüberstellung der Verläufe der FOS/TAC (FAL)-Werte und der normierten Gasproduktion des Verarmungsversuchs für Cobalt in den Versuchsbiogasreaktoren (mit Referenzreaktor).

Für die Erarbeitung des minimalen Schwellenwertes für Cobalt wurden neben den bereits erwähnten Verläufen der physikochemischen und gasanalytischen Parametern FOS (Kapp), FOS/TAC (FAL), pH-Wert, normierte Biogas- und Methanproduktion, auch die Ergebnisse der mikrobiologischen Analysen vom UFZ (vgl. Kapitel 4.3.7) mit den ermittelten Spurenelementkonzentrationen in Bezug gesetzt und miteinander verglichen. Abbildung 14 zeigt die Verläufe der Cobaltkonzentrationen des Verarmungsversuchs bis zum Zusammenbruch der beiden Reaktoren. 


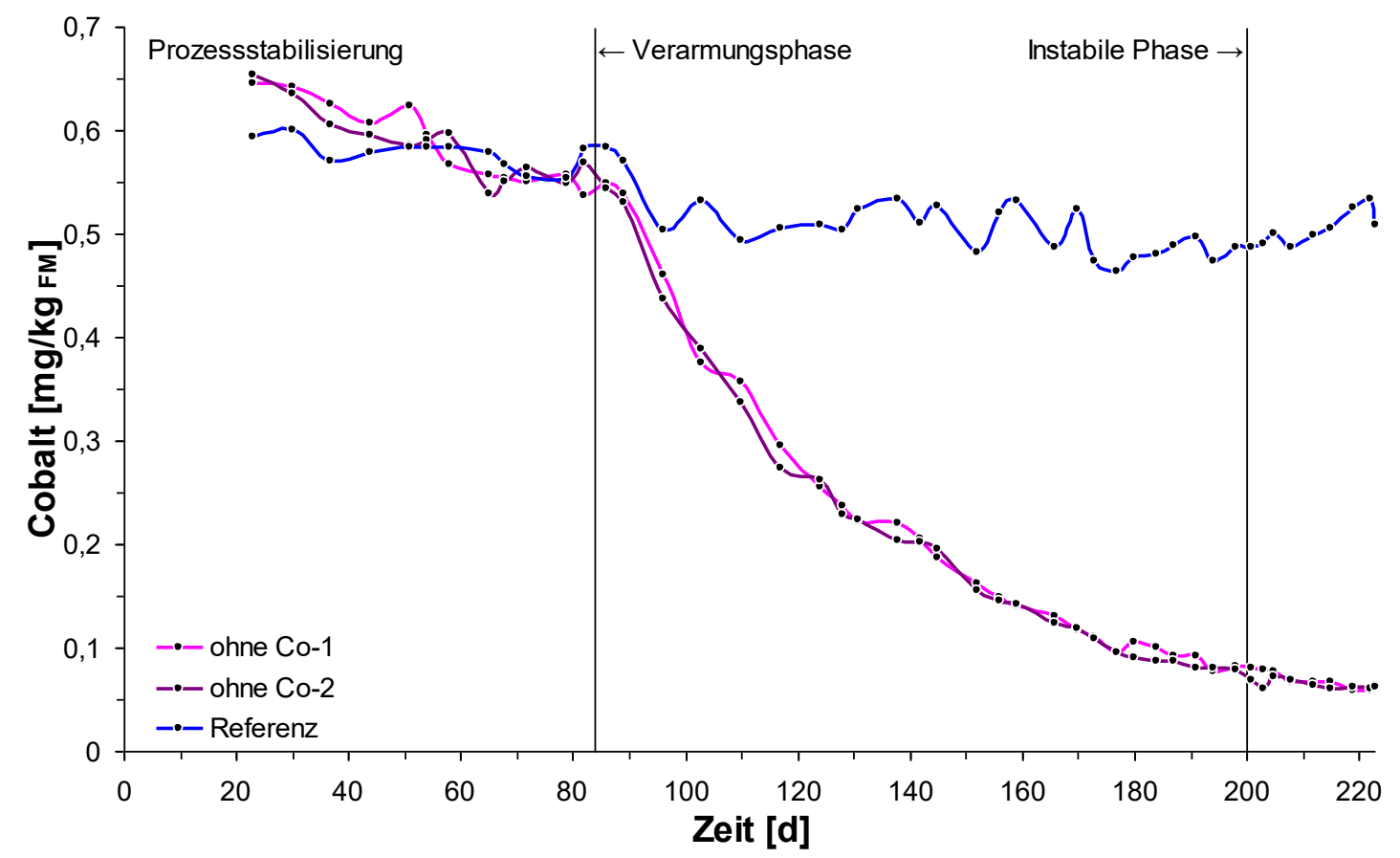

Abbildung 14: Verläufe der Cobaltkonzentrationen des Verarmungsversuchs für Cobalt in den Versuchsbiogasreaktoren (mit Referenzreaktor).

Bei der Versuchsreihe mit Cobaltmangel kann nach diesen Vergleichen der minimale Schwellenbereich bei Reaktor 4.7 (ohne Co-1) auf die Versuchstage 208 bis 214, bei Reaktor 4.8 (ohne Co-2) auf die Versuchstage 203 bis 214 eingegrenzt werden (Abbildung 14). Die in diesem Zeitraum in den Reaktorproben enthaltenen Cobaltgehalte wurden gemittelt und als untere Schwellenkonzentration für Cobalt festgelegt.

Unter den für die Projektphase gewählten Parametern, einer Raumbelastung von $5 \mathrm{~g}_{\mathrm{oTS}} /(\mathrm{L} \bullet \mathrm{d})$ bei einer mittleren hydraulischen Verweilzeit von 40 Tagen, in der Kombination mit den aufgeführten Instabilitätskriterien in der Doppelversuchsreihe

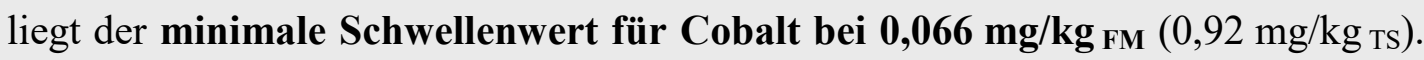
Im Vergleich zu den in Kapitel 2.3 in Tabelle 1 aufgeführten minimalen Bedarfswerten aus der Literatur harmoniert dieser ermittelte Wert sehr gut mit den Angaben von SCHERER \& SAHM (1981), SAUER (2010) und LINDORFER (2014).

\subsubsection{Verarmungsversuch für Nickel}

Die beiden Nickelmangel-Reaktoren gingen im Vergleich $\mathrm{zu}$ den beiden Reaktoren ohne Cobaltadditiv (vgl. Kapitel 4.3.2) 30 bzw. 58 Versuchstage später in die instabile Phase über. Abbildung 15 bis Abbildung 18 zeigen die Verläufe der FOS/TAC (FAL)- 
Werte, $\mathrm{pH}-$ Werte, der auf Normbedingungen umgerechneten Gasproduktion und der normierten Methangehalte in den Nickelmangel-Reaktoren (Reaktor 4.5 = ohne Ni-1, Reaktor 4.6 = ohne Ni-2), inklusive des Kontrollreaktors (Reaktor 4.9 = Referenz).

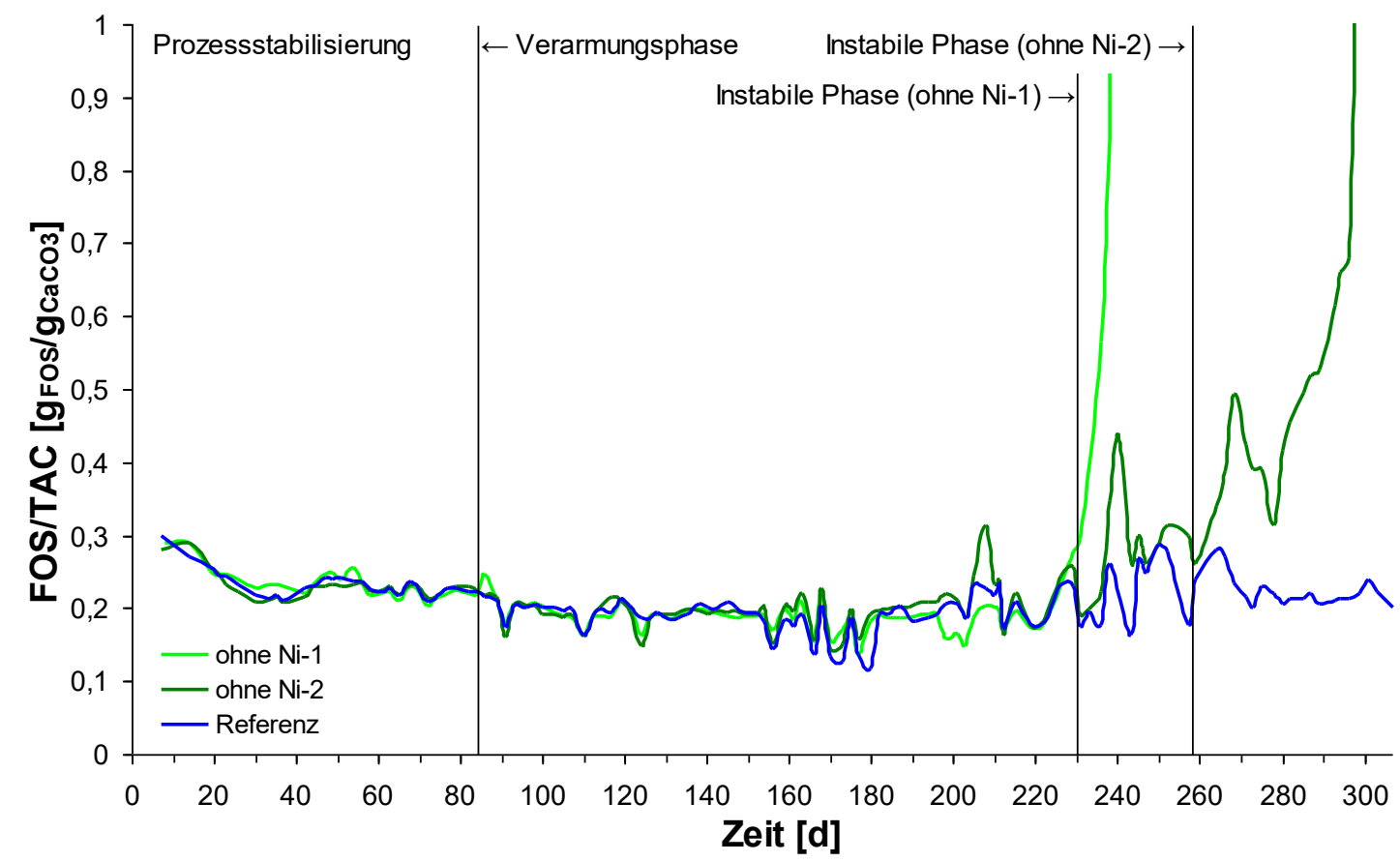

Abbildung 15: $\quad$ FOS/TAC (FAL)-Verläufe des Verarmungsversuchs für Nickel in den Versuchsbiogasreaktoren (mit Referenzreaktor).

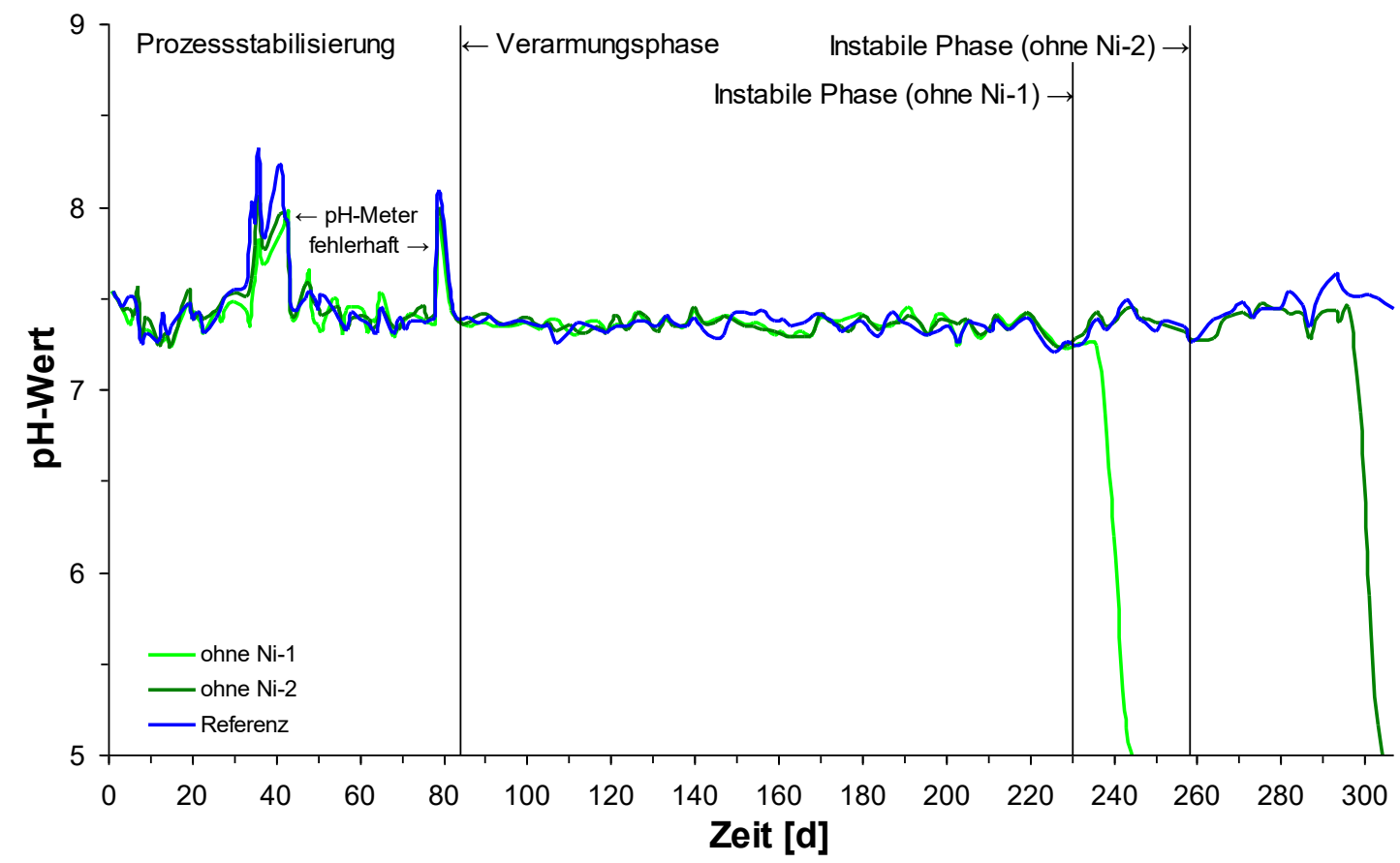

Abbildung 16: $\quad$ pH-Wert-Verläufe des Verarmungsversuchs für Nickel in den Versuchsbiogasreaktoren (mit Referenzreaktor). 


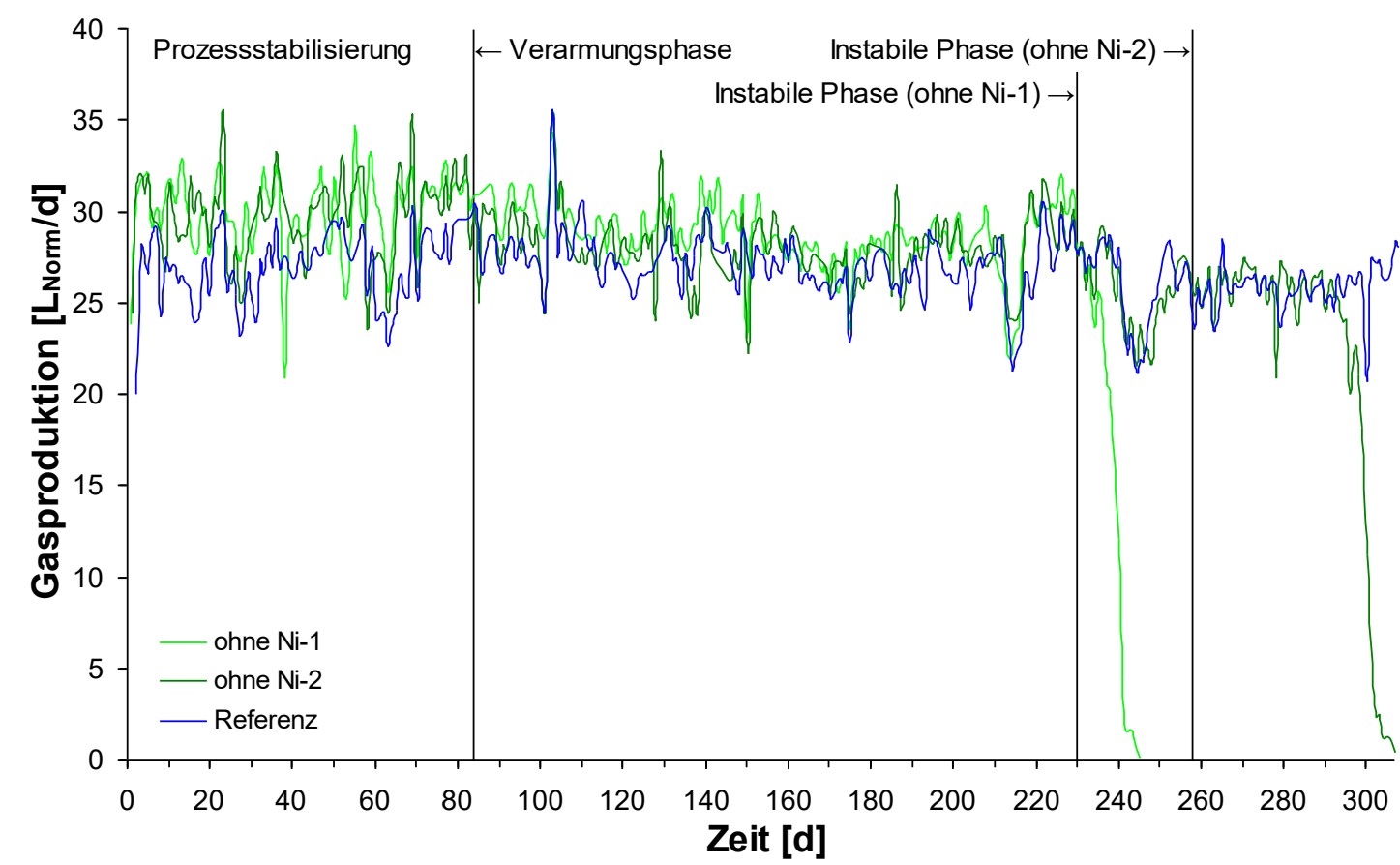

Abbildung 17: $\quad$ Verläufe der normierten Gasproduktion des Verarmungsversuchs für Nickel in den Versuchsbiogasreaktoren (mit Referenzreaktor).

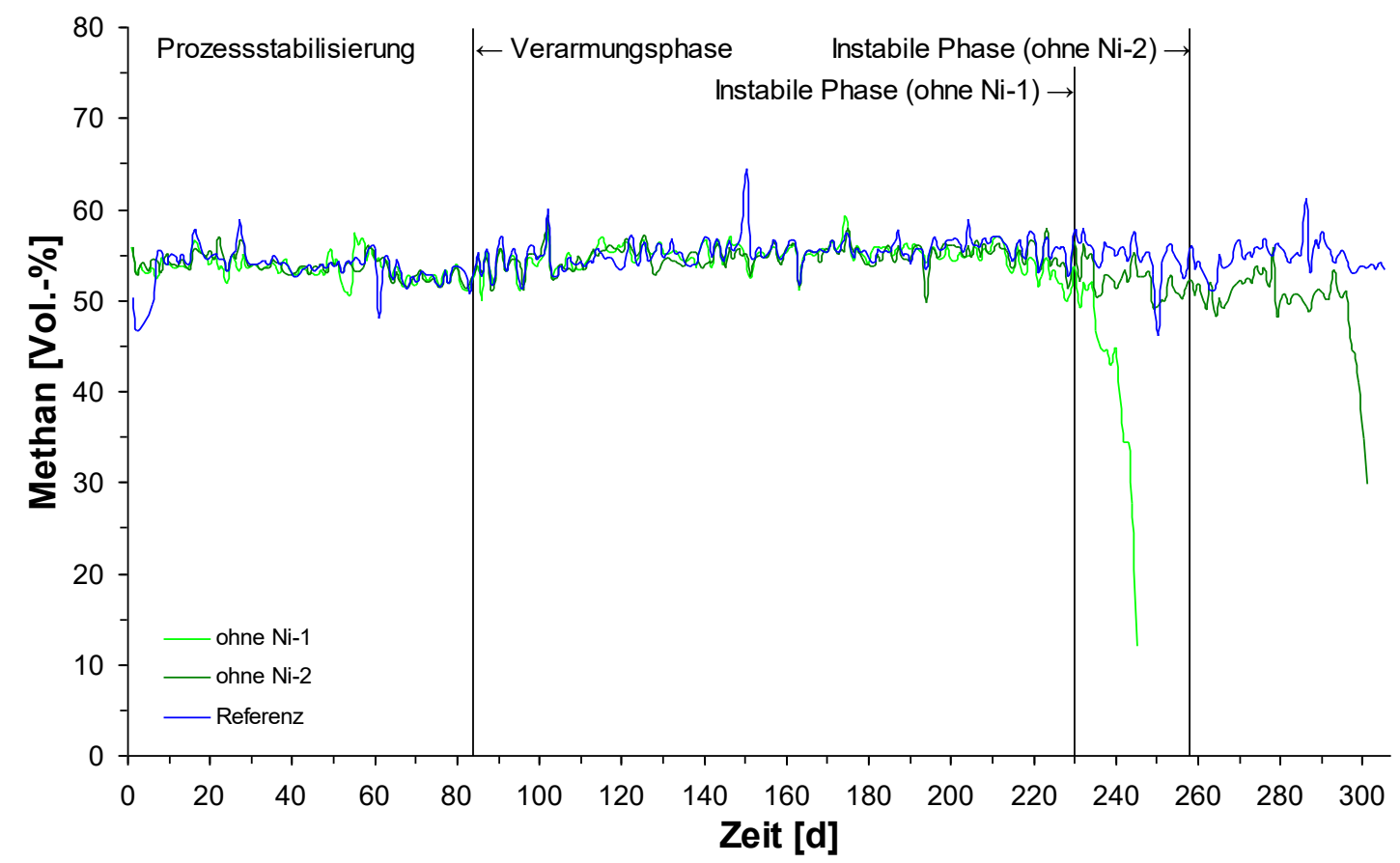

Abbildung 18: $\quad$ Verläufe des normierten Methangehalts des Verarmungsversuchs für Nickel in den Versuchsbiogasreaktoren (mit Referenzreaktor).

Auch bei den Reaktoren ohne zusätzliche Nickel-Zugabe zeigte sich in der Verarmungsphase ein ähnliches Bild wie bei den Reaktoren im Cobaltmangel. Die pH-Werte (Abbildung 16), die normierte Gasproduktion (Abbildung 17) und die normierten 
Methangehalte (Abbildung 18) verliefen im Wesentlichen stabil und wurden nicht von den kurzen Schwankungen der FOS/TAC (FAL)-Werte (Abbildung 15) zwischen den Versuchstagen 154 bis 182 sowie 205 bis 211 beeinträchtigt. Lediglich bei den Werten der normierten Gasproduktion war in der Verarmungsphase im Zeitraum von Versuchstag 213 bis 216 ein kurzzeitiger leichter Einbruch der Kurve zu verzeichnen, jedoch in allen Reaktoren des Versuchs, auch im Referenzreaktor 4.9. Die Ursache hierfür bleibt unklar

Der Nickelmangel-Reaktor 4.5 (ohne Ni-1) zeigte bei den FOS/TAC (FAL)-Werten (Abbildung 15) und der normierten Gasproduktion (Abbildung 17) ab Versuchstag 230 erste Auffälligkeiten. Die FOS/TAC (FAL)-Werte stiegen in den folgenden Tagen sprunghaft an; die pH-Werte (Abbildung 16) sowie die normierte Gasproduktion (Abbildung 17) und die normierten Methangehalte (Abbildung 18) fielen stark ab. Nach vier Verweilzeiten ohne zusätzliche Nickel-Zugabe kamen die Gasbildungsprozesse am Versuchstag 245 zum Erliegen.

Auch der zweite Nickelmangel-Reaktor 4.6 zeigte nur wenige Tage nach dem ersten Reaktor, am Versuchstag 238, erste Anzeichen eines beginnenden Absturzes bei den FOS/TAC (FAL)-Werten (Abbildung 15) an, wurde aber genau in diesem Zeitraum versehentlich mit einer zu niedrigen Inputsubstratmenge gefüttert. Dies führte dazu, dass sich die Mikroorganismen im Reaktor auf die veränderte Situation wahrscheinlich gut adaptieren konnten und der Reaktor deutlich länger lief als zu diesem Zeitpunkt erwartet. Die FOS/TAC (FAL)-Werte befanden sich dann bis Versuchstag 257 auf einem leicht höheren Niveau als zuvor während der Verarmungsphase, die normierten Methangehalte (Abbildung 18) zeigten leicht verringerte Volumenanteile gegenüber der Kontrolle im Referenzreaktor 4.9. Die pH-Werte (Abbildung 16) blieben hingegen unbeeinflusst auf einem konstanten, optimalen Niveau. Ab Versuchstag 258 begann auch in diesem Reaktor endgültig die instabile Phase, wiederum verbunden mit steigenden FOS/TAC (FAL)-Werten. Die pH-Werte, die normierte Gasproduktion (Abbildung 17) und die normierten Methangehalte sanken ab Versuchstag 296 sprunghaft ab, sodass die Gasbildungsprozesse nach fünfeinhalb Verweilzeiten ohne zusätzliche Nickel-Zugabe zum Erliegen kamen. In Abbildung 19 sind analog zu den Cobaltmangel-Reaktoren (vgl. Kapitel 4.3.2) zur Veranschaulichung die Verläufe der FOS/TAC (FAL)-Werte und der normierten Gasproduktion in den beiden NickelmangelReaktoren und dem Referenzreaktor direkt gegenübergestellt. 


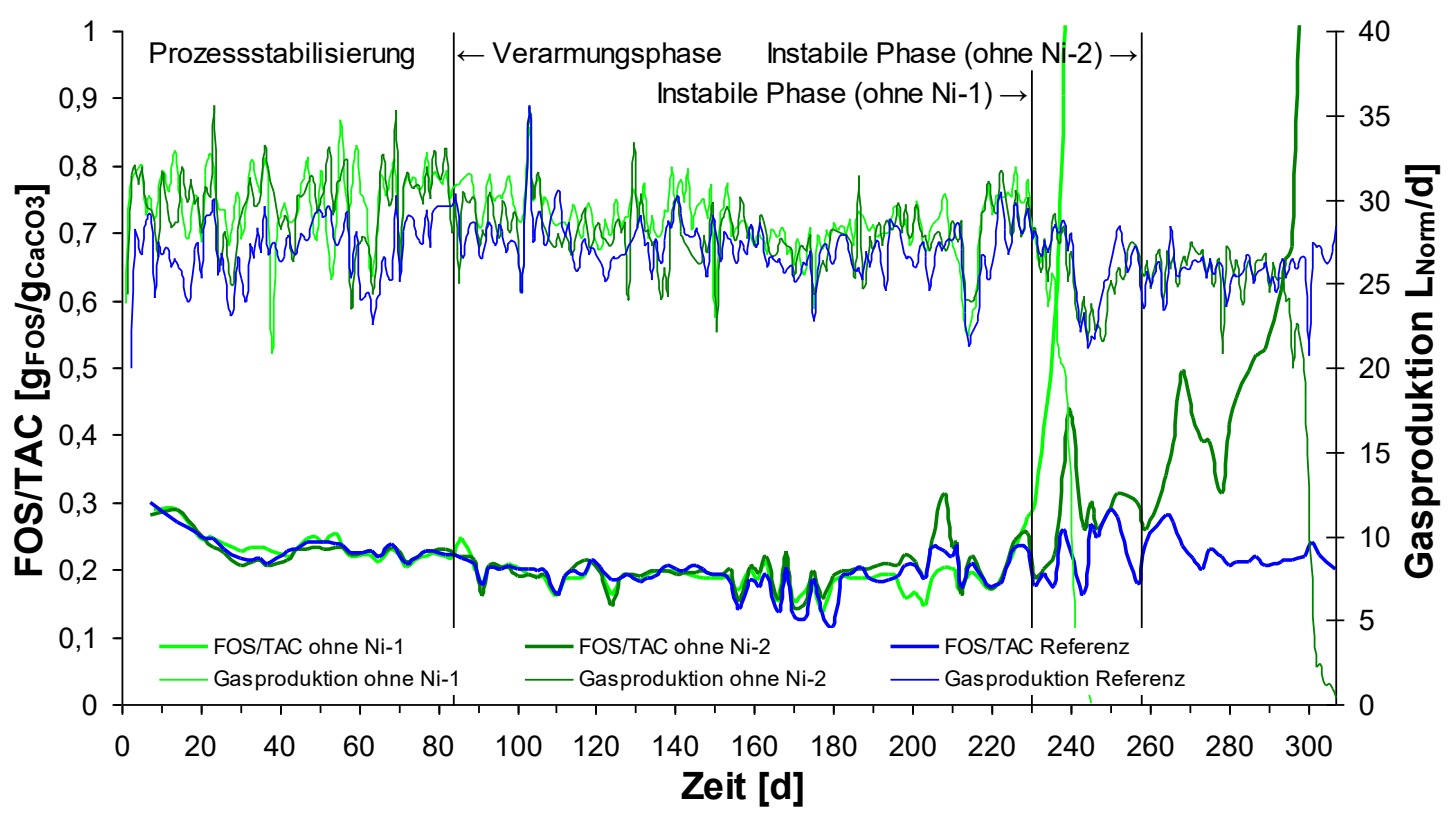

Abbildung 19: Gegenüberstellung der Verläufe der FOS/TAC (FAL)-Werte und der normierten Gasproduktion des Verarmungsversuchs für Nickel in den Versuchsbiogasreaktoren (mit Referenzreaktor).

Die Bestimmung des minimalen Schwellenwertes für Nickel erfolgte nach dem gleichen Vorgehen wie bei den Reaktoren ohne Cobaltadditiv (vgl. Kapitel 4.3.2). Abbildung 20 zeigt die Verläufe der Nickelkonzentrationen des Verarmungsversuchs.

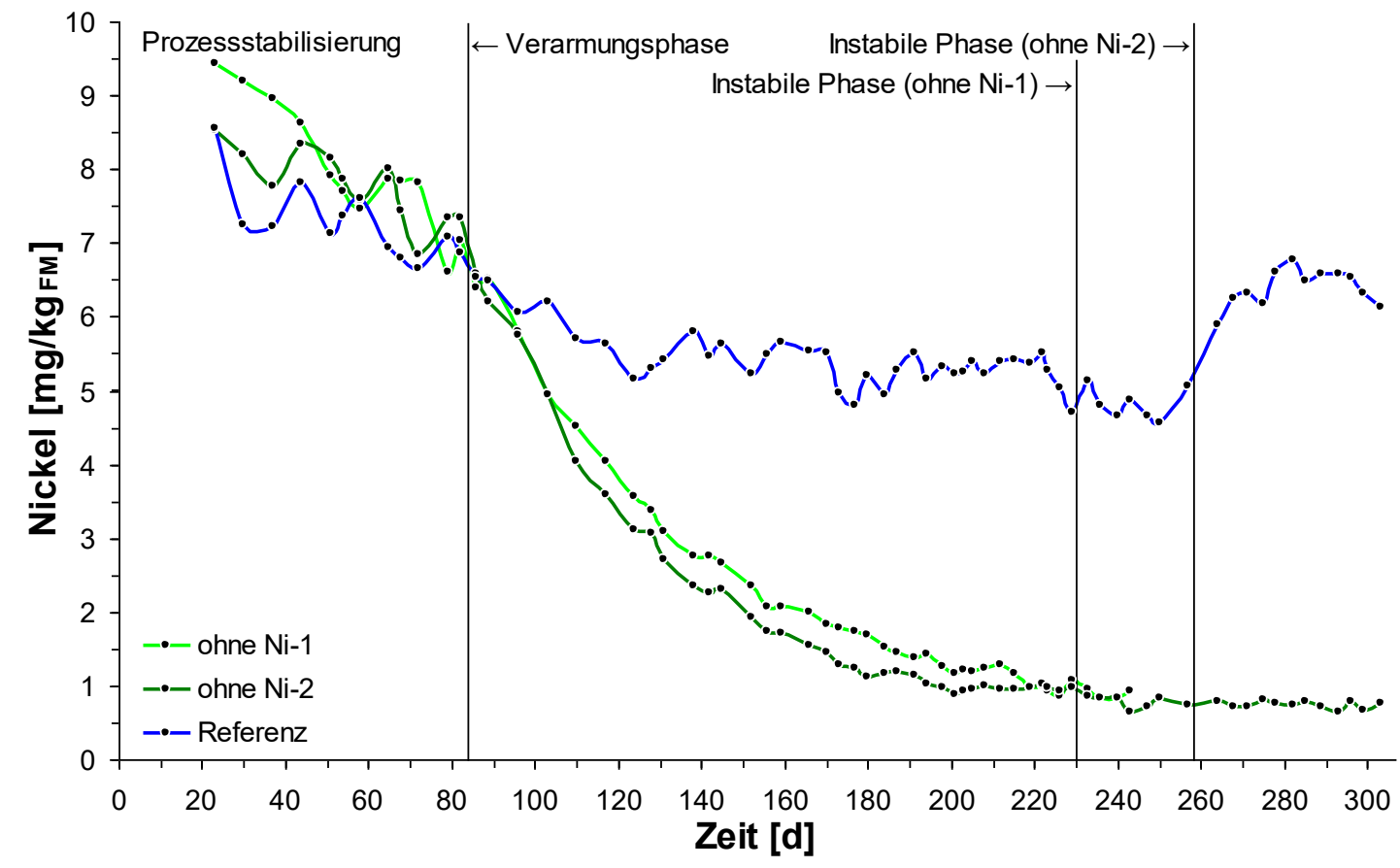

Abbildung 20: $\quad$ Verläufe der Nickelkonzentrationen des Verarmungsversuchs für Nickel in den Versuchsbiogasreaktoren (mit Referenzreaktor). 
Die unteren Schwellenbereiche liegen bei Reaktor 4.5 (ohne Ni-1) im Zeitraum der Versuchstage 231 bis 236, bei Reaktor 4.6 (ohne Ni-2) in einem langen Zeitfenster zwischen Versuchstag 238 und 296. Die Nickelgehalte der Proben aus diesen Zeitfenstern wurden ebenfalls gemittelt und die untere Schwellenkonzentration festgelegt.

Bei einer Raumbelastung von $5 \mathrm{~g}_{\mathrm{oTS}} /(\mathrm{L} \cdot \mathrm{d})$ und einer mittleren hydraulischen Verweilzeit von 40 Tagen in Verbindung mit den im Doppelversuch aufgeführten Instabilitätskriterien konnte der minimale Nickel-Schwellenwert bei $\mathbf{0 , 8 2} \mathbf{~ m g / k g ~ F M ~}$ $\left(9,5 \mathrm{mg} / \mathrm{kg}_{\mathrm{TS}}\right)$ festgelegt werden. Jedoch ist dieser Wert stark in Frage zu stellen (siehe Erläuterungen im folgenden Kapitel).

\subsubsection{Mögliche Zinkhemmung im Verarmungsversuch für Nickel}

In den beiden Reaktoren mit Nickelmangel (vgl. Kapitel 4.3.3) fiel mit Beginn der Verarmungsphase ab Versuchstag 84 eine kontinuierlich ansteigende Zinkkonzentration auf (Abbildung 21). Dieser Anstieg trat exakt ab dem Zeitpunkt ein, wo die dem Versuchsablauf angepasste Spurenelementlösung in die Reaktoren zugegeben wurde. So liegt die Vermutung nahe, dass in der Additivlösung ohne Nickel für die beiden Versuchsbiogasreaktoren das Element Zink fehlerhaft zugemischt wurde, es sich demnach um einen Einwaagefehler handelt.

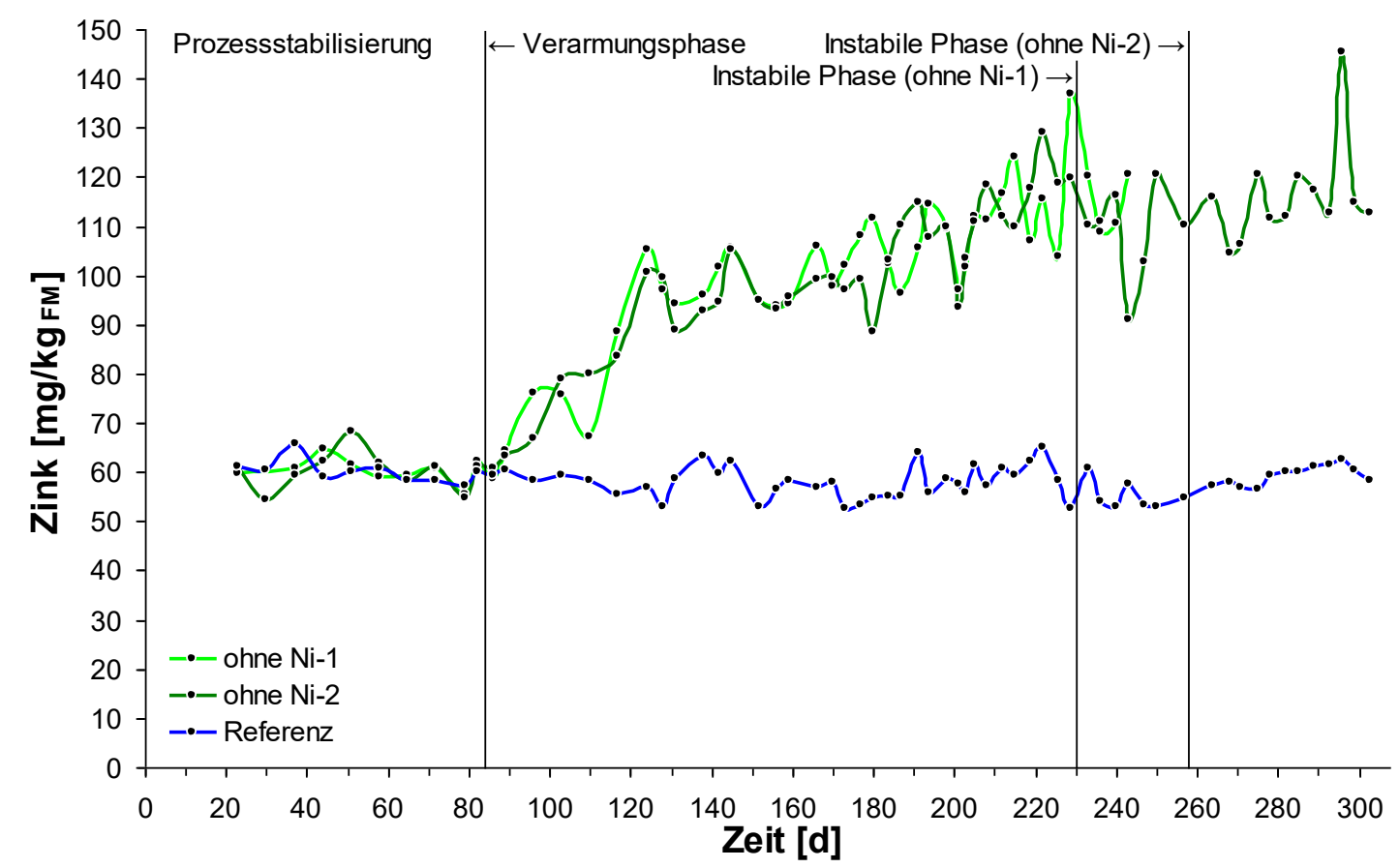

Abbildung 21: Verläufe der Zinkkonzentrationen des Verarmungsversuchs für Nickel in den Versuchsbiogasreaktoren (mit Referenzreaktor). 
Der Fehler wurde leider erst nach Abschluss der Projektphase erkannt, eine Analyse der Spurenelementlösung war nicht mehr möglich. Die Zinkkonzentration erreichte im Reaktor ohne Ni-1 direkt vor Beginn der instabilen Phase am Versuchstag 229 einen maximalen Wert von $137 \mathrm{mg} / \mathrm{kg}_{\mathrm{FM}}$ (Abbildung 21). Ab Versuchstag 203 lagen die Zinkgehalte durchgängig über $100 \mathrm{mg} / \mathrm{kg}_{\mathrm{FM}}$. Auch bei dem Reaktor ohne Ni-2 zeigte sich ein ähnliches Bild. Mit Ausnahme eines einmaligen geringen Einbruchs der Zinkkonzentration am Versuchstag 243 mit einem Wert von $91 \mathrm{mg} / \mathrm{kg}_{\mathrm{FM}}$, lagen die Zinkgehalte ab Versuchstag 203 konstant über $100 \mathrm{mg} / \mathrm{kg}_{\mathrm{FM}}$. Der Maximalwert im Reaktor ohne Ni-2 wurde während seiner instabilen Phase am Versuchstag 296 mit $145 \mathrm{mg} / \mathrm{kg}_{\mathrm{FM}}$ erreicht.

Die mit Beginn der Verarmungsphase neu angepasste Zusammensetzung der Spurenelementlösung für die Reaktoren im Cobaltmangel (vgl. Kapitel 4.3.2) sowie die unveränderte Additivlösung für den Referenzreaktor waren davon nicht betroffen (Abbildung 22), was die Vermutung, dass der Zinküberschuss aus der neuen Additivlösung für die Reaktorversuche mit Nickelmangel stammte, weiter bestätigt.

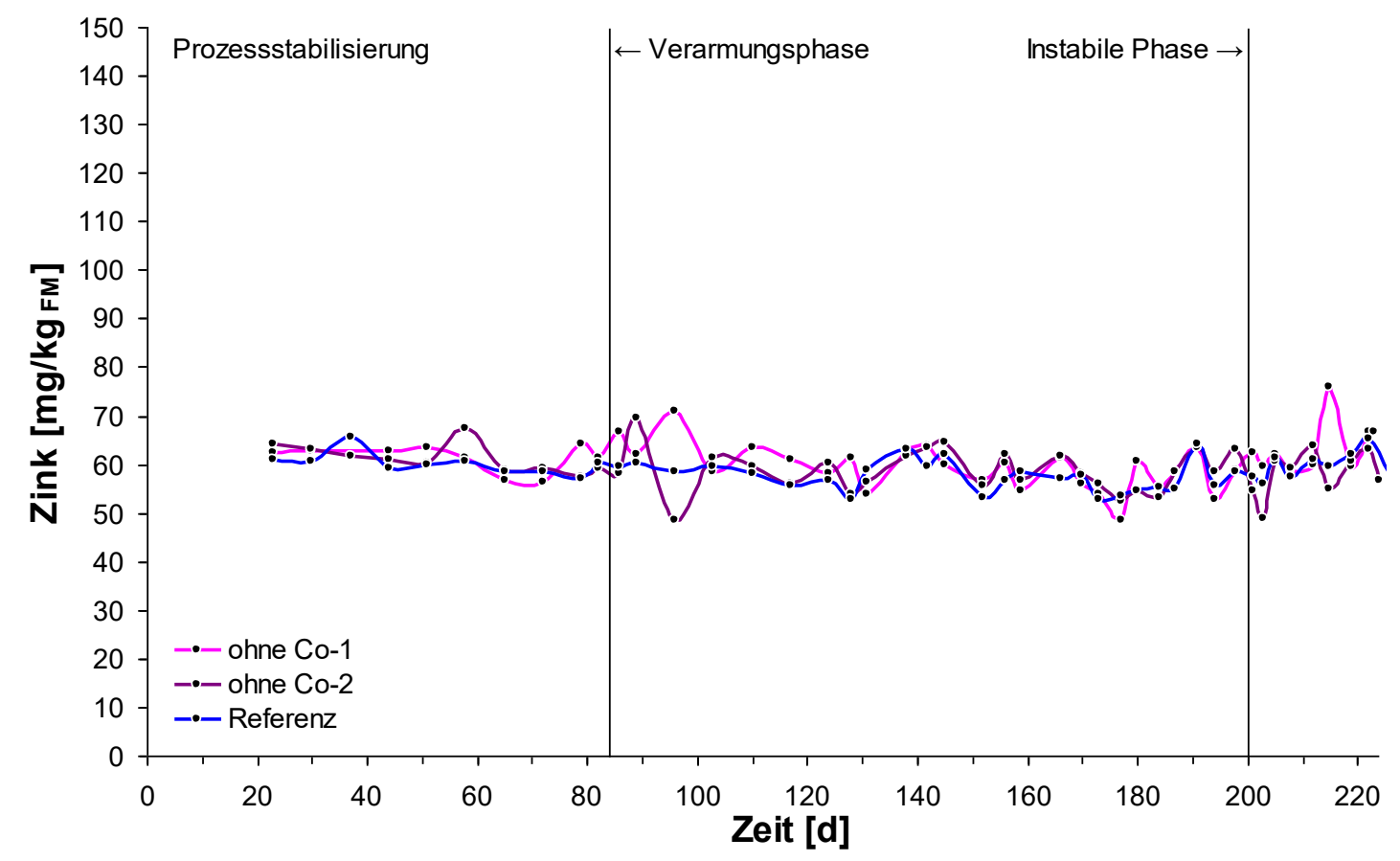

Abbildung 22: $\quad$ Verläufe der Zinkkonzentrationen des Verarmungsversuchs für Cobalt in den Versuchsbiogasreaktoren (mit Referenzreaktor).

Die Zinkkonzentrationen in den Cobaltmangel-Reaktoren und im Referenzreaktor lagen im Vergleich auf einem konstanten Niveau von durchschnittlich $60 \mathrm{mg} / \mathrm{kg}_{\mathrm{FM}}$. Dieser Wert liegt zwar auch in einem höheren Konzentrationsbereich für das Element Zink, 
LiNDORFER ET AL. (2012) gibt z.B. aus Analysen von 600 NawaRo-Fermentern Zinkgehalte von 0,83 bis $33 \mathrm{mg} / \mathrm{kg}_{\mathrm{FM}}$ (Medianwert 9,2 mg/ $/ \mathrm{kg}_{\mathrm{FM}}$ Zink) an, stellt jedoch kein Instabilitätskriterium dar, da alle physikochemischen und gasanalytischen Prozessparameter im Referenzreaktor stabil und optimal verliefen.

In geringeren Konzentrationen wirkt Zink wie in Kapitel 2.2.5 und 2.3 beschrieben stimulierend auf die Aktivität der Mikroorganismen, erfüllt wichtige Funktionen im anaeroben Gärprozess und dient als essenzieller Mikronährstoff. Zink kann im Biogasprozess aber auch als inhibierendes Schadelement auftreten (vgl. Kapitel 2.2.6), wenn es in höherer Konzentration in gelöster oder auch komplexierter Form mit organischen Liganden vorliegt (OleszKIEWICZ \& Sharma, 1990; KAISER ET AL., 2008; FrieHE ET AL., 2016). Ein Rückgang der Biogasproduktion, ein Absinken des pH-Wertes und damit eine Verstärkung der Mobilität des Schwermetalls können u.a. die Folgen einer Hemmwirkung sein (vgl. Kapitel 2.2.6).

In der Fachliteratur sind für nur wenige Angaben zu Zink-Hemmkonzentrationen bzw. Toxizitätsschwellenwerten in anaeroben (Biogas)prozessen zu finden, sie variieren zum Teil stark (vgl. Kapitel 2.2.6 und 5.9). WEILAND (2001) führt Hemmungen ab einer Zinkkonzentration von $150 \mathrm{mg} / \mathrm{L}$ auf. KOUZELI-KATSIRI \& KARTSONAS (1986) geben Zinkgehalte für Hemmungen ab $150 \mathrm{mg} / \mathrm{L}$, für Toxizität ab $250 \mathrm{mg} / \mathrm{L}$ an. Dagegen beschreiben ZAYED \& WINTER (2000) schon 40 mg/L Zink als hemmenden Schwellenwert bei anaeroben Abbauprozessen. Aber auch die Schwermetall-Sulfidfällung kann im Zusammenhang mit hohen Zinkgehalten von Bedeutung sein (vgl. Kapitel 2.2.6), da Elemente wie Blei, Cadmium, Eisen, Kupfer, Nickel oder Zink schwerlösliche Metallsulfide bilden können, die keinen direkt toxischen Einfluss auf die Mikroorganismen ausüben (KROISS \& SVARDAL, 2015).

Bei der hohen Raumbelastung von $5 \mathrm{~g}_{\mathrm{oTS}} /(\mathrm{L} \bullet \mathrm{d})$ sind vermutlich Hemmwirkungen durch Zink bereits bei $>100$ bzw. $120 \mathrm{mg} / \mathrm{kg}_{\mathrm{FM}}$ aufgetreten, die die Instabilität der Prozessverläufe in den Nickelmangel-Reaktoren beschleunigten, sodass sie letztendlich bei den Prozessparametern verfrüht größere Änderungen anzeigten. Das würde den ermittelten hohen Nickel-Minimalschwellenwert von $0,82 \mathrm{mg} / \mathrm{kg}_{\mathrm{FM}}$ (vgl. Kapitel 4.3.3) erklären, der im Kontrast zu den in Kapitel 2.3 in Tabelle 1 aufgeführten Literaturwerten zwischen 0,006 und 0,6 steht. So liegt die tatsächliche minimale Nickelkonzentration, bis $\mathrm{zu}$ der stabile Biogasprozesse aufrechterhalten werden können, wahrscheinlich deutlich unter dem erarbeiteten Wert. 
Es ist zu vermuten, dass die beiden mit Nickel unterversorgten Reaktoren wohl nicht wegen des induzierten Nickelmangels zu den im Verarmungsversuch ermittelten Zeitpunkten zusammenbrachen, sondern vor allem als Folge der fehlerhaften, zu hoch dosierten Zinkkonzentration. Bei alleinigem Nickelmangel wären sie vermutlich noch eine Zeit lang weitergelaufen bzw. erst bei niedrigeren Nickelkonzentrationen versäuert.

\subsubsection{Bewertung weiterer Elemente in den Verarmungsversuchen}

Neben den für den Biogasprozess wichtigen Spurenelementen Cobalt und Nickel fielen bei den Konzentrationsverläufen der essenziellen Grundelemente und Makronährstoffe (Gruppe I, vgl. Kapitel 2.2.5) mit Ausnahme von Natrium keine Schwankungen auf. Die Elemente Calcium, Kalium und Magnesium (Abbildung 23) sowie Phosphor und Schwefel (Abbildung 24) verliefen auf konstanten Niveaus. Beim Element Natrium (Abbildung 23) fielen die Gehalte in allen fünf Reaktoren jedoch stark über die gesamte Versuchsdauer ab und lagen am Versuchsende auf sehr niedrigen Stufen:

- Reaktor ohne Co-1: von 1000 auf $29 \mathrm{mg} / \mathrm{kg}_{\mathrm{FM}}$ Natrium,

- Reaktor ohne Co-2: von 1030 auf $27 \mathrm{mg} / \mathrm{kg}_{\mathrm{FM}}$,

- Reaktor ohne Ni-1: von 1000 auf $23 \mathrm{mg} / \mathrm{kg}_{\mathrm{FM}}$,

- Reaktor ohne Ni-2: von 1000 auf $11 \mathrm{mg} / \mathrm{kg}_{\mathrm{FM}}$,

- Referenzreaktor: von 1010 auf $12 \mathrm{mg} / \mathrm{kg}_{\mathrm{FM}}$.

Das lässt sich mit dem natriumarmen Substrat Mais-Silage erklären (vgl. Kapitel 4.2, Tabelle 8), da Natrium kein Additivelement in der zugesetzten Spurenelementlösung war (vgl. Kapitel 4.1, Tabelle 6). Die hohen Natriumwerte zu Beginn der Projektphase lassen sich auf hohe Gehalte im Inokulum zurückführen. Trotz der niedrigen Natriumkonzentrationen zeigten alle physikochemischen und gasanalytischen Parameter im Referenzreaktor bis Versuchsende kaum Auffälligkeiten, sodass zusätzliche Hemmwirkungen wahrscheinlich ausgeschlossen werden können. Dennoch könnte ein Zusammenspiel der sehr niedrigen Cobalt- bzw. Nickelgehalte in den instabilen Phasen (vgl. Kapitel 4.3.2 und 4.3.3), der hohen Zinkkonzentrationen in den NickelmangelReaktoren (vgl. Kapitel 4.3.4) und der niedrigen Natriumgehalte die Versäuerungsprozesse begünstigt haben. MUNK ET AL. (2010) beschreiben einen in Laborversuchen ermittelten minimalen Schwellenwert für Natrium von $10 \mathrm{mg} / \mathrm{L}$. Bedarfswerte sind in der Literatur kaum zu finden. LindoRfER ET AL. (2012) führt aus Analysen von 600 NawaRo-Fermentern einen Wertebereich von 20 bis 1100 mg/kg FM Natrium auf. 

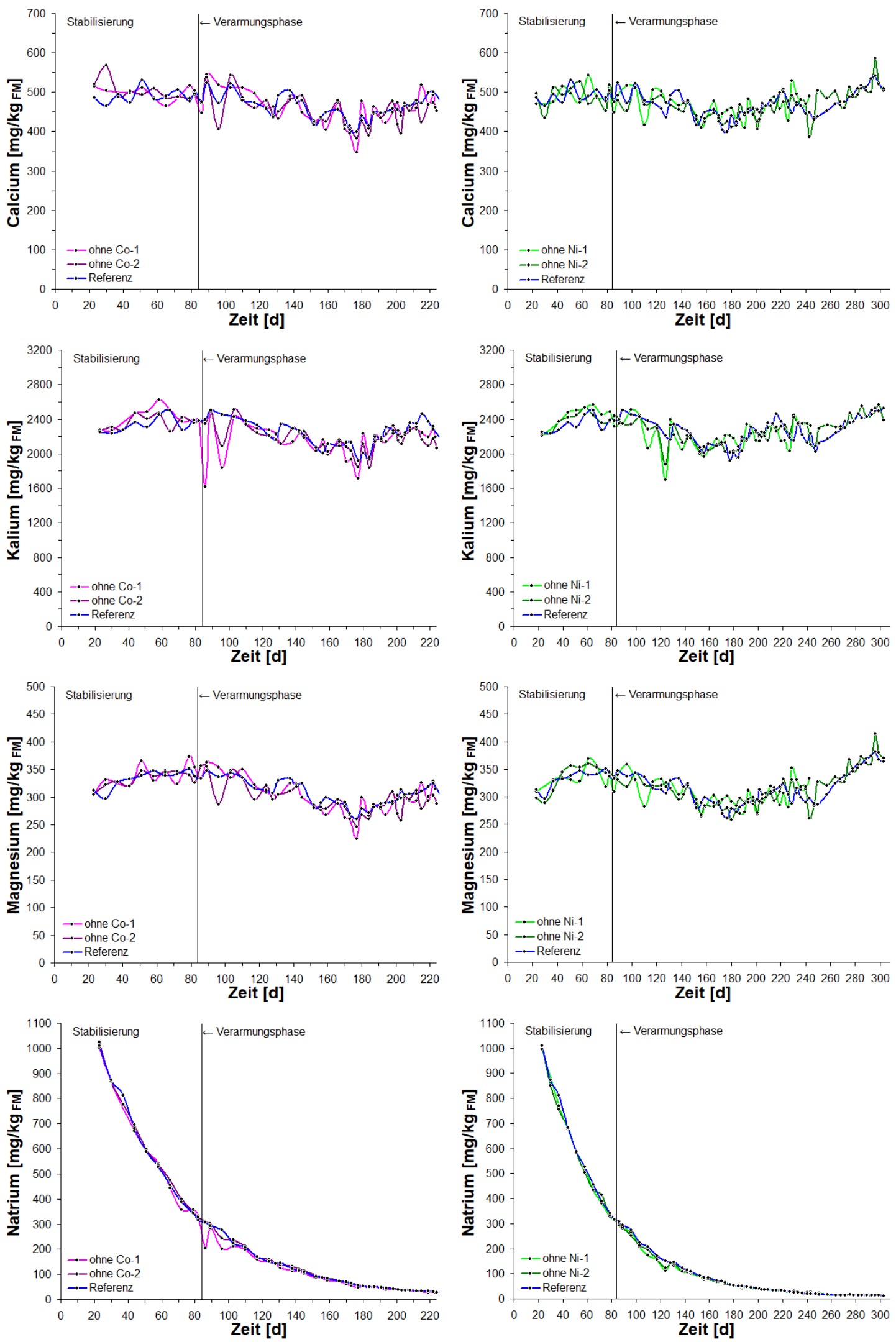

Abbildung 23: $\quad$ Verläufe der Calcium-, Kalium-, Magnesium- und Natriumgehalte der Verarmungsversuche für Cobalt und Nickel in den Versuchsbiogasreaktoren (mit Referenzreaktor). 

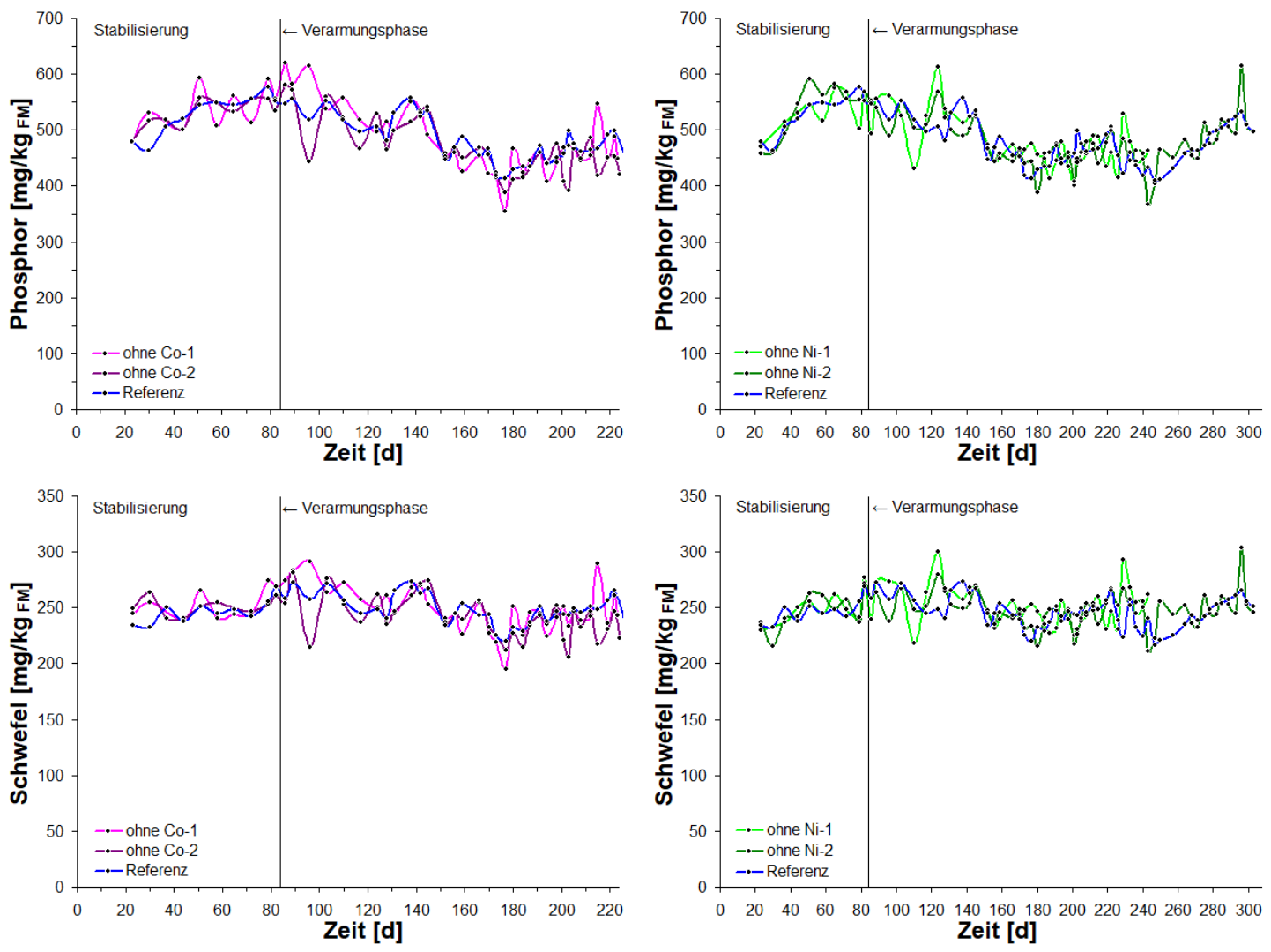

Abbildung 24: Verläufe der Phosphor- und Schwefelgehalte der Verarmungsversuche für Cobalt und Nickel in den Versuchsbiogasreaktoren (mit Referenzreaktor).

Die essenziellen Spurenelemente (Gruppe II) Eisen, Kupfer, Mangan und Molybdän (Abbildung 25) lagen mit Ausnahme von Kupfer alle auf stabilen Niveaus, in Konzentrationsbereichen weit über den in Kapitel 2.3 in Tabelle 1 angegebenen minimalen Bedarfswerten aus der Literatur, da sie Elemente der zugeführten Additivlösung waren (vgl. Kapitel 4.1, Tabelle 6). Kupfer war dagegen in der Spurenelementlösung nicht enthalten, sodass sich die Gehalte in den Reaktoren in den letzten beiden Verweilzeiten im Mittel bei $1,2 \mathrm{mg} / \mathrm{kg}_{\mathrm{FM}}$ bewegten, also auf einem niedrigen unteren Level, ähnlich den von SAUER (2010) und LINDORFER (2014) aufgeführten minimalen Bedarfswerten von 1,5 bzw. 1,1 mg/kg FM Kupfer (vgl. Kapitel 2.3, Tabelle 1). Für Biogasanlagen in Europa geben SCHATTAUER ET AL. (2011) hingegen Kupfergehalte zwischen 0,45 bis $15 \mathrm{mg} / \mathrm{kg}_{\mathrm{FM}}$ an; einen Optimalbereich von $0,1 \mathrm{bis} 10 \mathrm{mg} / \mathrm{kg}$ FM Kupfer nennen LeBuHn \&EFFEnBERger (2012). Die Prozessdaten des Referenzreaktors deuteten allerdings nicht auf Mangelzustände hin, sodass die Kupferkonzentrationen in diesen Versuchen für stabile Biogasprozesse ausreichend sind. 

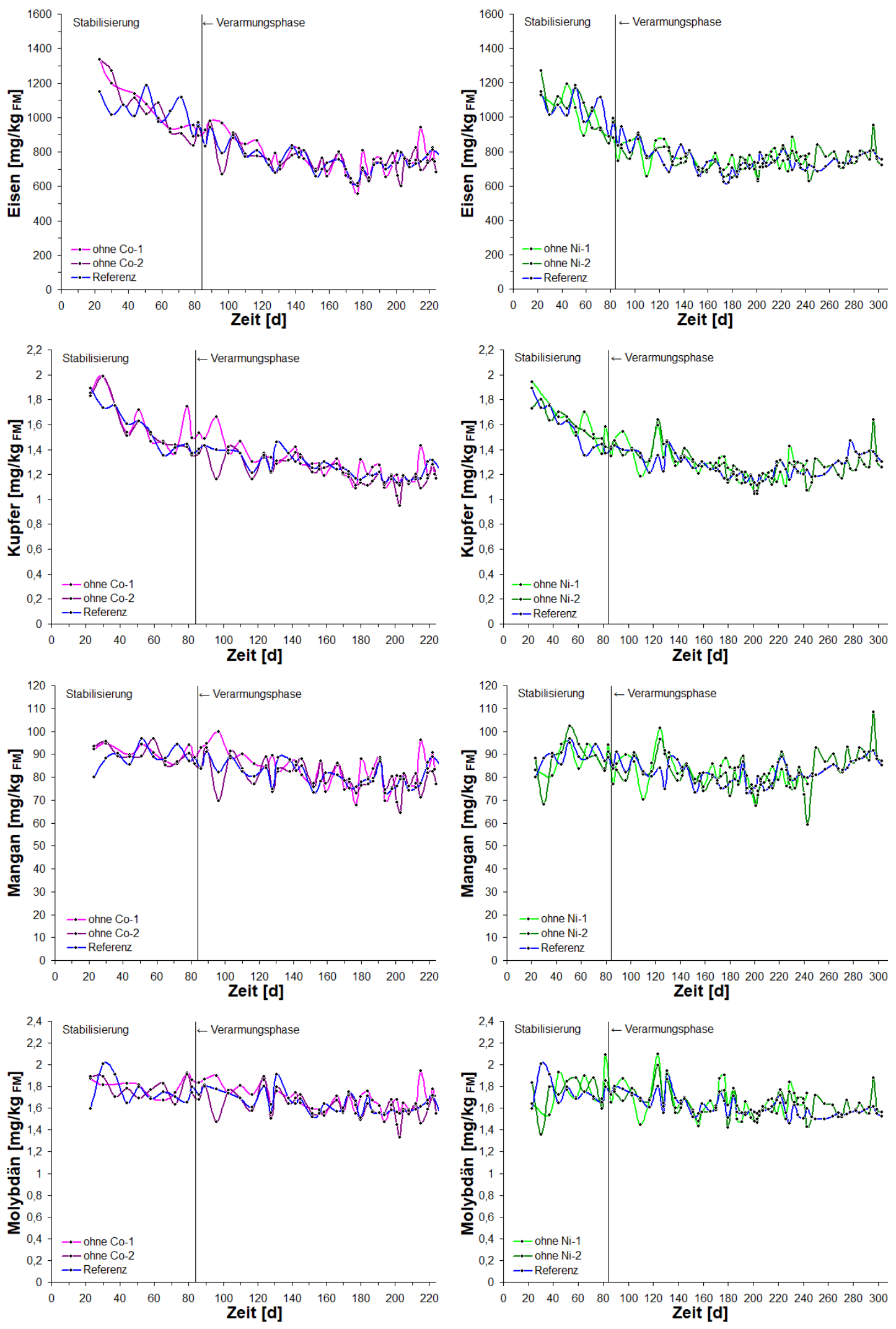

Abbildung 25: $\quad$ Verläufe der Eisen-, Kupfer-, Mangan- und Molybdängehalte der Verarmungsversuche für Cobalt und Nickel in den Versuchsbiogasreaktoren (mit Referenzreaktor). 
Die Elemente der Gruppe III (vgl. Kapitel 2.2.5) Cadmium, Chrom, Blei, Aluminium, Lanthan (als Vertreter der Lanthanoide/ Seltene Erdelemente), Titan und Strontium blieben ebenfalls auf stabilen Niveaus unauffällig (Abbildung 26 und Abbildung 27). Der Anstieg der Chrom- und Nickelgehalte im Referenzreaktor ab Versuchtag 250 lässt sich vermutlich auf Abrasions-/Ablösungsvorgänge an den metallischen Oberflächen des mittig im Reaktor fixierten Ankerrührers mit den Rührblättern zurückführen (vgl. Kapitel 3.5.3). Die höheren Konzentrationen zu Versuchsbeginn, v.a. bei Chrom, Blei und Lanthan, sind durch höhere Gehalte im Inokulum zu begründen.
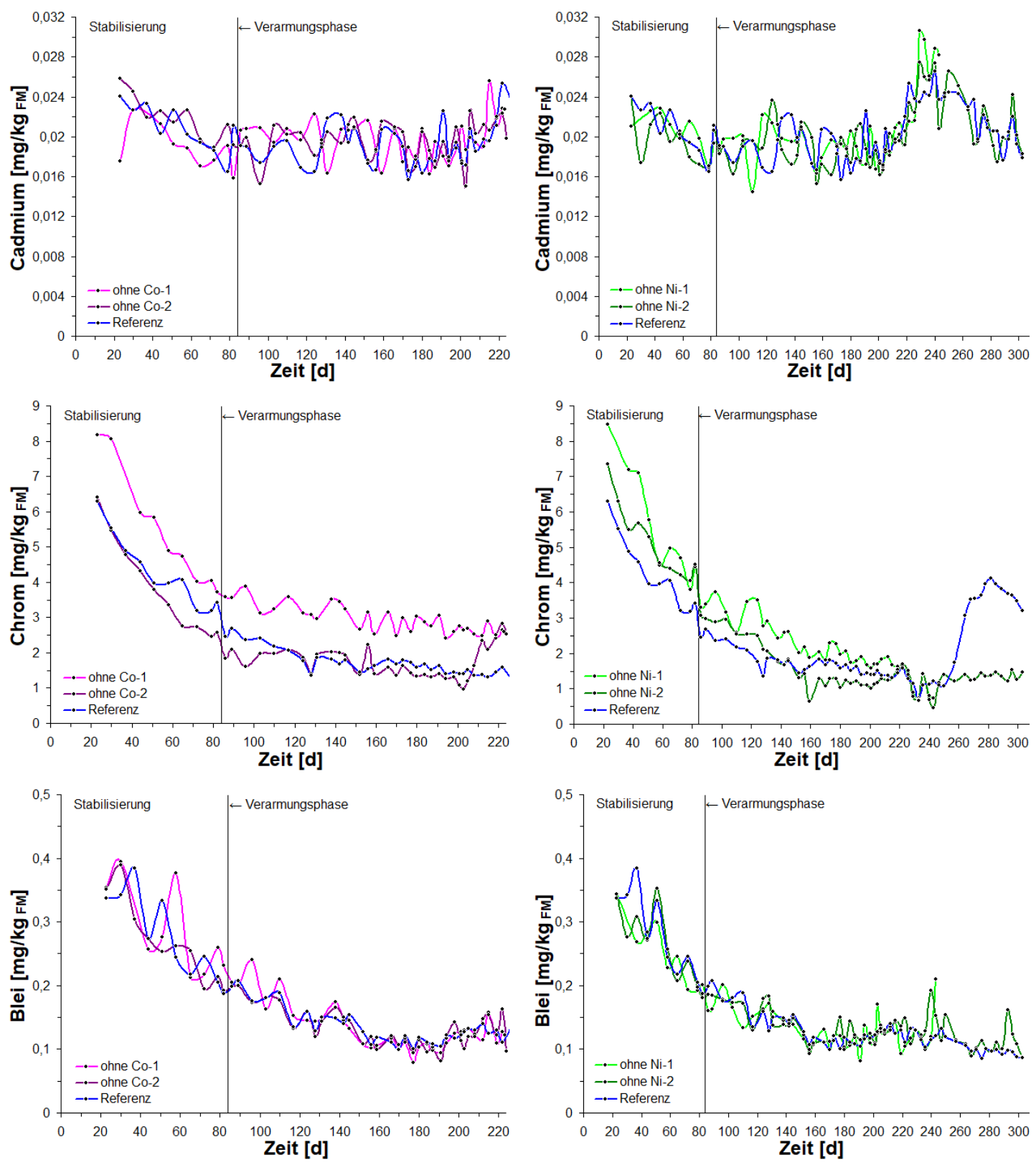

Abbildung 26: $\quad$ Verläufe der Cadmium-, Chrom- und Bleigehalte der Verarmungsversuche für Cobalt und Nickel in den Versuchsbiogasreaktoren (mit Referenzreaktor). 

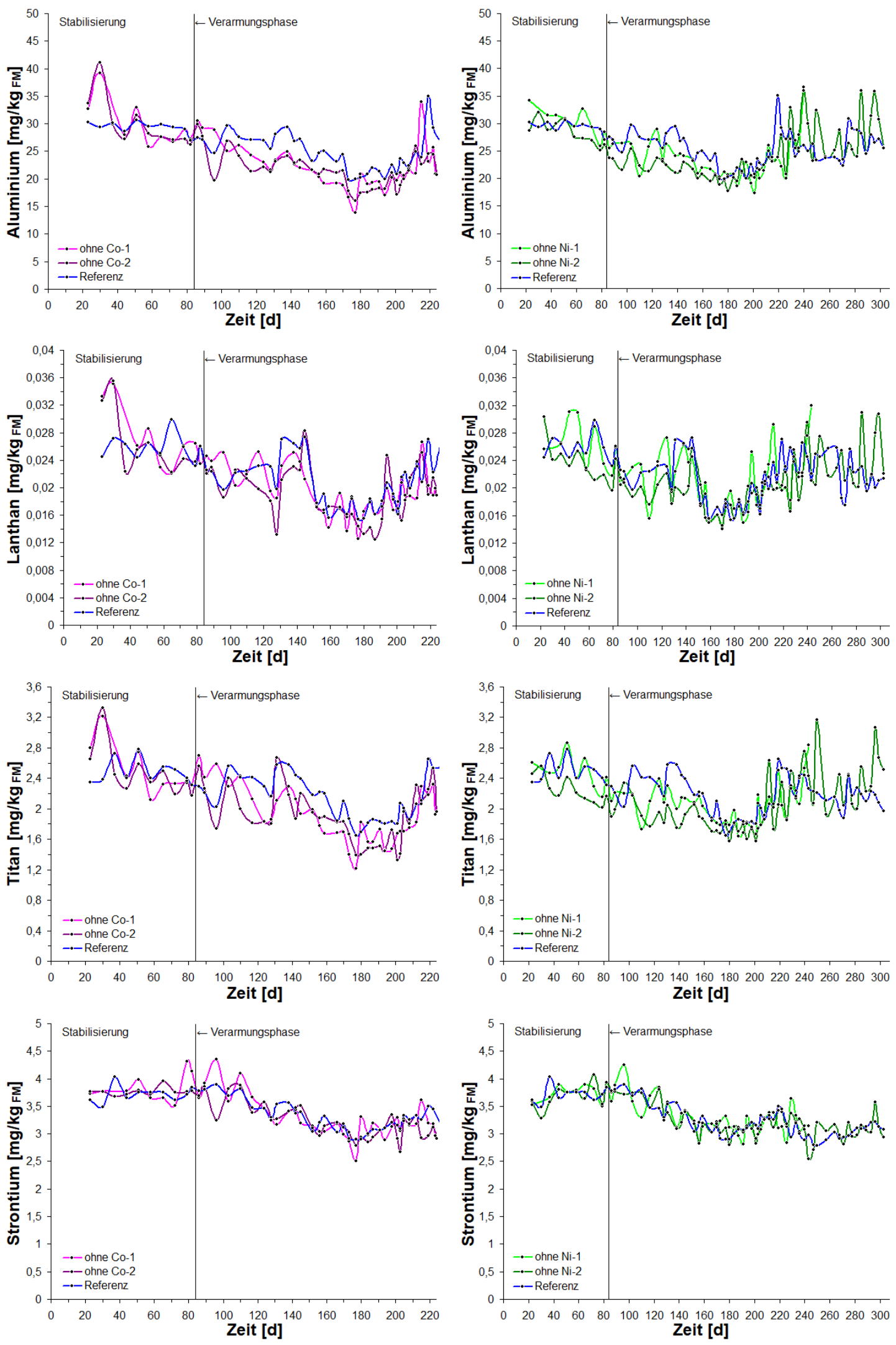

Abbildung 27: Verläufe der Aluminium-, Lanthan-, Titan- und Strontiumgehalte der Verarmungsversuche für Cobalt und Nickel in den Versuchsbiogasreaktoren (mit Referenzreaktor). 
Selen-, Vanadium- und Arsengehalte konnten für die erste Projektphase noch nicht erhoben werden, da diese Elemente erst im Herbst 2016 in das neue, angepasste Multielementkalibrationslösungsset „Pflanzen/Organische Proben“ für die ICP-OESMessungen aufgenommen wurden (vgl. Kapitel 3.4.3). Die Selen- und Arsengehalte konnten auch von den ICP-MS-Messungen noch nicht herangezogen werden, da die KED-Methode zur Entfernung von möglichen Interferenzen (vgl. Kapitel 3.4.4) erst mit Etablierung des neuen Massenspektrometers seit Sommer 2016 Anwendung fand.

\subsubsection{Eisen/Schwefel-Verhältnisse in den Verarmungsversuchen}

Als weiteres Kriterium zur Beurteilung von Stabilitäts- bzw. Spurenelementsituationen für optimale Biogasprozesse weist SAUER (2010) dem Eisen/Schwefel-Verhältnis eine übergeordnete Rolle zu. Er postuliert auf Basis der Daten aus zwei Praxisbiogasanlagen einen Schwellenwert für das Eisen/Schwefel-Verhältnis von 0,4, ab dem optimale Biogasausbeuten möglich sein sollten. Bei den Verarmungsversuchen für Cobalt und Nickel lagen die Eisen/Schwefel-Verhältnisse in allen Versuchsbiogasreaktoren über die kompletten Versuchszeiträume weit oberhalb dieses minimalen Schwellenwertes, in den Verarmungsphasen durchschnittlich bei 3,0 (Abbildung 28). Diese hohen Verhältnisse sind wiederum dadurch zu begründen, dass Eisen Additivelement in den zugesetzten Spurenelementlösungen war (vgl. Kapitel 4.1, Tabelle 6).

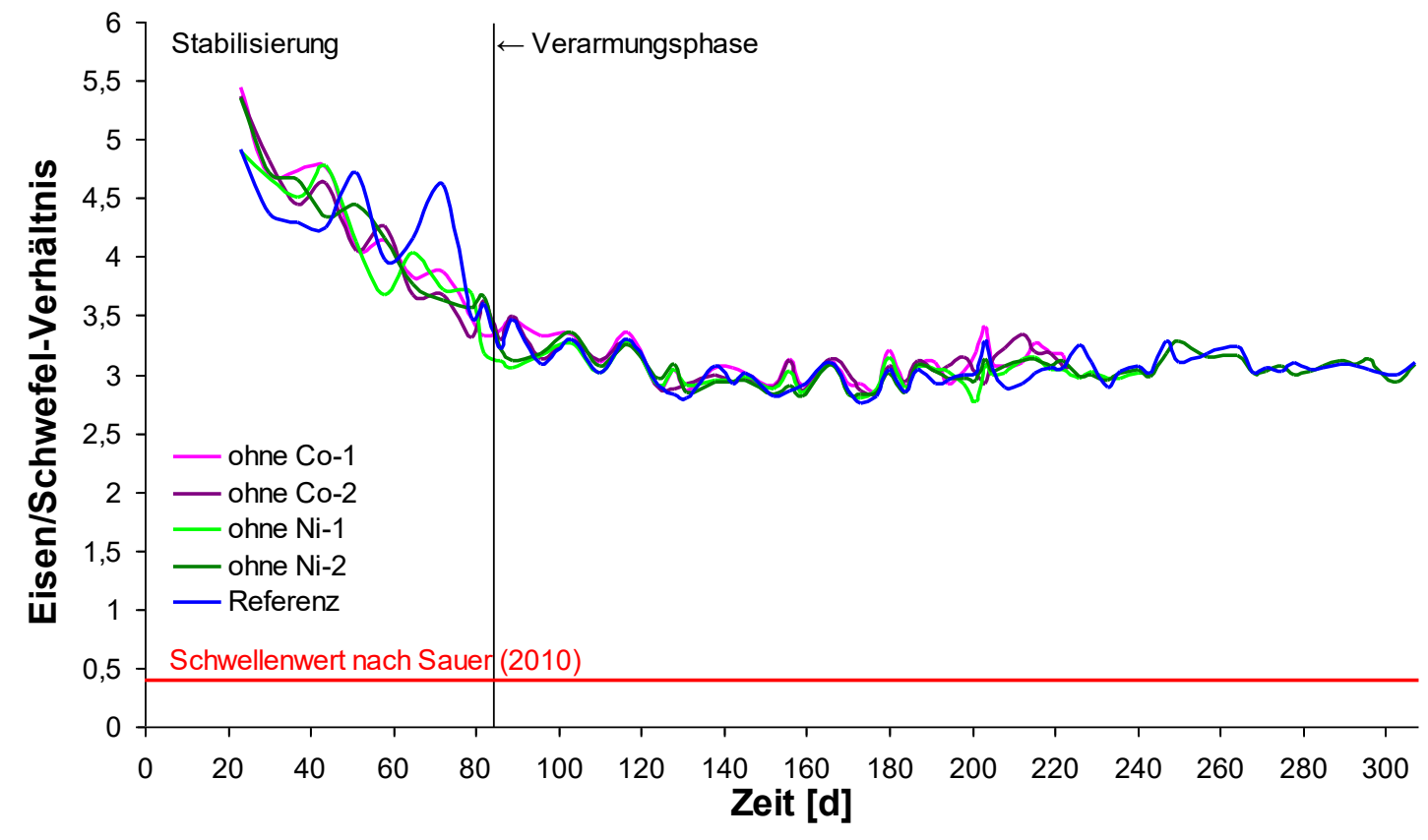

Abbildung 28: Verläufe der Eisen/Schwefel-Verhältnisse der Verarmungsversuche für Cobalt und Nickel in den Versuchsbiogasreaktoren (mit Referenzreaktor). 


\subsubsection{Mikrobiologischer Überblick zur Zusammensetzung, Dynamik und Aktivität der mikrobiellen Gemeinschaften in den Verarmungsversuchen}

Auf Basis der mikrobiologischen Untersuchungen am Reaktormaterial in der Arbeitsgruppe Mikrobiologie anaerober Systeme am UFZ konnten die Ergebnisse mit den Spurenelementdaten kombiniert betrachet werden. Die Nickelmangel-Reaktoren zeigten während der Stabilisierungs- und Verarmungsphase nahezu durchweg eine hohe Dominanz der Gattung Methanosarcina mit relativen Anteilen von ca. 75 bis 90 \%. Ab Versuchstag 199, circa drei Verweilzeiten nach Beginn der Verarmung, sanken die relativen Anteile auf etwa $60 \%$ deutlich ab, die Familie Methanobacteriaceael Methanomassiliicoccaceae trat verstärkt in Erscheinung. In den instabilen Phasen gingen die relativen Anteile von Methanosarcina dann noch weiter zurück auf $<20 \%$ bis $30 \%$, Methanobacteriaceae/Methanomassiliicoccaceae dominierte diese Phasen mit bis zu 77 \%. Im Referenzreaktor war der relative Anteil von Methanosarcina allgemein deutlich höher. So könnte geschlussfolgert werden, dass Methanosarcina auf Nickelmangel empfindlich reagiert und durch eine Verringerung der relativen Abundanz dieser Gattung ein Zusammenbruch der Biogasprozesse frühzeitig angezeigt wird (SAUER ET AL., 2018). Ob dieser Effekt durch Nickelmangelzustände oder die mögliche ZinkHemmwirkung (vgl. Kapitel 4.3.4) induziert ist, kann jedoch nicht verifiziert werden.

Die relative Aktivität von Methanosarcina war ab Beginn der Verarmungsphase mit ca. 15 bis $30 \%$ deutlich geringer als die relative Abundanz. Dagegen wiesen die Familie Methanobacteriaceae/Methanomassiliicoccaceae (bis zu 80\%) und die Gattung Methanoculleus (bis zu 20 \%) viel höhere relative Aktivitäten als relative Abundanzen auf. Allerdings waren keine signifikanten Auswirkungen in Folge eines Nickelmangels bzw. einer Zinkhemmung auf die relative Aktivität einzelner Methanbildner zu erkennen, die Veränderungen traten in gleicher Weise auch im Referenzreaktor auf.

Nach WINTSCHE ET AL. (2016) braucht der acetoklastische Weg der Methanbildung, der nur von der Ordnung Methanosarcinales umgesetzt werden kann, einen dreifach so hohen Nickelbedarf als der hydrogenotrophe Weg, da dort mehr nickelhaltige Enzyme beteiligt sind. So kann bei einem Nickelmangel die acetoklastische Methanbildung nicht ablaufen. Methanosarcina ist allerdings dazu fähig, seinen Stoffwechsel anzupassen und auf hydrogenotrophe Methanbildung zu wechseln. So könnte Methanosarcina ab Beginn der Verarmungsphase über circa drei Verweilzeiten versucht haben, durch eine Adaptation seines Stoffwechselweges den Nickelmangel auszugleichen. Dann war 
Methanosarcina der Konkurrenzsituation $\mathrm{zu}$ den hydrogenotrophen Methanbildnern vermutlich nicht mehr gewachsen, sodass die relativen Anteile absanken. Oder das Umstellen seines Stoffwechsels auf den hydrogenotrophen Weg stellt eventuell in Mangelsituationen lediglich eine Notlösung für Methanosarcina dar, die sich aber langfristig gegen rein hydrogenotrophe Methanbildner nicht durchsetzen kann. In der RNA-basierten T-RFLP-Analyse war dies nicht erkennbar, da der relative Anteil an mcrA-Transkripten von Methanosarcina recht gering war (SAUER ET AL., 2018).

Die beiden Cobaltmangel-Reaktoren zeigten in allen Prozessphasen eine deutliche Abundanz von Methanosarcina. Im Verlauf der Stabilisierungs- und Verarmungsphase schwankten die relativen Anteile von Methanobacteriaceae/Methanomassiliicoccaceae um ca. $10-20 \%$ und erreichten im Gegensatz zu den Nickelmangel-Reaktoren erst in der instabilen Phase eine deutlichere Abundanz von bis zu $50 \%$. Eine so deutliche Veränderung der Zusammensetzung der methanogenen Gemeinschaften infolge des Spurenelementmangels wie bei den beiden Nickelmangel-Reaktoren war jedoch nicht $\mathrm{zu}$ verzeichnen. Auch die RNA-basierten T-RFLP-Analysen wiesen ähnliche relative Aktivitäten der Methanbildner wie in den Nickelmangel-Reaktoren auf. Es zeigten sich im Verlauf der Verarmungsphase aber deutliche Zunahmen der relativen Aktivität von Methanosarcina, während Methanoculleus, Methanobacteriaceae und Methanomassiliicoccaceae relativ dazu abnahmen. So wird Methanosarcina durch einen Cobaltmangel also deutlich weniger beeinträchtigt als andere Methanbildner (SAUER ET AL., 2018).

In allen Reaktoren der Verarmungsversuche für Cobalt und Nickel war die Diversität der methanogenen Gemeinschaften als eher gering einzustufen. Die bakteriellen Gemeinschaften wiesen hingegen eine deutlich höhere Diversität als die Methanogenen auf. Obwohl sich die relativen Abundanzen in den Versuchsbiogasreaktoren zwar im Laufe der Prozessphasen immer wieder veränderten, konnte keine dieser Änderungen direkt auf Nickel- oder Cobaltmangelzustände zurückgeführt werden. Die Änderungen traten in allen fünf Reaktoren, also auch im Referenzreaktor mit gleichbleibenden Bedingungen, wenig spezifisch auf (SAUER ET AL., 2018).

Zusammenfassend ist festzuhalten, dass Methanosarcina als potenzieller Indikatororganismus für einen Spurenelementmangel herangezogen werden kann. Nickelmangel führt zu einer Verringerung der relativen Abundanz von Methanosarcina, Cobaltmangel zu einem Anstieg seiner relativen Aktivität. Inwiefern die mögliche Zinkhemmung dabei eine Rolle spielte, kann abschließend nicht geklärt werden. 


\subsection{Substitutionsversuche von Mais-Silage mit Silagen von Winterackerbohne und Amarant}

\subsection{1 Überblick}

In der zweiten Projektphase der Laborfermenterversuche am DBFZ wurden jeweils im Doppelversuch die ersten beiden Substitutionen für Mais durchgeführt, indem 30 \% Winterackerbohne- bzw. 30 \% Amarant-Silage zu 70 \% Mais-Silage (oTS-bezogen) den Reaktoren zugeführt wurden. Ziel war es zu prüfen, ob Zumischungen von spurenelementreicheren Energiepflanzen-Silagen ausreichend sind, einen stabilen Biogasprozess ohne separate Zusätze von Metallverbindungen aufrechtzuerhalten. Dafür wurden die Versuchsbiogasreaktoren aus einem stabilen Zustand heraus zusätzlich zu Mais mit Silagen von Winterackerbohne bzw. Amarant (vgl. Kapitel 4.2) gefüttert, die von der Abteilung Pflanzenbau der Universität Göttingen bereitgestellt wurden. Es erfolgte keine Zugabe zusätzlicher Spurenelementadditive. Das Stabilitätsverhalten dieser Silagemischungen sollte bei einer (quasi)kontinuierlichen Reaktorfahrweise über mindestens drei Verweilzeiten untersucht werden.

Im Vorfeld der Substitutionsversuche wurde das zu wählende Mischungsverhältnis der spurenelementreicheren Energiepflanzen-Silagen zur spurenelementarmen Mais-Silage (vgl. Kapitel 4.2) im Forschungsverbund diskutiert. Es war klar, dass, wenn ein Mischungsverhältnis beispielsweise von $25 \%$ Mais- zu 75 \% Winterackerbohne- oder Amarant-Silage erfolgreich getestet werden würde, diese Mischungen aufgrund von niedrigeren Biomasseerträgen der alternativen Energiepflanzen $\mathrm{zu}$ Mais keine Akzeptanz in der Biogaspraxis finden würden. So wurde entschieden, ein für die Anlagenbetreibenden vermeintlich gerade noch akzeptables Mischungsverhältnis von $30 \%$ Winterackerbohne- bzw. Amarant-Silage zu 70 \% Mais-Silage (oTS-bezogen) zu testen. Bei erfolgreichen Substitutionsversuchen mit diesen Pflanzenvarianten könnte so eher eine positive Handlungsempfehlung an die Biogaspraxis gegeben werden.

Nach der abgeschlossenen ersten Projektphase (vgl. Kapitel 4.3) erfolgte am DBFZ ein Neustart aller fünf Versuchsbiogasreaktoren. Die Reaktoren wurden abermals mit Gärmaterial aus dem 100-Liter-Inokulumsreaktor (Reaktor 3.2) befüllt, der gezielt mit den gleichen Betriebsparametern wie die Substitutionsversuche betrieben wurde. Tabelle 10 gibt einen Überblick über die einzelnen Prozessphasen der beiden Substitutionsversuche. 
Tabelle 10: $\quad$ Überblick über die zweite Projektphase der (quasi)kontinuierlichen Nassfermentationsversuche am DBFZ.

\begin{tabular}{|c|c|c|c|c|c|c|}
\hline \multicolumn{7}{|c|}{$\begin{array}{l}\text { Projektphase } 2 \quad \text { Substitutionsversuche } 1 \text { und } 2 \\
\text { Vergärung von } 30 \% \text { Silage aus Winterackerbohne bzw. Amarant zu } 70 \% \text { Mais-Silage (oTS-bezogen) }\end{array}$} \\
\hline Prozessphase & Reaktor & Kurzbeschreibung & Abkürzung & $\begin{array}{l}\text { Versuchs- } \\
\text { tag }\end{array}$ & bis & $\begin{array}{c}\text { Versuchs- } \\
\text { tag }\end{array}$ \\
\hline Prozessstabilisierung & $4.5-4.9$ & Mais-Silage + Spurenelementvollversorgung & & 0 & - & 56 \\
\hline \multirow[t]{4}{*}{ Substitutionsphase } & 4.5 & Substitution Winterackerbohne 1 & WAB-1 & 57 & - & 103 \\
\hline & 4.6 & Substitution Winterackerbohne 2 & WAB-2 & 57 & - & 103 \\
\hline & 4.7 & Substitution Amarant 1 & Amarant-1 & 57 & - & 103 \\
\hline & 4.8 & Substitution Amarant 2 & Amarant-2 & 57 & - & 103 \\
\hline \multirow[t]{4}{*}{ Versäuerungsphase } & 4.5 & Substitution Winterackerbohne 1 & WAB-1 & 104 & - & 128 \\
\hline & 4.6 & Substitution Winterackerbohne 2 & WAB-2 & 104 & - & 128 \\
\hline & 4.7 & Substitution Amarant 1 & Amarant-1 & 104 & - & 128 \\
\hline & 4.8 & Substitution Amarant 2 & Amarant-2 & 104 & - & 128 \\
\hline \multirow[t]{2}{*}{ Rettungsphase } & $4.5-4.8$ & \multicolumn{2}{|l|}{ Halbe Fütterung + Halbe Spurenelementlösung } & 129 & - & 133 \\
\hline & & \multicolumn{2}{|l|}{ Volle Fütterung + Halbe Spurenelementlösung } & 134 & - & 138 \\
\hline Erholungsphase & $4.5-4.8$ & \multicolumn{2}{|l|}{ Volle Fütterung + Viertel Spurenelementlösung } & 139 & - & 188 \\
\hline Kontrolle & 4.9 & \multicolumn{2}{|c|}{ Mais-Silage + Spurenelementvollversorgung Referenz } & 0 & - & 188 \\
\hline
\end{tabular}

Zusätzlich zur spurenelementarmen Mais-Silage als Start-Inputsubstrat wurde täglich in alle Biogasreaktoren abermals die synthetische Spurenelementadditivlösung nach OECHSNER ET AL. (2008; vgl. Kapitel 4.1) zugegeben. Nach einer 56-tägigen Phase der Prozessstabilisierung begann ab Versuchstag 57 die Umstellung der Substrate (Substitutionsphase), indem die synthetischen Additive durch die Silagemischungen Winterackerbohne (Reaktor 4.5 $=\mathrm{WAB}-1$ und Reaktor 4.6 $=\mathrm{WAB}-2$ ) bzw. Amarant (Reaktor 4.7 = Amarant-1 und Reaktor 4.8=Amarant-2) zu Lasten von Mais ersetzt wurden. Reaktor 4.9 (= Referenz) wurde erneut parallel dazu als Kontrolle mit MaisSilage als Alleinfuttermittel und zusätzlicher Spurenelementvollversorgung betrieben.

Es zeigte sich aber nach etwas mehr als einer Verweilzeit, dass die Reaktoren mit den Zumischungen von $30 \%$ Winterackerbohne- sowie $30 \%$ Amarant-Silage $\mathrm{zu} \quad 70 \%$ Mais-Silage (oTS-bezogen) bei einer Raumbelastung von $5 \mathrm{~g}_{\mathrm{oTS}} /(\mathrm{L} \bullet \mathrm{d})$ allmählich versäuerten (Versäuerungsphase). Somit waren die Ersatzsilagen, wie auch schon im Vorfeld durch Berechnungen erwartet, nicht in der Lage, genügend Spurenelemente für stabile Biogasprozesse bereitzustellen. Die Biogasprozesse in den Reaktoren konnten jedoch durch die zusätzliche Zugabe von nur noch einem Viertel der ursprünglichen Spurenelementadditivmenge stabilisiert werden. 


\subsubsection{Substitutionsversuch 1: Vergärung von $30 \%$ Silage aus Winterackerbohne zu $70 \%$ Mais-Silage (oTS-bezogen)}

Nach der Substratumstellung (ab Versuchstag 57) begannen in der Substitutionsphase in den beiden Versuchsbiogasreaktoren mit der Silagemischung Winterackerbohne zu Mais (Reaktor 4.5 = WAB-1 und Reaktor 4.6 =WAB-2) ab Versuchstag 104 die FOS (Kapp)- und FOS/TAC (FAL)-Werte anzusteigen. Letztere überstiegen dann ab Versuchstag 112 den kritischen Schwellenwert von $0,3 \mathrm{~g}_{\mathrm{FOS}} / \mathrm{g}_{\mathrm{CaCO}}$ mit starkem Trend nach oben in den Folgetagen (Abbildung 29). Die pH-Wert-Verläufe blieben in diesem Zeitraum in den beiden Versuchsbiogasreaktoren noch stabil. Sie zeigten ab Versuchstag 122 erstmals fallende Trends an (Abbildung 30). Mit Beginn der Versäuerungsphase waren in den auf Normbedingungen $(273,15 \mathrm{~K}, 101,325 \mathrm{kPa})$ umgerechneten Verläufen der Gasproduktion (Abbildung 31) und Methangehalte (Abbildung 32) ebenfalls fallende Trends ersichtlich. Ab Versuchstag 122 lagen die normierten Methangehalte in den beiden Reaktoren sogar unter 50 Vol.-\%.

Am Versuchstag 128, etwa zwei Verweilzeiten nach Substitutionsbeginn, standen die beiden Reaktoren kurz vor dem Zusammenbruch. Alle prozessanalytischen Parameter wiesen sehr hohe [FOS (Kapp) und FOS/TAC (FAL)] bzw. niedrige Werte bei der normierten Gasproduktion und den normierten Methangehalten auf. Der Kontrollreaktor 4.9 (= Referenz) lief über die komplette Versuchsdauer stabil.

Nach Absprache mit allen Partnern im Forschungsverbund wurde entschieden, zu versuchen, die sehr instabilen, kurz vor dem Zusammenbruch stehenden Reaktoren durch ein schnelles Eingreifen wieder zu stabilisieren (Rettungsphase). Hierfür wurden ab Versuchstag 129 für vier Tage die Beschickungsmengen der Pflanzenmischungen halbiert (halbe Raumbelastung), und es erfolgte für insgesamt zehn Tage bis zum Versuchstag 138 eine Zugabe der halben Menge $(0,4 \mathrm{~mL}$ pro Tag) der synthetischen Spurenelementlösung nach OECHSNER ET AL. (2008; vgl. Kapitel 4.1). Ab Versuchstag 139 wurde dann bis zum Versuchsende am Versuchstag 188 nur noch ein Viertel der ursprünglichen Spurenelementadditivmenge $(0,2 \mathrm{~mL}$ pro Tag) zusätzlich in die Substitutionsreaktoren zugegeben (Erholungsphase). Die physikochemischen und gasanalytischen Parameter in den beiden Reaktoren stabilisierten sich während der Rettungsphase schnell und lagen die gesamte Erholungsphase über, also über mehr als eine Verweilzeit, auf einem kontinuierlich stabilen Niveau. 


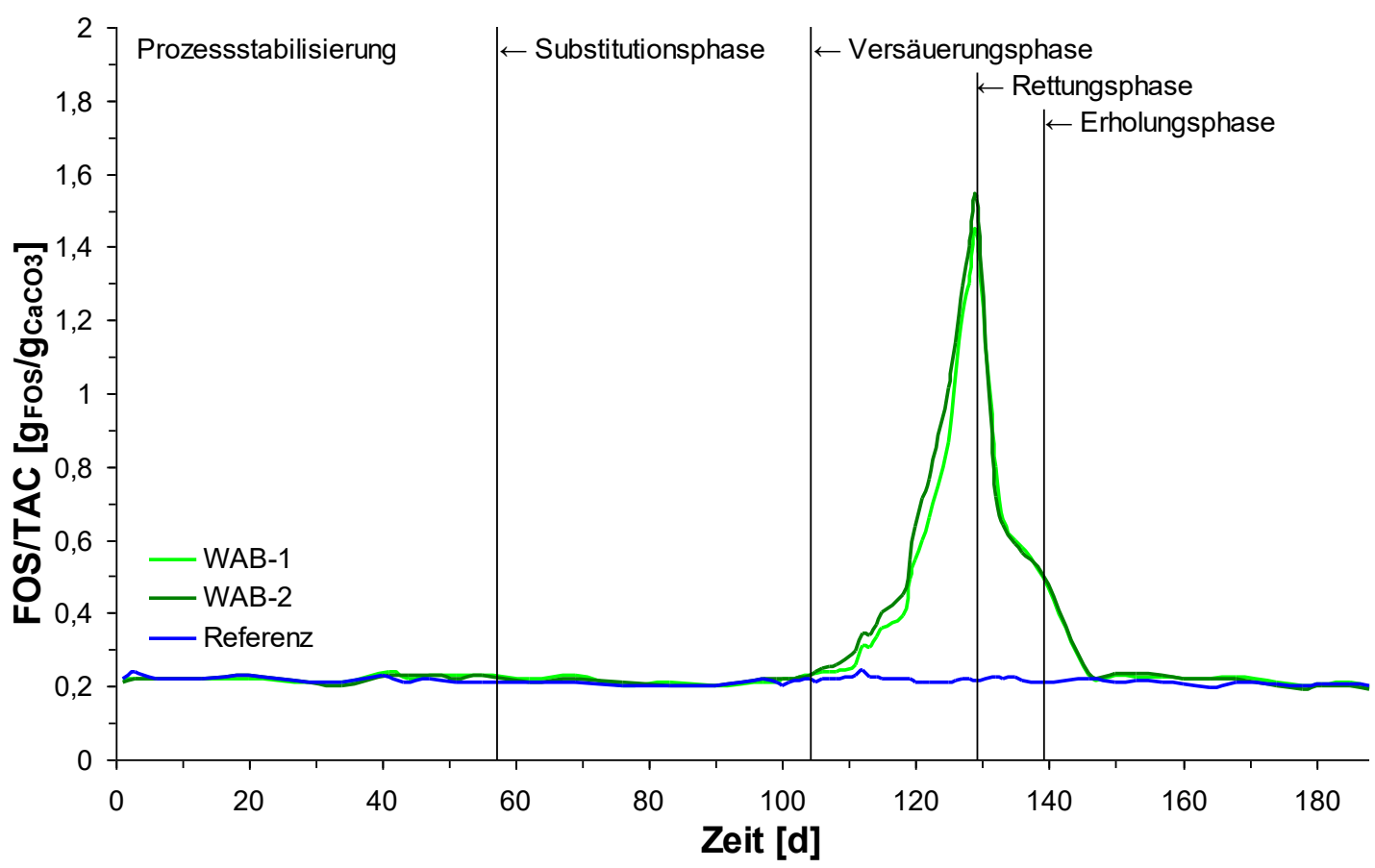

Abbildung 29: $\quad$ FOS/TAC (FAL)-Verläufe des Substitutionsversuchs 1 mit 30\% Winterackerbohne- zu $70 \%$ Mais-Silage (oTS-bezogen) in den Versuchsbiogasreaktoren (mit Referenzreaktor).

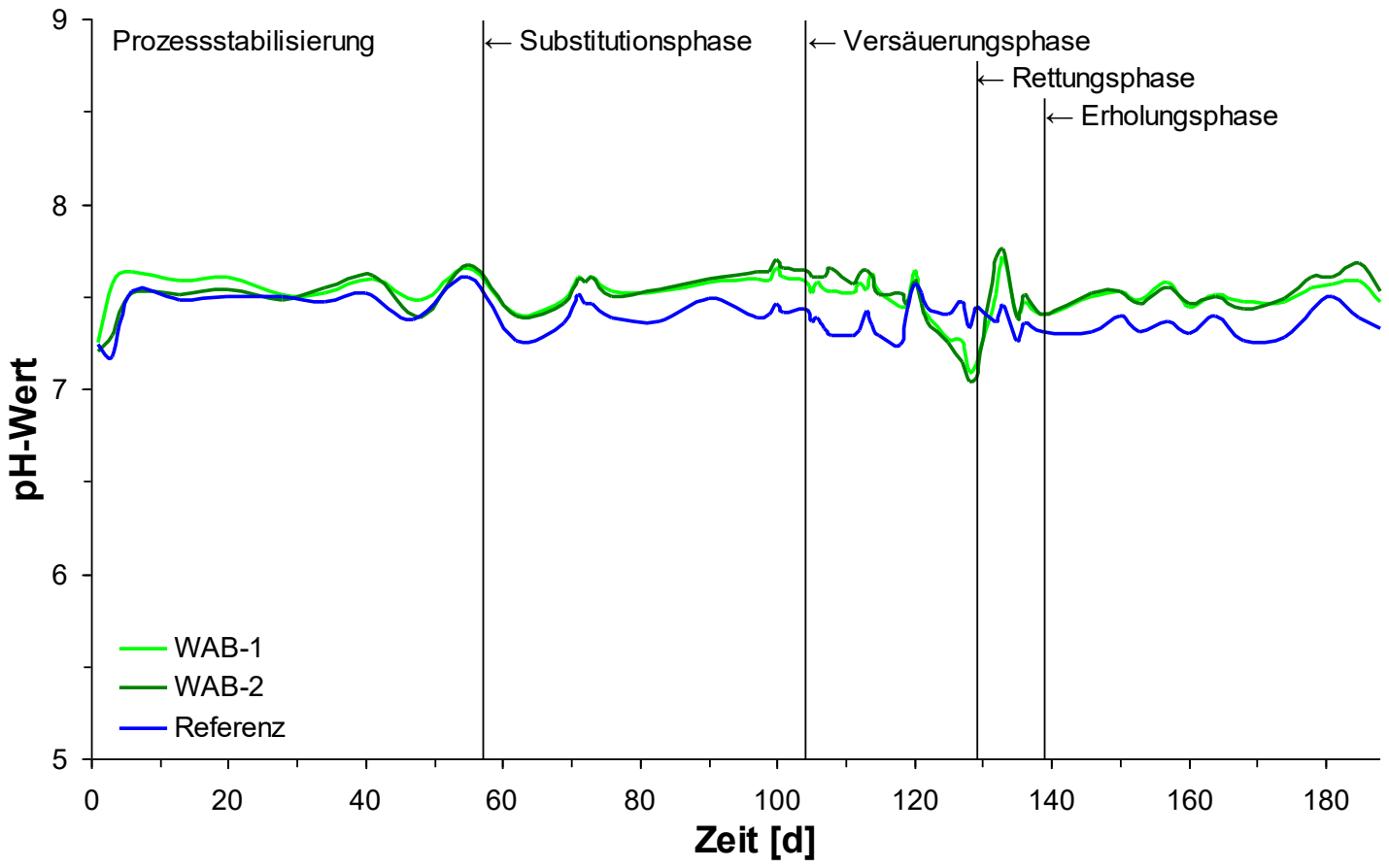

Abbildung 30: $\quad$ pH-Wert-Verläufe des Substitutionsversuchs 1 mit $30 \%$ Winterackerbohne- zu $70 \%$ Mais-Silage (oTS-bezogen) in den Versuchsbiogasreaktoren (mit Referenzreaktor). 


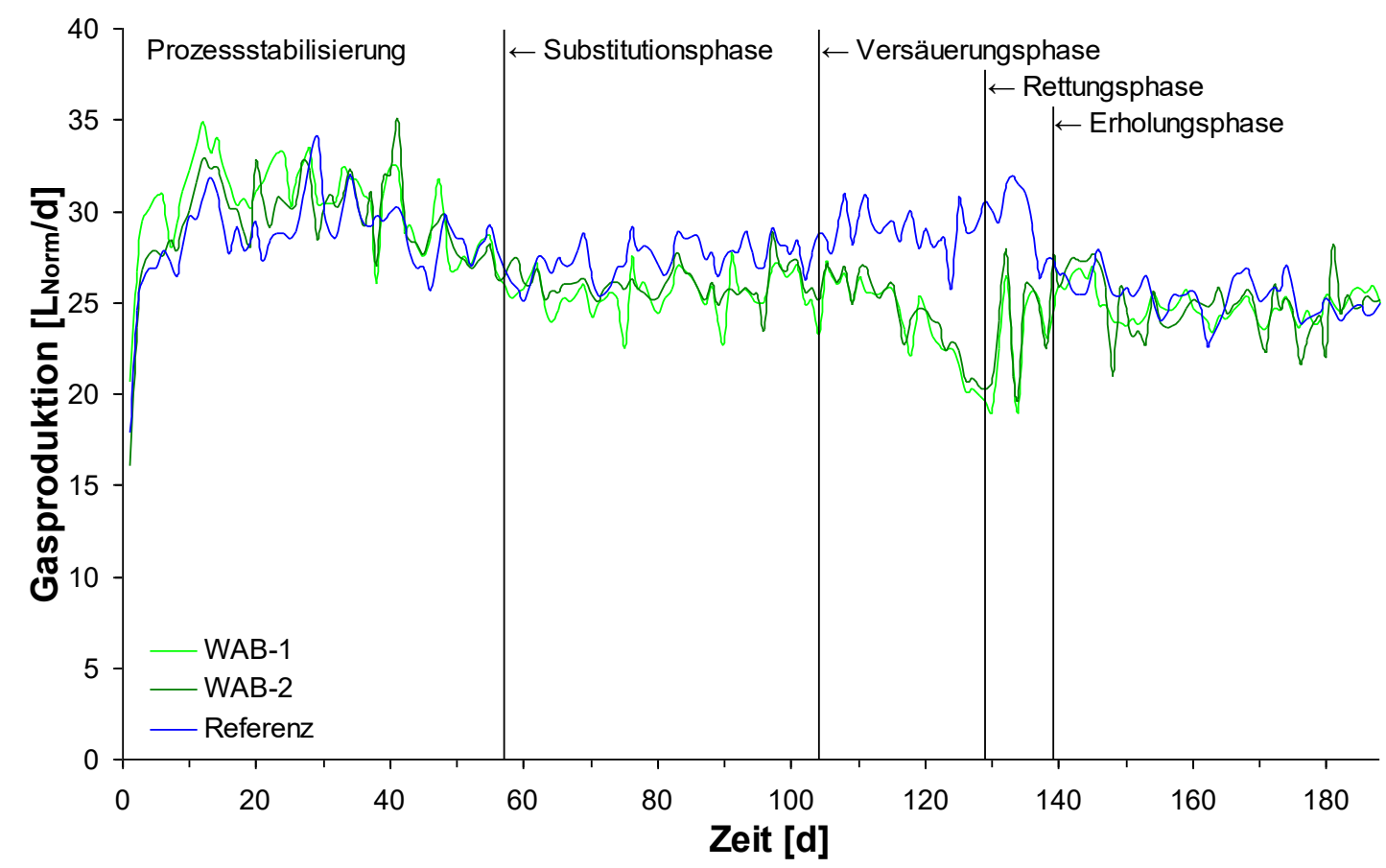

Abbildung 31: $\quad$ Verläufe der normierten Gasproduktion des Substitutionsversuchs 1 mit $30 \%$ Winterackerbohne-zu $70 \%$ Mais-Silage (oTS-bezogen) in den Versuchsbiogasreaktoren (mit Referenzreaktor).

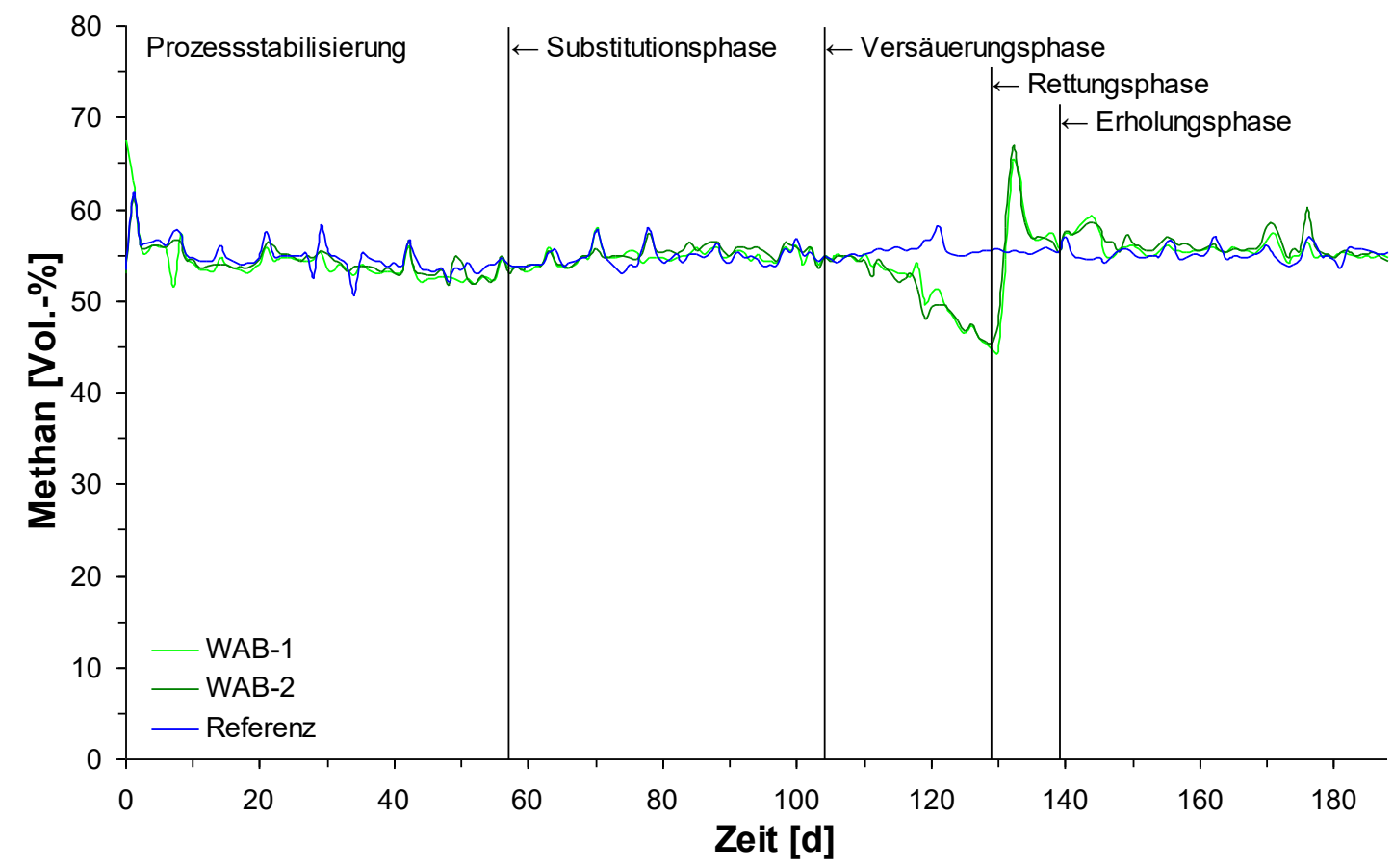

Abbildung 32: Verläufe des normierten Methangehalts des Substitutionsversuchs 1 mit $30 \%$ Winterackerbohne- zu $70 \%$ Mais-Silage (oTS-bezogen) in den Versuchsbiogasreaktoren (mit Referenzreaktor). 
Dass die Rettung der Substitutionsreaktoren gelang, bestätigt die Annahme, dass die Prozessinstabilitäten nicht durch die hohe Raumbelastung von $5 \mathrm{~g}_{\mathrm{oTS}} /(\mathrm{L} \cdot \mathrm{d})$ bedingt waren, sondern durch einen Spurenelementmangel. Lediglich die FOS (Kapp)-Werte blieben noch geringfügig erhöht. Dies könnte eventuell auf die bereits bekannten Effekte der Phenylessigsäure, einem Abbauprodukt des Phenylalanins, durch lysierte Mikroorganismen zurückgeführt werden (GRIEHL ET AL., 2010). Zum besseren Überblick sind für den ersten Substitutionsversuch in Abbildung 33 die Verläufe der FOS/TAC (FAL)-Werte und der normierten Gasproduktion direkt gegenübergestellt.

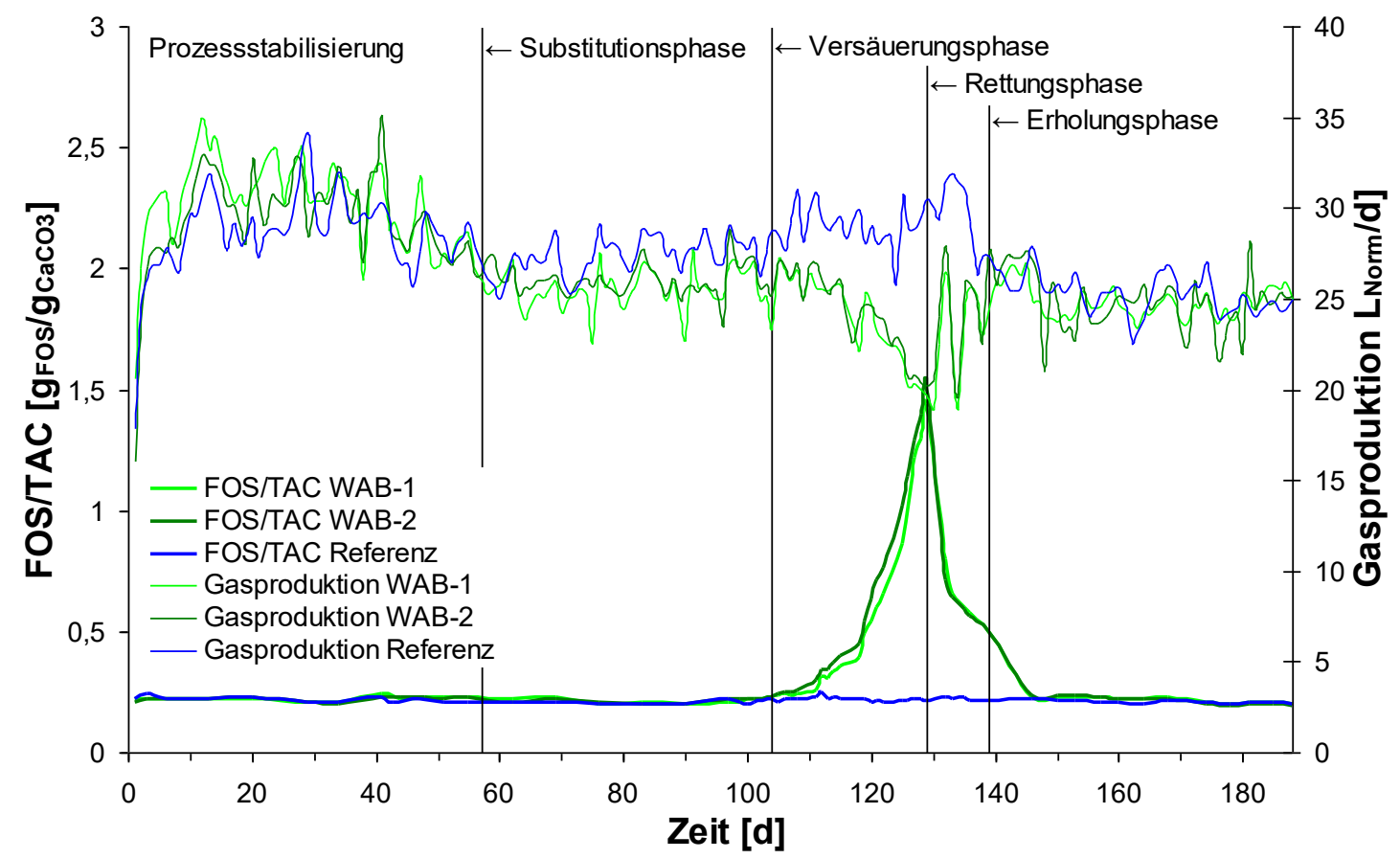

Abbildung 33: Gegenüberstellung der Verläufe der FOS/TAC (FAL)-Werte und der normierten Gasproduktion des Substitutionsversuchs 1 mit $30 \%$ Winterackerbohne- zu $70 \%$ Mais-Silage (oTS-bezogen) in den Versuchsbiogasreaktoren (mit Referenzreaktor).

Bei den Verläufen der normierten Gasproduktion (Abbildung 31 und Abbildung 33) fiel direkt mit Beginn der Erholungsphase ab Versuchstag 139 auf, dass die beiden Substitutionsreaktoren nahezu identische Biogasmengen gegenüber dem Referenzreaktor mit Spurenelementvollversorgung lieferten. Dies erwies sich zunächst als beachtlich, denn nach Ergebnissen aus den diskontinuierlichen Batchversuchen (vgl. Kapitel 4.2), wies die Winterackerbohne-Silage deutliche geringere Gaserträge gegenüber Mais-Silagen auf. Durch die Zumischung von $30 \%$ (oTS-bezogen) der spurenelementreichen, aber gasertragsärmeren Ersatzsilage zur Mais-Silage wäre zu 
erwarten gewesen, dass die Biogasausbeute des Referenzreaktors oberhalb der Ausbeuten der Substitutionsreaktoren liegt, wie es zuvor während der Substitutionsphase bis zur Versäuerungsphase der Fall war.

Die Hoffnung auf mögliche Synergieeffekte durch die Pflanzenmischung bei nur noch einem Viertel der ursprünglichen Spurenelementadditivmenge stellte sich jedoch als Fehleinschätzung heraus. Denn die mikrobiologischen Analysen am UFZ zeigten, dass sich die Mikroorganismengemeinschaften im Referenzreaktor 4.9 erheblich von denen in den beiden Substitutionsreaktoren unterschieden, vor allem in Hinsicht der methanogenen Archaeen (vgl. Kapitel 4.4.6).

Dadurch bedingt war ferner zu erwarten, dass in diesem Versuchsabschnitt im Vergleich $\mathrm{zu}$ einer „Normalsituation“ durch die divergente Mikroorganismengemeinschaft im Referenzreaktor auch der Volumenanteil Methan deutlich geringer sein müsste. Jedoch lagen die normierten Methangehalte (Abbildung 32) entgegen dieser Annahme über die komplette Versuchsdauer durchgängig auf einem konstant hohen Niveau. Es liegt die Vermutung nahe, dass Veränderungen der Mais-Silage-Qualität die Biogaserträge im Referenzreaktor deutlich stärker beeinflussten als in den Substitutionsreaktoren, weil diese nur 70 \% Mais-Silage (oTS-bezogen) enthielten. Während der gesamten Projektphase wurden insgesamt 13 unterschiedliche Mais-Silage-Chargen zur Beschickung verwendet. So kam ab Versuchstag 136 eine neue Charge Mais-Silage zum Einsatz, deren Qualität vermutlich eine geringere Gasausbeute zur Folge hatte. Genau ab diesem Zeitpunkt waren bei den Verläufen der normierten Gasproduktion (Abbildung 31 und Abbildung 33) deutliche Einbrüche zu erkennen.

Schlussfolgernd konnten bei den physikochemischen und gasanalytischen Prozessparametern beim Referenzreaktor mit Ausnahme des Verlaufs der normierten Gasproduktion zwar kaum Unterschiede erkannt werden, jedoch im Hinblick auf die methanogenen Archaeen (vgl. Kapitel 4.4.6) erwies sich der Kontrollreaktor in diesem letzten Abschnitt der Projektphase als nicht mehr geeignet für direkte Vergleiche mit den Substitutionsreaktoren. $\mathrm{Ob}$ dies ein Ausnahmeeffekt war, konnte nicht bestätigt werden, da aus Kostengründen der Referenzversuch in allen Projektphasen des Forschungsvorhabens nicht als Doppelversuch durchgeführt wurde. 
Ein ausschlaggebender Faktor für die Versäuerung bzw. den absehbaren potenziellen Zusammenbruch der beiden Versuchsbiogasreaktoren in dieser Doppelversuchsreihe war offenbar die niedrige Cobaltkonzentration (Abbildung 34). So lagen die Cobaltgehalte der Silagemischung 30\% Winterackerbohne- zu $70 \%$ Mais-Silage (oTSbezogen) in den Reaktoren kurz vor der Rettungsphase nur leicht oberhalb des in der ersten Projektphase erhobenen minimalen Schwellenwertes von 0,066 mg/kg FM (vgl. Kapitel 4.3.2) mit Werten von 0,088 (WAB-1) bzw. 0,082 (WAB-2) mg/kg FM Cobalt, trotz der um circa Faktor 3 höheren durchschnittlichen Cobaltgehalte in der Winterackerbohne-Silage (vgl. Kapitel 4.2, Tabelle 8).

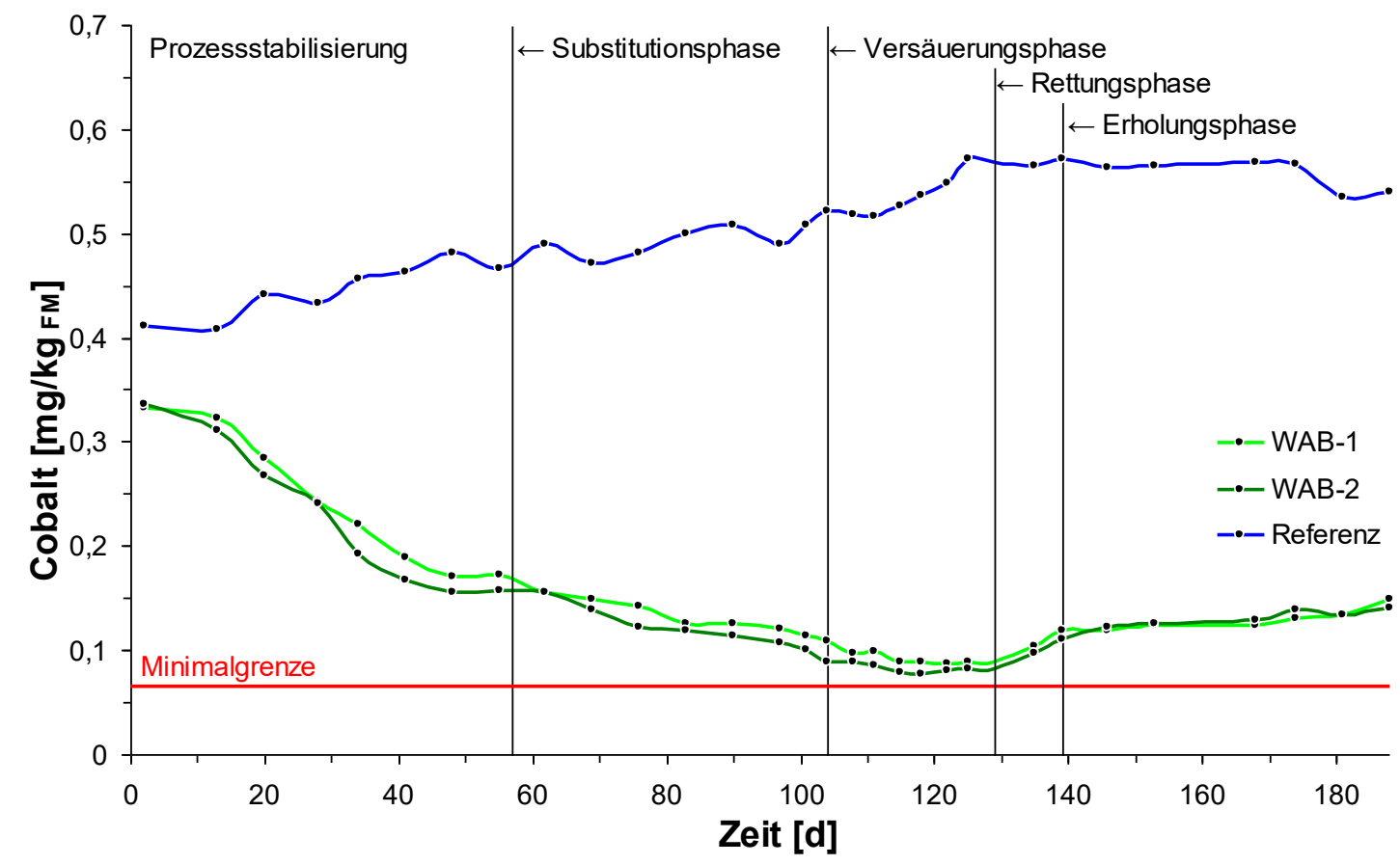

Abbildung 34: Verläufe der Cobaltkonzentrationen des Substitutionsversuchs 1 mit $30 \%$ Winterackerbohne- zu $70 \%$ Mais-Silage (oTS-bezogen) in den Versuchsbiogasreaktoren (mit Referenzreaktor).

Anhand der Verläufe der Nickelkonzentrationen in den beiden Substitutionsreaktoren über die gesamte Projektphase (Abbildung 35) kann nachvollzogen werden, dass die $30 \%$-Zumischung (oTS-bezogen) der Winterackerbohne-Silage ausreichend Nickel (vgl. Kapitel 4.2, Tabelle 8) unter diesen Versuchsbedingungen für einen stabilen Biogasprozess bereitstellte, um deutlich oberhalb des in der ersten Projektphase ermittelten, jedoch vermutlich zu hoch angegebenen minimalen Schwellenwertes von $0,82 \mathrm{mg} / \mathrm{kg}_{\mathrm{FM}} \mathrm{Nickel} \mathrm{zu}$ bleiben (vgl. Kapitel 4.3.3 und 4.3.4). In dieser Silagemischung ist Nickel kein limitierender Faktor. 


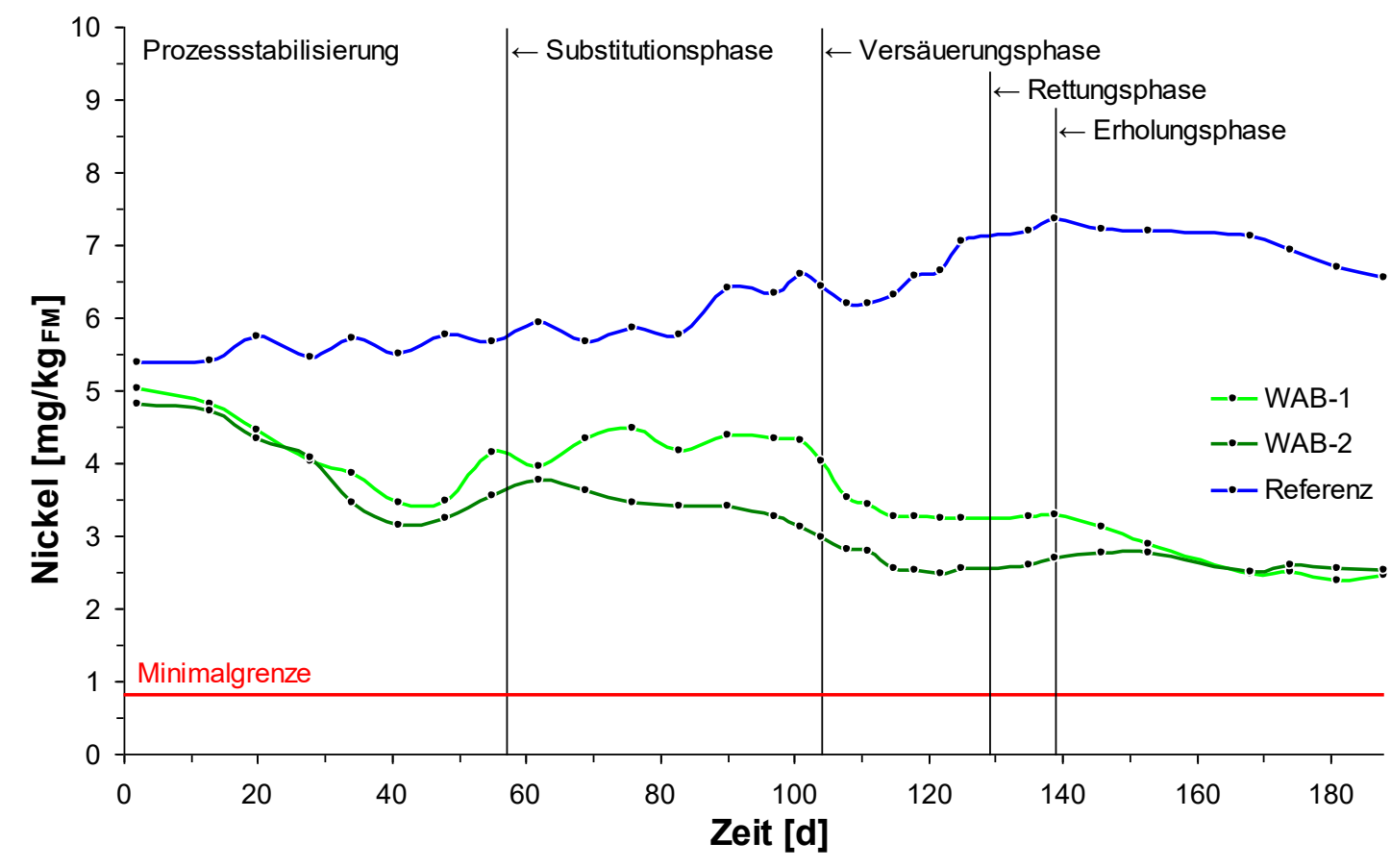

Abbildung 35: Verläufe der Nickelkonzentrationen des Substitutionsversuchs 1 mit $30 \%$ Winterackerbohne- zu $70 \%$ Mais-Silage (oTS-bezogen) in den Versuchsbiogasreaktoren (mit Referenzreaktor).

Das Versuchsende bzw. der Versuchsabbruch am Versuchstag 188 wurde aufgrund des fortgeschrittenen Zeitplanes notwendig, um die Biogasreaktoren für die dritte Projektphase (vgl. Kapitel 4.5) vorzubereiten. So konnte zwar die Langzeitstabilität dieser Pflanzensilagemischung mit einer Zugabe nur eines Viertels der ursprünglichen Spurenelementadditivmenge nach der Rettungsphase nicht über mindestens drei Verweilzeiten untersucht werden, jedoch zeigten alle Parameter in dieser 49-tägigen Erholungsphase, also über mehr als eine Verweilzeit, völlig unauffällige stabile Verläufe an.

Die Cobalt- und Nickelkonzentrationen in diesem ersten Substitutionsversuch lagen in der Erholungsphase über mehr als eine Verweilzeit bei stabil verlaufenden physikochemischen und gasanalytischen Parametern auf einem konstanten Niveau, deutlich über den minimalen Schwellenwerten. Somit hält die EnergiepflanzenSilagemischung mit $30 \%$ Winterackerbohne $\mathrm{zu} \quad 70 \%$ Mais (oTS-bezogen) in Kombination mit einer Zugabe von nur noch einem Viertel der ursprünglichen Spurenelementadditivmenge unter den gewählten Betriebsparametern stabile Biogasprozesse aufrecht. Ohne diese Additivzugabe brechen die Prozesse mit dieser Silagemischung jedoch zusammen. Cobalt stellt ein kritisches Mangelelement dar, Nickel ist bis zum Zusammenbruch kein limitierendes Element. 


\subsubsection{Substitutionsversuch 2: Vergärung von $30 \%$ Silage aus Amarant zu $70 \%$ Mais-Silage (oTS-bezogen)}

Nach der Substratumstellung (ab Versuchstag 57) begannen auch in diesem zweiten Substitutionsversuch in den beiden Versuchsbiogasreaktoren mit der Silagemischung Amarant $\mathrm{zu}$ Mais (Reaktor 4.7 = Amarant-1 und Reaktor 4.8 $=$ Amarant-2) in der Substitutionsphase ab Versuchstag 104 die FOS (Kapp)- und FOS/TAC (FAL)-Werte anzusteigen. Ab Versuchstag 112 überstiegen die FOS/TAC (FAL)-Werte analog zum ersten Substitutionsversuch (vgl. Kapitel 4.4.2) den kritischen Schwellenwert von 0,3 $\mathrm{g}_{\mathrm{FOS}} / \mathrm{g}_{\mathrm{CaCO}}$ (Abbildung 36). Die $\mathrm{pH}$-Wert-Verläufe blieben $\mathrm{zu}$ diesem Zeitpunkt noch stabil, begannen ab Versuchstag 120 aber auch zu fallen (Abbildung 37). Die Verläufe der normierten Gasproduktion (Abbildung 38) sowie der normierten Methangehalte (Abbildung 39) zeigten ebenfalls fallende Trends mit Beginn der Versäuerungsphase. Auch in diesen Reaktoren lagen ab Versuchstag 122 die normierten Methangehalte unter 50 Vol.-\%. Analog zum Substitutionsversuch von Mais-Silage mit Silage von Winterackerbohne wurden die Prozesse in den beiden Reaktoren mit Amarant-Silage nach circa zwei Verweilzeiten nach Substitutionsbeginn am Versuchstag 128 stark instabil und standen vor dem Zusammenbruch.

Die Rettungsphase ab Versuchstag 129 erfolgte in der gleichen Abfolge wie in Substitutionsversuch 1 (vgl. Kapitel 4.4.2). Ab Versuchstag 139 wurde danach bis Versuchsende am Versuchstag 188 ebenfalls nur noch ein Viertel der ursprünglichen Spurenelementadditivmenge $(0,2 \mathrm{~mL}$ pro Tag) zusätzlich in die beiden Reaktoren gegeben (Erholungsphase). Die physikochemischen und gasanalytischen Parameter stabilisierten sich auch in diesem Substitutionsversuch während der Rettungsphase sehr schnell und verliefen über mehr als eine Verweilzeit die komplette Erholungsphase über auf einem konstant stabilen Niveau. In Anlehnung an Kapitel 4.4.2 sind für diesen zweiten Substitutionsversuch zum besseren Überblick in Abbildung 40 die Verläufe der FOS/TAC (FAL)-Werte und der normierten Gasproduktion gegenübergestellt.

Das in Kapitel 4.4.2 bereits beschriebene Phänomen von nahezu identischen Biogasmengen bei der normierten Gasproduktion (Abbildung 38 und Abbildung 40) mit Beginn der Erholungsphase ab Versuchstag 139 trat in diesen Reaktoren ebenso auf. Die vermutete Ursache ist, dass Veränderungen der Mais-Silage-Qualität die Gaserträge im Referenzreaktor deutlich stärker beeinflussten als in den Substitutionsreaktoren. 


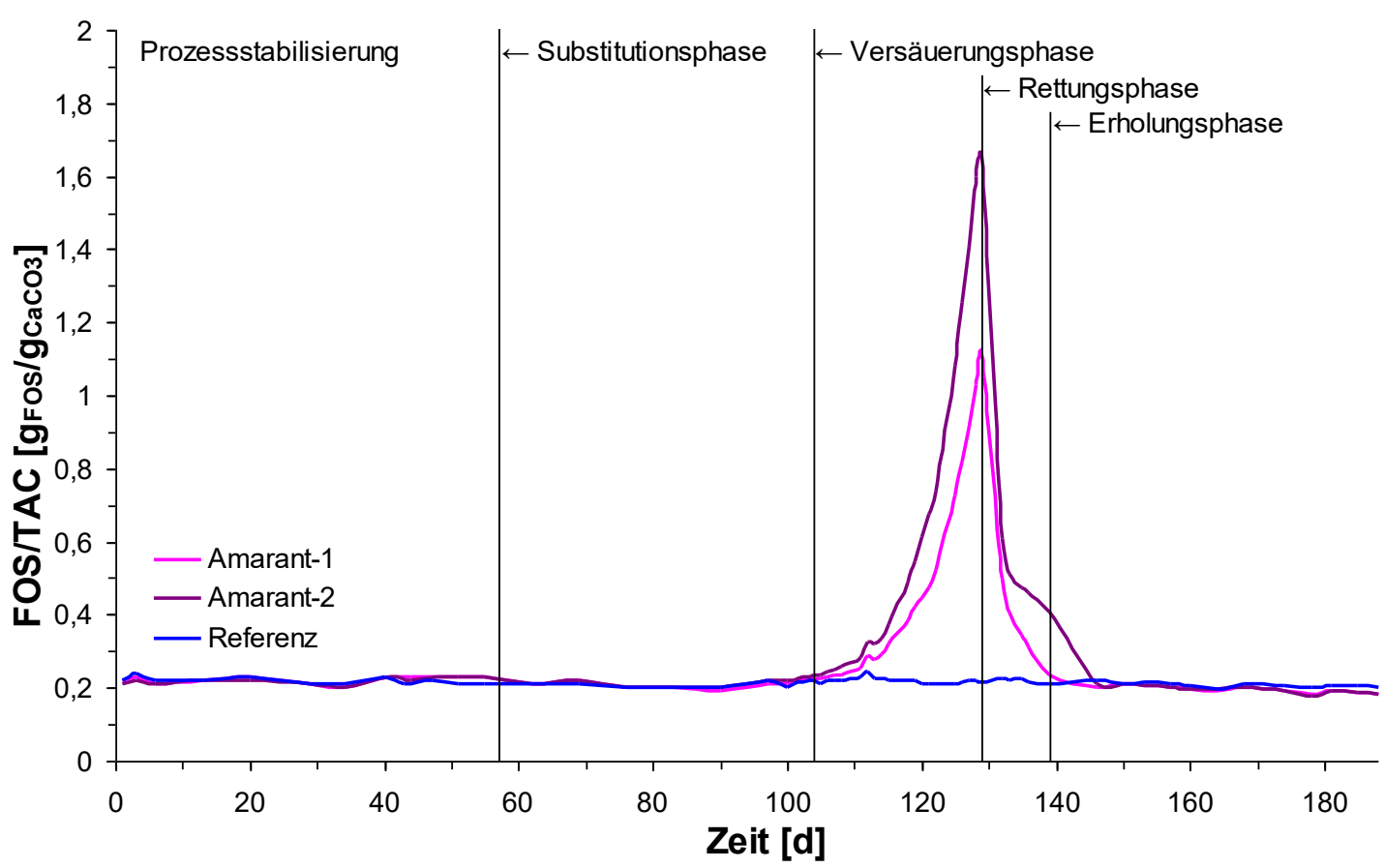

Abbildung 36: FOS/TAC (FAL)-Verläufe des Substitutionsversuchs 2 mit 30\% Amarant- zu $70 \%$ Mais-Silage (oTS-bezogen) in den Versuchsbiogasreaktoren (mit Referenzreaktor).

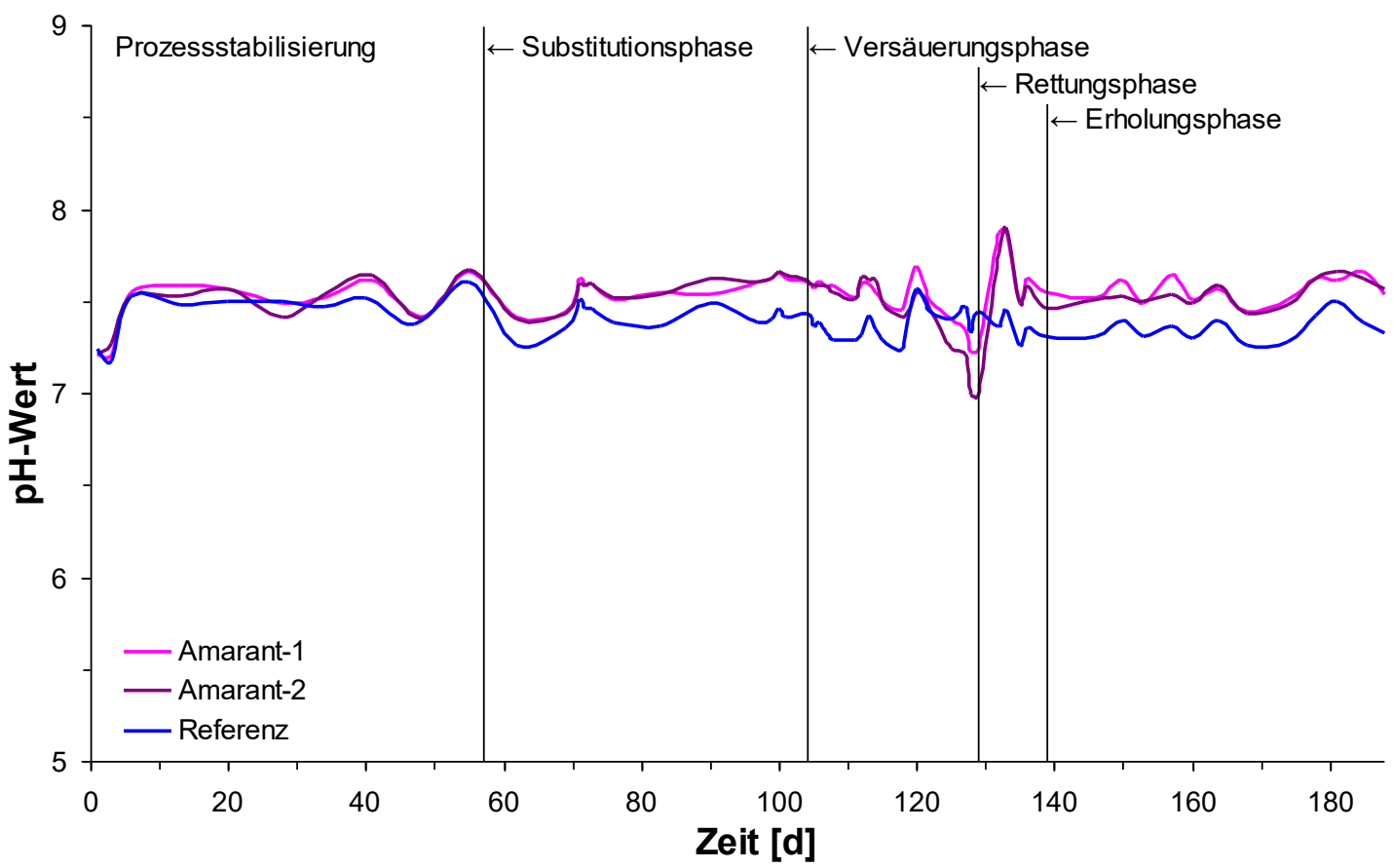

Abbildung 37: $\quad$ pH-Wert-Verläufe des Substitutionsversuchs 2 mit $30 \%$ Amarant-zu $70 \%$ Mais-Silage (oTS-bezogen) in den Versuchsbiogasreaktoren (mit Referenzreaktor). 


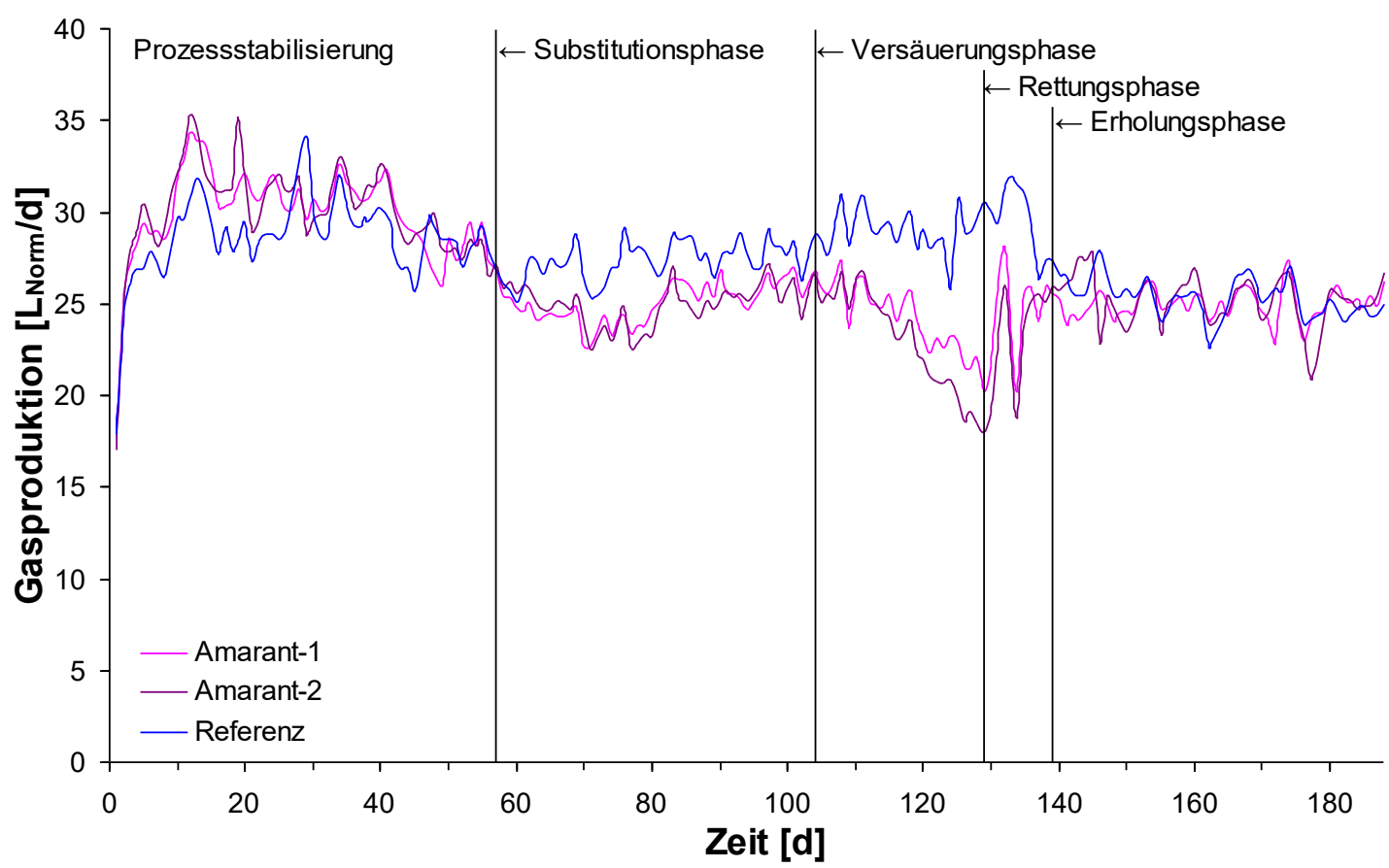

Abbildung 38: $\quad$ Verläufe der normierten Gasproduktion des Substitutionsversuchs 2 mit $30 \%$ Amarant-zu $70 \%$ Mais-Silage (oTS-bezogen) in den Versuchsbiogasreaktoren (mit Referenzreaktor).

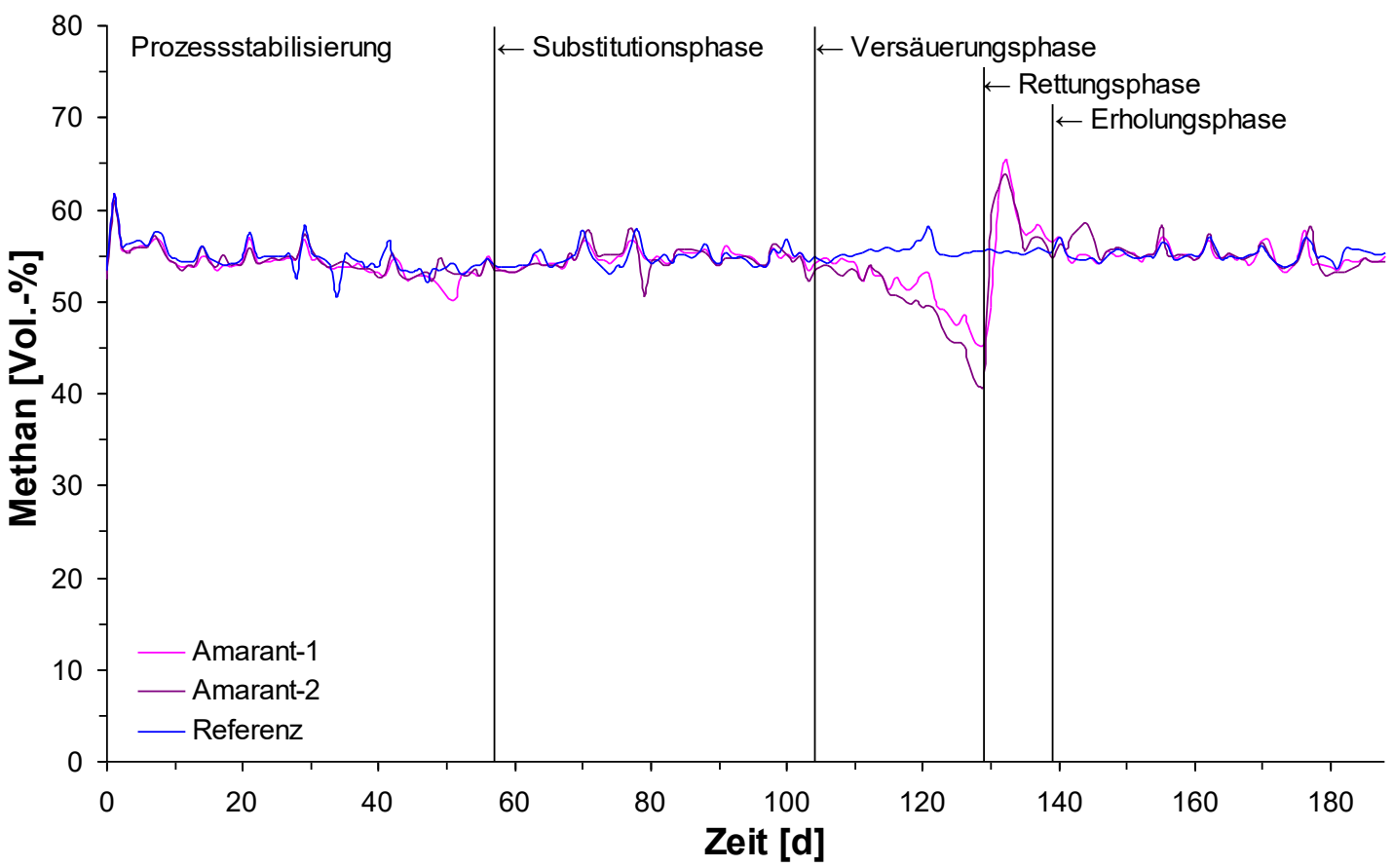

Abbildung 39: Verläufe des normierten Methangehalts des Substitutionsversuchs 2 mit $30 \%$ Amarant- zu $70 \%$ Mais-Silage (oTS-bezogen) in den Versuchsbiogasreaktoren (mit Referenzreaktor). 


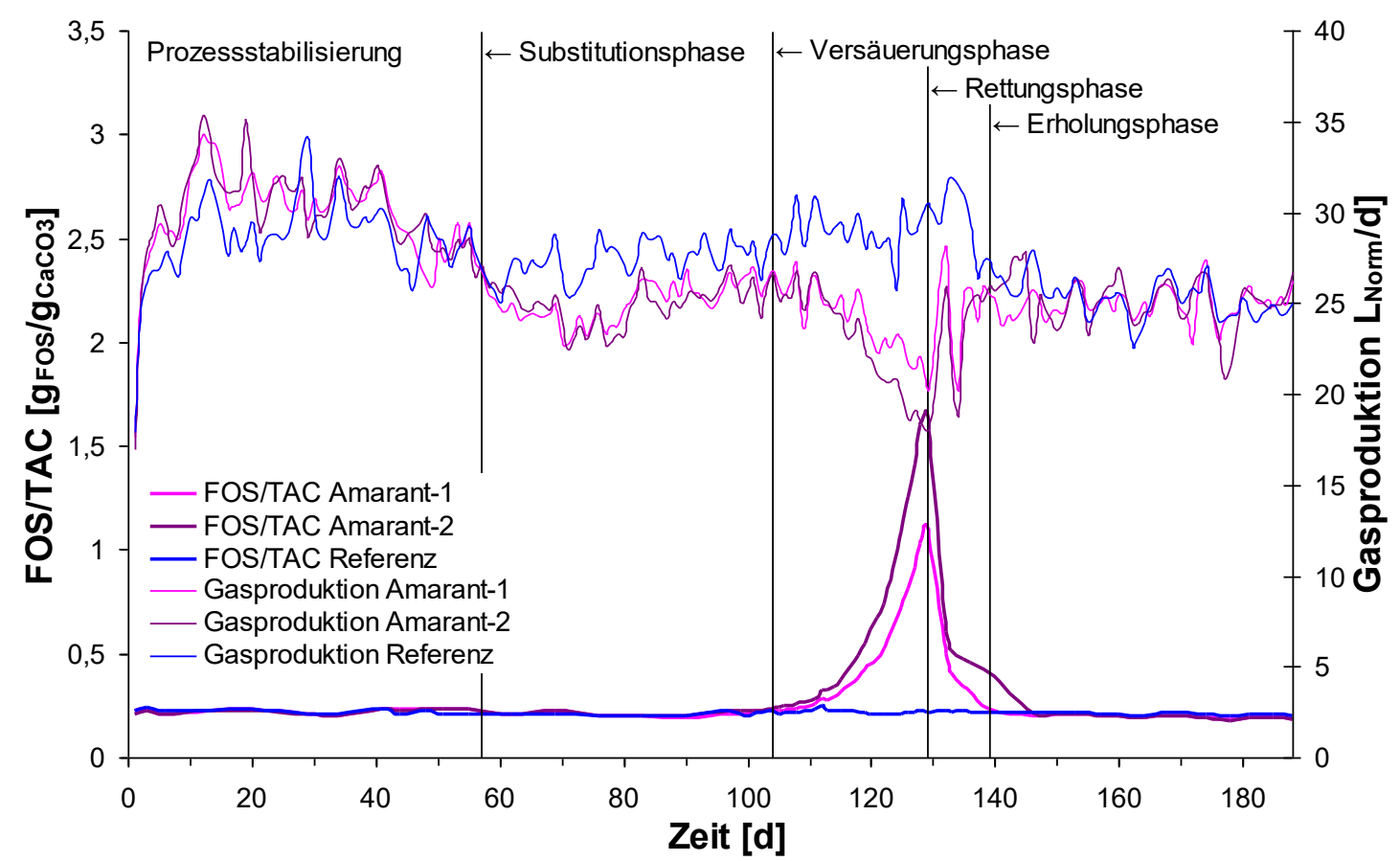

Abbildung 40: $\quad$ Gegenüberstellung der Verläufe der FOS/TAC (FAL)-Werte und der normierten Gasproduktion des Substitutionsversuchs 2 mit $30 \%$ Amarant- zu $70 \%$ Mais-Silage (oTS-bezogen) in den Versuchsbiogasreaktoren (mit Referenzreaktor).

Auch in dieser Doppelversuchsreihe war die sehr niedrige Cobaltkonzentration für die Versäuerung bzw. den abzusehenden potenziellen Zusammenbruch der beiden Versuchsbiogasreaktoren das offensichtlich entscheidende Kriterium (Abbildung 41). Bei der Mischung $30 \%$ Amarant- zu $70 \%$ Mais-Silage (oTS-bezogen) wurde der minimale Cobalt-Schwellenwert von $0,066 \mathrm{mg} / \mathrm{kg}_{\mathrm{FM}}$ (vgl. Kapitel 4.3.2) kurz vor Beginn der Rettungsphase in beiden Reaktoren exakt erreicht bzw. sogar noch leicht unterschritten mit jeweils einem Wert von $0,063 \mathrm{mg} / \mathrm{kg}$ FM am Versuchstag 125.

Die $30 \%$-Zumischung (oTS-bezogen) der Energiepflanzen-Silage Amarant lieferte unter diesen Versuchsbedingungen wie auch die $30 \%$-Zumischung (oTS-bezogen) der Winterackerbohne-Silage im ersten Substitutionsversuch ausreichend Nickel für stabile Biogasprozesse (Abbildung 42). Über die gesamte Projektphase lagen die Verläufe der Nickelkonzentrationen in den beiden Substitutionsreaktoren deutlich oberhalb des ermittelten, jedoch vermutlich zu hoch angegebenen minimalen Schwellenwertes von $0,82 \mathrm{mg} / \mathrm{kg}_{\mathrm{FM}}$ Nickel (vgl. Kapitel 4.3.3 und 4.3.4). D.h. auch in dieser Silagemischung ist Nickel kein limitierender Faktor bis zum Prozesszusammenbruch. 


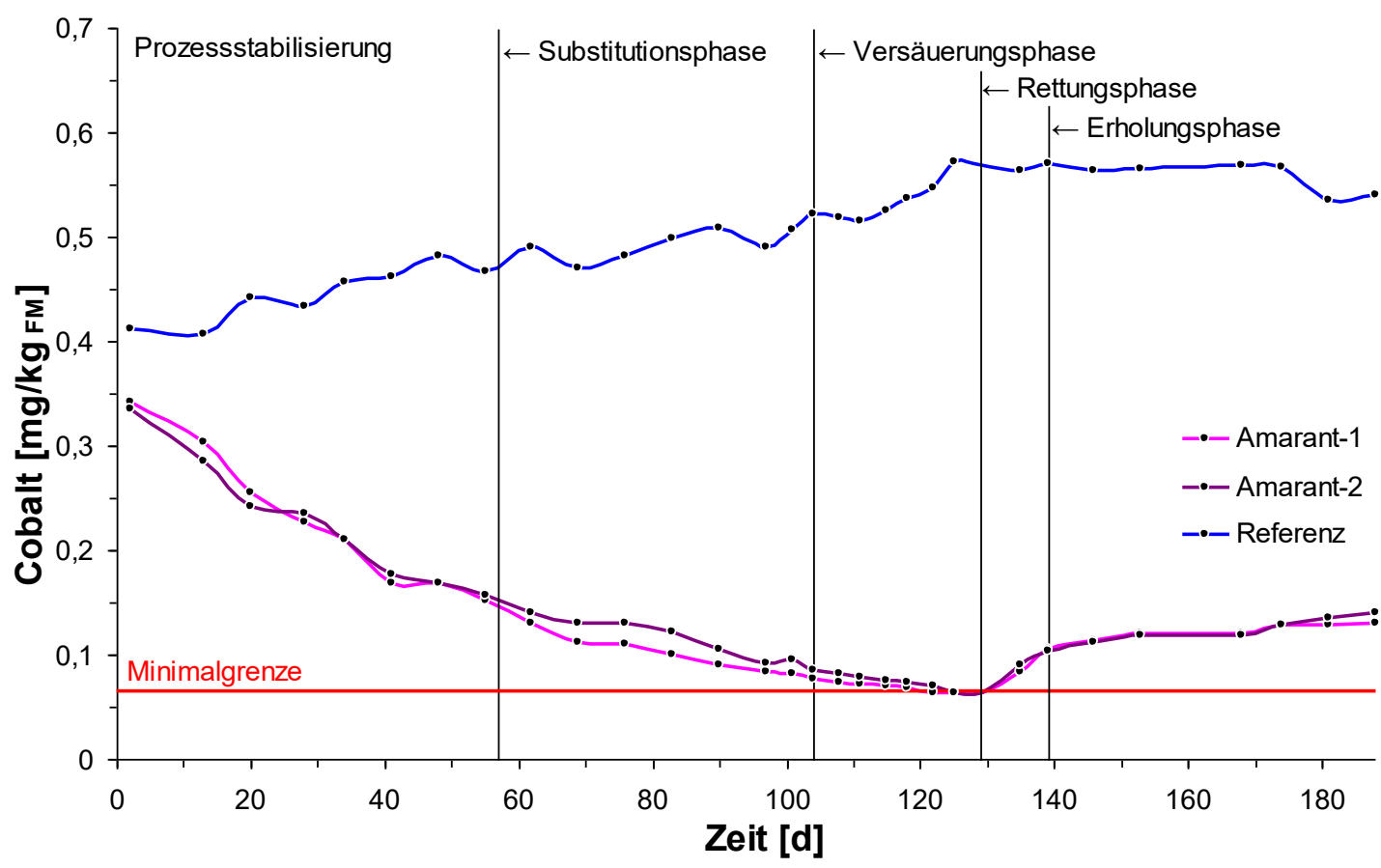

Abbildung 41: Verläufe der Cobaltkonzentrationen des Substitutionsversuchs 2 mit $30 \%$ Amarant- zu $70 \%$ Mais-Silage (oTS-bezogen) in den Versuchsbiogasreaktoren (mit Referenzreaktor).

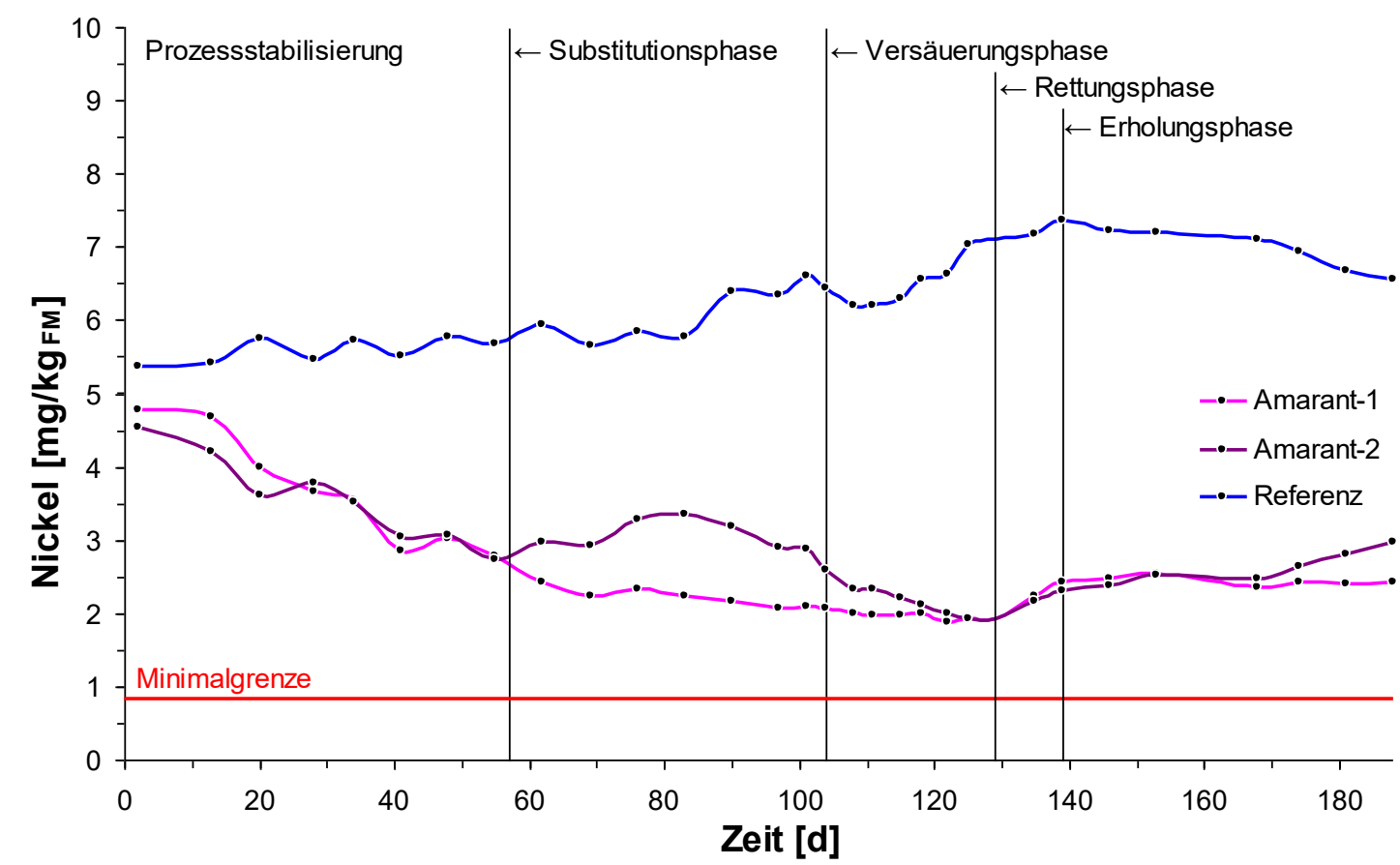

Abbildung 42: Verläufe der Nickelkonzentrationen des Substitutionsversuchs 2 mit $30 \%$ Amarant- zu $70 \%$ Mais-Silage (oTS-bezogen) in den Versuchsbiogasreaktoren (mit Referenzreaktor). 
Die Langzeitstabilität dieser Pflanzensilagemischung durch die Zugabe von nur einem Viertel der ursprünglichen Spurenelementadditivmenge nach der Rettungsphase konnte auch in diesem Doppelversuch nicht über mindestens drei Verweilzeiten (120 Versuchstage) untersucht werden, da wie in Kapitel 4.4.2 erwähnt, die Reaktoren für die dritte Projektphase (vgl. Kapitel 4.5) vorbereitet werden mussten. In der über mehr als eine Verweilzeit andauernden Erholungsphase (49 Versuchstage) zeigten die physikochemischen und gasanalytischen Parameter (vgl. Abbildung 36 bis Abbildung 40) jedoch auch in diesen beiden Substitutionsreaktoren konstant stabile Verläufe.

Die Cobalt- und Nickelgehalte lagen auch im zweiten Substitutionsversuch bei stabilen Prozessparametern die gesamte Erholungsphase über auf einem konstanten Niveau, deutlich über den minimalen Schwellenwerten. Unter den Versuchsbedingungen ist auch diese Silagemischung mit $30 \%$ Amarant zu $70 \%$ Mais (oTSbezogen) in Kombination einer Zugabe nur noch eines Viertels der ursprünglichen Spurenelementadditivmenge geeignet, stabile Biogasprozesse aufrechtzuerhalten. Ohne Zugabe dieser Additivlösung brechen die Prozesse jedoch zusammen. Cobalt ist das kritische Mangelelement und Nickel bis zum Zusammenbruch nicht limitierend.

\subsubsection{Bewertung weiterer Elemente in den Substitutionsversuchen 1 und 2}

Neben den für den Biogasprozess wichtigen Mikronährstoffen Cobalt und Nickel (vgl. Kapitel 4.4.2 und 4.4.3) zeigten sich bei den Konzentrationsverläufen der essenziellen Grundelemente und Makronährstoffe (Gruppe I, vgl. Kapitel 2.2.5) weitere positive Auffälligkeiten. Die beiden substituierten Silagen Winterackerbohne und Amarant stellten ab Versuchstag 57 gute Quellen für Makronährstoffe dar (Abbildung 43 und Abbildung 44). Vor allem die Mischung mit $30 \%$ Amarant-Silage (oTS-bezogen) wies hohe Anstiege bei den Calcium-, Kalium-, Magnesium- und Phosphorgehalten in den Reaktoren auf. Die Mischung mit 30 \% WinterackerbohneSilage (oTS-bezogen) zeigte in den Reaktoren ebenfalls erhöhte Gehalte bei Calcium und Kalium. Magnesium und Phosphor hingegen lagen konstant auf dem Niveau des Referenzreaktors. Die Schwefelkonzentrationen der beiden Pflanzenmischungen wiesen in den Substitutionsreaktoren gegenüber der Kontrolle keine höheren Gehalte auf und verliefen stabil (Abbildung 44). Dies passt gut mit den gegenüber Mais angereicherten Konzentrationen der betreffenden Elemente in den Energiepflanzen-Silagen überein (vgl. Kapitel 4.2, Tabelle 8). 
Kapitel 4: (Quasi)kontinuierliche Laborfermenterversuche
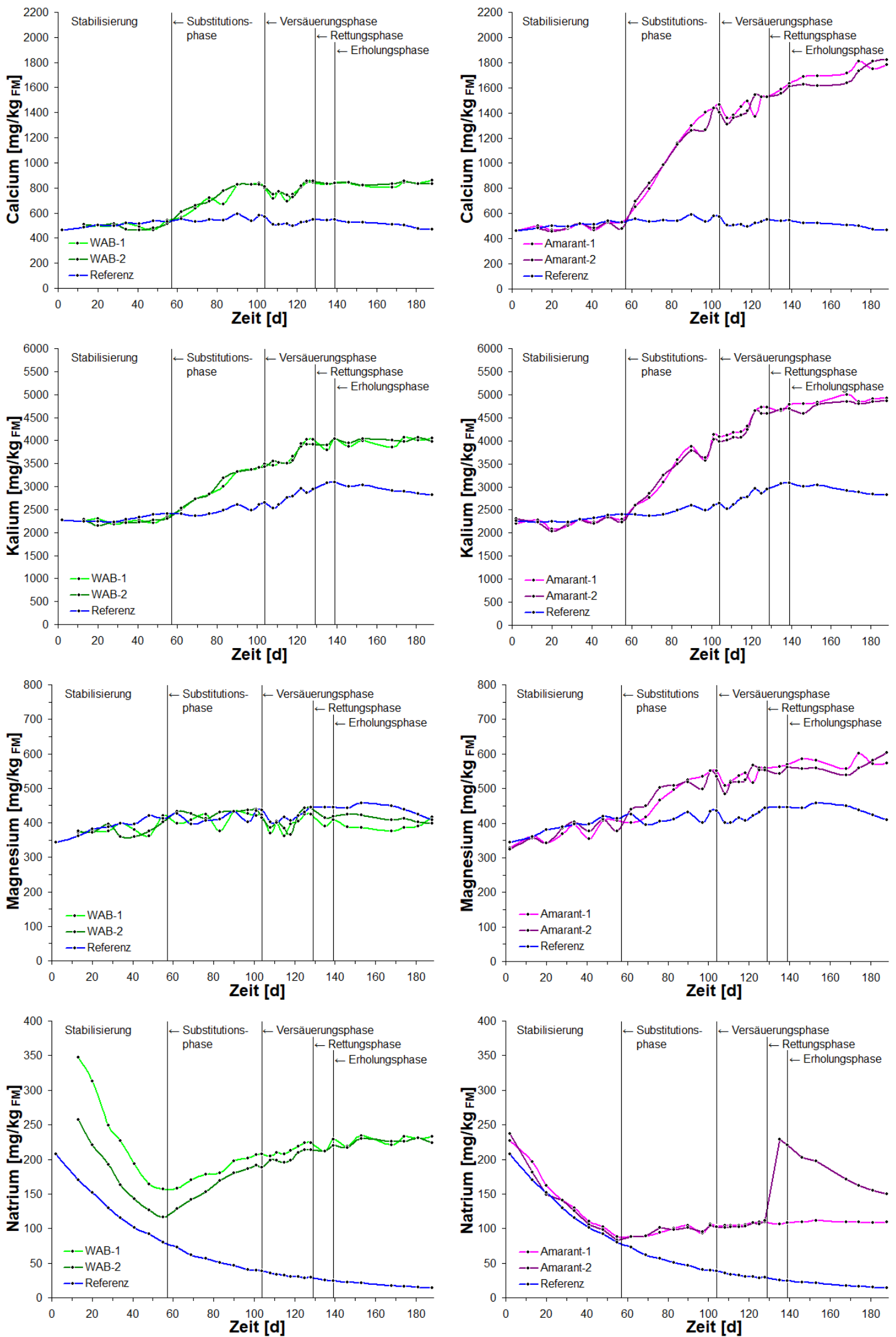

Abbildung 43: $\quad$ Verläufe der Calcium-, Kalium-, Magnesium- und Natriumgehalte der Substitutionsversuche 1 und 2 in den Versuchsbiogasreaktoren (mit Referenzreaktor). 

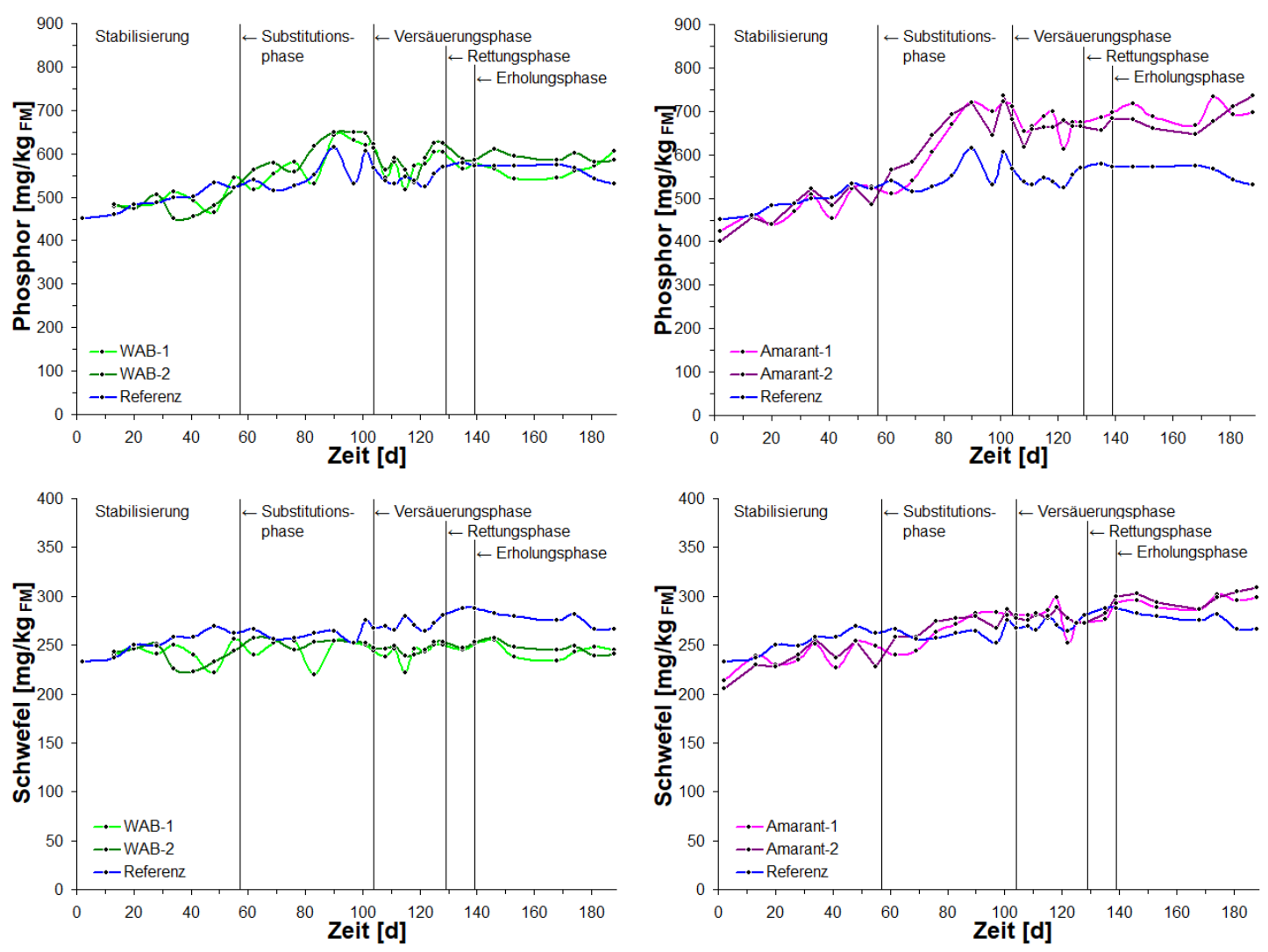

Abbildung 44: Verläufe der Phosphor- und Schwefelgehalte der Substitutionsversuche 1 und 2 in den Versuchsbiogasreaktoren (mit Referenzreaktor).

Die Natriumgehalte (Abbildung 43) fielen im Referenzreaktor analog zur ersten Projektphase (vgl. Kapitel 4.3.5) über die gesamte Versuchsdauer stark ab und lagen am Versuchsende mit $14-15 \mathrm{mg} / \mathrm{kg}_{\mathrm{FM}}$ auf einem sehr niedrigen Level. In den beiden Versuchsbiogasreaktoren mit Winterackerbohne-Silage stiegen mit Beginn der Substitution die Natriumkonzentrationen hingegen erfreulicherweise stark bzw. in den beiden Reaktoren mit Amarant-Silage leicht an und lagen in der Erholungsphase bei durchschnittlich ca. $230 \mathrm{mg} / \mathrm{kg}_{\text {FM }}$ (Reaktoren WAB-1 und WAB-2) bzw. $110 \mathrm{mg} / \mathrm{kg}$ FM (Reaktor Amarant-1). Im Reaktor Amarant-2 erfolgte am Versuchstag 129 zu Beginn der Rettungsphase ein Eingriff durch Zugabe von $4 \mathrm{~g}$ Natriumhydrogencarbonat $\left(\mathrm{NaHCO}_{3}\right)$, um den stärker als in Reaktor Amarant-1 gefallenen $\mathrm{pH}-$ Wert $(<7,0$, vgl. Abbildung 37) in Anlehnung an Burgstaler ET AL. (2011) zu puffern bzw. der Versäuerung entgegenzuwirken. Das erklärt den steilen Anstieg des Natriumwertes in dem Reaktor zu diesem Zeitpunkt. Die hohen Natrium-Durchschnittsgehalte in den substituierten Silagen (vgl. Kapitel 4.2, Tabelle 8) lassen sich v.a. auf die Verwendung des Silierhilfsmittels Kofasil ${ }^{\circledR}$ Liquid zurückführen. Es wurde aufgrund der niedrigen 
Trockensubstanzgehalte der Energiepflanzenvarianten (vgl. Kapitel 4.2, Tabelle 7) zur Vermeidung von Fehlgärungen eingesetzt (3 L/t Siliergut) und enthielt neben Hexamethylentetramin (Urotropin) das Salz Natriumnitrit $\left(\mathrm{NaNO}_{2}\right)$. Die Natriumgehalte in den unbehandelten Winterackerbohne-Pflanzen sind gegenüber Mais jedoch auch stark angereichert (Faktor 30 bis 40). Amarant enthält ähnliche Natriumkonzentrationen wie Mais (vgl. Analysen von FAHLBUSCH, 2018).

Bei den essenziellen Mikronährstoffen (Gruppe II) Eisen, Mangan, Molybdän, Zink und Selen fielen während der Prozessstabilisierung in allen Substitutionsreaktoren fallende Konzentrationsverläufe auf (Abbildung 45 und Abbildung 46). Hintergrund war, dass die Zugabe der Spurenelementlösung (vgl. Kapitel 4.1, Tabelle 6) bereits während der Stabilisierungsphase ausgesetzt wurde, um mit Beginn der Substitutionsphase am Versuchstag 57 von einem niedrigeren Niveau aus mit den Zumischungen der beiden Energiepflanzen-Silagen Winterackerbohne bzw. Amarant zu Mais-Silage zu starten. Andernfalls hätte die Versuchszeit um ein Vielfaches länger angedauert. Jedoch fielen auch während der Substitutions- und Versäuerungsphase die Konzentrationen dieser Spurenelemente in den Substitutionsreaktoren weiter ab, allerdings bis zum Beginn der Rettungsphase am Versuchstag 129 nicht in kritische Konzentrationsbereiche nach Tabelle 1 in Kapitel 2.3. Nur bei Selen (Abbildung 46) lagen die Gehalte in den vier Reaktoren auf einer niedrigen Stufe, mit Werten von 0,0036 bzw. $0,0032 \mathrm{mg} / \mathrm{kg}_{\mathrm{FM}}$ Selen (Reaktor WAB-1 bzw. WAB-2) und 0,0037 bzw. 0,0042 mg/kg FM Selen (Reaktor Amarant-1 bzw. Amarant-2) kurz vor Beginn der Rettungsphase. Der minimale Bedarfswert von 0,002 mg/kg FM Selen (nach MunK ET AL., 2010) wurde jedoch nicht unterschritten. In den Erholungsphasen lagen die Eisen-, Mangan-, Molybdän-, Zinkund Selengehalte dann wieder auf guten, stabilen Niveaus.

Die Anstiege der Molybdän- und Selengehalte im Referenzreaktor ab Versuchstag 108 lassen sich abschließend nicht stichhaltig erklären. Im Versuchszeitraum wurde die Additivlösung mit diesen Elementen unverändert in den Kontrollfermenter zugegeben. Auch waren keine entsprechenden Anstiege bei den anderen Elementen der Additivlösung (vgl. Kapitel 4.1, Tabelle 6) ersichtlich. Es gab ferner am Versuchstag 108 zwar auch einen Mais-Silage-Chargenwechsel, der höhere Elementeinträge hätte induzieren können, allerdings hätten dann auch in den vier Substitutionsreaktoren mit 70 \% MaisSilage (oTS-bezogen) Anstiege dieser beiden Elemente erkennbar sein müssen. 

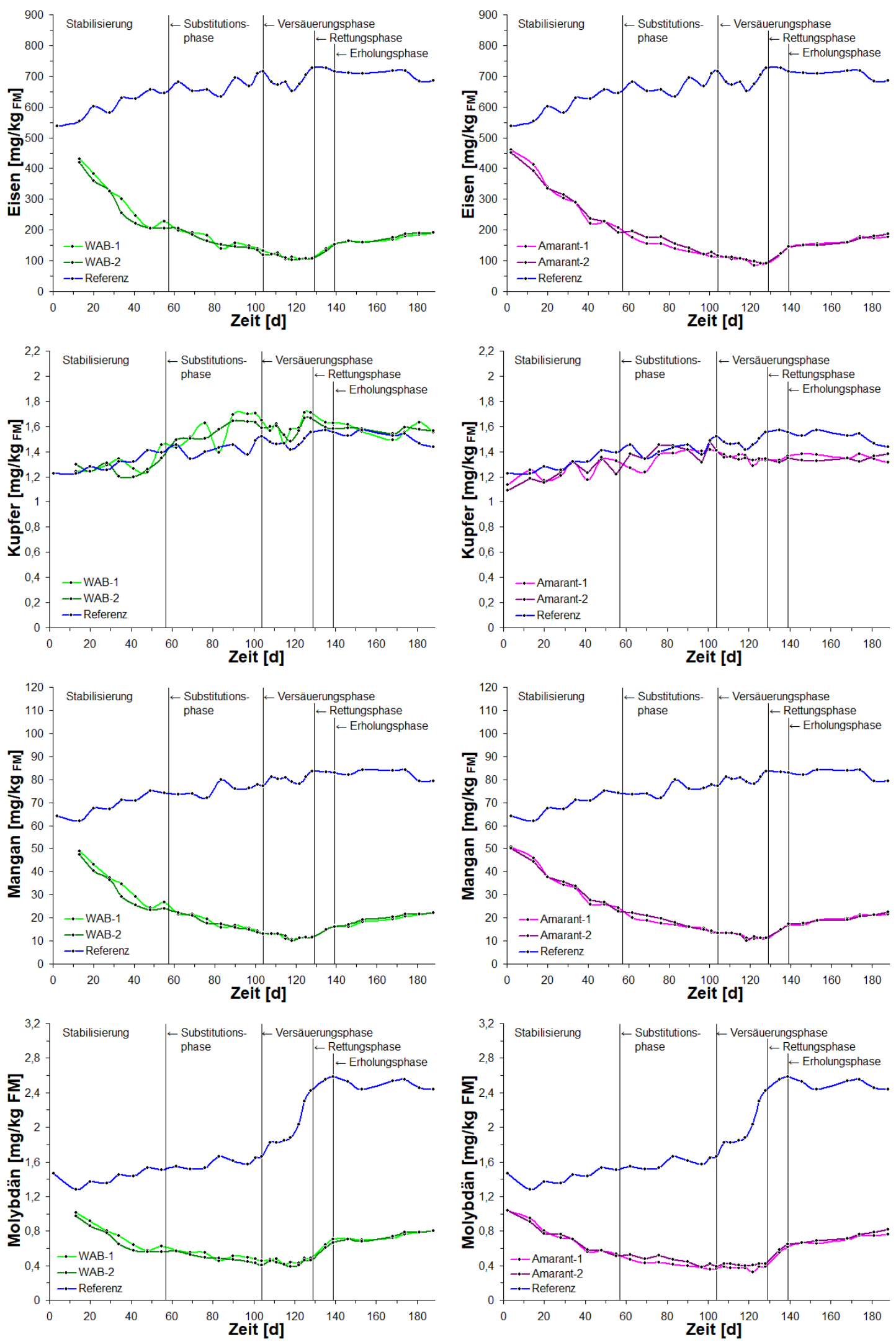

Abbildung 45: Verläufe der Eisen-, Kupfer-, Mangan- und Molybdängehalte der Substitutionsversuche 1 und 2 in den Versuchsbiogasreaktoren (mit Referenzreaktor). 

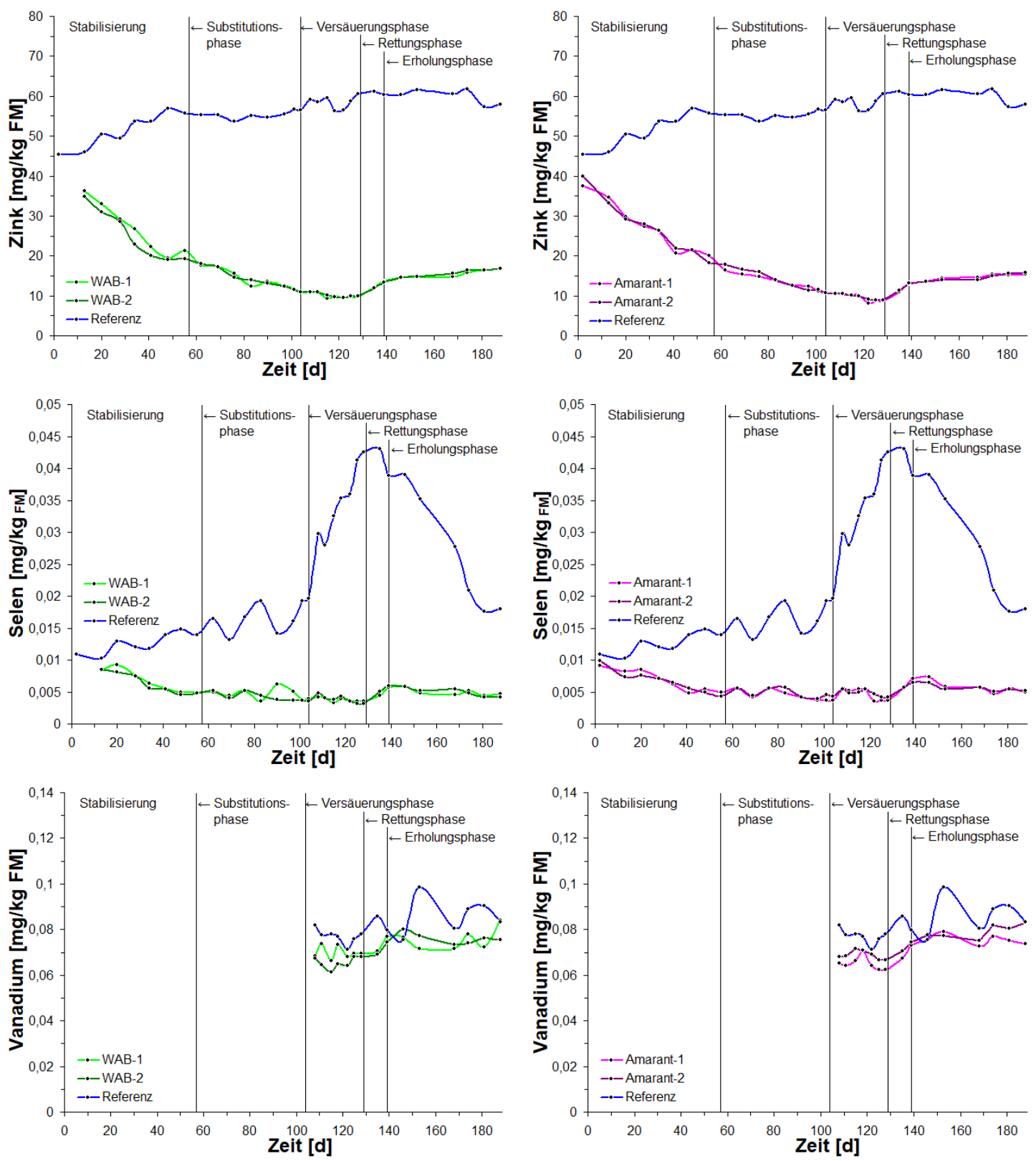

Abbildung 46: $\quad$ Verläufe der Zink-, Selen- und Vanadiumgehalte der Substitutionsversuche 1 und 2 in den Versuchsbiogasreaktoren (mit Referenzreaktor).

Die Kupfer- und Vanadiumgehalte (verlässliche Messungen von Vanadium waren seit Herbst 2016 möglich, vgl. Kapitel 3.4.3) zeigten keine Auffälligkeiten und verliefen in allen vier Substitutionsreaktoren über die gesamte Substitutionsdauer hinweg im Bereich des Referenzreaktors (Abbildung 45 und Abbildung 46). Kupfer war in der Spurenelementlösung nicht enthalten (vgl. Kapitel 4.1, Tabelle 6), sodass sich die Konzentrationen in den Reaktoren analog zur ersten Projektphase (vgl. Kapitel 4.3.5) auf einem niedrigen Level bewegten. Auch die durchschnittlichen Kupferkonzentrationen in der Winterackerbohne- bzw. Amarant-Silage sind gegenüber der 
Mais-Silage nicht angereichert, sondern liegen etwa im Bereich des Maiswertes (vgl. Kapitel 4.2, Tabelle 8). Auch hier deuteten die Prozessdaten des Referenzreaktors jedoch nicht auf Mangelzustände hin, sodass die Kupfergehalte in den Doppelversuchen für stabile Biogasprozesse ausreichend sind.

Die Konzentrationsverläufe der Elemente der Gruppe III (vgl. Kapitel 2.2.5), Arsen, Cadmium, Blei, Aluminium, Lanthan (als Vertreter der Lanthanoide/Seltene Erdelemente), Titan und Strontium liefen während der gesamten Versuchsdauer nicht in kritische hohe Bereiche (Abbildung 47 und Abbildung 48). Arsen und Cadmium sowie Strontium (in den Substitutionsreaktoren mit Winterackerbohne-Silage) lagen weitestgehend konstant. Dies ist zurückzuführen, dass sich die durchschnittlichen Konzentrationen dieser Elemente in der Winterackerbohne- bzw. Amarant-Silage gegenüber den Gehalten in der Mais-Silage kaum unterscheiden (vgl. Kapitel 4.2, Tabelle 8). Der Cadmiumgehalt in der Winterackerbohne-Silage liegt sogar niedriger gegenüber Mais.

Aluminium, Lanthan, Titan und Strontium (nur in den Reaktoren mit Amarant-Silage) wiesen mit Beginn der Substitutionsphase hingegen leichte bis stärkere Anstiege auf (Abbildung 48). Ursache dafür ist, dass die durchschnittlichen Elementkonzentrationen in der Winterackerbohne- bzw. Amarant-Silage um das Zwei- bis Vierfache gegenüber den Konzentrationen in Mais-Silagen angereichert sind (vgl. Kapitel 4.2, Tabelle 8).

Blei zeigte in allen Substitutionsreaktoren fallende Konzentrationsverläufe mit Beginn der Prozessstabilisierung, lag dann jedoch ab ca. Versuchstag 100 auf einem konstanten Level in niedrigen Konzentrationsbereichen. Die leicht erhöhten Bleikonzentrationen zu Versuchsbeginn stammten vermutlich aus dem verwendeten Inokulummaterial.

Auch in dieser zweiten Projektphase zeigten sich in vier der fünf Versuchsbiogasreaktoren auffällige Anstiege der Chromgehalte (Abbildung 47), korrelierend mit Anstiegen der Nickelkonzentrationen (Abbildung 35 und Abbildung 42). So stiegen die Chromkonzentrationen in den Reaktoren WAB-1 und WAB-2 ab Versuchstag 41, in Reaktor Amarant-2 ab Versuchstag 55 sowie im Referenzreaktor ab Versuchstag 76 kontinuierlich an. Die Gründe sind vermutlich die in Kapitel 3.5.3 erwähnten Abrasions- oder Ablösungsvorgänge an den metallischen Oberflächen der mittig in den Reaktoren fixierten Ankerrührer mit den Rührblättern. Nur der Reaktor Amarant-1 blieb über die gesamte Versuchsdauer bei den Chromwerten unauffällig. 
Kapitel 4: (Quasi)kontinuierliche Laborfermenterversuche
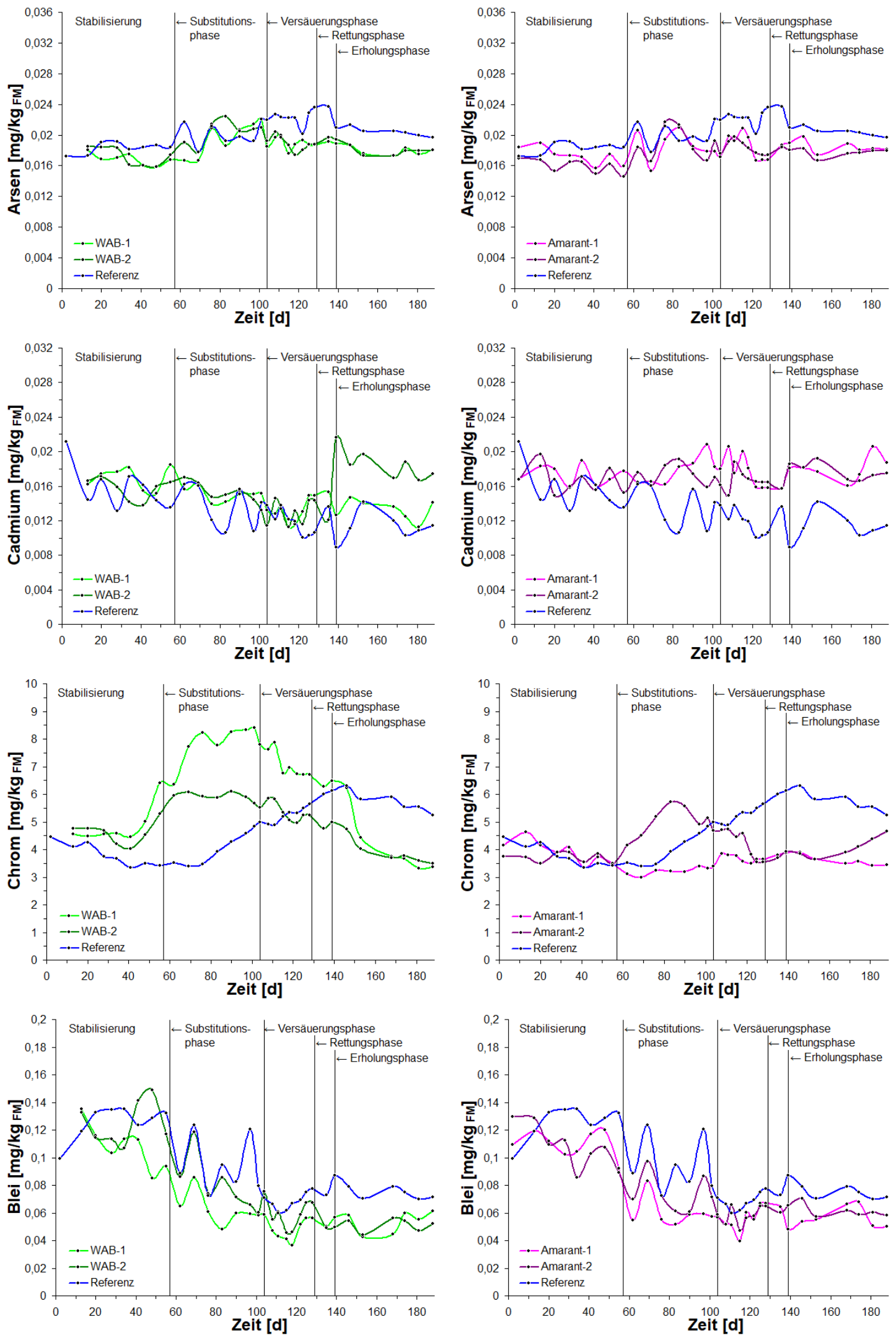

Abbildung 47: Verläufe der Arsen-, Cadmium-, Chrom- und Bleigehalte der Substitutionsversuche 1 und 2 in den Versuchsbiogasreaktoren (mit Referenzreaktor). 

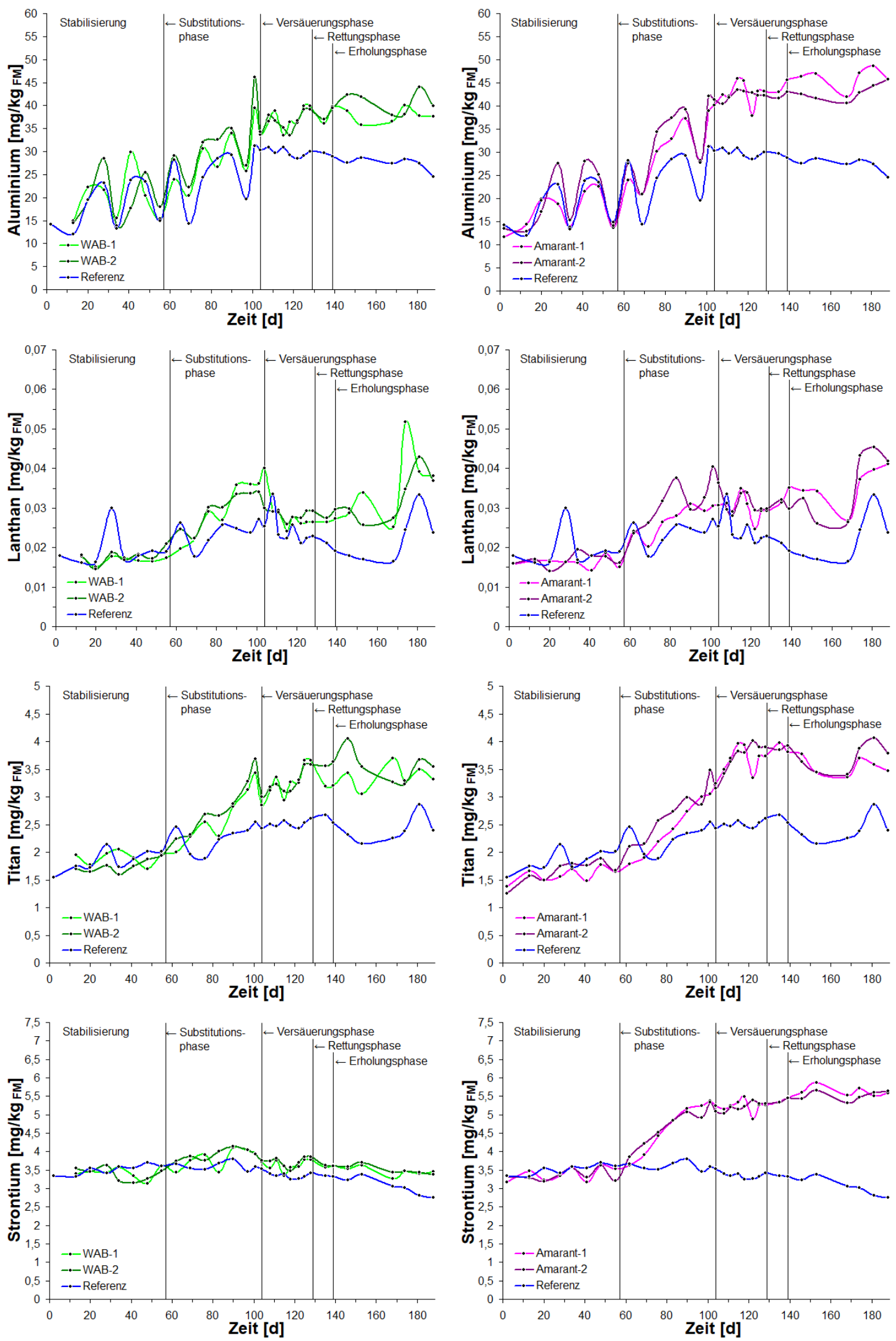

Abbildung 48: $\quad$ Verläufe der Aluminium-, Lanthan-, Titan- und Strontiumgehalte der Substitutionsversuche 1 und 2 in den Versuchsbiogasreaktoren (mit Referenzreaktor). 


\subsubsection{Eisen/Schwefel-Verhältnisse in den Substitutionsversuchen 1 und 2}

Die Eisen/Schwefel-Verhältnisse fielen mit Beginn der zweiten Projektphase in den für die beiden Substitutionsversuche vorgesehenen Versuchsbiogasreaktoren kontinuierlich ab (Abbildung 49). Ursache war die Aussetzung der Zugabe der Spurenelementlösung, die auch das Element Eisen enthielt (vgl. Kapitel 4.1, Tabelle 6), bereits während der Stabilisierungsphase, um mit Beginn der Substitutionsphase am Versuchstag 57 von einem niedrigeren Konzentrationsniveau aus mit den Zumischungen der Silagen Winterackerbohne bzw. Amarant zu Mais-Silage zu starten. Dem Referenzreaktor wurde durchgängig die volle Menge der Additivlösung mit Eisen zugegeben, sodass das Eisen/Schwefel-Verhältnis in diesem Reaktor stabil auf einem hohen Niveau lag.

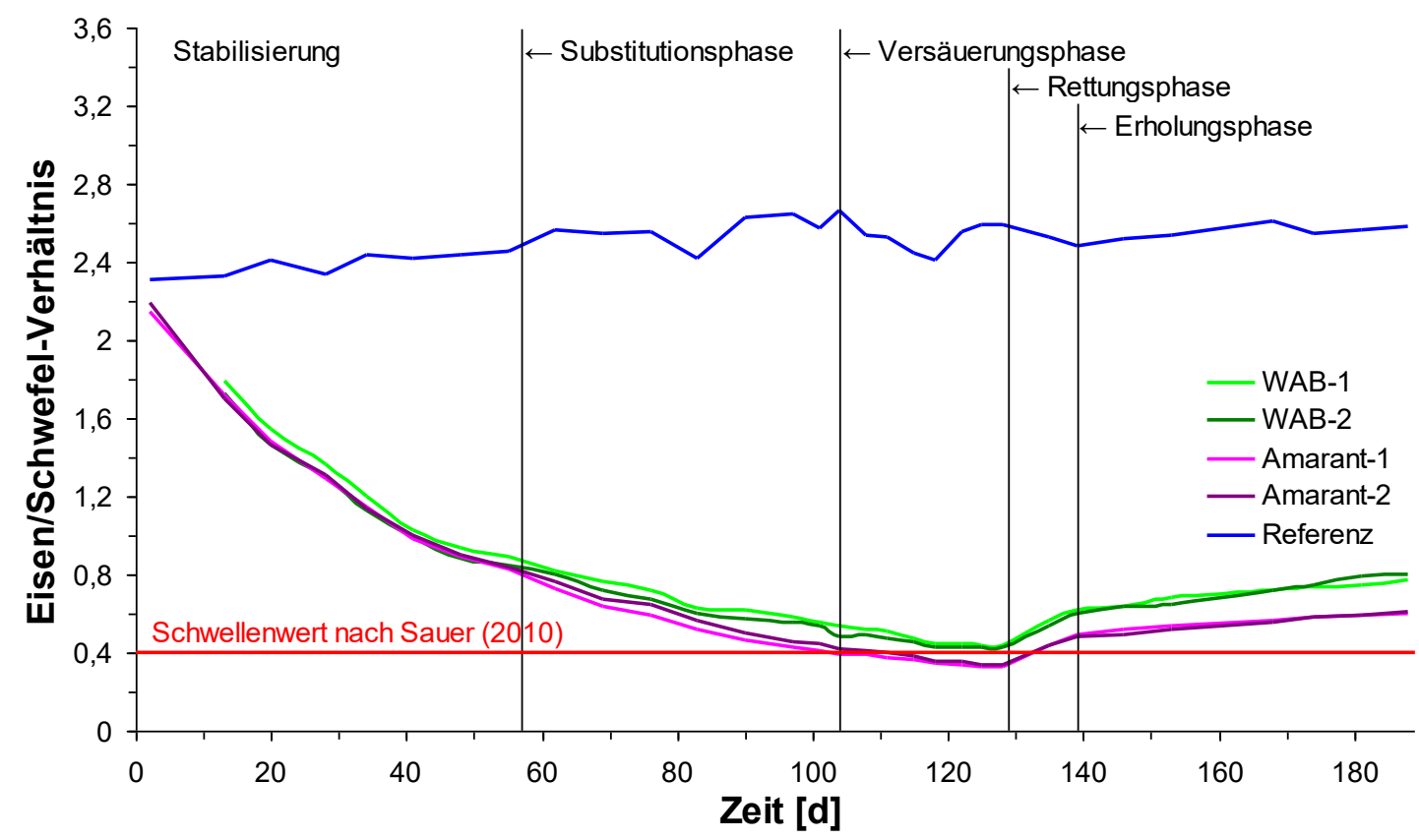

Abbildung 49: $\quad$ Verläufe der Eisen/Schwefel-Verhältnisse der Substitutionsversuche 1 und 2 in den Versuchsbiogasreaktoren (mit Referenzreaktor).

Während der Substitutionsphasen mit den Silagemischungen $30 \%$ Winterackerbohne bzw. $30 \%$ Amarant zu $70 \%$ Mais (oTS-bezogen) sanken die Eisen/SchwefelVerhältnisse dann weiter $a b$. In den jeweiligen Versäuerungsphasen erreichten bzw. unterschritten sie sogar den minimalen Schwellenwert nach SAUER (2010) von 0,4. Somit stellen die Eisen/Schwefel-Verhältnisse ein weiteres entscheidendes Instabilitätskriterium für die absehbaren Zusammenbrüche der ersten beiden Substitutionsversuche dar. 
Nach den Rettungsversuchen durch die Zugabe von nur noch einem Viertel der ursprünglichen Spurenelementadditivmenge lagen die Eisen/Schwefel-Verhältnisse in den vier Substitutionsreaktoren dann während der kompletten Erholungsphasen wieder auf stabilen und konstanten Niveaus bei 0,6 (Substitutionsreaktoren mit AmarantSilage) bzw. 0,8 (Substitutionsreaktoren mit Winterackerbohne-Silage).

\subsubsection{Mikrobiologischer Überblick zur Zusammensetzung und Dynamik der mikrobiellen Gemeinschaften in den Substitutionsversuchen 1 und 2}

In den Substitutionsreaktoren mit den Zumischungen von $30 \%$ Winterackerbohnebzw. 30 \% Amarant-Silage zu 70 \% Mais-Silage (oTS-bezogen) unterlagen die relativen Anteile der methanogenen Gemeinschaften im Laufe der Prozessphasen ständigen Schwankungen. Die relativen Anteile von Methanosarcina und Methanobacteriaceae/ Methanomassiliicoccaceae waren jedoch ähnlich und variierten zwischen 30 bis $50 \%$. In den Substitutionsphasen waren aber deutliche Unterschiede der Methanbildner zum Kontrollreaktor erkennbar. Die relativen Anteile der Methanogenen veränderten sich in den Reaktoren mit Winterackerbohne- bzw. Amarant-Silage durch die Substratumstellung kaum, im Referenzreaktor trat dagegen die Ordnung Methanomicrobiales mit bis zu 28 \% relativer Abundanz dominant auf. Da es jedoch keinen Doppelversuch der Kontrolle gab, kann nicht mit Sicherheit gesagt werden, ob es sich dabei nur um eine zufällige Veränderung handelte.

Insgesamt zeigte sich, dass durch den Ersatz der Mais-Silage durch $30 \%$ Winterackerbohne- bzw. 30 \% Amarant-Silage (oTS-bezogen) die bakteriellen Gemeinschaften in den Substitutionsphasen und den folgenden Versäuerungs- sowie Erholungsphasen in ihrer Zusammensetzung wesentlich mehr beeinflusst wurden als die methanogenen Gemeinschaften. Letztgenannte blieben in ihrer Zusammensetzung weitestgehend stabil oder unterlagen einer unspezifischen Dynamik.

Jedoch auch der Referenzreaktor wies Veränderungen der bakteriellen Gemeinschaften während der gesamten Projektphase auf. Die deutlich größeren Veränderungen in den vier Substitutionsreaktoren sind vermutlich auf die unterschiedlichen Nährstoffzusammensetzungen von Winterackerbohne- bzw. Amarant-Silage gegenüber MaisSilage zurückzuführen, die Variabilität im Referenzreaktor vermutlich auf größere Qualitätsunterschiede der verwendeten Mais-Silagen (SAUER ET AL., 2018). 
Eine charakteristische Veränderung wie bei Nickelmangelzuständen bzw. einer möglichen Zinkhemmung (vgl. Kapitel 4.3.7) durch eine Verringerung der relativen Abundanz von Methanosarcina wurde in den Substitutionsreaktoren nicht beobachtet. Somit kann abermals bestätigt werden, dass der Zusammenbruch der Biogasprozesse nicht auf einen Nickelmangel (oder mögliche Hemmwirkung durch Zink), sondern auf einen Cobaltmangel zurückzuführen ist (SAUER ET AL., 2018). 


\subsection{Substitutionsversuche von Mais-Silage mit Silagen von Deutsches Weidelgras und Durchwachsene Silphie}

\subsection{1 Überblick}

In der dritten Projektphase am DBFZ wurden die dritten und vierten Substitutionsversuche für Mais durchgeführt, indem 30 \% Deutsches Weidelgras- bzw. $30 \%$ Durchwachsene Silphie-Silage zu 70 \% Mais-Silage (oTS-bezogen) den Reaktoren zugeführt wurden, um gleichermaßen zu überprüfen, ob diese Zumischungen spurenelementreicherer Energiepflanzen-Silagen ausreichend sind, stabile Biogasprozesse aufrechtzuerhalten. Hierfür wurden die Reaktoren im Doppelversuch abermals aus einer stabilen Phase heraus auf die von der Abteilung Pflanzenbau der Universität Göttingen bereitgestellten Energiepflanzen-Silagen Deutsches Weidelgras bzw. Durchwachsene Silphie (vgl. Kapitel 4.2) umgestellt, bei gleichzeitigem Wegfall der Zugaben der zusätzlichen synthetischen Spurenelementlösungen. Die Langzeitstabilität dieser Varianten alternativer Pflanzensilagemischungen sollte analog $\mathrm{zu}$ den ersten beiden Substitutionsversuchen (vgl. Kapitel 4.4) bei einer (quasi)kontinuierlichen Reaktorfahrweise über mindestens drei Verweilzeiten getestet werden.

Bei nicht erfolgreichem Verlauf ganz ohne zusätzliche Additivzugabe, waren ebenfalls Rettungsmaßnahmen unter Zugabe eines Viertels der ursprünglichen Spurenelementadditivmenge bei den Reaktoren mit Durchwachsene Silphie-Silage geplant. Für die beiden Reaktoren mit Deutsches Weidelgras-Silage wurde nach Absprache im Forschungsverbund festgelegt, statt eines Viertels der ursprünglichen kompletten Spurenelementlösung, ausschließlich nur ein Viertel der Menge der synthetischen Cobaltchloridlösung als Monoelementadditiv zuzuschlagen. Denn die gemittelten Elementgehalte der Deutsches Weidelgras-Silage (vgl. Kapitel 4.2, Tabelle 8) ließen vermuten, dass in den beiden Versuchsbiogasreaktoren nur eine Unterversorgung des Spurenelements Cobalt vorliegt, Nickel im Substitut aber ausreichend vorhanden ist. So sollte überprüft werden, ob lediglich auch eine reduzierte Zugabe einer MonoelementCobaltlösung einen stabilen Reaktorbetrieb bei einer Zumischung von 30 \% Deutsches Weidelgras- zu $70 \%$ Mais-Silage (oTS-bezogen) aufrechterhalten kann. Die dritte Projektphase begann parallel zum Zusatzversuch mit Mais-Silage-Monovergärung unter reduzierter Spurenelementzugabe (vgl. Kapitel 4.6). Tabelle 11 gibt einen Überblick über die einzelnen Phasen der beiden Substitutionsversuche. 
Tabelle 11: $\quad$ Überblick über die dritte Projektphase der (quasi)kontinuierlichen Nassfermentationsversuche am DBFZ.

\begin{tabular}{|c|c|c|c|c|c|c|}
\hline \multicolumn{7}{|c|}{$\begin{array}{l}\text { Projektphase } 3 \quad \text { Substitutionsversuche } 3 \text { und } 4 \\
\text { Vergärung von } 30 \% \text { Silage aus Deutsches Weidelgras bzw. Durchwachsene Silphie zu } 70 \% \text { Mais-Silage } \\
\text { (oTS-bezogen) }\end{array}$} \\
\hline Prozessphase & Reaktor & Kurzbeschreibung & Abkürzung & $\begin{array}{c}\text { Versuchs- } \\
\text { tag }\end{array}$ & bis & $\begin{array}{c}\text { Versuchs- } \\
\text { tag }\end{array}$ \\
\hline Prozessstabilisierung & $4.5-4.9$ & Mais-Silage + Spurenelementvollversorgung & & 0 & - & 37 \\
\hline \multirow[t]{4}{*}{ Substitutionsphase } & 4.5 & Substitution Deutsches Weidelgras 1 & Weidelgras- 1 & 38 & - & 111 \\
\hline & 4.6 & Substitution Deutsches Weidelgras 2 & Weidelgras-2 & 38 & - & 111 \\
\hline & 4.7 & Substitution Durchwachsene Silphie 1 & Silphie-1 & 38 & - & 111 \\
\hline & 4.8 & Substitution Durchwachsene Silphie 2 & Silphie-2 & 38 & - & 111 \\
\hline \multirow[t]{4}{*}{ Versäuerungsphase } & 4.5 & Substitution Deutsches Weidelgras 1 & Weidelgras-1 & 112 & - & 182 \\
\hline & 4.6 & Substitution Deutsches Weidelgras 2 & Weidelgras-2 & 112 & - & 182 \\
\hline & 4.7 & Substitution Durchwachsene Silphie 1 & Silphie-1 & 112 & - & 146 \\
\hline & 4.8 & Substitution Durchwachsene Silphie 2 & Silphie-2 & 112 & - & 146 \\
\hline \multirow[t]{4}{*}{ Rettungsphase 1} & 4.5 & Diverse Eingriffe (siehe Textkapitel) & Weidelgras-1 & 183 & - & 231 \\
\hline & 4.6 & Diverse Eingriffe (siehe Textkapitel) & Weidelgras-2 & 183 & - & 231 \\
\hline & 4.7 & Diverse Eingriffe (siehe Textkapitel) & Silphie-1 & 147 & - & 231 \\
\hline & 4.8 & Diverse Eingriffe (siehe Textkapitel) & Silphie-2 & 147 & - & 154 \\
\hline Rettungsphase 2 & 4.5 & Futterreduktion & Weidelgras-1 & 332 & - & 350 \\
\hline \multirow[t]{4}{*}{ Erholungsphase 1} & 4.5 & Volle Fütterung + Viertel Cobalt-Lösung & Weidelgras-1 & 207 & - & 387 \\
\hline & 4.6 & Volle Fütterung + Viertel Cobalt-Lösung & Weidelgras-2 & 207 & - & 387 \\
\hline & 4.7 & Volle Fütterung + Viertel Spurenelementlösung & Silphie-1 & 210 & - & 387 \\
\hline & 4.8 & Volle Fütterung + Viertel Spurenelementlösung & Silphie-2 & 163 & - & 387 \\
\hline \multirow[t]{2}{*}{ Erholungsphase 2} & 4.5 & Volle Fütterung + Viertel Spurenelementlösung & Weidelgras-1 & 388 & - & 428 \\
\hline & 4.6 & Volle Fütterung + Viertel Spurenelementlösung & Weidelgras-2 & 388 & - & 428 \\
\hline \multirow[t]{2}{*}{ Indizienphase } & 4.5 & 5 Tage Cobalt-, danach Cobalt-Selen-Lösung & Weidelgras-1 & 429 & - & 493 \\
\hline & 4.6 & 5 Tage Cobalt-, danach Cobalt-Wolfram-Lösung & Weidelgras-2 & 429 & - & 493 \\
\hline Kontrolle & 4.9 & Mais-Silage + Spurenelementvollversorgung & Referenz & 0 & - & 238 \\
\hline
\end{tabular}

Alle fünf Reaktoren wurden abermals mit Gärmaterial aus dem 100-Liter-Inokulumsreaktor (Reaktor 3.2) angeimpft, der gezielt mit den gleichen Betriebsparametern wie die Substitutionsversuche betrieben wurde. Erneut wurde täglich zusätzlich zum StartInputsubstrat Mais-Silage zur Spurenelementvollversorgung in alle Reaktoren die synthetische Additivlösung nach OECHSNER ET AL. (2008; vgl. Kapitel 4.1) zugegeben. Nach der Prozessstabilisierung begannen ab Versuchstag 38 die Substitutionsversuche mit der Umstellung der Substrate (Substitutionsphase) von Mais-Silage durch $30 \%$ (oTS-bezogen) Deutsches Weidelgras- (Reaktor 4.5 = Weidelgras-1 und Reaktor 4.6= Weidelgras-2) bzw. $30 \%$ Durchwachsene Silphie-Silage (Reaktor $4.7=$ Silphie-1 und 
Reaktor 4.8 = Silphie-2). Der Reaktor 4.9 (= Referenz) wurde wie schon in den ersten beiden Projektphasen parallel dazu als Kontrollreaktor mit Mais-Silage-Monovergärung und zusätzlicher synthetischer Spurenelementvollversorgung betrieben.

Etwa zwei Verweilzeiten nach Beginn der Substitution, fingen die Reaktoren mit den Zumischungen von $30 \%$ Deutsches Weidelgras- bzw. $30 \%$ Durchwachsene SilphieSilage zu $70 \%$ Mais-Silage (oTS-bezogen) jedoch allmählich an zu versäuern (Versäuerungsphase). Es zeigte sich auch bei diesen beiden Zumischungen, wie schon im Vorfeld durch Berechnungen erwartet, dass bei einer Raumbelastung von $5 \mathrm{~g}_{\mathrm{oTS}} /(\mathrm{L} \bullet \mathrm{d})$ keine stabilen Biogasprozesse gewährleistet werden können. Durch die zusätzliche Zugabe eines Viertels der ursprünglichen Spurenelementadditivmenge konnten die Biogasprozesse in diesen Substitutionsversuchen stabilisiert werden.

\subsubsection{Substitutionsversuch 3: Vergärung von 30 \% Silage aus Deutsches Weidelgras zu $70 \%$ Mais-Silage (oTS-bezogen)}

Ab Versuchstag 112, knapp zwei Verweilzeiten nach der Umstellung der Substrate ab Versuchstag 38 begannen in den beiden Versuchsbiogasreaktoren mit der Silagemischung Deutsches Weidelgras zu Mais die FOS (Kapp)- und FOS/TAC (FAL)-Werte (Abbildung 50) allmählich anzusteigen. Wenige Tage später fingen auch die $\mathrm{pH}-\mathrm{Werte}$ in den Substitutionsreaktoren sukzessive an zu fallen (Abbildung 51) sowie die Werte der auf Normbedingungen $(273,15 \mathrm{~K}, 101,325 \mathrm{kPa})$ umgerechneten Biogasproduktion abzusinken (Abbildung 52). Die normierten Methangehalte (Abbildung 53) zeigten mit Beginn der Substitutionsphase über die Versäuerungsphase hinweg gegenüber dem Kontrollreaktor kontinuierlich leicht fallende Trends.

Die Rettungsversuche (Rettungsphase 1) starteten kurz vor dem Zusammenbruch der beiden Reaktoren ab Versuchstag 183, etwa dreieinhalb Verweilzeiten nach Substitutionsbeginn. In den Substitutionsreaktoren waren $\mathrm{zu}$ diesem Zeitpunkt die FOS/TAC (FAL)-Werte bereits auf 1,3 (Reaktor Weidelgras-1) bzw. 1,4 $\mathrm{g}_{\mathrm{FOS}} / \mathrm{g}_{\mathrm{CaCO} 3}$ (Reaktor Weidelgras-2) angestiegen (Abbildung 50). Die pH-Werte waren mit 6,9 bzw. 6,6 (Abbildung 51), die Werte der normierten Gasproduktion mit 19 bzw. 12 L $\mathrm{Norm} / \mathrm{d}$ (Abbildung 52) sowie die Werte des normierten Methangehalts mit 51 bzw. 46 Vol.-\% (Abbildung 53) ebenfalls während der letzten Versuchstage in der Versäuerungsphase deutlich abgesunken. Die Verläufe der FOS/TAC (FAL)-Werte und der normierten Gasproduktion sind in Abbildung 54 direkt gegenübergestellt. 


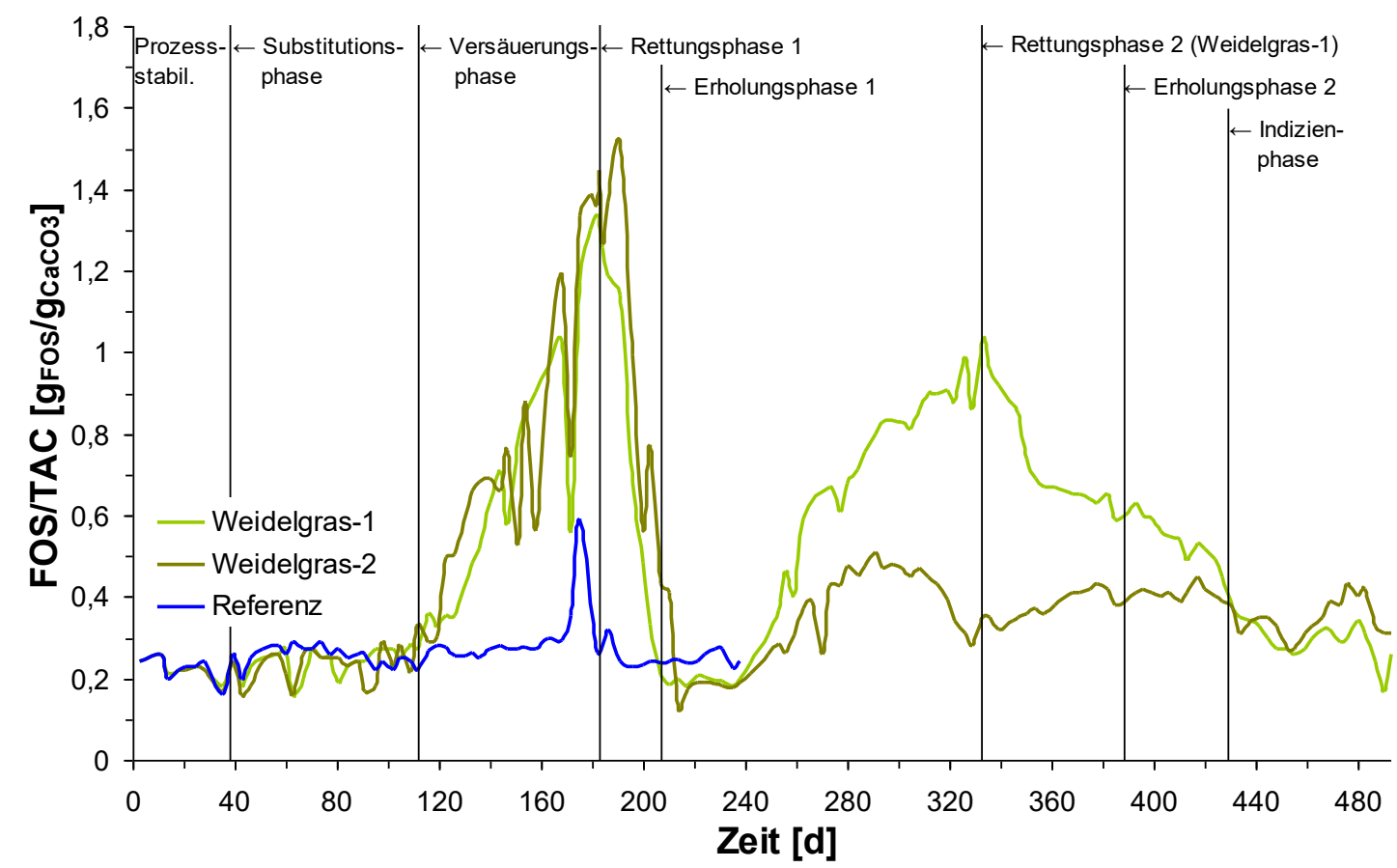

Abbildung 50: $\quad$ FOS/TAC (FAL)-Verläufe des Substitutionsversuchs 3 mit 30\% Deutsches Weidelgras- zu $70 \%$ Mais-Silage (oTS-bezogen) in den Versuchsbiogasreaktoren (mit Referenzreaktor).

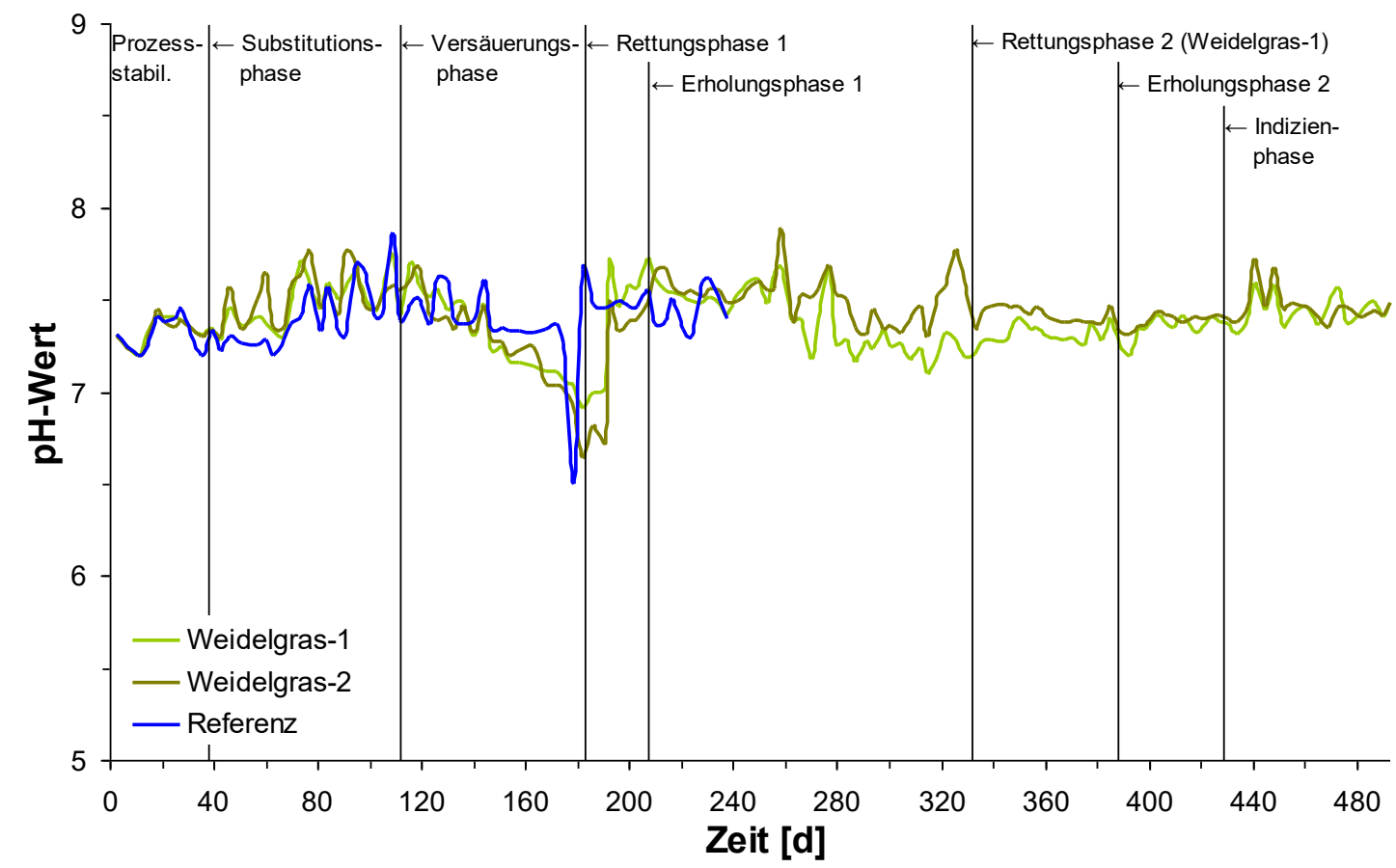

Abbildung 51: $\quad$ pH-Wert-Verläufe des Substitutionsversuchs 3 mit $30 \%$ Deutsches Weidelgras- zu $70 \%$ Mais-Silage (oTS-bezogen) in den Versuchsbiogasreaktoren (mit Referenzreaktor). 


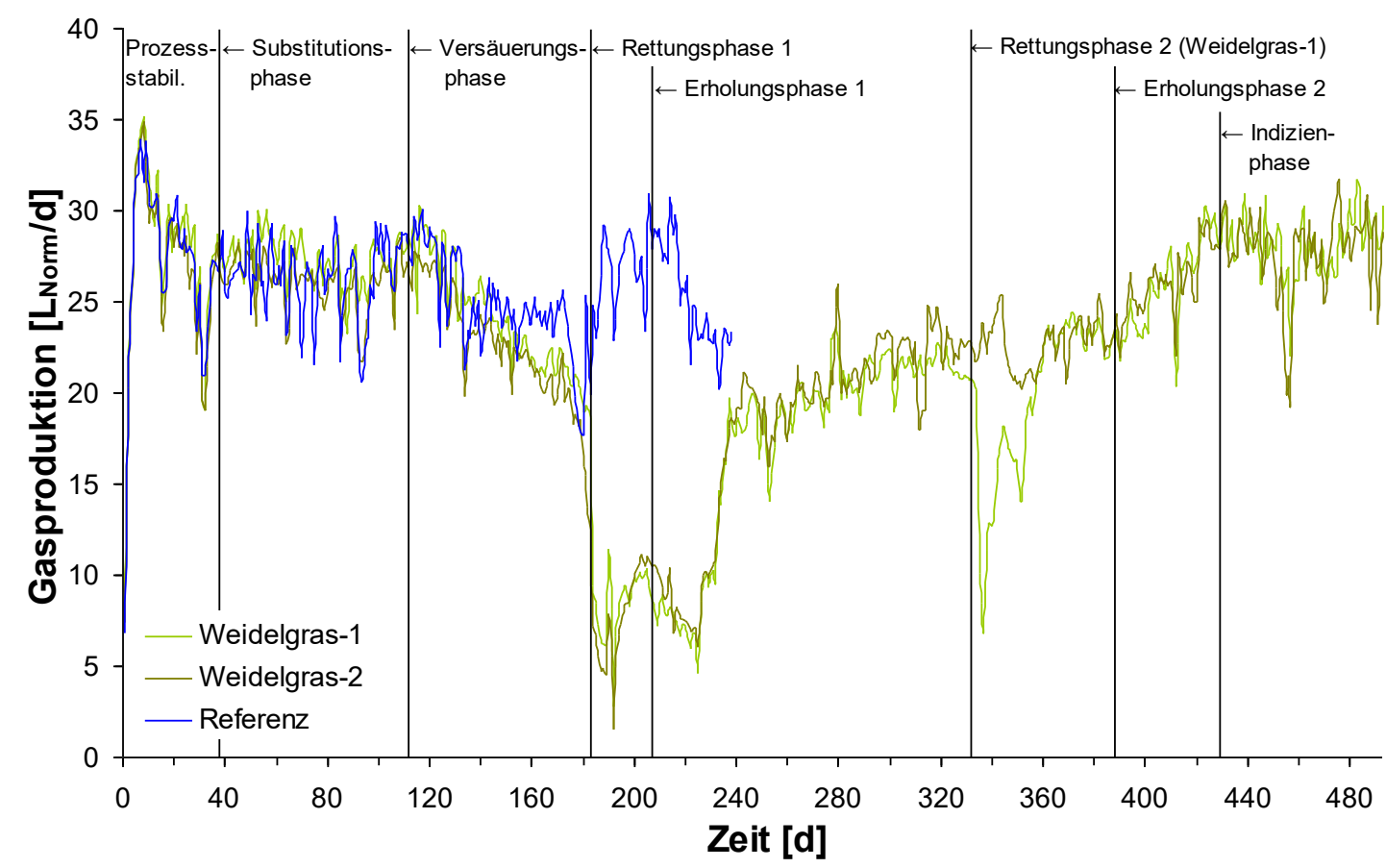

Abbildung 52: $\quad$ Verläufe der normierten Gasproduktion des Substitutionsversuchs 3 mit $30 \%$ Deutsches Weidelgras-zu $70 \%$ Mais-Silage (oTS-bezogen) in den Versuchsbiogasreaktoren (mit Referenzreaktor).

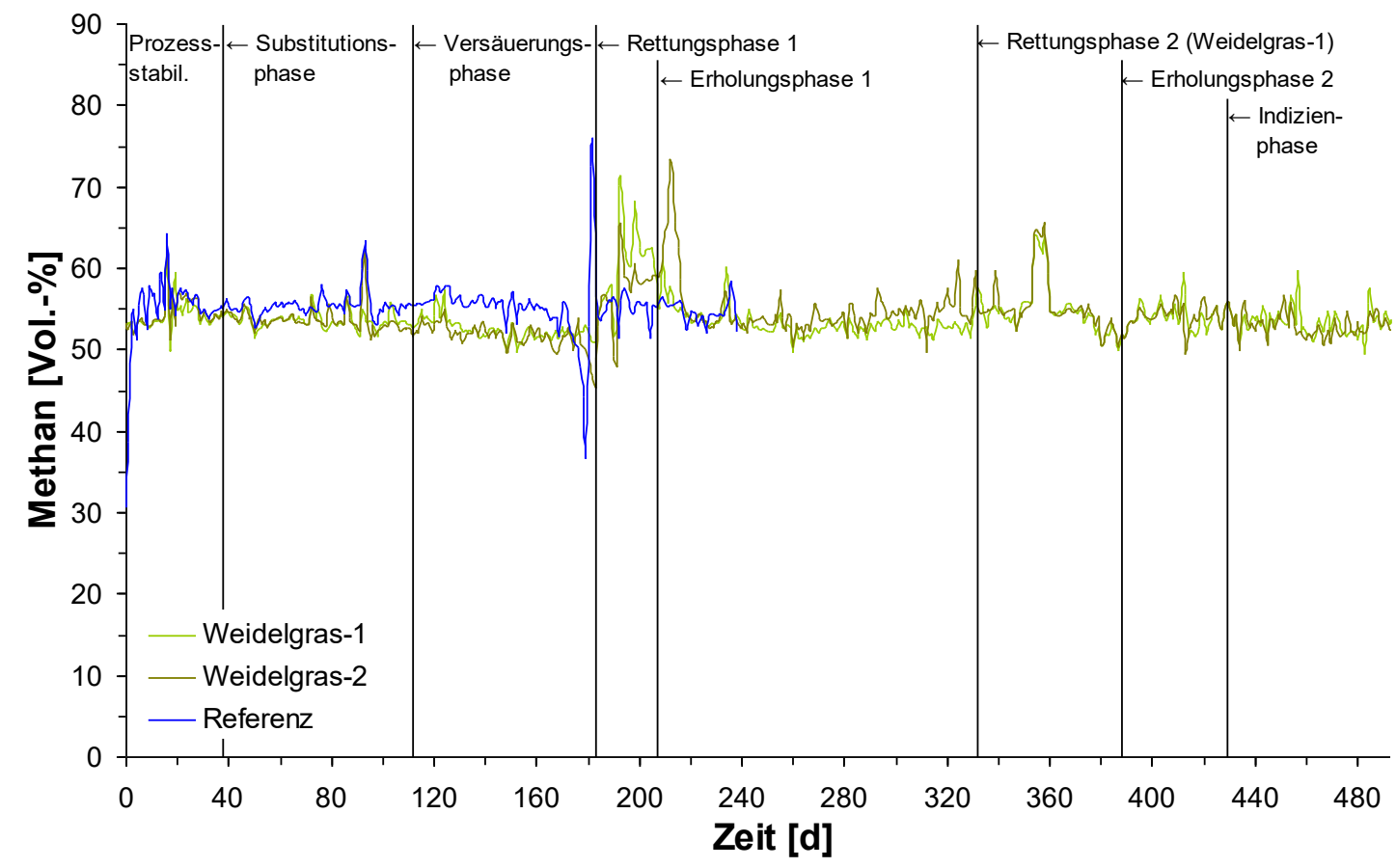

Abbildung 53: Verläufe des normierten Methangehalts des Substitutionsversuchs 3 mit $30 \%$ Deutsches Weidelgras-zu $70 \%$ Mais-Silage (oTS-bezogen) in den Versuchsbiogasreaktoren (mit Referenzreaktor). 


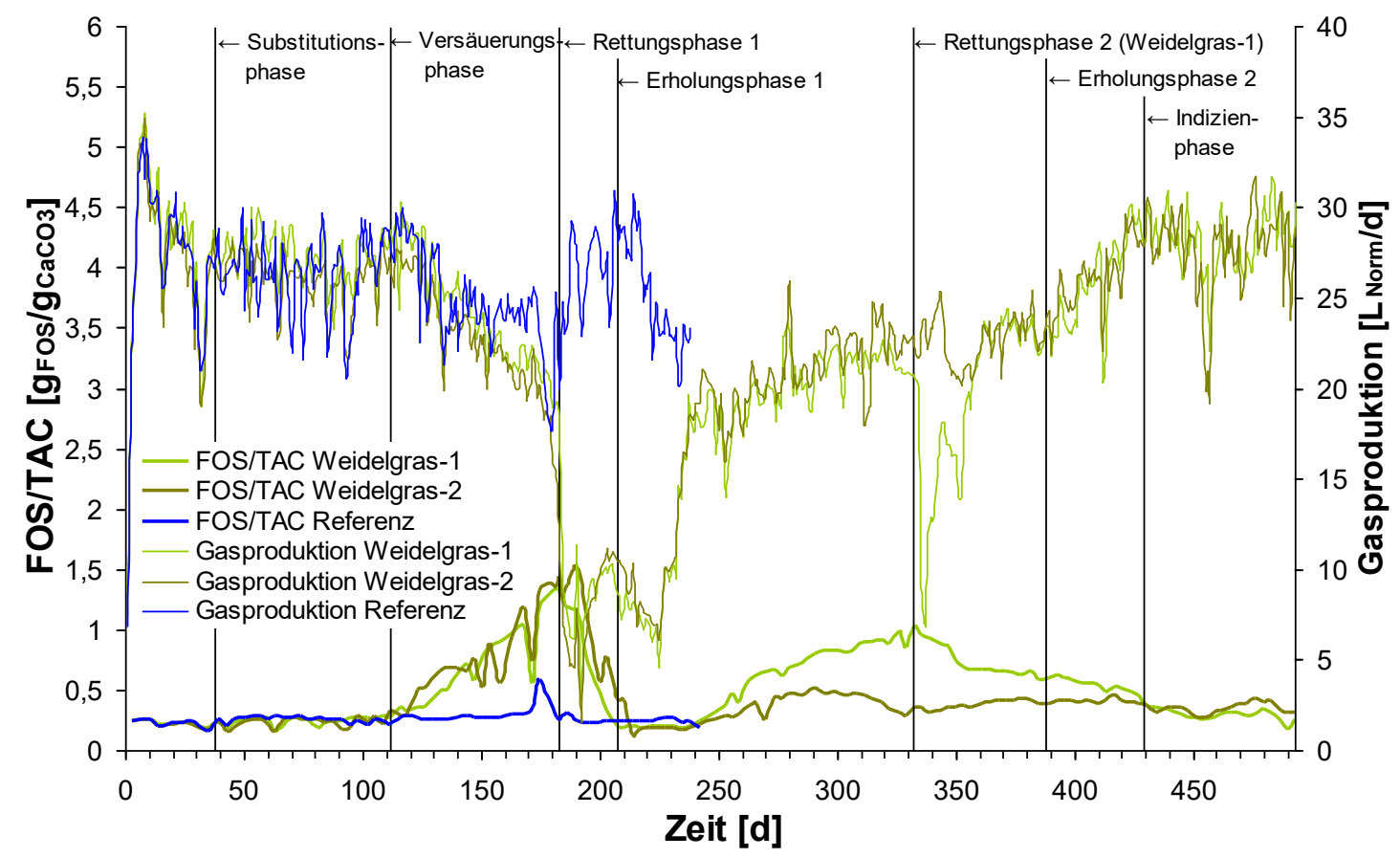

Abbildung 54: $\quad$ Gegenüberstellung der Verläufe der FOS/TAC (FAL)-Werte und der normierten Gasproduktion des Substitutionsversuchs 3 mit 30\% Deutsches Weidelgras- zu $70 \%$ Mais-Silage (oTS-bezogen) in den Versuchsbiogasreaktoren (mit Referenzreaktor).

Mit Beginn der Rettungsphase 1 wurde den beiden Substitutionsreaktoren wie erwähnt nur ein Viertel der ursprünglichen Menge der synthetischen Cobaltchloridlösung $\left(\mathrm{CoCl}_{2} \bullet 6 \mathrm{H}_{2} \mathrm{O}\right)$ als Monoelementadditiv zugeschlagen, Durch Änderungen der Substratzufuhr - jeweils neun Versuchstage mit Aussetzen, 33 Versuchstage mit Zugabe eines Viertels und sieben Versuchstage mit Zugabe der Hälfte der Subtratmengen - sowie der Zugabe der reduzierten Cobaltchloridlösung konnten die beiden Reaktoren wieder stabilisiert werden. Die Rettungsmaßnahmen wurden am Versuchstag 231 beendet.

Ab Versuchstag 207 lagen die Prozessparameter der beiden Reaktoren (Abbildung 50, Abbildung 51 und Abbildung 53) mit Ausnahme der normierten Gasproduktion wieder auf dem Niveau wie vor der Versäuerungsphase (Erholungsphase 1). Die Biogasproduktion erreichte hingegen erst ab Versuchstag 237 wieder stabilere normierte Werte zwischen 20 und $22 \mathrm{~L}_{\mathrm{Norm}} / \mathrm{d}$ (Abbildung 52 und Abbildung 54), die jedoch niedriger lagen als in der Substitutionsphase vor Beginn der Versäuerung mit durchschnittlich $27 \mathrm{~L}_{\text {Norm }} / \mathrm{d}$. 
Während der Erholungsphase 1 stiegen die FOS/TAC (FAL)-Werte nach Erreichen der ursprünglichen Prozessbedingungen mit voller Substratzufuhr ab Versuchstag 238 wieder stetig an (Abbildung 50 und Abbildung 54). Zwar sind kurzzeitige Anstiege der FOS/TAC (FAL)-Werte durchaus nicht ungewöhnlich, da sich die Mikroorganismengemeinschaften auf die erhöhten Substratmengen erst wieder einstellen müssen, jedoch wäre nach wenigen Versuchstagen eine Abnahme oder Stabilisierung zu erwarten. Allerdings entwickelten sich die Verläufe der beiden Substitutionsreaktoren weiter auseinander. Die Ursache hierfür bleibt unklar, da alle anderen Prozessparameter in der Erholungsphase 1 wieder stabile Werte aufwiesen.

Die FOS/TAC (FAL)-Werte in Reaktor Weidelgras-2 stabilisierten sich jedoch ohne weitere Einflussnahme ab Versuchstag 291 wieder auf einem leicht erhöhten Niveau von durchschnittlich $0,39 \mathrm{~g}_{\mathrm{FOS}} / \mathrm{g}_{\mathrm{CaCO} 3}$. Bei Reaktor Weidelgras-1 musste dagegen ab Versuchstag 332 bei einem Wert von 1,0 $\mathrm{g}_{\mathrm{FOS}} / \mathrm{g}_{\mathrm{CaCO} 3}$ erneut durch Futterreduktion (fünf Versuchstage mit Aussetzen, vier Versuchstage mit Zugabe der Hälfte und zehn Versuchstage mit Zugabe von $75 \%$ der Subtratmenge) bis zum Versuchstag 350 eingegriffen werden (Rettungsphase 2). Diese neunzehntägige Reduktion der Substratmenge genügte, um die FOS/TAC (FAL)-Werte wieder abzusenken und die eingebrochene normierte Gasproduktion wieder auf das gleiche Niveau des Reaktors Weidelgras-2 anzuheben (Abbildung 52 und Abbildung 54). Die normierte Gasproduktion lag jedoch in den beiden Reaktoren mit durchschnittlich $23 \mathrm{~L}_{\mathrm{Norm}} / \mathrm{d}$ weiterhin auf einem signifikant niedrigeren Niveau als während der Substitutionsphase.

Die instabilen Verläufe der FOS/TAC (FAL)-Werte während der Erholungsphase 1 und v.a. die geminderte normierte Biogasproduktion in den beiden Reaktoren zeigten, dass Cobalt doch nicht nur das alleinige Spurenelement für eine Unterversorgung bei dieser Silagemischung ist. Um dies zu überprüfen, wurde ab Versuchstag 388 über eine komplette Verweilzeit bis Versuchstag 428 ein Viertel der ursprünglichen Menge der synthetische Spurenelementlösung mit allen Elementen zugegeben (Erholungsphase 2), analog zu den Rettungsversuchen der Substitutionen mit Winterackerbohneund Amarant-Silage (vgl. Kapitel 4.4.2 und 4.4.3). Die normierte Gasproduktion (Abbildung 52 und Abbildung 54) stieg deutlich an bis auf das Niveau der Werte wie in der Substitutionsphase mit etwa $27 \mathrm{~L}_{\text {Norm }} / \mathrm{d}$. Gegen Ende der Erholungsphase 2 pegelten sich ab Versuchstag 421 die Werte sogar auf einem Level von durchschnittlich $29 \mathrm{~L}_{\text {Norm }} / \mathrm{d}$ ein. 
Ausschlaggebend für die Versäuerung bzw. den frühzeitig absehbaren potenziellen Zusammenbruch der beiden Reaktoren in dieser Doppelversuchsreihe waren wiederum die sehr niedrigen Cobaltkonzentrationen (Abbildung 55). Sie lagen kurz vor Beginn der Rettungsphase 1 erneut genau im Bereich des in der ersten Projektphase erhobenen minimalen Schwellenwertes von $0,066 \mathrm{mg} / \mathrm{kg}_{\mathrm{FM}}$ Cobalt (vgl. Kapitel 4.3.2) mit Konzentrationen von 0,068 (Weidelgras-1) bzw. 0,062 mg/kg FM (Weidelgras-2).

Nach Beendigung der Rettungsphase 1 lagen die Cobaltkonzentrationen ab Versuchstag 231 bis zur letzten Probenahme am Versuchstag 364, also die komplette Erholungsphase 1 hinweg, auf einem konstanten, guten Niveau mit durchschnittlichen Gehalten von 0,17 (Weidelgras-1) bzw. $0,14 \mathrm{mg} / \mathrm{kg}_{\mathrm{FM}}$ Cobalt (Weidelgras-2). Sie verliefen demnach weit oberhalb des in der ersten Projektphase ermittelten minimalen Schwellenwertes. Auch die kurzzeitige Substratreduktion in Reaktor Weidelgras-1 in der Rettungsphase 2 von Versuchstag 332 bis Versuchstag 350 beeinträchtigte den Verlauf der Cobaltkonzentration in diesem Reaktor nicht.

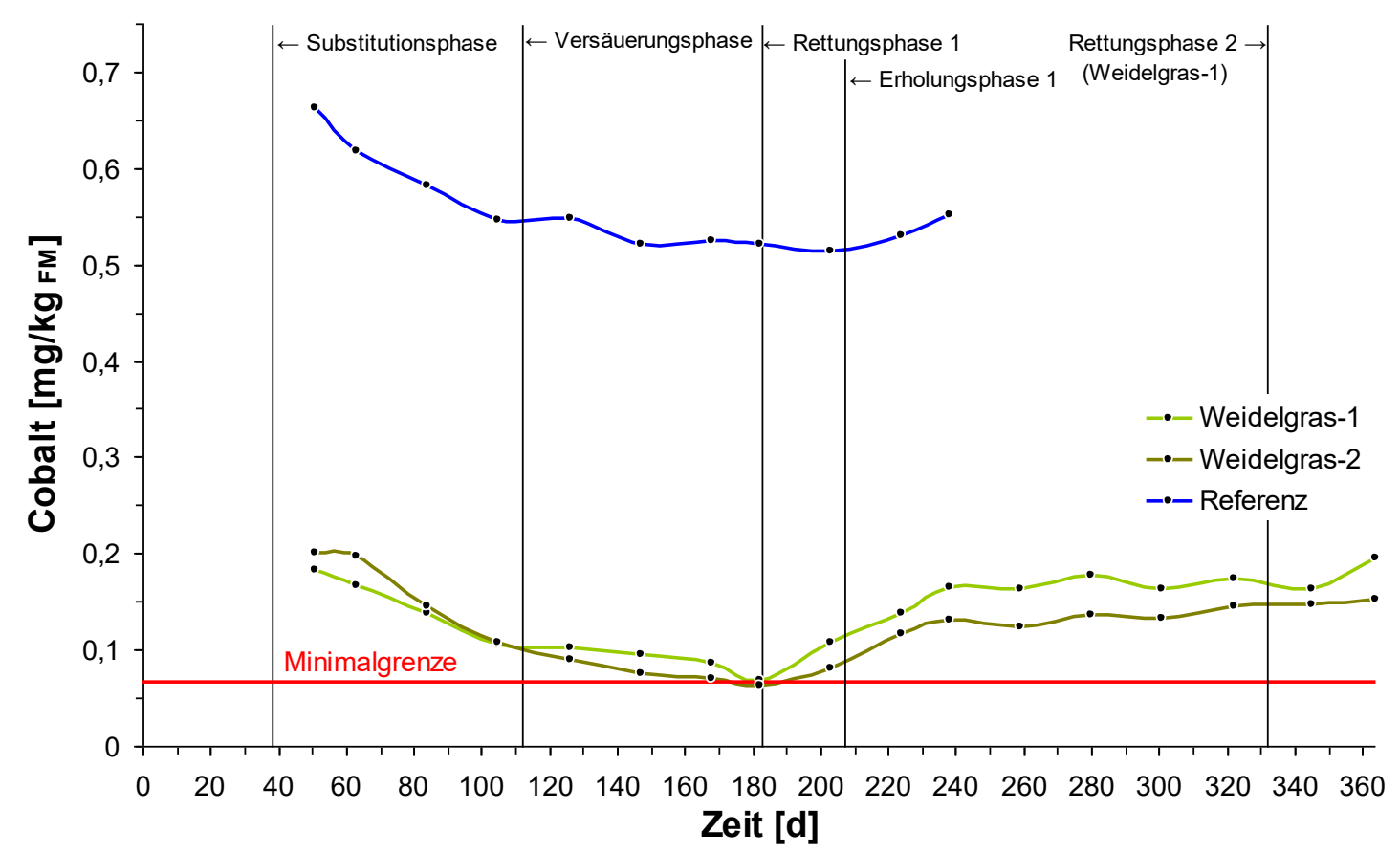

Abbildung 55: Verläufe der Cobaltkonzentrationen des Substitutionsversuchs 3 mit $30 \%$ Deutsches Weidelgras- zu $70 \%$ Mais-Silage (oTS-bezogen) in den Versuchsbiogasreaktoren (mit Referenzreaktor).

Die Nickelgehalte (Abbildung 56) lagen die gesamte Projektphase hinweg sehr deutlich oberhalb des in der ersten Projektphase ermittelten, jedoch wahrscheinlich zu hoch angegebenen, minimalen Schwellenwertes von $0,82 \mathrm{mg} / \mathrm{kg}$ FM (vgl. Kapitel 4.3.3 und 
Kapitel 4.3.4). Sie sanken zwar im Verlaufe der Erholungsphase 1 weiter ab, da mit Beginn der Rettungsphase 1 keine zusätzliche Nickellösung, sondern nur ein Viertel der Menge einer Monoelement-Cobaltlösung in die Substitutionsreaktoren zugegeben wurde, pegelten sich ab Versuchstag 301 aber auf einem konstanten Level bis zur letzten Probenahme am Versuchstag 364 ein, mit Durchschnittswerten von 2,0 (Weidelgras-1) bzw. $1,0 \mathrm{mg} / \mathrm{kg}_{\mathrm{FM}}$ Nickel in Reaktor Weidelgras-2. In Reaktor Weidelgras-1 bestand eine Problematik mit Nickel und Chrom (Abrieb erzeugte zusätzlichen Input, vgl. hierzu Kapitel 3.5.3 und 4.5.4), daher sind die Nickelgehalte in diesem Reaktor mit Vorsicht zu betrachten. Reaktor Weidelgras-2 war von diesem Problem jedoch nicht betroffen.

Unter den Versuchsbedingungen bei der Substitution mit dieser Silagemischung ist Nickel demnach kein limitierender Faktor. Dies bestätigt die Vermutung, dass Nickel im Substitut ausreichend vorhanden ist. Denn die Durchschnittsgehalte der Deutsches Weidelgras-Silage wiesen die höchsten Nickel-Werte aller untersuchten Silagen auf (vgl. Kapitel 4.2, Tabelle 8). Sie lagen mit $0,30 \mathrm{mg} / \mathrm{kg}_{\text {FM N}}$ Nickel um Faktor 5 höher als die Durchschnittsgehalte von Mais-Silage mit $0,06 \mathrm{mg} / \mathrm{kg}$ FM.

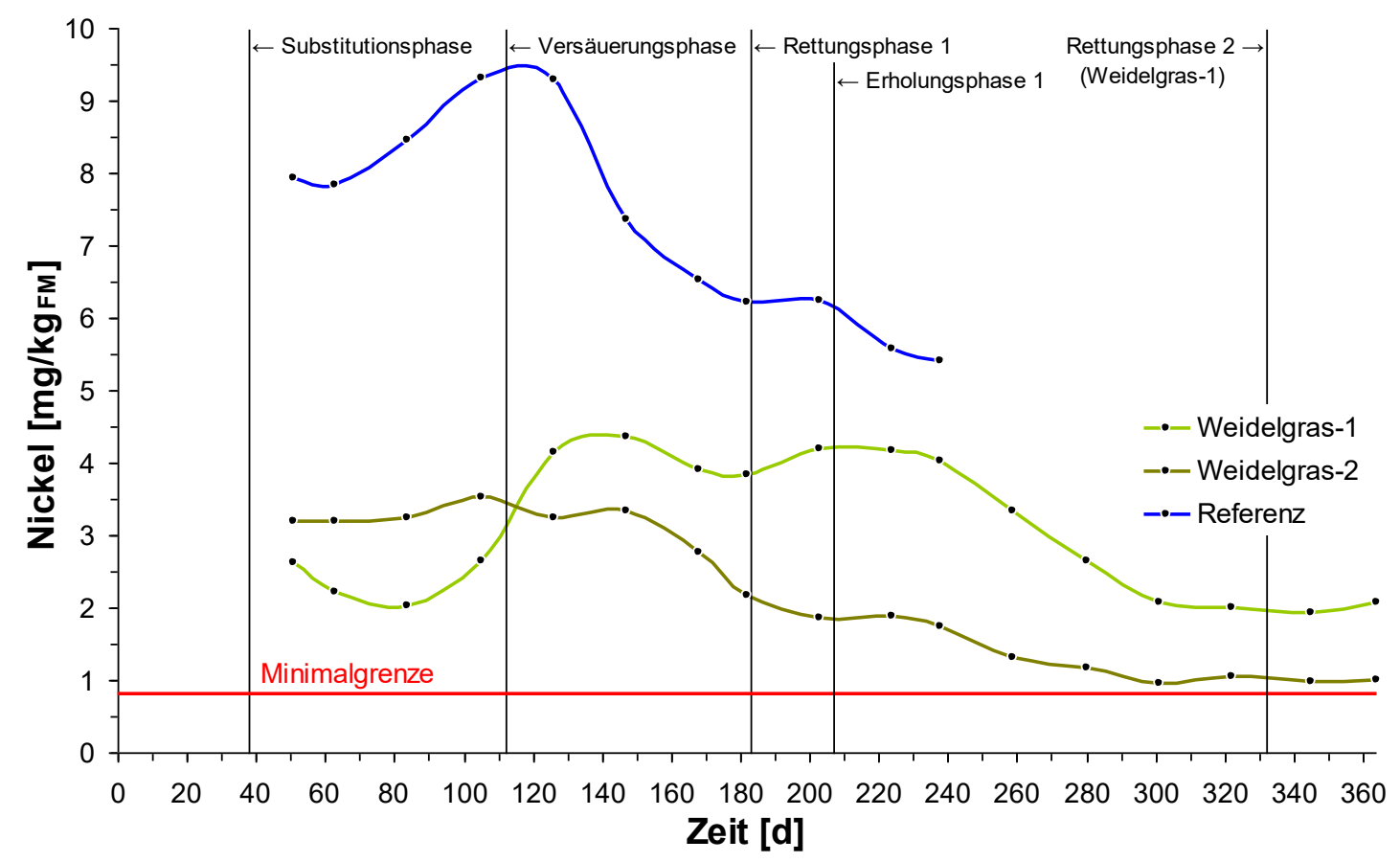

Abbildung 56: Verläufe der Nickelkonzentrationen des Substitutionsversuchs 3 mit $30 \%$ Deutsches Weidelgras- zu $70 \%$ Mais-Silage (oTS-bezogen) in den Versuchsbiogasreaktoren (mit Referenzreaktor). 
Der Kontrollreaktor 4.9 lief während der gesamten dritten Projektphase weitestgehend stabil, mit Ausnahme einer Auffälligkeit ab Versuchstag 172 mit plötzlich ansteigenden bzw. fallenden Werten der physikochemischen und gasanalytischen Prozessdaten (Abbildung 50 bis Abbildung 54). Die genaue Ursache konnte zunächst nicht ermittelt werden, da alle Betriebsparameter bis zu diesem Zeitpunkt unauffällig und im Normalbereich waren. Eine schlechte Mais-Silage-Qualität konnte ausgeschlossen werden, da der letzte Wechsel der Mais-Silage-Charge bereits am Versuchstag 155 stattfand, demnach 17 Tage vorher. Der Grund liegt wiederum an den sehr niedrigen Natriumgehalten im Reaktor (vgl. Kapitel 4.4.4). Nachdem die Fütterung für drei Versuchstage (Versuchstag 179 bis 181) ausgesetzt wurde normalisierten sich die Prozesswerte jedoch schnell und liefen stabil weiter. Nach der Umstellung auf die Rettungskonfigurationen in den Reaktoren mit Deutsches Weidelgras-Silage ab Versuchstag 183 (Rettungsphase 1) wurde der Kontrollversuch im Referenzreaktor noch bis Versuchstag 238 auf einem stabilen Niveau weiterbetrieben und dann beendet. Der Nachweis, dass eine Monovergärung von Mais-Silage unter den Prozessbedingungen mit nur einem Viertel der ursprünglichen Spurenelementadditivmenge nicht stabil betrieben werden kann, wurde im Zusatzversuch erbracht (vgl. Kapitel 4.6).

In der letzten Prozessphase, als Indizienphase bezeichnet, wurde ab Versuchstag 429 eine Aufsplittung des Doppelversuchs vollzogen, um nach der Erholungsphase 2 im verbleibenden Zeitraum bis zur Abschaltung der Versuchsbiogasreaktoren am Versuchstag 493 noch Hinweise zu finden, welche der Spurenelementkonzentrationen bei dem Substitutionsversuch von $30 \%$ Deutsches Weidelgras- zu $70 \%$ Mais-Silage (oTS-bezogen) noch nicht für stabile Biogasprozesse bzw. optimale Biogaserträge ausreicht. Der Reaktor Weidelgras-1 bekam fünf Versuchstage lang zusätzlich zur Pflanzenmischung wiederum nur eine Monoelementadditivlösung mit Cobaltchlorid $\left(\mathrm{CoCl}_{2} \cdot 6 \mathrm{H}_{2} \mathrm{O}\right)$ und danach bis Versuchsende die volle Menge $(0,8 \mathrm{~mL}$ pro Tag) einer Spurenelementlösung mit Cobaltchlorid und Calciumselenid (CaSe), um zusätzlich das Spurenelement Selen (Se) zu testen. Der Reaktor Weidelgras-2 erhielt ebenfalls fünf Versuchstage zusätzlich zur Pflanzenmischung nur eine Monoelementadditivlösung mit Cobaltchlorid, danach bis Versuchsende die volle Menge eines Spurenelementadditivs mit Cobaltchlorid und Ammoniummetawolframathydrat $\left[\left(\mathrm{NH}_{4}\right)_{6} \mathrm{H}_{2} \mathrm{~W}_{12} \mathrm{O}_{40} \bullet \mathrm{xH}_{2} \mathrm{O}\right]$, um zusätzlich das Spurenelement Wolfram (W) zu testen. Die Wahl von Selen und Wolfram wurde getroffen, da die Elemente in der ursprünglichen Spurenelementlösung 
am geringsten konzentriert waren (vgl. Kapitel 4.1, Tabelle 6) und die Reaktoren bereits seit der Substitutionsphase am Limit der Spurenelementkonzentrationen betrieben wurden, sodass diese minimalen Einflussnahmen die besten Erkenntnisse versprachen.

Die Indizienphase lief ab Versuchstag 434 über insgesamt 60 Versuchstage (anderthalb Verweilzeiten) bis zum Versuchsende am Versuchstag 493. Die stabilen und prozesstechnisch guten Werte der physikochemischen und gasanalytischen Parameter (Abbildung 50, Abbildung 51 und Abbildung 53) sowie vor allem die auf einem sehr hohen Niveau gebliebenen Ausbeuten der normierten Biogasproduktion mit durchschnittlich $28 \mathrm{~L}_{\mathrm{Norm}} / \mathrm{d}$ in beiden Reaktoren (Abbildung 52) zeigten deutlich, dass sowohl Selen als auch Wolfram, wie Cobalt und Nickel, eine entscheidende Rolle für optimale Biogasprozesse spielen. Aufgrund des schon überschrittenen Endes des Verbundvorhabens konnten jedoch keine weiteren Versuche hierzu durchgeführt als auch die Elementkonzentrationen dieser Indizienphase in den Reaktorproben mittels Multielementanalysen nicht bestimmt werden. ICP-MS-Messungen lagen nur bis Versuchstag 224 vor. Auch eine Abschätzung der Selen- und Wolframwerte war aufgrund des unbekannten Konzentrationsniveaus $\mathrm{zu}$ Beginn der Rettungs- bzw. Erholungsphase 2 durch die diversen Eingriffe nicht möglich. Es kann jedoch davon ausgegangen werden, dass sich durch die Zugabe von einem Viertel der ursprünglichen Menge der synthetischen Spurenelementlösung in Erholungsphase 2 die Selen- und Wolframgehalte zu Beginn der Indizienphase wieder auf stabilen Niveaus über den minimalen Schwellenwerten befanden und während der Indizienphase konstant blieben.

Die Energiepflanzen-Silagemischung mit $30 \%$ Deutsches Weidelgras zu 70 \% Mais (oTS-bezogen) in Kombination mit der Zugabe eines Viertels der ursprünglichen Menge der synthetischen Cobaltchloridlösung als Monoelementadditiv ist für stabile Cobaltgehalte im Fermenter ausreichend. Ohne Cobaltadditiv reichen die in den Fermentern eingebrachten Cobaltmengen dieser Silagemischung für stabile Biogasprozesse nicht aus. Nickel stellt bei dieser Kombination kein Mangelelement dar.

Allerdings zeigten Instabilitäten bei den FOS/TAC (FAL)-Werten und geminderte normierte Biogaserträge an, dass eine reduzierte Cobaltadditiv-Zugabe allein nicht genügt, stabile Biogasprozesse über mindestens drei Verweilzeiten in den Substitutionsreaktoren aufrechtzuerhalten. Wird der Silagemischung hingegen eine auf ein Viertel reduzierte Spurenelementlösung mit allen enthaltenen Elementen zugegeben, können optimale Biogasprozesse gewährleistet werden. 


\subsubsection{Substitutionsversuch 4: Vergärung von 30 \% Silage aus Durchwachsene Silphie zu $70 \%$ Mais-Silage (oTS-bezogen)}

Nach der Substratumstellung ab Versuchstag 38 begannen nach etwa zwei Verweilzeiten, analog zum dritten Substitutionsversuch (vgl. Kapitel 4.5.2), in den beiden Versuchsbiogasreaktoren mit der Silagemischung Durchwachsene Silphie zu Mais im Verhältnis 30:70 (oTS-bezogen) ab Versuchstag 112 (Versäuerungsphase) die FOS (Kapp)- und FOS/TAC (FAL)-Werte (Abbildung 57) kontinuierlich anzusteigen. Die $\mathrm{pH}-$ Werte sowie die Werte der normierten Biogasproduktion fingen wenige Tage danach an, allmählich einzubrechen (Abbildung 58 und Abbildung 59). Die Verläufe des normierten Methangehalts (Abbildung 60) zeigten mit Beginn der Versäuerungsphase ab Versuchstag 112 gegenüber dem Kontrollreaktor fallende Kurven.

Die Biogasprozesse in den beiden Substitutionsreaktoren waren etwa zweieinhalb Verweilzeiten nach Substitutionsbeginn so stark instabil, dass ab Versuchstag 147 Rettungsmaßnahmen (Rettungsphase) beginnen mussten, da die FOS/TAC (FAL)Werte bereits auf 3,0 (Reaktor Silphie-1) bzw. 1,7 $\mathrm{g}_{\mathrm{FOS}} / \mathrm{g}_{\mathrm{CaCO} 3}$ (Reaktor Silphie-2) angestiegen waren (Abbildung 57). Zu diesem Zeitpunkt drastisch eingebrochen waren auch die pH-Werte mit 5,9 bzw. 6,8 (Abbildung 58), die Werte der normierten Gasproduktion mit 4 bzw. $14 \mathrm{~L}_{\mathrm{Norm}} / \mathrm{d}$ (Abbildung 59) sowie die Werte des normierten Methangehalts mit 40 bzw. 45 Vol.-\% (Abbildung 60). Über tageweise vollständige Aussetzungen bzw. partielle Änderungen mit einem Viertel, der Hälfte oder der vollen Substratzufuhr sowie kurzzeitigen Variationen bei der Zugabe der synthetischen Spurenelementlösung (volle oder auf nur ein Viertel reduzierte Zugabe) konnten die beiden Substitutionsreaktoren erfolgreich stabilisiert werden. Bei dem Reaktor Silphie-2 wurden die Rettungsmaßnahmen bereits am Versuchstag 154 abgeschlossen. Bei dem Reaktor Silphie-1 dauerten die Eingriffe aufgrund der gravierend schlechten Prozesswerte zu Beginn der Rettungsphase länger an, bis zum Versuchstag 231.

Um wie geplant nach der Rettungsphase stabile Biogasprozesse über mindestens drei Verweilzeiten zu realisieren, wurde den beiden Substitutionsreaktoren während der gesamten Erholungsphase ab Versuchstag 163 (Reaktor Silphie-2) bzw. ab Versuchstag 210 (Reaktor Silphie-1) bis Versuchsende am Versuchstag 387 täglich zusätzlich zu der Silagemischung 30 \% Durchwachsene Silphie zu 70 \% Mais (oTS-bezogen) erneut nur ein Viertel der ursprünglichen Spurenelementadditivmenge zugegeben. 


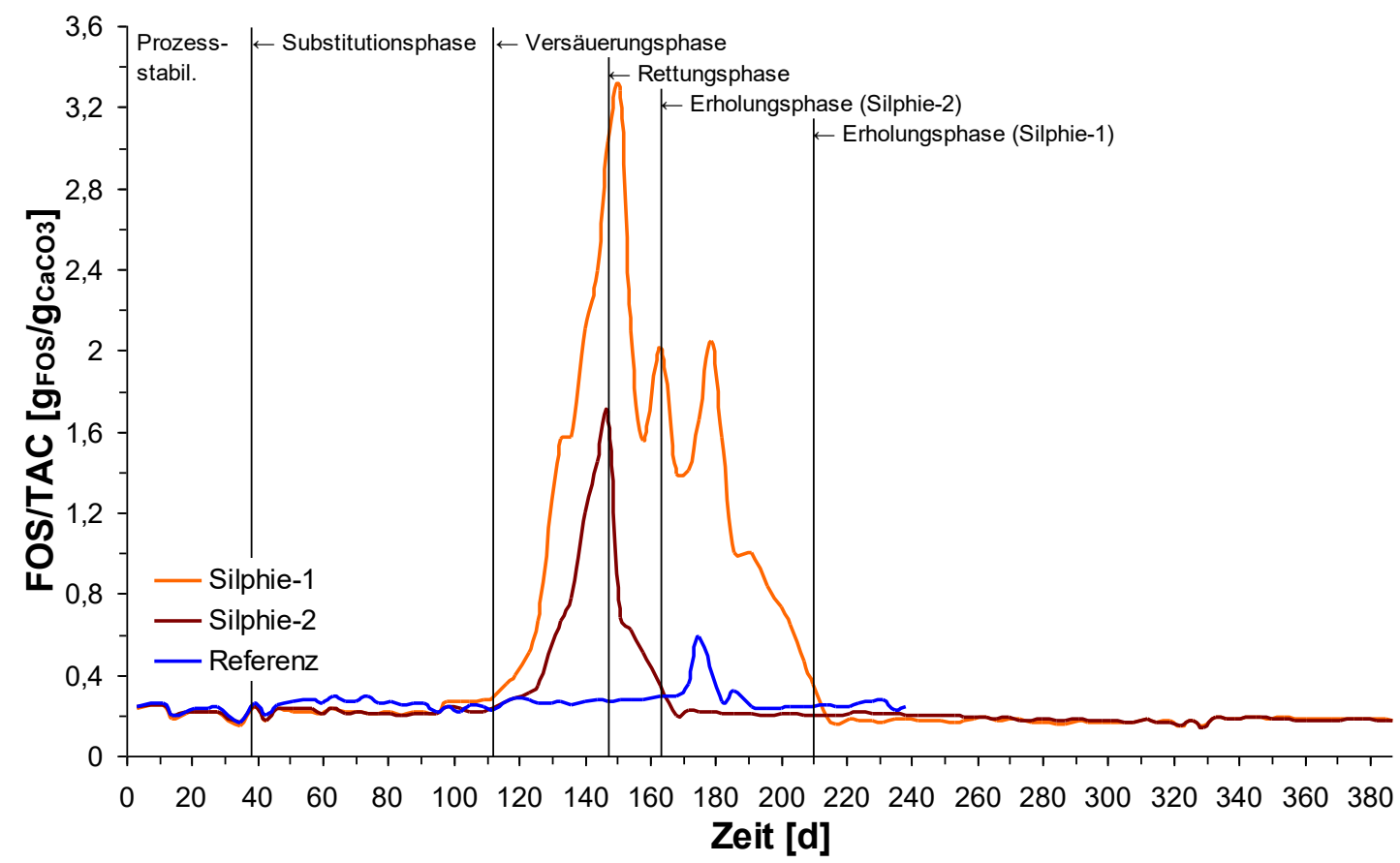

Abbildung 57: $\quad$ FOS/TAC (FAL)-Verläufe des Substitutionsversuchs 4 mit $30 \%$ Durchwachsene Silphie- zu $70 \%$ Mais-Silage (oTS-bezogen) in den Versuchsbiogasreaktoren (mit Referenzreaktor).

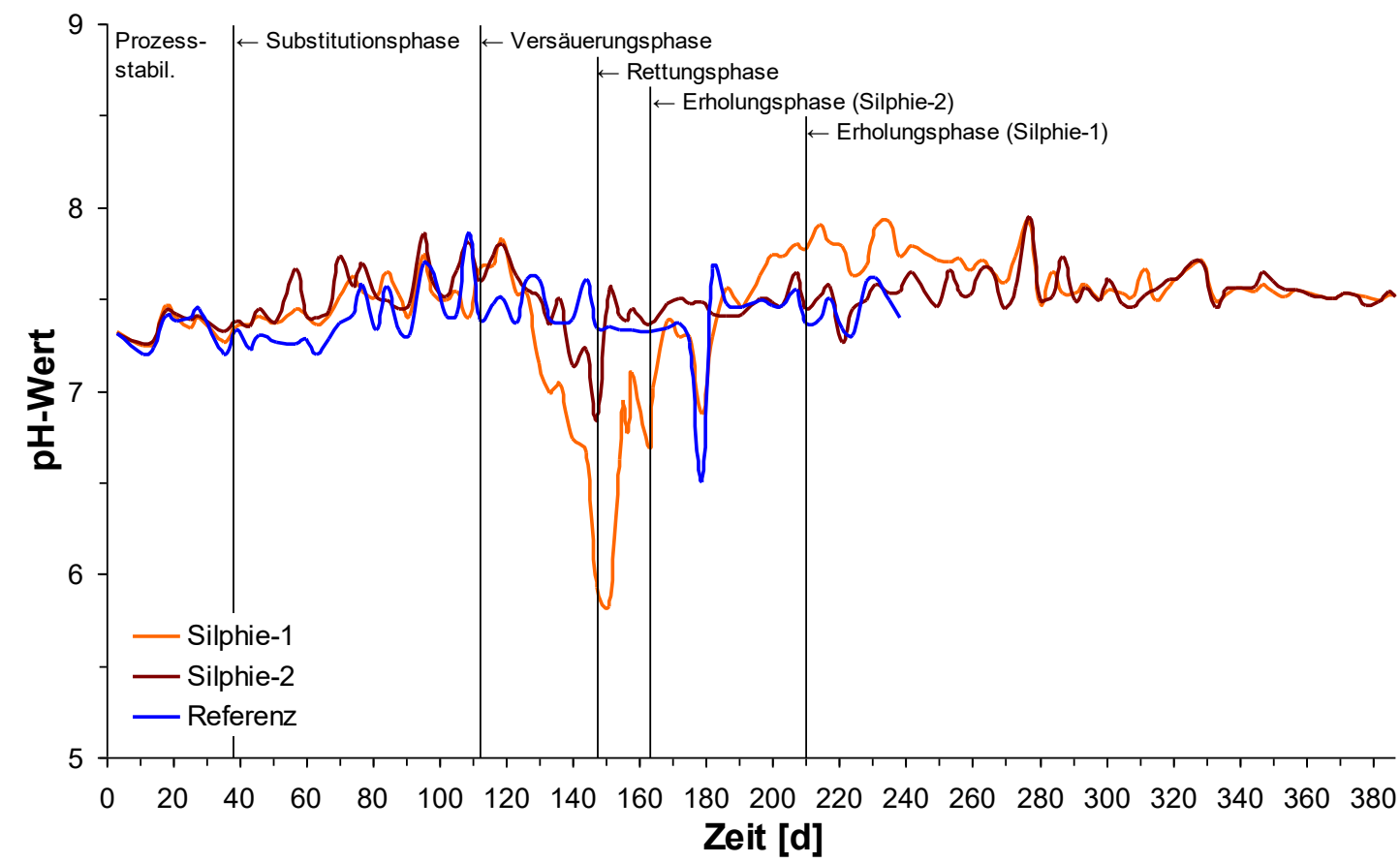

Abbildung 58: $\quad$ pH-Wert-Verläufe des Substitutionsversuchs 4 mit $30 \%$

Durchwachsene Silphie- zu $70 \%$ Mais-Silage (oTS-bezogen) in den Versuchsbiogasreaktoren (mit Referenzreaktor). 


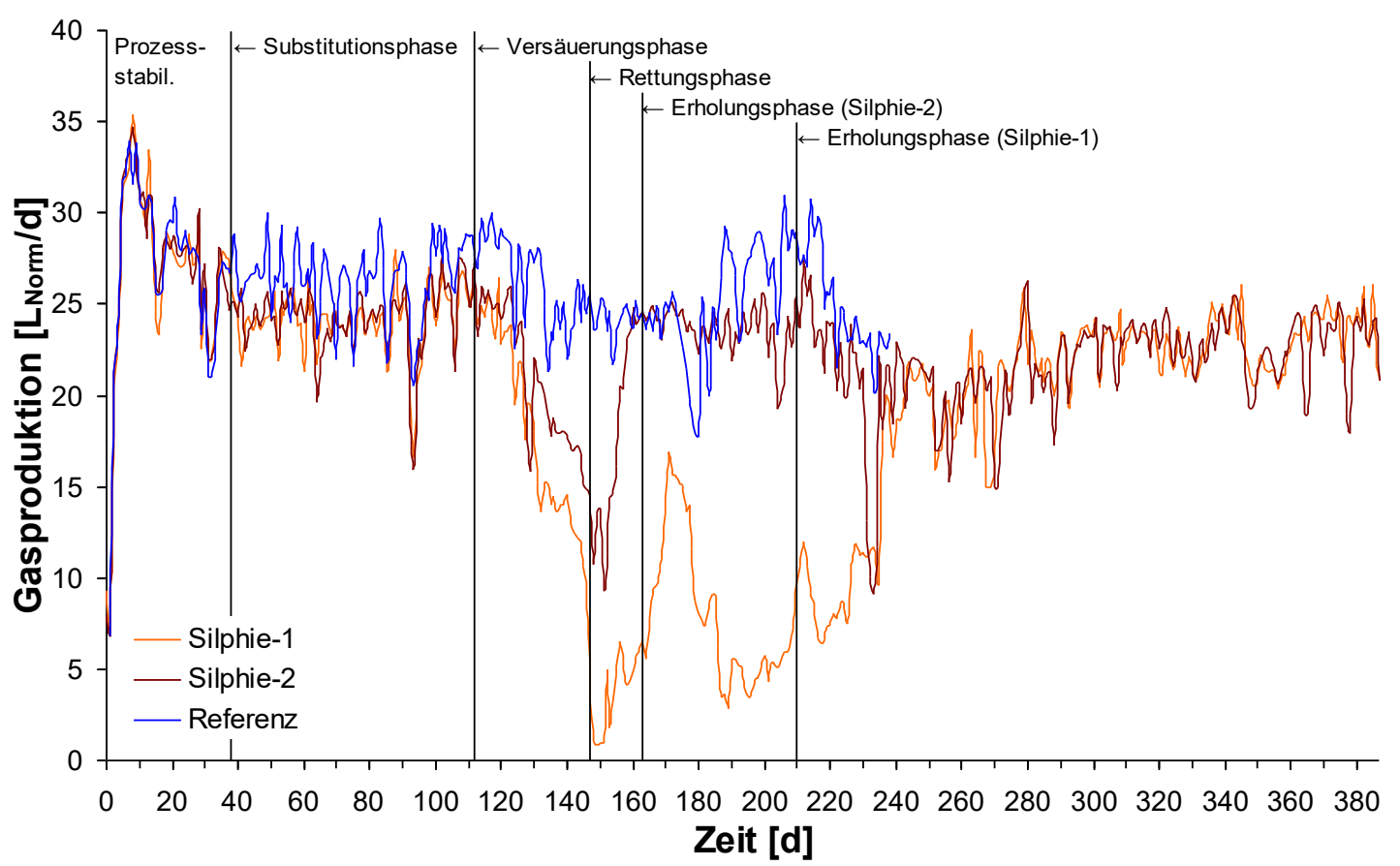

Abbildung 59: $\quad$ Verläufe der normierten Gasproduktion des Substitutionsversuchs 4 mit $30 \%$ Durchwachsene Silphie- zu $70 \%$ Mais-Silage (oTS-bezogen) in den Versuchsbiogasreaktoren (mit Referenzreaktor).

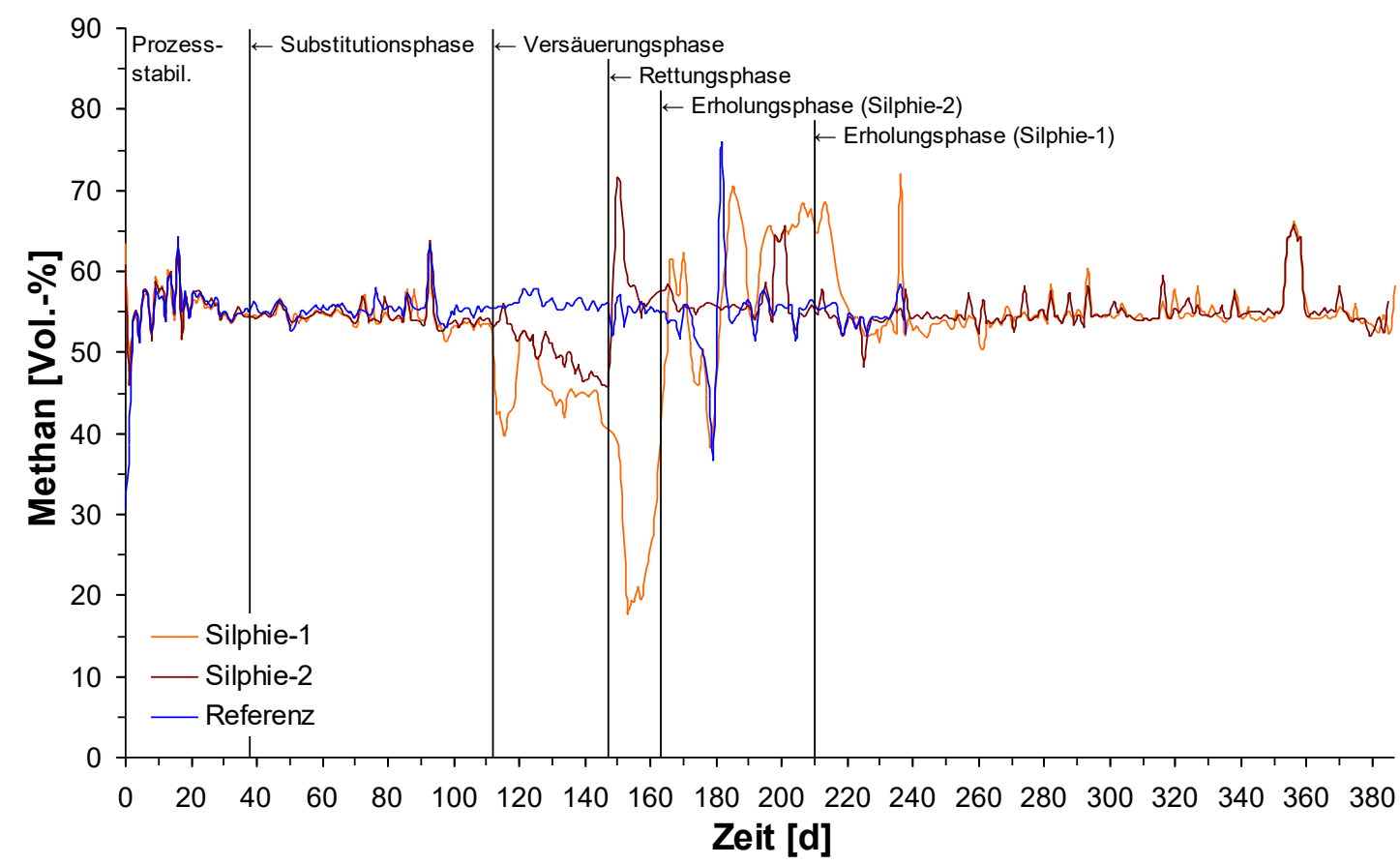

Abbildung 60: $\quad$ Verläufe des normierten Methangehalts des Substitutionsversuchs 4 mit $30 \%$ Durchwachsene Silphie- zu $70 \%$ Mais-Silage (oTS-bezogen) in den Versuchsbiogasreaktoren (mit Referenzreaktor). 
Auch in diesem Kapitel sind zum besseren Überblick in Abbildung 61 die Verläufe der FOS/TAC (FAL)-Werte und der normierten Gasproduktion direkt gegenübergestellt. Die physikochemischen und gasanalytischen Prozessparameter lagen in den Erholungsphasen über einen Zeitraum von etwa viereinhalb (Reaktor Silphie-1) bzw. fünfeinhalb Verweilzeiten (Reaktor Silphie-2) auf einem guten, kontinuierlichen Niveau. Die stabilisierende Langzeitwirkung dieser Silagemischung in Kombination mit der Zugabe eines Viertels der synthetischen Spurenelementlösung nach OECHSNER ET AL. (2008; vgl. Kapitel 4.1) konnte damit nachgewiesen werden.

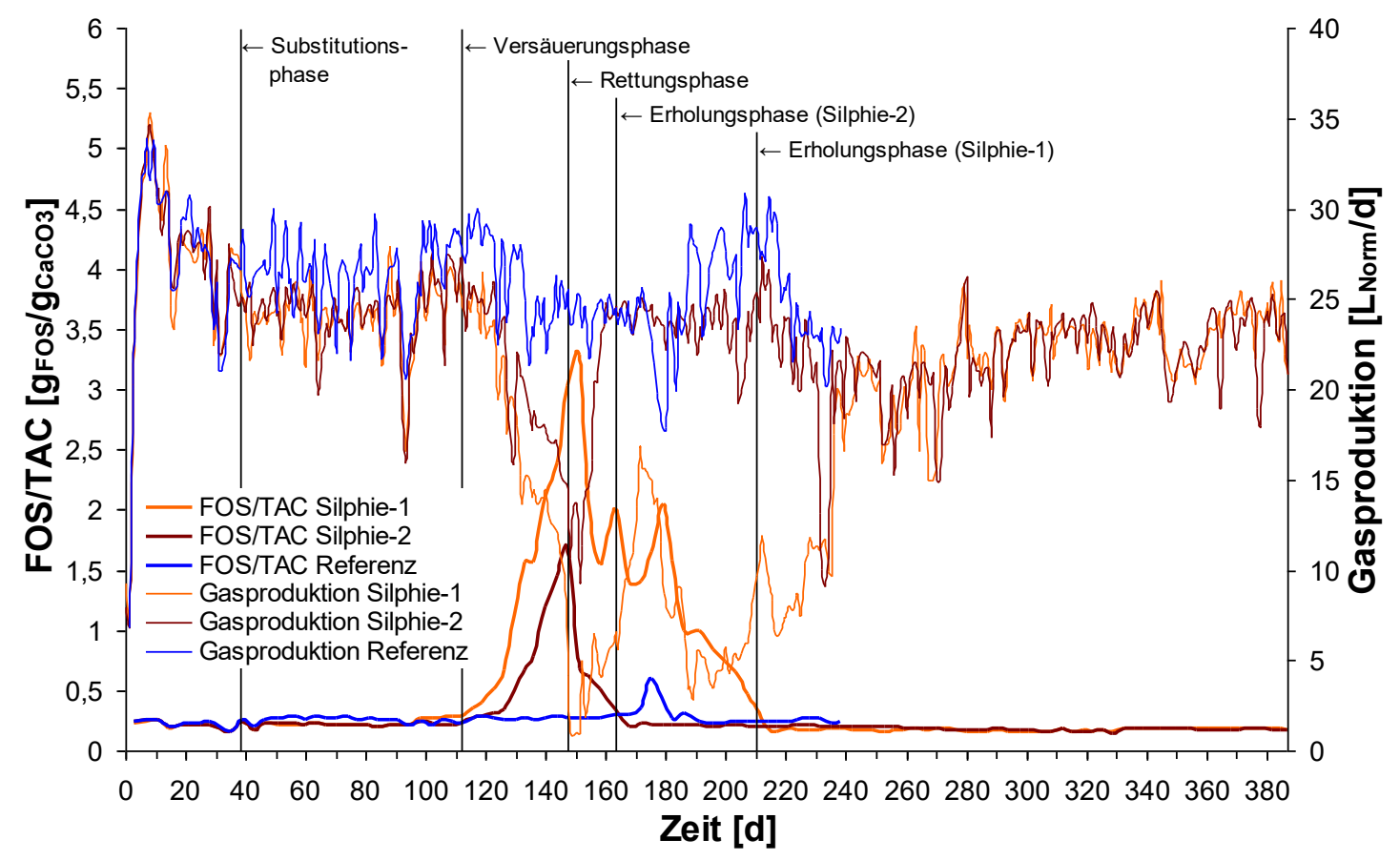

Abbildung 61: Gegenüberstellung der Verläufe der FOS/TAC (FAL)-Werte und der normierten Gasproduktion des Substitutionsversuchs 4 mit $30 \%$ Durchwachsene Silphie- zu $70 \%$ Mais-Silage (oTS-bezogen) in den Versuchsbiogasreaktoren (mit Referenzreaktor).

Für die Versäuerung der beiden Reaktoren bzw. den absehbaren potenziellen Prozesszusammenbruch ausschlaggebend waren in dieser Doppelversuchsreihe erneut die sehr niedrigen Cobaltkonzentrationen (Abbildung 62). Sie lagen kurz vor Beginn der Rettungsphase mit Gehalten von 0,064 (Reaktor Silphie-1) bzw. 0,066 mg/kg FM Cobalt (Reaktor Silphie-2) direkt im Bereich des in der ersten Projektphase bestimmten minimalen Schwellenwertes von $0,066 \mathrm{mg} / \mathrm{kg}_{\text {FM }}$ Cobalt (vgl. Kapitel 4.3.2). In der jeweiligen Erholungsphase nach der Rettungsphase verliefen die Cobaltgehalte in den beiden Reaktoren auf konstant hohen Niveaus, fern des minimalen Schwellenwertes. 


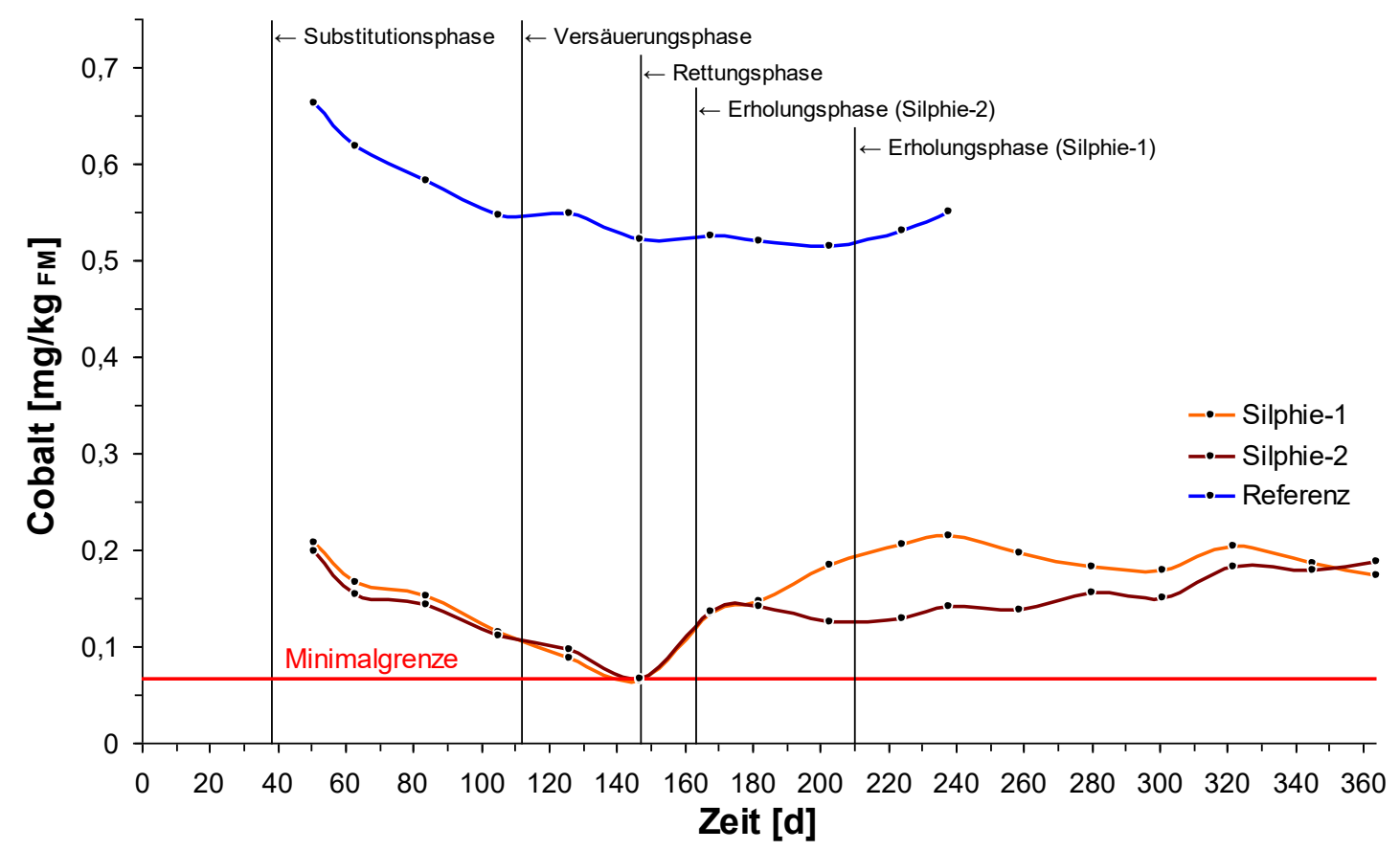

Abbildung 62: Verläufe der Cobaltkonzentrationen des Substitutionsversuchs 4 mit $30 \%$ Durchwachsene Silphie- zu $70 \%$ Mais-Silage (oTS-bezogen) in den Versuchsbiogasreaktoren (mit Referenzreaktor).

Dagegen lagen die Nickelgehalte (Abbildung 63) im gesamten Substitutionsversuch deutlich oberhalb des in der ersten Projektphase ermittelten, jedoch wahrscheinlich zu hoch angegebenen, minimalen Schwellenwertes von $0,82 \mathrm{mg} / \mathrm{kg}_{\mathrm{FM}}$ (vgl. Kapitel 4.3.3 und 4.3.4). In der jeweiligen Erholungsphase nach der Rettungsphase zeigten die Verläufe jedoch die gleiche Nickel-Chrom-Problematik wie der Reaktor Weidelgras-1 im Substitutionsversuch 3 (vgl. Kapitel 4.5.2). Reaktor Silphie-1 wies mehrere deutliche Konzentrationsschwankungen während der Erholungsphase auf, Reaktor Silphie-2 einen auffälligen Anstieg ab Versuchstag 280.

Aufgrund der Nickel-Chrom-Problematik (vgl. hierzu Kapitel 3.5.3 und 4.5.4) sind die Nickelkonzentrationen in diesen beiden Substitutionsreaktoren zwar auch mit Vorsicht $\mathrm{zu}$ betrachten, durch das generell hohe Konzentrationsniveau von Nickel wäre im Doppelversuch der Schwellenwert allerdings nicht erreicht worden. Bei der Substitution mit dieser Silagemischung ist unter den Versuchsbedingungen Nickel daher auch kein limitierendes Element. 


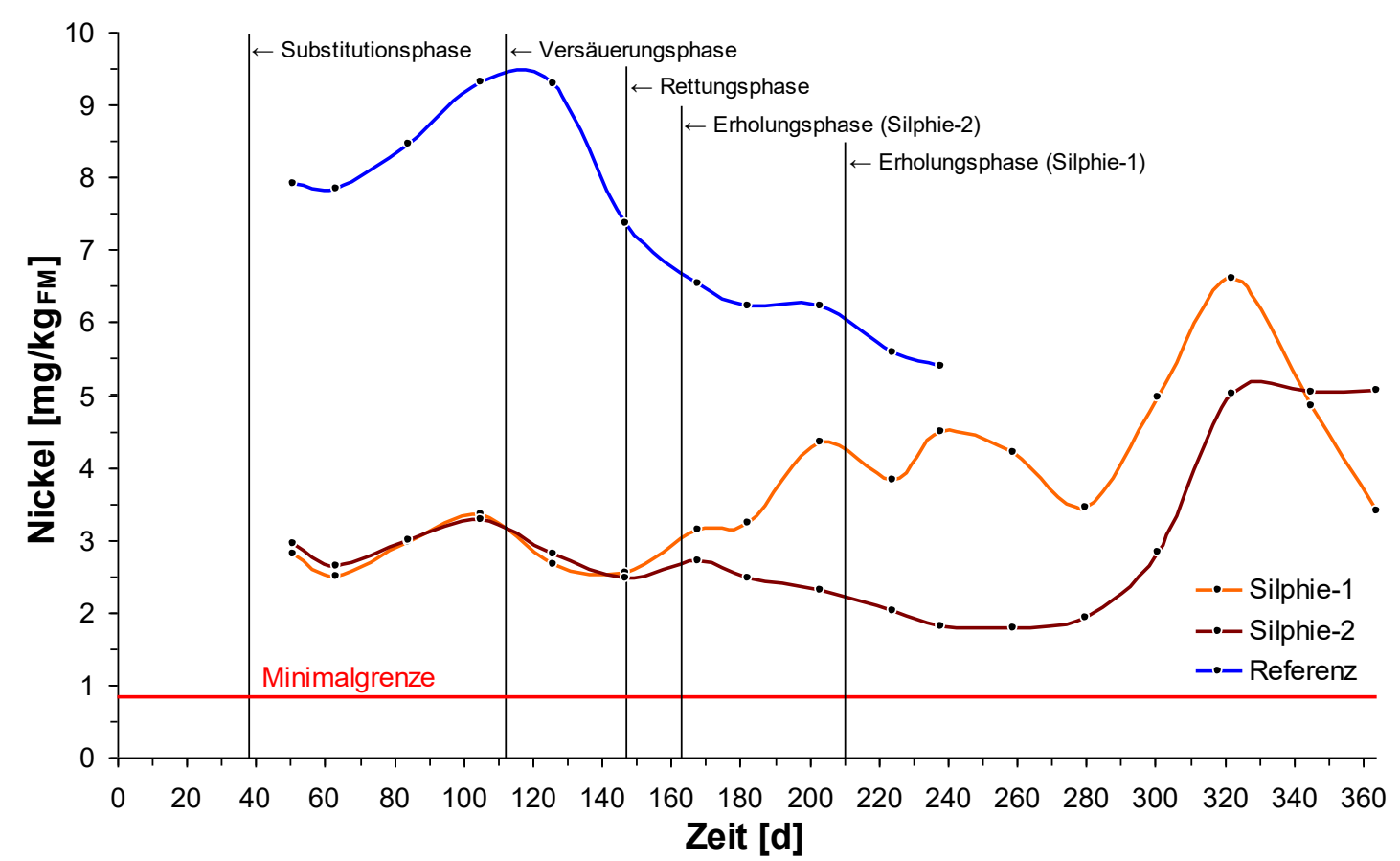

Abbildung 63: Verläufe der Nickelkonzentrationen des Substitutionsversuchs 4 mit $30 \%$ Durchwachsene Silphie- zu $70 \%$ Mais-Silage (oTS-bezogen) in den Versuchsbiogasreaktoren (mit Referenzreaktor).

Die Langzeitstabilität der Biogasprozesse bei Verwendung der EnergiepflanzenSilagemischung mit $30 \%$ Durchwachsene Silphie zu 70 \% Mais (oTS-bezogen) in Kombination mit der Zugabe nur noch eines Viertels der ursprünglichen Spurenelementadditivmenge konnte in der Doppelversuchsreihe unter den gewählten Betriebsparametern nachgewiesen werden. Die Cobalt- und Nickelkonzentrationen lagen auch in diesem vierten Substitutionsversuch bei stabilen physikochemischen und gasanalytischen Parametern in den Erholungsphasen deutlich über den in der ersten Projektphase ermittelten minimalen Schwellenwerten.

Ohne die Zugabe der reduzierten synthetischen Spurenelementadditivlösung reichen die in die Reaktoren eingebrachten Cobaltmengen dieser Silagemischung allerdings nicht aus. Der Cobaltmangel ist also wiederum indirekt Ursache für Versäuerungsprozesse und Minderausbeuten an Biogas unter den Versuchsbedingungen. Nickel hingegen war im gesamten Substitutionsversuch ausreichend vorhanden. 


\subsubsection{Nickel-Chrom-Problematik in den Substitutionsversuchen 3 und 4}

Bei den Nickelkonzentrationen der Substitutionsversuche 3 und 4 (vgl. Kapitel 4.5.2 und 4.5.3) fielen stark steigende bzw. variierende Verläufe in vier der fünf Versuchsbiogasreaktoren auf. Nach einer Überprüfung aller Elementkonzentrationen in den Reaktorproben wurde festgestellt, dass in den verschiedenen Reaktoren eine stark positive Korrelation zwischen den stark erhöhten Gehalten an Nickel und Chrom feststellbar ist (Abbildung 64, Abbildung 65 und Abbildung 66).

Die Ursache für diese hohen Konzentrationen sind wahrscheinlich Abrasions- oder Ablösungsvorgänge an den metallischen Oberflächen der mittig in den Reaktoren fixierten Ankerrührer mit den Rührblättern. Da die Nickel-Chrom-Problematik in allen Projektphasen der Laborfermenterversuche am DBFZ auftrat, ist sie in Kapitel 3.5.3, einem Unterkapitel zur Fehlerbetrachtung und Qualitätssicherung, näher beschrieben.

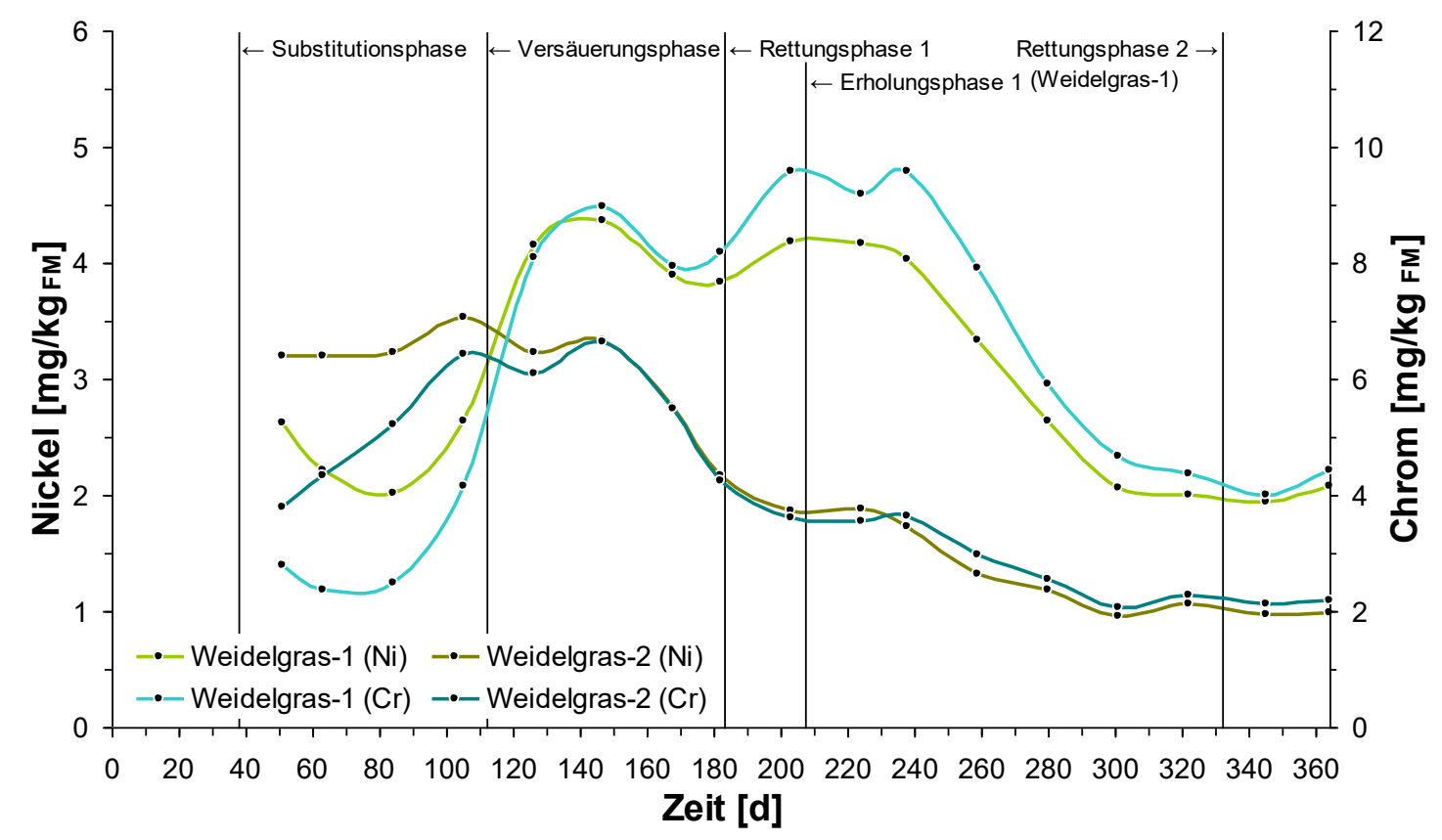

Abbildung 64: Verläufe der Nickel- und Chromkonzentrationen des Substitutionsversuchs 3 mit $30 \%$ Deutsches Weidelgras-zu $70 \%$ Mais-Silage (oTS-bezogen) in den Versuchsbiogasreaktoren. 


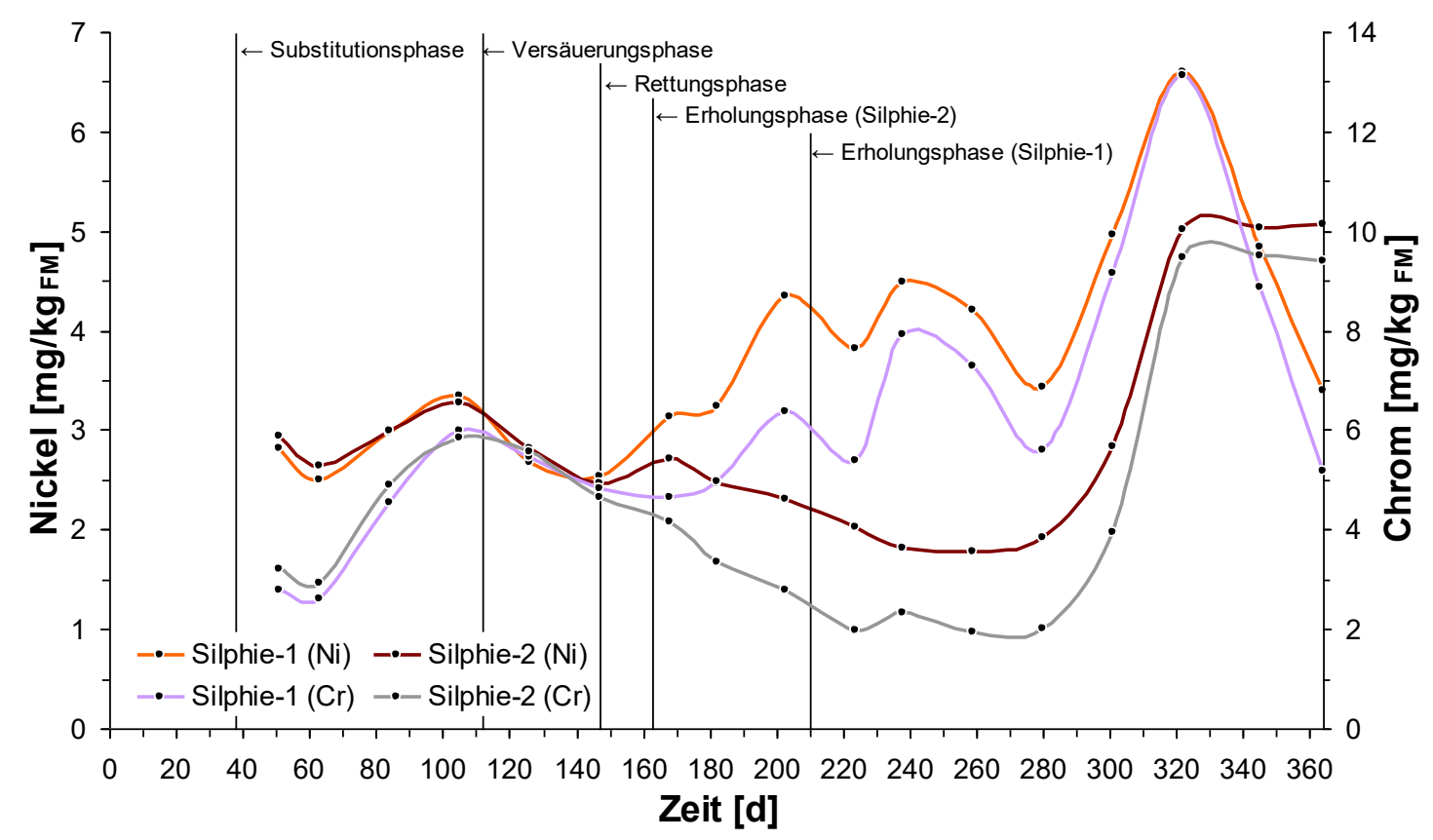

Abbildung 65: Verläufe der Nickel- und Chromkonzentrationen des Substitutionsversuchs 4 mit $30 \%$ Durchwachsene Silphie- zu $70 \%$ Mais-Silage (oTS-bezogen) in den Versuchsbiogasreaktoren.

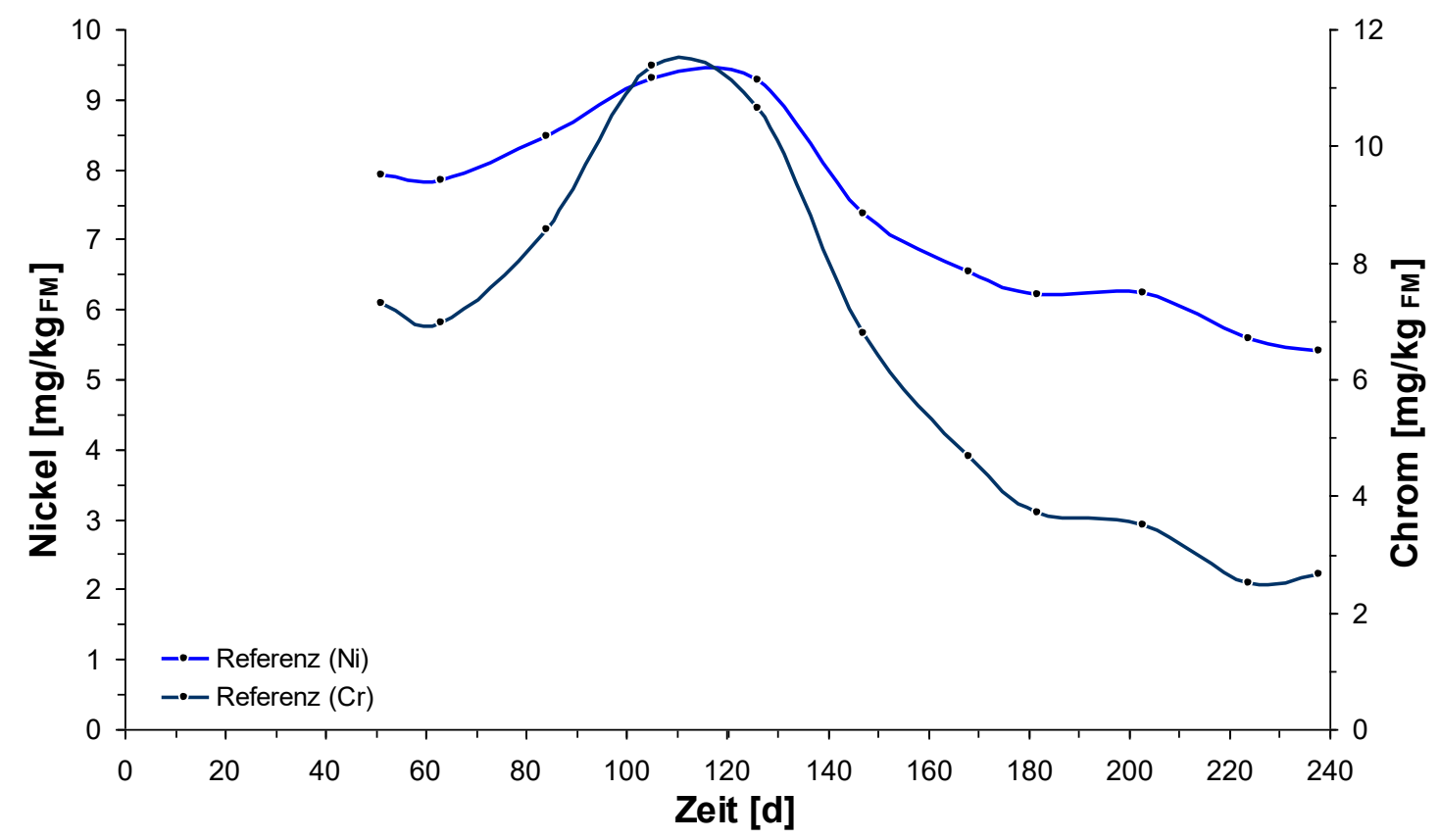

Abbildung 66: Verläufe der Nickel- und Chromkonzentrationen im Referenzreaktor während der Substitutionsversuche 3 und 4. 


\subsubsection{Bewertung weiterer Elemente in den Substitutionsversuchen 3 und 4}

Bei den essenziellen Grundelementen und Makronährstoffen (Gruppe I, vgl. Kapitel 2.2.5) zeigten sich Parallelen zu den ersten beiden Substitutionsversuchen mit Winterackerbohne- und Amarant-Silage (vgl. Kapitel 4.4.4). Die beiden zugemischten Silagen von Deutsches Weidelgras und Durchwachsene Silphie stellten mit Beginn der Substitutionsphasen ab Versuchstag 38 gute Quellen für einige Makronährstoffe dar, bedingt durch höhere Elementgehalte gegenüber der Mais-Silage. Deutliche Anstiege konnten bei den Reaktoren mit Durchwachsene Silphie-Silage bei Calcium-, Kalium-, Magnesium- und Natrium nachgewiesen werden (Abbildung 67). In den Substitutionsreaktoren mit Deutsches Weidelgras-Silage stiegen die Kalium- und Natriumgehalte an. Die Konzentrationen von Calcium, Magnesium (Abbildung 67), Phosphor und Schwefel (Abbildung 68). lagen gegenüber dem Referenzreaktor mit Mais-Silage-Monovergärung auf ähnlichen Niveaus. Die hohen Natrium-Durchschnittsgehalte in den substituierten Silagen (vgl. Kapitel 4.2, Tabelle 8) lassen sich auch hier wiederum v.a. auf den Einsatz des Silierhilfsmittels Kofasil ${ }^{\circledR}$ Liquid zurückführen (siehe hierzu die Erläuterungen in Kapitel 4.4.4). Gegenüber Mais sind die Natriumgehalte in unbehandelten Deutsches Weidelgras-Pflanzen mit Faktor 25 bis 30 jedoch auch stark angereichert. Unbehandelte Durchwachsene Silphie-Pflanzen weisen zum Erntezeitpunkt gegenüber Mais sehr ähnliche Natriumgehalte auf (vgl. Analysen von FAHLBUSCH, 2018).

Im Referenzreaktor fiel der Natriumgehalt (Abbildung 67) über die Versuchsdauer abermals stark ab, analog zur ersten und zweiten Projektphase (vgl. Kapitel 4.3.5 und 4.4.4), und lag mit nur noch $10,2 \mathrm{mg} / \mathrm{kg}_{\mathrm{FM}}$ Natrium am Versuchstag 168 auf einem sehr kritischen niedrigen Level nach dem minimalen Schwellenwert von $10 \mathrm{mg} / \mathrm{L}$ nach MunK ET AL. (2010). Ab Versuchstag 172 zeigten sich als Folge ungünstig verändernde Werte bei den physikochemischen und gasanalytischen Prozessdaten (nähere Erläuterungen hierzu in Kapitel 4.5.2), sodass am Versuchstag 179 eine Zugabe von $25 \mathrm{~g}$ Natriumhydrogencarbonat $\left(\mathrm{NaHCO}_{3}\right)$ erfolgte, um einer $\mathrm{zu}$ erwartenden Versäuerung entgegenzuwirken. Eingriffe mit Natriumhydrogencarbonat wurden auch in den Rettungsphasen der beiden Reaktoren mit Deutsches Weidelgras-Silage am Versuchstag $191\left(25 \mathrm{~g} \mathrm{NaHCO}_{3}\right)$ sowie im Substitutionsreaktor Silphie-1 an den Versuchstagen $151(60 \mathrm{~g}), \quad 154(30 \mathrm{~g}), 156(50 \mathrm{~g})$ und $157(5 \mathrm{~g})$ vollzogen. Beim letztgenannten Reaktor stiegen die Natriumgehalte dadurch bis über $4000 \mathrm{mg} / \mathrm{kg}$ FM an (in Abbildung 67 abgeschnitten). 
Kapitel 4: (Quasi)kontinuierliche Laborfermenterversuche
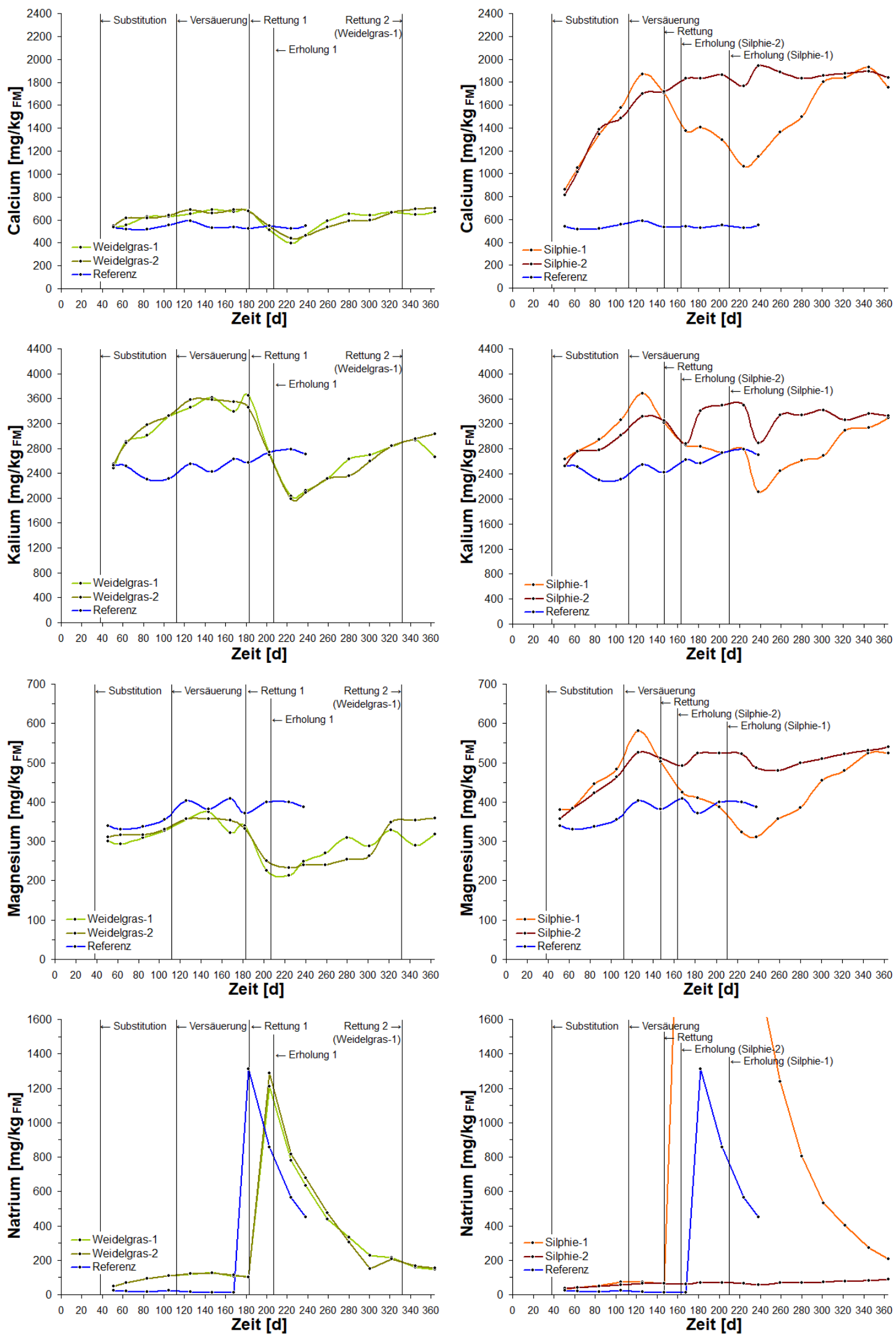

Abbildung 67: Verläufe der Calcium-, Kalium-, Magnesium- und Natriumgehalte der Substitutionsversuche 3 und 4 in den Versuchsbiogasreaktoren (mit Referenzreaktor). 

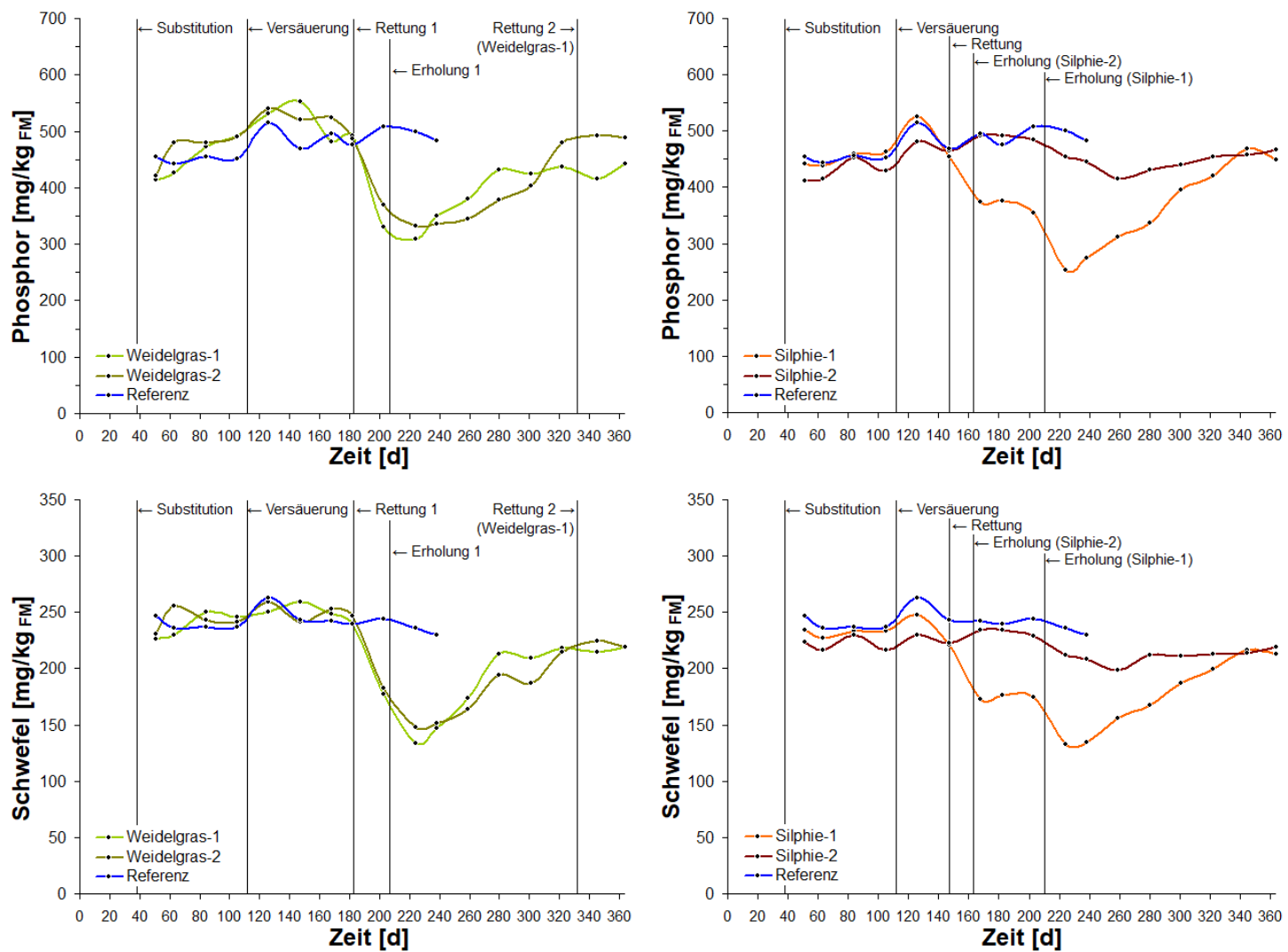

Abbildung 68: Verläufe der Phosphor-und Schwefelgehalte der Substitutionsversuche 3 und 4 in den Versuchsbiogasreaktoren (mit Referenzreaktor).

Die essenziellen Mikronährstoffe (Gruppe II) Eisen, Kupfer, Mangan, Molybdän, Zink und Selen (Abbildung 69 und Abbildung 70) zeigten wie in den Substitutionsversuchen mit Winterackerbohne- und Amarant-Silage (mit Ausnahme von Kupfer, vgl. hierzu Kapitel 4.4.4) durchweg fallende Konzentrationsverläufe mit Beginn der Substitutionsphasen. Der Grund dafür ist, dass auch hier die Zugabe der Spurenelementlösung während der Stabilisierungsphase bereits ausgesetzt wurde, um dann am Versuchstag 38 von einem niedrigeren Niveau aus mit den Substratzumischungen zu starten.

Das Ausgangsniveau war dennoch hoch, sodass während der jeweiligen Substitutionsund Versäuerungsphasen die Spurenelementgehalte in den Substitutionsreaktoren weiter abfielen, allerdings bis zum Beginn der Rettungsphasen am Versuchstag 147 (Reaktoren mit Durchwachsene Silphie-Silage) bzw. Versuchstag 183 (Reaktoren mit Deutsches Weidelgras-Silage) in keine kritischen Konzentrationsbereiche nach den in Kapitel 2.3 in Tabelle 1 zusammengestellten minimalen Bedarfswerten. Das bestätigt, dass Cobalt in beiden Versuchen mit Silagen von Deutsches Weidelgras bzw. Durchwachsene Silphie zum Zeitpunkt des Beginns der Rettungsversuche das alleinige kritische Element für die Versäuerung der Biogasprozesse war. 

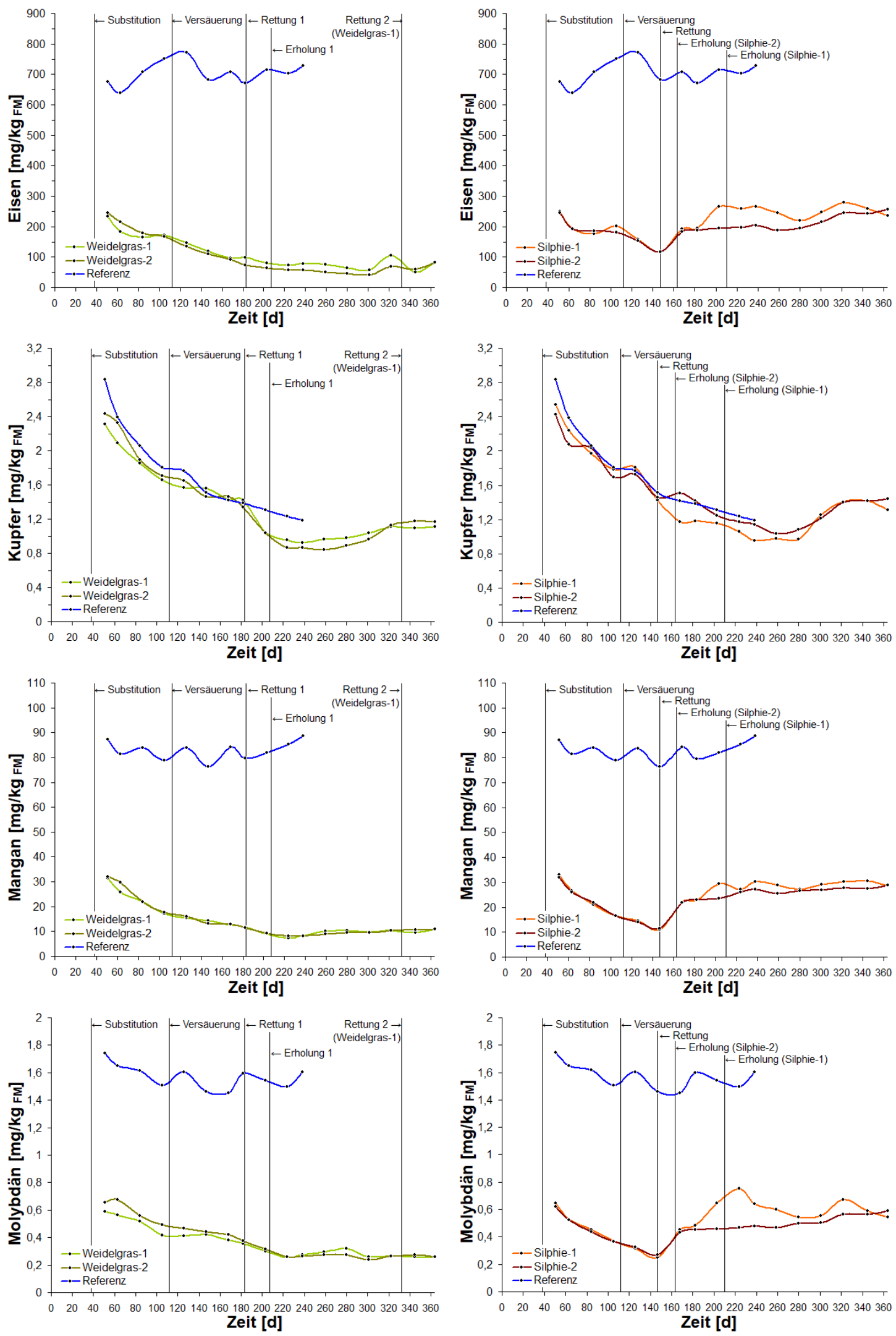

Abbildung 69: Verläufe der Eisen-, Kupfer-, Mangan- und Molybdängehalte der Substitutionsversuche 3 und 4 in den Versuchsbiogasreaktoren (mit Referenzreaktor). 

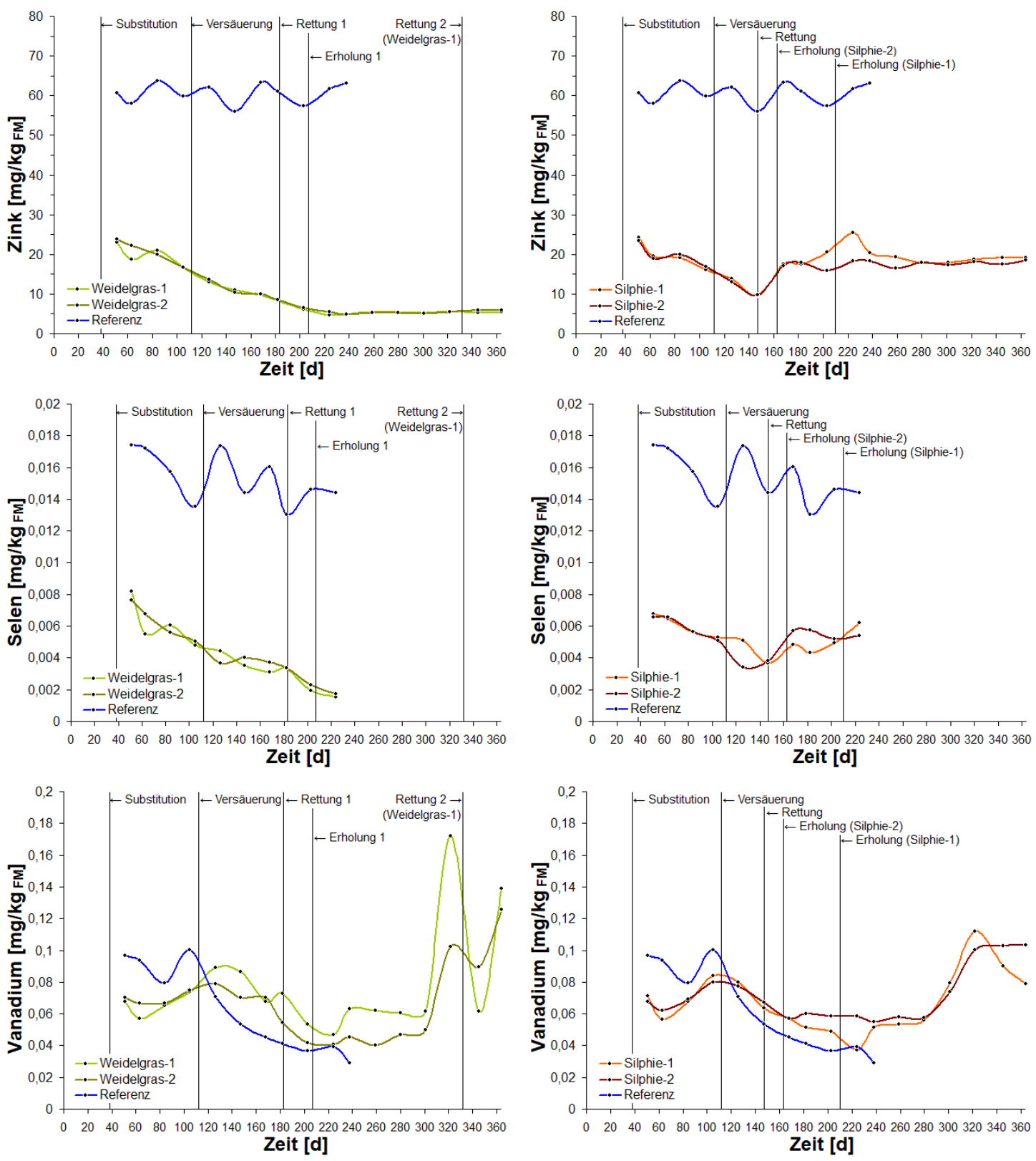

Abbildung 70: $\quad$ Verläufe der Zink-, Selen- und Vanadiumgehalte der Substitutionsversuche 3 und 4 in den Versuchsbiogasreaktoren (mit Referenzreaktor).

In den Erholungsphasen der Reaktoren mit 30 \% Durchwachsene Silphie-Silage (oTS-bezogen) und der Zugabe eines Viertels der ursprünglichen Spurenelementadditivmenge lagen alle in Abbildung 69 und Abbildung 70 aufgeführten Spurenelemente nach den Rettungsmaßnahmen wieder auf stabilen optimalen Niveaus, weit über den minimalen Schwellenwerten. Nur Kupfer, das nicht in der Spurenelementlösung enthalten war (vgl. Kapitel 4.1, Tabelle 6), zeigte Konzentrationsverläufe auf einem niedrigen unteren Level. Auch die Vanadiumkonzentrationen blieben weitestgehend unauffällig mit Ausnahme eines kurzzeitigen Anstiegs in der letzten Verweilzeit der 
Erholungsphasen, vermutlich bedingt durch variierende Gehalte in den eingesetzten Mais-Silagen durch anhaftende Boden- und Staubpartikel (vgl. POSPIECH ET AL., 2017b). Aluminium, Lanthan und Titan zeigen die gleichen Anstiege (vgl. Abbildung 72).

In der Erholungsphase 1 der Reaktoren mit 30 \% Deutsches Weidelgras-Silage (oTSbezogen) in Kombination mit der Zugabe eines Viertels der ursprünglichen Menge der synthetischen Cobaltchloridlösung als Monoelementadditiv lagen nur die Manganund Molybdängehalte (Abbildung 69) über den minimalen Bedarfswerten. Für Vanadium (Abbildung 70) liegen keine unteren Schwellenwerte in der Literatur vor. Es kann jedoch davon ausgegangen werden, dass Vanadium in den Substitutionsversuchen mit Deutsches Weidelgras-Silage kein Mangelelement, sondern für die Biogasprozesse ausreichend in den verwendeten Silagen vorhanden ist. Die beiden Substitutionsreaktoren zeigten gegenüber dem Referenzreaktor ähnliche Verläufe. Die kurzzeitigen Anstiege an den Versuchstagen 322 und 364 waren vermutlich wiederum durch variierende Gehalte in den verwendeten Silagen durch anhaftende Boden- und Staubpartikel (vgl. hierzu POSPIECH ET AL., 2017b). Gleiche Effekte zeigten sich auch bei Aluminium, Titan und Lanthan (vgl. Abbildung 72).

Die in die beiden Reaktoren eingebrachten Mengen der essenziellen Spurenelemente Eisen, Kupfer, Zink und Selen aus der Silagemischung $30 \%$ Deutsches Weidelgras zu 70 \% Mais (oTS-bezogen) reichten im Gegensatz zu Mangan und Molybdän (vgl. Kapitel 4.2, Tabelle 8) für stabile Niveaus über den unteren Schwellenwerten nicht aus. Die Konzentrationsverläufe dieser vier Spurenelemente (Abbildung 69 und Abbildung 70) bewegten sich im Bereich der in Kapitel 2.3 in Tabelle 1 angegebenen minimalen Bedarfswerte oder lagen darunter. Das bestätigt die Aussage in Kapitel 4.5.2, dass eine alleinige reduzierte Cobaltadditiv-Zugabe nicht genügt, stabile Biogasprozesse über mindestens drei Verweilzeiten in den Substitutionsreaktoren aufrechtzuerhalten.

Eisen, Kupfer, Zink, Selen (und höchstwahrscheinlich auch Wolfram) stellen neben Cobalt weitere Mangelelemente bei dieser Silagemischung unter den gewählten Betriebsparametern und ohne Zugabe der synthetischen Spurenelementlösung (mit allen Elementen) dar.

Bei den Verläufen der Elemente der Gruppe III (vgl. Kapitel 2.2.5), Arsen, Cadmium, Blei, Aluminium, Lanthan (als Vertreter der Lanthanoide / Seltene Erdelemente), Titan und Strontium waren zum Teil starke Konzentrationsschwankungen ersichtlich, die sich jedoch weithin in niedrigen Bereichen bewegten (Abbildung 71 und Abbildung 72). 

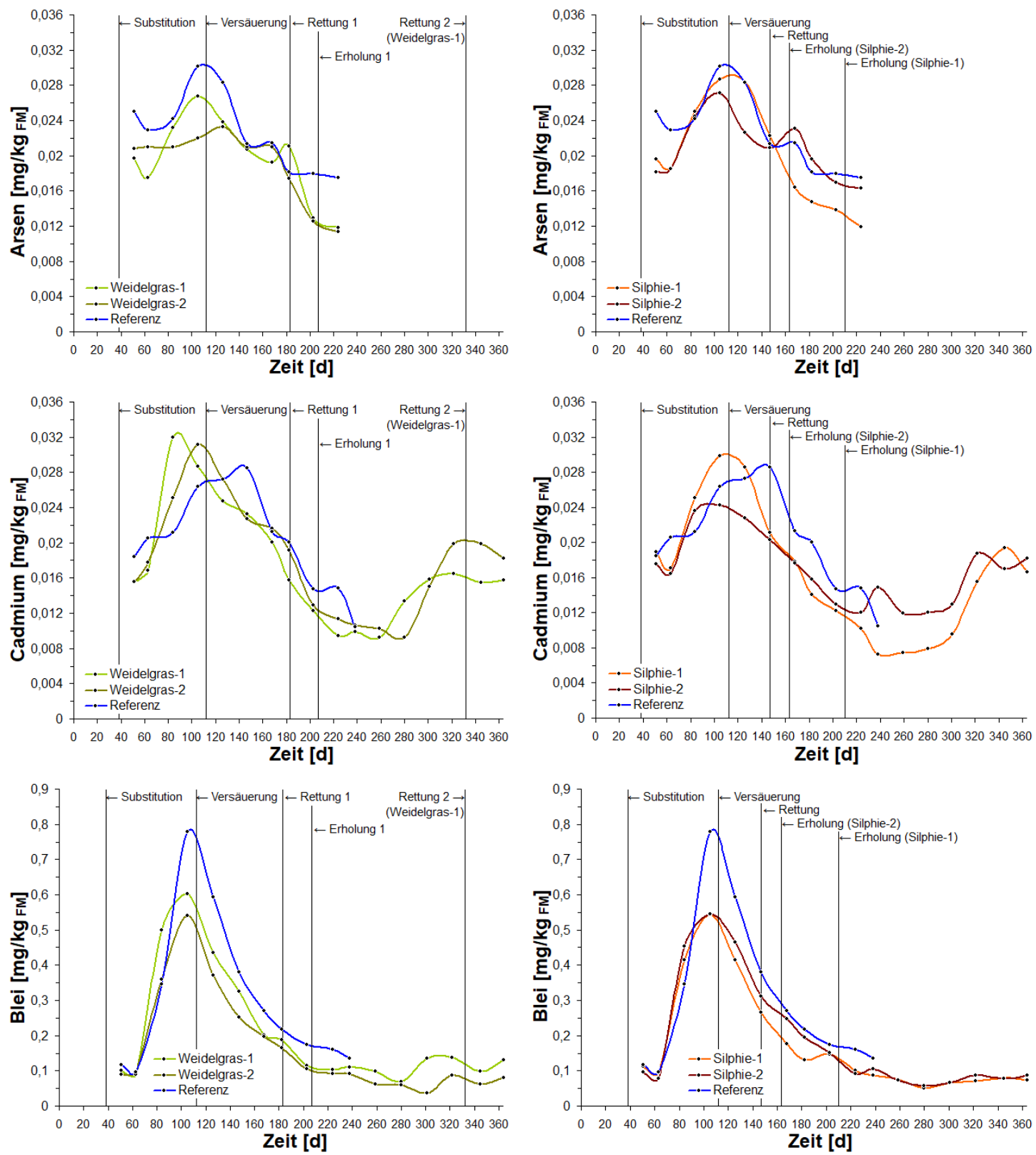

Abbildung 71: Verläufe der Arsen-, Cadmium-und Bleigehalte der Substitutionsversuche 3 und 4 in den Versuchsbiogasreaktoren (mit Referenzreaktor).

Auch hier waren die variierenden Gehalte durch Chargenwechsel der Mais-Silagen bedingt, da die Referenz von diesen Schwankungen in gleichem Maße betroffen war. Unterschiedlich große Mengen anhaftender Bodenpartikel in den Silagen könnten die Schwankungen hervorgerufen haben. Die Strontiumgehalte in den beiden Reaktoren mit Deutsches Weidelgras-Silage lagen auf einem konstanten Level und wiesen keine Unterschiede gegenüber der Referenz auf (Abbildung 72). In die beiden Reaktoren mit Durchwachsene Silphie-Silage wurden durch hohe Durchschnittsgehalte in der Silage (etwa Faktor 5 höher gegenüber Mais-Silage, vgl. Kapitel 4.2, Tabelle 8) größere Strontiummengen eingetragen. 

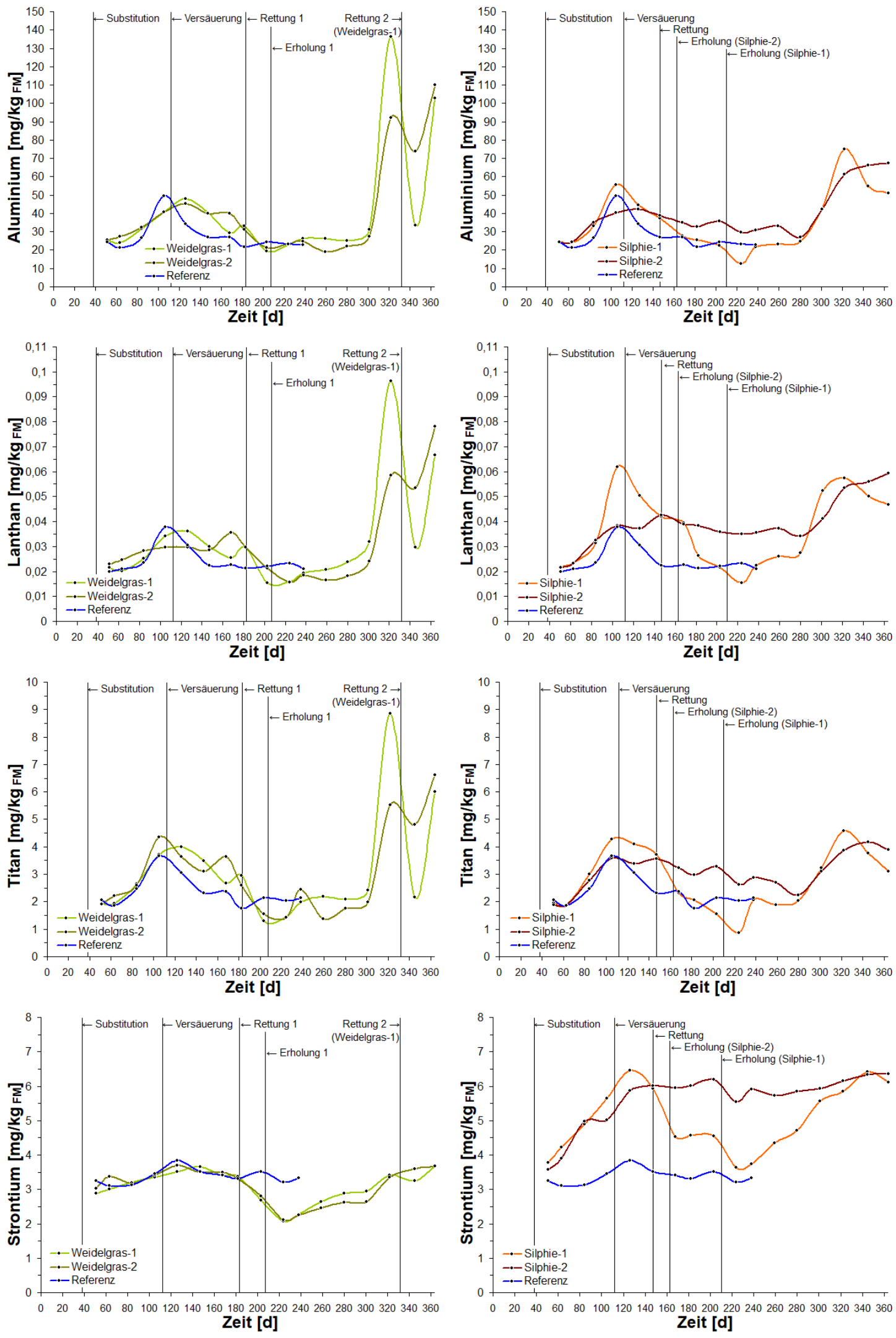

Abbildung 72: $\quad$ Verläufe der Aluminium-, Lanthan-, Titan- und Strontiumgehalte der Substitutionsversuche 3 und 4 in den Versuchsbiogasreaktoren (mit Referenzreaktor). 


\subsubsection{Eisen/Schwefel-Verhältnisse in den Substitutionsversuchen 3 und 4}

Die Eisen/Schwefel-Verhältnisse in den Versuchsbiogasreaktoren zeigten auch bei diesen dritten und vierten Substitutionsversuchen kontinuierlich fallende Verläufe (Abbildung 73 und Abbildung 74). Ursache war wiederum die Aussetzung der Zugabe der Spurenelementlösung bereits während der Stabilisierungsphase, um von einem niedrigeren Niveau aus mit den beiden Substratzumischungen zu starten. Trotz dieses niedrigeren Startniveaus fielen während der Substitutions- und Versäuerungsphasen die Verhältnisse in den Substitutionsreaktoren stetig weiter ab. Der Referenzreaktor als Kontrolle erhielt über die gesamte Projektphase die volle Menge der Spurenelementadditivlösung, sodass das Eisen/Schwefel-Verhältnis stabil auf einem hohen Level lag.

Vor Beginn der Rettungsphase 1 wurde in den beiden Substitutionsreaktoren mit Deutsches Weidelgras-Silage der Schwellenwert nach SAUER (2010) von 0,4 erreicht (Reaktor Weidelgras-1) bzw. mit 0,30 (Reaktor Weidelgras-2) sogar unterschritten (Abbildung 73). Die Eisen/Schwefel-Verhältnisse blieben dann die Erholungsphase 1 über auf sehr niedrigen, kritischen Niveaus, da mit dem Rettungsversuch durch die Zugabe eines Viertels der ursprünglichen Menge der synthetischen Cobaltchloridlösung als Monoelementadditiv keine neuen größeren Mengen an Eisen im Biogasbildungsprozess hinzukamen. Diese ungünstigen Eisen/Schwefel-Verhältnisse liefern neben den kritischen Cobaltkonzentrationen somit ein weiteres maßgebliches Instabilitätskriterium in den Substitutionsreaktoren mit Deutsches WeidelgrasSilage und bestätigen zudem, dass eine alleinige Zugabe einer reduzierten Cobaltadditivlösung für diese Silagemischung nicht ausreicht. Eisen muss unter den Versuchsbedingungen ebenfalls supplementiert werden.

Die Eisen/Schwefel-Verhältnisse in den Substitutionsreaktoren mit Durchwachsene Silphie-Silage erreichten vor Beginn der Rettungsphase beide einen Wert von 0,53 (Abbildung 74). Sie lagen zu diesem Zeitpunkt damit oberhalb des von SAUER (2010) angegebenen minimalen Schwellenwertes von 0,4 in einem noch nicht kritischen Stabilitätsbereich. Somit ist abschließend nachgewiesen, dass in den Substitutionsreaktoren mit Durchwachsene Silphie-Silage das alleinige entscheidende Element für die Versäuerung bzw. den absehbaren Prozesszusammenbruch Cobalt war. In den jeweiligen Erholungsphasen nach den Rettungsmaßnahmen mit der Zugabe nur eines Viertels der ursprünglichen synthetischen Spurenelementlösung lagen die Eisen/Schwefel-Verhältnisse wieder auf höheren, unkritischen Niveaus. 


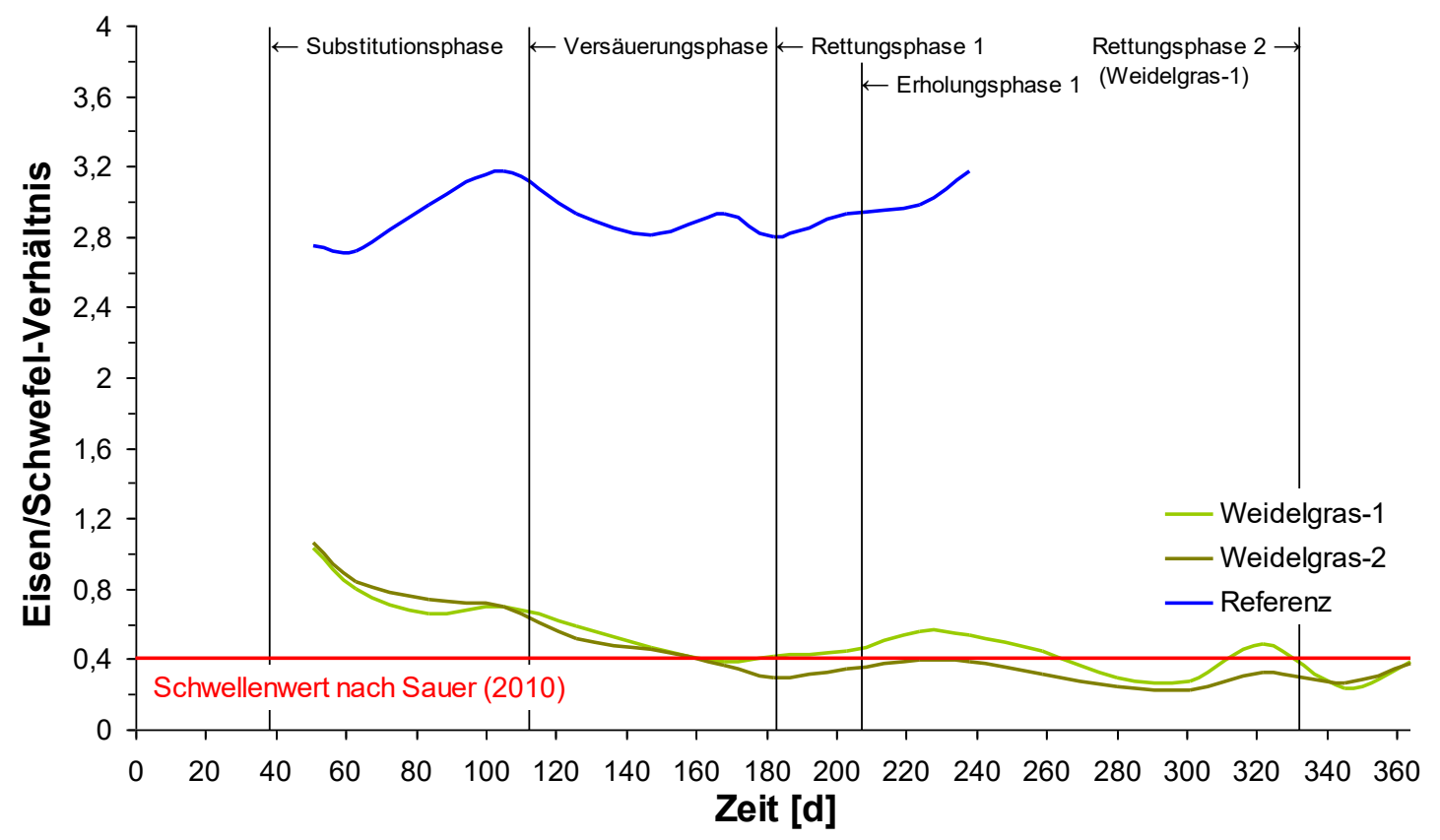

Abbildung 73: $\quad$ Verläufe der Eisen/Schwefel-Verhältnisse des Substitutionsversuchs 3 mit $30 \%$ Deutsches Weidelgras- zu $70 \%$ Mais-Silage (oTS-bezogen) in den Versuchsbiogasreaktoren (mit Referenzreaktor).

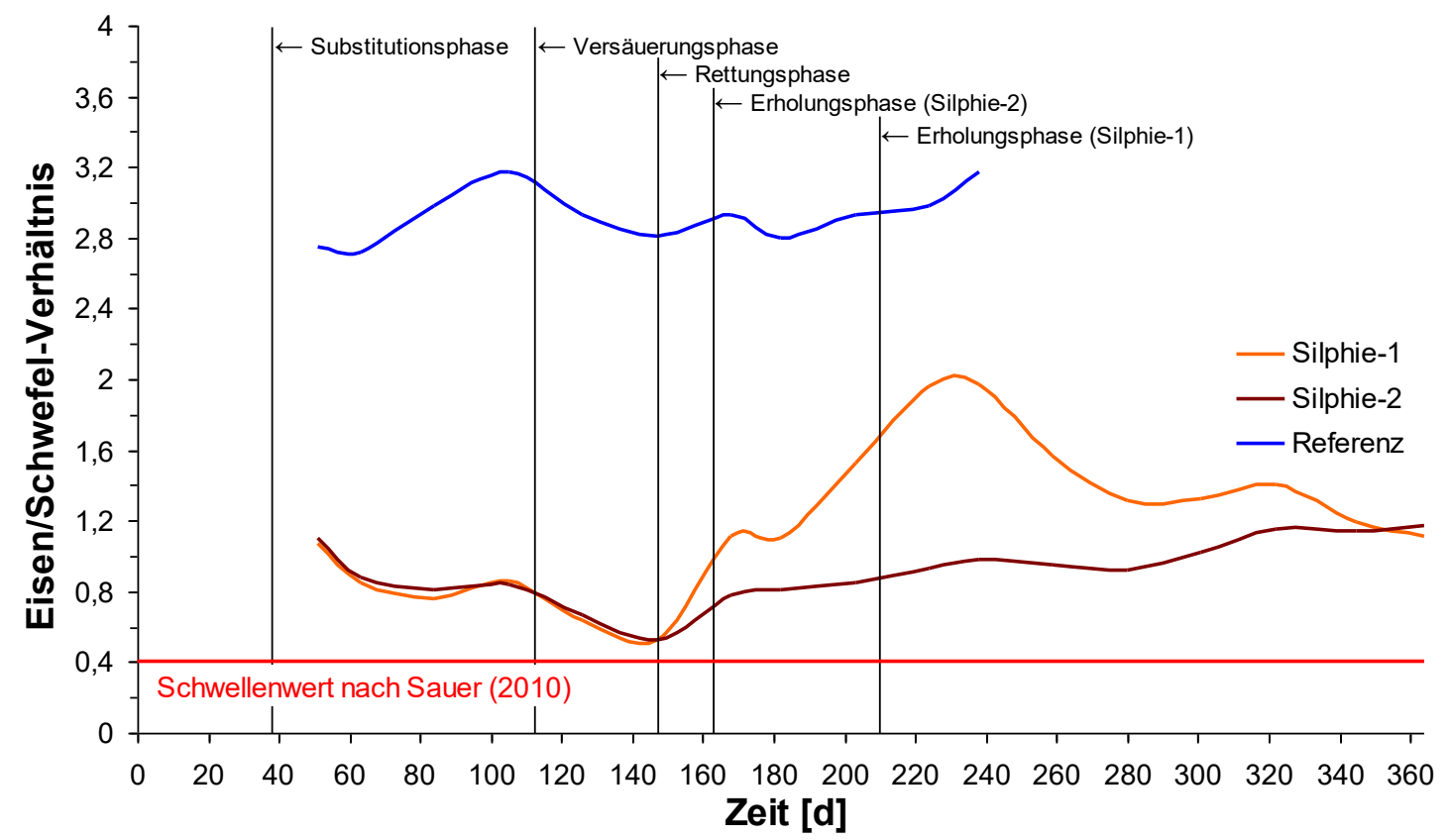

Abbildung 74: Verläufe der Eisen/Schwefel-Verhältnisse des Substitutionsversuchs 4 mit $30 \%$ Durchwachsene Silphie-zu $70 \%$ Mais-Silage (oTS-bezogen) in den Versuchsbiogasreaktoren (mit Referenzreaktor). 


\subsubsection{Mikrobiologischer Überblick zur Zusammensetzung und Dynamik der mikrobiellen Gemeinschaften in den Substitutionsversuchen 3 und 4}

Analog zu den Substitutionsversuchen mit Winterackerbohne- bzw. Amarant-Silage (vgl. Kapitel 4.4.6) unterlagen die relativen Anteile der methanogenen Gemeinschaften in den Substitutionsreaktoren mit den Zumischungen von $30 \%$ Deutsches Weidelgrasbzw. $30 \%$ Durchwachsene Silphie-Silage zu $70 \%$ Mais-Silage (oTS-bezogen) im Laufe der Prozessphasen ständigen Schwankungen. Methanosarcina dominierte alle Prozessphasen mit relativen Anteilen von 40 bis $85 \%$. Von Methanobacteriaceae/ Methanomassiliicoccaceae, Methanobacterium sowie Methanoculleus wurden nur geringere relative Anteile detektiert. Insgesamt zeigte sich auch bei diesen Substitutionsversuchen, dass die bakteriellen Gemeinschaften durch die Substratumstellungen und den später folgenden instabilen Phasen in ihrer Zusammensetzung wesentlich mehr beeinflusst wurden als die Methanbildner (vgl. Kapitel 4.4.6).

Auch bei diesen Substitutionsversuchen konnte eine charakteristische Veränderung wie bei Nickelmangel oder der möglicherweise aufgetretenen Hemmwirkung durch Zink (vgl. Kapitel 4.3.7) durch eine Verringerung der relativen Abundanz von Methanosarcina in den Substitutionsreaktoren nicht beobachtet werden. Somit kann wiederum bestätigt werden, dass der Zusammenbruch der Biogasprozesse in den Reaktoren durch den Cobaltmangel hervorgerufen wurde (SAUER ET AL., 2018). 


\subsection{Zusatzversuch mit Mais-Silage-Monovergärung unter reduzierter Spurenelementzugabe}

\subsection{1 Überblick}

Bei den Substitutionsversuchen mit Winterackerbohne- bzw. Amarant-Silage in der zweiten Projektphase (vgl. Kapitel 4.4) erwies sich der Referenzreaktor ab Beginn der Rettungs- bzw. Erholungsphase als Kontrolle ungeeignet, da dieser die volle Menge der synthetischen Spurenelementlösung erhielt und nicht in der gleichen Konfiguration wie die Substitutionsreaktoren mit nur einem Viertel der synthetischen Additivlösung betrieben werden konnte. So blieb ungeklärt, ob die verwendete Spurenelementmenge nicht zu hoch dosiert war oder ob beispielsweise Cobalt in den Energiepflanzen-Silagen von Winterackerbohne bzw. Amarant für die Mikroorganismen verfügbarer vorlag oder eventuell beides.

Um zu prüfen, ob eine reduzierte Spurenelementkonfiguration nur mit Mais-SilageMonovergärung stabil verläuft, wurden in einem Zusatzversuch gegen Ende der zweiten Projektphase zwei weitere Laborfermenter (Reaktor 4.11= Mais-1, Reaktor 4.12= Mais-2) mit Gärmaterial aus dem Inokulumsreaktor (Reaktor 3.2) angeimpft und mit Mais-Silage als Standard-Inputsubstrat und zusätzlich einem Viertel der synthetischen Spurenelementlösung nach OECHSNER ET AL. (2008; vgl. Kapitel 4.1) beschickt (Stabile Phase). Alle Betriebsparameter wie Raumbelastung, Verweilzeit, Reaktorgeometrie oder Rührleistung wurden für diesen Doppelversuch analog zu den Reaktoren der Verarmungs- bzw. Substitutionsversuche gewählt. Die Gaszusammensetzung und die Zusammensetzung und Dynamik der mikrobiellen Gemeinschaften wurden nicht analysiert. Tabelle 12 gibt einen Überblick über die beiden Prozessphasen des Zusatzversuchs.

Tabelle 12: $\quad$ Überblick über den Zusatzversuch der (quasi)kontinuierlichen Nassfermentationsversuche am DBFZ.

\begin{tabular}{|c|c|c|c|c|c|c|}
\hline \multicolumn{7}{|c|}{$\begin{array}{l}\text { Zusatzversuch } \\
\text { Mais-Silage-Monovergärung mit reduzierter Spurenelemente-Zugabe }\end{array}$} \\
\hline Prozessphase & Reaktor & Kurzbeschreibung & Abkürzung & $\begin{array}{l}\text { Versuchs- } \\
\text { tag }\end{array}$ & bis & $\begin{array}{c}\text { Versuchs } \\
\text { tag }\end{array}$ \\
\hline \multirow[t]{2}{*}{ Stabile Phase } & 4.11 & Mais-Silage + Viertel Spurenelementlösung & Mais-1 & 0 & - & 93 \\
\hline & 4.12 & Mais-Silage + Viertel Spurenelementlösung & Mais-2 & 0 & - & 93 \\
\hline \multirow[t]{2}{*}{ Versäuerungsphase } & 4.11 & Mais-Silage + Viertel Spurenelementlösung & Mais-1 & 94 & - & 119 \\
\hline & 4.12 & Mais-Silage + Viertel Spurenelementlösung & Mais-2 & 94 & - & 133 \\
\hline
\end{tabular}


Nach etwas weniger als zweieinhalb Verweilzeiten zeigte sich jedoch, dass die beiden Versuchsbiogasreaktoren bei einer Raumbelastung von $5 \mathrm{~g}_{\mathrm{oTS}} /(\mathrm{L} \cdot \mathrm{d})$ zu versäuern begannen und allmählich zusammenbrachen (Versäuerungsphase). Die Zugabe eines Viertels der ursprünglich eingesetzten synthetischen Spurenelementlösung scheint bei Mais-Silage-Monovergärung nicht ausreichend zu sein.

\subsubsection{Charakterisierung der Prozessparameter und Cobalt- und Nickelgehalte}

Mit Beginn der Versäuerungsphase ab Versuchstag 94 stiegen in beiden Reaktoren die FOS/TAC (FAL)-Werte über den kritischen Wert von $0,3 \mathrm{~g}_{\mathrm{FOS}} / \mathrm{g}_{\mathrm{CaCO} 3}$ und in der Folge rasant an (Abbildung 75). Ab Versuchstag 98 begannen die $\mathrm{pH}$-Werte $\mathrm{zu}$ fallen (Abbildung 76). Auch bei der normierten Gasproduktion wurden erste Anzeichen fallender Trends nach zwei Verweilzeiten ab Versuchstag 82 deutlich (Abbildung 77). In Abbildung 78 sind die drei Parameter zum besseren Überblick gegenübergestellt.

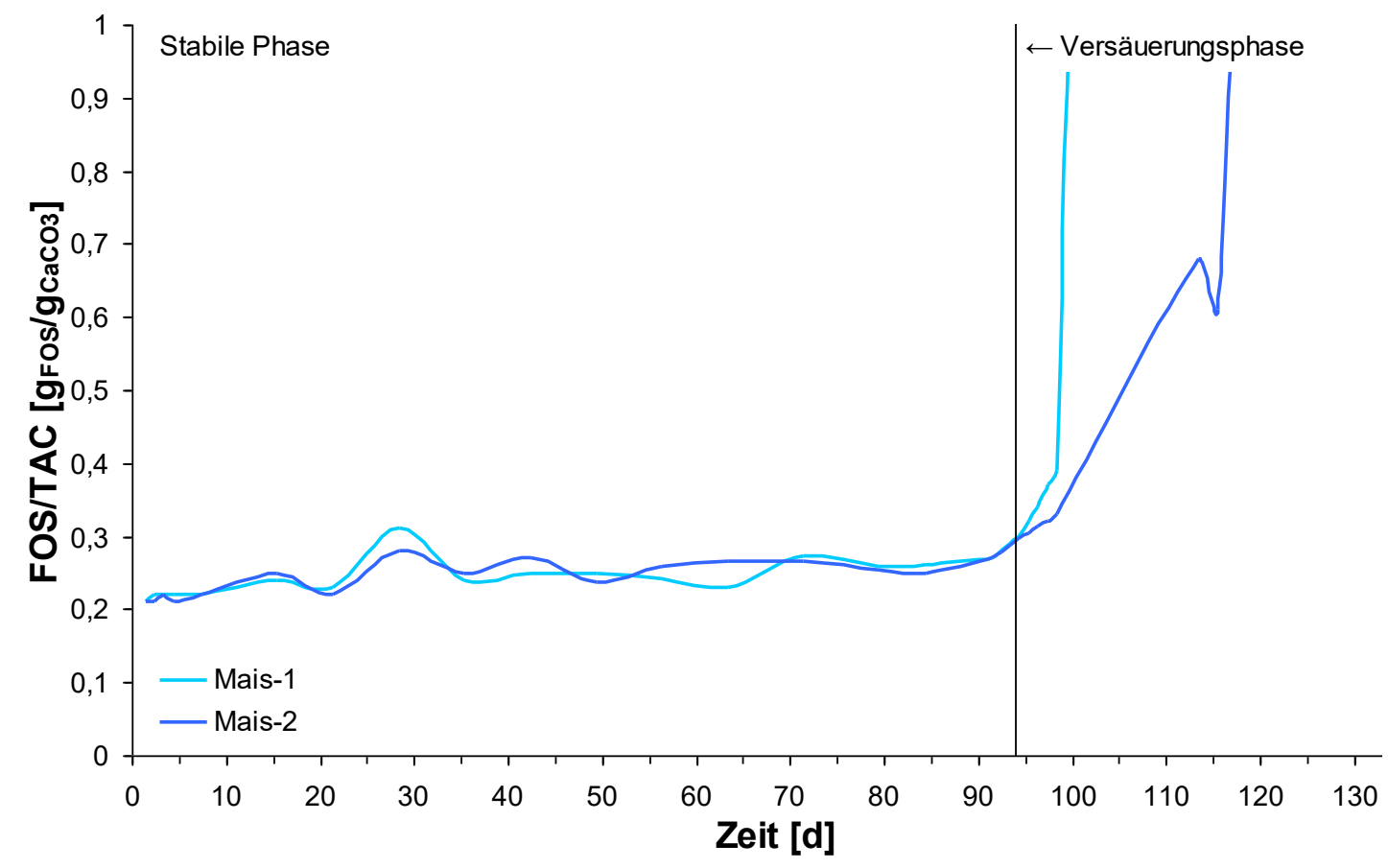

Abbildung 75: $\quad$ FOS/TAC (FAL)-Verläufe des Zusatzversuchs mit Mais-Silage-Monovergärung unter reduzierter Spurenelementzugabe in den Versuchsbiogasreaktoren. 


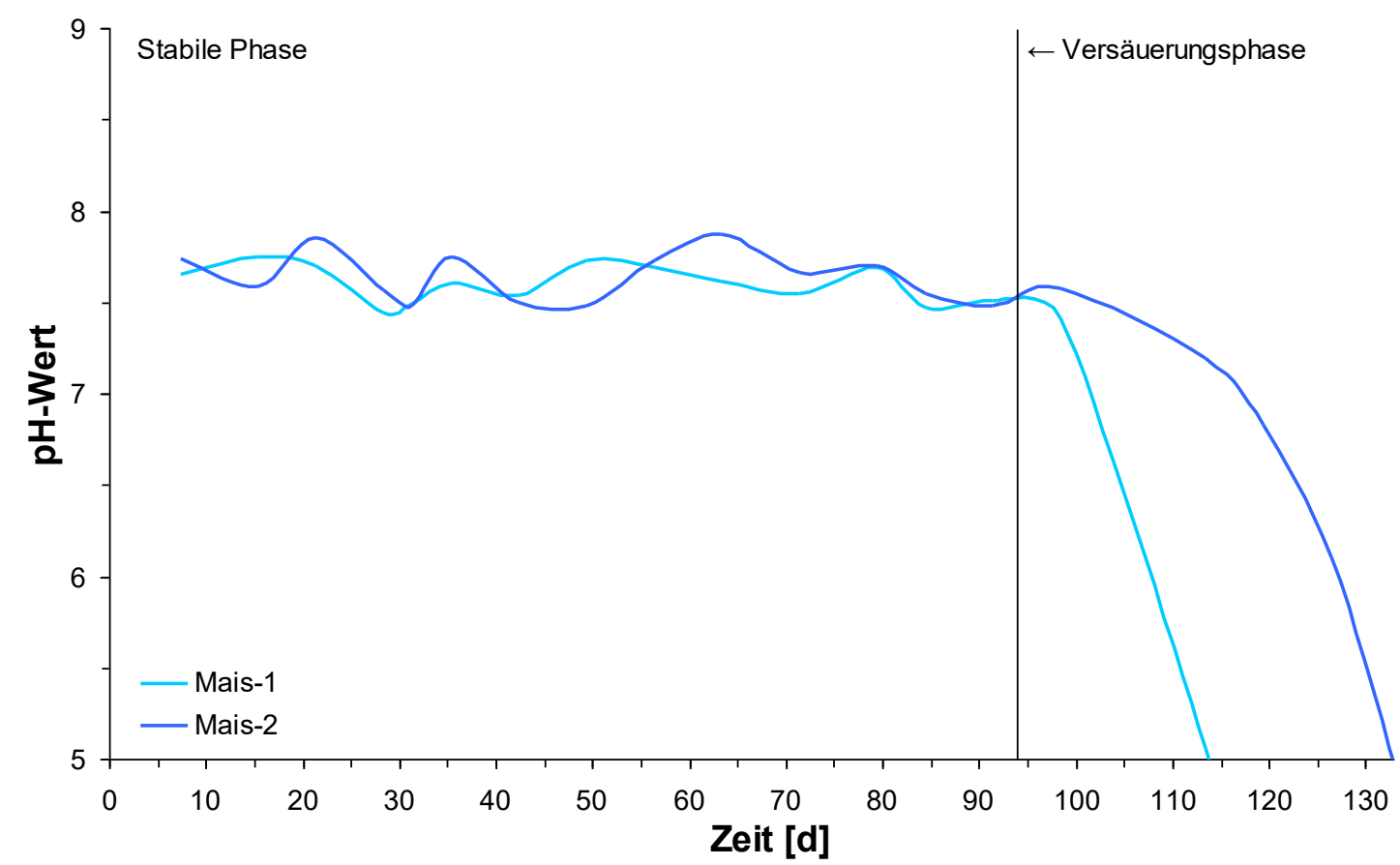

Abbildung 76: $\quad$ pH-Wert-Verläufe des Zusatzversuchs mit Mais-Silage-Monovergärung unter reduzierter Spurenelementzugabe in den Versuchsbiogasreaktoren.

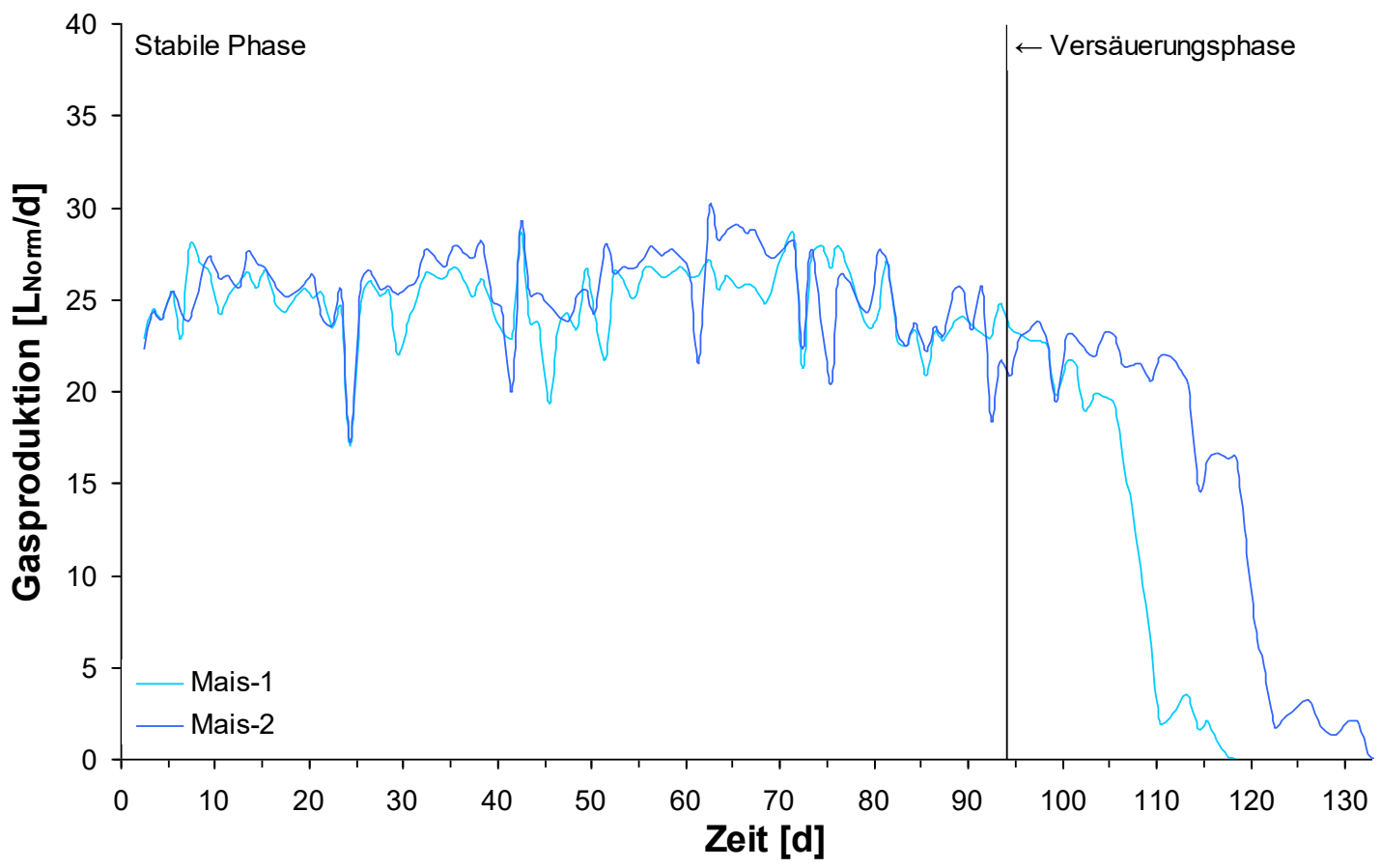

Abbildung 77: $\quad$ Verläufe der normierten Gasproduktion des Zusatzversuchs mit MaisSilage-Monovergärung unter reduzierter Spurenelementzugabe in den Versuchsbiogasreaktoren. 


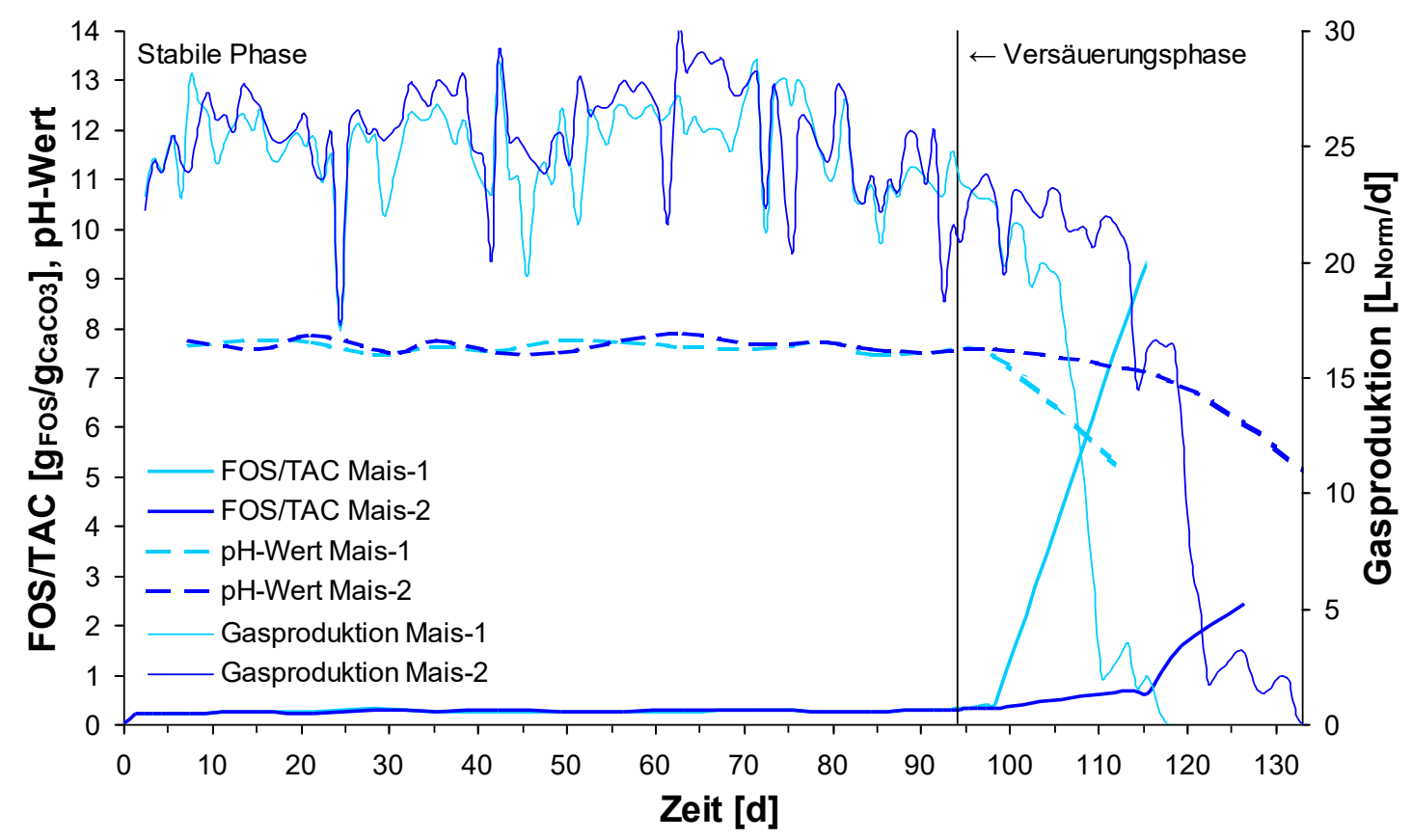

Abbildung 78: $\quad$ Gegenüberstellung der pH-Wert- und FOS/TAC (FAL)-Verläufe sowie der normierten Gasproduktion des Zusatzversuchs mit Mais-SilageMonovergärung unter reduzierter Spurenelementzugabe in den Versuchsbiogasreaktoren.

In der Versäuerungsphase lagen die Werte der normierten Gasproduktion ab Versuchstag 102 in Reaktor Mais-1 bzw. ab Versuchstag 114 in Reaktor Mais-2 unter $20 \mathrm{~L}_{\mathrm{Norm}} / \mathrm{d}$ (Abbildung 77 und Abbildung 78). Die Biogasproduktion in beiden Reaktoren brach in den folgenden Versuchstagen komplett zusammen. Am Versuchstag 119 wurde der Zusatzversuch bei Reaktor Mais-1, am Versuchstag 133 bei Reaktor Mais-2 beendet.

Jedoch waren weder die Cobalt- noch die Nickelkonzentrationen ausschlaggebende Faktoren für die Versäuerung bzw. den Zusammenbruch der beiden Versuchsbiogasreaktoren. Denn wie Abbildung 79 und Abbildung 80 zeigen, lagen sowohl die Cobaltals auch die Nickelgehalte in den beiden zusätzlichen Reaktoren weit über dem in der ersten Projektphase ermittelten minimalen Schwellenwert von $0,066 \mathrm{mg} / \mathrm{kg}$ FM Cobalt (vgl. Kapitel 4.3.2) bzw. dem durch die mögliche Zinkhemmung vermutlich zu hoch angegebenen minimalen Schwellenwert von $0,82 \mathrm{mg} / \mathrm{kg}_{\mathrm{FM}}$ Nickel (vgl. Kapitel 4.3.3 und 4.3.4). Die Spurenelemente Nickel und Cobalt waren also im Zusatzversuch für stabile Biogasprozesse ausreichend vorhanden und stellten keine limitierenden Faktoren dar. 


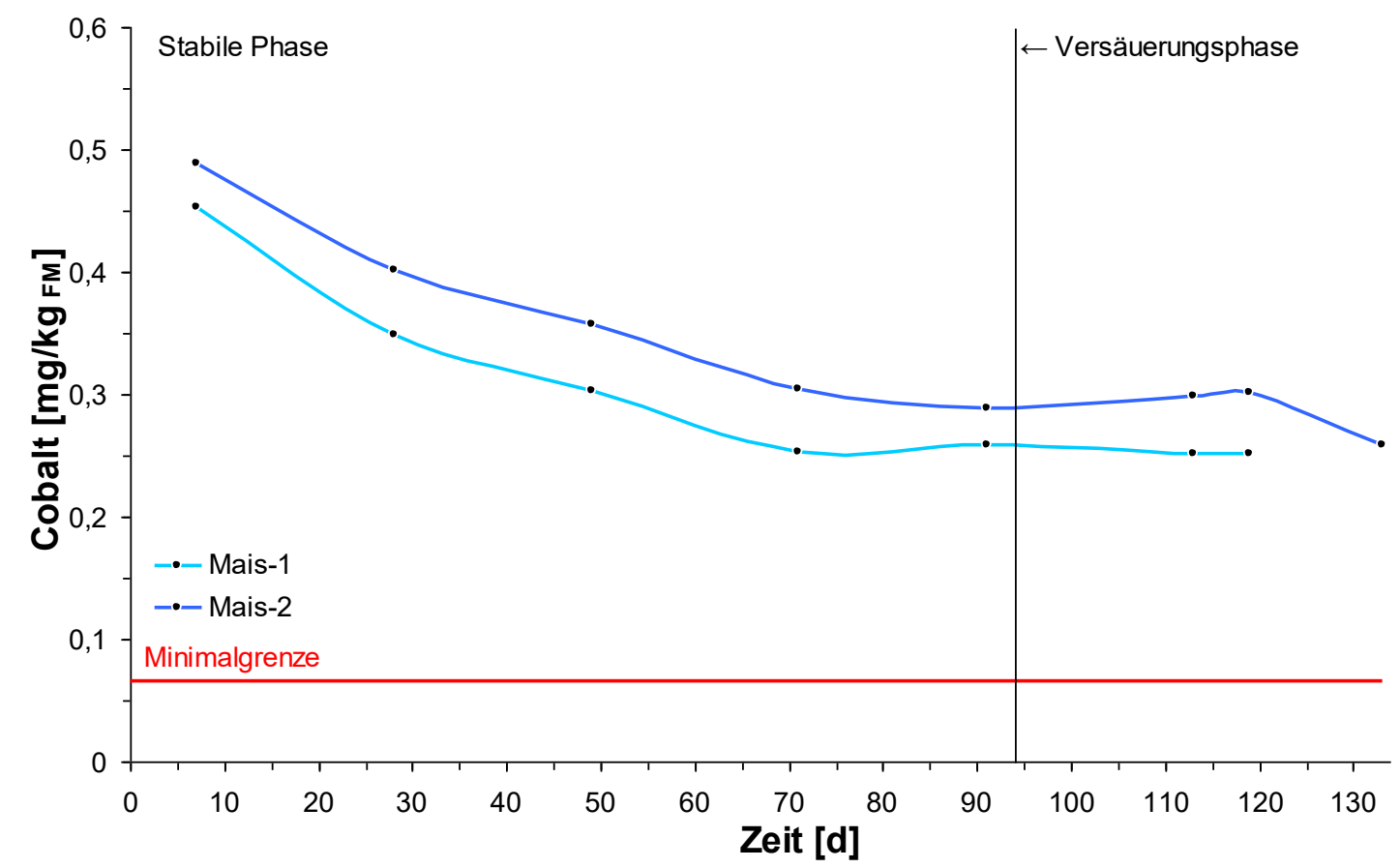

Abbildung 79: Verläufe der Cobaltkonzentrationen des Zusatzversuchs mit MaisSilage-Monovergärung unter reduzierter Spurenelementzugabe in den Versuchsbiogasreaktoren.

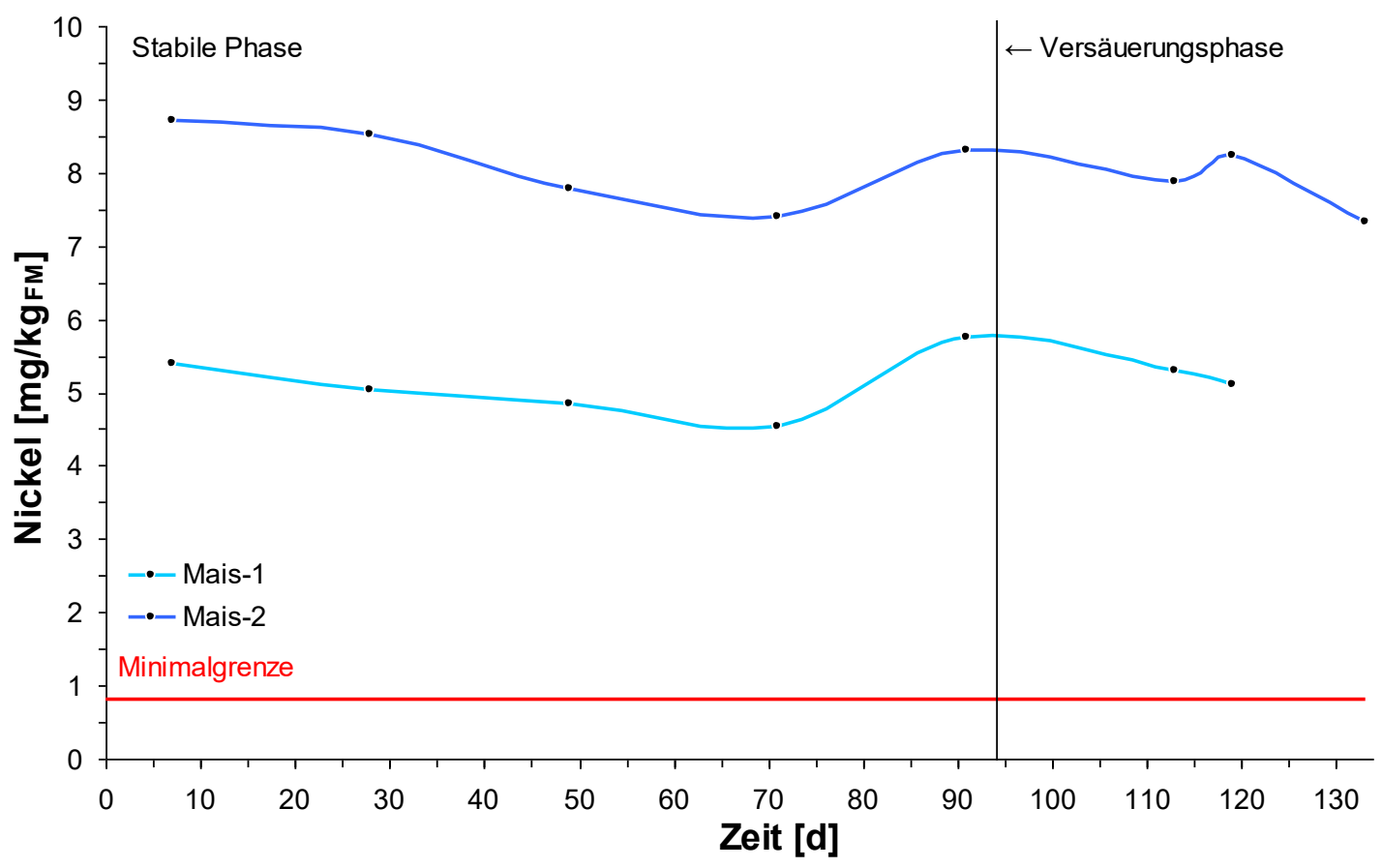

Abbildung 80: $\quad$ Verläufe der Nickelkonzentrationen des Zusatzversuchs mit MaisSilage-Monovergärung unter reduzierter Spurenelementzugabe in den Versuchsbiogasreaktoren. 
Es konnte nachgewiesen werden, dass die Zugabe eines Viertels der ursprünglich eingesetzten synthetischen Spurenelementlösung unter diesen Versuchsbedingungen nicht ausreichend ist, bei Mais-Silage-Monovergärung stabile Biogasprozesse zu gewährleisten. Die Cobalt- und Nickelgehalte lagen über die gesamte Versuchsdauer auf einem Niveau weit über den minimalen Schwellenwerten und waren somit bis zum Zusammenbruch der Reaktoren nicht limitierend. Dies belegt die Wirksamkeit der alternativen spurenelementreicheren Energiepflanzen-Silagen als unterstützende Spurenelementlieferanten im Betrieb von Biogasreaktoren, wie es in der zweiten Projektphase mit Winterackerbohne- und Amarant-Silage (vgl. Kapitel 4.4) bzw. in der dritten Projektphase mit Silagen von Deutsches Weidelgras und Durchwachsene Silphie (vgl. Kapitel 4.5) erfolgreich getestet wurde.

\subsubsection{Bewertung weiterer Elemente im Zusatzversuch}

Bei den essenziellen Grundelementen und Makronährstoffen (Gruppe I, vgl. Kapitel 2.2.5) Calcium, Kalium, Magnesium, Natrium, Phosphor und Schwefel waren über die komplette Dauer des Zusatzversuchs fallende Konzentrationsverläufe zu beobachten (Abbildung 81). Sie erreichten bis zum Zusammenbruch der beiden Reaktoren jedoch keine kritischen Bereiche, verglichen mit Durchschnittswerten von LINDORFER ET AL. (2012) aus Analysen von 600 NawaRo-Fermentern, mit Ausnahme von Natrium.

Bedingt durch das natriumarme Inputsubstrat Mais-Silage (vgl. Kapitel 4.2, Tabelle 8) und dadurch, dass Natrium kein zugesetztes Element in der Spurenelementadditivlösung war (vgl. Kapitel 4.1, Tabelle 6) fielen die Natriumkonzentrationen im Reaktor Mais-1 bis auf $14 \mathrm{mg} / \mathrm{kg}_{\mathrm{FM}}$ bzw. im Reaktor Mais-2 bis auf $12 \mathrm{mg} / \mathrm{kg}_{\mathrm{FM}}$. Sie lagen damit nur sehr knapp über dem von MUNK ET AL. (2010) in Laborversuchen ermittelten kritischen Minimalwert von $10 \mathrm{mg} / \mathrm{L}$ Natrium und scheinen für die Versäuerung der beiden Reaktoren verantwortlich gewesen zu sein. Allerdings lagen die Natriumgehalte im Referenzreaktor der ersten und zweiten Projektphase gegen Versuchsende jeweils in vergleichbaren Bereichen (vgl. Kapitel 4.3.5 und 4.4.4), ohne dass Störungen auftraten. In der dritten Projektphase hingegen traten im Referenzreaktor Instabilitäten auf (vgl. Kapitel 4.5.5). 

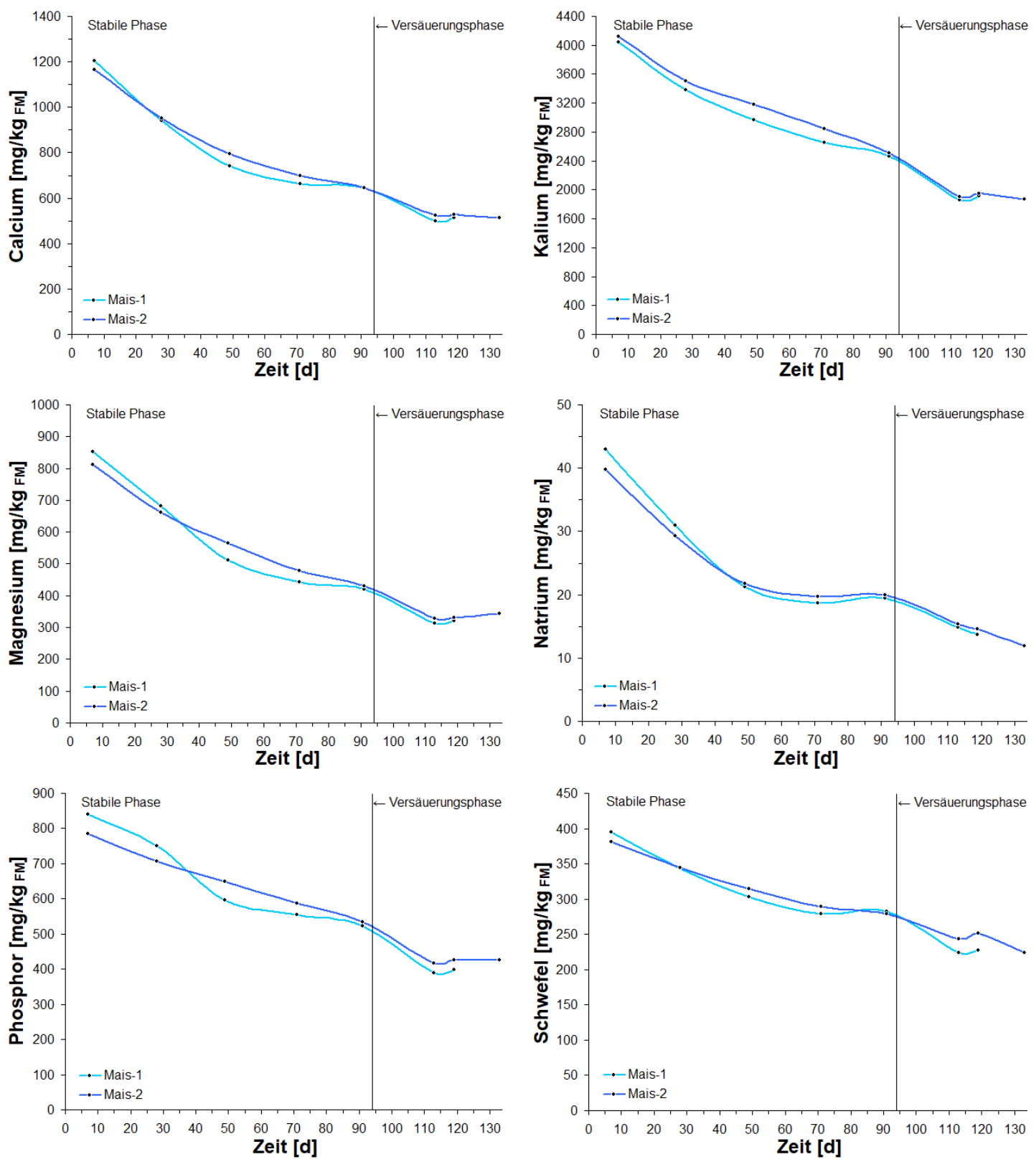

Abbildung 81: Verläufe der Calcium-, Kalium-, Magnesium-, Natrium-, Phosphorund Schwefelgehalte des Zusatzversuchs mit Mais-Silage-Monovergärung unter reduzierter Spurenelementzugabe in den Versuchsbiogasreaktoren.

Auch die essenziellen Mikronährstoffe (Gruppe II) Eisen, Mangan und Molybdän wiesen trotz der Zugabe eines Viertels der ursprünglichen synthetischen Spurenelementlösung, in der diese Elemente enthalten waren (vgl. Kapitel 4.1, Tabelle 6) fallende Konzentrationsverläufe auf (Abbildung 82). Bis zum Prozesszusammenbruch in den beiden zusätzlichen Reaktoren liefen sie jedoch in keine kritischen minimalen Bereiche der unteren Schwellenwerte. 

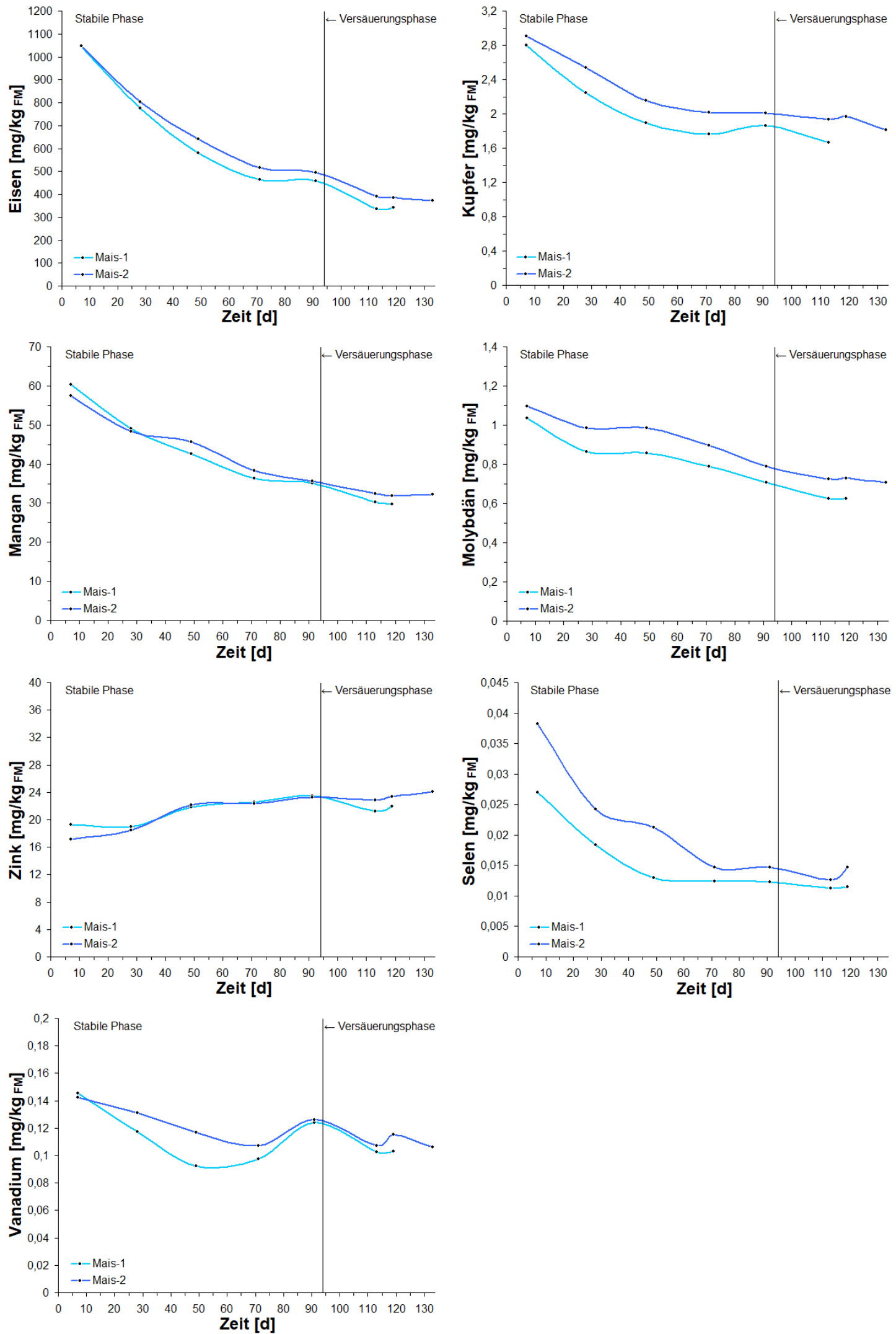

Abbildung 82: $\quad$ Verläufe der Eisen-, Kupfer-, Mangan-, Molybdän-, Zink-, Selenund Vanadiumgehalte des Zusatzversuchs mit Mais-Silage-Monovergärung unter reduzierter Spurenelementzugabe in den Versuchsbiogasreaktoren. 
Die Gehalte des Spurenelements Selen, das Bestandteil der Additivlösung war, zeigten zunächst ebenfalls fallende Trends an, konnten sich aber nach etwa der Hälfte des Zusatzversuchs auf einem konstanten Level stabilisieren (Abbildung 82). Sie lagen weit über dem minimalen Bedarfswert von 0,002 mg/kg FM Selen nach MUNK ET AL. (2010). In gleicher Weise verliefen die Konzentrationen von Kupfer (Abbildung 82), welches nicht in der Spurenelementlösung enthalten war.

Die Elementkonzentrationen von Zink und Vanadium lagen über die gesamte Dauer des Zusatzversuchs auf stabilen Niveaus (Abbildung 82). Ob der Mikronährstoff Wolfram beim Zusammenbruch eine Rolle spielte, konnte aufgrund der Nichtberücksichtigung in der ICP-MS-Multielementauswahl durch aufgetretene zu große Streuwerte und qualitativ nicht abgesicherte Messergebnisse für die Pflanzenreferenzmaterialien (für diese liegen keine zertifizierten Werte vor, vgl. Kapitel 3.5.2), nicht ermittelt werden. Es kann jedoch davon ausgegangen werden, dass die Wolframkonzentrationen durch die Zugabe der synthetischen Spurenelementlösung (Wolfram war Bestandteil der Additivlösung) nicht in einen kritischen Bereich fielen.

Die Konzentrationsverläufe der Elemente der Gruppe III (vgl. Kapitel 2.2.5), Arsen, Cadmium, Blei, Aluminium, Lanthan, Titan und Strontium zeigten alle fallende oder weitgehend konstante Trends und wiesen keine kritischen Konzentrationsbereiche auf (Abbildung 83). Höhere Konzentrationen zu Versuchsbeginn sind durch höhere Gehalte der Elemente im Inokulum zu begründen. Leichte Schwankungen in den Verläufen, v.a. bei Cadmium, Aluminium, Lanthan und Titan sind bedingt durch die Verwendung mehrerer Mais-Silagen über die Versuchsdauer, mit leicht variierenden Konzentrationen v.a. bei den genannten pedogenen Elementen. Diese stammen von unterschiedlichen Mengen an anhaftenden Boden- und Staubpartikeln (vgl. hierzu POSPIECH ET AL., 2017b) oder von anderen Einträgen, z.B. Materialabrieb. Während des Zusatzversuchs kamen fünf unterschiedliche Chargen Mais-Silage zum Einsatz.

In den beiden Reaktoren waren die Chromgehalte sehr hoch (Abbildung 83). So wies der Reaktor Mais-1 im Mittel 9,2 mg/kg FM, Reaktor Mais-2 sogar 17 mg/kg FM Chrom auf. Die Ursache kann nicht nur mit höheren Gehalten im eingesetzten Inokulum analog zur ersten Projektphase (vgl. Kapitel 4.3.5) begründet werden, da die Verläufe während der Versuchsdauer hätten fallen müssen. Die hohen Gehalte sind wiederum vermutlich durch Abrasions- oder Ablösungsvorgänge an den metallischen Oberflächen des mittig im Reaktor fixierten Ankerrührers verursacht worden (vgl. Kapitel 3.5.3). 

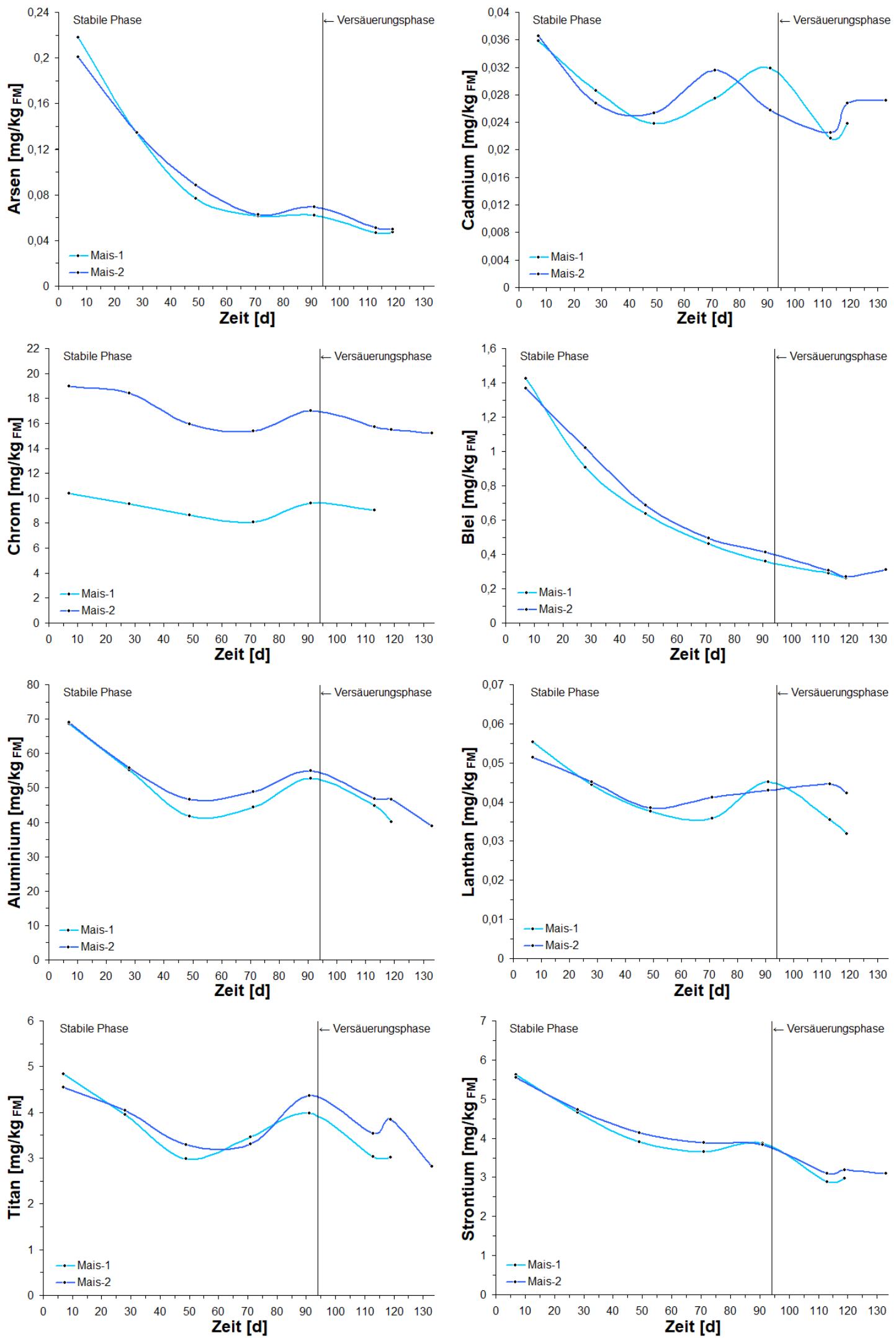

Abbildung 83: Verläufe der Arsen-, Cadmium-, Chrom-, Blei-, Aluminium-,

Lanthan-, Titan- und Strontiumgehalte des Zusatzversuchs mit MaisSilage-Monovergärung unter reduzierter Spurenelementzugabe in den Versuchsbiogasreaktoren. 
In den Laborfermenterversuchen allein mit Mais-Silage und einem Viertel der Spurenelementadditivzugabe stellten die Konzentrationen der Makronährstoffe Calcium, Kalium, Magnesium, Phosphor und Schwefel sowie aller wichtigen Spurenelemente wie Eisen, Kupfer, Mangan, Molybdän, Zink, Selen und Vanadium, einschließlich Cobalt und Nickel (vgl. Kapitel 4.6.2), keine auschlaggebenden limitierenden Faktoren für den Prozesszusammenbruch der beiden Reaktoren dar. Die Rolle des essenziellen Spurenelementes Wolfram beim Zusammenbruch blieb unklar. Die Wolframgehalte waren aber höchstwahrscheinlich unproblematisch.

Auch die Gehalte weiterer Elemente zeigten mit Ausnahme von Chrom keine Auffälligkeiten. Dass jedoch die hohen Chromkonzentrationen entscheidend für die Versäuerung waren, scheint sehr unwahrscheinlich. Sie stiegen seit Versuchsstart nicht an, sondern blieben weitgehend auf einem hohen konstanten Niveau.

Für die Versäuerung bzw. den erfolgten Zusammenbruch der beiden Reaktoren war vermutlich maßgeblich der Mangel des essenziellen Makronährstoffs Natrium verantwortlich, der von der Maispflanze nur wenig aufgenommen wird und nicht in der Spurenelementadditivlösung vorhanden war (vgl. Kapitel 4.2, Tabelle 8). Unter Einbeziehung der Betrachtungen der Natriumgehalte im Referenzreaktor in den drei Projektphasen der Laborfermenterversuche (vgl. Kapitel 4.3.5, 4.4.4 und 4.5.5) sollte schlussfolgernd eine minimale Konzentration für Natrium von 20 mg/kg FM in Fermentern nicht unterschritten werden, um eine optimale Biogasbildung bzw. stabile Biogasprozesse aufrechtzuerhalten.

\subsubsection{Eisen/Schwefel-Verhältnisse im Zusatzversuch}

In den beiden zusätzlichen Versuchsbiogasreaktoren mit Mais-Silage-Monovergärung und einem Viertel der ursprünglich in den drei Projektphasen eingesetzten synthetischen Spurenelementlösung fielen die Eisen/Schwefel-Verhältnisse im Verlauf der Versuchsdauer leicht kontinuierlich ab (Abbildung 84). Sie lagen in der Versäuerungsphase jedoch bei konstant hohen Werten von 1,5 (Reaktor Mais-1) bzw. 1,6 (Reaktor Mais-2) und damit weit oberhalb des von SAUER (2010) angegebenen minimalen Schwellenwertes von 0,4 D.h. auch die Eisen/Schwefel-Verhältnisse lieferten kein Instabilitätskriterium für den Zusammenbruch der beiden Reaktoren. 


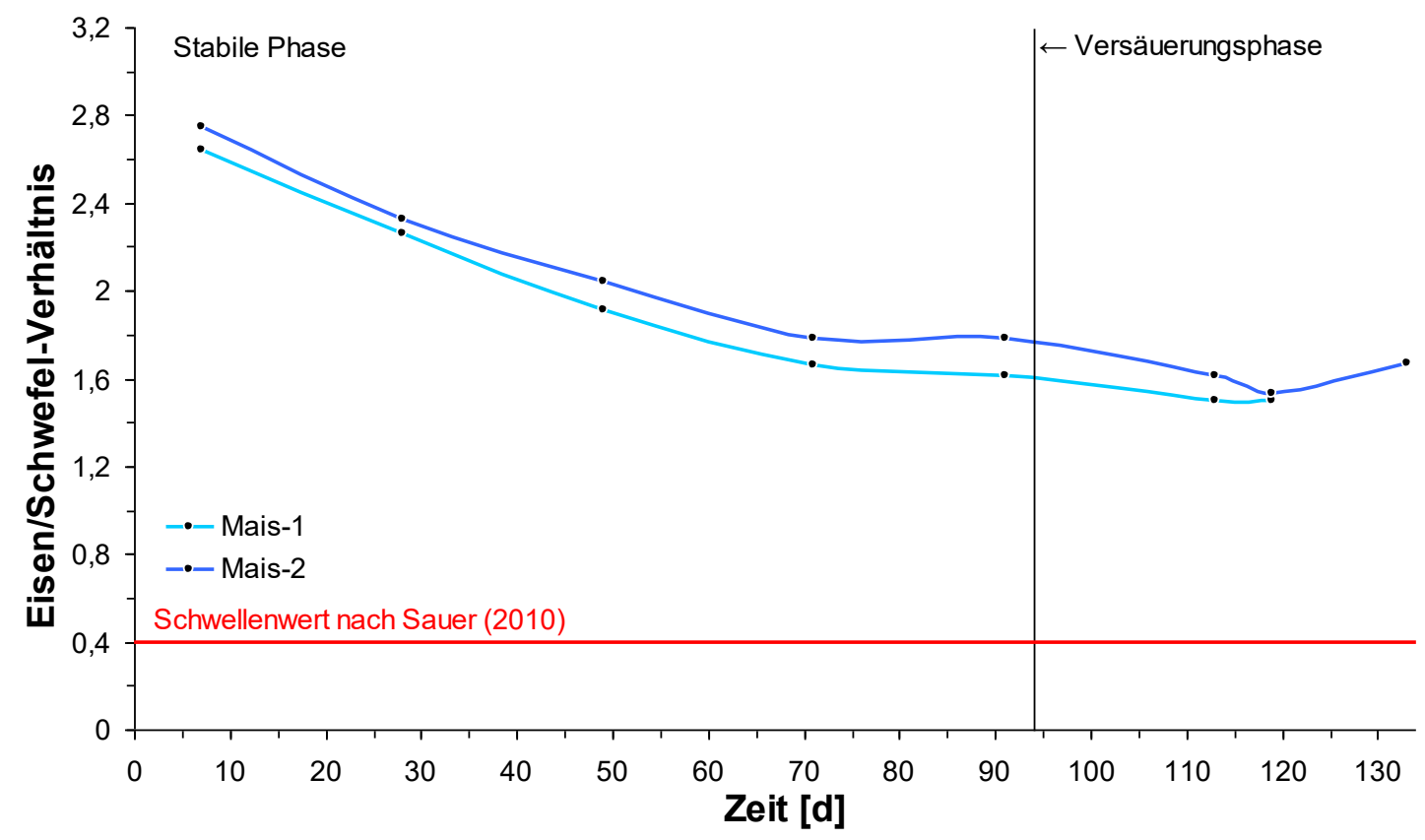

Abbildung 84: Verläufe der Eisen/Schwefel-Verhältnisse des Zusatzversuchs mit Mais-Silage-Monovergärung unter reduzierter Spurenelementzugabe in den Versuchsbiogasreaktoren. 


\section{Kapitel 5: Diskontinuierliche Gärversuche (ORGA-Tests)}

\section{1 Überblick}

Als weiterer Bestandteil dieser Arbeit wurden mit Hilfe von diskontinuierlichen Gärversuchen systematische Testreihen $\mathrm{zu}$ oberen hemmenden bzw. toxischen Konzentrationsbereichen für verschiedene Spurenelemente durchgeführt, um neben den erarbeiteten minimalen Schwellenwerten (vgl. Kapitel 4) maximale Schwellenwerte zu bestimmen, ab denen der Biogasprozess beeinträchtigt wird bzw. bei weiterer Konzentrationserhöhung zusammenbricht. Die Zeitversuche wurden auf Basis des Oberhausen-Rostock-Göttinger Aktivitätstests (ORGA-Test) für die Spurenelemente Nickel, Cobalt, Molybdän, Kupfer, Cadmium und Blei realisiert.

Dieser Aktivitätstest wurde als standardisiertes Verfahren an drei Forschungsinstituten in Oberhausen, Rostock und Göttingen entwickelt, als Akronym wird das Kurzwort „ORGA-Test“ verwendet (Engler et Al., 2011; Merrettig-Bruns et AL., 2011, MERRETTIG-BRUNS ET AL., 2012). Er ermöglicht eine vergleichende Quantifizierung der biologischen Abbaukinetik von Substraten in Biogasfermentern. Mit diesem Aktivitätstest waren für die hier durchgeführten Experimente eine hohe zeitliche Auflösung und bei allen Zeitreihen standardisierte Versuchsbedingungen möglich. Die Testvarianten der Spurenelementzugaben konnten quantitativ und vergleichend betrachtet und die oberen hemmenden bzw. toxischen Konzentrationsbereiche gut eingegrenzt werden.

\subsection{Versuchsaufbau und Durchführung}

Beim ORGA-Test wurde in mehrtägigen Versuchsreihen mit Mehrfachwiederholungen mit dem Gasbildungssystem der Firma ANKOM Technology (ANKOM ${ }^{R F}$ Gas Production System) in festgelegten Zeitintervallen systematisch der Druckanstieg in Gasbildungsmodulen bei konstantem Volumen und konstanter Temperatur erfasst, an eine Software übertragen und aufgezeichnet. Die Kommunikation und Übertragung des Druckanstiegs und weiterer Parameter, wie die aktuelle Temperatur oder die Akkuspannung, erfolgte mittels Radiofrequenz (RF)-Technologie zwischen den Gasbildungsmodulen und einem Referenzmodul über einen Basis-Koordinator (RF-Modem) an eine Datenaufzeichnungssoftware (ANKOM Gas Pressure Monitor). 
Jedes Gasbildungsmodul bestand aus einer Gärflasche, auf die ein Druckmesskopf aufgeschraubt wurde. Dieser enthielt u.a. einen Sensor zur Druckaufnahme, einen Akku-Pack, einen Luer-Anschluss sowie ein Entlüftungsventil. Das Referenzmodul diente der Messung und Übertragung des Atmosphärendrucks.

Die ORGA-Testreihen wurden mit jeweils 17 Enghalsflaschen durchgeführt, die ein Nennvolumen von $1000 \mathrm{~mL}$ aufwiesen. Pro Versuch wurden in der Regel vier Testvarianten in dreifacher Wiederholung untersucht. Jede Testvariante enthielt ein bestimmtes Spurenelement als Zusatzstoff in einer für den Versuch festgelegten Konzentrationsstufe. Abbildung 85 zeigt Fotoaufnahmen eines ORGA-Tests.

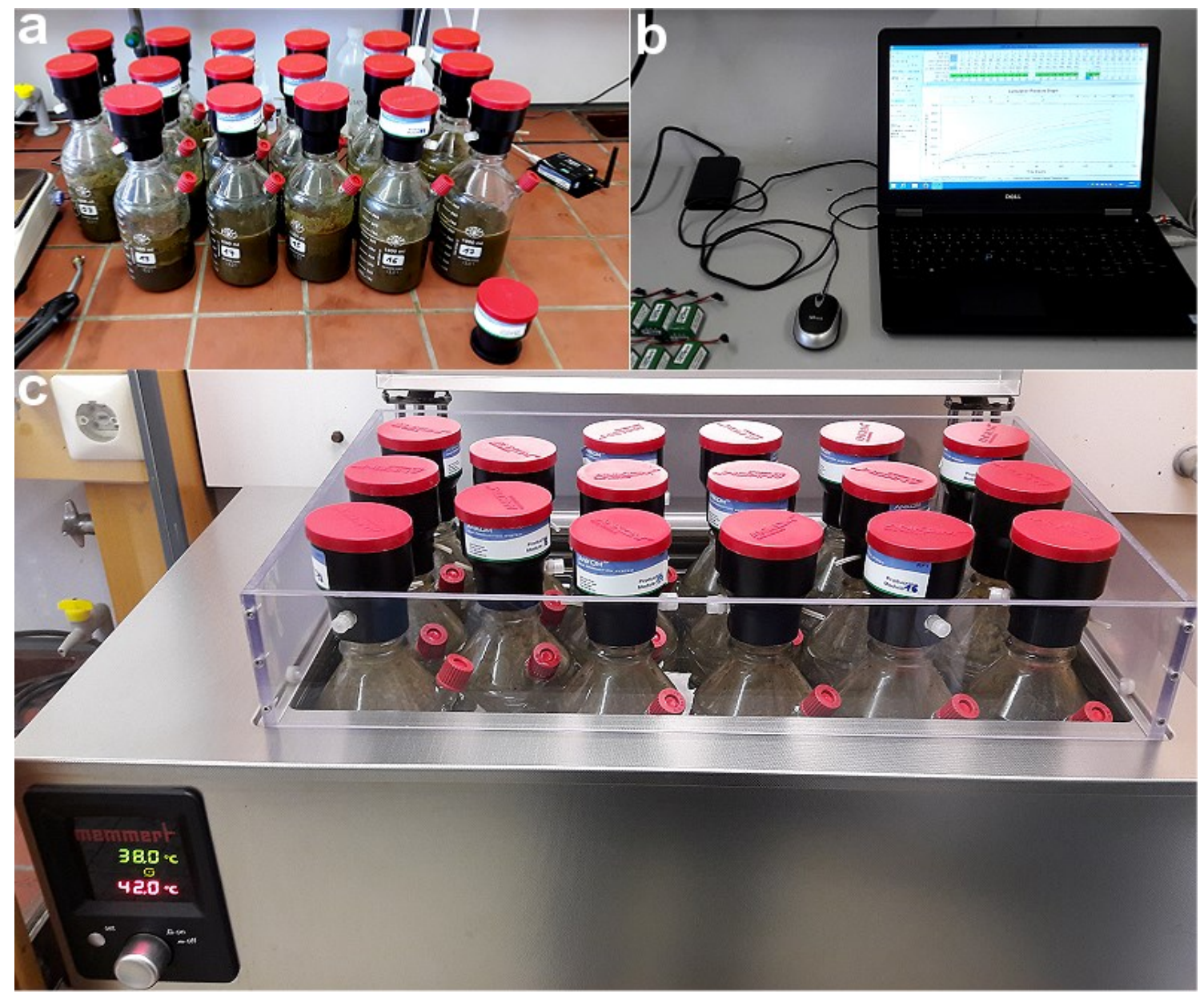

Abbildung 85: $\quad$ Fotoaufnahmen eines diskontinuierlichen Gärversuchs auf Basis des Oberhausen-Rostock-Göttinger Aktivitätstests (ORGA-Test).

a) Für eine Testreihe vorbereitete Gasbildungsmodule, vorne rechts: Referenzmodul zur Überwachung und Aufzeichnung des aktuellen Atmosphärendrucks, hinten rechts: Basis-Koordinator (RF-Modem) zur Kommunikation zwischen den Gasbildungsmodulen, dem Referenzmodul und der Aufzeichnungssoftware; b) Software zur Datenaufzeichnung; c) Schrägansicht auf die Gasbildungsmodule im Wasserbad. 
Die Gärflaschen wurden mit einer definierten Menge an Inokulum, demineralisiertem Wasser, dem Standard-Inputsubstrat sowie steigenden Testelementzugaben befüllt und abschließend mit Stickstoff inertisiert. Für die Auswertung der Versuchsreihen wurden zudem Nullproben (Blankproben) in Doppelwiederholung (Gärflaschen nur mit Inokulum und demineralisiertem Wasser befüllt) sowie Kontrollproben in Dreifachwiederholung (Gärflaschen mit Inokulum, demineralisiertem Wasser und Standard-Inputsubstrat befüllt) mitgeführt. Über die in jeder Versuchsreihe durch gravimetrische Bestimmung stets gleichbleibend gehaltenen Kopfraumvolumina in den Gärflaschen waren konstante Volumina als standardisierte Versuchsbedingung gewährleistet. Alle Versuchsreihen wurden unter mesophilen Bedingungen bei einer konstanten Temperatur von $38^{\circ} \mathbf{C}$ in einem Wasserbad (Memmert WPE 45) durchgeführt. Die Versuchszeit jeder Reihe betrug zehn Tage.

Um ferner bei allen Testreihen gleichbleibende, standardisierte Versuchsbedingungen zu gewährleisten, wurde als Standard-Inputsubstrat Mais-Silage gewählt, das in landwirtschaftlichen Biogasanlagen am häufigsten verwendete Substrat. Hierfür wurde im Vorfeld der Versuchsreihen eine größere Charge an Mais-Silage von der Biogasanlage in Verliehausen genommen, bei $105^{\circ} \mathrm{C}$ bis zur Gewichtskonstanz getrocknet und mit der Planeten-Kugelmühle feingemahlen (vgl. Kapitel 3.2). Als Inokulum kam bei allen Testreihen jeweils über 10 bis 15 Tage ausgefaultes Fermentermaterial zum Einsatz, das ebenfalls von der Praxisbiogasanlage in Verliehausen stammte. Proben vom Inokulum und der Silage wurden parallel zu den Versuchsreihen aufgeschlossen sowie die Element-, TS- und oTS-Gehalte ermittelt (vgl. Kapitel 3.3 und 3.4). Für eine bessere Rührfähigkeit wurde dem Inokulum im Verhältnis 1:1 demineralisiertes Wasser beigemischt. Aus jeweils einer Stammlösung mit hohem Spurenelementgehalt wurden entsprechend berechnete Mengen in die Gärflaschen zugegeben, um die gewünschten Testkonzentrationen zu erhalten (Tabelle 13). Für alle Versuchsreihen wurde ein Aufzeichnungsintervall von 30 Minuten mit 60 Sekunden-Live-Intervallen (bzw. Kommunikation) gewählt. Die Durchmischung der Gasbildungsmodule erfolgte manuell einmal täglich durch circa 30-sekündiges Schwenken der Gärflaschen.

Nach dem Aufbau des Systems sowie diversen Geräte-, Vor- und Validierungstests mit Backhefe (Saccharomyces cerevisiae) sowie Fermenter- und Silagematerial begannen die einzelnen Versuchsreihen im Spätsommer 2015. Tabelle 13 zeigt einen Überblick über die durchgeführten Versuchsreihen mit den entsprechenden Testlösungen. 
Tabelle 13: $\quad$ Überblick über die diskontinuierlichen Gärversuche (ORGA-Tests) zur Erarbeitung von Hemm- und Toxizitätskonzentrationen verschiedener Spurenelemente.

\begin{tabular}{|c|c|c|c|c|c|c|}
\hline \multirow[t]{2}{*}{ Versuch } & \multirow{2}{*}{$\begin{array}{l}\text { Stammlösung } \\
\text { Basisstoff }\end{array}$} & \multirow{2}{*}{$\begin{array}{c}\text { Stammlösung } \\
\text { Konzentration } \\
{[\mathrm{mg} / \mathrm{L}]}\end{array}$} & \multicolumn{4}{|c|}{ Testkonzentrationen in den Gasbildungsmodulen } \\
\hline & & & $\begin{array}{c}\text { Variante } 1 \\
\text { [mg/L] }\end{array}$ & $\begin{array}{c}\text { Variante } 2 \\
\text { [mg/L] }\end{array}$ & $\begin{array}{c}\text { Variante } 3 \\
\text { [mg/L] }\end{array}$ & $\begin{array}{c}\text { Variante } 4 \\
{[\mathrm{mg} / \mathrm{L}]}\end{array}$ \\
\hline Nickel 1 & $\mathrm{NiCl}_{2} \cdot 6 \mathrm{H}_{2} \mathrm{O}$ & 200 & 1,5 & 2,5 & 5,0 & 15 \\
\hline Nickel 2 & $\mathrm{NiCl}_{2} \cdot 6 \mathrm{H}_{2} \mathrm{O}$ & 1000 & 30 & 60 & 125 & 500 \\
\hline Nickel 3 & $\mathrm{NiCl}_{2} \cdot 6 \mathrm{H}_{2} \mathrm{O}$ & 1000 & 15 & 20 & 25 & - \\
\hline Nickel 4 & $\mathrm{NiCl}_{2} \cdot 6 \mathrm{H}_{2} \mathrm{O}$ & 1000 & 20 & 25 & 30 & 35 \\
\hline Cobalt 1 & $\mathrm{Co}\left(\mathrm{NO}_{3}\right)_{2}$ & 10000 & 100 & 500 & 800 & 1200 \\
\hline Cobalt 2 & $\mathrm{Co}\left(\mathrm{NO}_{3}\right)_{2}$ & 10000 & 25 & 50 & 75 & 100 \\
\hline Molybdän & $\left(\mathrm{NH}_{4}\right)_{2} \mathrm{MoO}_{4}$ & 10000 & 20 & 40 & 100 & 200 \\
\hline Kupfer 1 & $\mathrm{CuCl}_{2} \cdot 2 \mathrm{H}_{2} \mathrm{O}$ & 1000 & 15 & 25 & 50 & 100 \\
\hline Kupfer 2 & $\mathrm{CuCl}_{2} \cdot 2 \mathrm{H}_{2} \mathrm{O}$ & 1000 & 30 & 40 & 50 & 60 \\
\hline Cadmium 1 & $\mathrm{CdCl}_{2}$ & 1000 & 10 & 20 & 50 & 100 \\
\hline Cadmium 2 & $\mathrm{Cd}\left(\mathrm{NO}_{3}\right)_{2}$ & 10000 & 200 & 250 & 300 & 400 \\
\hline Blei & $\mathrm{Pb}\left(\mathrm{NO}_{3}\right)_{2}$ & 1000 & 20 & 50 & 100 & 200 \\
\hline
\end{tabular}

Ein wichtiger Parameter für gleichbleibende, standardisierte Versuchsbedingungen in den Testreihen ist zudem das Substrat/Inokulum-Verhältnis bzw. oTS-Verhältnis [gots (Substrat) / gots (Inokulum)]. MerRetTig-Bruns ET AL. (2012) stellten in Gärversuchen mit Stärke als Substrat ab einem oTS-Verhältnis von 0,3 bis 0,4 einen verzögerten Verlauf der Biogasproduktion fest. Ab einem oTS-Verhältnis von 0,5 trat eine Hemmung der Gasbildung durch Versäuerung auf. So wurde für den ORGA-Test die optimale Substratdosierung bei Stärke und Mais-Silage auf ein Substrat/InokulumVerhältnis von $\mathbf{0 , 2}$ bis $\mathbf{0 , 3}$ festgelegt, um eine gute Verwertung des Substrates zu gewährleisten bzw. Hemmungwirkungen durch Versäuerungsprozesse zu vermeiden (MerRetTig-Bruns et AL., 2012).

Die Zunahme der Biogasmenge ist bei konstanter Temperatur $\left(38^{\circ} \mathrm{C}\right)$ und kontantem Volumen (Kopfraumvolumina in den Gärflaschen) direkt proportional zum gemessenen Druckanstieg. So wurde der aufgezeichnete kumulierte Druckanstieg über das Ideale Gasgesetz und das Gesetz von Avogadro in kumuliertes Gasvolumen bzw. gebildete Biogasmenge umgerechnet. Für eine Vergleichbarkeit der Versuchsreihen erfolgen die Darstellungen im Zeitverlauf als gemittelte Summenkurven des spezifischen Biogasertrags in $\mathbf{m L}_{\mathbf{N}} / \mathbf{g}_{\mathbf{o}}$ Ts. Als Hemmwirkungskriterium wurden um mindestens $4 \%$ geminderte spezifische Biogasausbeuten gegenüber der Kontrolle festgelegt, basierend auf der von ENGLER ET AL. (2011) beschriebenen Messunsicherheit des Testverfahrens. 
In Anlehnung an Merrettig-Bruns ET AL. (2012) wurde als zusätzlicher Parameter zur Bewertung der biologischen Aktivität in den Biogasreaktoren bzw. Gasbildungsmodulen der Zeitparameter $\mathbf{t}_{\mathbf{2 5 0}}$ herangezogen. Er kennzeichnet den jeweiligen Zeitpunkt in den Testreihen, bei dem ein spezifischer Biogasertrag von $250 \mathrm{~mL}_{\mathrm{N}} / \mathrm{g}_{\mathrm{oTS}}$ erreicht wird und erwies sich als gut geeignetes Kriterium für eine Beurteilung der einzelnen Testvarianten in den Versuchsreihen.

Tabelle 14 zeigt einen Überblick über die einzelnen Parameter der durchgeführten diskontinuierlichen Gärversuche (ORGA-Tests). In einer zusätzlichen Versuchsreihe wurden die spezifischen Biogaserträge der in den (quasi)kontinuierlichen Laborfermenterversuchen eingesetzten Energiepflanzen-Silagen ermittelt und mit den Werten von Batchtests am DBFZ verglichen (vgl. Kapitel 4.2).

Tabelle 14: $\quad$ Überblick über die Parameter der diskontinuierlichen Gärversuche (ORGA-Tests).

\begin{tabular}{|c|c|}
\hline Parameter & Beschreibung \\
\hline System & ANKOM-Gasbildungssystem (ANKOM ${ }^{\mathrm{RF}}$ Gas Production System) \\
\hline Versuchsdauer & $10 \mathrm{~d}$ \\
\hline Zeitliche Auflösung & 30 min Messwerte (60 s Live-Intervalle) \\
\hline Atmosphäre & Stickstoff (Kopfraumbegasung) \\
\hline Temperatur & $38^{\circ} \mathrm{C}$ im Wasserbad \\
\hline Durchmischung & ca. alle $24 \mathrm{~h}$ täglich, manuell \\
\hline Gärgefäße & 17 Enghalsflaschen aus Glas, beschichtet, mit Septum \\
\hline Nennvolumen & $1000 \mathrm{~mL}$ \\
\hline Arbeitsvolumen & Kopfraumvolumen (gravimetrisch bestimmt) \\
\hline Inokulum & Fermentermaterial, ausgefault \\
\hline Herkunft & Praxisbiogasanlage Verliehausen \\
\hline Ausfaulungsgrad & $10-15 d$ \\
\hline Verdünnung & $1: 1$ mit demineralisiertem Wasser \\
\hline Substrat & Mais-Silage \\
\hline Herkunft & Praxisbiogasanlage Verliehausen, Probenahme: 29.05.2015 \\
\hline Bearbeitung & getrocknet $\left(105^{\circ} \mathrm{C}\right)$ und feingemahlen \\
\hline TS; oTS & 30,9; 96,5 Gew.-\% \\
\hline Optimales oTS-Verhältnis & $0,2-0,3$ (Substrat/Inokulum-Verhältnis) \\
\hline Aktivitätskriterium & Zeitparameter $\mathrm{t}_{250}$ \\
\hline Testvarianten & Dreifachwiederholung \\
\hline Kontrolle & Dreifachwiederholung \\
\hline Nullproben (Blankproben) & Doppelwiederholung \\
\hline
\end{tabular}




\subsection{Obere Hemm- und Toxizitätswirkung von Nickel}

Für das Spurenelement Nickel wurden vier Versuchsreihen durchgeführt, bei denen jeweils in Dreifachwiederholung eine $\mathrm{NiCl}_{2} \cdot 6 \mathrm{H}_{2} \mathrm{O}$-Lösung in verschiedenen Konzentrationsstufen als Zusatzstoff in die Gasbildungsmodule zugegeben wurde. Die Nickelgehalte im eingebrachten Substrat $\left(0,051 \mathrm{mg} / \mathrm{kg}_{\mathrm{FM}}\right)$ und Inokulum $(0,48 \mathrm{bis}$ $0,66 \mathrm{mg} / \mathrm{kg}_{\mathrm{FM}}$ ) erwiesen sich als gering und waren somit vernachlässigbar für die Ermittlung der Hemm- und Toxizitätskonzentration. In den Tabellen zu den Testreihen (Tabelle 15 bis Tabelle 18) sind die Messparameter des Substrates und Inokulums sowie die Ergebnisse der jeweiligen Testvarianten aufgeführt, Abbildung 86 bis Abbildung 89 zeigen die gemittelten Summenkurven der spezifischen Biogasausbeute in $\mathrm{mL}_{\mathrm{N}} / \mathrm{g}_{\mathrm{oTS}}$ sowie den Zeitparameter $\mathrm{t}_{250}$.

Die Reihe „Nickel 1“ galt als ein erster Testversuch (Tabelle 15). Das verwendete Fermentermaterial als Inokulum war jedoch zu lange ausgefault (50 d) und entsprach nicht dem optimalen Ausfaulungsgrad von 10 bis 15 Tagen. Der dadurch bedingte Trockensubstanzgehalt von 6,2 Gew.-\% war gegenüber der frischen Probe deutlich niedriger als erwartet, sodass das Substrat/Inokulum-Verhältnis mit 0,38 über dem optimalen Dosierungswert von 0,2 bis 0,3 nach MERRETTIG-BRUnS ET AL. (2012) lag. Die Biogasproduktion in den Gasbildungsmodulen verlief verzögert und auf einem niedrigeren Level als unter Optimalbedingungen ab (Abbildung 86), was wiederum die Befunde von MERretTig-Bruns ET AL. (2012) bestätigt.

Aber auch die Nickelkonzentrationen in den Gasbildungsmodulen (1,5, 2,5, 5,0 und $15 \mathrm{mg} / \mathrm{L})$ erwiesen sich als zu niedrig, die spezifischen Biogaserträge aller Varianten lagen über der spezifischen Gasausbeute der Kontrolle (Abbildung 86). Dies zeigte sich auch beim Bewertungskriterium, dem Zeitparameter $\mathrm{t}_{250}$ (Zeitpunkt in den Testreihen, bei dem ein spezifischer Biogasertrag von $250 \mathrm{~mL}_{\mathrm{N}} / \mathrm{g}_{\mathrm{oTS}}$ erreicht wird). Die $\mathrm{t}_{250}$-Zeitpunkte aller Varianten waren mit 2,7 und 3,0 sowie 3,4 und 3,5 Versuchstagen bereits vor der Kontrolle (3,8 Tage) erreicht (Abbildung 86). Die gewählten Konzentrationen der Testvarianten fördern also unter diesen nicht optimalen Bedingungen eher den Biogasbildungsprozess anstatt ihn $\mathrm{zu}$ hemmen, die biologische Aktivität scheint gegenüber der Kontrolle erhöht. Aufgrund der zu hohen Anfangsbelastung bzw. des zu hohen Substrat/Inokulum-Verhältnisses sind die Ergebnisse dieses ersten Testversuchs jedoch von begrenzter Aussagekraft. 
Tabelle 15: ORGA-Versuchsreihe „Nickel 1“.

\begin{tabular}{|c|c|c|c|c|c|c|c|c|c|}
\hline \multirow{2}{*}{$\begin{array}{l}\text { Nickel } 1^{\circ} \\
\text { Substrat: }\end{array}$} & \multirow[b]{2}{*}{ Einwaage } & \multirow{3}{*}{$\begin{array}{l}{[\mathrm{g}]} \\
{\left[\mathrm{mg} / \mathrm{kg}_{\mathrm{FM}}\right]}\end{array}$} & \multirow[b]{2}{*}{7,0} & & \multirow{2}{*}{$\begin{array}{c}\text { Testvariante, } \\
\text { Konzentration } \\
\text { im Gärreaktor } \\
{[\mathrm{mg} / \mathrm{L}]}\end{array}$} & \multirow{2}{*}{$\begin{array}{c}\begin{array}{c}\text { Zeit- } \\
\text { kriterium } \\
\mathbf{t}_{\mathbf{2 5 0}} \\
{[\mathrm{d}]}\end{array} \\
3,8\end{array}$} & \multicolumn{3}{|c|}{$\begin{array}{c}\text { Spezifische } \\
\text { Biogasausbeute } \\
\text { am Versuchsende } \\
{\left[\mathrm{mL}_{\mathrm{N}} / \mathrm{g}_{\mathrm{oTs}}\right]}\end{array}$} \\
\hline & & & & & & & 379 & \pm & 11 \\
\hline & Nickel & & 0,051 & & 1,5 & 3,4 & 385 & \pm & 6 \\
\hline \multirow[t]{4}{*}{ Inokulum: } & Ausfaulungsgrad & [d] & 50 & & 2,5 & 3,5 & 395 & \pm & 6 \\
\hline & TS; oTS & [Gew.-\%] & 6,2 & 70,8 & 5,0 & 3,0 & 426 & \pm & 5 \\
\hline & Einwaage & [g] & 400 & & 15 & 2,7 & 459 & \pm & 3 \\
\hline & Nickel & {$\left[\mathrm{mg} / \mathrm{kg}_{\mathrm{FM}}\right]$} & 0,52 & & Nullversuch & - & 25 & \pm & 9 \\
\hline \multicolumn{3}{|c|}{ S/l-Verhältnis*: } & 0,38 & & & & & & \\
\hline
\end{tabular}

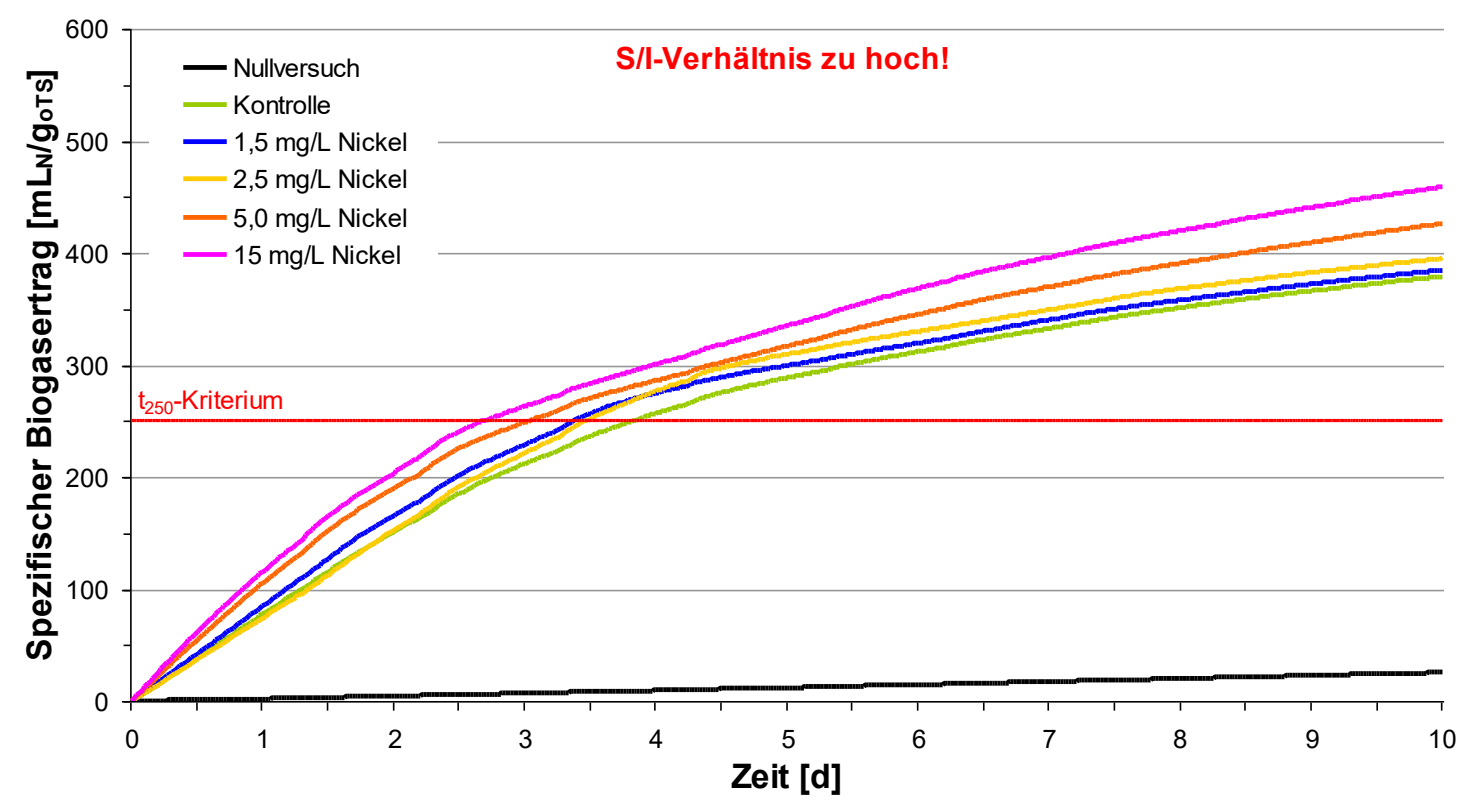

Abbildung 86: Verlauf der gemittelten Gassummenkurven der ORGA-Versuchsreihe „Nickel 1 “ zur Überprüfung der oberen Hemm- und Toxizitätswirkung von Nickel.

Bei der Reihe „Nickel 2“ (Tabelle 16) zeigte sich, dass der gemittelte spezifische Biogasertrag der Testvariante mit den Nickelgehalten von $30 \mathrm{mg} / \mathrm{L}\left(554 \pm 10 \mathrm{~mL} / \mathrm{g}_{\mathrm{oTs}}\right)$ nahezu der Kontrolle $\left(564 \pm 1 \mathrm{~mL} / \mathrm{g}_{\mathrm{oTS}}\right)$ entsprach, die gemittelten Gassummenkurven verliefen konstant parallel auf gleichem Niveau (Abbildung 87). Auch die $\mathrm{t}_{250^{-}}$ Zeitpunkte unterschieden sich mit 2,0 d (Kontrolle) bzw. 2,1 d (30 mg/L Nickel) kaum voneinander. Bei dieser Konzentration ist also noch keine signifikante Hemmwirkung auf den Biogasbildungsprozess abzuleiten. 
Tabelle 16: ORGA-Versuchsreihe „Nickel 2“.

\begin{tabular}{|c|c|c|c|c|c|c|c|c|c|}
\hline \multirow[t]{2}{*}{$\begin{array}{l}\text { Nickel } 2 \\
\text { Substrat: }\end{array}$} & \multirow[b]{2}{*}{ Einwaage } & \multirow{3}{*}{$\begin{array}{l}{[\mathrm{g}]} \\
{\left[\mathrm{mg} / \mathrm{kg}_{\mathrm{FM}}\right]}\end{array}$} & \multirow[b]{2}{*}{7,0} & & \multirow{2}{*}{$\begin{array}{c}\text { Testvariante, } \\
\text { Konzentration } \\
\text { im Gärreaktor } \\
{[\mathrm{mg} / \mathrm{L}]}\end{array}$} & \multirow{2}{*}{$\begin{array}{c}\begin{array}{c}\text { Zeit- } \\
\text { kriterium } \\
\mathbf{t}_{\mathbf{2 5 0}} \\
{[\mathrm{d}]}\end{array} \\
2,0\end{array}$} & \multicolumn{3}{|c|}{$\begin{array}{c}\text { Spezifische } \\
\text { Biogasausbeute } \\
\text { am Versuchsende } \\
{\left[\mathrm{mL}_{\mathrm{N}} / \mathrm{g}_{\mathrm{oTs}}\right]}\end{array}$} \\
\hline & & & & & & & 564 & \pm & 1 \\
\hline & Nickel & & 0,051 & & 30 & 2,1 & 554 & \pm & 10 \\
\hline \multirow[t]{4}{*}{ Inokulum: } & Ausfaulungsgrad & [d] & 15 & & 60 & 4,2 & 355 & \pm & 18 \\
\hline & TS; oTS & [Gew.-\%] & 8,4 & 68,7 & 125 & - & 217 & \pm & 7 \\
\hline & Einwaage & [g] & 400 & & 500 & - & 3 & \pm & 2 \\
\hline & Nickel & {$\left[\mathrm{mg} / \mathrm{kg}_{\mathrm{FM}}\right]$} & 0,48 & & Nullversuch & - & 56 & \pm & 1 \\
\hline S/I-Verhältnis*: & & & 0,29 & & & & & & \\
\hline
\end{tabular}

*: Substrat/Inokulum-Verhältnis (oTS-Verhältnis): goTs (Substrat) / goTs (Inokulum)

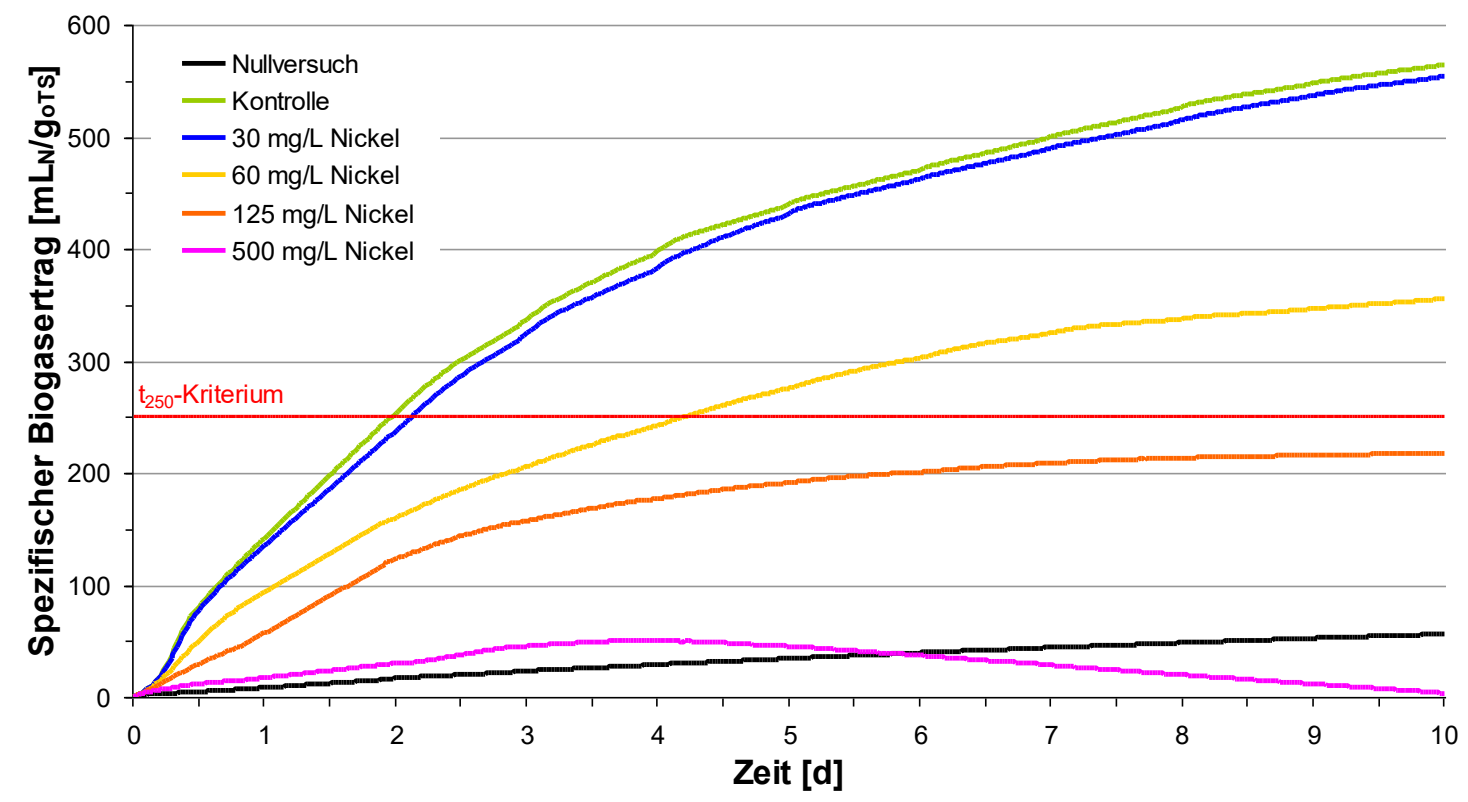

Abbildung 87: Verlauf der gemittelten Gassummenkurven der ORGA-Versuchsreihe „Nickel 2“ zur Überprüfung der oberen Hemm- und Toxizitätswirkung von Nickel.

Die Module mit den Konzentrationen von 60 und $125 \mathrm{mg} / \mathrm{L}$ Nickel wiesen hingegen deutlich geringere gemittelte spezifische Biogaserträge $\left(355 \pm 18 \mathrm{~mL}_{\mathrm{N}} / \mathrm{g}_{\mathrm{oTS}}\right.$ bzw. $\left.217 \pm 7 \mathrm{~mL}_{\mathrm{N}} / \mathrm{g}_{\mathrm{oTS}}\right)$ gegenüber der Kontrolle auf. Der $\mathrm{t}_{250}$-Zeitpunkt der $60 \mathrm{mg} / \mathrm{L} \mathrm{Nickel-}$ Testvariante lag mit 4,2 d weit entfernt von der Kontrolle $(2,0 \mathrm{~d})$, bei der $125 \mathrm{mg} / \mathrm{L}$ Nickel-Testvariante wurde er nicht mehr erreicht. Bei diesen hohen Nickelgehalten traten folglich starke Hemmwirkungen auf. Bei der Testvariante mit den Nickelgehalten von $500 \mathrm{mg} / \mathrm{L}$ lag der gemittelte spezifische Biogasertrag nach etwa sechs Versuchstagen sogar unterhalb der Nullprobe. Daraus ist zu schlussfolgern, dass diese Nickelkonzentration toxisch auf die Umsetzungsprozesse im Aktivitätstest wirkt. 
Im Versuch „Nickel 3“ wurden Testvarianten mit 15, 20 und 25 mg/L Nickelgehalten geprüft, um bei einer mittleren optimalen Anfangsbelastung (Substrat/InokulumVerhältnis) von 0,25 herauszufinden, ob diese Nickelkonzentrationen noch eine fördernde Wirkung auf den Biogasbildungsprozess zeigen oder ob sie ähnlich der $30 \mathrm{mg} / \mathrm{L}$ Nickel-Testvariante in Reihe „Nickel 2“ wirkungslos bleiben. Letzteres war der Fall, es konnten gegenüber der Kontrolle weder positive noch negative Effekte sowohl bei den gemittelten spezifischen Biogaserträgen als auch bei dem $t_{250^{-}}$ Aktivitätskriterium beobachtet werden (Tabelle 17 und Abbildung 88). Die Ergebnisse unterschieden sich nicht signifikant voneinander.

Tabelle 17: ORGA-Versuchsreihe „Nickel 3“.

\begin{tabular}{|c|c|c|c|c|c|c|c|c|c|}
\hline \multirow{2}{*}{$\begin{array}{l}\text { Nickel } 3 \\
\text { Substrat: }\end{array}$} & \multirow[b]{2}{*}{ Einwaage } & \multirow{3}{*}{$\begin{array}{l}{[\mathrm{g}]} \\
{\left[\mathrm{mg} / \mathrm{kg}_{\mathrm{FM}}\right]}\end{array}$} & \multirow[b]{2}{*}{6,0} & & \multirow{2}{*}{$\begin{array}{c}\text { Testvariante, } \\
\text { Konzentration } \\
\text { im Gärreaktor } \\
{[\mathrm{mg} / \mathrm{L}]}\end{array}$} & \multirow{2}{*}{$\begin{array}{c}\begin{array}{c}\text { Zeit- } \\
\text { kriterium } \\
\mathbf{t}_{\mathbf{2 5 0}} \\
{[\mathrm{d}]}\end{array} \\
1,5\end{array}$} & \multicolumn{3}{|c|}{$\begin{array}{c}\text { Spezifische } \\
\text { Biogasausbeute } \\
\text { am Versuchsende } \\
{\left[\mathrm{mL}_{\mathrm{N}} / \mathrm{g}_{\mathrm{oTS}}\right]}\end{array}$} \\
\hline & & & & & & & 568 & \pm & 4 \\
\hline & Nickel & & 0,051 & & 15 & 1,6 & 562 & \pm & 20 \\
\hline \multirow[t]{4}{*}{ Inokulum: } & Ausfaulungsgrad & [d] & 12 & & 20 & 1,6 & 570 & \pm & 12 \\
\hline & TS; oTS & [Gew.-\%] & 8,0 & 71,4 & 25 & 1,7 & 561 & \pm & 8 \\
\hline & Einwaage & [g] & 400 & & Nullversuch & - & 115 & \pm & 5 \\
\hline & Nickel & {$\left[\mathrm{mg} / \mathrm{kg}_{\mathrm{FM}}\right]$} & 0,66 & & & & & & \\
\hline S/I-Verhältnis*: & & & 0,25 & & & & & & \\
\hline
\end{tabular}

*: Substrat/Inokulum-Verhältnis (oTS-Verhältnis): goTs (Substrat) / goTs (Inokulum)

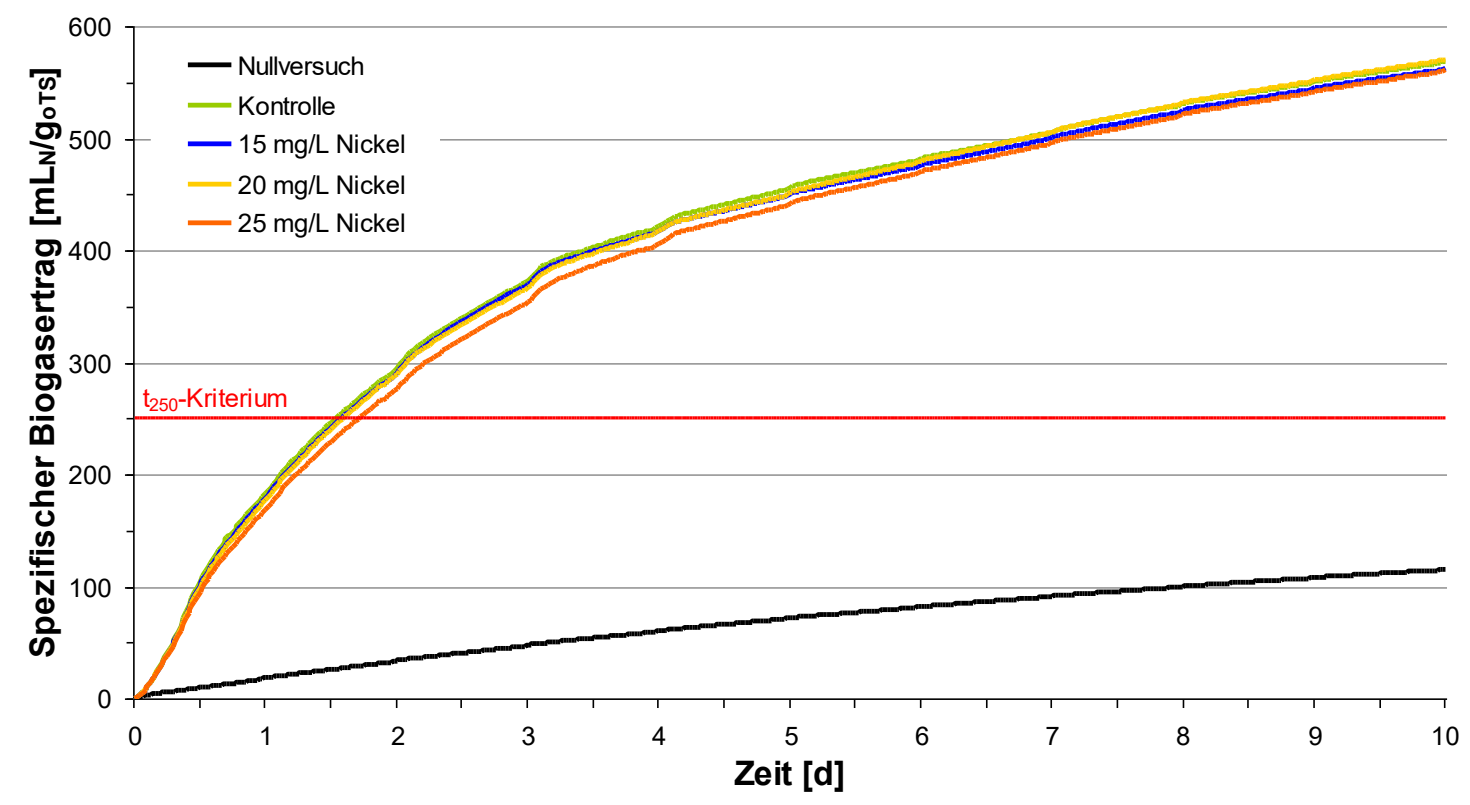

Abbildung 88: Verlauf der gemittelten Gassummenkurven der ORGA-Testreihe „Nickel 3“ zur Überprüfung der oberen Hemm- und Toxizitätswirkung von Nickel. 
Die Testreihe „Nickel 4“ bestätigte die Ergebnisse aus den Versuchen „Nickel 2“ und „Nickel 3“ bei den Nickelgehalten von 20, 25 und $30 \mathrm{mg} / \mathrm{L}$, die gemittelten spezifischen Biogasausbeuten als auch die $t_{250}$-Zeitpunkte wiesen keine signifikanten Unterschiede gegenüber der Kontrolle auf (Tabelle 18 und Abbildung 89). Die Testvariante mit Nickelgehalten von $35 \mathrm{mg} / \mathrm{L}$ zeigte hingegen eine beginnende Hemmwirkung auf die biologische Aktivität in den Modulen, der gemittelte spezifische Biogasertrag war mit $522 \pm 5 \mathrm{~mL}_{\mathrm{N}} / \mathrm{g}_{\mathrm{oTS}}$ gegenüber der Kontrolle $\left(554 \pm 10 \mathrm{~mL} / \mathrm{g}_{\mathrm{oTS}}\right)$ um $6 \%$ gemindert. Auch zeichnete sich bereits nach 14 Stunden eine im Gassummenkurvenverlauf sichtbare geminderte Gasproduktion $\mathrm{ab}$, das $\mathrm{t}_{250}$-Zeitkriterium war nach 2,3 Tagen erreicht, einen halben Tag später als in der Kontrolle (1,8 d).

Tabelle 18: ORGA-Versuchsreihe „Nickel 4“.

\begin{tabular}{|c|c|c|c|c|c|c|c|c|c|}
\hline \multirow{2}{*}{$\begin{array}{l}\text { Nickel } 4 \\
\\
\text { Substrat: }\end{array}$} & \multirow[b]{2}{*}{ Einwaage } & \multirow{3}{*}{$\begin{array}{l}{[\mathrm{g}]} \\
{\left[\mathrm{mg} / \mathrm{kg}_{\mathrm{FM}}\right]}\end{array}$} & \multirow[b]{2}{*}{7,0} & & \multirow{2}{*}{$\begin{array}{c}\text { Testvariante, } \\
\text { Konzentration } \\
\text { im Gärreaktor } \\
{[\mathrm{mg} / \mathrm{L}]}\end{array}$} & \multirow{2}{*}{$\begin{array}{c}\begin{array}{c}\text { Zeit- } \\
\text { kriterium } \\
\mathbf{t}_{\mathbf{2 5 0}} \\
{[\mathrm{d}]}\end{array} \\
1,8\end{array}$} & \multicolumn{3}{|c|}{$\begin{array}{c}\text { Spezifische } \\
\text { Biogasausbeute } \\
\text { am Versuchsende } \\
{\left[\mathrm{mL}_{\mathrm{N}} / \mathrm{g}_{\mathrm{oTS}}\right]}\end{array}$} \\
\hline & & & & & & & 554 & \pm & 10 \\
\hline & Nickel & & 0,051 & & 20 & 1,9 & 553 & \pm & 6 \\
\hline \multirow[t]{4}{*}{ Inokulum: } & Ausfaulungsgrad & [d] & 15 & & 25 & 2,0 & 549 & \pm & 6 \\
\hline & TS; oTS & [Gew.-\%] & 8,2 & 73,4 & 30 & 2,1 & 546 & \pm & 5 \\
\hline & Einwaage & [g] & 400 & & 35 & 2,3 & 522 & \pm & 5 \\
\hline & Nickel & {$\left[\mathrm{mg} / \mathrm{kg}_{\mathrm{FM}}\right]$} & 0,58 & & Nullversuch & - & 91 & \pm & 1 \\
\hline \multicolumn{3}{|c|}{ S/I-Verhältnis*: } & 0,28 & & & & & & \\
\hline
\end{tabular}

*: Substrat/Inokulum-Verhältnis (oTS-Verhältnis): gots (Substrat) / goTs (Inokulum)

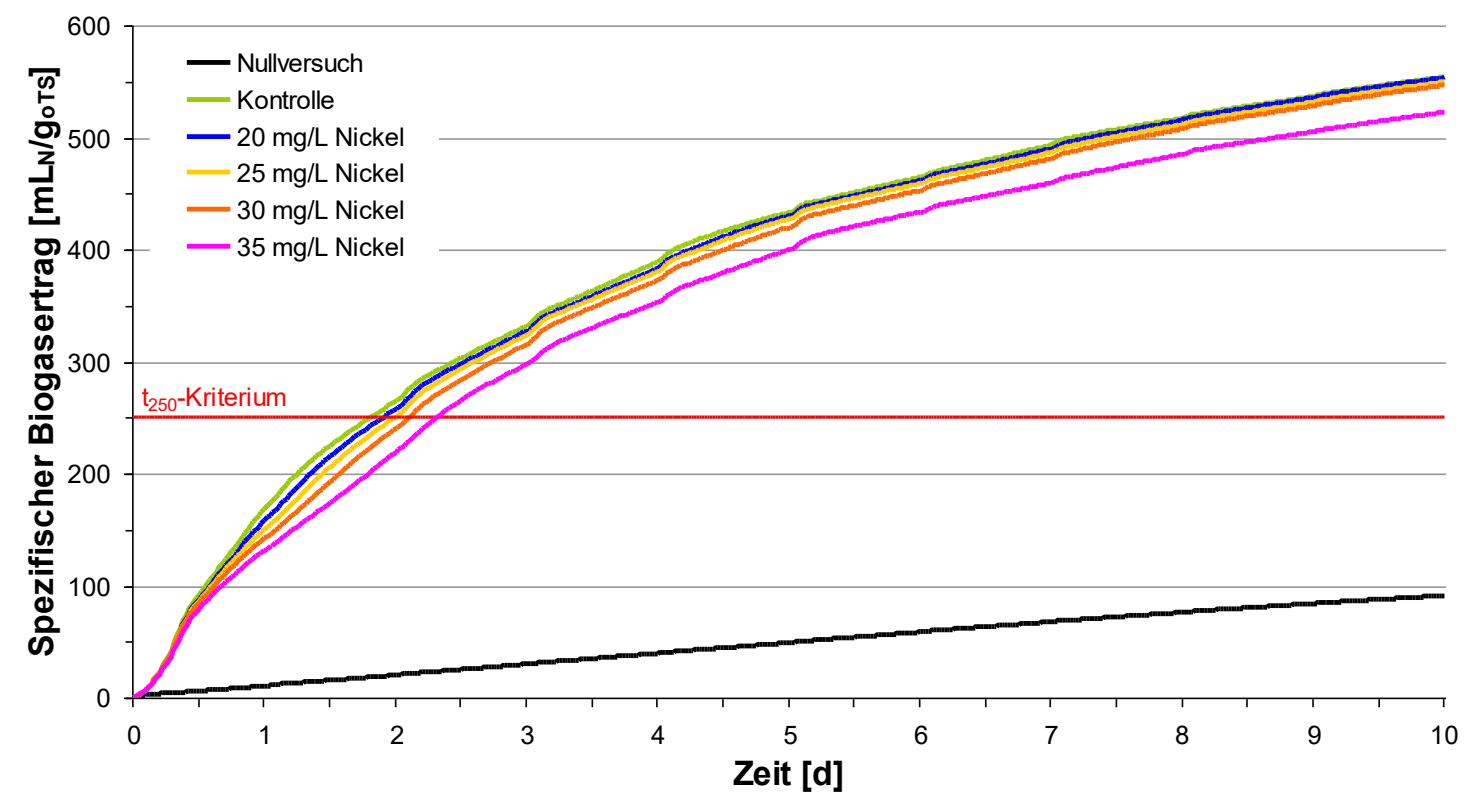

Abbildung 89: Verlauf der gemittelten Gassummenkurven der ORGA-Testreihe „,Nickel 4 “ zur Überprüfung der oberen Hemm- und Toxizitätswirkung von Nickel. 
Die Abbaukinetiken in den drei Nickel-Versuchsreihen mit optimaler Anfangsbelastung (Reihe „Nickel 2“ bis „Nickel 4“) zeigten typische ausgeglichene Gassummenverläufe für komplexe Substrate wie Mais-Silage: Nach sehr kurzen Anlaufphasen (lag-Phasen) $\mathrm{zu}$ Versuchsbeginn folgten nur wenige Stunden andauernde, sehr steil ansteigende Phasen starker Gasbildung, die dann in weniger steil ansteigende Phasen übergingen. Diese gestuften Substratabbauverläufe waren jedoch nicht immer deutlich ausgeprägt. In Abbildung 90 sind die Kontrollen und alle Testvarianten der Versuchsreihen „Nickel 2“ bis „Nickel 4“ zusammengestellt.

Bei den hohen Konzentrationen von 125 und 500 mg/L Nickel traten in den Verläufen zudem stationäre Phasen (Plateauphasen) nach 9,5 Tagen (125 mg/L Nickel) bzw. von 3,7 bis 4,2 Tagen ( $500 \mathrm{mg} / \mathrm{L}$ Nickel) auf, die durch die sehr niedrige, stark gehemmte Abbauaktivität der gasbildenden Mikroorganismen zu begründen sind. In diesen Phasen waren keine Zunahmen der spezifischen Biogaserträge mehr $\mathrm{zu}$ verzeichnen, die biologische Abbauaktivität war demnach eingestellt. Letztgenannte Nickelkonzentration wies nach der stationären Phase nach 4,2 Tagen dann sogar eine Phase des Rückgangs des spezifischen Biogasertrags auf. Das bedeutet, dass die Umsetzungsprozesse in den Gasbildungsmodulen vollständig zum Erliegen kamen und diese sehr hohe Nickelkonzentration eine toxische Wirkung auf die gasbildenden Mikroorganismen ausübt.

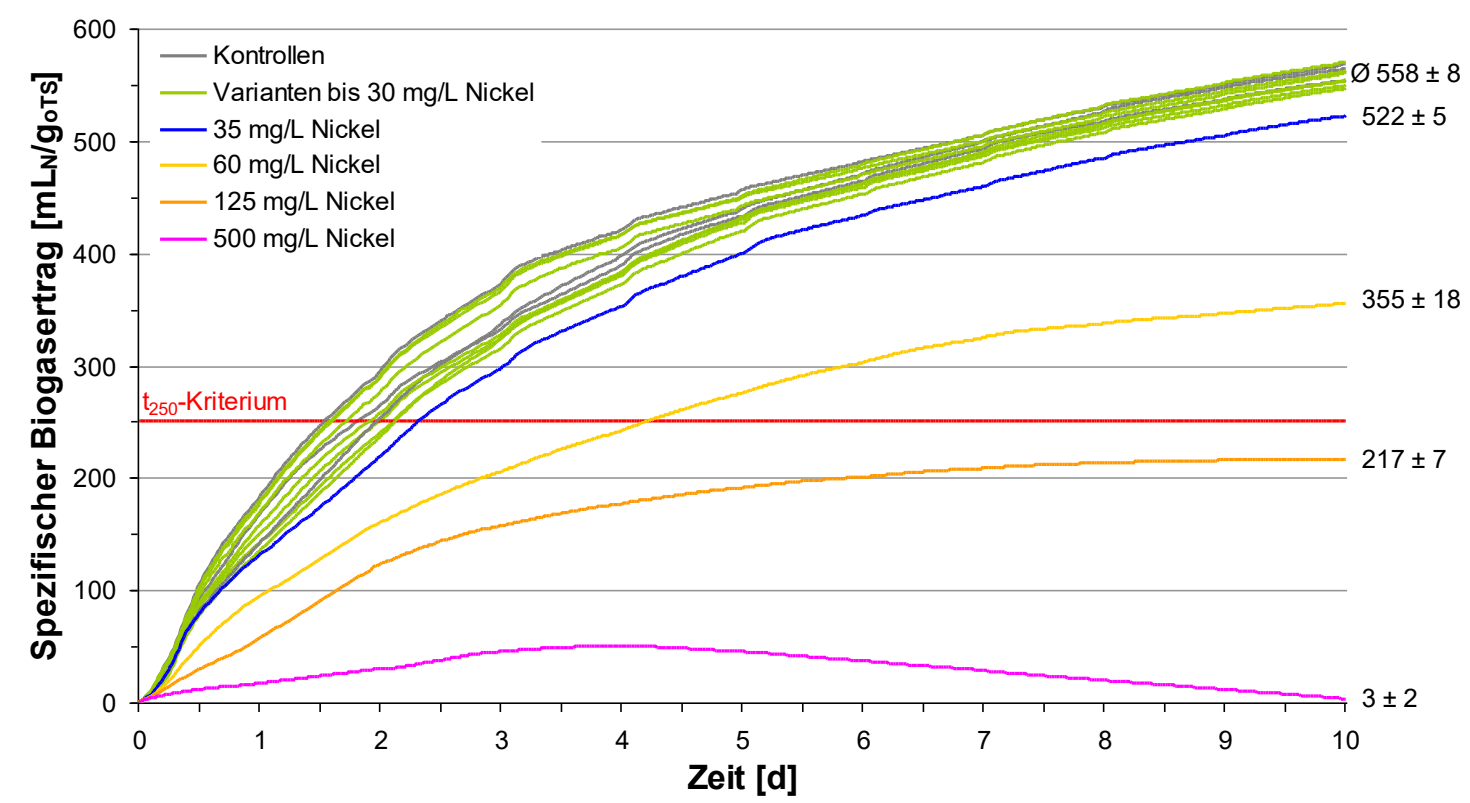

Abbildung 90: Verlauf der gemittelten Gassummenkurven der ORGA-Testreihen „Nickel 2 “bis „,Nickel 4“ zur Überprüfung der oberen Hemm- und Toxizitätswirkung von Nickel. 


\subsection{Obere Hemm- und Toxizitätswirkung von Cobalt}

Für das Spurenelement Cobalt wurden zwei ORGA-Testreihen durchgeführt, bei denen eine $\mathrm{Co}\left(\mathrm{NO}_{3}\right)_{2}$-Lösung in verschiedenen Konzentrationsstufen jeweils in Dreifachwiederholung als Zusatzstoff in die Gasbildungsmodule zugegeben wurde. Auch bei diesen beiden Versuchsreihen waren die eingebrachten Cobaltgehalte des Substrates $\left(0,010 \mathrm{mg} / \mathrm{kg}_{\mathrm{FM}}\right)$ und Inokulums $\left(0,13 \mathrm{bzw} .0,11 \mathrm{mg} / \mathrm{kg}_{\mathrm{FM}}\right)$ so gering, dass sie für die Hemm- und Toxizitätskonzentrationsbestimmung unberücksichtigt bleiben konnten (Tabelle 19 und Tabelle 20).

Im Versuch „Cobalt 1“ wurden Testvarianten mit 100, 500, 800 und 1200 mg/L Cobalt (Tabelle 19 und Abbildung 91) geprüft. Der Zeitparameter $t_{250}$ wurde bei allen Konzentrationen nicht erreicht, die drei höchsten Testvarianten erwiesen sich als stark überdosiert und damit toxisch für die gasbildenden Mikroorganismen. Die gemittelten spezifischen Biogaserträge dieser drei Varianten stiegen zwar im ersten 30 MinutenAufzeichnungsintervall rasant an, gingen dann jedoch direkt in kurze Plateauphasen über, gefolgt von sehr flach ansteigenden Phasen bis zum Ende des ersten Versuchstages. Die kurzfristigen Anstiege nach 22,5 Stunden waren bedingt durch die tägliche Durchmischung. Nach 24 Stunden (800 und $1200 \mathrm{mg} / \mathrm{L}$ Cobalt) bzw. 49 Stunden (500 mg/L Cobalt) begann die Biogasproduktion in den Gasbildungsmodulen dann allmählich komplett zusammenzubrechen mit keinerlei Biogasausbeute mehr nach 2,4 bzw. 3,1 Tagen.

Die Variante mit den Cobaltgehalten von $100 \mathrm{mg} / \mathrm{L}$ zeigte in den ersten 23 Stunden zunächst eine sehr flach ansteigende Phase, bedingt durch die sehr starke Hemmwirkung dieser Konzentration auf die biologische Abbauaktivität. Danach trat ebenfalls eine Phase des Rückgangs der gemittelten spezifischen Biogasausbeute ein. Nach 49 Stunden lag der spezifische Gasertrag dann erstmals unterhalb des Nullversuchs und bewegte sich bis zum Versuchsende fortan konstant etwa auf dem gleichen Niveau der Nullvariante (Abbildung 91). Nach zehn Tagen betrug die gemittelte spezifische Gasausbeute lediglich $109 \pm 5 \mathrm{~mL}_{\mathrm{N}} / \mathrm{g}_{\mathrm{oTS}}$ gegenüber $121 \pm 8 \mathrm{~mL}_{\mathrm{N}} / \mathrm{g}_{\mathrm{oTS}}$ im Nullversuch bzw. $608 \pm 13 \mathrm{~mL}_{\mathrm{N}} / \mathrm{g}_{\mathrm{oTS}}$ bei der Kontrolle. Sie war somit um $82 \%$ gegenüber dem Kontrollversuch gemindert. Demnach stellt die Cobaltkonzentration von 100 mg/L genau den Schwellenbereich einer toxischen Wirkung auf die Abbauprozesse im Aktivitätstest dar. 
Tabelle 19: ORGA-Versuchsreihe „Cobalt 1“.

\begin{tabular}{|c|c|c|c|c|c|c|c|c|c|}
\hline \multirow[t]{2}{*}{$\begin{array}{l}\text { Cobalt } 1 \\
\text { Substrat: }\end{array}$} & \multirow[b]{2}{*}{ Einwaage } & \multirow{3}{*}{$\begin{array}{l}\text { [g] } \\
{\left[\mathrm{mg} / \mathrm{kg}_{\mathrm{FM}}\right]}\end{array}$} & \multirow[b]{2}{*}{3,5} & & \multirow{2}{*}{$\begin{array}{c}\text { Testvariante, } \\
\text { Konzentration } \\
\text { im Gärreaktor } \\
{[\mathrm{mg} / \mathrm{L}]}\end{array}$} & \multirow{2}{*}{$\begin{array}{c}\begin{array}{c}\text { Zeit- } \\
\text { kriterium } \\
\mathbf{t}_{\mathbf{2 5 0}} \\
{[\mathrm{d}]}\end{array} \\
1,2\end{array}$} & \multicolumn{3}{|c|}{$\begin{array}{c}\text { Spezifische } \\
\text { Biogasausbeute } \\
\text { am Versuchsende } \\
{\left[\mathrm{mL}_{\mathrm{N}} / \mathrm{g}_{\mathrm{oTs}}\right]}\end{array}$} \\
\hline & & & & & & & 608 & \pm & 13 \\
\hline & Cobalt & & 0,010 & & 100 & - & 109 & \pm & 5 \\
\hline \multirow[t]{4}{*}{ Inokulum: } & Ausfaulungsgrad & [d] & 10 & & 500 & - & - & & \\
\hline & TS; oTS & [Gew.-\%] & 7,4 & 76,8 & 800 & - & - & & \\
\hline & Einwaage & [g] & 300 & & 1200 & - & - & & \\
\hline & Cobalt & {$\left[\mathrm{mg} / \mathrm{kg}_{\mathrm{FM}}\right]$} & 0,13 & & Nullversuch & - & 121 & \pm & 8 \\
\hline S/I-Verhältnis*: & & & 0,20 & & & & & & \\
\hline
\end{tabular}

*: Substrat/Inokulum-Verhältnis (oTS-Verhältnis): goTs (Substrat) / goTs (Inokulum)

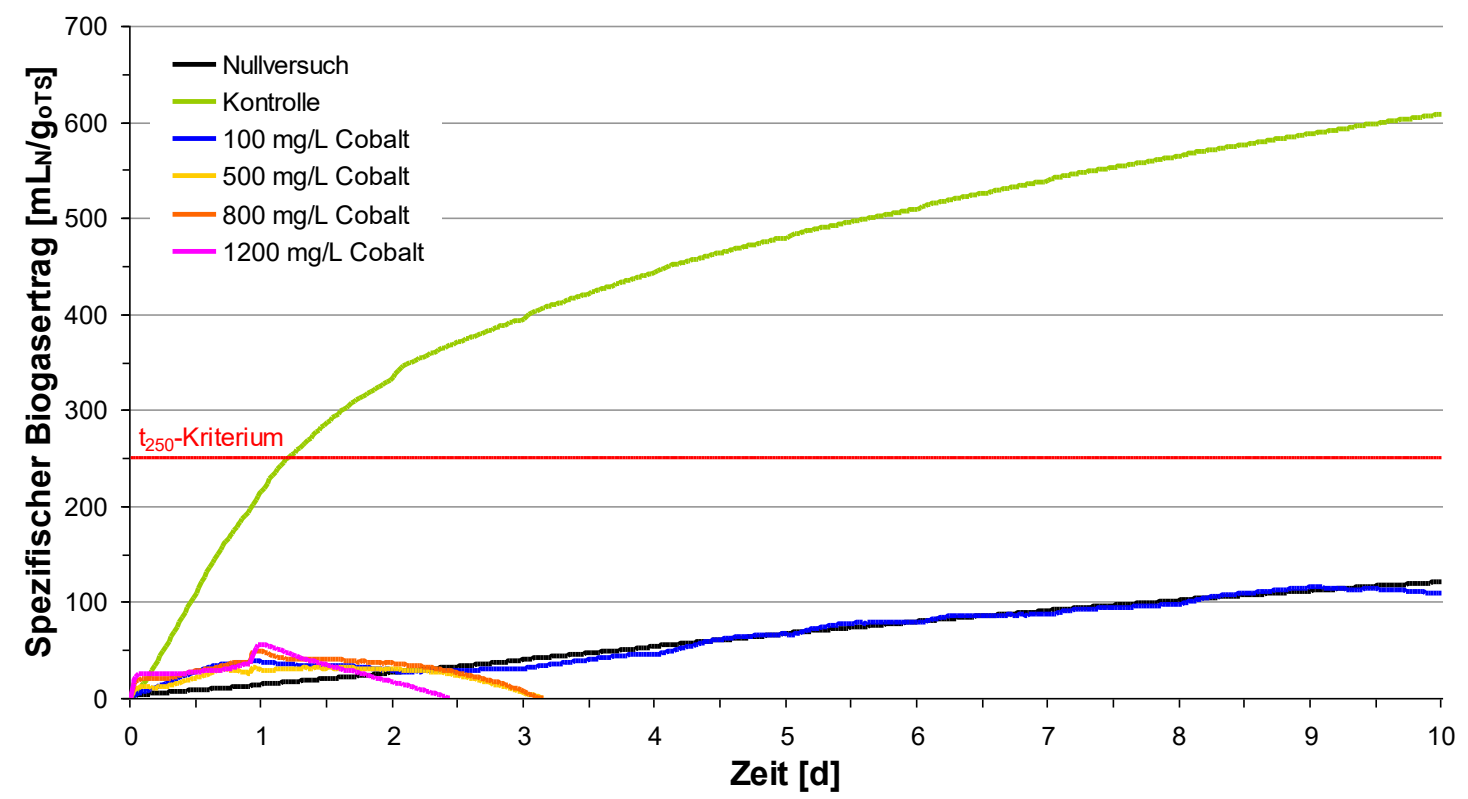

Abbildung 91: Verlauf der gemittelten Gassummenkurven der ORGA-Testreihe „, Cobalt 1 “ zur Überprüfung der oberen Hemm- und Toxizitätswirkung von Cobalt.

Im Versuch „Cobalt 2“ zeigten die Testvarianten mit 50 und $75 \mathrm{mg} / \mathrm{L}$ Cobalt starke Hemmwirkungen auf die biologische Aktivität in den Gasbildungsmodulen mit leicht fallenden gemittelten spezifischen Biogaserträgen nach 5,4 bzw. 6,7 Versuchstagen. Die $50 \mathrm{mg} / \mathrm{L}$ Cobalt-Testvariante konnte das $\mathrm{t}_{250}$-Aktivitätskriterium erst nach 2,8 Tagen überschreiten, 1,7 Tage später als in der Kontrolle. Bei den $75 \mathrm{mg} / \mathrm{L}$ Cobaltgehalten wurde das $\mathrm{t}_{250}$-Zeitkriterium nicht erreicht. Eine Hemmwirkung auf die Umsetzungsprozesse im Aktivitätstest war bei der niedrigsten Testvariante mit $25 \mathrm{mg} / \mathrm{L}$ Cobalt deutlich zu erkennen. Gegenüber der Kontrolle $\left(659 \pm 14 \mathrm{~mL} / \mathrm{g}_{\mathrm{oTS}}\right)$ fiel der gemittelte spezifische Biogasertrag mit $542 \pm 30 \mathrm{~mL}_{\mathrm{N}} / \mathrm{g}_{\mathrm{oTS}}$ am Versuchsende $18 \%$ niedriger aus. 
Der $t_{250}$-Zeitpunkt wurde nach 1,5 Versuchstagen erreicht, acht Stunden später als im Kontrollversuch $(1,1 \mathrm{~d})$. Eine beginnende Hemmwirkung ist demnach bei einer Cobaltkonzentration von etwa $20 \mathrm{mg} / \mathrm{L}$ zu erwarten.

Der Schwellenbereich mit einer beginnenden toxischen Wirkung bei der Variante mit den Cobaltkonzentrationen von $100 \mathrm{mg} / \mathrm{L}$ konnte in dieser zweiten Cobalt-Versuchsreihe bestätigt werden (Tabelle 20 und Abbildung 92). Der gemittelte spezifische Biogasertrag $\left(124 \pm 3 \mathrm{~mL}_{\mathrm{N}} / \mathrm{g}_{\mathrm{oTS}}\right)$ lag abermals im Bereich des Nullversuchs $\left(109 \pm 6 \mathrm{~mL}_{\mathrm{N}} / \mathrm{g}_{\mathrm{oTS}}\right)$. Auch der Gassummenkurvenverlauf wies ein sehr ähnliches Bild wie in der Versuchsreihe „Cobalt 1“ auf.

Tabelle 20: ORGA-Versuchsreihe ,Cobalt 2“.

\begin{tabular}{|c|c|c|c|c|c|c|c|c|c|}
\hline \multirow{2}{*}{$\begin{array}{l}\text { Cobalt } 2 \\
\text { Substrat: }\end{array}$} & \multirow[b]{2}{*}{ Einwaage } & \multirow{3}{*}{$\begin{array}{l}{[\mathrm{g}]} \\
{\left[\mathrm{mg} / \mathrm{kg}_{\mathrm{FM}}\right]}\end{array}$} & \multirow[b]{2}{*}{3,5} & & \multirow[t]{2}{*}{$\begin{array}{c}\text { Testvariante, } \\
\text { Konzentration } \\
\text { im Gärreaktor } \\
{[\mathrm{mg} / \mathrm{L}]} \\
\text { Kontrolle }\end{array}$} & \multirow[t]{2}{*}{$\begin{array}{c}\begin{array}{c}\text { Zeit- } \\
\text { kriterium } \\
\mathbf{t}_{\mathbf{2 5 0}} \\
{[\mathrm{d}]}\end{array} \\
1,1\end{array}$} & \multicolumn{3}{|c|}{$\begin{array}{c}\text { Spezifische } \\
\text { Biogasausbeute } \\
\text { am Versuchsende } \\
{\left[\mathrm{mL}_{\mathrm{N}} / \mathrm{g}_{\mathrm{OTS}}\right]}\end{array}$} \\
\hline & & & & & & & 659 & \pm & 14 \\
\hline & Cobalt & & 0,010 & & 25 & 1,5 & 542 & \pm & 30 \\
\hline \multirow[t]{4}{*}{ Inokulum: } & Ausfaulungsgrad & [d] & 12 & & 50 & 2,8 & 291 & \pm & 3 \\
\hline & TS; oTS & [Gew.-\%] & 7,4 & 76,9 & 75 & - & 218 & \pm & 16 \\
\hline & Einwaage & [g] & 300 & & 100 & - & 124 & \pm & 3 \\
\hline & Cobalt & {$\left[\mathrm{mg} / \mathrm{kg}_{\mathrm{FM}}\right]$} & 0,11 & & Nullversuch & - & 109 & \pm & 6 \\
\hline S/I-Verhältnis*: & & & 0,20 & & & & & & \\
\hline
\end{tabular}

*: Substrat/Inokulum-Verhältnis (oTS-Verhältnis): gots (Substrat) / goTs (Inokulum)

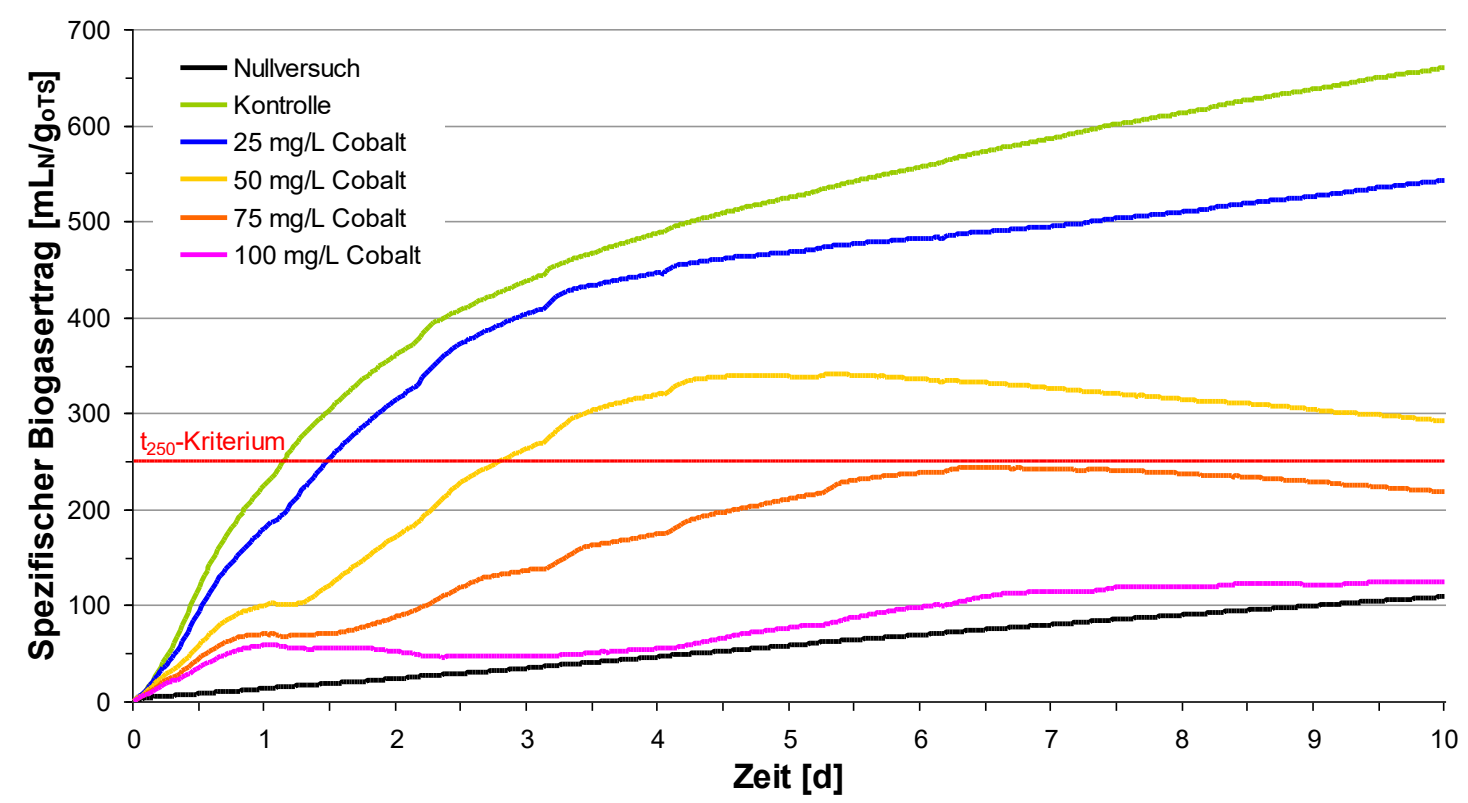

Abbildung 92: Verlauf der gemittelten Gassummenkurven der ORGA-Testreihe „Cobalt 2“ zur Überprüfung der oberen Hemm- und Toxizitätswirkung von Cobalt. 


\subsection{Obere Hemmwirkung von Molybdän}

Der obere hemmende Konzentrationsbereich für das Spurenelement Molybdän wurde durch eine Versuchsreihe eruiert. Jeweils in Dreifachwiederholung wurden zusätzlich Testlösungen in verschiedenen Konzentrationsstufen mit $\left(\mathrm{NH}_{4}\right)_{2} \mathrm{MoO}_{4}$ als Basisstoff in die Gasbildungsmodule gegeben. Auch bei diesem Versuch waren die eingebrachten Molybdängehalte des Substrates $\left(0,14 \mathrm{mg} / \mathrm{kg}_{\mathrm{FM}}\right)$ und Inokulums $\left(0,30 \mathrm{mg} / \mathrm{kg}_{\mathrm{FM}}\right) \mathrm{sehr}$ gering und somit für die Ermittlung der Hemmkonzentration vernachlässigbar (Tabelle 21).

Die gemittelten spezifischen Biogaserträge der Testvarianten mit den Molybdänkonzentrationen $20 \mathrm{mg} / \mathrm{L}\left(529 \pm 4 \mathrm{~mL}_{\mathrm{N}} / \mathrm{g}_{\mathrm{oTS}}\right)$ und $40 \mathrm{mg} / \mathrm{L}\left(528 \pm 9 \mathrm{~mL}_{\mathrm{N}} / \mathrm{g}_{\mathrm{oTS}}\right)$ zeigten gegenüber der Kontrolle $\left(542 \pm 2 \mathrm{~mL}_{\mathrm{N}} / \mathrm{g}_{\mathrm{oTS}}\right)$ nur sehr leichte Minderausbeuten (Abbildung 93). Auch die $t_{250}$-Zeitpunkte lagen eng beieinander und waren gegenüber der Kontrolle (3,0 d) nicht (20 mg/L Molybdän: 3,0 d) bzw. nicht wesentlich (40 mg/L Molybdän: 3,3 d) auffällig.

Die Testvariante mit Molybdängehalten von $100 \mathrm{mg} / \mathrm{L}$ wies eine Minderausbeute um $4 \%\left(520 \pm 5 \mathrm{~mL} / \mathrm{g}_{\mathrm{oTS}}\right)$ gegenüber der Kontrolle $\left(542 \pm 2 \mathrm{~mL} / \mathrm{g}_{\mathrm{oTS}}\right)$ auf und lag somit genau im Bereich des festgelegten Vier-Prozent-Kriteriums für Mindererträge (Tabelle 21 und Abbildung 93). So kann bei dieser hohen Molybdänkonzentration eine beginnende obere Hemmwirkung auf den Biogasprozess bzw. die biologische Aktivität abgeleitet werden. Das $t_{250}$-Aktivitätskriterium war nach 3,2 Versuchstagen erreicht, nur etwa fünf Stunden später als in der Kontrolle (3,0 d).

Tabelle 21: ORGA-Versuchsreihe „Molybdän“.

\begin{tabular}{|c|c|c|c|c|c|c|c|c|c|}
\hline \multirow{2}{*}{$\begin{array}{l}\text { Molybdän } \\
\text { Substrat: }\end{array}$} & \multirow[b]{2}{*}{ Einwaage } & \multirow{3}{*}{$\begin{array}{l}\text { [g] } \\
{\left[\mathrm{mg} / \mathrm{kg}_{\mathrm{FM}}\right]}\end{array}$} & \multirow[b]{2}{*}{5,0} & & \multirow[t]{2}{*}{$\begin{array}{c}\text { Testvariante, } \\
\text { Konzentration } \\
\text { im Gärreaktor } \\
{[\mathrm{mg} / \mathrm{L}]} \\
\text { Kontrolle }\end{array}$} & \multirow[t]{2}{*}{$\begin{array}{c}\begin{array}{c}\text { Zeit- } \\
\text { kriterium } \\
\mathbf{t}_{\mathbf{2 5 0}} \\
{[\mathrm{d}]}\end{array} \\
3,0\end{array}$} & \multicolumn{3}{|c|}{$\begin{array}{c}\text { Spezifische } \\
\text { Biogasausbeute } \\
\text { am Versuchsende } \\
{\left[\mathrm{mL}_{\mathrm{N}} / \mathrm{g}_{\mathrm{oTs}}\right]}\end{array}$} \\
\hline & & & & & & & 542 & \pm & 2 \\
\hline & Molybdän & & 0,14 & & 20 & 3,0 & 529 & \pm & 4 \\
\hline \multirow[t]{4}{*}{ Inokulum: } & Ausfaulungsgrad & [d] & 11 & & 40 & 3,3 & 528 & \pm & 9 \\
\hline & TS; oTS & [Gew.-\%] & 9,0 & 73,1 & 100 & 3,2 & 520 & \pm & 5 \\
\hline & Einwaage & [g] & 300 & & 200 & 3,5 & 482 & \pm & 7 \\
\hline & Molybdän & {$\left[\mathrm{mg} / \mathrm{kg}_{\mathrm{FM}}\right]$} & 0,30 & & Nullversuch & - & 103 & \pm & 2 \\
\hline \multicolumn{3}{|c|}{ S/I-Verhältnis*: } & 0,24 & & & & & & \\
\hline
\end{tabular}




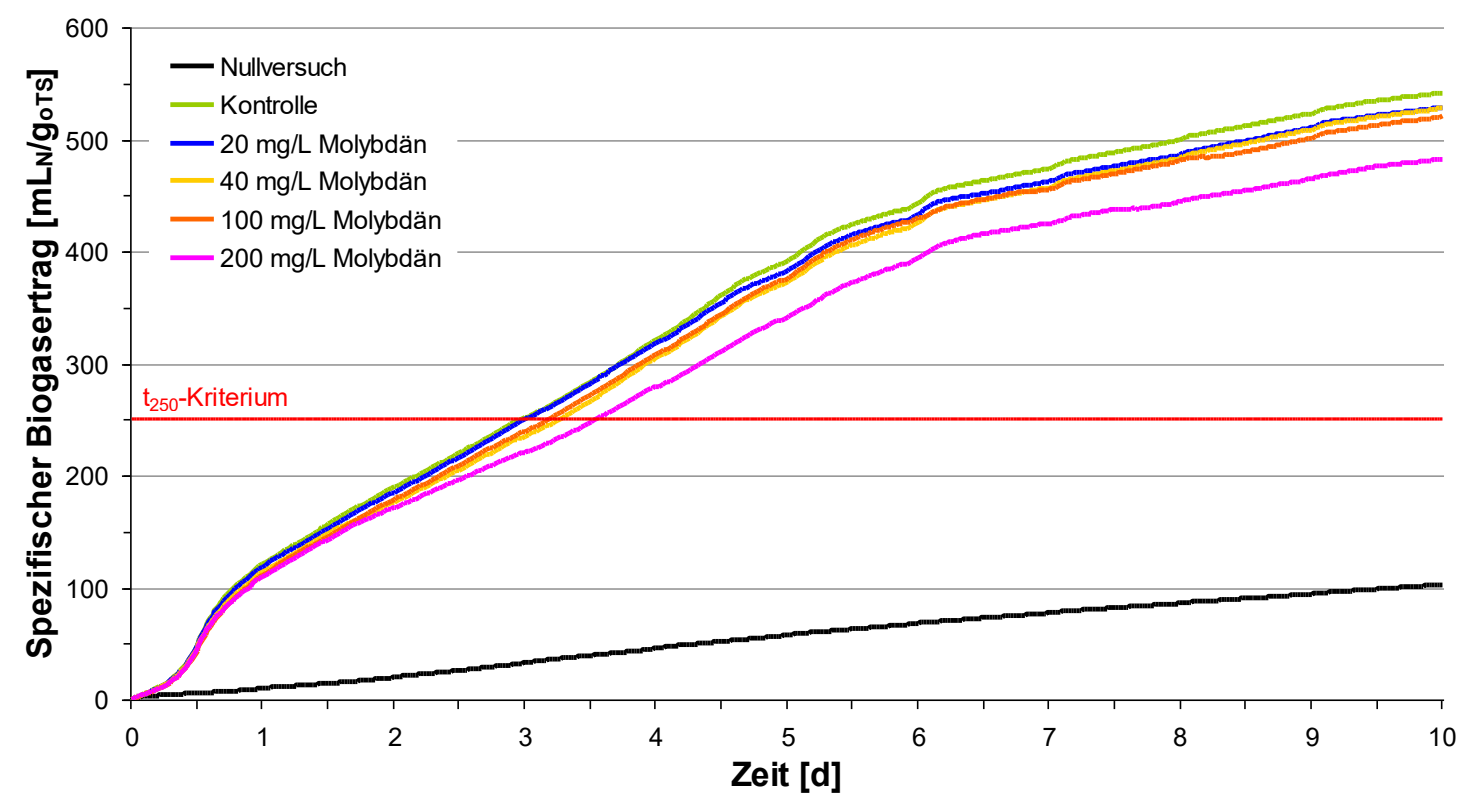

Abbildung 93: Verlauf der gemittelten Gassummenkurven der ORGA-Testreihe zur Überprüfung der oberen Hemmwirkung von Molybdän.

\subsection{Obere Hemm- und Toxizitätswirkung von Kupfer}

Für das Spurenelement Kupfer wurden zwei Versuchsreihen durchgeführt, bei denen jeweils in Dreifachwiederholung eine $\mathrm{CuCl}_{2} \cdot 2 \mathrm{H}_{2} \mathrm{O}$-Lösung in verschiedenen Konzentrationsstufen zusätzlich in die Gärreaktoren zugegeben wurde. Auch bei diesen beiden Testreihen waren die in die Gärprozesse eingebrachten Kupfergehalte des Substrates $\left(1,10 \mathrm{mg} / \mathrm{kg}_{\mathrm{FM}}\right)$ und Inokulums (4,1 bzw. 2,2 mg/ $\left.\mathrm{kg}_{\mathrm{FM}}\right)$ niedrig, dass sie für die Bestimmung der Hemm- und Toxizitätskonzentrationen unberücksichtigt bleiben konnten (Tabelle 22 und Tabelle 23).

Bei der Reihe „Kupfer 1“ gaben die gewählten Kupfer-Konzentrationen 15, 25, 50 und $100 \mathrm{mg} / \mathrm{L}$ gleich einen guten Überblick über die oberen hemmenden bzw. toxischen Konzentrationsbereiche (Abbildung 94). Beim Zeitparameter $\mathrm{t}_{250}$ lagen die Varianten mit 15 und $25 \mathrm{mg} / \mathrm{L}$ Kupfer wie die Kontrolle bei 1,4 Tagen. Auch die gemittelten spezifischen Biogaserträge wiesen mit $556 \pm 26 \mathrm{~mL}_{\mathrm{N}} / \mathrm{g}_{\mathrm{oTs}}(15 \mathrm{mg} / \mathrm{L}$ Kupfer$)$ bzw. $572 \pm 8 \mathrm{~mL} / \mathrm{g}_{\mathrm{oTS}}\left(25 \mathrm{mg} / \mathrm{L}\right.$ Kupfer) gegenüber der Kontrolle mit $559 \pm 18 \mathrm{~mL}_{\mathrm{N}} / \mathrm{g}_{\mathrm{oTS}}$ keine signifikanten Unterschiede auf. Die Testvariante mit 50 mg/L Kupfer erreichte hingegen den $t_{250}$-Zeitpunkt erst nach 2,0 Versuchstagen, 13,5 Stunden später als in der Kontrolle (1,4 d). Eine beginnende Hemmwirkung auf die Umsetzungsprozesse im Aktivitätstest war zu erkennen (Tabelle 22 und Abbildung 94). 
Bei der Testvariante mit $\mathbf{1 0 0} \mathbf{~ m g / L ~ K u p f e r ~ k o n n t e ~ d e r ~ S c h w e l l e n b e r e i c h ~ e i n e r ~}$ toxischen Wirkung auf die gasbildenden Mikroorganismen im Aktivitätstest identifiziert werden. Die Gassummenkurve zeigte durch die starke Hemmwirkung dieser Konzentration auf die biologische Abbauaktivität zunächst einen verzögerten sehr flachen Anstieg. Nach 30 Stunden stagnierte die spezifische Biogasausbeute, begann dann nach 42 Stunden leicht abzusinken und lag nach 5,2 Versuchstagen unterhalb des Nullversuchs. Danach bewegte sie sich konstant etwa im Bereich der Nullvariante und stieg erst gegen Versuchsende wieder leicht an (Abbildung 94).

Tabelle 22: ORGA-Versuchsreihe „Kupfer 1“.

\begin{tabular}{|c|c|c|c|c|c|c|c|c|c|}
\hline \multirow{2}{*}{$\begin{array}{l}\text { Kupfer } 1 \\
\text { Substrat: }\end{array}$} & \multirow[b]{2}{*}{ Einwaage } & \multirow{3}{*}{$\begin{array}{l}{[\mathrm{g}]} \\
{\left[\mathrm{mg} / \mathrm{kg}_{\mathrm{FM}}\right]}\end{array}$} & \multirow[b]{2}{*}{5,0} & & \multirow[t]{2}{*}{$\begin{array}{c}\begin{array}{c}\text { Testvariante, } \\
\text { Konzentration } \\
\text { im Gärreaktor } \\
{[\mathrm{mg} / \mathrm{L}]}\end{array} \\
\text { Kontrolle }\end{array}$} & \multirow[t]{2}{*}{$\begin{array}{c}\begin{array}{c}\text { Zeit- } \\
\text { kriterium } \\
\mathbf{t}_{\mathbf{2 5 0}} \\
{[\mathrm{d}]}\end{array} \\
1,4\end{array}$} & \multicolumn{3}{|c|}{$\begin{array}{c}\text { Spezifische } \\
\text { Biogasausbeute } \\
\text { am Versuchsende } \\
{\left[\mathrm{mL}_{\mathrm{N}} / \mathrm{g}_{\mathrm{oTs}}\right]}\end{array}$} \\
\hline & & & & & & & 559 & \pm & 18 \\
\hline & Kupfer & & 1,10 & & 15 & 1,4 & 556 & \pm & 26 \\
\hline \multirow[t]{4}{*}{ Inokulum: } & Ausfaulungsgrad & {$[\mathrm{d}]$} & 12 & & 25 & 1,4 & 572 & \pm & 8 \\
\hline & TS; oTS & [Gew.-\%] & 6,6 & 73,7 & 50 & 2,0 & 553 & \pm & 5 \\
\hline & Einwaage & [g] & 400 & & 100 & - & 132 & \pm & 9 \\
\hline & Kupfer & {$\left[\mathrm{mg} / \mathrm{kg}_{\mathrm{FM}}\right]$} & 4,1 & & Nullversuch & - & 95 & \pm & 2 \\
\hline S/I-Verhältnis*: & & & 0,25 & & & & & & \\
\hline
\end{tabular}

*: Substrat/Inokulum-Verhältnis (oTS-Verhältnis): gots (Substrat) / gots (Inokulum)

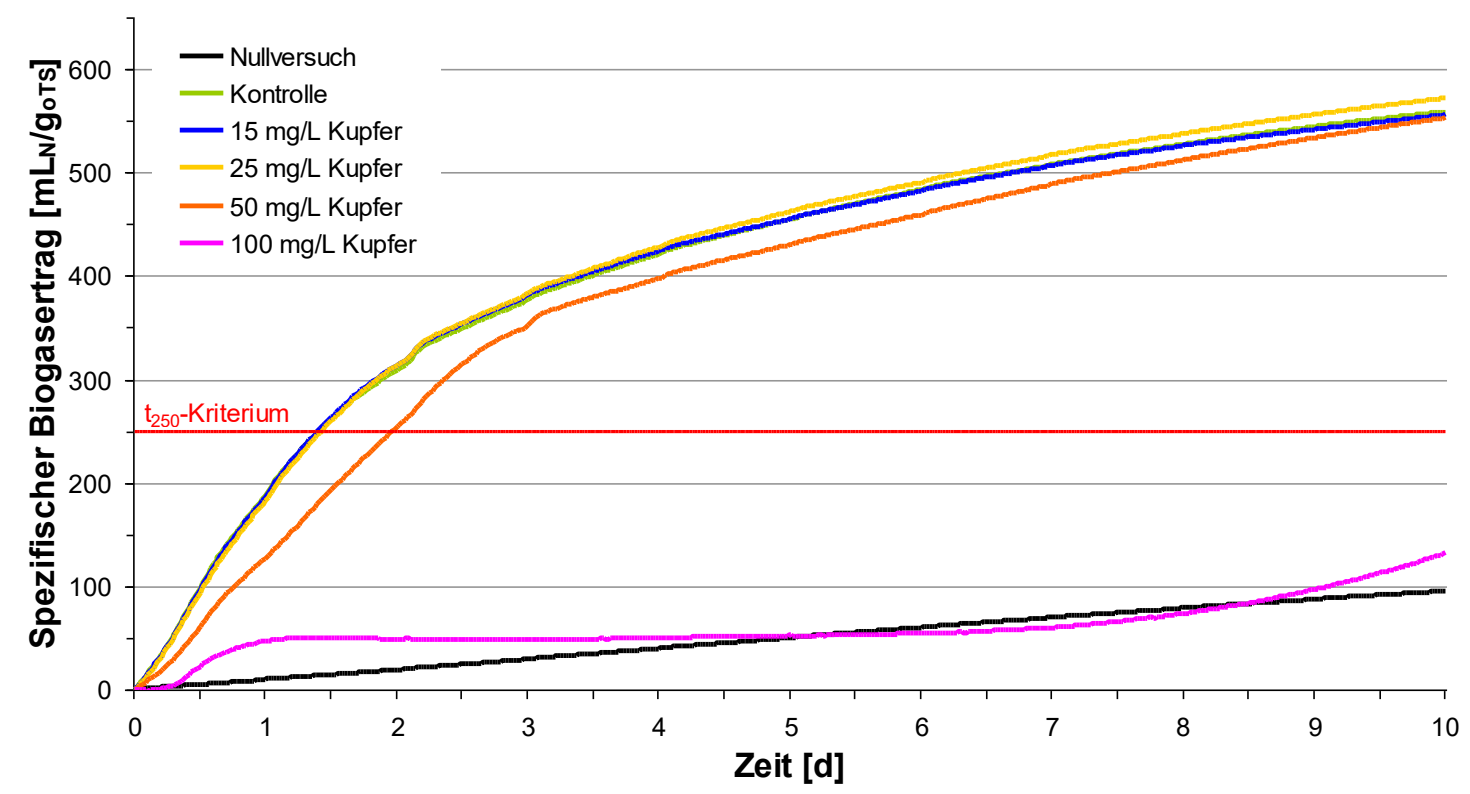

Abbildung 94: Verlauf der gemittelten Gassummenkurven der ORGA-Testreihe „, Kupfer 1 “ zur Überprüfung der oberen Hemm- und Toxizitätswirkung von Kupfer. 
Die Reihe „Kupfer 2“ (Tabelle 23 und Abbildung 95) bestätigte das Ergebnis aus dem Versuch „Kupfer 1“. Die Testvariante mit Kupfergehalten von 50 mg/L zeigte eine beginnende Hemmwirkung auf die biologische Abbauaktivität in den Gasbildungsmodulen, die gemittelte spezifische Biogasausbeute war mit $568 \pm 13 \mathrm{~mL}_{\mathrm{N}} / \mathrm{g}_{\mathrm{oTS}}$ gegenüber der Kontrolle $\left(611 \pm 6 \mathrm{~mL}_{\mathrm{N}} / \mathrm{g}_{\mathrm{oTS}}\right)$ um $7 \%$ gemindert. Das $\mathrm{t}_{250}$-Zeitkriterium war nach 1,7 Tagen erreicht, sechs Stunden später als in der Kontrolle (1,5 d). Einen sehr ähnlichen gehemmten Verlauf zeigte die Testvariante mit Kupfergehalten von $60 \mathrm{mg} / \mathrm{L}$. Die anderen beiden Varianten mit Kupferkonzentrationen von 30 und $40 \mathrm{mg} / \mathrm{L}$ wiesen sowohl bei den gemittelten spezifischen Biogaserträgen als auch den $t_{250^{-}}$ Zeitpunkten keine signifikanten Unterschiede gegenüber der Kontrolle auf.

Tabelle 23: ORGA-Versuchsreihe „Kupfer 2“.

\begin{tabular}{|c|c|c|c|c|c|c|c|c|c|}
\hline \multirow{2}{*}{$\begin{array}{l}\text { Kupfer } 2 \\
\\
\text { Substrat: }\end{array}$} & \multirow[b]{2}{*}{ Einwaage } & \multirow[b]{2}{*}{ [g] } & \multirow[b]{2}{*}{5,0} & & \multirow[t]{2}{*}{$\begin{array}{c}\text { Testvariante, } \\
\text { Konzentration } \\
\text { im Gärreaktor } \\
{[\mathrm{mg} / \mathrm{L}]}\end{array}$} & \multirow{2}{*}{$\begin{array}{c}\begin{array}{c}\text { Zeit- } \\
\text { kriterium } \\
\mathbf{t}_{\mathbf{2 5 0}} \\
{[\mathrm{d}]}\end{array} \\
1,5\end{array}$} & \multicolumn{3}{|c|}{$\begin{array}{c}\text { Spezifische } \\
\text { Biogasausbeute } \\
\text { am Versuchsende } \\
{\left[\mathrm{mL}_{\mathrm{N}} / \mathrm{g}_{\mathrm{oTS}}\right]}\end{array}$} \\
\hline & & & & & & & 611 & \pm & 6 \\
\hline & Kupfer & {$\left[\mathrm{mg} / \mathrm{kg}_{\mathrm{FM}}\right]$} & 1,10 & & 30 & 1,5 & 602 & \pm & 10 \\
\hline \multirow[t]{4}{*}{ Inokulum: } & Ausfaulungsgrad & [d] & 10 & & 40 & 1,5 & 598 & \pm & 11 \\
\hline & TS; oTS & [Gew.-\%] & 5,8 & 72,5 & 50 & 1,7 & 568 & \pm & 13 \\
\hline & Einwaage & [g] & 400 & & 60 & 1,7 & 556 & \pm & 2 \\
\hline & Kupfer & {$\left[\mathrm{mg} / \mathrm{kg}_{\mathrm{FM}}\right]$} & 2,2 & & Nullversuch & - & 103 & \pm & 6 \\
\hline \multicolumn{3}{|c|}{ S/I-Verhältnis*: } & 0,29 & & & & & & \\
\hline
\end{tabular}

*: Substrat/Inokulum-Verhältnis (oTS-Verhältnis): goTs (Substrat) / goTs (Inokulum)

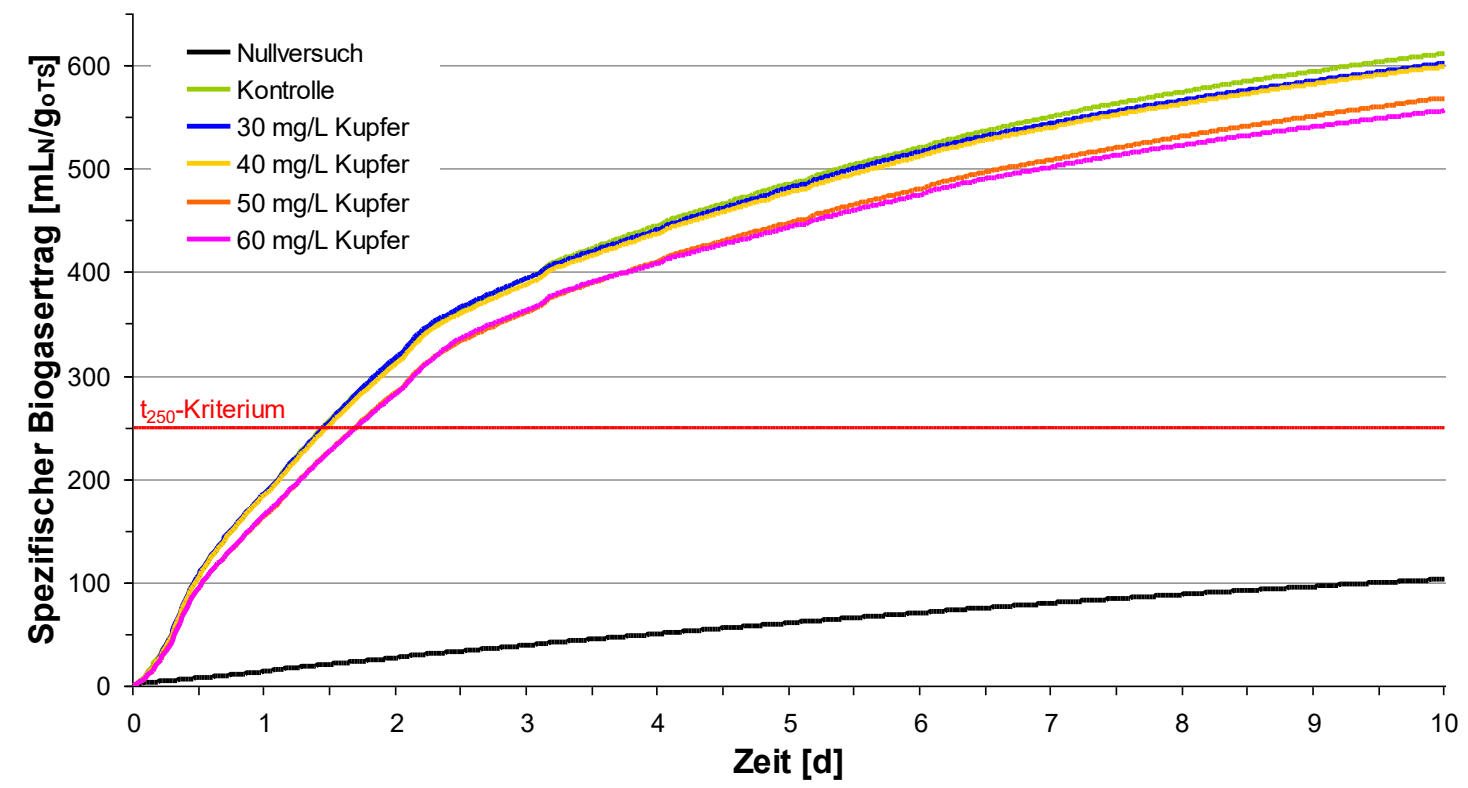

Abbildung 95: Verlauf der gemittelten Gassummenkurven der ORGA-Testreihe „Kupfer 2“ zur Überprüfung der oberen Hemm- und Toxizitätswirkung von Kupfer. 


\subsection{Obere Hemm- und Toxizitätswirkung von Cadmium}

In Deutschland sind schätzungsweise bis zu $10 \%$ der landwirtschaftlichen Flächen mit Schadelementen belastet (SAUER \& RUPPERT, 2013; SAUER ET AL., 2017). Für einige stärker kontaminierte Standorte scheidet eine Weiternutzung für die Nahrungs- oder Futtermittelproduktion aus. Solche Areale sollten bevorzugt für den Energiepflanzenanbau genutzt werden. Das kritischste Element beim Transfer vom Boden in die Pflanze stellt nach SAUER \& RUPPERT (2013) das stark mobile und leicht pflanzenverfügbare Schwermetall Cadmium dar. Daher sollte getestet werden, ab welcher Konzentration dieses potenziell toxische Spurenelement hemmend bzw. toxisch auf die Biogasproduktion wirkt.

Hierfür wurden zwei ORGA-Versuchsreihen durchgeführt. In der ersten Reihe wurde eine $\mathrm{CdCl}_{2}$-Lösung, in der zweiten Testreihe eine $\mathrm{Cd}\left(\mathrm{NO}_{3}\right)_{2}$-Lösung in verschiedenen Konzentrationsstufen jeweils in Dreifachwiederholung als Zusatzstoff in die Module zugegeben. Die eingebrachten Cadmiumgehalte des Substrates $\left(0,022 \mathrm{mg} / \mathrm{kg}_{\mathrm{FM}}\right)$ und Inokulums $\left(0,032\right.$ bzw. $\left.0,028 \mathrm{mg} / \mathrm{kg}_{\mathrm{FM}}\right)$ waren in diesen beiden Versuchsreihen erneut sehr niedrig, dass sie für die Bestimmung der Hemm- und Toxizitätskonzentration keine Rolle spielten (Tabelle 24 und Tabelle 25).

Im ersten Versuch „Cadmium 1“ wurden Testvarianten mit 10, 20, 50 und 100 mg/L Cadmium geprüft (Tabelle 24 und Abbildung 96). Der Zeitparameter $\mathrm{t}_{250}$ zeigte bei den Konzentrationen von $10(2,5 \mathrm{~d})$ und $20 \mathrm{mg} / \mathrm{L}$ Cadmium (2,6 d) keine signifikante Abweichung gegenüber der Kontrolle (2,5 d).

Tabelle 24: ORGA-Versuchsreihe „Cadmium 1“.

\begin{tabular}{|c|c|c|c|c|c|c|c|c|c|}
\hline \multirow{2}{*}{$\begin{array}{l}\text { Cadmium } 1 \\
\\
\text { Substrat: }\end{array}$} & \multirow[b]{2}{*}{ Einwaage } & \multirow{3}{*}{$\begin{array}{l}\text { [g] } \\
{\left[\mathrm{mg} / \mathrm{kg}_{\mathrm{FM}}\right]}\end{array}$} & \multirow[b]{2}{*}{6,0} & & \multirow[t]{2}{*}{$\begin{array}{c}\text { Testvariante, } \\
\text { Konzentration } \\
\text { im Gärreaktor } \\
{[\mathrm{mg} / \mathrm{L}]} \\
\text { Kontrolle }\end{array}$} & \multirow{2}{*}{$\begin{array}{c}\begin{array}{c}\text { Zeit- } \\
\text { kriterium } \\
\mathbf{t}_{\mathbf{2 5 0}} \\
{[\mathrm{d}]}\end{array} \\
2,5\end{array}$} & \multicolumn{3}{|c|}{$\begin{array}{c}\text { Spezifische } \\
\text { Biogasausbeute } \\
\text { am Versuchsende } \\
{\left[\mathrm{mL}_{\mathrm{N}} / \mathrm{g}_{\mathrm{oTs}}\right]}\end{array}$} \\
\hline & & & & & & & 543 & \pm & 18 \\
\hline & Cadmium & & 0,022 & & 10 & 2,5 & 556 & \pm & 4 \\
\hline \multirow[t]{4}{*}{ Inokulum: } & Ausfaulungsgrad & {$[\mathrm{d}]$} & 10 & & 20 & 2,6 & 548 & \pm & 18 \\
\hline & TS; oTS & [Gew.-\%] & 9,2 & 69,7 & 50 & 3,0 & 517 & \pm & 12 \\
\hline & Einwaage & [g] & 400 & & 100 & 4,3 & 485 & \pm & 17 \\
\hline & Cadmium & {$\left[\mathrm{mg} / \mathrm{kg}_{\mathrm{FM}}\right]$} & 0,032 & & Nullversuch & - & 110 & \pm & 4 \\
\hline S/I-Verhältnis*: & & & 0,23 & & & & & & \\
\hline
\end{tabular}




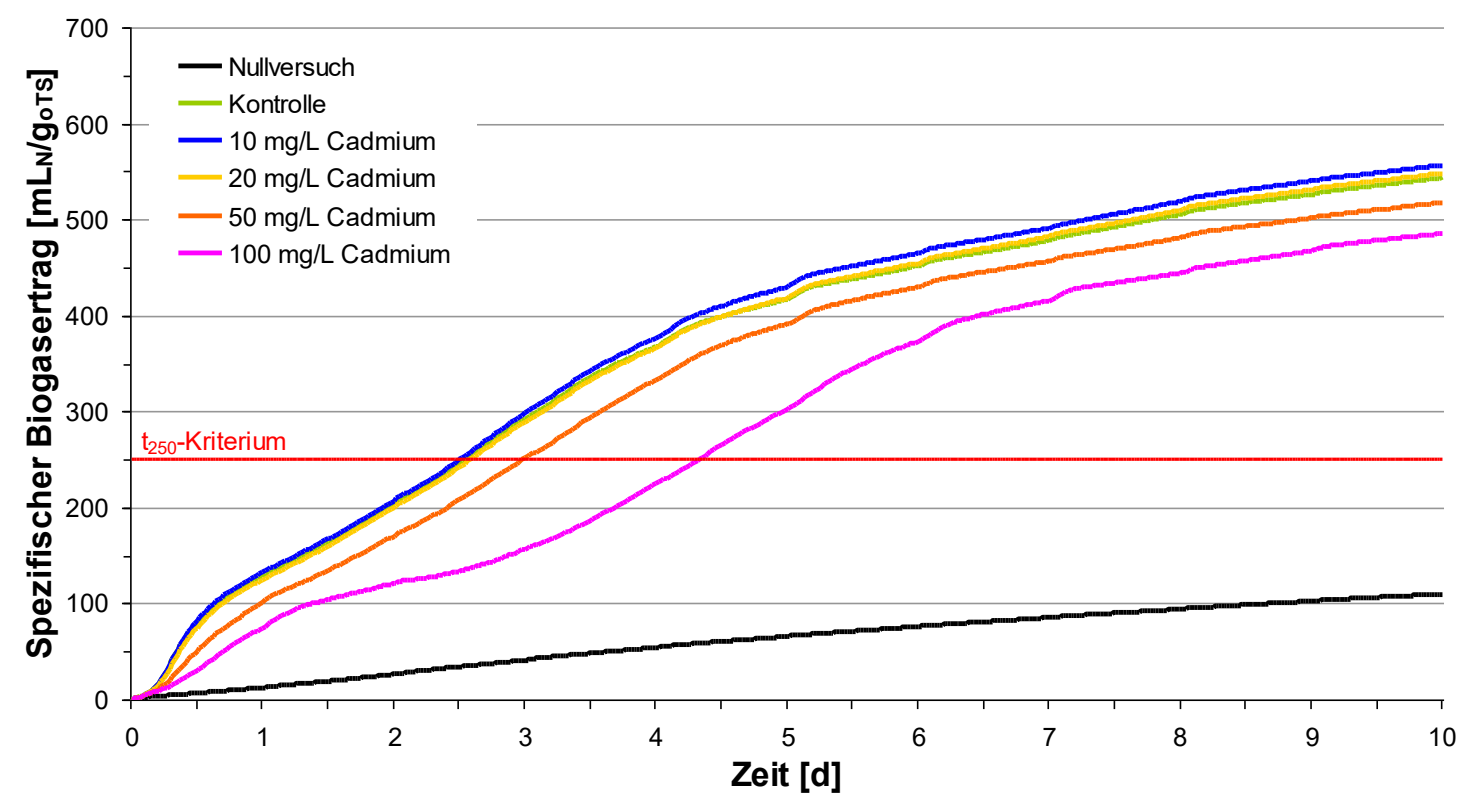

Abbildung 96: Verlauf der gemittelten Gassummenkurven der ORGA-Testreihe , Cadmium 1“ zur Überprüfung der Hemm- und Toxizitätswirkung von Cadmium.

Bei der Variante mit 50 mg/L Cadmium war eine beginnende obere Hemmwirkung auf die Umsetzungsprozesse im Aktivitätstest zu erkennen, der $t_{250}$-Zeitpunkt lag bei 3,0 Versuchstagen und damit elf Stunden später als im Kontrollversuch. Gegenüber der Kontrolle $\left(543 \pm 18 \mathrm{~mL}_{\mathrm{N}} / \mathrm{g}_{\mathrm{oTS}}\right)$ fiel auch der gemittelte spezifische Biogasertrag mit $517 \pm 12 \mathrm{~mL}_{\mathrm{N}} / \mathrm{g}_{\mathrm{oTs}}$ am Versuchsende $5 \%$ niedriger aus. Die Testvariante mit $100 \mathrm{mg} / \mathrm{L}$ Cadmium wies eine noch stärkere Hemmung des Biogasprozesses auf, der $\mathrm{t}_{250}$-Zeitpunkt wurde erst nach 4,3 Versuchstagen erreicht und die gemittelte spezifische Biogasausbeute war mit $485 \pm 17 \mathrm{~mL}_{\mathrm{N}} / \mathrm{g}_{\mathrm{oTS}}$ um $11 \%$ gegenüber der Kontrolle gemindert.

Im Versuch „Cadmium 2“ wurden Testvarianten mit 200, 250, 300 und $400 \mathrm{mg} / \mathrm{L}$ Cadmium geprüft, um die Toxizitätswirkung von Cadmium zu bestimmen (Tabelle 25 und Abbildung 97). Das Aktivitätskriterium $t_{250}$ wurde bei allen Konzentrationsstufen nicht erreicht, die vier Testvarianten erwiesen sich als sehr stark hemmend bzw. toxisch für die gasbildenden Mikroorganismen. Die gemittelten spezifischen Biogasausbeuten stiegen am ersten Versuchstag nur sehr flach an und gingen dann nach kurzen Plateauphasen in fallende Verläufe über (Abbildung 97). Nach 2,7 (200 mg/L Cadmium), 2,9 (250 mg/L Cadmium) und 3,2 (200 mg/L Cadmium) sowie 4,9 Versuchstagen (500 mg/L Cadmium) kamen die Biogasbildungsprozesse komplett zum Erliegen. 
Die Testvariante mit Cadmiumgehalten von 200 mg/L zeigte dann nach 7,4 Versuchstagen wieder eine minimale Gasbildung. Am Versuchsende betrug der gemittelte spezifische Biogasertrag $61 \pm 27 \mathrm{~mL} / \mathrm{g}_{\mathrm{oTS}}$ gegenüber $112 \pm 1 \mathrm{~mL}_{\mathrm{N}} / \mathrm{g}_{\mathrm{oTS}}$ im Nullversuch bzw. $636 \pm 19 \mathrm{~mL} / \mathrm{g}_{\text {oTs }}$ bei der Kontrolle. Er war gegenüber dem Nullversuch um $45 \%$ bzw. gegenüber der Kontrolle um $90 \%$ gemindert. Diese Cadmiumkonzentration bewegte sich demnach im Schwellenbereich einer toxischen Wirkung auf die Umsetzungsprozesse im Aktivitätstest.

Tabelle 25: ORGA-Versuchsreihe „Cadmium 2“.

\begin{tabular}{|c|c|c|c|c|c|c|c|c|c|}
\hline \multirow{2}{*}{$\begin{array}{l}\text { Cadmium } 2 \\
\text { Substrat: }\end{array}$} & \multirow[b]{2}{*}{ Einwaage } & \multirow{3}{*}{$\begin{array}{l}{[\mathrm{g}]} \\
{\left[\mathrm{mg} / \mathrm{kg}_{\mathrm{FM}}\right]}\end{array}$} & \multirow[b]{2}{*}{4,5} & & \multirow{2}{*}{$\begin{array}{c}\text { Testvariante, } \\
\text { Konzentration } \\
\text { im Gärreaktor } \\
{[\mathrm{mg} / \mathrm{L}]} \\
\text { Kontrolle }\end{array}$} & \multirow{2}{*}{$\begin{array}{c}\begin{array}{c}\text { Zeit- } \\
\text { kriterium } \\
\mathbf{t}_{\mathbf{2 5 0}} \\
{[\mathrm{d}]}\end{array} \\
1,3\end{array}$} & \multicolumn{3}{|c|}{$\begin{array}{c}\text { Spezifische } \\
\text { Biogasausbeute } \\
\text { am Versuchsende } \\
{\left[\mathrm{mL}_{\mathrm{N}} / \mathrm{g}_{\mathrm{oTs}}\right]}\end{array}$} \\
\hline & & & & & & & 636 & \pm & 19 \\
\hline & Cadmium & & 0,022 & & 200 & - & 61 & \pm & 27 \\
\hline \multirow[t]{4}{*}{ Inokulum: } & Ausfaulungsgrad & [d] & 10 & & 250 & - & - & & \\
\hline & TS; oTS & [Gew.-\%] & 8,7 & 75,0 & 300 & - & - & & \\
\hline & Einwaage & [g] & 300 & & 400 & - & - & & \\
\hline & Cadmium & {$\left[\mathrm{mg} / \mathrm{kg}_{\mathrm{FM}}\right]$} & 0,028 & & Nullversuch & - & 112 & \pm & 1 \\
\hline S/I-Verhältnis*: & & & 0,22 & & & & & & \\
\hline
\end{tabular}

*: Substrat/Inokulum-Verhältnis (oTS-Verhältnis): goTs (Substrat) / goTs (Inokulum)

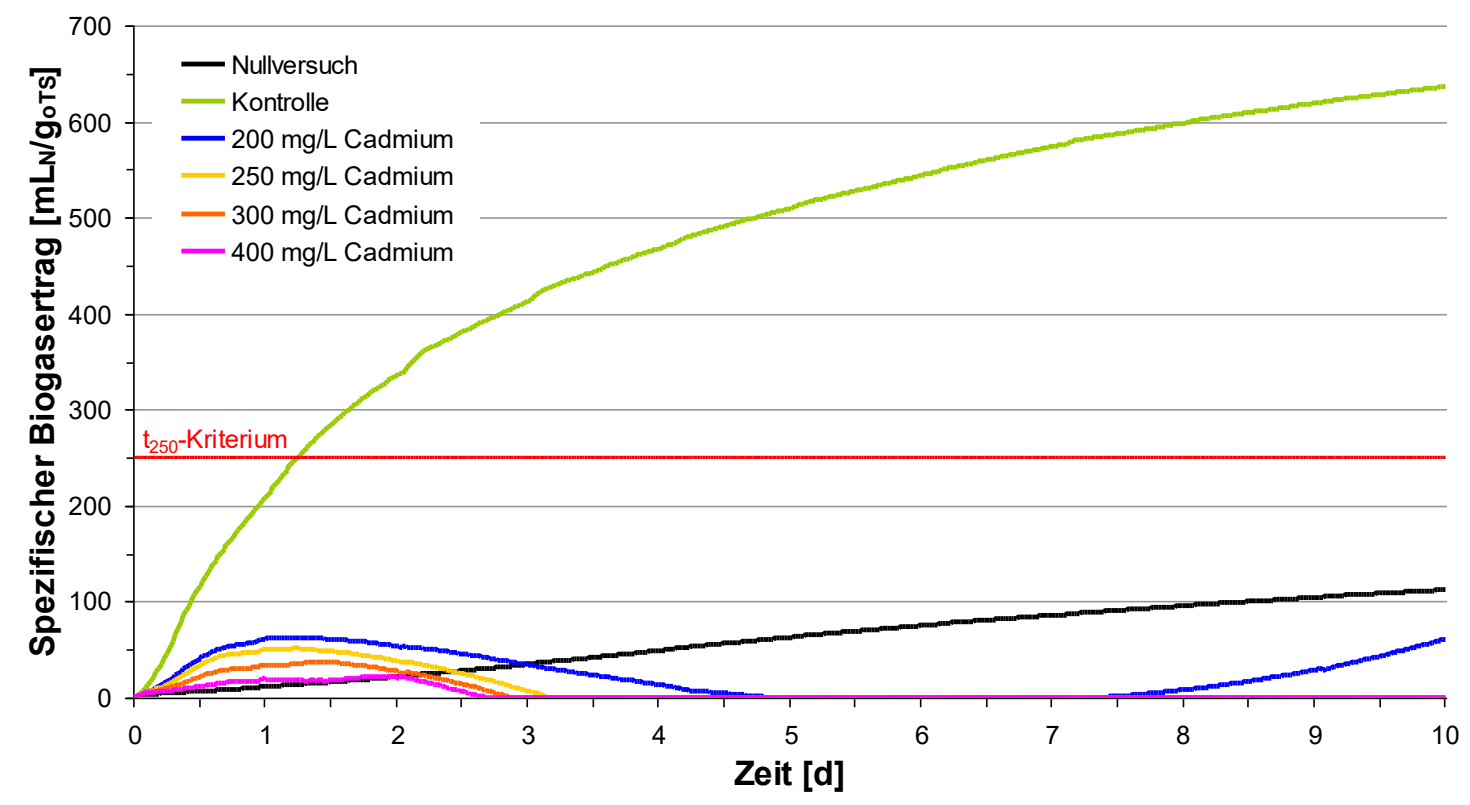

Abbildung 97: Verlauf der gemittelten Gassummenkurven der ORGA-Testreihe „, Cadmium 2“ zur Überprüfung der Hemm- und Toxizitätswirkung von Cadmium. 


\subsection{Obere Hemmwirkung von Blei}

Für das inessenzielle, aber ebenfalls in höherer Dosis potenziell toxisch wirkende Schwermetall Blei wurde der obere hemmende Konzentrationsbereich in einer Versuchsreihe bestimmt. Blei ist beim Transfer vom Boden in die Pflanze eher immobil und stark im Boden gebunden. Allerdings sind Verunreinigungen der EnergiepflanzenSilage durch anhaftendes Bodenmaterial möglich und könnten v.a. beim Anbau auf kontaminierten Standorten zu einer Belastung der Silage führen (SAUER ET AL., 2017).

Jeweils in Dreifachwiederholung wurden in die Gasbildungsmodule zusätzlich Testlösungen in verschiedenen Konzentrationsstufen mit $\mathrm{Pb}\left(\mathrm{NO}_{3}\right)_{2}$ als Basisstoff gegeben. Die eingebrachten Bleigehalte des Substrat- $\left(0,051 \mathrm{mg} / \mathrm{kg}_{\mathrm{FM}}\right)$ und Inokulummaterials $\left(1,0 \mathrm{mg} / \mathrm{kg}_{\mathrm{FM}}\right)$ waren abermals gering und blieben für die Ermittlung der Hemmkonzentration unberücksichtigt (Tabelle 26).

Die gemittelte spezifische Biogasausbeute der Variante mit Bleigehalten von 20 mg/L zeigte mit $466 \pm 6 \mathrm{~mL} / \mathrm{g}_{\mathrm{oTS}}$ gegenüber der Kontrolle $\left(501 \pm 10 \mathrm{~mL} / \mathrm{g}_{\mathrm{oTS}}\right)$ am Testende einen Minderertrag von $7 \%$ (Tabelle 26 und Abbildung 98) und somit eine beginnende obere Hemmwirkung auf die Biogasproduktion bzw. die biologische Aktivität an. Der $\mathrm{t}_{250}$-Zeitpunkt war mit 2,4 Tagen erst acht Stunden nach der Kontrolle (2,0 d) erreicht. Die Varianten mit stärkerer oberer Hemmung $50 \mathrm{mg} / \mathrm{L}\left(421 \pm 4 \mathrm{~mL}_{\mathrm{N}} / \mathrm{g}_{\mathrm{oTS}}\right), 100 \mathrm{mg} / \mathrm{L}$ $\left(415 \pm 5 \mathrm{~mL} / \mathrm{g}_{\mathrm{oTS}}\right)$ sowie $200 \mathrm{mg} / \mathrm{L}$ Blei $\left(414 \pm 11 \mathrm{~mL} / \mathrm{g}_{\mathrm{oTS}}\right)$ wiesen ähnliche Minderausbeuten von 16 bis $17 \%$ gegenüber der Kontrolle $\left(501 \pm 10 \mathrm{~mL}_{\mathrm{N}} / \mathrm{g}_{\mathrm{oTS}}\right)$ auf. Das $\mathrm{t}_{250^{-}}$ Zeitkriterium wurde nach 2,6 (50 mg/L Blei), 2,9 (100 mg/L Blei) bzw. 3,6 Versuchstagen (200 mg/L Blei) erreicht.

Tabelle 26: ORGA-Versuchsreihe „Blei“.

\begin{tabular}{|c|c|c|c|c|c|c|c|c|}
\hline \multicolumn{4}{|l|}{ Blei } & \multirow[t]{2}{*}{$\begin{array}{c}\text { Testvariante, } \\
\text { Konzentration } \\
\text { im Gärreaktor } \\
{[\mathrm{mg} / \mathrm{L}]} \\
\text { Kontrolle }\end{array}$} & \multirow[t]{2}{*}{ 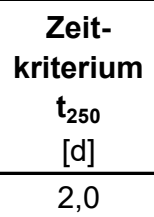 } & \multicolumn{3}{|c|}{$\begin{array}{c}\text { Spezifische } \\
\text { Biogasausbeute } \\
\text { am Versuchsende } \\
{\left[\mathrm{mL}_{\mathrm{N}} / \mathrm{g}_{\mathrm{oTs}}\right]}\end{array}$} \\
\hline \multirow[t]{2}{*}{ Substrat: } & Einwaage & [g] & 6,0 & & & 501 & \pm & 10 \\
\hline & Blei & {$\left[\mathrm{mg} / \mathrm{kg}_{\mathrm{FM}}\right]$} & 0,051 & 20 & 2,4 & 466 & \pm & 6 \\
\hline \multirow[t]{4}{*}{ Inokulum: } & Ausfaulungsgrad & [d] & 14 & 50 & 2,6 & 421 & \pm & 4 \\
\hline & TS; oTS & [Gew.-\%] & 10,$0 ; 71,7$ & 100 & 2,9 & 415 & \pm & 5 \\
\hline & Einwaage & [g] & 400 & 200 & 3,6 & 414 & \pm & 11 \\
\hline & Blei & {$\left[\mathrm{mg} / \mathrm{kg}_{\mathrm{FM}}\right]$} & 1,0 & Nullversuch & - & 95 & \pm & 1 \\
\hline \multicolumn{3}{|c|}{ S/I-Verhältnis*: } & 0,20 & & & & & \\
\hline
\end{tabular}

*: Substrat/Inokulum-Verhältnis (oTS-Verhältnis): goTs (Substrat) / goTs (Inokulum) 


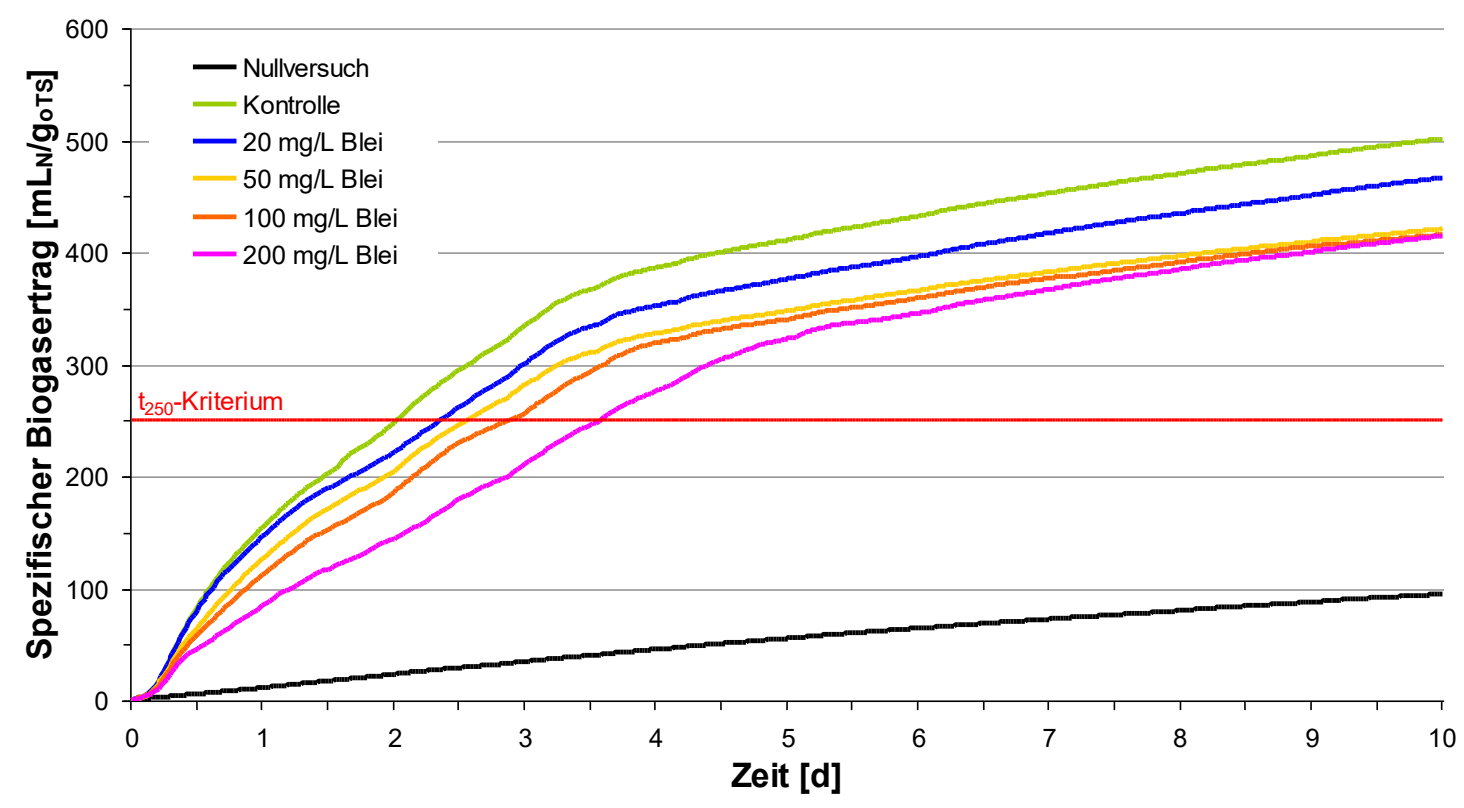

Abbildung 98: Verlauf der gemittelten Gassummenkurven der ORGA-Testreihe zur Überprüfung der Hemm- und Toxizitätswirkung von Blei.

\subsection{Diskussion und Schlussfolgerung}

In Tabelle 27 sind die oberen Hemm- und Toxizitätskonzentrationen der untersuchten Spuren- und potenziellen Schadelemente zusammenfassend dargestellt. Neben der hohen zeitlichen Auflösung, den standardisierten Versuchsbedingungen sowie den vergleichsweise kurzen Versuchszeiträumen ist ein weiterer Vorteil des Testverfahrens, dass einzelne Umsetzungsphasen während des Biogasbildungsprozesses detaillierter betrachtet werden können als in vergleichbaren diskontinuierlichen Gasertragstests wie z.B. in Batchversuchen im Eudiometer oder im Hohenheimer Biogasertragstest.

Tabelle 27: $\quad$ Obere Hemm- und Toxizitätskonzentrationen [mg/L] der mit Hilfe von ORGA-Tests untersuchten Spuren- und potenziellen Schadelemente.

\begin{tabular}{lccc}
\hline Spurenelement & Elementsymbol & $\begin{array}{c}\text { Hemmkonzentration } \\
\text { [mg/L] }\end{array}$ & $\begin{array}{c}\text { Toxizitätskonzentration } \\
\text { [mg/L] }\end{array}$ \\
\hline Cobalt & $\mathrm{Co}$ & 20 & 100 \\
Nickel & $\mathrm{Ni}$ & 35 & 500 \\
Molybdän & $\mathrm{Mo}$ & 100 & n.b. \\
Kupfer & $\mathrm{Cu}$ & 50 & 100 \\
Cadmium & $\mathrm{Cd}$ & 50 & 200 \\
Blei & $\mathrm{Pb}$ & 20 & n.b. \\
\hline n.b.: nicht bestimmt & & &
\end{tabular}


Ein Nachteil ist hingegen, dass lediglich die Menge an Gesamtbiogas erfasst werden kann. Mit dem hier angewandten Gasbildungssystem war eine kontinuierliche aussagekräftige Messung der Gaszusammensetzung nicht möglich. Besonders die Anteile und Verhältnisse von Kohlenstoffdioxid und Methan würden jedoch noch weitere vertiefende Interpretationsmöglichkeiten eröffnen.

Wie bereits in Kapitel 2.2.6 beschrieben, ist es aufgrund der hohen Vielfalt an Bakterien und Archaeen im Biogasbildungsprozess und deren unterschiedlich stark ausgeprägten Adaptionsfähigkeiten an höhere Schwermetallkonzentrationen sowie der Abhängigkeit von den jeweiligen Betriebs- und Prozessparametern schwierig, allgemeingültige Maximalkonzentrationen festzulegen. In der Fachliteratur lassen sich nur wenige Angaben über inhibierende oder toxische Gehalte verschiedener Schwermetalle in anaeroben (Biogas)prozessen finden. Auch variieren diese aufgrund der unterschiedlich betrachteten Medien bzw. eingesetzten Substrate und/oder Testlösungen stark und liegen zum Teil um mehrere Größenordnungen auseinander. Eine vergleichende Auflistung von Literaturwerten oberer Hemm- und Toxizitätskonzentrationen für ausgewählte Schwermetalle in anaeroben Prozessen zeigt Tabelle 28.

Tabelle 28: Zusammenstellung oberer Hemm- und Toxizitätskonzentrationen [mg/L] ausgewählter Schwermetalle in anaeroben Prozessen.

\begin{tabular}{|c|c|c|c|c|c|c|c|c|}
\hline $\begin{array}{l}\text { [mg/L] } \\
\text { Quelle }\end{array}$ & & $\begin{array}{r}\text { Blei } \\
\mathrm{Pb}\end{array}$ & $\begin{array}{r}\text { Cadmium } \\
\mathrm{Cd}\end{array}$ & $\begin{array}{r}\text { Chrom (III) } \\
\text { Cr (III) }\end{array}$ & $\begin{array}{r}\text { Chrom (VI) } \\
\mathrm{Cr}(\mathrm{VI})\end{array}$ & $\begin{array}{r}\text { Kupfer } \\
\mathrm{Cu}\end{array}$ & $\begin{array}{r}\text { Nickel } \\
\mathrm{Ni}\end{array}$ & $\begin{array}{r}\text { Zink } \\
\text { Zn }\end{array}$ \\
\hline \multirow[t]{2}{*}{$a$} & hemmend: & 20 & 50 & & & 50 & 35 & $120^{*}$ \\
\hline & toxisch: & & 200 & & & 100 & 500 & \\
\hline b & hemmend: & & & \multicolumn{2}{|c|}{100 (Chrom) } & 50 & & 150 \\
\hline c & hemmend: & & & & & 10 & 60 & 40 \\
\hline \multirow[t]{2}{*}{ d } & hemmend: & 300 & & 120 & 100 & 40 & 10 & 150 \\
\hline & toxisch: & 340 & 20 & 200 & 200 & 170 & 30 & 250 \\
\hline \multirow[t]{2}{*}{ e } & hemmend: & & & 130 & 110 & 40 & 10 & 400 \\
\hline & toxisch: & 340 & 20 & 260 & 420 & 70 & 30 & 600 \\
\hline
\end{tabular}

a: vorliegende Arbeit ( ${ }^{*}$ : abgeschätzter Wert aus dem Verarmungsversuch für Nickel, vgl. Kapitel 4.3.4); b: Weiland, 2001; c: Zayed \& Winter, 2000; d: Kouzeli-Katsiri \& Kartsonas, 1986; e: Hayes \& Theis, 1978.

In Tabelle 29 wird an den für diese Arbeit verwendeten Energiepflanzenvarianten beispielhaft einmal aufgezeigt, dass bei sehr hohen Inputsubstratmengen eine Hemmwirkung durch zu hohe Konzentrationen im Fermenter nicht möglich ist bzw. welche maximalen Gehalte die Silagen aufweisen müssten, um die oberen Hemmschwellenwerte zu erreichen. Eine Relevanz bestünde ggf. nur für stark kontaminierte Standorte. 
Tabelle 29: Berechnungen zu den verwendeten Energiepflanzen-Silagen für die (quasi)kontinuierlichen Nassfermentationsversuche am DBFZ

(Konzentrationsangaben in $\mathrm{mg} / \mathrm{kg}_{F M}$ ).

\begin{tabular}{lccccc}
\hline [mg/kg & Mais & $\begin{array}{c}\text { Winter- } \\
\text { ackerbohne }\end{array}$ & Amarant & $\begin{array}{c}\text { Durchwachsene } \\
\text { Silphie }\end{array}$ & $\begin{array}{c}\text { Deutsches } \\
\text { Weidelgras* }\end{array}$ \\
\hline 1. Durchschnittsgehalte der im & NiCo-Verbundprojekt verwendeten Energiepflanzen-Silagen: \\
$\mathbf{C o}$ & 0,010 & 0,032 & 0,020 & 0,025 & 0,023 \\
$\mathbf{N i}$ & 0,06 & 0,19 & 0,08 & 0,18 & 0,31 \\
$\mathbf{M o}$ & 0,20 & 0,20 & 0,26 & 0,05 & 1,0 \\
$\mathbf{C u}$ & 1,4 & 1,7 & 1,1 & 1,6 & 1,5 \\
$\mathbf{Z n}$ & 8,5 & 4,9 & 4,0 & 4,1 & 8,2 \\
$\mathbf{C d}$ & 0,019 & 0,005 & 0,026 & 0,009 & 0,008 \\
$\mathbf{P b}$ & 0,06 & 0,05 & 0,09 & 0,11 & 0,12
\end{tabular}

2. Theoretische Konzentrationen im Fermenter bei Monovergärung von $100 \%$ Pflanzensilage ohne weitere Zusatz- oder Prozesshilfsstoffe:

$\begin{array}{llllll}\text { Co } & 0,013 & 0,035 & 0,022 & 0,030 & 0,030 \\ \mathbf{N i} & 0,08 & 0,21 & 0,09 & 0,21 & 0,41 \\ \mathbf{M o} & 0,26 & 0,22 & 0,29 & 0,06 & 1,3 \\ \mathbf{C u} & 1,8 & 1,9 & 1,2 & 1,9 & 2,0 \\ \mathbf{Z n} & 11 & 5,3 & 4,4 & 4,9 & 10,8 \\ \mathbf{C d} & 0,025 & 0,005 & 0,029 & 0,011 & 0,010 \\ \mathbf{P b} & 0,08 & 0,05 & 0,10 & 0,13 & 0,16\end{array}$

3. Theoretische Gehalte, die die Pflanzensilagen aufweisen müssten, um die oberen Hemmkonzentrationen bei Monovergärung von $100 \%$ Pflanzensilage zu erreichen:

\begin{tabular}{llllll}
$\mathbf{C o}$ & 15 & 18 & 18 & 17 & 15 \\
$\mathbf{N i}$ & 27 & 32 & 32 & 29 & 27 \\
$\mathbf{M o}$ & 77 & 92 & 90 & 84 & 76 \\
$\mathbf{C u}$ & 38 & 46 & 45 & 42 & 38 \\
$\mathbf{Z n}$ & 92 & 110 & 108 & 101 & 91 \\
$\mathbf{C d}$ & 38 & 46 & 45 & 42 & 38 \\
$\mathbf{P b}$ & 15 & 18 & 18 & 17 & 15 \\
\hline$*$ & Erster Schnitt & & & &
\end{tabular}

In Punkt 1 sind die Durchschnittsgehalte der im NiCo-Verbundprojekt verwendeten Energiepflanzen-Silagen für die (quasi)kontinuierlichen Nassfermentationsversuche am DBFZ für die sechs mit Hilfe der ORGA-Tests untersuchten Spuren- und potenziellen Schadelemente aufgeführt (vgl. Kapitel 4.2, Tabelle 8). Ergänzt ist die Liste durch das Element Zink, dessen abgeschätzter oberer Hemmschwellenwert aus dem Verarmungsversuch für Nickel stammt (vgl. Kapitel 4.3.4).

Punkt 2 zeigt die über die FM-bezogenen Aufkonzentrationsfaktoren (1,30 für Mais-, 1,09 für Winterackerbohne-, 1,11 für Amarant-, 1,19 für Durchwachsene Silphie- und 1,32 für Deutsches Weidelgras-Silage; vgl. Kapitel 4.2, Tabelle 7) berechneten Gehalte im Fermenter bei einer ausschließlichen Monovergärung von $100 \%$ der jeweiligen Energiepflanzen-Silage. Es wird deutlich, dass alle sieben Elemente sehr weit unter den oberen Hemmschwellenwerten liegen. Selbst das nickel- und molybdänreiche Substrat Deutsches Weidelgras-Silage erreicht „nur“ eine theoretische Konzentration von

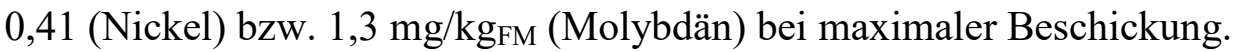


In Punkt 3 ist zusammengestellt, welche maximalen Gehalte (in $\mathrm{mg} / \mathrm{kg}_{\mathrm{FM}}$ ) die Pflanzensilagen unter Beachtung der Aufkonzentrationsfaktoren aufweisen müssten, um bei Monovergärung von 100 \% Inputsubstrat die oberen Hemmschwellenwerte zu erreichen. Die Gehalte in den Pflanzensilagen lägen allerdings so hoch, dass selbst bei einem Anbau auf sehr stark belasteten Flächen lediglich das Spurenelement Zink kritische Werte erreichen könnte (vgl. Elementdaten in SAUER \& RUPPERT, 2013).

Ferner zu erwähnen, dass die mit Hilfe der ORGA-Tests untersuchten Spuren- und potenziellen Schadelemente Cobalt, Nickel, Molybdän, Kupfer, Cadmium und Blei bei dem im Gärsubstrat vorhandenen Schwefel- und Phosphorüberschuss zur Ausfällung als Sulfide und Phosphate bzw. zur Sorption und/oder Komplexierung neigen. Mögliche inhibierende oder toxische Wirkungen auf die Mikroorganismen können so kompensiert werden (vgl. Kapitel 6.3.5 und 6.3.6).

Einen weiteren Diskussionspunkt stellt die Frage dar, inwieweit die ermittelten oberen Hemmkonzentrationen bzw. Toxizitätsschwellenwerte aus diskontinuierlichen Batchversuchen auf die realen Verhältnisse in landwirtschaftlichen Praxisbiogasanlagen übertragbar sind. Um eine gute Vergleichbarkeit quantifizierbarer Größen in den Batchversuchen $\mathrm{zu}$ erreichen, sind zwar die zugrundeliegenden Betriebsparameter und Versuchsbedingungen in den Tests selbst weitestgehend standardisiert, jedoch ist die Anwendbarkeit im Größenmaßstab von Praxisfermentern kaum möglich.

Die Mikroorganismen haben in Batchtests reichlich Zeit, sich an die vorherrschenden Umgebungsbedingungen in den Gärbehältnissen anzupassen. Sie werden nicht ausgespült, sondern nur einmal zu Versuchsbeginn in die Behältnisse gefüllt. Das bedeutet, dass die Schwellenwerte beginnender oberer Hemmwirkungen in den Praxisbiogasanlagen durch den austragsbedingten höheren „Vermehrungsdruck“ der Mikroorganismen vermutlich etwas niedriger als in den Batchversuchen liegen.

Außerdem können die vielschichtigen Wechselwirkungen der Bakterien und Archaeen im Biogasprozess, die bereits erwähnten unterschiedlich langen Adaptionszeiten, aber auch z.B. Komplexbildungen in Abhängigkeit von den jeweiligen Substraten, Prozessund Milieubedingungen großen Einfluss auf die inhibierende Wirkung von Spuren- und Schadelementen ausüben. Dennoch liefern die Ergebnisse aus den diskontinuierlichen Gärversuchen sehr wichtige Richtwerte für Praxisfermenter zur Beurteilung der Prozessstabilität im extremen Konzentrationsbereich. 


\section{Kapitel 6: Vereinfachtes Bestimmungsverfahren}

\section{1 Überblick}

In Rahmen des Forschungsvorhabens wurden als weiterer Bestandteil Verfahren zur Bestimmung der für den Biogasprozess wichtigen Spurenelementgehalte im Fermentermaterial getestet mit dem Ziel einer Vereinfachung und zeitlicher Einsparungen. Es wurden zudem Trennungs- und Filtrationsversuche mit verschiedenen Fraktionen des Fermentermaterials durchgeführt, um den reaktiven Spurenelementanteil im Fermenter besser charakterisieren zu können und Rückschlüsse auf die Verfügbarkeit der Spurenelemente zu gewinnen.

Hintergrund ist, dass bislang die Ermittlung der Elementgehalte im Fermentermaterial oft anhand von genommenen Festproben zeitaufwändig über die Schritte der Probenaufbereitung und eines Säureaufschlussverfahrens mit anschließender Multielementbestimmung (vgl. Kapitel 3.4.3 und 3.4.4) erfolgt. Mit der vereinfachten Methode kann der in dieser Arbeit angewandte ,lange Weg“ in Form der Trocknung repräsentativer Probenaliquote, einer anschließenden Vorzerkleinerung und Feinmahlung und eines mehrstufigen Verfahrens des Säuretotalaufschlusses (vgl. Kapitel 3.2 und 3.3) deutlich verkürzt und somit der nötige Zeitraum zwischen der Probenahme, Aufbereitung, Analyse, Interpretation und Anwendung in der Praxis wesentlich reduziert werden, von etwa zwei Wochen auf nur wenige Tage. Für Biogasanlagenbetreiber und Analyselabore brächte das erhebliche Vorteile, z.B. die Einsparung von Arbeitszeit und Kosten sowie ein deutlich frühzeitigeres Erkennen und Gegensteuern bei kritischen Spurenelementmangelsituationen im Fermenter (vgl. Kapitel 2.3).

\subsection{Durchführung von Versuchen mit verschiedenen Fraktionen von Fermentermaterialproben}

In ersten Filtrationstests wurde versucht, jeweils unverdünnte sowie 1:10 bzw. 1:20 verdünnte Fermentermaterialproben aus der Praxisbiogasanlage in Verliehausen über $50 \mathrm{~mL}-$ Spritzen mit Spritzenvorsatzfiltern durch eine 0,45 $\mu \mathrm{m}$-PFTE-Membran zu filtrieren. Diese Versuche scheiterten, da sich die Vorsatzfilter sofort zusetzten und kein Tropfen die Filter passierte. Ebenso waren Versuche des Zentrifugierens zur Trennung der Fest- und Flüssigphasen von unverdünnten sowie 1:10 bzw. 1:20 verdünnten 
Fermenterproben mit der Universal-Tischzentrifuge Heraeus Multifuge $3 S$ nicht erfolgreich, bedingt durch den sehr geringen Dichteunterschied des organischen Reaktormaterials gegenüber Wasser.

Hiernach wurden Unterdruckfiltrationsversuche zur Trennung der Fest- und Flüssigphasen von unverdünnten sowie 1:5 bzw. 1:10 verdünnten Fermenterproben aus der Praxisbiogasanlage in Verliehausen über verschiedene Feinfiltergrößen (von 0,45 bis $40 \mu \mathrm{m})$ getestet. Die Proben wurden hierfür im Vorfeld der Tests zunächst durch ein Kunststoffsieb (Maschenweite ca. $2 \mathrm{~mm}$ ) gegeben, um gröbere Bestandteile im Fermentermaterial zu separieren. Die Filtrationsversuche wurden mit einer MembranVakuumpumpe durchgeführt, im Filtriersystem konnte ein Unterdruck bis minimal 1 bar $(100 \mathrm{kPa})$ erzeugt werden. Bei den getesteten unverdünnten Fermenterproben setzten sich die sehr feinen Filtergrößen (bis $5 \mu \mathrm{m}$ ) jedoch sofort zu, bei den anderen Feinfiltergrößen (bis $40 \mu \mathrm{m}$ ) passierten die Proben nur anfangs über einen sehr kurzen Zeitraum die Feinfilter, bis sich auch diese zusetzten. Von den verdünnten Fermenterproben konnten in einem Zeitraum von mehr als sechs Stunden nur sehr wenige Milliliter gewonnen werden, und das auch nur über die gröberen Filtergrößen. Zusammenfassend reichte in allen Versuchen der Unterdruck nicht aus, um in einem zufriedenstellenden Zeitraum eine für die Multielementbestimmung ausreichende Probenmenge abzufiltrieren.

Nach den Unterdruckfiltrationsversuchen wurden Trennungsversuche und Testreihen in Teflon-Filtrationsapparaturen durchgeführt. Die Fermenterproben wurden zunächst ebenfalls durch ein grobmaschigeres Kunststoffsieb (Maschenweite ca. $2 \mathrm{~mm}$ ) gerührt und fest gepresst, um gröbere Bestandteile im Fermentermaterial abzutrennen. Anschließend wurden die grob separierten Proben durch ein weiteres Prüfsieb aus Aluminium mit einer definierten Maschenweite von $63 \mu \mathrm{m}$ (Rhewum Typ DIN 4188) gepresst. Dieser Vorgang wurde mit den Rückständen in den Sieben mehrmals wiederholt, um die Proben möglichst vollständig zu separieren.

Neben den Gesamtproben lagen somit zwei Fraktionen vor: die Siebrückstände, im Folgenden als „Pressrückstände ( $>63 \mu \mathrm{m}$ )“ bezeichnet, und die $<63 \mu \mathrm{m}$-Fraktionen, im Folgenden „Presssäfte $(<\mathbf{6 3} \boldsymbol{\mu m}$ )“ genannt. Pressrückstände $(>63 \mu \mathrm{m})$ und Presssäfte $(<63 \mu \mathrm{m})$ wurden von Fermenterproben aus der Praxisbiogasanlage in Verliehausen sowie von Proben des Reaktormaterials aus den Laborfermentern der dritten Projektphase am DBFZ (vgl. Kapitel 4.5) gewonnen und getestet. 
Aliquote der Gesamtproben sowie aller in den Versuchsreihen getesteten Pressrückstände $(>63 \mu \mathrm{m})$ wurden bei $105^{\circ} \mathrm{C}$ bis zur Gewichtskonstanz getrocknet, mit der Planeten-Kugelmühle analysenfein gemahlen (vgl. Kapitel 3.2), mittels des Säuretotalaufschlussverfahrens vollständig in Lösung gebracht (vgl. Kapitel 3.3) und die Elementgehalte mittels ICP-OES-Messungen (vgl. Kapitel 3.4.3) bestimmt.

Presssäfte $(<63 \mu \mathrm{m})$ von ausgewählten Materialproben wurden dann in verschiedenen Verdünnungsstufen (1:5, 1:10, 1:20 und 1:50) mit den von Prof. Dr. Hans Ruppert in der Arbeitsgruppe Umweltgeochemie entwickelten Teflon-Filtrationsapparaturen, in der alle Teile, die mit den Probenlösungen in Berührung kommen, aus reinem Polytetrafluorethylen (PTFE, Teflon) bestehen, filtriert. Die Apparaturen waren an das Druckluftsystem der Labore am GZG angeschlossen, das einen maximalen Druck bis 4,5 bar (450 kPa) ermöglicht. Die Porenweite der Cellulose-Acetat-Filter betrug 0,45 $\mu \mathrm{m}$. Im Folgenden werden diese Fraktionen als „Filtrate $(<\mathbf{0 , 4 5} \boldsymbol{\mu m})$ “ bezeichnet.

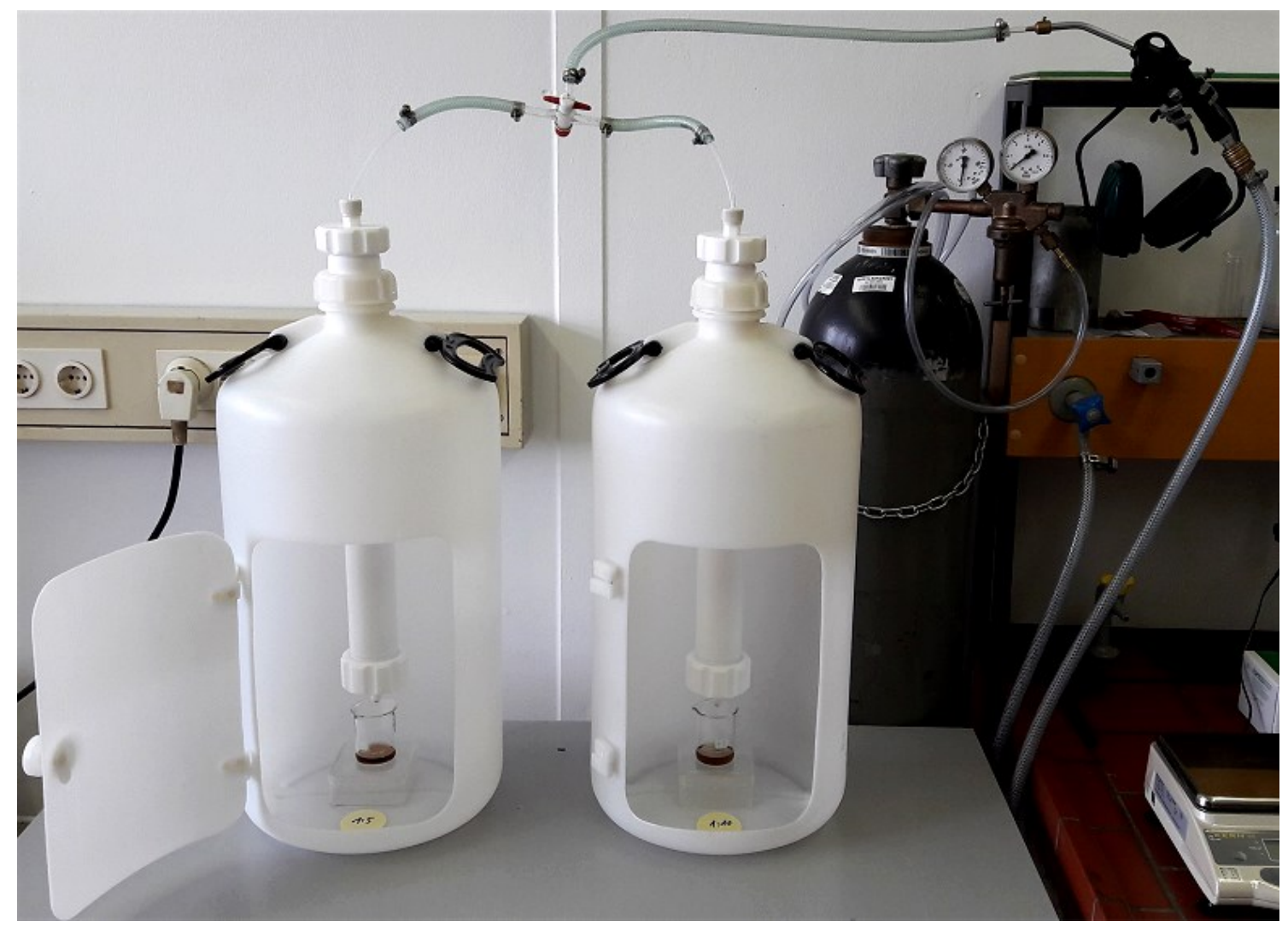

Abbildung 99: Fotoaufnahme von zwei Teflon-Filtrationsapparaturen mit Filtraten $(<0,45 \mu \mathrm{m})$ einer Fermenterprobe in den beiden Verdünnungsstufen 1:5 (links) und 1:10 (rechts). 
Auch bei diesen Versuchen war jeweils eine mehrstündige Filtrationsdauer erforderlich, um eine ausreichende Probenmenge (Zielvorgabe: mindestens $20 \mathrm{~mL}$ Filtrat) für die Multielementbestimmung mittels ICP-OES (vgl. Kapitel 3.4.3) zu erhalten. Die Filtrationsdauer der 1:5-Verdünnungen lag durchschnittlich bei ca. 300 Minuten. Im Vergleich dazu waren die Filtrationszeiten der anderen Verdünnungsstufen kürzer und lagen bei den 1:10-Verdünnungen im Schnitt bei 250 Minuten bzw. 190 (1:20) und 100 Minuten (1:50-Verdünnungen).

Anschließend wurden in die PTFE-Becher des Druckaufschluss-Systems (PicoTrace $D A S$ 30, vgl. Kapitel 3.3) jeweils $9 \mathrm{~mL}$ der Filtrate $(<\mathbf{0 , 4 5} \boldsymbol{\mu m})$ der ausgewählten Fermenterproben in den jeweils vier verschiedenen Verdünnungsstufen mit $1 \mathrm{~mL}$ 65 \%iger hochreiner Salpetersäure $\left(\mathrm{HNO}_{3}\right)$ versetzt (Matrix: 6,5\% \% ige $\left.\mathrm{HNO}_{3}\right)$ und ein interner Standard mit definierter Elementkonzentration (50 ppm Beryllium) zugegeben. Das interne Standard-Element Beryllium diente bei den folgenden ICP-OES-Messungen zur Überprüfung der Richtigkeit und Verlustfreiheit des $\mathrm{HNO}_{3}$-Säureaufschlusses bzw. der Qualität der Messergebnisse (vgl. Kapitel 3.5.2). Danach wurden die Becherdeckel aufgelegt und zwei verschiedene Heizprogramme getestet:

\section{Variante 1 - Langsames Aufkochen mit $\mathrm{HNO}_{3}$ mit langer Haltephase bei $100{ }^{\circ} \mathrm{C}$ :}

$6 \mathrm{~h}$ hochheizen auf $30^{\circ} \mathrm{C}$ und $6 \mathrm{~h}$ halten dieser Temperatur, dann hochheizen in $6 \mathrm{~h}$ von $30{ }^{\circ} \mathrm{C}$ auf $50{ }^{\circ} \mathrm{C}$ und $6 \mathrm{~h}$ halten dieser Temperatur, danach hochheizen in $6 \mathrm{~h}$ von $50{ }^{\circ} \mathrm{C}$ auf $100^{\circ} \mathrm{C}$ und $12 \mathrm{~h}$ halten dieser Temperatur, abschließend auf Raumtemperatur abkühlen lassen.

\section{Variante 2 - Schnelles Aufkochen mit $\mathrm{HNO}_{3}$ mit kurzer Haltephase bei $100{ }^{\circ} \mathrm{C}$ :}

$2 \mathrm{~h}$ hochheizen auf $100^{\circ} \mathrm{C}$ und $2 \mathrm{~h}$ halten dieser Temperatur, dann auf Raumtemperatur abkühlen lassen.

Filtrate $(<0,45 \mu \mathrm{m})$ in den jeweiligen Verdünnungsstufen 1:5, 1:10, 1:20 und 1:50 sowie den beiden $\mathrm{HNO}_{3}$-Aufschlussvarianten wurden an zwei Fermenter-, einer Nachgärersowie einer Gärrestprobe aus der Praxisbiogasanlage in Verliehausen getestet. Die Praxisbiogasanlage wird hauptsächlich mit Energiepflanzen-Silagen (v.a. Mais und GPS), aber auch zum Teil mit Gülle (Rindergülle) und synthetischen Spurenelementadditiven beschickt. 
Die Presssäfte $(<\mathbf{6 3} \boldsymbol{\mu m})$ der ausgewählten Fermenterproben wurden in den PTFEBechern mit Reinstwasser (Milli-Q) und 65\%iger Salpetersäure (Matrix: 6,5\%ige $\mathrm{HNO}_{3}$, entsprechend der $<0,45 \mu \mathrm{m}$-Filtratproben) mit einem Verdünnungsfaktor von 1:20 verdünnt, ebenfalls mit einem internen Standard mit $50 \mathrm{ppm}$ Beryllium versetzt und die Becherdeckel aufgelegt. Als Heizprogramm wurde die Variante 1 gewählt (langsames Aufkochen mit $\mathrm{HNO}_{3}$ mit langer Haltephase bei $100{ }^{\circ} \mathrm{C}$ ).

Abschließend wurden die leicht gelblichen, aber rückstandsfreien, klaren Aufschlusslösungen der Filtrate $(<0,45 \mu \mathrm{m})$ bzw. Presssäfte $(<63 \mu \mathrm{m})$ aus den PTFE-Bechern in Polyethylen (PE)-Fläschchen für die Multielementanalysen mittels ICP-OES überführt. Für die spätere Blindwertkorrektur und Berechnung der Nachweisgrenzen wurden bei allen Salpetersäureaufschlüssen zudem Blindproben $\left(6,5 \%\right.$ ige $\left.\mathrm{HNO}_{3}\right)$ in Mehrfachwiederholung mitgeführt (vgl. Kapitel 3.4.3).

\subsection{Ergebnisse und Interpretation der Trennungs- und Filtrations- versuche}

\subsubsection{Elementmengenvergleiche zur Abschätzung der Aufschlusseffizienz}

Um die Effizienz der in den Versuchen durchgeführten Salpetersäureaufschlüsse zu beurteilen, wurden von allen Fraktionen (mit Ausnahme der Filtrate $<0,45 \mu \mathrm{m}$ ) und den Gesamtproben gravimetrisch die Massen bestimmt. Die jeweiligen Elementmengen (in $\mathrm{mg}$ ) in den Presssäften $(<63 \mu \mathrm{m})$ wurden aus den Massen (in $\mathrm{kg}$ ) und den gemessenen Elementkonzentrationen (in $\mathrm{mg} / \mathrm{kg}_{\mathrm{FM}}$ ) berechnet. Aus den Massen und Elementgehalten der Pressrückstände $(>63 \mu \mathrm{m})$ und Gesamtproben, die mittels des etablierten mehrstufigen Säuretotalaufschlussverfahrens (vgl. Kapitel 3.3) vollständig in Lösung gebracht wurden, wurden ebenfalls die jeweiligen Elementmengen (in mg) berechnet.

In Tabelle 30 sind beispielhaft die prozentualen Werte des Vergleichs der Summen der Elementmengen in den Pressrückständen $(>63 \mu \mathrm{m})$ plus den Presssäften $(<63 \mu \mathrm{m})$ gegenüber den Elementmengen in den Gesamtproben der Materialproben aus dem Kontrollreaktor (Referenz) der dritten Projektphase am DBFZ (vgl. Kapitel 4.1 und 4.5) sowie der Fermentermaterialprobe aus der Praxisbiogasanlage in Verliehausen (Fermenter BGA 07/17) zusammengestellt. Zum besseren Überblick der Abweichungen zwischen den Summenwerten gegenüber den Gesamtmengen liegt den Prozentwerten in der Tabelle eine Farbskala in den Ampelfarben zugrunde: 0 bis $15 \%$ Abweichung von $100 \%$ grün, 16 bis $30 \%$ gelb und $>30 \%$ rot. 
Tabelle 30: $\quad$ Vergleich der Summe der Elementmengen im Pressrückstand ( $>63 \mu \mathrm{m})$ plus Presssaft $(<63 \mu \mathrm{m})$ gegenüber der Elementmenge in der Gesamtprobe ausgewählter Referenzreaktorproben der dritten Projektphase am DBFZ und einer Fermentermaterialprobe (Fermenter BGA 07/17) aus der Praxisbiogasanlage in Verliehausen.

\begin{tabular}{|c|c|c|c|c|c|c|c|c|c|c|}
\hline \multirow[b]{2}{*}{ Probe } & \multicolumn{6}{|c|}{ Makronährstoffe } & \multicolumn{4}{|c|}{ Mikronährstoffe } \\
\hline & $\mathbf{P}$ & $\mathbf{s}$ & $\mathbf{C a}$ & $\mathbf{K}$ & $\mathrm{Mg}$ & $\mathrm{Na}$ & Co & $\mathrm{Ni}$ & Mo & $\mathrm{Fe}$ \\
\hline Referenz DBFZ VP3 (VT105) & $98 \%$ & $105 \%$ & $117 \%$ & $121 \%$ & $114 \%$ & $77 \%$ & $109 \%$ & $96 \%$ & $110 \%$ & $110 \%$ \\
\hline Referenz DBFZ VP3 (VT126) & $87 \%$ & $97 \%$ & $103 \%$ & $114 \%$ & $105 \%$ & $89 \%$ & $104 \%$ & $94 \%$ & $104 \%$ & $97 \%$ \\
\hline Referenz DBFZ VP3 (VT147) & $91 \%$ & $96 \%$ & $78 \%$ & $113 \%$ & $104 \%$ & $85 \%$ & $100 \%$ & $92 \%$ & $103 \%$ & $103 \%$ \\
\hline Referenz DBFZ VP3 (VT203) & $92 \%$ & $91 \%$ & $97 \%$ & $105 \%$ & $103 \%$ & $111 \%$ & $101 \%$ & $91 \%$ & $101 \%$ & $105 \%$ \\
\hline Referenz DBFZ VP3 (VT224) & $90 \%$ & $93 \%$ & $109 \%$ & $106 \%$ & $100 \%$ & $114 \%$ & $99 \%$ & $97 \%$ & $104 \%$ & $104 \%$ \\
\hline \multirow[t]{2}{*}{ Fermenter BGA 07/17 } & $78 \%$ & $92 \%$ & $97 \%$ & $111 \%$ & $76 \%$ & $99 \%$ & $99 \%$ & $82 \%$ & $101 \%$ & $85 \%$ \\
\hline & \multicolumn{3}{|c|}{ Mikronährstoffe } & \multicolumn{3}{|c|}{ Weitere Elemente } & & & & \\
\hline Probe & $\mathrm{Cu}$ & Mn & Zn & Al & $\mathrm{Ba}$ & $\mathrm{Cr}$ & Li & $\mathrm{Pb}$ & $\mathrm{Sr}$ & $\mathrm{Ti}$ \\
\hline Referenz DBFZ VP3 (VT105) & $124 \%$ & $109 \%$ & $124 \%$ & $64 \%$ & $98 \%$ & $92 \%$ & $82 \%$ & $108 \%$ & $116 \%$ & $55 \%$ \\
\hline Referenz DBFZ VP3 (VT126) & $122 \%$ & $94 \%$ & $119 \%$ & $77 \%$ & $93 \%$ & $94 \%$ & $89 \%$ & $114 \%$ & $102 \%$ & $50 \%$ \\
\hline Referenz DBFZ VP3 (VT147) & $117 \%$ & $76 \%$ & $118 \%$ & $81 \%$ & $103 \%$ & $97 \%$ & $80 \%$ & $112 \%$ & $98 \%$ & $56 \%$ \\
\hline Referenz DBFZ VP3 (VT203) & $110 \%$ & $111 \%$ & $115 \%$ & $75 \%$ & $99 \%$ & $94 \%$ & $86 \%$ & $109 \%$ & $104 \%$ & $43 \%$ \\
\hline Referenz DBFZ VP3 (VT224) & $112 \%$ & $104 \%$ & $104 \%$ & $78 \%$ & $102 \%$ & $107 \%$ & $83 \%$ & $95 \%$ & $113 \%$ & $44 \%$ \\
\hline Fermenter BGA 07/17 & $106 \%$ & $96 \%$ & $104 \%$ & $63 \%$ & $79 \%$ & $53 \%$ & $84 \%$ & $93 \%$ & $95 \%$ & $39 \%$ \\
\hline
\end{tabular}

Es wird deutlich, dass die Summenmengen in den Pressrückständen $(>63 \mu \mathrm{m})$ und Presssäften $(<63 \mu \mathrm{m})$ gegenüber den Elementmengen in den Gesamtproben für alle Makronährstoffe und essenziellen Spurenelemente sowie einige weitere Elemente gut übereinstimmen, was die Konsistenz der Probenahme, Probenaufbereitung und Analytik bedeutet. Das heißt, dass diese Elemente in den nur mit Salpetersäure aufgeschlossenen Presssäften $(<63 \mu \mathrm{m})$ eine gute Aufschlusseffizienz aufweisen. Sie konnten vollständig in Lösung gebracht werden, trotz der schwächeren Salpetersäurekonzentration in den Lösungen bzw. noch vorhandener organischer Rückstände in den überführten Aufschlusslösungen, auf die die gelblichen Farben hinweisen. Es wurden visuell keinerlei Trübungen in diesen Lösungen festgestellt (vgl. Kapitel 6.2).

Die niedrigen Konzentrationsverhältnisse in den Presssaftproben $(<63 \mu \mathrm{m})$ von Aluminium und Titan sowie teilweise Chrom (Fermentermaterialprobe aus der Praxisbiogasanlage in Verliehausen) bzw. die Unterschiede der Summenmengen der beiden Fraktionen zur Gesamtprobe (Tabelle 30) sind jedoch vermutlich aufschlussbedingt induziert. Die im Aufschluss verwendete Salpetersäure konnte unter den gewählten Versuchsbedingungen (vgl. Kapitel 6.2) diese Elemente in den Presssäften $(<63 \mu \mathrm{m})$ nicht vollständig aufschließen. Eine Ursache der Minderbefunde sind vermutlich schwerlösliche Phasen im Reaktor- bzw. Fermentermaterial, z.B. Oxide oder Silikate 
von Aluminium, Titan oder Chrom, die von anhaftenden Boden- und Staubpartikeln in den als Inputsubstrat verwendeten (Mais-)Silagen (vgl. POSPIECH ET AL., 2017b) oder anderen Einträgen (z.B. Materialabrieb) stammen.

\subsubsection{Elementkonzentrationen der Filtrate $(<0,45 \mu \mathrm{m})$}

Die Elementgehalte der Verdünnungsvarianten der getesteten Filtrate $(<0,45 \mu \mathrm{m})$ waren nach den Salpetersäureaufschlüssen mit der ICP-OES gut nachweis-, mess- und vergleichbar. Für die Dateninterpretation wurden 20 Elemente verwendet. Lediglich die 1:50-Verdünnungen zeigten aufgrund sehr niedriger Lösungskonzentrationen im Bereich der Nachweisgrenzen zum Teil größere Abweichungen gegenüber den drei anderen Verdünnungsstufen. In Tabelle 31 sind beispielhaft die Elementgehalte der Filtratproben $(<0,45 \mu \mathrm{m})$ der 1:20-Verdünnungen der Aufschlussvariante 1 (langsames Aufkochen mit $\mathrm{HNO}_{3}$ mit langer Haltephase bei $100{ }^{\circ} \mathrm{C}$ ) bzw. der Aufschlussvariante 2 (schnelles Aufkochen mit $\mathrm{HNO}_{3}$ mit kurzer Haltephase bei $100{ }^{\circ} \mathrm{C}$ ) sowie die Elementkonzentrationen der zugehörigen Gesamtproben (Säuretotalaufschluss) aufgeführt.

Es kann festgestellt werden, dass sich die beiden Aufschlussvarianten der Filtratproben $(<0,45 \mu \mathrm{m})$ nur geringfügig voneinander unterscheiden. D.h. ein langsames Aufkochen mit Salpetersäure mit langen Zwischenstufen und langer Haltephase bei $100{ }^{\circ} \mathrm{C}$ (Variante 1) liefert gegenüber einem schnellen Aufkochen mit $\mathrm{HNO}_{3}$ und kurzer Haltephase bei $100{ }^{\circ} \mathrm{C}$ (Variante 2 ) bei den mittels ICP-OES gut bestimmbaren Elementen keine verbesserte Aufschlusseffizienz. Jedoch besteht bei der schnelleren Aufschlussvariante eine potenziell höhere Gefahr des Überschäumens bzw. Überkochens der Proben. So sind entsprechend große Probengefäße bzw. entsprechend angepasste Proben- und Säurevolumina zu wählen.

Um die bei den Trennungs- und Filtrationsversuchen gewonnenen Fraktionen besser miteinander vergleichen $\mathrm{zu}$ können, wurden die Elementkonzentrationen in den Fraktionen in Bezug zu den Elementkonzentrationen in der Gesamtprobe gesetzt. D.h. ein Wert von 1 entspricht der Elementkonzentration in der zugehörigen Gesamtprobe. Ein Wert $>1$ spiegelt einen höheren bzw. ein Wert $<1$ einen niedrigeren Elementgehalt gegenüber der Gesamtprobe wider. Die Elementkonzentrationsverhältnisse der 1:20Verdünnungen der getesteten Filtratproben $(<0,45 \mu \mathrm{m})$ der Aufschlussvariante 1 bzw. 2 in Bezug zu den Gesamtproben sind in Abbildung 100 und Abbildung 101, angeordnet in drei wesentlichen Gruppen (vgl. Kapitel 2.2.5), grafisch dargestellt. 
Tabelle 31: $\quad$ Übersicht der Elementkonzentrationen [mg/ $\left./ \mathrm{kg}_{F M}\right]$ der Filtratproben (<0,45 $\mu \mathrm{m}, 1: 20$-Verdünnungen, Aufschlussvarianten V1 und V2) und der Gesamtproben verschiedener Gärmaterialien aus der Praxisbiogasanlage (BGA) in Verliehausen.

\begin{tabular}{|c|c|c|c|c|c|c|c|c|c|c|c|}
\hline \multirow[t]{2}{*}[\mathrm{mg}/\mathrm{kg}_{\mathrm{FM}}]{} & \multicolumn{6}{|c|}{ Variante Makronährstoffe } & \multicolumn{5}{|c|}{ Mikronährstoffe } \\
\hline & & $\mathbf{P}$ & $\mathbf{s}$ & $\mathrm{Ca}$ & $\mathbf{K}$ & $\mathbf{M g}$ & $\mathrm{Na}$ & Co & $\mathbf{N i}$ & Mo & $\mathbf{F e}$ \\
\hline \multirow[t]{3}{*}{ Fermenter BGA 07/17 } & Filtrat (V1) & 294 & 81 & 123 & 4777 & 85 & 267 & 0,02 & 0,16 & 0,13 & 17 \\
\hline & Filtrat (V2) & 292 & 81 & 124 & 4756 & 85 & 272 & 0,03 & 0,16 & 0,13 & 17 \\
\hline & Gesamtprobe & 1065 & 483 & 1427 & 5048 & 659 & 319 & 0,17 & 0,93 & 0,37 & 466 \\
\hline \multirow[t]{3}{*}{ Fermenter BGA 05/16 } & Filtrat (V1) & 154 & 57 & 118 & 3190 & 62 & 207 & 0,03 & 0,20 & 0,05 & 17 \\
\hline & Filtrat (V2) & 150 & 55 & 113 & 3032 & 60 & 197 & 0,02 & 0,24 & 0,05 & 17 \\
\hline & Gesamtprobe & 790 & 360 & 1199 & 3283 & 513 & 208 & 0,15 & 0,67 & 0,25 & 306 \\
\hline \multirow[t]{3}{*}{ Nachgärer BGA 05/16 } & Filtrat (V1) & 179 & 66 & 137 & 3064 & 72 & 181 & 0,04 & 0,24 & 0,07 & 28 \\
\hline & Filtrat (V2) & 173 & 63 & 132 & 2894 & 70 & 172 & 0,04 & 0,23 & 0,06 & 27 \\
\hline & Gesamtprobe & 807 & 350 & 1148 & 3199 & 521 & 215 & 0,12 & 0,57 & 0,24 & 236 \\
\hline \multirow[t]{5}{*}{ Gärrest BGA 03/16 } & Filtrat (V1) & 250 & 71 & 145 & 3684 & 80 & 181 & 0,03 & 0,26 & 0,07 & 25 \\
\hline & Filtrat (V2) & 253 & 70 & 145 & 3645 & 81 & 180 & 0,04 & 0,27 & 0,06 & 25 \\
\hline & Gesamtprobe & 787 & 374 & 1084 & 3667 & 451 & 188 & 0,13 & 0,64 & 0,24 & 200 \\
\hline & \multicolumn{4}{|c|}{ Mikronährstoffe } & \multicolumn{3}{|c|}{ Weitere Elemente } & & & & \\
\hline & & $\mathrm{Cu}$ & Mn & $\mathrm{Zn}$ & Al & $\mathbf{B a}$ & $\mathrm{Cr}$ & Li & $\mathbf{P b}$ & $\mathrm{Sr}$ & $\mathrm{Ti}$ \\
\hline \multirow[t]{3}{*}{ Fermenter BGA 07/17 } & Filtrat (V1) & 0,10 & 2,0 & 0,31 & 0,58 & 0,35 & 0,06 & 0,03 & 0,01 & 0,63 & 0,23 \\
\hline & Filtrat (V2) & 0,09 & 2,0 & 0,30 & 0,63 & 0,35 & 0,06 & 0,02 & 0,01 & 0,62 & 0,24 \\
\hline & Gesamtprobe & 4,7 & 43 & 21 & 362 & 9,4 & 1,7 & 0,23 & 0,43 & 5,4 & 37 \\
\hline \multirow[t]{3}{*}{ Fermenter BGA 05/16 } & Filtrat (V1) & 0,05 & 1,2 & 0,17 & 0,78 & 0,18 & 0,04 & 0,02 & 0,02 & 0,49 & 0,15 \\
\hline & Filtrat (V2) & 0,05 & 1,1 & 0,19 & 0,29 & 0,17 & 0,12 & 0,02 & 0,01 & 0,46 & 0,13 \\
\hline & Gesamtprobe & 3,3 & 28 & 17 & 252 & 5,5 & 1,1 & 0,16 & 0,19 & 3,9 & 27 \\
\hline \multirow[t]{3}{*}{ Nachgärer BGA 05/16 } & Filtrat (V1) & 0,20 & 1,1 & 0,31 & 0,60 & 0,26 & 0,06 & 0,01 & 0,07 & 0,62 & 0,21 \\
\hline & Filtrat (V2) & 0,20 & 1,1 & 0,34 & 0,46 & 0,25 & 0,05 & 0,01 & 0,06 & 0,58 & 0,20 \\
\hline & Gesamtprobe & 4,0 & 25 & 18 & 213 & 5,0 & 0,84 & 0,15 & 0,17 & 3,7 & 23 \\
\hline \multirow[t]{3}{*}{ Gärrest BGA 03/16 } & Filtrat (V1) & 0,85 & 2,0 & 1,08 & 0,49 & 0,34 & 0,07 & 0,01 & 0,13 & 0,72 & 0,22 \\
\hline & Filtrat (V2) & 0,84 & 2,0 & 1,13 & 0,43 & 0,32 & 0,06 & 0,02 & 0,14 & 0,70 & 0,19 \\
\hline & Gesamtprobe & 22 & 25 & 23 & 211 & 5,8 & 0,84 & 0,14 & 0,76 & 3,8 & 22 \\
\hline
\end{tabular}

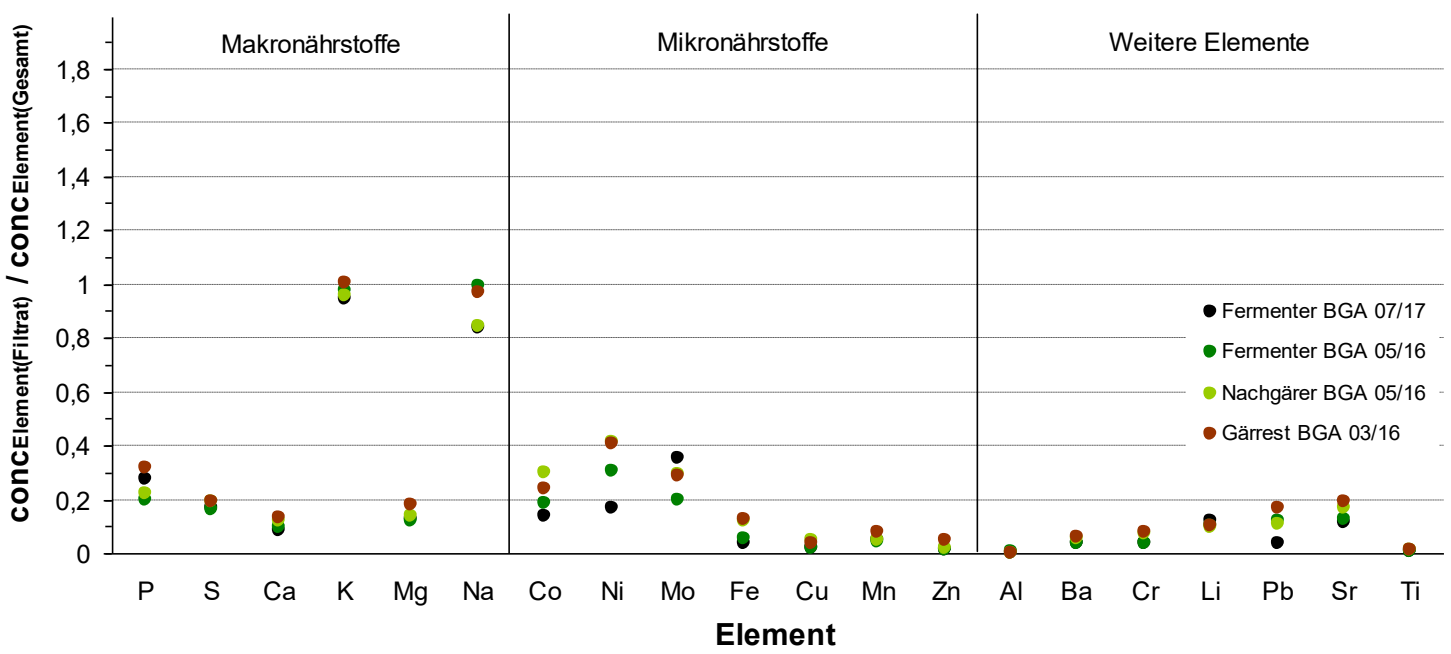

Abbildung 100: Elementkonzentrationsverhältnisse der Filtratproben $(<0,45 \mu \mathrm{m}$, 1:20-Verdünnungen, Aufschlussvariante 1) verschiedener Gärmaterialien aus der Praxisbiogasanlage in Verliehausen. 


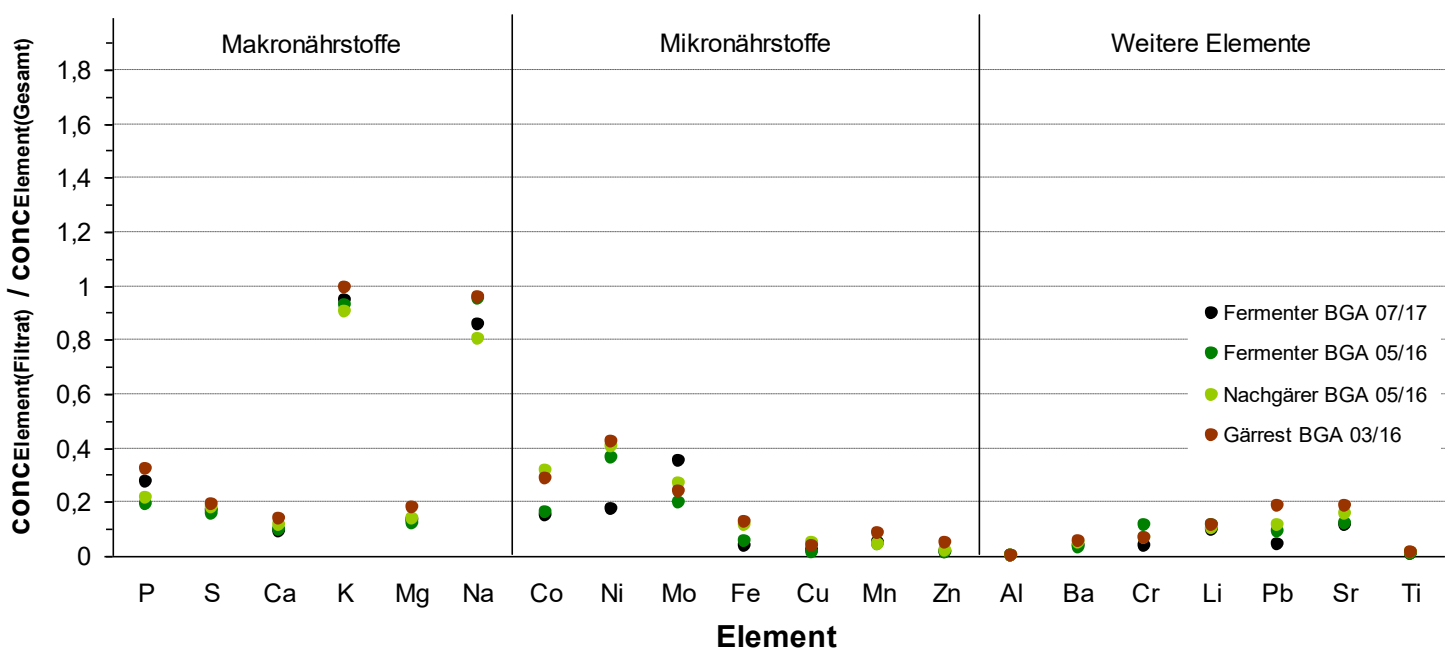

Abbildung 101: Elementkonzentrationsverhältnisse der Filtratproben $(<0,45 \mu \mathrm{m}$, 1:20-Verdünnungen, Aufschlussvariante 2) verschiedener Gärmaterialien aus der Praxisbiogasanlage in Verliehausen.

Insgesamt variieren die Verhältnisse auf einem sehr niedrigen Niveau. Auffällig sind aber die Kalium- und Natrium-Verhältnisse, die mit Werten zwischen 0,8 und 1 nahezu im Bereich der Gesamtprobe liegen. Die weiteren Makronährstoffe variieren zwischen 0,09 (Calcium) und 0,32 (Phosphor) auf einem geringeren Niveau. Gleiches gilt für die für den Biogasprozess wichtigen Spurenelemente Cobalt, Nickel und Molybdän, die zwischen 0,14 und 0,42 liegen. Alle anderen Mikronährstoffe und weiteren Elemente liegen unterhalb von 0,2 und finden sich in den Filtraten $(<0,45 \mu \mathrm{m})$ kaum wieder. Weitere Betrachtungen sind in Kapitel 6.3.5 aufgeführt.

\subsubsection{Elementkonzentrationen der Presssäfte $(<63 \mu \mathrm{m})$}

Die Presssäfte $(<63 \mu \mathrm{m})$ waren nach den $\mathrm{HNO}_{3}$-Säureaufschlüssen ebenfalls mittels ICP-OES elementanalytisch gut nachweis- und messbar. Analog zu den Filtraten $(<0,45 \mu \mathrm{m})$ wurden 20 Elemente verglichen. In Tabelle 32 sind beispielhaft die Elementkonzentrationen der Presssäfte $(<63 \mu \mathrm{m})$ und zugehörigen Gesamtproben (Säuretotalaufschluss) von Fermentermaterial aus dem Referenzreaktor der dritten Projektphase am DBFZ von fünf Versuchstagen mit kontinuierlich stabilem Prozessverlauf (Versuchstage 105, 126, 147, 203 und 224) dargestellt. Dieser Reaktor wurde über die gesamte Projektdauer (quasi)kontinuierlich mit Mais-Silage-Monovergärung und einer zusätzlichen synthetischen Spurenelementvollversorgung (Co, Ni, Mo, Fe, Mn, Zn, Se und W) betrieben (vgl. Kapitel 4.1 und 4.5). Als Praxisvergleich ist zudem eine Fermentermaterialprobe aus der Biogasanlage in Verliehausen aufgeführt. Abbildung 102 zeigt die Elementkonzentrationsverhältnisse. 
Tabelle 32: $\quad$ Übersicht der Elementkonzentrationen [mg/kg $\left.{ }_{F M}\right]$ der Presssäfte $(<63 \mu \mathrm{m}, P S)$ und der Gesamtproben $(G)$ ausgewählter Referenzreaktorproben der dritten Projektphase am DBFZ und einer Fermentermaterialprobe aus der Praxisbiogasanlage (BGA) in Verliehausen.

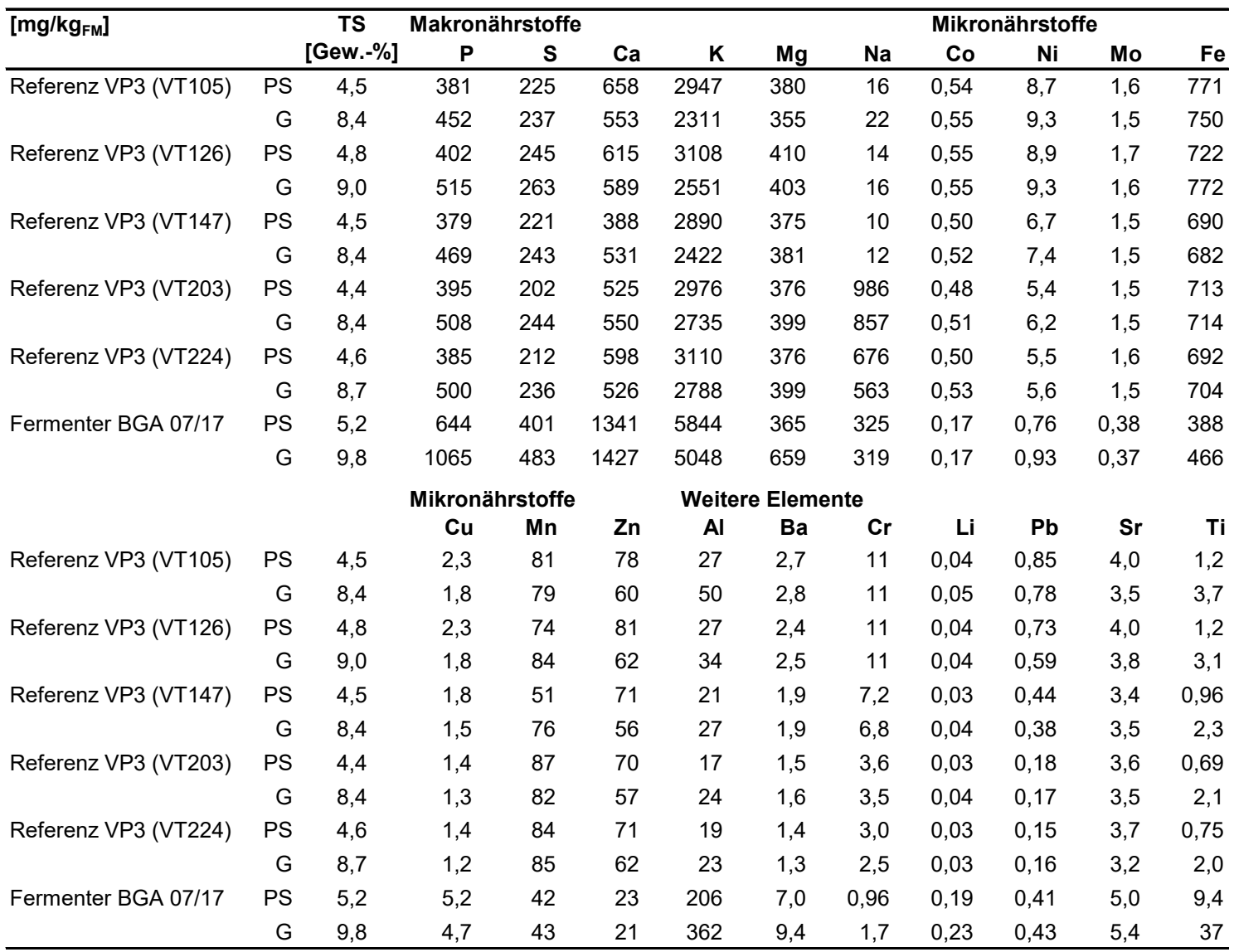

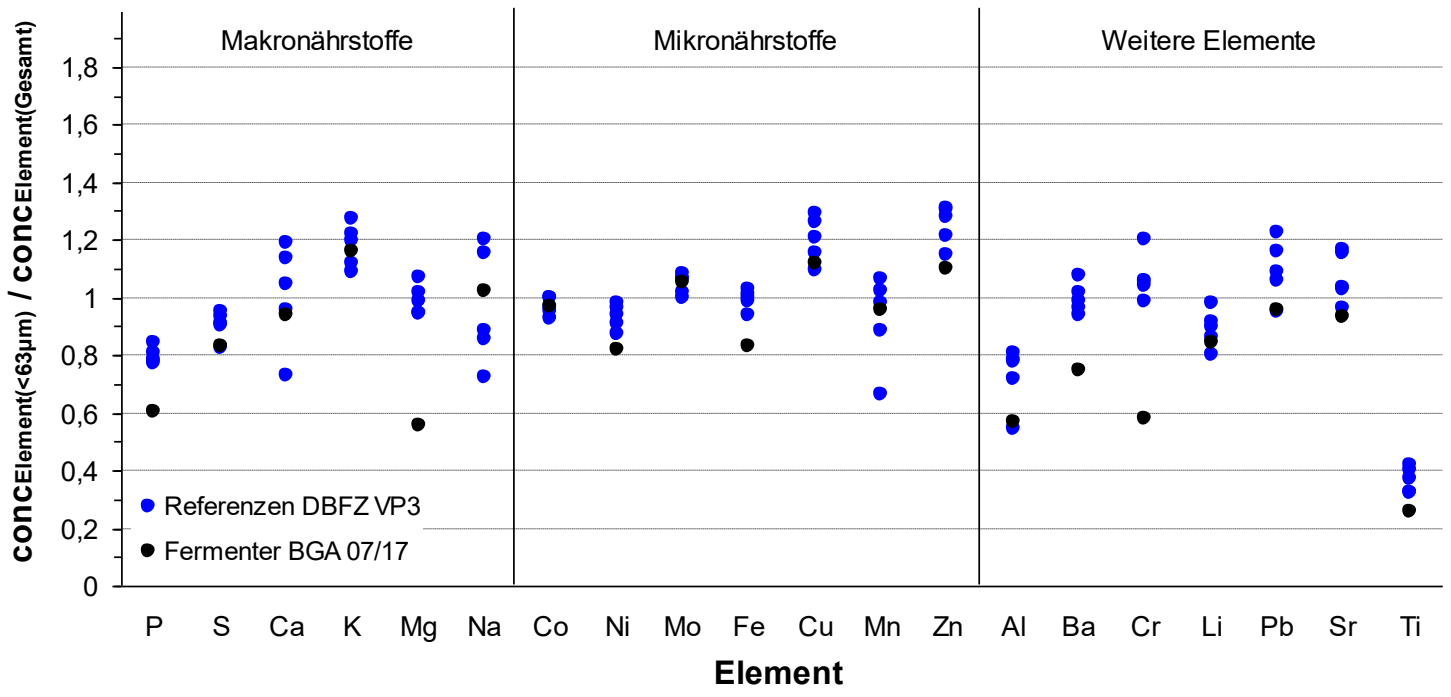

Abbildung 102: Elementkonzentrationsverhältnisse der Presssaftproben $(<63 \mu \mathrm{m})$ ausgewählter Referenzreaktorproben der dritten Projektphase am $D B F Z$ und einer Fermentermaterialprobe aus der Praxisbiogasanlage $(B G A)$ in Verliehausen. 
Die gewonnenen Presssäfte $(<63 \mu \mathrm{m})$ dieser Reaktormaterialproben weisen gegenüber den Gesamtproben vergleichbare Elementkonzentrationen für einige Makronährstoffe (Calcium, Magnesium und Natrium), Mikronährstoffe (Cobalt, Molybdän, Eisen und Mangan) und weitere Elemente (Barium, Chrom, Blei und Strontium) auf. Schwefel, Nickel und Lithium zeigen geringfügig niedrigere Elementverhältnisse gegenüber den Gesamtproben. Die Elemente Phosphor, Aluminium und Titan sind in den Presssaftproben $(<63 \mu \mathrm{m})$ deutlich niedriger konzentriert. Kalium, Kupfer und Zink weisen dagegen höhere Elementkonzentrationsverhältnisse in den Presssäften $(<63 \mu \mathrm{m})$ auf. Das bedeutet, dass diese drei Elemente mehr zur „feineren“ Fraktion $(<63 \mu \mathrm{m})$ hin tendieren. Eine mögliche Kontamination durch das in den Pressversuchen verwendete Prüfsieb kann ausgeschlossen werden, da das Material des Siebs aus Aluminium bestand und sonst keine weiteren Metallgegenstände beim Rühren und Pressen eingesetzt wurden (vgl. Kapitel 6.2).

Auch die in der Abbildung 102 zum Vergleich dargestellten Verhältnisse der Elementkonzentrationen der Fermentermaterialprobe aus der Biogasanlage in Verliehausen liegen mit Ausnahme von Phosphor, Magnesium, Eisen, Barium und Chrom, die noch geringere Konzentrationsverhältnisse gegenüber der Gesamtprobe aufweisen, im Bereich der Elementkonzentrationsverhältnisse der Reaktormaterialproben vom DBFZ. Weitere vergleichende Betrachtungen sind in Kapitel 6.3.5 dargestellt.

\subsubsection{Elementkonzentrationen der Pressrückstände (> $63 \mu \mathrm{m})$}

Wie in Kapitel 6.2 beschrieben, wurden die Pressrückstände ( $>63 \mu \mathrm{m})$ aller getesteten Fermentermaterialproben wie auch die zugehörigen Gesamtproben bei $105^{\circ} \mathrm{C}$ bis zur Gewichtskonstanz getrocknet, mit der Planeten-Kugelmühle analysenfein gemahlen, mittels des mehrstufigen Säuretotalaufschlussverfahrens vollständig in Lösung gebracht und anschließend die Elementgehalte mittels ICP-OES-Messungen bestimmt. Für die Dateninterpretation wurden entsprechend $\mathrm{zu}$ den Filtrat- $(<0,45 \mu \mathrm{m})$ und Presssaftproben $(<63 \mu \mathrm{m}) 20$ Elemente verglichen.

In Tabelle 33 sind analog zu Kapitel 6.3.3 die gemessenen Elementkonzentrationen der Pressrückstände $(>63 \mu \mathrm{m})$ und zugehörigen Gesamtproben der Reaktormaterialproben aus dem Kontrollreaktor (Referenz) der dritten Projektphase am DBFZ sowie einer Fermentermaterialprobe aus der Praxisbiogasanlage in Verliehausen dargestellt. Abbildung 103 zeigt die Elementkonzentrationsverhältnisse. 
Tabelle 33: $\quad$ Übersicht der Elementkonzentrationen $\left[\mathrm{mg} / \mathrm{kg}_{F M}\right]$ der Pressrückstände $(>63 \mu \mathrm{m}, P R)$ und der Gesamtproben $(G)$ ausgewählter Referenzreaktorproben der dritten Projektphase am DBFZ und einer Fermentermaterialprobe aus der Praxisbiogasanlage (BGA) in Verliehausen.

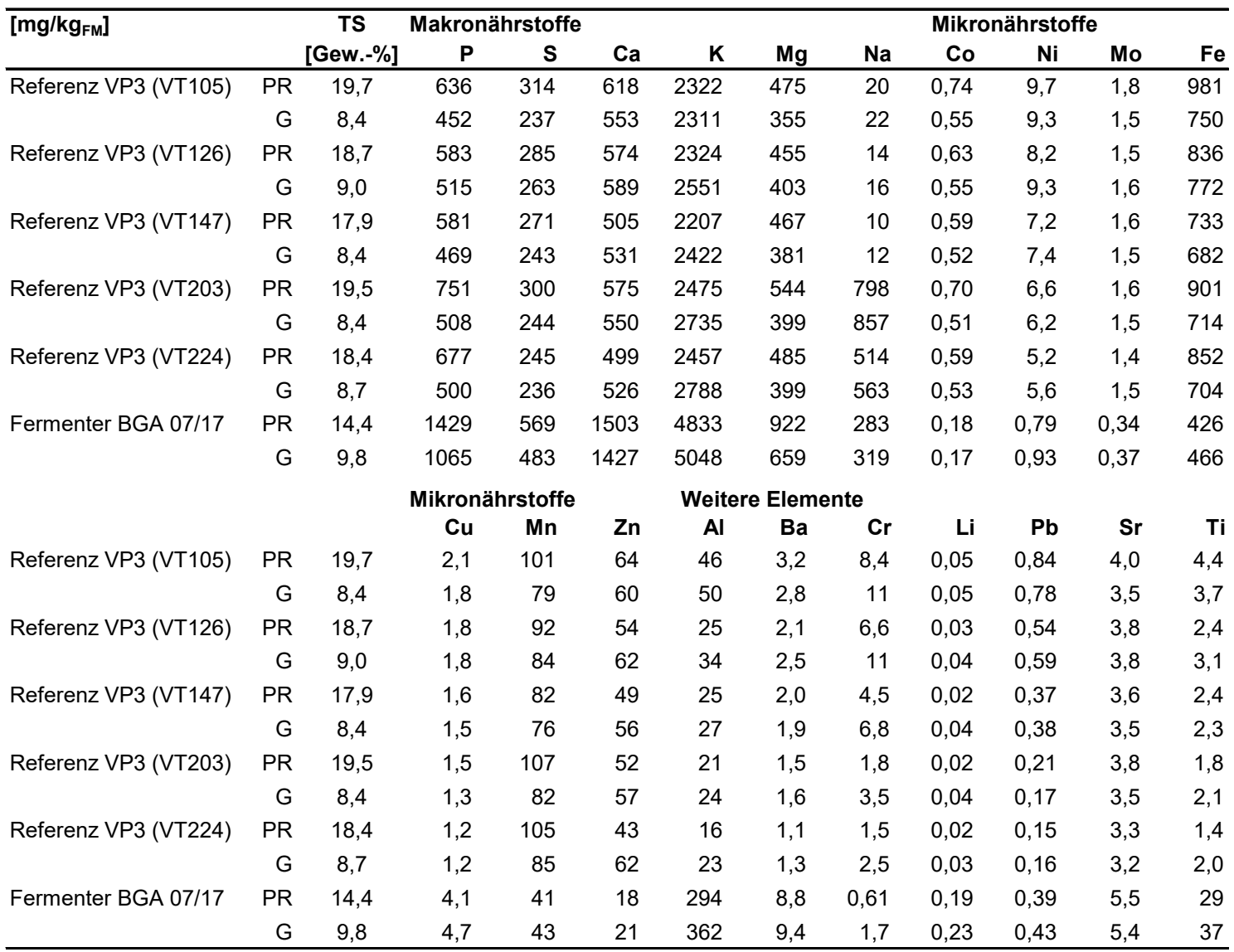

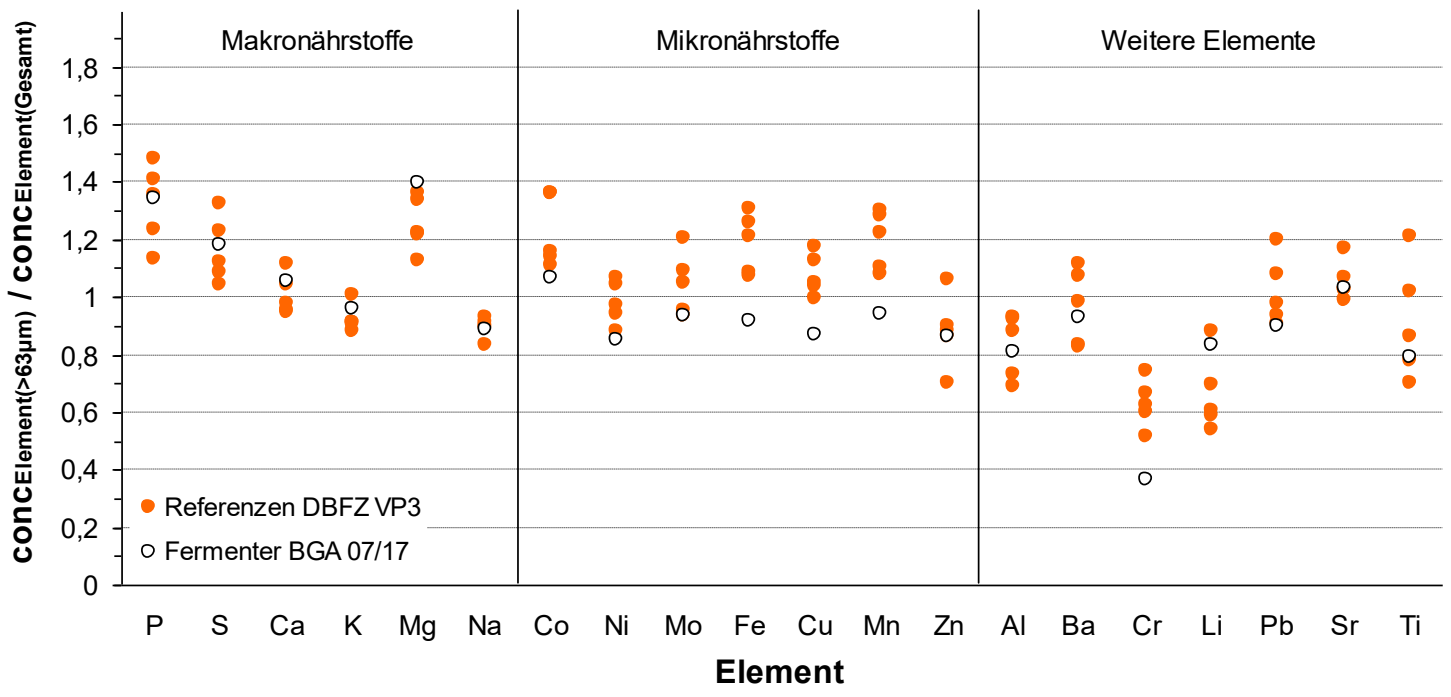

Abbildung 103: Elementkonzentrationsverhältnisse der Pressrückstände (> $63 \mu \mathrm{m})$ ausgewählter Referenzreaktorproben der dritten Projektphase am $D B F Z$ und einer Fermentermaterialprobe aus der Praxisbiogasanlage (BGA) in Verliehausen. 
Die Pressrückstände $(>63 \mu \mathrm{m})$ der Materialproben aus dem Kontrollreaktor am DBFZ zeigen gegenüber den Gesamtproben ähnliche Konzentrationen für die Makronährstoffe Calcium und Kalium und für die Mikronährstoffe Nickel, Molybdän und Kupfer sowie für die weiteren Elemente Barium, Blei, Strontium und Titan. Die essenziellen Makronährelemente Phosphor, Schwefel und Magnesium sowie die Spurenelemente Cobalt, Eisen und Mangan weisen höhere Konzentrationen in den Pressrückständen ( $>63 \mu \mathrm{m})$ gegenüber den Gesamtproben auf. D.h. diese sechs Elemente tendieren mehr zur „gröberen“" Fraktion ( $>\mathbf{6 3} \boldsymbol{\mu m})$. Natrium, Zink und Aluminium zeigen etwas niedrigere Elementverhältnisse gegenüber den Gesamtproben. Die Elemente Chrom und Lithium sind in den Pressrückstandsproben $(>63 \mu \mathrm{m})$ deutlich niedriger konzentriert.

Die Elementkonzentrationsverhältnisse der Fermentermaterialprobe aus der Praxisbiogasanlage in Verliehausen (Fermenter BGA 07/17) liegen bei den Pressrückständen $(>63 \mu \mathrm{m})$ weitestgehend im Bereich der Verhältnisse der Elementgehalte der Reaktormaterialproben vom DBFZ. Nur bei den Mikronährstoffen Eisen, Kupfer und Mangan sowie bei Chrom liegen etwas niedrigere Verhältnisse vor.

\subsubsection{Vergleich der Elementkonzentrationen sowie der Elementanteile in der Festsubstanz und flüssigen Phase der verschiedenen Fraktionen}

Zur Veranschaulichung der Unterschiede in den einzelnen Fraktionen sind in Abbildung 104 beispielhaft die Elementkonzentrationsverhältnisse der drei Fraktionen Filtrat $(<0,45 \mu \mathrm{m})$, Presssaft $(<63 \mu \mathrm{m})$ und Pressrückstand $(>63 \mu \mathrm{m}) \mathrm{zu}$ der Gesamtprobe des in Kapitel 6.3.2, 6.3.3 und 6.3.4 aufgeführten Fermentermaterials aus der Praxisbiogasanlage in Verliehausen zusammengestellt.

Ähnliche Konzentrationsverhältnisse im Pressrückstand $(>63 \mu \mathrm{m})$ und Presssaft $(<63 \mu \mathrm{m})$ gegenüber der Gesamtprobe zeigen Calcium, Natrium, Cobalt, Molybdän, Mangan, Blei und Strontium. Deutlich höhere Elementkonzentrationsverhältnisse weist der Pressrückstand $(>63 \mu \mathrm{m})$ für die Makronährstoffe Phosphor, Schwefel und Magnesium auf. Die Verhältnisse dieser Elemente im Presssaft $(<63 \mu \mathrm{m})$ sind dagegen deutlich niedriger. Ebenfalls etwas höher gegenüber der Gesamtprobe liegen im Presssaft $(<63 \mu \mathrm{m})$ die Verhältnisse von Kalium $(1,16)$ sowie der essenziellen Spurenelemente Kupfer $(1,12)$ und Zink $(1,10)$. Im Pressrückstand $(>63 \mu \mathrm{m})$ sind dagegen die Elementkonzentrationsverhältnisse bei diesen Elementen geringfügig niedriger. Die Konzentrationsverhältnisse im Pressrückstand $(>63 \mu \mathrm{m})$ und 
Presssaft $(<63 \mu \mathrm{m})$ der Mikronährstoffe Nickel und Eisen sowie der Elemente Barium und Lithium fallen gegenüber der Gesamtprobe etwas niedriger aus $(0,75$ bis 0,93$)$. Für Aluminium, Chrom und Titan sind die Elementkonzentrationsverhältnisse in den beiden Fraktionen deutlich niedriger $(0,26$ bis 0,81$)$ gegenüber der Gesamtprobe.

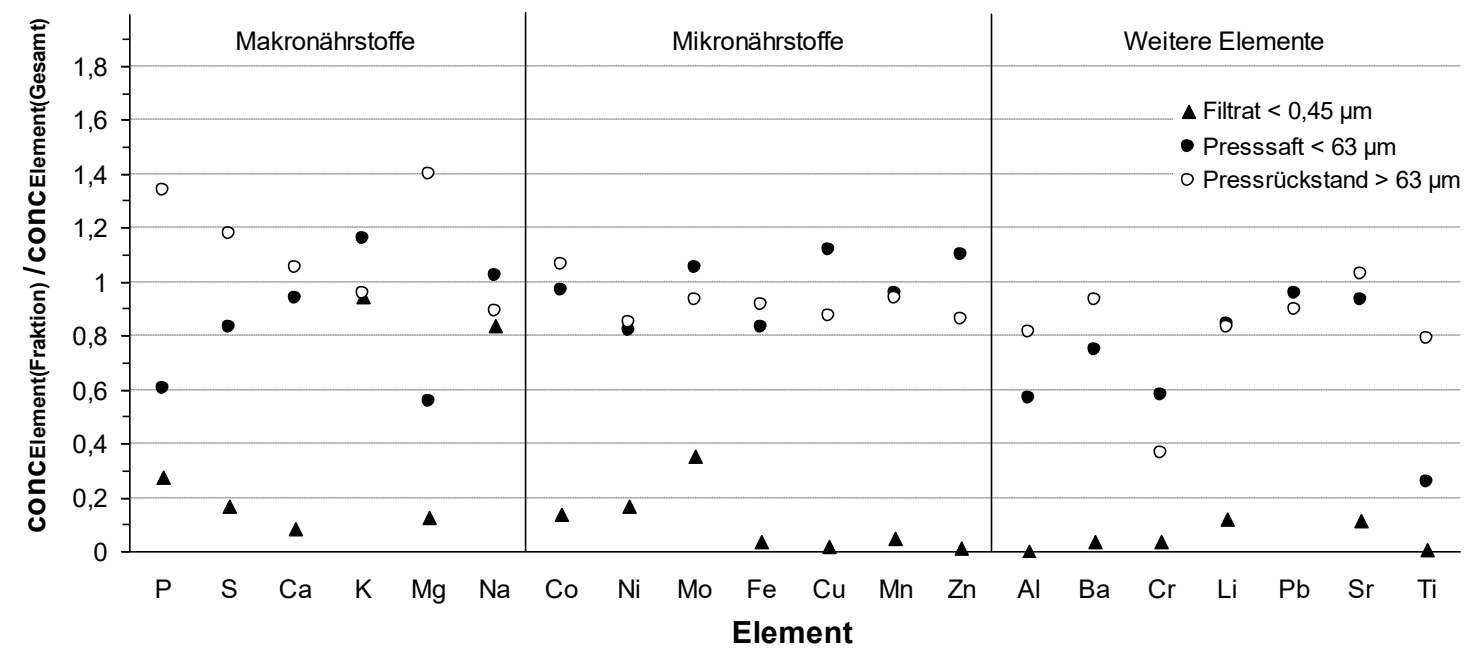

Abbildung 104: Vergleich der Elementkonzentrationsverhältnisse der Fraktionen Filtrat $(<0,45 \mu \mathrm{m})$, Presssaft $(<63 \mu \mathrm{m})$ und Pressrückstand (> $63 \mu \mathrm{m}$ ) einer Fermentermaterialprobe (Fermenter BGA 07/17) aus der Praxisbiogasanlage in Verliehausen.

In Abbildung 105 sind als weiterer Vergleich die Elementkonzentrationsverhältnisse in den Presssäften $(<63 \mu \mathrm{m})$ und Pressrückständen $(>63 \mu \mathrm{m})$ zu den Gesamtproben der in Kapitel 6.3.3 und 6.3.4 aufgeführten Materialproben aus dem Kontrollreaktor (Referenz) der dritten Projektphase am DBFZ sowie einer Fermentermaterialprobe aus der Praxisbiogasanlage in Verliehausen (Fermenter BGA 07/17) dargestellt.

Die Elementkonzentrationsverhältnisse der Reaktormaterialproben aus dem Referenzfermenter am DBFZ stimmen insgesamt gut mit den Trends der Verhältnisse der Fermentermaterialprobe aus der Praxisbiogasanlage in Verliehausen überein. Lediglich das essenzielle Spurenelement Eisen sowie Chrom weisen in beiden Fraktionen etwas höhere Verhältnisse als in der Fermenterprobe aus der Praxisbiogasanlage auf. In den Presssäften $(<63 \mu \mathrm{m})$ liegen die Verhältnisse für Phosphor, Magnesium und Barium höher als in der Praxisfermenterprobe und variieren bei den beiden letztgenannten Elementen im Bereich um den Wert 1. Die Pressrückstände $(>63 \mu \mathrm{m})$ zeigen gegenüber der Fermenterprobe aus der Biogasanlage in Verliehausen für Kupfer und Mangan etwas höhere Verhältnisse an. 


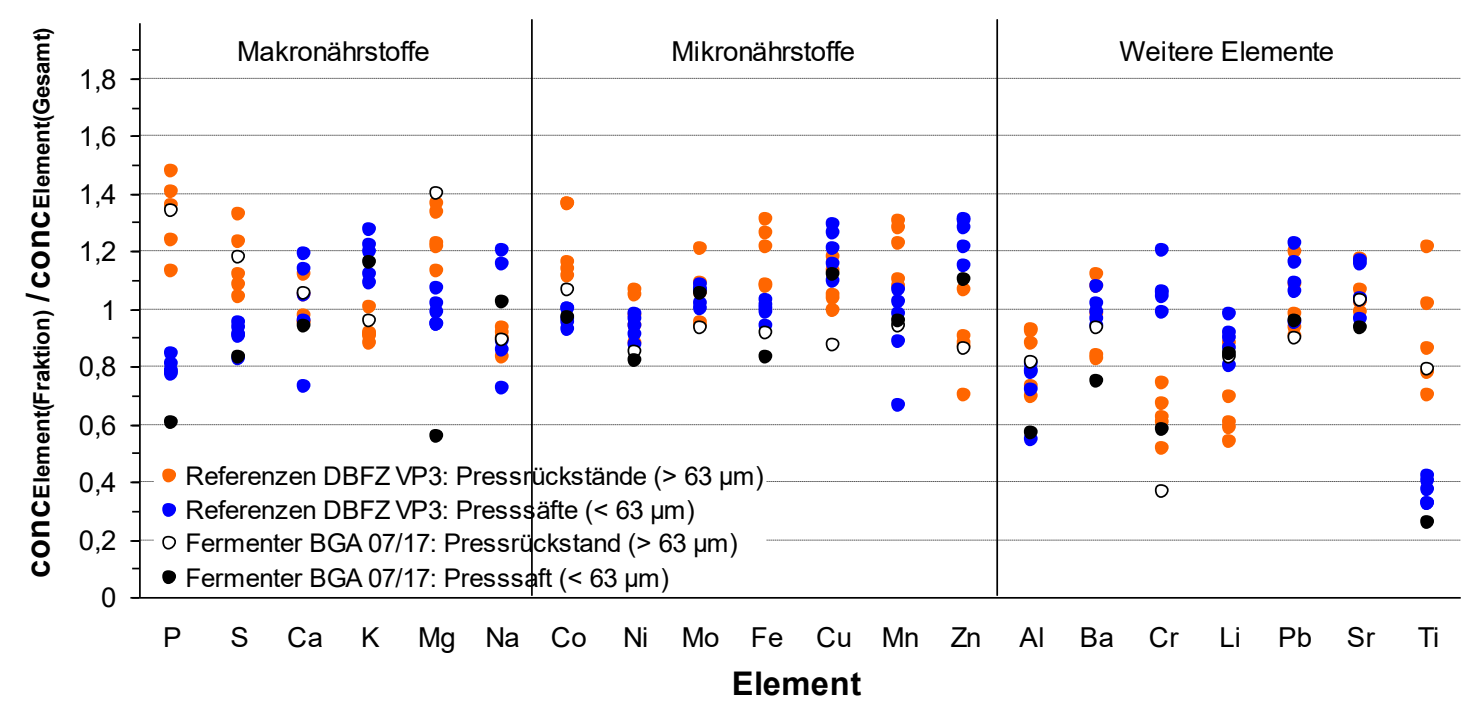

Abbildung 105: Vergleich der Elementkonzentrationsverhältnisse der Fraktionen Presssäfte $(<63 \mu \mathrm{m})$ und Pressrückstände (> $63 \mu \mathrm{m})$ ausgewählter Referenzreaktorproben der dritten Projektphase am DBFZ und einer Fermentermaterialprobe (Fermenter BGA 07/17) aus der Praxisbiogasanlage in Verliehausen.

Im Folgenden wird kurz näher betrachtet, wie stark die Elementanteile in der Festsubstanz und der flüssigen Phase in der Gesamtprobe, im Pressrückstand ( $>63 \mu \mathrm{m})$ sowie im Presssaft $(<63 \mu \mathrm{m})$ variieren, um weitere Anhaltspunkte über die Bioverfügbarkeit durch die Spurenelementverteilung zwischen den flüssigen und festen Phasen zu erhalten. Unter der Annahme, dass die Elementkonzentrationen in den Filtraten $(<0,45 \mu \mathrm{m})$ auch den Gehalten im wässrigen Teil (flüssige Phase) der Gesamtproben bzw. der einzelnen Fraktionen entsprechen, kann die jeweilige Verteilung der Elemente in der Festsubstanz und flüssigen Phase bestimmt werden. Die Wasseranteile in den Pressrückständen $(>63 \mu \mathrm{m})$, Presssäften $(<63 \mu \mathrm{m})$ und Gesamtproben wurden über die jeweiligen Messungen der prozentualen Trockensubstanzgehalte (vgl. Kapitel 3.4.1) ermittelt (Wasseranteil [Gew.-\%] = 100 - Trockensubstanzgehalt [TS, Gew.-\%]). Die Anteile in der Festsubstanz stellen die Differenz der Elementkonzentrationen in der gesamten Probe bzw. gesamten Fraktion und den ermittelten Gehalten in der flüssigen Phase dar.

Abbildung 106 zeigt anhand der Fermentermaterialprobe aus der Praxisbiogasanlage in Verliehausen (Fermenter BGA 07/17) beispielhaft die Elementanteile in den beiden Phasen der verschiedenen Fraktionen. Alle weiteren betrachteten Reaktormaterialproben weisen eine ähnliche Verteilung auf und werden hier nicht näher dargestellt. 

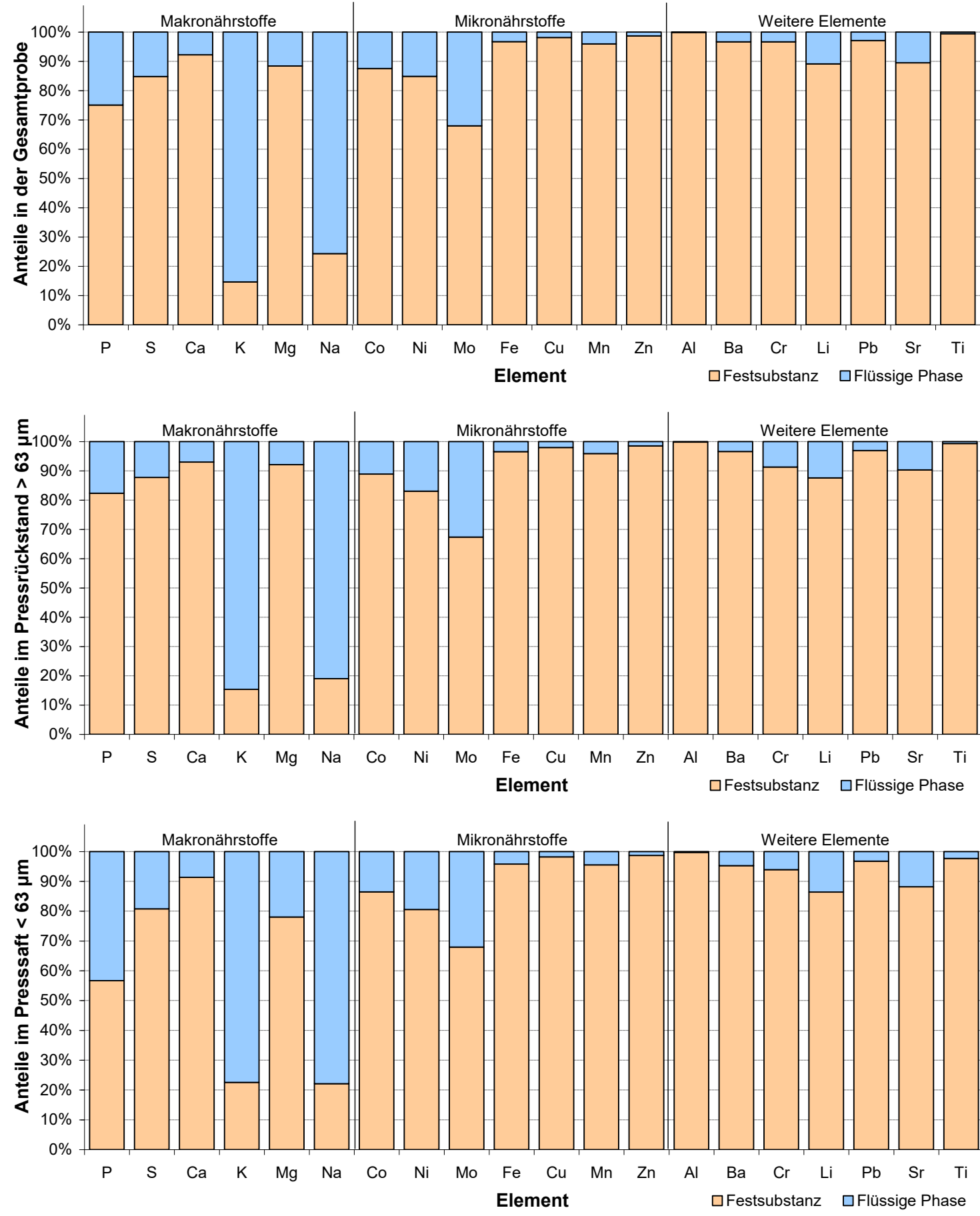

Abbildung 106: Verteilung der Elemente in der Festsubstanz und der flüssigen Phase in der Gesamtprobe (oben), im Pressrückstand (> $63 \mu \mathrm{m}$, Mitte) sowie im Presssaft $(<63 \mu \mathrm{m}$, unten) einer Fermentermaterialprobe (Fermenter BGA 07/17) aus der Praxisbiogasanlage in Verliehausen.

Die Gesamtprobe und die beiden Fraktionen Pressrückstand ( $>63 \mu \mathrm{m})$ sowie Presssaft $(<63 \mu \mathrm{m})$ zeigen bei der Verteilung der Elemente in der Festsubstanz und der flüssigen Phase der Fermentermaterialprobe insgesamt ähnliche Trends (Abbildung 106). Die höchsten Anteile in der Flüssigphase weisen die Makronährstoffe Kalium (85, 85 und 
$78 \%$ ) und Natrium (76, 81 und $78 \%$ ) auf. Zurückzuführen ist dies auf die sehr hohen

„Wiederfindungen“ der Kalium- bzw. Natrium-Elementkonzentrationen im Filtrat $(<0,45 \mu \mathrm{m})$ der Probe (vgl. Kapitel 6.3.2). Die weiteren Makronährelemente Phosphor, Schwefel, Calcium und Magnesium variieren in der flüssigen Phase der Gesamtprobe zwischen 7,8 und $25 \%$, im Pressrückstand (> $63 \mu \mathrm{m}$ ) zwischen 7,0 und $18 \%$ und im Presssaft $(<63 \mu \mathrm{m})$ zwischen 8,7 und $43 \%$, wobei Calcium den geringsten Anteil, Phosphor den höchsten Anteil in der Flüssigphase zeigt.

Bei den essenziellen Mikronährstoffen variieren die Elementanteile nur sehr gering und liegen in den flüssigen Phasen bei Molybdän im Durchschnitt bei $32 \%$, gefolgt von Nickel (durchschnittlich $17 \%$ ) und Cobalt (durchschnittlich $12 \%$ ). Eisen, Kupfer, Mangan und Zink weisen sehr niedrige Anteile in den Flüssigphasen auf. Ein sehr ähnliches Bild zeigen auch die weiteren Elemente.

Insgesamt deuten die hohen Elementanteile in den Festsubstanzen darauf hin, dass die für die Mikroorganismen essenziellen Nährstoffe (mit Ausnahme von Kalium und Natrium) unter der getroffenen Annahme (die Elementkonzentrationen in den Filtraten $(<0,45 \mu \mathrm{m})$ entsprechen den Gehalten im wässrigen Teil der Gesamtproben bzw. der einzelnen Fraktionen) sich eher in der Festsubstanz vorfinden als dass sie in der flüssigen Phase vorliegen. Sie können ferner wie ausführlicher in Kapitel 2.3 beschrieben durch eine Vielzahl von biochemischen Umsetzungsprozessen aus ihren ursprünglichen Bindungsformen in der Silage gelöst und mikrobiell verfügbar werden. Zwar können sich im Fermenter mit Sulfiden, Carbonaten und Phosphaten auch unlösliche Präzipitate bilden, aber besonders durch Komplexierung mit organischen Liganden können die Nährelemente auch löslich gehalten werden.

\subsection{6 Überlegungen zu Löslichkeiten von Schwermetallen im Fermenter}

In diesem Unterkapitel wird der Frage nachgegangen, ob möglicherweise Sulfide oder Phosphate die Konzentrationen von Schwermetallen im Fermenter bestimmen. Die nachfolgenden Betrachtungen basieren auf Überlegungen von Prof. Dr. Hans Ruppert, die im Zusammenhang dieser Arbeit im Hinblick auf Schwermetallfällungsreaktionen diskutiert wurden. Grundlage bilden die Elementgehalte der Filtratprobe $(<0,45 \mu \mathrm{m})$ der Fermenterprobe aus der Biogasanlage in Verliehausen (Fermenter BGA 07/17). 
Folgende Annahmen wurden getroffen: Wenn die Schwermetallkonzentrationen im Filtrat $(<0,45 \mu \mathrm{m})$ einer Fermenterprobe aus einer Biogasanlage höher sind als die aus den Löslichkeiten ihrer Sulfide oder Phosphate errechneten Gehalte, sollten diese Elemente komplexiert sein oder an Feinstkolloide $(<0,45 \mu \mathrm{m})$ gebunden vorliegen. Diese Interaktion ist vermutlich durch organische Bindungen verursacht.

Vorgehensweise und Randbedingungen: Es werden die Elementdaten der Filtratprobe rein anorganisch für fermentertypische pH-Werte von 7 und 8 (vgl. Kapitel 2.2.3) betrachtet. Es wird hypothetisch davon ausgegangen, dass die Schwermetalle in rein anorganischen Reaktionen ausfallen, die Metalle wie auch die Sulfid- und PhosphatIonen also anorganisch gelöst vorliegen. Die Berechnungen erfolgten bei 1 bar (Atmosphärendruck) und $25^{\circ} \mathrm{C}$ unter Vernachlässigung des Elektrolytgehaltes der Lösung (die Aktivität wurde gleich der Konzentration gesetzt) und der wahrscheinlichen Komplexbildung der Schwermetalle. Des Weiteren blieb auch die Sorption an (organische) Kolloide im sub- $\mu \mathrm{m}$-Bereich unbeachtet. Eine weitere Problematik besteht darin, dass vor allem bei den Sulfiden die 2. Dissoziationskonstante $\mathrm{HS}^{-} \rightleftharpoons \mathrm{S}^{2-}+\mathrm{H}^{+}$sehr unsicher ist, weil bei älteren $\mathrm{S}^{2-}$-Gleichgewichtsbestimmungen zur Ermittlung der Dissoziationskonstanten Polysulfid-Spezies nicht berücksichtigt wurden (RICKARD \& LUTHER, 2007; RICKARD, 2012). Schwefel und Phosphor können ferner gleichzeitig noch Bestandteile organischer Kolloide sein.

Phosphate: Die Berechnungen der Phosphat-Spezies auf Basis einfacher Phosphate $\left(\mathrm{Me}_{3}\left(\mathrm{PO}_{4}\right)_{2}\right)$ erbrachten folgende Reihung für die Filtratprobe (von Untersättigung zu zunehmender Übersättigung): Nickel ist bei einem pH-Wert von 7 und 8 untersättigt, Zink und Cobalt bei einem pH-Wert von 7. Übersättigt sind Zink (pH-Wert 8), Cobalt $($ pH-Wert 8$)<$ Kupfer $<$ Eisen $<$ Mangan $<$ Blei. Nach Löslichkeitswerten von www.periodensystem-online.de sind bei einem $\mathrm{pH}$-Wert von 8 alle Phosphate übersättigt.

Sulfide: Als einfache Sulfide (MeS, sowie $\mathrm{Cu}_{2} \mathrm{~S}$ und $\mathrm{MoS}_{2}, \mathrm{NiS}_{2}$ und $\mathrm{FeS}_{2}$ ) sind nahezu alle Elemente übersättigt (in zunehmender Übersättigung geordnet auf Basis von $\left.\mathrm{Me}^{2+}=\mathrm{K}_{\mathrm{sp}} \cdot \mathrm{H}^{+} / \mathrm{HS}^{-}\right): \quad$ Mangan $<$ Eisen $<$ Cobalt $<$ Nickel $<$ Zink $<$ Blei $<$ Molybdän $\left(\mathrm{MoS}_{2}\right)<\operatorname{Kupfer}\left(\mathrm{Cu}_{2} \mathrm{~S}\right)<\mathrm{Fe}($ Markasit$)<\operatorname{Nickel}\left(\mathrm{NiS}_{2}\right)<$ Kupfer $<$ Eisen (Pyrit). Die generelle Übersättigung gilt auch für Berechnungen auf Basis $\mathrm{Me}^{2+}=\mathrm{K}_{\mathrm{sp}} / \mathrm{S}^{2-}$, wobei die $\mathrm{S}^{2-}$-Gehalte aus dem Verhältnis $\mathrm{HS}^{-} / \mathrm{S}^{2-}$ aus Abbildung 6 von RICKARD \& LUTHER (2007) für pH-Wert 7 und 8 abgeschätzt wurden. 
Mit Ausnahme von Mangan sind alle Elemente bezüglich Sulfid um Größenordnungen übersättigt. Das bedeutet, dass der weitaus größte Teil der Elemente in Komplexen oder an kolloidale organische Substanzen in der flüssigen Phase gebunden sein muss. Die Frage, in welcher Speziesform die Schwermetalle (oder auch die Liganden) wirklich vorliegen, kann nicht beantwortet werden.

Weitere Gedanken: Da mit Ausnahme von Kalium, Natrium, Phosphor und Molybdän (vgl. Kapitel 6.3.5) die meisten Elemente zu weniger als $20 \%$ in der flüssigen Phase vorliegen, sondern an die organischen Substanzen sorbiert oder dort eingebaut sind, erscheint folgender Mechanismus der Verfügbarmachung der essenziellen Elemente für die Gasproduktion aus Biomasse möglich zu sein:

Die Mikroorganismen wandeln die Biomasse teilweise in Methan und Kohlenstoffdioxid (und andere Produkte) um. Dabei stehen die in der durch die Pflanzensilage schon teilweise aufbereitete Pflanzenstruktur eingebauten oder sorbierten Elemente direkt den biogasbildenden Mikroorganismen zur Verfügung und zwar genau dort, wo sich die Mehrzahl der Mikroben befindet. Werden nun synthetische Spurenelementadditive zugesetzt, werden diese essenziellen Elemente mit großer Wahrscheinlichkeit bei den $\mathrm{pH}-$ Werten von 7 bis 8 im Fermenter an den organischen Substanzen sorbiert und stehen somit für die physiologischen Prozesse ebenfalls zur Verfügung. Die Konzentrationen an freien Metallionen dürften extrem gering sein, da die Elemente sonst als Sulfide (oder teilweise Phosphate) ausfallen müssten.

Auch die Verdünnungsstufen, mit denen die Filtrate des Fermentermaterials gewonnen wurden (vgl. Kapitel 6.2), geben einen weiteren Hinweis. Aus technischen Gründen (schnelles Zusetzen der Filter) wurde vor dem Filtrieren das Fermentermaterial 5-, 10-, 20- und 50-fach verdünnt. Nach Rückrechnung mit dem Verdünnungsfaktor ergaben sich für die verschiedenen Verdünnungsstufen ausgezeichnete Übereinstimmungen der Elementgehalte des Filtrates (Ausnahme waren die 1:50-Verdünnungen aufgrund sehr niedriger Lösungskonzentrationen im Bereich der Nachweisgrenzen bei den Messungen). Auch bei stärkeren Verdünnungen erfolgte also keine Rücklösung der Elemente von dem organischen Material, was für eine starke Elementbindung an die organischen Substanzen spricht und nicht für eine Nachdissoziation von der Oberfläche. 


\subsection{Methodenvorschlag}

Um deutlich frühzeitiger auf problematische Spurenelementmangelsituationen im Fermenter reagieren zu können, wird als vereinfachte Methode zur Spurenelementcharakterisierung folgende Vorgehensweise vorgeschlagen:

(1) Probenahme eines repräsentativen Probenaliquotes des Fermentermaterials,

(2) Gute Homogenisierung dieses Aliquotes,

(3) Je nach Dringlichkeit kurzes oder längeres Aufkochen einer ausreichenden Teilmenge mit konzentrierter Salpetersäure $\left(\mathrm{HNO}_{3}\right)$,

[Anmerkung: Ein langsames Aufkochen mit Salpetersäure mit längeren Zwischenstufen und einer längeren Haltephase bei $100^{\circ} \mathrm{C}$ liefert gegenüber einem schnellen Aufkochen mit Salpetersäure und kurzer Haltephase bei $100{ }^{\circ} \mathrm{C}$ nach den in dieser Arbeit erhobenen Ergebnissen keine verbesserte Aufschlusseffizienz (vgl. hierzu Kapitel 6.2 und 6.3.1). Bei dem schnelleren Aufkochen besteht jedoch eine potenziell höhere Gefahr des Überschäumens bzw. Überkochens der Proben. Es sind ggf. entsprechend große Probengefäße bzw. angepasste Proben- und Säurevolumina zu wählen.]

(4) Multielementanalyse mittels ICP-OES,

(5) Datenauswertung vor dem Hintergrund der Mindestgehalte der essenziellen Spurenelemente.

Mit dieser vorgeschlagenen vereinfachten Methode kann unter Beachtung einer Qualitätssicherung der nötige Zeitraum zwischen Probenahme, Aufbereitung, Analyse und Interpretation auf wenige Tage reduziert werden. 


\section{Zusammenfassung}

In dieser Arbeit wurden zunächst auf Basis von Nassfermentationsversuchen im Labormaßstab in einer ersten Projektphase für die beiden essenziellen Spurenelemente Cobalt und Nickel die Minimalgehalte im Biogasprozess erarbeitet. Bei einer Raumbelastung von $5 \mathrm{~g}_{\mathrm{oTS}} /(\mathrm{L} \bullet \mathrm{d})$ und einer mittleren hydraulischen Verweilzeit von 40 Tagen liegt der minimale Cobalt-Schwellenwert für eine optimale Biogasbildung bei $0,066 \mathrm{mg} / \mathrm{kg}$ FM. Dieser Wert harmonisiert sehr gut mit minimalen Bedarfswerten aus der Literatur. Der ermittelte minimale Schwellenwert für Nickel liegt aufgrund methodischer Probleme wahrscheinlich deutlich unter $0,82 \mathrm{mg} / \mathrm{kg}_{\mathrm{FM}}$ und ist vermutlich durch eine Zinkzugabe verursacht, die den oberen Hemmschwellenwert von etwa $120 \mathrm{mg} / \mathrm{kg}_{\mathrm{FM}}$ überschritt.

In vier Substitutionsversuchen in einer zweiten und dritten Projektphase wurde getestet, wie durch eine Zumischung von spurenelementreichen anbauwürdigen Energiepflanzen bei der Vergärung von Mais-Silage die Zugabe von synthetischen Spurenelementadditiven in Biogasanlagen minimiert werden kann. Jeweils $30 \%$ Silage von Winterackerbohne, Amarant, Deutsches Weidelgras und Durchwachsene Silphie wurde mit $70 \%$ Mais-Silage (oTS-bezogen) gemischt. Die Biogasbildungsprozesse in allen vier Substitutionsversuchen verliefen instabil und wären nach zwei bis dreieinhalb Verweilzeiten zusammengebrochen. Cobalt stellt das kritische Mangelelement dar, Nickel war nicht limitierend. Jedoch konnten alle vier Doppelversuche durch die Zugabe von nur noch einem Viertel der ursprünglichen Spurenelementadditivlösung mit Co, Ni, Mo, Fe, $\mathrm{Mn}, \mathrm{Zn}$, Se und W gerettet werden. Die Biogasprozesse stabilisierten sich und alle physikochemischen und gasanalytischen Parameter sowie die essenziellen Elementkonzentrationen, inklusive Cobalt und Nickel, lagen wieder auf konstant guten Niveaus über mehrere Verweilzeiten.

Die niedrigen Eisen/Schwefel-Verhältnisse liefern neben den kritischen Cobaltgehalten ein weiteres maßgebliches Instabilitätskriterium in den Substitutionsversuchen mit Silagen von Winterackerbohne, Amarant und Deutsches Weidelgras. Nur im Doppelversuch mit Durchwachsene Silphie-Silage lagen die Eisen/Schwefel-Verhältnisse oberhalb des prognostizierten minimalen Schwellenwertes von 0,4 von SAUER (2010). Nach Rettungsversuchen durch die Zugabe eines Viertels der ursprünglich eingesetzten synthetischen Additivlösung konnten sich die Eisen/Schwefel-Verhältnisse und damit die Biogasausbeuten in allen Versuchen wieder auf unkritischen Niveaus stabilisieren. 
Im dritten Substitutionsversuch mit Deutsches Weidelgras-Silage wurde statt eines Viertels der ursprünglichen kompletten Spurenelementlösung nur eine reduzierte synthetische Cobaltchloridlösung als Monoelementadditiv zuzugeben, da die Durchschnittsgehalte der Deutsches Weidelgras-Silage vermuten ließen, dass in beiden Reaktoren ausreichend Nickel im Substitut vorhanden war. Eine reduzierte Cobaltadditiv-Zugabe genügt allerdings nicht, stabile Biogasprozesse über mindestens drei Verweilzeiten aufrechtzuerhalten. Eisen, Kupfer, Zink und Selen stellen neben Cobalt weitere Mangelelemente bei dieser Silagemischung unter den gewählten Betriebsparametern dar. Auch die Eisen/Schwefel-Verhältnisse bleiben unter dem minimalen Schwellenwert.

In einem Zusatzversuch konnte außerdem nachgewiesen werden, dass die Zugabe nur eines Viertels der ursprünglich eingesetzten synthetischen Spurenelementlösung bei Mais-Silage-Monovergärung für stabile Biogasprozesse nicht ausreicht. Dies bestätigt die erhöhte Wirksamkeit der elementreicheren Pflanzensilagen als verfügbarkeitssteigernde Spurenelementlieferanten im Fermenterbetrieb. Das kritische Element für den Prozesszusammenbruch war allerdings Natrium, dessen minimaler Schwellenwert nach MunK ET AL. (2010) bei $10 \mathrm{mg} / \mathrm{L}$ liegt. Ursache ist das geringe NatriumAufnahmevermögen von Mais. Schlussfolgernd sollte eine minimale Konzentration für Natrium von $20 \mathrm{mg} / \mathrm{kg}_{\mathrm{FM}}$ in Praxisfermentern nicht unterschritten werden, um eine optimale Biogasbildung bzw. stabile Biogasprozesse zu garantieren. Kann das nicht gewährleistet werden, sollte Natrium neben Cobalt, Nickel, Molybdän, Eisen, Kupfer, Mangan, Zink, Selen, Vanadium und Wolfram einer potenziellen Spurenelementadditivlösung zugesetzt werden.

Nach den Auswertungen aller Substitutionsversuche, der Verarmungsversuche sowie des Zusatzversuchs unter Einbeziehung der Elementdaten der Praxisbiogasanlage in Verliehausen ist in Tabelle 34 als Empfehlung zusammenfassend aufgeführt, welcher Wertebereich und welche Minimal- und Optimalwerte einzuhalten wären, um optimale Biogasbildungsprozesse zu gewährleisten. Mit Ausnahme von Wolfram sind in der Tabelle alle essenziellen Mikronährstoffe sowie das essenzielle Makronährelement Natrium angegeben. Es sollte dabei für potenziell toxische Elemente immer das Minimierungsgebot gelten, d.h. minimaler Einsatz bei optimaler Biogasausbeute. Dies entlastet den Geldbeutel und reduziert die Kontamination der Böden, auf die die Gärreste aufgetragen werden. 
Tabelle 34: $\quad$ Empfohlener Wertebereich sowie Minimal- und Optimalwerte [mg/ $\left./ \mathrm{kg}_{F M}\right]$ wichtiger essenzieller Mikronährstoffe und Natrium für optimale Biogasbildungsprozesse.

\begin{tabular}{llccc}
\hline Symbol & Element & $\begin{array}{c}\text { empfohlener } \\
\text { Wertebereich } \\
{\left[\mathrm{mg} / \mathrm{kg}_{\mathrm{FM}}\right]}\end{array}$ & $\begin{array}{c}\text { Minimalwert } \\
{\left[\mathrm{mg} / \mathrm{kg}_{\mathrm{FM}}\right]}\end{array}$ & $\begin{array}{c}\text { Optimalwert } \\
{\left[\mathrm{mg} / \mathrm{kg}_{\mathrm{FM}}\right]}\end{array}$ \\
\hline $\mathbf{C o}$ & Cobalt & $0,10-0,25$ & 0,08 & 0,13 \\
$\mathbf{N i}$ & Nickel & $0,4-2,0$ & 0,3 & 1,0 \\
$\mathbf{M o}$ & Molybdän & $0,2-0,7$ & 0,18 & 0,3 \\
$\mathbf{F e}$ & Eisen & $150-300$ & 100 & 200 \\
$\mathbf{C u}$ & Kupfer & $1,2-4,0$ & 1,1 & 1,8 \\
$\mathbf{M n}$ & Mangan & $8,0-30$ & 7,0 & 20 \\
$\mathbf{Z n}$ & Zink & $8,0-25$ & 7,0 & 15 \\
$\mathbf{S e}$ & Selen & $0,005-0,03$ & 0,003 & 0,015 \\
$\mathbf{V}$ & Vanadium & $0,02-0,15$ & 0,01 & 0,08 \\
$\mathbf{N a}$ & Natrium & $>25$ & 20 & $>50$ \\
\hline
\end{tabular}

Über diskontinuierliche Gärversuche auf Basis von ORGA-Tests wurden zudem für die essenziellen Spurenelemente Cobalt, Nickel, Molybdän und Kupfer sowie die weiteren potenziellen Schadelemente Cadmium und Blei obere Schwellenwerte erarbeitet, die eine beginnende Hemm- bzw. Toxizitätswirkung auf die Umsetzungsprozesse bzw. biologische Abbauaktivität in den Gärreaktoren anzeigen. Hierfür wurde der aufgezeichnete kumulierte Druckanstieg in den einzelnen Gasbildungsmodulen über das Ideale Gasgesetz und das Gesetz von Avogadro in kumuliertes Biogasvolumen bzw. gebildete Biogasmenge umgerechnet. Eine sehr gute Vergleichbarkeit der in den Versuchsreihen getesteten Konzentrationen wurde über die Berechnung der spezifischen Biogaserträge in $\mathrm{mL}_{\mathrm{N}} / \mathrm{g}_{\mathrm{oTS}}$ und deren Darstellung als gemittelte Gassummenkurven erreicht. Als weiterer Parameter zur Bewertung der biologischen Aktivität in den Gasbildungsmodulen wurde der Zeitparameter $\mathrm{t}_{250}$ in Anlehnung an MERRETTIG-BRUNS ET AL. (2012) herangezogen, der sich bei den ORGA-Tests als sehr gut geeignet erwies. Durch die hohe zeitliche Auflösung, die standardisierten Versuchsbedingungen bei allen durchgeführten Testreihen sowie die vergleichsweise kurzen Versuchszeiträume von hier zehn Tagen eignet sich der ORGA-Test sehr gut zur Bestimmung von oberen Hemm- bzw. Toxizitätswirkungen von Substraten und Zusatzstoffen in anaeroben Gärprozessen. Abbildung 107 zeigt zusammenfassend eine schematische Darstellung des Einflusses von Spurenelementen auf den Biogasprozess bei zu geringen, optimalen, hemmenden und toxischen Konzentrationen sowie jeweilige Schwellenwerte für jede Prozessstufe. 


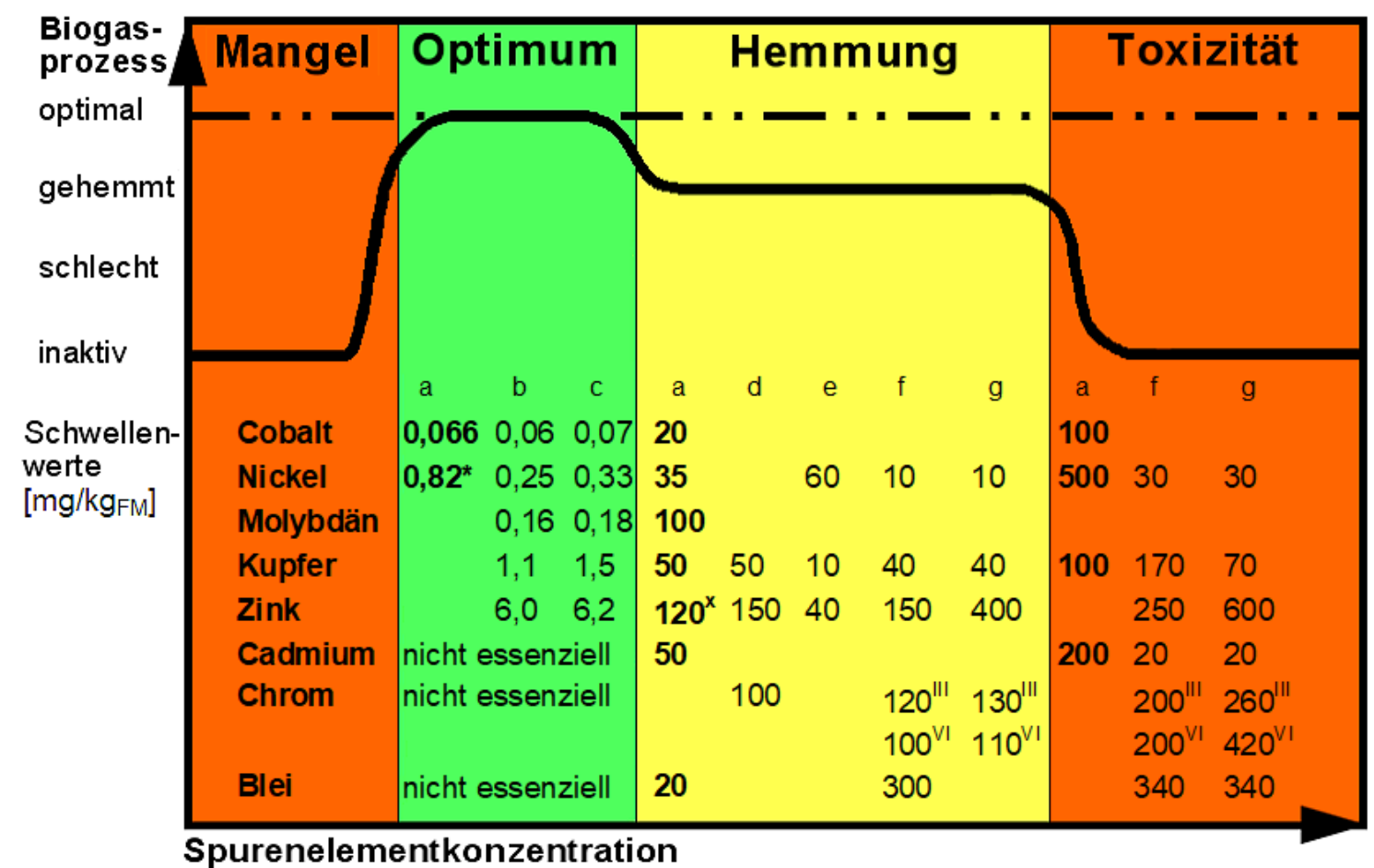

Abbildung 107: Schematische Darstellung des Einflusses von Spurenelementen auf den Biogasprozess bei zu geringen, optimalen, hemmenden und toxischen Konzentrationen mit Angabe von jeweiligen Schwellenwerten, stark modifiziert nach VAN HULLEBUSCH ET AL. (2016). a) vorliegende Arbeit; b) Lindorfer (2014); c) Sauer (2010); d) Weiland (2001); e) Zayed \& Winter (2000); f) Kouzeli-Katsiri \& Kartsonas (1986); g) Hayes \& Theis (1978); *: Wert zu hoch aufgrund möglicher Zinkhemmung; ${ }^{x}$ : abgeschätzter Wert aus NickelVerarmungsversuch (Kapitel 4.3.4); ${ }^{I I I,}{ }^{V I}$ : Chrom (III) und (VI).

Um den reaktiven Spurenelementanteil im Fermenter besser charakterisieren zu können, wurden außerdem Trennungs- und Filtrationsversuche mit verschiedenen Fraktionen des Fermentermaterials durchgeführt. Hohe Elementanteile in den Festsubstanzen der Fraktionen deuten darauf hin, dass die essenziellen Nährstoffe (mit Ausnahme von Kalium und Natrium) sich deutlich mehr in der organischen Festsubstanz als in der flüssigen Phase befinden. Der größte Teil der Elemente in der flüssigen Phase scheint zudem zusätzlich komplexiert vorzuliegen oder ist an kolloidale organische Substanzen gebunden. Direkt dort, wo sich die Mehrzahl der biogasbildenden Mikroben befindet, stehen die in die Inputsubstrate eingebauten oder sorbierten Elemente den Mikroben zur Verfügung. Bei der Zugabe von synthetischen Spurenelementlösungen werden die essenziellen Nährelemente mit großer Wahrscheinlichkeit an den festen organischen Substanzen sorbiert und stehen ebenfalls für die Biogasbildungsprozesse zur Verfügung. Ein vereinfachtes Verfahren zur schnelleren Spurenelementcharakterisierung basierend auf einer Extraktion mit konzentrierter Salpetersäure wird vorgeschlagen. 


\section{Literatur}

Aquino, S.F., Stuckey, D.C. (2007): Bioavailability and Toxicity of Metal Nutrients during Anaerobic Digestion. Journal of Environmental Engineering 133 (1), 28-35.

Link: https://doi.org/10.1061/(ASCE)0733-9372(2007)133:1(28)

BANKS, C.J., HEAVEN, S. (2013): Optimisation of biogas yields from anaerobic digestion by feedstock type. In: Wellinger, A., Murphy, J., Baxter, D. (Hrsg.): The biogas handbook - Science, production and applications. Woodhead Publishing Series in Energy 52, Woodhead Publishing Limited, Cambridge u.a., 131-165.

Link: https://doi.org/10.1533/9780857097415.1.131

Bauer, C., Korthals, M., Gronauer, A., Lebuhn, M. (2008): Methanogens in biogas production from renewable resources - a novel molecular population analysis approach. Water Science and Technology 58 (7), 1433-1439.

Link: https://doi.org/10.2166/wst.2008.514

Bauer, C., Lebuhn, M., Gronauer, A. (2009): Mikrobiologische Prozesse in landwirtschaftlichen Biogasanlagen. In: Bayerische Landesanstalt für Landwirtschaft (Hrsg.): Schriftenreihe der Bayerischen Landesanstalt für Landwirtschaft 12/2009, 38 S. Link:

https://www.lfl.bayern.de/mam/cms07/publikationen/daten/schriftenreihe/p_36964.pdf

BRAUN, E. (1982): Biogas - Methangärung organischer Abfallstoffe: Grundlagen und Anwendungsbeispiele. Springer, Wien, 206 S.

Link: https://doi.org/10.1007/978-3-7091-8675-6

BRENDA: BRENDA - The Comprehensive Enzyme Information System. OnlineVolltextdatenbank, Technische Universität Braunschweig.

Link: https://www.brenda-enzymes.org

Burgstaler, J., Wiedow, D., Godlinski, F., Kanswohl, N. (2011): Einsatz von Natriumhydrogencarbonat in landwirtschaftlichen Biogasanlagen. Landbauforschung vTI Agriculture and Forestry Research 4 (61), 343-352.

Link: https://d-nb.info/1017873259/34

CAllander, I.J., BARFord, J.P. (1983): Precipitation, chelation, and the availability of metals as nutrients in anaerobic digestion: II. Applications. Biotechnology and Bioengineering 25 (8), 1959-1972. Link: https://doi.org/10.1002/bit.260250806

Chen, Y., Cheng, J.J., Creamer, K.S. (2008): Inhibition of anaerobic digestion process: A review. Bioresource Technology 99 (10), 4044-4064.

Link: https://doi.org/10.1016/j.biortech.2007.01.057 
Choong, Y.Y., Norli, I., Abdullah, A.Z., Yhaya, M.F. (2016): Impacts of trace element supplementation on the performance of anaerobic digestion process: A critical review. Bioresource Technology 209, 369-379.

Link: https://doi.org/10.1016/j.biortech.2016.03.028

CONRAD, R., WetTER, B. (1990): Influence of temperature on energetics of hydrogen metabolism in homoacetogenic, methanogenic, and other anaerobic bacteria. Archives of Microbiology 155 (1), 94-98. Link: https://doi.org/10.1007/BF00291281

DEMIREL, B., SCHERER, P. (2011): Trace element requirements of agricultural biogas digesters during biological conversion of renewable biomass to methane. Biomass and Bioenergy 35 (3), 992-998. Link: https://doi.org/10.1016/j.biombioe.2010.12.022

DIN EN 10088-1:2005 (2005): Nichtrostende Stähle - Teil 1: Verzeichnis der nichtrostenden Stähle. Deutsche Fassung EN 10088-1:2005, Deutsches Institut für Normung, Berlin, 49 S. Link: https://www.beuth.de/de/norm/din-en-10088-1/65991201

DIN EN 12879:2001 (2001): Charakterisierung von Schlämmen - Bestimmung des Glühverlustes der Trockenmasse. Deutsche Fassung EN 12879:2000, Deutsches Institut für Normung, Berlin, 7 S. Link: https://www.beuth.de/de/norm/din-en-12879/36017972

DIN EN 12880:2001 (2001): Charakterisierung von Schlämmen - Bestimmung des Trockenrückstandes und des Wassergehalts. Deutsche Fassung EN 12880:2000, Deutsches Institut für Normung, Berlin, $7 \mathrm{~S}$.

Link: https://www.beuth.de/de/norm/din-en-12880/36017907

DüMV (2012): Düngemittelverordnung vom 5. Dezember 2012. BGBl. I, S. 2482, zuletzt geändert am 2. Oktober 2019 (BGB1. I, S. 1414), 117 S.

Link: https://www.gesetze-im-internet.de/d_mv_2012/D\%C3\%BCMV.pdf

Engler, N., Merrettig-Bruns, U., Feher, A., Fritz, T., Nelles, M. (2011): Der ORGA-Test: Entwicklung eines Testverfahrens zur praxisnahen Beurteilung der Fermenterbiologie von NawaRo-Biogasanlagen. In: Fachagentur Nachwachsende Rohstoffe e.V. (Hrsg.): Einsatz von Hilfsmitteln zur Steigerung der Effizienz und Stabilität des Biogasprozesses. Gülzower Fachgespräche 35, 32-44.

Link: https://www.fnr-server.de/ftp/pdf/literatur/pdf_445-gfghilfsmittelbiogas_web.pdf

FAHLBUSCH, W. (2018): Transfer of Main and Trace Elements from Soil to Plant with an Emphasis on Trace Element Supply for Biogas Digestion Plants. Dissertation, Göttingen, 157 S. Link: http://hdl.handle.net/11858/00-1735-0000-002E-E4B1-4

Fahlbusch, W., Hey, K., Sauer, B., RupPert, H. (2018): Trace element delivery for biogas production enhanced by alternative energy crops: results from two-year field trials. Energy, Sustainability and Society 8 (38), 11 S.

Link: https://doi.org/10.1186/s13705-018-0180-1 
FAthePuRE, B.Z. (1987): Factors Affecting the Methanogenic Activity of Methanothrix soehngenii VNBF. Applied and Environmental Microbiology 53 (12), 2978-2982.

Link: https://www.ncbi.nlm.nih.gov/pmc/articles/PMC204234/

Feher, A., Fritz, T., Loewen, A., Nelles, M. (2013): Bedarfsgerechter Einsatz von Spurenelement-Biogasadditiven unter Berücksichtigung der Bioverfügbarkeit. In: ProFair Consult+Project GmbH (Hrsg.): Tagungsband zum 6. Biogas-Innovationskongress 2013, Osnabrück, 55-62.

Feng, X.M., Karlsson, A., Svensson, B.H., Bertilsson, S. (2010): Impact of trace element addition on biogas production from food industrial waste - linking process to microbial communities. FEMS Microbiology Ecology 74 (1), 226-240.

Link: https://doi.org/10.1111/j.1574-6941.2010.00932.x

Friehe, J., Schattauer, A., Weiland, P. (2016): Grundlagen der anaeroben Fermentation. In: Fachagentur Nachwachsende Rohstoffe e.V. (Hrsg.): Leitfaden Biogas - Von der Gewinnung zur Nutzung. 7. Aufl., 11-20. Link:

https://www.fnr.de/fileadmin/allgemein/pdf/broschueren/Leitfaden_Biogas_web_V01.pdf

GAllert, C., Winter, J.(2015): Mikrobielle Grundlagen der Methangärung. In: Rosenwinkel, K.-H., Kroiss, H., $\quad$ Dichtl, N., Seyfried, C.-F., Weiland, P. (Hrsg.): Anaerobtechnik. 3. Aufl., Springer, Berlin u.a., 20-53.

Link: https://doi.org/10.1007/978-3-642-24895-5_2

GonZalez-Gil, G., Kleerebezem, R., Lettinga, G. (1999): Effects of Nickel and Cobalt on Kinetics of Methanol Conversion by Methanogenic Sludge as Assessed by On-Line $\mathrm{CH}_{4}$ Monitoring. Applied and Environmental Microbiology 65 (4), 1789-1793. Link: https://doi.org/10.1128/AEM.65.4.1789-1793.1999

Gustavsson, J., Shakeri Yekta, S., Sundberg, C., Karlsson, A., Ejlertsson, J., SKYllBERG, U., Svensson, B.H. (2013): Bioavailability of cobalt and nickel during anaerobic digestion of sulfur-rich stillage for biogas formation. Applied Energy 112, 473-477. Link: https://doi.org/10.1016/j.apenergy.2013.02.009

Griehl, C., Hecht, C., Streuber, S., Ganß, Y., Kremp, H. (2010): Bestimmung von Phenylessigsäure zur Früherkennung von Prozessstörungen in Biogasanlagen. Chemie Ingenieur Technik 82 (12), 2223-2229. Link: https://doi.org/10.1002/cite.200900064

GrimM, D. (2016): Untersuchung der Auswirkungen von Spurenelementen auf den Biogasprozess. Masterarbeit, Hochschule Merseburg (FH), 102 S.

HAYES, T.D., ThEIS, T.L. (1978): The distribution of heavy metals in anaerobic digestion. Journal Water Pollution Control Federation 50 (1), 61-72.

Link: https://www.jstor.org/stable/25039507

Heinrichs, H., Herrmann, A.G. (1990): Praktikum der Analytischen Geochemie. Springer, Berlin u.a., 669 S. Link: https://doi.org/10.1007/978-3-642-61286-2 
HEYNER, T. (2017): Substitution von Spurenelementzusätzen durch alternative Energiepflanzen in Biogasanlagen. Masterarbeit, Brandenburgische Technische Universität Cottbus-Senftenberg, $116 \mathrm{~S}$.

Hinken, L., Urban, I., Haun, E., Urban, I., Weichgrebe, D., Rosenwinkel, K.-H. (2008): The valuation of malnutrition in the mono-digestion of maize silage by anaerobic batch tests. Water Science and Technology 58 (7), 1453-1459.

Link: https://doi.org/10.2166/wst.2008.491

Jarvis, A., Nordberg, A., Jarlsvik, T., Mathisen, B., Svensson, B.H. (1997): Improvement of a grass-clover silage-fed biogas process by the addition of cobalt. Biomass and Bioenergy 12 (6), 453-460.

Link: https://doi.org/10.1016/S0961-9534(97)00015-9

Kaiser, F., Metzner, T., Effenberger, M., Gronauer, A. (2008): Sicherung der Prozessstabilität in landwirtschaftlichen Biogasanlagen. In: Bayerische Landesanstalt für Landwirtschaft (Hrsg.): LfL-Information der Bayerischen Landesanstalt für Landwirtschaft. September 2008, 2. Aufl., 14 S. Link:

https://www.lfl.bayern.de/mam/cms07/publikationen/daten/informationen/p_32448.pdf

KAYHANIAN, M., Rich, D. (1995): Pilot-scale high solids thermophilic anaerobic digestion of municipal solid waste with an emphasis on nutrient requirements. Biomass and Bioenergy 8 (6), 433-444. Link: https://doi.org/10.1016/0961-9534(95)00043-7

Kida, K., Shigematsu, T., Kijima, J., Numaguchi, M., Mochinaga, Y., Abe, N., MorimurA, S. (2001): Influence of $\mathrm{Ni}^{2+}$ and $\mathrm{Co}^{2+}$ on Methanogenic Activity and the Amounts of Coenzymes Involved in Methanogenesis. Journal of Bioscience and Bioengineering 91 (6), 590-595. Link: https://doi.org/10.1016/S1389-1723(01)80179-1

KLETZIN, A., AdAms, M.W.W. (1996): Tungsten in biological systems. FEMS Microbiology Reviews 18 (1), 5-63. Link: https://doi.org/10.1016/0168-6445(95)00025-9

Koch, K., Post, M., $\quad$ Auer, M., $\quad$ LeBuhn, M. (2017): Einsatzstoffspezifische Besonderheiten in der Prozessführung. In: Arbeitsgemeinschaft Landtechnik und landwirtschaftliches Bauwesen in Bayern e.V. (Hrsg.): Biogas Forum Bayern Nr. III 18/2017. $13 \mathrm{~S}$.

Link: http://www.biogas-forum-bayern.de/media/files/0003/einsatzstoffspezifischebesonderheiten-in-der-prozessf-hrung.pdf

Koch, K., Gepperth, S., Andrade, D., Ebertseder, F., Gronauer, A. (2011): Hilfsmitteleinsatz bei der Biogaserzeugung - Überblick und Erfahrungen aus Labor und Praxis. In: Fachagentur Nachwachsende Rohstoffe e.V. (Hrsg.): Einsatz von Hilfsmitteln zur Steigerung der Effizienz und Stabilität des Biogasprozesses. Gülzower Fachgespräche 35, 16-31.

Link: https://www.fnr-server.de/ftp/pdf/literatur/pdf_445-gfghilfsmittelbiogas_web.pdf 
Kouzeli-Katsiri, A., Kartsonas, N. (1986): Inhibition of Anaerobic Digestion by Heavy Metals. In: Bruce, A.M, Kouzeli-Katsiri, A., Newman, P.J. (Hrsg): Anaerobic Digestion Of Sewage Sludge And Organic Agricultural Wastes. Elsevier, London u.a., 104-119.

Koster, I.W., Rinzema, A., De Vegt, A.L., LettingA, G. (1986): Sulfide inhibition of the methanogenic activity of granular sludge at various $\mathrm{pH}-$ levels. Water Research 20 (12), 1561-1567. Link: https://doi.org/10.1016/0043-1354(86)90121-1

Kroiss, H., Svardal, K. (2005): Einflussfaktoren auf die anaeroben biologischen Abbauvorgänge. In: Bischofsberger, W., Dichtl, N., Rosenwinkel, K.-H., Seyfried, C.F., Böhnke, B. (Hrsg.): Anaerobtechnik. 2. Aufl., Springer, Berlin u.a., 49-86.

Link: https://doi.org/10.1007/3-540-26593-7_3

KroIss, H., SVARDAL, K. (2015): Einflussfaktoren auf die anaeroben biologischen Abbauvorgänge. In: Rosenwinkel, K.-H., Kroiss, H., Dichtl, N., Seyfried, C.F., Weiland, P. (Hrsg.): Anaerobtechnik. 3. Aufl., Springer, Berlin u.a., 81-112.

Link: https://doi.org/10.1007/978-3-642-24895-5_3

Kunst, S. (2005): Mikrobiologische Grundlagen. In: Bischofsberger, W., Dichtl, N., Rosenwinkel, K.-H., Seyfried, C.F., Böhnke, B. (Hrsg.): Anaerobtechnik. 2. Aufl., Springer, Berlin u.a., 23-48. Link: https://doi.org/10.1007/3-540-26593-7_2

LeBuhn, M, EFFEnBERger, M. (2012): Hygienisierung durch Biogastechnologie. $K W-$ Korrespondenz Wasserwirtschaft 8, 419-424.

Lebuhn, M, Gronauer, A. (2009): Microorganisms in the biogas-process - the unknown beings. Landtechnik 64 (2), 127-130.

Link: https://doi.org/10.15150/lt.2009.651

Lebuhn, M.; LiU, F.; Heuwinkel, H.; Gronauer, A. (2008): Biogas production from mono-digestion of maize silage-long-term process stability and requirements. Water Science and Technology 58 (8), 1645-1651. Link: https://doi.org/10.2166/wst.2008.495

LeE, C.C., Hu, Y., RIBBE, M.W. (2010): Vanadium Nitrogenase Reduces CO. Science 326 (5992), 642. Link: https://doi.org/10.1126/science.1191455

Lemmer, A., Vintiloiu, A., Preißler, D., Bastam, C., Bäuerle, L., Oechsner, H., MATHIES, E., RAMHOLD, D. (2011): Untersuchungen zum Einsatz von Mineralstoffen in Biogasanlagen - Bedeutung der Mineralstoffe für die anaeroben Mikroorganismen und Ursachen für Konzentrationsunterschiede in Biogasfermentern. In: Fachagentur Nachwachsende Rohstoffe e.V. (Hrsg.): Einsatz von Hilfsmitteln zur Steigerung der Effizienz und Stabilität des Biogasprozesses. Gülzower Fachgespräche 35, 45-77. Link: https://www.fnr-server.de/ftp/pdf/literatur/pdf_445-gfghilfsmittelbiogas_web.pdf 
Liebetrau, J., PfeIfFER, D., ThrÄN, D. [Hrsg.] (2013): Messmethodensammlung Biogas - Methoden zur Bestimmung von analytischen und prozessbeschreibenden Parametern im Biogasbereich. Schriftenreihe des BMU-Förderprogramms ,Energetische Biomassenutzung', Band 7, 2. Aufl., DBFZ, Leipzig, 212 S.

Link: https://www.energetische-biomassenutzung.de/fileadmin/media/6_Publikationen/ 07_MMS_Biogas_2014.pdf

LINDORFER, H. (2014): Analyse und Bewertung der Spurenelementversorgung von Mikroorganismen. Präsentation zur Fachtagung: Prozessmesstechnik in Biogasanlagen, 25-26.03.2014, Leipzig, $22 \mathrm{~S}$.

LiNDORFER, H., RAMHOLD, D., FraUZ, B. (2012): Nutrient and trace element supply in anaerobic digestion plants and effect of trace element application. Water Science and Technology 66 (9), 1923-1929. Link: https://doi.org/10.2166/wst.2012.399

Merrettig-Bruns, U., Kabasci, S., Loewen, A., Feher, A., Engler, N., Nelles, M. (2011): ORGA-Test-Standardisiertes Testverfahren zur Bestimmung der biologischen Aktivität in Biogasfermentern zur Ermittlung von Hemmwirkungen und Mangelerscheinungen. In: ProFair Consult+Project GmbH (Hrsg.): Tagungsband zum 4. Biogas-Innovationskongress 2011, Osnabrück, 35-44.

Merrettig-Bruns, U., Kabasci, S., Loewen, A., Feher, A., Nelles, M., Engler, N. (2012): Entwicklung eines Testsystems zur Bestimmung der biologischen Aktivität in Biogasfermentern und Bestimmung des Einflusses von Minorkomponenten auf die Biogasbildung - Minorkomponententest. Schlussbericht zum Vorhaben, AiF/Programm zur Förderung der Industriellen Gemeinschaftsforschung und -entwicklung (IGF), Institut für Energie- und Umwelttechnik e.V. (AiF 16103 BG), $96 \mathrm{~S}$.

Link: https://www.iuta.de/igf-docs/schlussbericht_16103bg_minorkomponenten.pdf

Munk, B., Bauer, C., Gronauer, A., Lebuhn, M. (2010): Population dynamics of methanogens during acidification of biogas fermenters fed with maize silage. Engineering in Life Sciences 10 (6), 496-508.

Link: https://doi.org/10.1002/elsc.201000056

NRC: NATIONAL ReSEARCH COUNCIL [Hrsg.] (2003): Bioavailability of Contaminants in Soils and Sediments: Processes, Tools, and Applications. The National Academies Press, Washington, DC, 432 S. Link: https://doi.org/10.17226/10523

Oechsner, H., Lemmer, A., Ramhold, D., Mathies, E., MAyrhuber, E., Preißler,D. (2008): Verfahren zur Biogaserzeugung. EP 1997901 A2, Patentblatt 2008/49, Europäisches Patentamt, $18 \mathrm{~S}$.

Link: https://data.epo.org/publication-server/getpdf.jsp?cc=EP\&pn=1997901\&ki=A2

OleszKIEwiCZ, J.A., Sharma, V.K. (1990): Stimulation and Inhibition of Anaerobic Processes by Heavy 'Metals - A Review. Biological Wastes 31 (1), 45-67.

Link: https://doi.org/10.1016/0269-7483(90)90043-R 
Pobeheim, H., Munk, B., Johansson, J., Guebitz, G.M. (2010): Influence of trace elements on methane formation from a synthetic model substrate for maize silage. Bioresource Technology 101 (2), 836-839.

Link: https://doi.org/10.1016/j.biortech.2009.08.076

Pobeheim, H., Munk, B., Lindorfer, H., Guebitz, G.M. (2011): Impact of nickel and cobalt on biogas production and process stability during semi-continuous anaerobic fermentation of a model substrate for maize silage. Water Research 45 (2), 781-787.

Link: https://doi.org/10.1016/j.watres.2010.09.001

Pospiech, S., Fahlbusch, W., Pasold, T., SAuer, B., Ruppert, H. (2017a): Elementanalytik an ICP-OES und ICP-MS - Handbuch für die Probenpräparation, Messung und Auswertung. Interne Wissenssammlung der Arbeitsgruppe Umweltgeochemie (Skript), unveröffentlicht, Göttingen, 75 S.

Pospiech, S., Fahlbusch, W., Sauer, B., Pasold, T., RupPert, H. (2017b): Alteration of trace element concentrations in plants by adhering particles - Methods of correction. Chemosphere 182, 501-508. Link: https://doi.org/10.1016/j.chemosphere.2017.05.038

REHDER, D. (2000): Vanadium nitrogenase. Journal of Inorganic Biochemistry 80 (1-2), 133-136. Link: https://doi.org/10.1016/s0162-0134(00)00049-0

RICKARD, D. (2012): Sulfidic Sediments and Sedimentary Rocks. In: Rickard, D. (Hrsg.): Developments in Sedimentology 65. Elsevier, Amsterdam u.a., $816 \mathrm{~S}$.

Link: https://www.elsevier.com/books/sulfidic-sediments-and-sedimentary-rocks/ rickard/978-0-444-52989-3

RickARD, D., Luther, G.W. (2007): Chemistry of Iron Sulfides. Chemical Reviews 107 (2), 514-562. Link: https://doi.org/10.1021/cr0503658

RUPPERT, H. (2007): Serieller Totalaufschluß von Gesteinen und Böden mit Säuren zur nachfolgenden Bestimmung der Element-Gesamtgehalte. Einführung in die chemische Analytik von Feststoffen und Fluiden (Skript), unveröffentlicht, Göttingen, 12 S.

SAHM, H. (1981): Biologie der Methan-Bildung. Chemie Ingenieur Technik 53 (11), 854-863. Link: https://doi.org/10.1002/cite.330531105

SAUER, B. (2010): Elementgehalte und Stoffströme bei der Strom- und Wärmegewinnung im Bioenergiedorf Jühnde. Dissertation. Schriftenreihe Fortschritt neu denken, Heft 2, Göttingen, $124 \mathrm{~S}$.

SAUER, B., FAHLBUSCH, W., RUPPERT, H. (2014): Möglichkeiten einer ausgeglichenen Spurenelementversorgung von Biogasanlagen durch Mischungen unterschiedlicher Energiepflanzen. Schlussbericht zum Vorhaben, Fachagentur Nachwachsende Rohstoffe e.V. (Förderkennzeichen: 22041611), $37 \mathrm{~S}$.

Link: https://www.fnr-server.de/ftp/pdf/berichte/22041611.pdf 
SAUER, B., FAHLBUSCH, W., RUPPERT, H. (2017): Bioenergetische Nutzungskonzepte für kontaminierte Standorte. In: Ruppert, H., Ibendorf, J. (Hrsg.): Bioenergie im Spannungsfeld - Wege zu einer nachhaltigen Bioenergieversorgung. Universitätsverlag Göttingen, 333-353. Link: https://doi.org/10.17875/gup2017-1042

SAUER, B., RupPert, H. (2013): Bioenergy Production as an Option for Polluted SoilsA Non-phytoremediation Approach. In: Ruppert, H., Kappas, M., Ibendorf, J. (Hrsg.): Sustainable Bioenergy Production - An Integrated Approach. Springer, Berlin u.a., 425-444. Link: https://doi.org/10.1007/978-94-007-6642-6_14

Sauer, B., Sträuber, H., Fahlbusch, W., Hey, K., Pasold, T., Pröter, J., Rauber, R., RUPPERT, H., ZECHENDORF, M. (2018): Spurenelemente durch Energiepflanzen - Stoffströme und Handlungsempfehlungen für eine optimierte Prozessbiologie in Biogasanlagen (NiCo). Schlussbericht zum Vorhaben, Fachagentur Nachwachsende Rohstoffe e.V. (Förderkennzeichen: 22014813, 22019014, 22019114, 22019214), 85 S.

Link: https://www.fnr-server.de/ftp/pdf/berichte/22014813.pdf

Schattauer, A., Abdoun, E., Weiland, P., Plöchl, M., Heiermann, M. (2011): Abundance of trace elements in demonstration biogas plants. Biosystems Engineering 108 (1), 57-65. Link: https://doi.org/10.1016/j.biosystemseng.2010.10.010

SCHERER, P., SAHM, H. (1981): Effect of trace elements and vitamins on the growth of Methanosarcina barkeri. Acta Biotechnologica 1 (1), 57-65.

Link: https://doi.org/10.1002/abio.370010108

Schieder, D., Gronauer, A., Lebuhn, M., Bayer, K., Beck, J., Hiepr, G., Binder, S. (2010): Prozessmodell Biogas. In: Arbeitsgemeinschaft Landtechnik und landwirtschaftliches Bauwesen in Bayern e.V. (Hrsg.): Biogas Forum Bayern Nr. III - 03/2010. $39 \mathrm{~S}$.

Link: https://www.biogas-forum-bayern.de/publikationen/Prozessmodell_Biogas.pdf

SCHIERITZ, R. (2016): Untersuchung der Konzentration flüchtiger organischer Säuren (FOS) im Biogasprozess in Abhängigkeit der Gehalte von Nickel und Cobalt und die Entwicklung einer Methode zur semiquantitativen FOS-Analytik mittels Nahinfrarotspektroskopie (NIRS). Masterarbeit, Technische Universität Dresden, 99 S.

SCHINK, B. (1997): Energetics of Syntrophic Cooperation in Methanogenic Degradation Microbiology and Molecular Biology Reviews 61 (2), 262-280.

Link: https://mmbr.asm.org/content/61/2/262

SChNÜRER, A., ZEllner, G., Svensson, B.H. (1999): Mesophilic syntrophic acetate oxidation during methane formation in biogas reactors. FEMS Microbiology Ecology 29 (3), 249-261. Link: https://doi.org/10.1111/j.1574-6941.1999.tb00616.x

Scholwin, F., Liebetrau, J., Edelmann, W., RitzKowski, M., KöRner, I. (2009): Biogaserzeugung und -nutzung. In: Kaltschmitt, M., Hartmann, H., Hofbauer, H. (Hrsg.): Energie aus Biomasse - Grundlagen, Techniken und Verfahren. 2. Aufl., Springer, Berlin u.a., 851-931. Link: https://doi.org/10.1007/978-3-540-85095-3_16 
Schönheit, P., Mold, J., Thauer, R.K. (1979): Nickel, Cobalt, and Molybdenum Requirement for Growth of Methanobacterium thermoautotrophicum. Archives of Microbiology 123 (1), 105-107. Link: https://doi.org/10.1007/BF00403508

SimON, K. (2009): Einführung in die Geochemische Analytik. Instrumentelle Analytik (Skript), unveröffentlicht, Göttingen, $75 \mathrm{~S}$.

Skoog, D.A., Holler, F.J., Crouch, S.R. (2017): Principles of Instrumental Analysis. 7. Aufl., Cengage Learning, Boston, $992 \mathrm{~S}$.

Takashima, M., SPeece, R.E., Parkin, G.F. (1990): Mineral requirements for methane fermentation. Critical Reviews in Environmental Control 19 (5), 465-479.

Link: https://doi.org/10.1080/10643389009388378

Thanh, P.M., Ketheesan, B., Yan, Z., Stuckey, D. (2016): Trace metal speciation and bioavailability in anaerobic digestion: A review. Biotechnology Advances 34 (2), 122-136. Link: https://doi.org/10.1016/j.biotechadv.2015.12.006

Thauer, R.K., Kaster, A.-K., Goenrich, M., Schick, M., Hiromoto, T., Shima, S. (2010): Hydrogenases from methanogenic archaea, nickel, a novel cofactor, and $\mathrm{H}_{2}$ storage. Annual Review of Biochemistry 79, 507-536.

Link: https://doi.org/10.1146/annurev.biochem.030508.152103

van Hullebusch, E.D., Guibaud, G., Simon, S., Lenz, M., Yekta, S.S., Fermoso, F.G., Jain, R., Duester, L., Roussel, J., Guillon, E., Skyllberg, U., Almeida, C.M.R., Pechaud, Y., Garuti, M., Frunzo, L., Esposito, G., Carliell-Marquet, C., ORTNER, M., COLlins, G. (2016): Methodological approaches for fractionation and speciation to estimate trace element bioavailability in engineered anaerobic digestion ecosystems: An overview. Critical Reviews in Environmental Science and Technology 46 (16), 1324-1366. Link: https://doi.org/10.1080/10643389.2016.1235943

Wackett, L.P., Dodge, A.G., Ellis, L.B.M. (2004): Microbial Genomics and the Periodic Table. Applied and Environmental Microbiology 70 (2), 647-655.

Link: https://doi.org/10.1128/AEM.70.2.647-655.2004

WANDREY, C., AIVASIDIS, A. (1983): Zur Reaktionskinetik der anaeroben Fermentation. Chemie Ingenieur Technik 55 (7), 516-524. Link: https://doi.org/10.1002/cite.330550705

WeIland, P. (2000): Stand und Perspektiven der Biogasnutzung und -erzeugung in Deutschland. In: Fachagentur Nachwachsende Rohstoffe e.V. (Hrsg.): Energetische Nutzung von Biogas - Stand der Technik und Optimierungspotenzial. Gülzower Fachgespräche 15, 8-27.

Link: https://www.fnr-server.de/ftp/pdf/literatur/pdf_94gfg_bd15.pdf

WeILAND, P. (2001): Grundlagen der Methangärung - Biologie und Substrate. In: VDI-Gesellschaft Energietechnik (Hrsg.): Biogas als regenerative Energie - Stand und Perspektiven. VDI Berichte 1620, 19-32. 
WeIland, P. (2008): Wichtige Messdaten für den Prozessablauf und Stand der Technik in der Praxis. In: Fachagentur Nachwachsende Rohstoffe e.V. (Hrsg.): Messen, Steuern, Regeln bei der Biogaserzeugung. Gülzower Fachgespräche 27, 17-31. Link:

https://www.fnr-server.de/ftp/pdf/literatur/pdf_328-gf_band_27_biogaserzeugung_bf.pdf

WeIland, P. (2010): Biogas production: current state and perspectives. Applied Microbiology and Biotechnology 85 (4), 849-860.

Link: https://doi.org/10.1007/s00253-009-2246-7

WeINRICH, S. (2017): Praxisnahe Modellierung von Biogasanlagen. Dissertation. Schriftenreihe Umweltingenieurwesen 76, Rostock, 259 S. Link: http://rosdok.uni-rostock.de/file/rosdok disshab 0000001838/rosdok derivate 0000038195/

Dissertation_Weinrich_2018.pdf

Wintsche, B., Glaser, K., Sträuber, H., Centler, F., Liebetrau, J., Harms, H., Kleinsteuber, S. (2016): Trace Elements Induce Predominance among Methanogenic Activity in Anaerobic Digestion. Frontiers in Microbiology 7:2034.

Link: https://doi.org/10.3389/fmicb.2016.02034

Wittmann, C., Zeng, A.P., DeCKWER, W.D. (1995): Growth inhibition by ammonia and use of a pH-controlled feeding strategy for the effective cultivation of Mycobacterium chlorophenolicum. Applied Microbiology and Biotechnology 44 (3-4), 519-525. Link: https://doi.org/10.1007/BF00169954

Zandvoort, M.H., VAn Hullebusch, E.D., Fermoso, F.G., Lens, P.N.L. (2006): Trace Metals in Anaerobic Granular Sludge Reactors: Bioavailability and Dosing Strategies. Engineering in Life Sciences 6 (3), 293-301.

Link: https://doi.org/10.1002/elsc.200620129

Zandvoort, M.H., van Hullebusch, E.D., Gieteling, J., Lettinga, G., Lens, P.N.L. (2005): Effect of Sulfur Source on the Performance and Metal Retention of MethanolFed UASB Reactors. Biotechnology Progress 21 (3), 839-850.

Link: https://doi.org/10.1021/bp0500462

ZAYED, G, WINTER, J. (2000): Inhibition of methane production from whey by heavy metals - protective effect of sulfide. Applied Microbiology and Biotechnology 53, 726-731. Link: https://doi.org/10.1007/s002530000336 


\section{Anhang}

(1) Projektphase 1: Versuchs- und Probenahmetage (grau unterlegt) -

(Quasi)kontinuierliche Laborfermenterversuche am DBFZ. ...

(2) Projektphase 2: Versuchs- und Probenahmetage (grau unterlegt)-

(Quasi)kontinuierliche Laborfermenterversuche am DBFZ, mit Zusatzversuch

(3) Projektphase 3: Versuchs- und Probenahmetage (grau unterlegt) -

(Quasi)kontinuierliche Laborfermenterversuche am DBFZ.

(4) Projektphase 1: Elementgehalte der Makro- und Mikronährstoffe -

(Quasi)kontinuierliche Laborfermenterversuche am DBFZ -

Reaktor 4.5: Reaktor ohne Nickel-Zugabe 1

(5) Projektphase 1: Elementgehalte der Makro- und Mikronährstoffe -

(Quasi)kontinuierliche Laborfermenterversuche am DBFZ -

Reaktor 4.6: Reaktor ohne Nickel-Zugabe 2

(6) Projektphase 1: Elementgehalte der Makro- und Mikronährstoffe -

(Quasi)kontinuierliche Laborfermenterversuche am DBFZ -

Reaktor 4.7: Reaktor ohne Cobalt-Zugabe 1

(7) Projektphase 1: Elementgehalte der Makro- und Mikronährstoffe -

(Quasi)kontinuierliche Laborfermenterversuche am DBFZ -

Reaktor 4.8: Reaktor ohne Cobalt-Zugabe 2

(8) Projektphase 1: Elementgehalte der Makro- und Mikronährstoffe -

(Quasi)kontinuierliche Laborfermenterversuche am DBFZ -

Reaktor 4.9: Referenzreaktor (Spurenelementvollversorgung)

(9) Projektphase 2: Elementgehalte der Makro- und Mikronährstoffe -

(Quasi)kontinuierliche Laborfermenterversuche am DBFZ -

Reaktor 4.5 und 4.6: Substitutionsversuch 1: Winterackerbohne 1 und 2.

(10) Projektphase 2: Elementgehalte der Makro- und Mikronährstoffe -

(Quasi)kontinuierliche Laborfermenterversuche am DBFZ -

Reaktor 4.7 und 4.8: Substitutionsversuch 2: Amarant 1 und 2

(11) Projektphase 2: Elementgehalte der Makro- und Mikronährstoffe -

(Quasi)kontinuierliche Laborfermenterversuche am DBFZ -

Reaktor 4.9: Referenzreaktor (Spurenelementvollversorgung)

(12) Projektphase 3: Elementgehalte der Makro- und Mikronährstoffe -

(Quasi)kontinuierliche Laborfermenterversuche am DBFZ -

Reaktor 4.5 und 4.6: Substitutionsversuch 3: Deutsches Weidelgras 1 und 2

(13) Projektphase 3: Elementgehalte der Makro- und Mikronährstoffe -

(Quasi)kontinuierliche Laborfermenterversuche am DBFZ -

Reaktor 4.7 und 4.8: Substitutionsversuch 4: Durchwachsene Silphie 1 und 2

(14) Projektphase 3: Elementgehalte der Makro- und Mikronährstoffe -

(Quasi)kontinuierliche Laborfermenterversuche am DBFZ -

Reaktor 4.9: Referenzreaktor (Spurenelementvollversorgung)

(15) Zusatzversuch: Elementgehalte der Makro- und Mikronährstoffe -

(Quasi)kontinuierliche Laborfermenterversuche am DBFZ -

Reaktor 4.11 und 4.12: Mais-Silage-Monovergärung mit reduzierter Spurenelemente-Zugabe ....216 


\section{Anhang Daten-Datei}

Microsoft Excel - Tabellenblätter:

Probenliste

Referenzmessungen ICP-OES und ICP-MS

Elementgehalte $\quad$ 1. Projektphase DBFZ: Reaktor 4.5 bis Reaktor 4.9

Elementgehalte $\quad$ 2. Projektphase DBFZ: Reaktor 4.5 bis Reaktor 4.9

Elementgehalte 3. Projektphase DBFZ: Reaktor 4.5 bis Reaktor 4.9

Elementgehalte Zusatzversuch DBFZ: Reaktor 4.11 und Reaktor 4.12

Elementgehalte Inputsubstrate: Diverse Energiepflanzen-Silagen und sonstige Substrate

Elementgehalte Praxisfermenter: Biogasanlage Verliehausen

Elementgehalte ORGA-Tests: Inokula und Stammlösungen

Elementgehalte Versuche mit verschiedenen Fraktionen von Fermentermaterialproben

Reaktorprotokolle 1. Projektphase DBFZ: Reaktor 4.5 bis Reaktor 4.9

Reaktorprotokolle 2. Projektphase DBFZ: Reaktor 4.5 bis Reaktor 4.9

Reaktorprotokolle 3. Projektphase DBFZ: Reaktor 4.5 bis Reaktor 4.9

Reaktorprotokolle Zusatzversuch DBFZ: Reaktor 4.11 und Reaktor 4.12

Reaktorprotokolle ORGA-Tests: Nickel

Reaktorprotokolle ORGA-Tests: Cobalt

Reaktorprotokoll ORGA-Test: Molybdän

Reaktorprotokolle ORGA-Tests: Kupfer

Reaktorprotokolle ORGA-Tests: Cadmium

Reaktorprotokoll ORGA-Test: Blei

Reaktorprotokoll ORGA-Test: Energiepflanzen-Silagen 
(1) Projektphase 1: Versuchs- und Probenahmetage (grau unterlegt) (Quasi)kontinuierliche Laborfermenterversuche am DBFZ

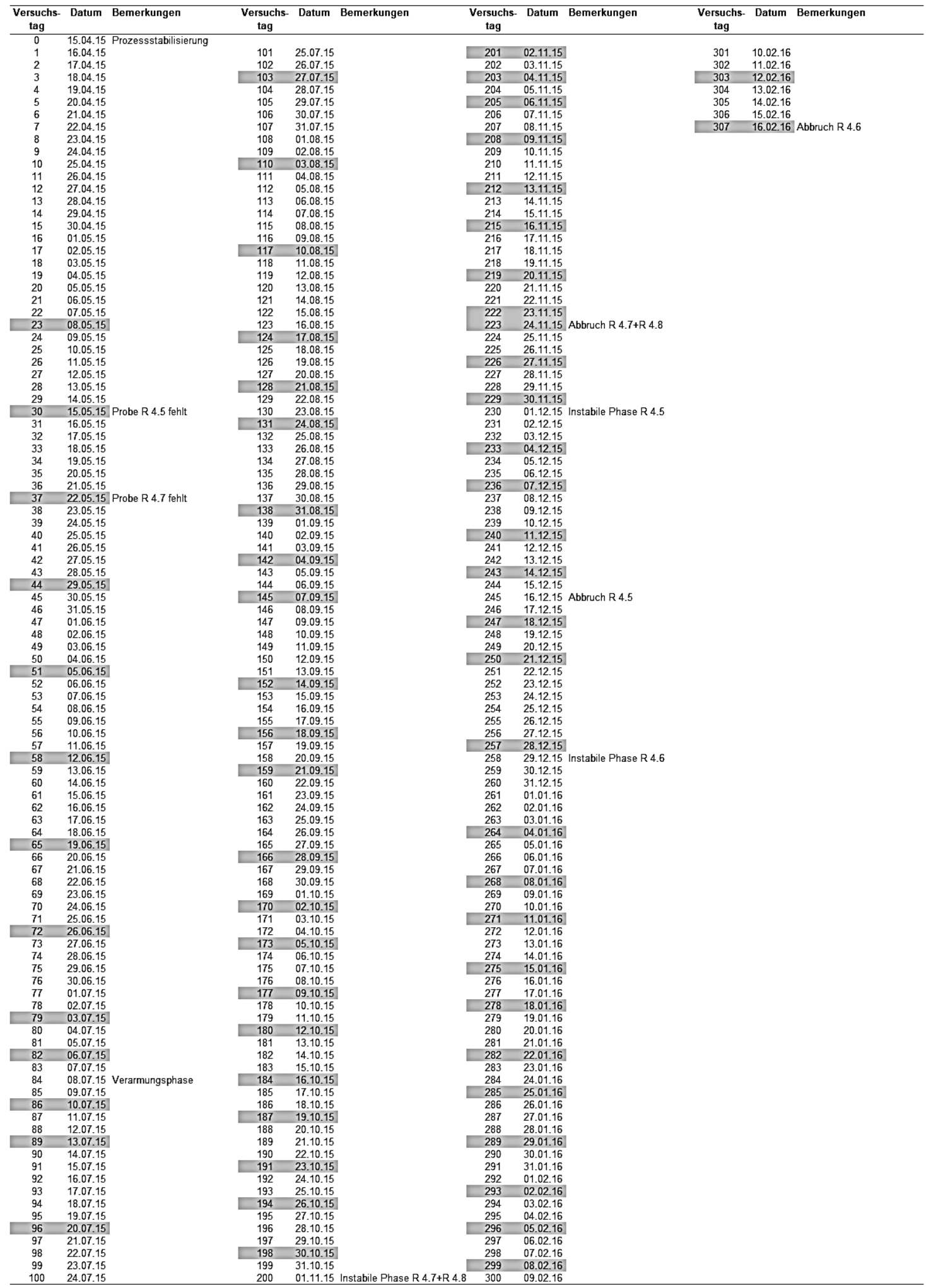


(2) Projektphase 2: Versuchs- und Probenahmetage (grau unterlegt) (Quasi)kontinuierliche Laborfermenterversuche am DBFZ, mit Zusatzversuch

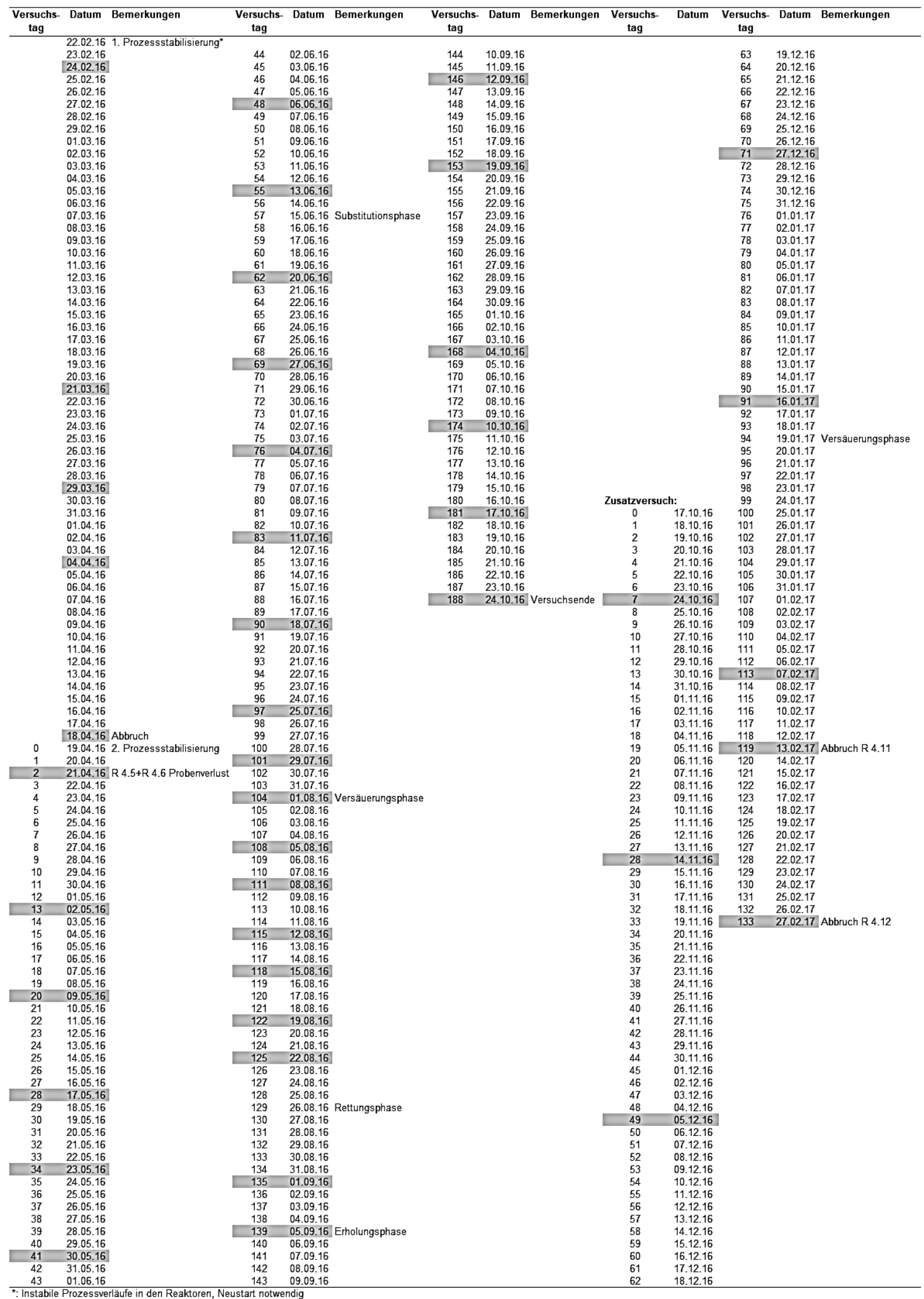


(3) Projektphase 3: Versuchs- und Probenahmetage (grau unterlegt) (Quasi)kontinuierliche Laborfermenterversuche am DBFZ

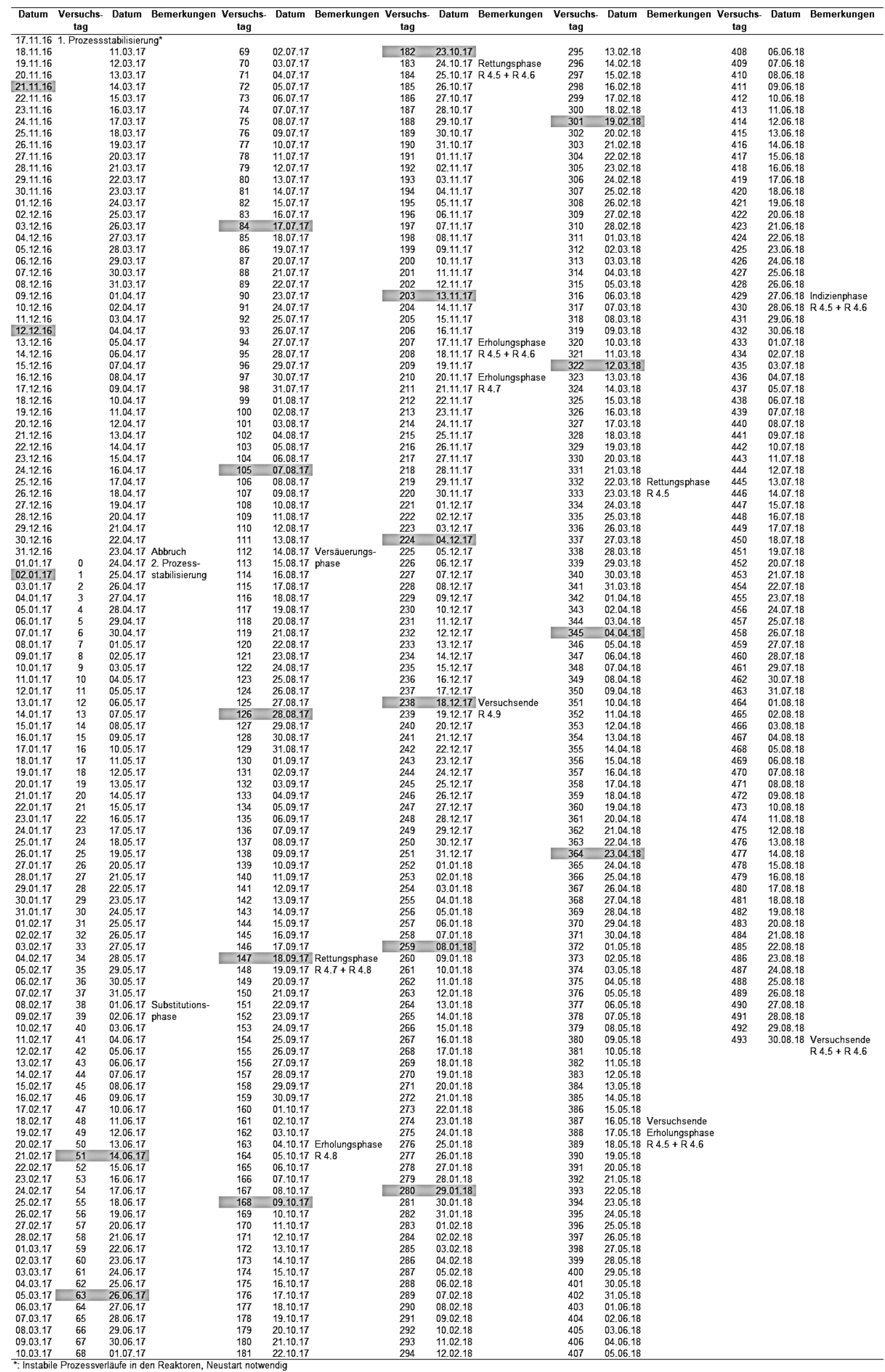


(4) Projektphase 1: Elementgehalte der Makro- und Mikronährstoffe (Quasi)kontinuierliche Laborfermenterversuche am DBFZ Reaktor 4.5: Reaktor ohne Nickel-Zugabe 1

\begin{tabular}{|c|c|c|c|c|c|c|c|c|c|c|c|c|c|c|c|c|c|c|}
\hline \multicolumn{4}{|c|}{ Verarmungsversuche } & \multicolumn{6}{|c|}{ Makronährstoffe } & \multicolumn{9}{|c|}{ Mikronährstoffe } \\
\hline & \multirow{2}{*}{$\begin{array}{l}\text { essmethode: } \\
\text { Element: }\end{array}$} & : ICP-OES & \multirow{2}{*}{\multicolumn{2}{|c|}{$\begin{array}{l}\text { ICP-OES ICP-OES } \\
\text { S } 181.972 \text { Ca } 317.933 r\end{array}$}} & \multirow{2}{*}{\multicolumn{2}{|c|}{$\begin{array}{l}\text { ICP-OES ICP-OES } \\
\text { K 766.491r Mg 279.078r }\end{array}$}} & \multirow{2}{*}{$\begin{array}{r}\text { ICP-OES } \\
\text { Na 588.995r }\end{array}$} & \multirow{3}{*}{$\begin{array}{r}\text { ICP-OES } \\
\text { Co } 230.786 \\
\text { Co }\end{array}$} & \multirow{3}{*}{$\begin{array}{r}\text { ICP-OES } \\
\text { Ni 231.604 } \\
\mathbf{N i}\end{array}$} & \multirow{2}{*}{$\begin{array}{l}\text { ICP-OES } \\
\text { Mo 202.032 }\end{array}$} & ICP-OES & ICP-OES & ICP-OES & ICP-OES & ICP-MS & ICP-OES \\
\hline \multicolumn{2}{|c|}{ mg/kg FM } & \multirow[b]{2}{*}{$\begin{array}{c}\text { TS } \\
\text { [Gew.-\%] }\end{array}$} & & P 178.222 & & & & & & & & & Fe 234.350 & Си 327.395 & Mn 257.610 & Zn 206.200 & Se 77 KED & v 292.401 \\
\hline $\begin{array}{l}\text { Versuchs- } \\
\text { tag }\end{array}$ & $\begin{array}{l}\text { Probe- } \\
\text { nahme }\end{array}$ & & $\begin{array}{c}\text { OTS } \\
\text { [Gew.-\%] }\end{array}$ & $\mathbf{P}$ & $\mathbf{s}$ & $\mathrm{Ca}$ & $\mathrm{K}$ & Mg & $\mathrm{Na}$ & & & & $\mathrm{Fe}$ & $\mathrm{Cu}$ & Mn & $\mathrm{Zn}$ & Se & $\mathbf{v}$ \\
\hline 23 & 08.05 .2015 & 6,1 & 78,4 & 470 & 230 & 471 & 2212 & 309 & 997 & 0,61 & 9,4 & 1,64 & 1128 & 1,94 & 83 & 60 & na & $\mathrm{na}$ \\
\hline 37 & 22.05 .2015 & 5,9 & 78,0 & 515 & 237 & 475 & 2363 & 331 & 771 & 0,60 & 8,9 & 1,54 & 1066 & 1,76 & 81 & 61 & na & na \\
\hline 44 & 29.05 .2015 & 6,0 & 77,9 & 531 & 250 & 515 & 2479 & 335 & 677 & 0,64 & 8,6 & 1,93 & 1193 & 1,66 & 94 & 65 & na & na \\
\hline 51 & 05.06 .2015 & 8,8 & 85,6 & 546 & 255 & 497 & 2504 & 330 & 586 & 0,59 & 7,9 & 1,77 & 1054 & 1,65 & 95 & 61 & na & na \\
\hline 58 & 12.06 .2015 & 6,1 & 79,1 & 517 & 244 & 473 & 2523 & 329 & 522 & 0,62 & 7,5 & 1,70 & 893 & 1,51 & 84 & 59 & 0,022 & 0,056 \\
\hline 65 & 19.06 .2015 & 7,4 & 81,9 & 575 & 262 & 542 & 2566 & 369 & 434 & 0,60 & 7,9 & 1,90 & 1056 & 1,70 & 94 & 59 & na & na \\
\hline 72 & 26.06 .2015 & 5,7 & 78,7 & 568 & 249 & 486 & 2454 & 356 & 379 & 0,58 & 7,8 & 1,69 & 924 & 1,52 & 89 & 61 & na & na \\
\hline 79 & 03.07.2015 & 6,5 & 81,3 & 503 & 241 & 486 & 2486 & 318 & 328 & 0,53 & 7,0 & 1,69 & 889 & 1,36 & 84 & 56 & 0,0163 & na \\
\hline 82 & 06.07 .2015 & 7,7 & 83,9 & 567 & 277 & 499 & 2315 & 338 & 313 & 0,59 & 7,0 & 2,1 & 879 & 1,58 & 94 & 62 & 0,0182 & 0,054 \\
\hline 86 & 10.07 .2015 & 6,0 & 80,5 & 493 & 240 & 448 & 2344 & 309 & 293 & 0,55 & 6,4 & 1,65 & 745 & 1,35 & 77 & 59 & 0,0167 & 0,031 \\
\hline 89 & 13.07 .2015 & 7,9 & 85,3 & 555 & 274 & 492 & 2339 & 339 & 281 & 0,60 & 6,5 & 1,77 & 837 & 1,47 & 86 & 64 & 0,0180 & 0,039 \\
\hline 96 & 20.07 .2015 & 7,3 & 84,6 & 560 & 273 & 516 & 2506 & 358 & 265 & 0,51 & 5,8 & 1,87 & 861 & 1,55 & 90 & 76 & 0,0162 & 0,042 \\
\hline 103 & 27.07 .2015 & 7,4 & 85,4 & 525 & 267 & 511 & 2444 & 331 & 208 & 0,55 & 4,9 & 1,72 & 872 & 1,39 & 87 & 76 & na & na \\
\hline 110 & 03.08 .2015 & 7,1 & 85,8 & 432 & 218 & 416 & 2065 & 282 & 173 & 0,41 & 4,5 & 1,45 & 654 & 1,19 & 70 & 67 & 0,0147 & 0,021 \\
\hline 117 & 10.08 .2015 & 6,5 & 83,0 & 526 & 264 & 507 & 2272 & 327 & 154 & 0,54 & 4,0 & 1,68 & 864 & 1,32 & 86 & 88 & na & na \\
\hline 124 & 17.08 .2015 & 8,5 & 87,7 & 613 & 300 & 502 & 1697 & 333 & 111 & 0,56 & 3,6 & 2,1 & 873 & 1,59 & 101 & 105 & 0,0155 & 0,027 \\
\hline 128 & 21.08 .2015 & 7,4 & 85,3 & 521 & 265 & 474 & 2396 & 318 & 142 & 0,53 & 3,4 & 1,62 & 803 & 1,33 & 90 & 97 & na & na \\
\hline 131 & 24.08 .2015 & 7,6 & 86,3 & 532 & 265 & 481 & 2221 & 328 & 131 & 0,55 & 3,1 & 1,94 & 769 & 1,45 & 91 & 94 & 0,0144 & 0,047 \\
\hline 138 & 31.08 .2015 & 7,5 & 86,2 & 512 & 258 & 466 & 2048 & 304 & 106 & 0,52 & 2,8 & 1,60 & 758 & 1,27 & 82 & 96 & na & na \\
\hline 142 & 04.09 .2015 & 7,1 & 85,0 & 525 & 268 & 475 & 2208 & 317 & 105 & 0,53 & 2,8 & 1,67 & 785 & 1,34 & 84 & 102 & na & na \\
\hline 145 & 07.09.2015 & 6,6 & 84,4 & 524 & 268 & 474 & 2178 & 318 & 100 & 0,53 & 2,7 & 1,68 & 798 & 1,35 & 86 & 106 & na & na \\
\hline 152 & 14.09 .2015 & 7,4 & 87,7 & 473 & 247 & 440 & 1997 & 295 & 87 & 0,52 & 2,4 & 1,58 & 710 & 1,30 & 79 & 95 & 0,0141 & 0,026 \\
\hline 156 & 18.09 .2015 & 7,7 & 87,0 & 441 & 231 & 407 & 1966 & 265 & 76 & 0,47 & 2,1 & 1,43 & 700 & 1,20 & 82 & 94 & na & na \\
\hline 159 & 21.09 .2015 & 7,5 & 86,7 & 449 & 239 & 425 & 2039 & 282 & 76 & 0,51 & 2,1 & 1,56 & 678 & 1,26 & 76 & 94 & 0,0115 & 0,0177 \\
\hline 166 & 28.09 .2015 & 7,6 & 86,6 & 473 & 256 & 478 & 2170 & 302 & 68 & 0,53 & 2,0 & 1,66 & 791 & 1,29 & 86 & 106 & na & na \\
\hline 170 & 02.10 .2015 & 7,8 & 88,1 & 452 & 240 & 425 & 2108 & 284 & 64 & 0,53 & 1,82 & 1,58 & 679 & 1,23 & 77 & 98 & 0,0125 & 0,0161 \\
\hline 173 & 05.10 .2015 & 8,6 & 89,1 & 465 & 248 & 446 & 2209 & 295 & 63 & 0,53 & 1,79 & 1,87 & 692 & 1,33 & 85 & 102 & 0,0122 & 0,020 \\
\hline 177 & 09.10 .2015 & 8,2 & 86,5 & 476 & 253 & 455 & 2212 & 301 & 59 & 0,54 & 1,73 & 1,91 & 725 & 1,34 & 88 & 108 & 0,0132 & 0,039 \\
\hline 180 & 12.10 .2015 & 7,0 & 85,1 & 456 & 247 & 460 & 2174 & 292 & 52 & 0,53 & 1,70 & 1,63 & 777 & 1,25 & 84 & 112 & na & na \\
\hline 184 & 16.10.2015 & 7,2 & 86,6 & 448 & 235 & 428 & 2080 & 283 & 48 & 0,51 & 1,51 & 1,78 & 676 & 1,22 & 83 & 102 & 0,0121 & 0,0171 \\
\hline 187 & 19.10 .2015 & 7,4 & 86,6 & 414 & 227 & 432 & 2029 & 270 & 43 & 0,47 & 1,44 & 1,50 & 695 & 1,16 & 77 & 96 & na & na \\
\hline 191 & 23.10 .2015 & 7,8 & 87,5 & 446 & 231 & 409 & 2338 & 272 & 42 & 0,48 & 1,39 & 1,47 & 702 & 1,13 & 83 & 105 & na & na \\
\hline 194 & 26.10 .2015 & 8,1 & 88,0 & 478 & 256 & 483 & 2310 & 311 & 42 & 0,52 & 1,42 & 1,66 & 776 & 1,22 & 81 & 115 & na & na \\
\hline 198 & 30.10 .2015 & 7,1 & 86,9 & 435 & 239 & 443 & 2150 & 287 & 36 & 0,50 & 1,25 & 1,51 & 697 & 1,12 & 73 & 109 & na & na \\
\hline 201 & 02.11 .2015 & 8,3 & 89,7 & 401 & 226 & 426 & 2145 & 269 & 33 & 0,46 & 1,16 & 1,48 & 625 & 1,06 & 68 & 97 & na & na \\
\hline 203 & 04.11 .2015 & 7,7 & 87,4 & 449 & 230 & 433 & 2252 & 291 & 35 & 0,49 & 1,20 & 1,50 & 706 & 1,07 & 74 & 102 & na & na \\
\hline 205 & 06.11 .2015 & 6,7 & 85,0 & 446 & 237 & 447 & 2191 & 288 & 33 & 0,50 & 1,20 & 1,55 & 709 & 1,14 & 77 & 112 & na & na \\
\hline 208 & 09.11 .2015 & 7,0 & 86,1 & 458 & 244 & 462 & 2298 & 299 & 33 & 0,51 & 1,22 & 1,59 & 732 & 1,15 & 78 & 111 & na & na \\
\hline 212 & 13.11 .2015 & 7,6 & 87,0 & 491 & 254 & 481 & 2323 & 319 & 32 & 0,54 & 1,29 & 1,67 & 797 & 1,21 & 82 & 117 & na & na \\
\hline 215 & 16.11.2015 & 7,5 & 86,7 & 487 & 260 & 488 & 2308 & 317 & 30 & 0,54 & 1,16 & 1,68 & 819 & 1,23 & 85 & 124 & na & na \\
\hline 219 & 20.11 .2015 & 7,8 & 86,8 & 435 & 231 & 458 & 2145 & 286 & 27 & 0,49 & 0,97 & 1,55 & 700 & 1,14 & 79 & 107 & na & na \\
\hline 222 & 23.11.2015 & 8,0 & 89,4 & 459 & 246 & 475 & 2149 & 307 & 27 & 0,52 & 1,01 & 1,65 & 747 & 1,22 & 83 & 116 & na & na \\
\hline 226 & 27.11 .2015 & 8,5 & 86,1 & 415 & 231 & 427 & 2034 & 281 & 24 & 0,47 & 0,86 & 1,50 & 683 & 1,10 & 76 & 104 & na & na \\
\hline 229 & 30.11 .2015 & 8,3 & 87,3 & 528 & 293 & 529 & 2448 & 352 & 27 & 0,59 & 1,06 & 1,84 & 883 & 1,43 & 80 & 137 & na & na \\
\hline 233 & 04.12 .2015 & 8,7 & 88,2 & 478 & 268 & 495 & 2367 & 331 & 25 & 0,53 & 0,94 & 1,74 & 793 & 1,30 & 75 & 120 & na & na \\
\hline 236 & 07.12 .2015 & 8,7 & 88,7 & 443 & 245 & 464 & 2342 & 313 & 23 & 0,49 & 0,83 & 1,63 & 724 & 1,21 & 81 & 109 & na & na \\
\hline 240 & 11.12 .2015 & 9,1 & 88,4 & 446 & 250 & 470 & 2349 & 314 & 24 & 0,49 & 0,83 & 1,65 & 752 & 1,26 & 80 & 111 & na & na \\
\hline 243 & 14.12.2015 & 9,5 & 90,1 & 462 & 262 & 490 & 2321 & 333 & 28 & 0,53 & 0,93 & 1,74 & 784 & 1,31 & 77 & 120 & na & na \\
\hline
\end{tabular}


(5) Projektphase 1: Elementgehalte der Makro- und Mikronährstoffe (Quasi)kontinuierliche Laborfermenterversuche am DBFZ Reaktor 4.6: Reaktor ohne Nickel-Zugabe 2

\begin{tabular}{|c|c|c|c|c|c|c|c|c|c|c|c|c|c|c|c|c|c|c|}
\hline \multicolumn{4}{|c|}{ Verarmungsversuche } & \multicolumn{6}{|c|}{ Makronährstoffe } & \multicolumn{9}{|c|}{ Mikronährstoffe } \\
\hline & essmethode: & : ICP-OES & ICP-OES & ICP-OES & ICP-OES & ICP-OES & ICP-OES & ICP-OES & ICP-OES & ICP-OES & ICP-OES & ICP-OES & ICP-OES & ICP-OES & ICP-MS & ICP-OES \\
\hline \multicolumn{2}{|c|}{$\mathrm{mg} / \mathrm{kg} \mathrm{FM}$} & & Element: & : P 178.222 & S 181.972 & Са 317.93зr & $\mathrm{K} 766.491 \mathrm{r}$ & Mg 279.078r & Na $588.995 \mathrm{r}$ & Co 230.786 & Ni 231.604 & Mo 202.032 & $\mathrm{Fe} 234.350$ & Си 327.395 & Mn 257.610 & Zn 206.200 & Se 77 KED & V 292.401 \\
\hline $\begin{array}{l}\text { Versuchs- } \\
\text { tag }\end{array}$ & $\begin{array}{l}\text { Probe- } \\
\text { nahme }\end{array}$ & $\begin{array}{c}\text { TS } \\
\text { [Gew.-\%] }\end{array}$ & $\begin{array}{c}\text { OTS } \\
\text { [Gew.-\%] }\end{array}$ & $\mathbf{P}$ & $\mathbf{s}$ & $\mathrm{Ca}$ & $\mathbf{K}$ & Mg & $\mathrm{Na}$ & Co & $\mathbf{N i}$ & Mo & $\mathrm{Fe}$ & $\mathrm{Cu}$ & Mn & $\mathrm{Zn}$ & Se & $\mathbf{v}$ \\
\hline 23 & 08.05 .2015 & 6,5 & 80,1 & 458 & 237 & 497 & 2237 & 298 & 1008 & 0,63 & 8,5 & 1,83 & 1271 & 1,73 & 88 & 61 & na & $\mathrm{na}$ \\
\hline 30 & 15.05 .2015 & 6,8 & 80,7 & 459 & 216 & 434 & 2236 & 289 & 850 & 0,53 & 8,2 & 1,36 & 1017 & 1,80 & 68 & 54 & na & na \\
\hline 37 & 22.05 .2015 & 7,6 & 84,1 & 493 & 241 & 511 & 2368 & 312 & 756 & 0,59 & 7,8 & 1,79 & 1119 & 1,63 & 89 & 59 & na & na \\
\hline 44 & 29.05 .2015 & 6,0 & 78,9 & 546 & 242 & 494 & 2424 & 345 & 682 & 0,59 & 8,3 & 1,72 & 1052 & 1,70 & 91 & 62 & na & na \\
\hline 51 & 05.06 .2015 & 7,8 & 82,8 & 591 & 263 & 510 & 2447 & 356 & 583 & 0,62 & 8,1 & 1,85 & 1169 & 1,66 & 102 & 68 & na & na \\
\hline 58 & 12.06 .2015 & 7,2 & 82,3 & 563 & 261 & 526 & 2537 & 353 & 503 & 0,61 & 7,6 & 1,88 & 1084 & 1,58 & 94 & 62 & na & na \\
\hline 65 & 19.06.2015 & 7,3 & 83,3 & 582 & 249 & 470 & 2443 & 360 & 435 & 0,58 & 8,0 & 1,75 & 936 & 1,55 & 88 & 58 & na & na \\
\hline 72 & 26.06 .2015 & 7,0 & 82,0 & 555 & 257 & 490 & 2359 & 354 & 413 & 0,51 & 7,3 & 1,88 & 934 & 1,48 & 90 & 61 & 0,0178 & 0,042 \\
\hline 79 & 03.07.2015 & 6,1 & 81,4 & 553 & 237 & 453 & 2372 & 344 & 333 & 0,54 & 7,3 & 1,59 & 845 & 1,49 & 83 & 55 & na & na \\
\hline 82 & 06.07 .2015 & 7,3 & 84,2 & 569 & 272 & 518 & 2435 & 345 & 320 & 0,59 & 7,3 & 1,86 & 992 & 1,42 & 91 & 61 & na & na \\
\hline 86 & 10.07 .2015 & 7,5 & 84,9 & 552 & 258 & 475 & 2404 & 336 & 303 & 0,58 & 6,6 & 1,74 & 831 & 1,42 & 85 & 61 & 0,0178 & 0,029 \\
\hline 89 & 13.07.2015 & 8,0 & 85,0 & 541 & 263 & 479 & 2351 & 331 & 285 & 0,58 & 6,2 & 1,75 & 820 & 1,47 & 83 & 63 & 0,0172 & 0,028 \\
\hline 96 & 20.07 .2015 & 7,0 & 85,0 & 489 & 238 & 452 & 2337 & 318 & 252 & 0,46 & 5,8 & 1,67 & 756 & 1,35 & 78 & 67 & 0,0142 & 0,026 \\
\hline 103 & 27.07 .2015 & 7,7 & 84,6 & 550 & 271 & 523 & 2418 & 344 & 213 & 0,56 & 4,9 & 1,79 & 908 & 1,41 & 91 & 79 & na & na \\
\hline 110 & 03.08.2015 & 7,0 & 84,9 & 504 & 248 & 468 & 2284 & 323 & 196 & 0,46 & 4,0 & 1,67 & 760 & 1,34 & 81 & 80 & 0,0139 & 0,026 \\
\hline 117 & 10.08 .2015 & 6,5 & 83,7 & 510 & 251 & 481 & 2292 & 319 & 158 & 0,53 & 3,6 & 1,67 & 816 & 1,31 & 82 & 84 & na & na \\
\hline 124 & 17.08 .2015 & 7,7 & 86,4 & 568 & 279 & 492 & 1874 & 320 & 124 & 0,55 & 3,1 & 2,0 & 827 & 1,64 & 96 & 101 & 0,0140 & 0,043 \\
\hline 128 & 21.08 .2015 & 7,2 & 85,4 & 538 & 267 & 474 & 2333 & 325 & 144 & 0,54 & 3,1 & 1,65 & 822 & 1,44 & 92 & 100 & na & na \\
\hline 131 & 24.08 .2015 & 8,6 & 87,0 & 501 & 253 & 467 & 2331 & 316 & 139 & 0,54 & 2,7 & 1,87 & 719 & 1,47 & 84 & 89 & 0,0134 & 0,041 \\
\hline 138 & 31.08 .2015 & 7,1 & 85,2 & 490 & 250 & 455 & 2125 & 296 & 110 & 0,49 & 2,4 & 1,55 & 732 & 1,30 & 78 & 93 & na & na \\
\hline 142 & 04.09 .2015 & 7,0 & 85,2 & 503 & 254 & 451 & 2170 & 304 & 107 & 0,50 & 2,3 & 1,60 & 743 & 1,33 & 81 & 94 & na & na \\
\hline 145 & 07.09.2015 & 6,9 & 85,1 & 528 & 270 & 480 & 2225 & 320 & 106 & 0,54 & 2,3 & 1,70 & 796 & 1,41 & 86 & 105 & na & na \\
\hline 152 & 14.09 .2015 & 7,0 & 86,2 & 467 & 245 & 431 & 2019 & 288 & 88 & 0,51 & 1,93 & 1,56 & 693 & 1,32 & 77 & 95 & 0,0142 & 0,0182 \\
\hline 156 & 18.09 .2015 & 8,2 & 88,2 & 443 & 235 & 409 & 2083 & 267 & 82 & 0,46 & 1,74 & 1,48 & 682 & 1,26 & 81 & 93 & na & na \\
\hline 159 & 21.09 .2015 & 7,6 & 87,3 & 458 & 246 & 432 & 2036 & 284 & 79 & 0,52 & 1,70 & 1,57 & 692 & 1,30 & 74 & 95 & 0,0125 & 0,0183 \\
\hline 166 & 28.09 .2015 & 7,3 & 86,1 & 443 & 240 & 447 & 2105 & 282 & 67 & 0,48 & 1,53 & 1,56 & 740 & 1,25 & 80 & 99 & na & na \\
\hline 170 & 02.10 .2015 & 8,2 & 88,5 & 464 & 249 & 439 & 2140 & 291 & 67 & 0,53 & 1,44 & 1,62 & 701 & 1,29 & 79 & 100 & 0,0122 & 0,0193 \\
\hline 173 & 05.10 .2015 & 6,9 & 86,6 & 440 & 231 & 417 & 2021 & 275 & 60 & 0,49 & 1,29 & 1,74 & 653 & 1,27 & 80 & 97 & 0,0121 & 0,032 \\
\hline 177 & 09.10 .2015 & 7,2 & 86,5 & 443 & 233 & 426 & 2018 & 280 & 56 & 0,50 & 1,24 & 1,76 & 664 & 1,29 & 82 & 99 & 0,0127 & 0,032 \\
\hline 180 & 12.10 .2015 & 7,6 & 87,3 & 389 & 215 & 411 & 2031 & 258 & 49 & 0,44 & 1,13 & 1,42 & 649 & 1,13 & 72 & 88 & na & na \\
\hline 184 & 16.10 .2015 & 7,2 & 86,9 & 458 & 241 & 426 & 2040 & 284 & 50 & 0,51 & 1,17 & 1,78 & 684 & 1,27 & 84 & 103 & 0,0126 & 0,033 \\
\hline 187 & 19.10.2015 & 7,2 & 86,3 & 460 & 248 & 469 & 2145 & 297 & 48 & 0,52 & 1,20 & 1,62 & 765 & 1,24 & 84 & 110 & na & na \\
\hline 191 & 23.10.2015 & 7,4 & 87,1 & 475 & 248 & 441 & 2149 & 291 & 45 & 0,51 & 1,14 & 1,56 & 750 & 1,22 & 89 & 115 & na & na \\
\hline 194 & 26.10 .2015 & 7,4 & 86,9 & 448 & 242 & 456 & 2234 & 295 & 43 & 0,49 & 1,03 & 1,56 & 719 & 1,18 & 75 & 108 & na & na \\
\hline 198 & 30.10 .2015 & 7,6 & 87,1 & 460 & 248 & 461 & 2247 & 299 & 39 & 0,50 & 0,98 & 1,57 & 737 & 1,19 & 77 & 110 & na & na \\
\hline 201 & 02.11 .2015 & 7,3 & 86,8 & 407 & 217 & 406 & 2190 & 271 & 37 & 0,45 & 0,88 & 1,48 & 641 & 1,05 & 68 & 94 & na & na \\
\hline 203 & 04.11 .2015 & 6,2 & 84,9 & 441 & 226 & 431 & 2182 & 291 & 38 & 0,48 & 0,92 & 1,47 & 708 & 1,04 & 72 & 103 & na & na \\
\hline 205 & 06.11 .2015 & 6,8 & 85,3 & 459 & 241 & 458 & 2200 & 297 & 35 & 0,50 & 0,94 & 1,53 & 728 & 1,13 & 78 & 111 & na & na \\
\hline 208 & 09.11 .2015 & 7,3 & 86,2 & 479 & 254 & 467 & 2149 & 300 & 32 & 0,50 & 1,00 & 1,62 & 776 & 1,21 & 81 & 118 & na & na \\
\hline 212 & 13.11.2015 & 7,3 & 86,9 & 476 & 248 & 465 & 2332 & 309 & 33 & 0,51 & 0,95 & 1,58 & 771 & 1,18 & 78 & 112 & na & na \\
\hline 215 & 16.11 .2015 & 7,0 & 86,2 & 440 & 235 & 449 & 2257 & 293 & 30 & 0,50 & 0,95 & 1,56 & 735 & 1,12 & 75 & 110 & na & na \\
\hline 219 & 20.11 .2015 & 7,6 & 86,5 & 477 & 254 & 495 & 2317 & 309 & 30 & 0,53 & 0,98 & 1,69 & 777 & 1,27 & 86 & 118 & na & na \\
\hline 222 & 23.11.2015 & 7,5 & 87,0 & 505 & 267 & 507 & 2334 & 332 & 31 & 0,56 & 1,03 & 1,77 & 833 & 1,31 & 91 & 129 & na & na \\
\hline 226 & 27.11.2015 & 7,4 & 86,5 & 455 & 252 & 462 & 2229 & 307 & 26 & 0,52 & 0,94 & 1,65 & 752 & 1,24 & 83 & 119 & na & na \\
\hline 229 & 30.11 .2015 & 8,6 & 88,9 & 485 & 267 & 492 & 2422 & 331 & 30 & 0,54 & 0,96 & 1,75 & 800 & 1,29 & 76 & 120 & na & na \\
\hline 233 & 04.12 .2015 & 8,0 & 88,8 & 446 & 252 & 467 & 2328 & 306 & 24 & 0,49 & 0,85 & 1,63 & 742 & 1,27 & 75 & 110 & na & na \\
\hline 236 & 07.12 .2015 & 8,6 & 87,8 & 463 & 255 & 481 & 2346 & 319 & 23 & 0,50 & 0,84 & 1,69 & 768 & 1,30 & 84 & 111 & na & na \\
\hline 240 & 11.12 .2015 & 8,4 & 87,7 & 458 & 255 & 471 & 2351 & 318 & 26 & 0,51 & 0,84 & 1,69 & 775 & 1,27 & 72 & 116 & na & na \\
\hline 243 & 14.12.2015 & 8,8 & 88,3 & 367 & 211 & 387 & 2100 & 260 & 21 & 0,41 & 0,65 & 1,43 & 628 & 1,07 & 59 & 91 & na & na \\
\hline 247 & 18.12.2015 & 8,3 & 87,7 & 404 & 223 & 437 & 2084 & 284 & 18 & 0,48 & 0,72 & 1,51 & 706 & 1,14 & 80 & 103 & na & na \\
\hline 250 & 21.12 .2015 & 8,3 & 87,5 & 465 & 256 & 504 & 2288 & 327 & 21 & 0,54 & 0,83 & 1,72 & 840 & 1,32 & 93 & 121 & na & na \\
\hline 257 & 28.12.2015 & 8,5 & 88,1 & 451 & 244 & 484 & 2332 & 321 & 16 & 0,50 & 0,73 & 1,64 & 768 & 1,26 & 87 & 110 & na & na \\
\hline 264 & 04.01.2016 & 8,3 & 87,9 & 482 & 252 & 502 & 2303 & 335 & 15 & 0,51 & 0,78 & 1,63 & 797 & 1,28 & 90 & 116 & na & na \\
\hline 268 & 08.01.2016 & 8,3 & 87,0 & 455 & 236 & 468 & 2318 & 329 & 16 & 0,47 & 0,70 & 1,52 & 711 & 1,20 & 83 & 105 & na & na \\
\hline 271 & 11.01 .2016 & 8,1 & 87,1 & 448 & 233 & 460 & 2295 & 327 & 14 & 0,47 & 0,71 & 1,52 & 704 & 1,17 & 82 & 106 & na & na \\
\hline 275 & 15.01.2016 & 8,6 & 88,1 & 512 & 261 & 513 & 2470 & 367 & 15 & 0,53 & 0,80 & 1,67 & 800 & 1,33 & 93 & 121 & na & na \\
\hline 278 & 18.01.2016 & 8,6 & 87,5 & 476 & 245 & 489 & 2381 & 346 & 14 & 0,49 & 0,75 & 1,57 & 732 & 1,25 & 86 & 111 & na & na \\
\hline 282 & 22.01.2016 & 8,5 & 87,6 & 482 & 244 & 494 & 2460 & 352 & 13 & 0,49 & 0,74 & 1,59 & 740 & 1,24 & 87 & 112 & na & na \\
\hline 285 & 25.01 .2016 & 8,6 & 86,9 & 519 & 260 & 517 & 2551 & 372 & 17 & 0,52 & 0,78 & 1,65 & 800 & 1,33 & 93 & 120 & na & na \\
\hline 289 & 29.01 .2016 & 8,3 & 87,1 & 506 & 253 & 505 & 2418 & 361 & 13 & 0,49 & 0,71 & 1,58 & 788 & 1,28 & 91 & 117 & na & na \\
\hline 293 & 02.02 .2016 & 8,3 & 86,7 & 494 & 245 & 500 & 2450 & 359 & 15 & 0,48 & 0,65 & 1,55 & 753 & 1,26 & 87 & 113 & na & na \\
\hline 296 & 05.02.2016 & 9,5 & 87,4 & 615 & 304 & 586 & 2493 & 415 & 15 & 0,55 & 0,79 & 1,88 & 952 & 1,64 & 108 & 145 & na & na \\
\hline 299 & 08.02.2016 & 8,6 & 87,8 & 508 & 253 & 523 & 2569 & 380 & 14 & 0,50 & 0,67 & 1,59 & 759 & 1,31 & 89 & 115 & na & na \\
\hline 303 & 12.02 .2016 & 9,1 & 89,1 & 497 & 246 & 504 & 2386 & 370 & 13 & 0,48 & 0,77 & 1,56 & 721 & 1,26 & 87 & 113 & na & na \\
\hline 307 & 16.02 .2016 & 9,1 & 88,6 & 527 & 249 & 534 & 2430 & 391 & 11 & 0,50 & 0,98 & 1,59 & 766 & 1,28 & 92 & 116 & na & na \\
\hline
\end{tabular}


(6) Projektphase 1: Elementgehalte der Makro- und Mikronährstoffe (Quasi)kontinuierliche Laborfermenterversuche am DBFZ Reaktor 4.7: Reaktor ohne Cobalt-Zugabe 1

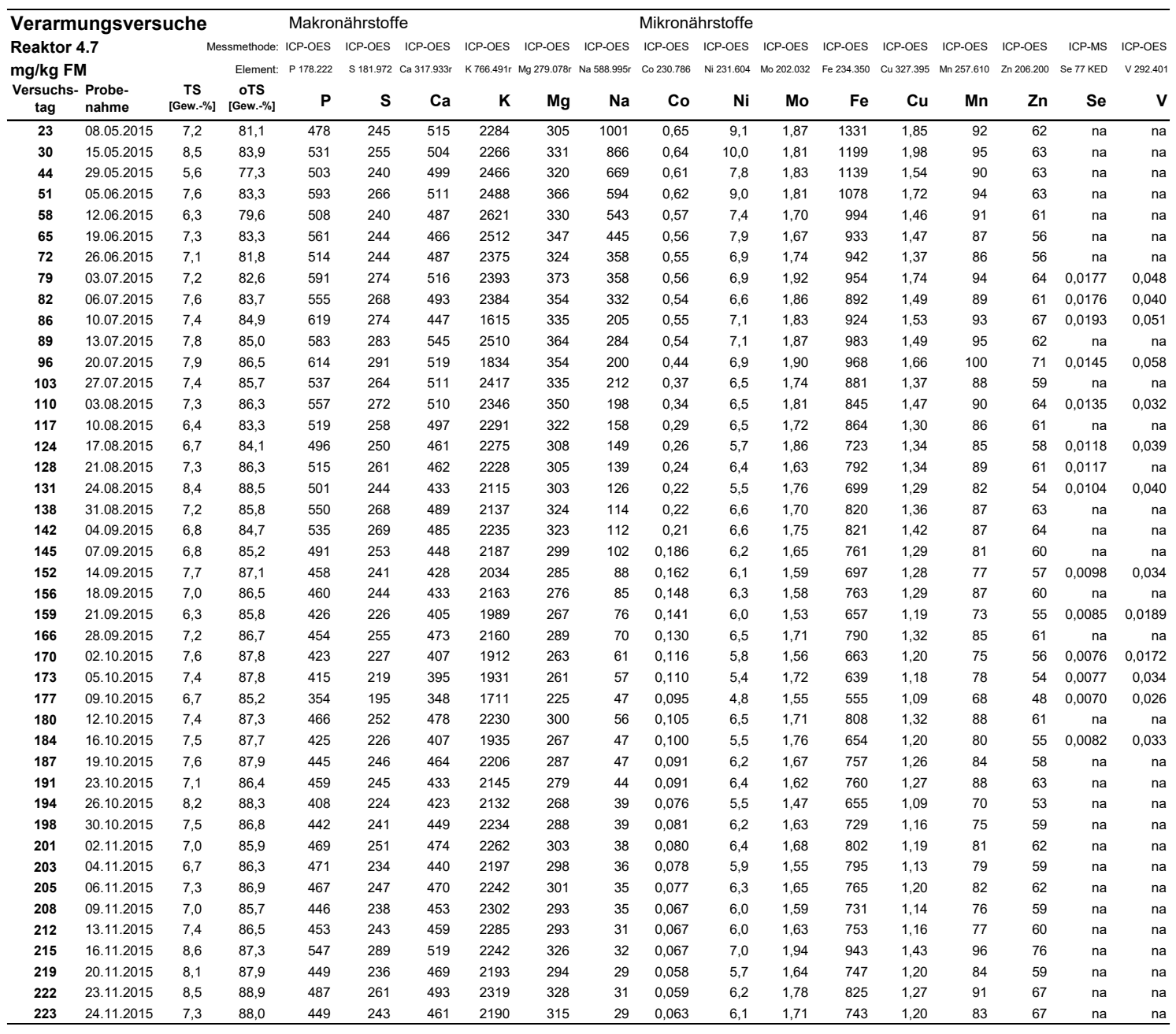


(7) Projektphase 1: Elementgehalte der Makro- und Mikronährstoffe (Quasi)kontinuierliche Laborfermenterversuche am DBFZ Reaktor 4.8: Reaktor ohne Cobalt-Zugabe 2

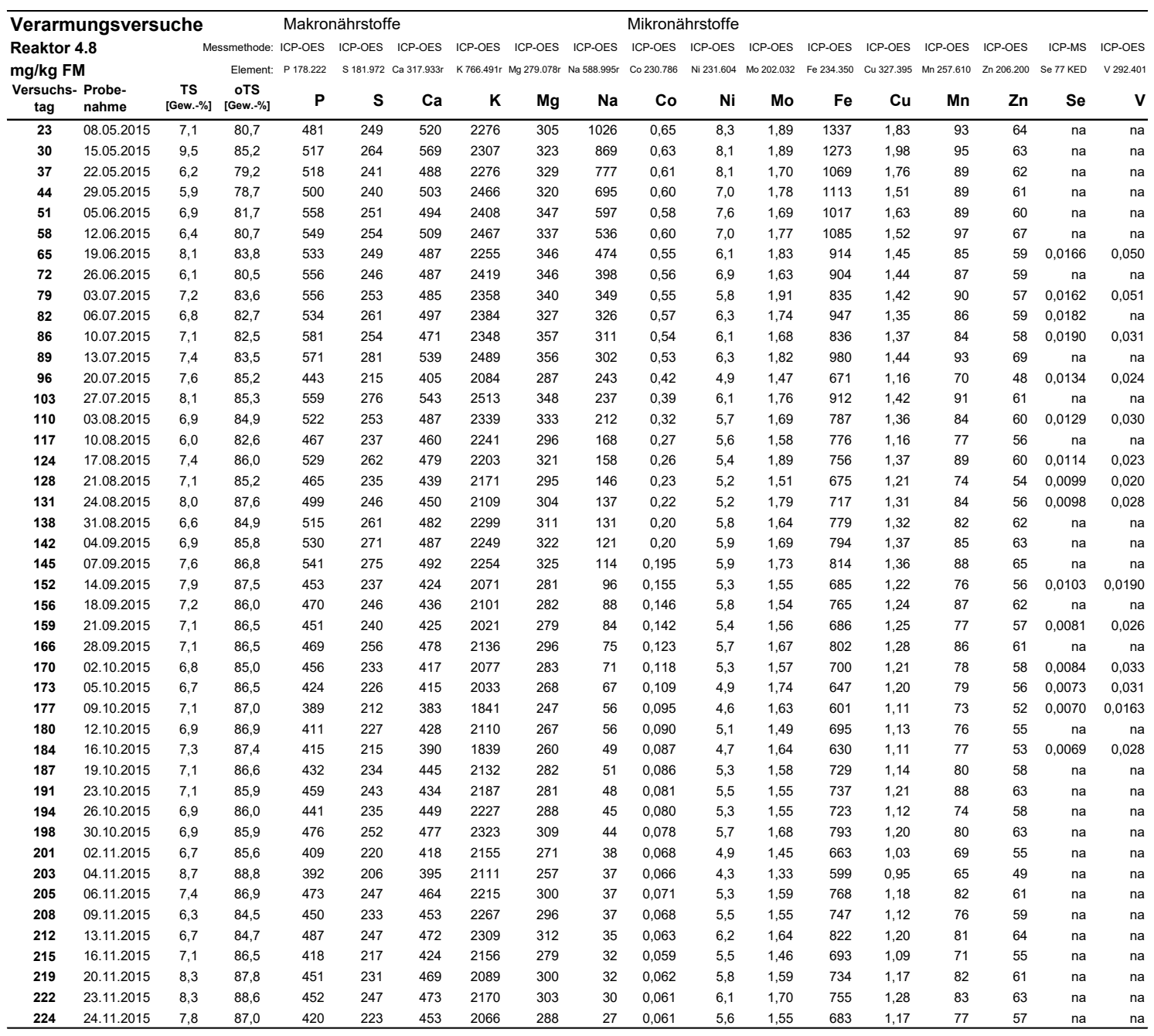


(8) Projektphase 1: Elementgehalte der Makro- und Mikronährstoffe (Quasi)kontinuierliche Laborfermenterversuche am DBFZ Reaktor 4.9: Referenzreaktor (Spurenelementvollversorgung)

\begin{tabular}{|c|c|c|c|c|c|c|c|c|c|c|c|c|c|c|c|c|c|c|}
\hline \multicolumn{4}{|c|}{ Verarmungsversuche } & \multicolumn{6}{|c|}{ Makronährstoffe } & \multicolumn{9}{|c|}{ Mikronährstoffe } \\
\hline & & lessmethode: & ICP-OES & ICP-OES & ICP-OES & ICP-OES & ICP-OES & ICP-OES & ICP-OES & ICP-OES & ICP-OES & ICP-OES & ICP-OES & ICP-OES & ICP-OES & ICP-MS & ICP-OES \\
\hline $\mathrm{mg} / \mathrm{kg} \mathrm{Fn}$ & & & Element: & : P 178.222 & S 181.972 & Са 317.933r & K $766.491 \mathrm{r}$ & Mg 279.078r & Na 588.995r & Co 230.786 & Ni 231.604 & Mo 202.032 & Fe 234.350 & Си 327.395 & Mn 257.610 & $\mathrm{Zn} 206.200$ & Se 77 KED & v 292.401 \\
\hline $\begin{array}{c}\text { Versuchs- } \\
\text { tag }\end{array}$ & $\begin{array}{l}\text { Probe- } \\
\text { nahme }\end{array}$ & $\begin{array}{c}\text { TS } \\
\text { [Gew.-\%] }\end{array}$ & $\begin{array}{c}\text { oTS } \\
\text { [Gew.-\%] }\end{array}$ & $\mathbf{P}$ & $\mathbf{s}$ & $\mathrm{Ca}$ & K & $\mathbf{M g}$ & $\mathrm{Na}$ & Co & $\mathrm{Ni}$ & Mo & $\mathrm{Fe}$ & $\mathrm{Cu}$ & $M n$ & $\mathrm{Zn}$ & Se & v \\
\hline 23 & 08.05 .2015 & 6,5 & 80,0 & 479 & 234 & 486 & 2249 & 312 & 1011 & 0,59 & 8,6 & 1,59 & 1148 & 1,89 & 80 & 61 & na & na \\
\hline 30 & 15.05.2015 & 6,2 & 78,7 & 463 & 232 & 464 & 2232 & 297 & 874 & 0,60 & 7,3 & 2,0 & 1013 & 1,73 & 88 & 60 & 0,023 & 0,048 \\
\hline 37 & 22.05.2015 & 7,2 & 82,2 & 505 & 250 & 496 & 2278 & 328 & 812 & 0,58 & 7,2 & 1,91 & 1072 & 1,75 & 90 & 66 & 0,026 & 0,049 \\
\hline 44 & 29.05.2015 & 6,3 & 80,5 & 520 & 237 & 473 & 2360 & 332 & 682 & 0,58 & 7,8 & 1,64 & 1008 & 1,60 & 85 & 59 & na & na \\
\hline 51 & 05.06 .2015 & 8,7 & 85,6 & 545 & 252 & 530 & 2306 & 339 & 589 & 0,58 & 7,1 & 1,80 & 1185 & 1,63 & 97 & 60 & na & na \\
\hline 58 & 12.06.2015 & 6,1 & 79,7 & 548 & 245 & 482 & 2454 & 347 & 527 & 0,58 & 7,6 & 1,68 & 975 & 1,54 & 89 & 61 & na & na \\
\hline 65 & 19.06.2015 & 6,4 & 80,8 & 546 & 249 & 490 & 2500 & 339 & 456 & 0,58 & 6,9 & 1,75 & 1035 & 1,35 & 88 & 58 & na & na \\
\hline 72 & 26.06.2015 & 7,0 & 82,6 & 556 & 242 & 505 & 2273 & 342 & 388 & 0,55 & 6,7 & 1,71 & 1116 & 1,42 & 94 & 58 & na & na \\
\hline 79 & 03.07.2015 & 7,1 & 83,3 & 577 & 255 & 477 & 2388 & 352 & 343 & 0,55 & 7,1 & 1,65 & 888 & 1,44 & 87 & 57 & na & na \\
\hline 82 & 06.07 .2015 & 7,2 & 83,9 & 552 & 269 & 505 & 2379 & 337 & 316 & 0,58 & 6,9 & 1,79 & 970 & 1,37 & 89 & 60 & na & na \\
\hline 86 & 10.07.2015 & 7,0 & 83,8 & 547 & 259 & 475 & 2397 & 333 & 308 & 0,58 & 6,5 & 1,73 & 833 & 1,40 & 84 & 59 & 0,0194 & 0,031 \\
\hline 89 & 13.07.2015 & 7,6 & 84,3 & 556 & 272 & 524 & 2500 & 347 & 294 & 0,57 & 6,5 & 1,80 & 944 & 1,43 & 91 & 60 & na & na \\
\hline 96 & 20.07 .2015 & 7,5 & 86,2 & 518 & 257 & 473 & 2451 & 337 & 275 & 0,55 & 6,1 & 1,78 & 793 & 1,40 & 82 & 58 & 0,0160 & 0,033 \\
\hline 103 & 27.07 .2015 & 7,8 & 84,7 & 551 & 271 & 521 & 2428 & 344 & 224 & 0,53 & 6,2 & 1,74 & 896 & 1,39 & 89 & 59 & na & na \\
\hline 110 & 03.08 .2015 & 7,6 & 86,5 & 519 & 257 & 480 & 2379 & 335 & 207 & 0,51 & 5,7 & 1,69 & 772 & 1,37 & 82 & 58 & 0,0143 & 0,029 \\
\hline 117 & 10.08.2015 & 6,1 & 82,4 & 497 & 245 & 474 & 2333 & 315 & 171 & 0,51 & 5,6 & 1,61 & 807 & 1,21 & 80 & 56 & na & na \\
\hline 124 & 17.08 .2015 & 6,6 & 84,5 & 506 & 249 & 457 & 2210 & 312 & 151 & 0,51 & 5,2 & 1,80 & 722 & 1,35 & 84 & 57 & 0,0153 & 0,027 \\
\hline 128 & 21.08 .2015 & 7,0 & 85,4 & 481 & 240 & 436 & 2160 & 307 & 143 & 0,50 & 5,3 & 1,56 & 679 & 1,22 & 75 & 53 & 0,0157 & 0,027 \\
\hline 131 & 24.08.2015 & 9,2 & 87,7 & 531 & 265 & 491 & 2336 & 329 & 146 & 0,52 & 5,4 & 1,91 & 740 & 1,46 & 88 & 59 & 0,0175 & 0,046 \\
\hline 138 & 31.08 .2015 & 6,7 & 84,5 & 558 & 273 & 504 & 2287 & 333 & 124 & 0,53 & 5,8 & 1,69 & 838 & 1,37 & 88 & 63 & na & na \\
\hline 142 & 04.09.2015 & 7,1 & 85,3 & 525 & 263 & 472 & 2265 & 318 & 116 & 0,51 & 5,5 & 1,64 & 768 & 1,30 & 83 & 60 & na & na \\
\hline 145 & 07.09.2015 & 6,7 & 84,8 & 535 & 267 & 480 & 2207 & 325 & 109 & 0,53 & 5,6 & 1,68 & 806 & 1,33 & 85 & 62 & na & na \\
\hline 152 & 14.09.2015 & 8,0 & 87,1 & 448 & 234 & 417 & 2068 & 280 & 92 & 0,48 & 5,2 & 1,52 & 659 & 1,25 & 73 & 53 & 0,0148 & 0,024 \\
\hline 156 & 18.09.2015 & 7,2 & 86,9 & 469 & 245 & 437 & 2008 & 289 & 85 & 0,52 & 5,5 & 1,55 & 699 & 1,27 & 78 & 57 & 0,0146 & 0,023 \\
\hline 159 & 21.09.2015 & 7,5 & 87,1 & 488 & 254 & 449 & 2113 & 299 & 84 & 0,53 & 5,7 & 1,64 & 735 & 1,30 & 82 & 58 & 0,0137 & 0,025 \\
\hline 166 & 28.09.2015 & 7,2 & 86,9 & 454 & 243 & 456 & 2088 & 288 & 71 & 0,49 & 5,5 & 1,57 & 755 & 1,24 & 81 & 57 & na & na \\
\hline 170 & 02.10 .2015 & 7,6 & 87,5 & 466 & 244 & 435 & 2130 & 291 & 70 & 0,52 & 5,5 & 1,60 & 698 & 1,25 & 79 & 58 & 0,0139 & 0,021 \\
\hline 173 & 05.10 .2015 & 7,8 & 86,8 & 419 & 226 & 404 & 2129 & 270 & 65 & 0,47 & 5,0 & 1,75 & 621 & 1,22 & 75 & 53 & 0,0128 & 0,024 \\
\hline 177 & 09.10 .2015 & 6,5 & 85,9 & 414 & 220 & 399 & 1914 & 260 & 55 & 0,46 & 4,8 & 1,66 & 618 & 1,17 & 76 & 53 & 0,0133 & 0,033 \\
\hline 180 & 12.10.2015 & 7,7 & 87,3 & 428 & 232 & 439 & 2007 & 279 & 54 & 0,48 & 5,2 & 1,51 & 705 & 1,16 & 78 & 55 & na & na \\
\hline 184 & 16.10 .2015 & 7,2 & 87,3 & 434 & 229 & 415 & 1960 & 272 & 50 & 0,48 & 4,9 & 1,71 & 650 & 1,20 & 79 & 55 & 0,0147 & 0,022 \\
\hline 187 & 19.10.2015 & 7,0 & 86,3 & 435 & 237 & 448 & 2190 & 282 & 50 & 0,49 & 5,3 & 1,56 & 721 & 1,19 & 78 & 55 & na & na \\
\hline 191 & 23.10.2015 & 7,7 & 86,8 & 472 & 251 & 447 & 2169 & 288 & 47 & 0,50 & 5,5 & 1,56 & 734 & 1,22 & 87 & 64 & na & na \\
\hline 194 & 26.10 .2015 & 7,9 & 87,1 & 440 & 238 & 449 & 2304 & 290 & 44 & 0,47 & 5,2 & 1,54 & 700 & 1,14 & 73 & 56 & na & na \\
\hline 198 & 30.10 .2015 & 7,1 & 86,6 & 450 & 246 & 457 & 2270 & 292 & 41 & 0,49 & 5,3 & 1,58 & 736 & 1,18 & 75 & 59 & na & na \\
\hline 201 & 02.11 .2015 & 7,6 & 87,5 & 458 & 244 & 456 & 2221 & 295 & 38 & 0,49 & 5,2 & 1,56 & 733 & 1,14 & 76 & 57 & na & na \\
\hline 203 & 04.11 .2015 & 7,9 & 87,4 & 498 & 243 & 449 & 2197 & 313 & 37 & 0,49 & 5,2 & 1,55 & 795 & 1,11 & 80 & 56 & na & na \\
\hline 205 & 06.11 .2015 & 6,5 & 85,0 & 475 & 249 & 471 & 2241 & 305 & 37 & 0,50 & 5,4 & 1,57 & 759 & 1,19 & 79 & 61 & na & na \\
\hline 208 & 09.11 .2015 & 7,7 & 86,4 & 462 & 246 & 465 & 2355 & 306 & 36 & 0,49 & 5,2 & 1,58 & 712 & 1,15 & 74 & 57 & na & na \\
\hline 212 & 13.11.2015 & 6,9 & 85,4 & 465 & 251 & 478 & 2345 & 306 & 34 & 0,50 & 5,4 & 1,59 & 732 & 1,18 & 75 & 61 & na & na \\
\hline 215 & 16.11.2015 & 7,7 & 86,3 & 466 & 248 & 472 & 2461 & 311 & 34 & 0,50 & 5,4 & 1,62 & 745 & 1,17 & 77 & 60 & na & na \\
\hline 219 & 20.11 .2015 & 7,8 & 86,8 & 492 & 256 & 499 & 2374 & 318 & 34 & 0,52 & 5,4 & 1,67 & 783 & 1,30 & 86 & 62 & na & na \\
\hline 222 & 23.11.2015 & 7,8 & 87,0 & 499 & 266 & 500 & 2280 & 325 & 31 & 0,53 & 5,5 & 1,72 & 813 & 1,31 & 89 & 65 & na & na \\
\hline 226 & 27.11.2015 & 7,3 & 86,6 & 455 & 238 & 477 & 2181 & 304 & 27 & 0,48 & 5,0 & 1,56 & 774 & 1,24 & 85 & 58 & na & na \\
\hline 229 & 30.11 .2015 & 7,9 & 87,0 & 423 & 223 & 447 & 2149 & 287 & 26 & 0,46 & 4,7 & 1,46 & 693 & 1,16 & 79 & 53 & na & na \\
\hline 233 & 04.12 .2015 & 8,0 & 89,3 & 459 & 257 & 474 & 2350 & 316 & 26 & 0,49 & 5,1 & 1,66 & 744 & 1,26 & 79 & 61 & na & na \\
\hline 236 & 07.12 .2015 & 8,5 & 87,7 & 436 & 232 & 464 & 2219 & 298 & 23 & 0,47 & 4,8 & 1,54 & 703 & 1,22 & 82 & 54 & na & na \\
\hline 240 & 11.12.2015 & 8,3 & 86,9 & 419 & 224 & 445 & 2125 & 291 & 21 & 0,45 & 4,7 & 1,51 & 688 & 1,20 & 80 & 53 & na & na \\
\hline 243 & 14.12.2015 & 8,3 & 88,9 & 433 & 240 & 448 & 2233 & 298 & 21 & 0,47 & 4,9 & 1,60 & 721 & 1,23 & 80 & 58 & na & na \\
\hline 247 & 18.12.2015 & 7,7 & 87,6 & 410 & 217 & 432 & 2025 & 287 & 19 & 0,46 & 4,6 & 1,52 & 710 & 1,18 & 81 & 53 & na & na \\
\hline 250 & 21.12.2015 & 9,0 & 88,6 & 411 & 221 & 438 & 2122 & 286 & 17 & 0,44 & 4,6 & 1,50 & 683 & 1,19 & 81 & 53 & na & na \\
\hline 257 & 28.12.2015 & 8,1 & 87,4 & 432 & 225 & 452 & 2172 & 304 & 17 & 0,47 & 5,1 & 1,50 & 712 & 1,20 & 83 & 55 & na & na \\
\hline 264 & 04.01 .2016 & 7,6 & 86,5 & 458 & 235 & 470 & 2242 & 324 & 14 & 0,48 & 5,9 & 1,53 & 758 & 1,28 & 86 & 57 & na & na \\
\hline 268 & 08.01 .2016 & 8,1 & 87,7 & 465 & 243 & 476 & 2286 & 332 & 15 & 0,49 & 6,2 & 1,56 & 731 & 1,31 & 84 & 58 & na & na \\
\hline 271 & 11.01 .2016 & 7,9 & 87,2 & 464 & 239 & 476 & 2372 & 338 & 15 & 0,48 & 6,3 & 1,54 & 731 & 1,29 & 83 & 57 & na & na \\
\hline 275 & 15.01 .2016 & 8,8 & 88,4 & 472 & 242 & 488 & 2358 & 341 & 17 & 0,49 & 6,2 & 1,54 & 730 & 1,31 & 84 & 56 & na & na \\
\hline 278 & 18.01.2016 & 7,8 & 86,5 & 493 & 248 & 494 & 2375 & 351 & 15 & 0,50 & 6,6 & 1,56 & 770 & 1,47 & 87 & 59 & na & na \\
\hline 282 & 22.01 .2016 & 8,1 & 87,3 & 498 & 253 & 509 & 2460 & 359 & 15 & 0,49 & 6,8 & 1,58 & 767 & 1,38 & 87 & 60 & na & na \\
\hline 285 & 25.01 .2016 & 8,3 & 86,9 & 504 & 254 & 509 & 2415 & 359 & 14 & 0,49 & 6,5 & 1,56 & 776 & 1,36 & 88 & 60 & na & na \\
\hline 289 & 29.01.2016 & 8,1 & 86,8 & 517 & 257 & 514 & 2432 & 369 & 13 & 0,50 & 6,6 & 1,59 & 791 & 1,37 & 89 & 61 & na & na \\
\hline 293 & 02.02 .2016 & 8,1 & 86,4 & 523 & 261 & 534 & 2500 & 375 & 13 & 0,51 & 6,6 & 1,61 & 801 & 1,38 & 91 & 61 & na & na \\
\hline 296 & 05.02.2016 & 8,2 & 86,6 & 533 & 266 & 541 & 2527 & 382 & 13 & 0,52 & 6,5 & 1,62 & 806 & 1,38 & 92 & 62 & na & na \\
\hline 299 & 08.02.2016 & 8,2 & 86,5 & 509 & 256 & 519 & 2491 & 367 & 13 & 0,49 & 6,3 & 1,55 & 769 & 1,35 & 88 & 61 & na & na \\
\hline 303 & 12.02.2016 & 7,8 & 86,4 & 496 & 251 & 509 & 2526 & 364 & 12 & 0,48 & 6,1 & 1,52 & 753 & 1,30 & 85 & 58 & na & na \\
\hline 307 & 16.02 .2016 & 11,8 & 89,1 & 727 & 360 & 705 & 2325 & 462 & 14 & 0,65 & 8,2 & 2,1 & 1118 & 1,89 & 127 & 87 & na & na \\
\hline
\end{tabular}


(9) Projektphase 2: Elementgehalte der Makro- und Mikronährstoffe (Quasi)kontinuierliche Laborfermenterversuche am DBFZ Reaktor 4.5 und 4.6: Substitutionsversuch 1: Winterackerbohne 1 und 2

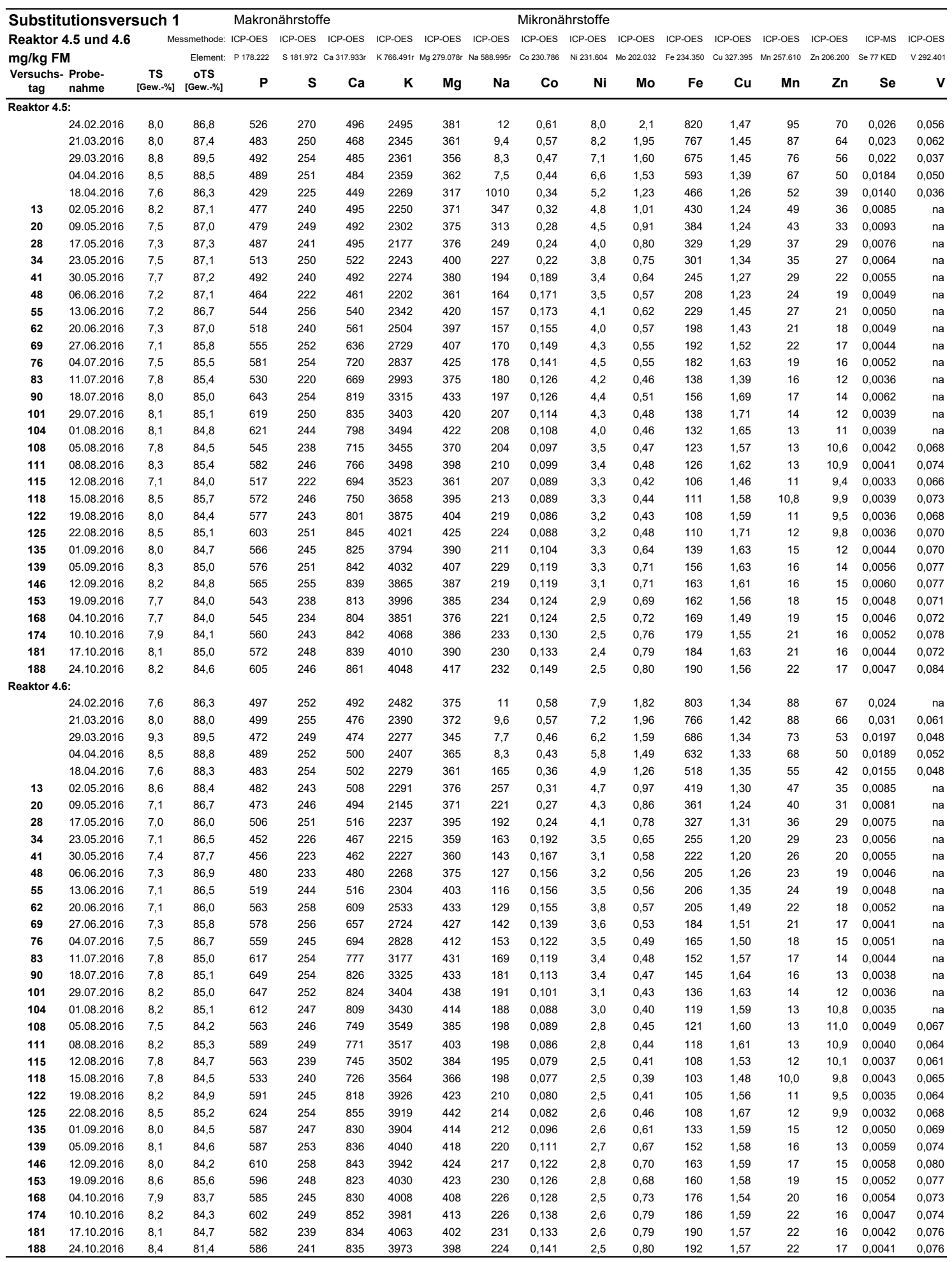


(10) Projektphase 2: Elementgehalte der Makro- und Mikronährstoffe (Quasi)kontinuierliche Laborfermenterversuche am DBFZ Reaktor 4.7 und 4.8: Substitutionsversuch 2: Amarant 1 und 2

\begin{tabular}{|c|c|c|c|c|c|c|c|c|c|c|c|c|c|c|c|c|c|c|}
\hline \multicolumn{4}{|c|}{ Substitutionsversuch 2} & \multicolumn{6}{|c|}{ Makronährstoffe } & Mikronä & àrstoffe & & & & & & & \\
\hline Reaktor 4 & 4.7 und 4.8 & & essmethode: & : ICP-OES & ICP-OES & ICP-OES & ICP-OES & ICP-OES & ICP-OES & ICP-OES & ICP-OES & ICP-OES & ICP-OES & ICP-OES & ICP-OES & ICP-OES & ICP-MS & ICP-OES \\
\hline $\mathrm{mg} / \mathrm{kg} \mathrm{Fn}$ & & & Element: & : P 178.222 & S 181.972 & Са $317.933 \mathrm{r}$ & K $766.491 r$ & Mg 279.078r & Na $588.995 r$ & Co 230.786 & $\mathrm{Ni} 231.604$ & Mo 202.032 & $\mathrm{Fe} 234.350$ & Сu 327.395 & Mn 257.610 & Zn 206.200 & Se 77 KED & V 292.401 \\
\hline $\begin{array}{c}\text { Versuchs- } \\
\text { tag }\end{array}$ & $\begin{array}{l}\text { Probe- } \\
\text { nahme }\end{array}$ & $\begin{array}{c}\text { TS } \\
\text { [Gew.-\%] }\end{array}$ & $\begin{array}{c}\text { oTS } \\
\text { [Gew..\%] }\end{array}$ & $\mathbf{P}$ & s & $\mathrm{Ca}$ & $K$ & Mg & $\mathrm{Na}$ & Co & $\mathrm{Ni}$ & Mo & $\mathrm{Fe}$ & $\mathrm{Cu}$ & $\mathrm{Mn}$ & $\mathrm{Zn}$ & Se & v \\
\hline Reaktor 4.7 & & & & & & & & & & & & & & & & & & \\
\hline & 24.02.2016 & 7,6 & 86,4 & 464 & 240 & 470 & 2258 & 348 & 10,9 & 0,54 & 7,3 & 1,70 & 753 & 1,26 & 83 & 63 & 0,023 & na \\
\hline & 21.03 .2016 & 9,0 & 89,4 & 484 & 249 & 472 & 2120 & 374 & 10,0 & 0,51 & 7,2 & 1,76 & 743 & 1,39 & 83 & 62 & 0,024 & 0,041 \\
\hline & 29.03 .2016 & 8,1 & 87,7 & 469 & 250 & 499 & 2227 & 366 & 10,0 & 0,45 & 6,7 & 1,51 & 690 & 1,38 & 71 & 53 & 0,0198 & 0,040 \\
\hline & 04.04 .2016 & 8,7 & 89,1 & 410 & 220 & 433 & 2249 & 311 & 7,1 & 0,38 & 5,4 & 1,29 & 555 & 1,19 & 56 & 42 & 0,0152 & 0,044 \\
\hline & 18.04.2016 & 7,7 & 88,0 & 400 & 217 & 432 & 2002 & 300 & 147 & 0,31 & 4,6 & 1,07 & 445 & 1,41 & 46 & 34 & 0,0134 & 0,052 \\
\hline 2 & 21.04 .2016 & 8,8 & 89,2 & 424 & 214 & 461 & 2198 & 328 & 226 & 0,34 & 4,8 & 1,04 & 460 & 1,14 & 51 & 37 & 0,0092 & na \\
\hline 13 & 02.05 .2016 & 8,3 & 88,8 & 463 & 239 & 500 & 2284 & 363 & 196 & 0,30 & 4,7 & 0,95 & 411 & 1,26 & 46 & 35 & 0,0082 & na \\
\hline 20 & 09.05 .2016 & 7,6 & 87,6 & 438 & 230 & 465 & 2088 & 343 & 162 & 0,25 & 4,0 & 0,80 & 340 & 1,17 & 38 & 30 & 0,0085 & na \\
\hline 28 & 17.05.2016 & 7,3 & 87,4 & 469 & 235 & 482 & 2150 & 368 & 141 & 0,23 & 3,7 & 0,72 & 303 & 1,21 & 34 & 27 & 0,0072 & na \\
\hline 34 & 23.05 .2016 & 7,8 & 87,5 & 509 & 251 & 523 & 2290 & 395 & 130 & 0,21 & 3,6 & 0,70 & 288 & 1,32 & 33 & 26 & 0,0062 & na \\
\hline 41 & 30.05 .2016 & 7,5 & 88,1 & 454 & 227 & 467 & 2230 & 355 & 110 & 0,168 & 2,9 & 0,56 & 222 & 1,17 & 26 & 21 & 0,0048 & na \\
\hline 48 & 06.06 .2016 & 7,3 & 86,7 & 521 & 253 & 532 & 2358 & 408 & 102 & 0,169 & 3,0 & 0,57 & 225 & 1,34 & 26 & 21 & 0,0054 & na \\
\hline 55 & 13.06 .2016 & 7,1 & 87,0 & 526 & 249 & 529 & 2292 & 408 & 88 & 0,152 & 2,8 & 0,53 & 206 & 1,32 & 24 & 20 & 0,0049 & na \\
\hline 62 & 20.06 .2016 & 7,5 & 86,7 & 511 & 240 & 652 & 2592 & 402 & 88 & 0,129 & 2,4 & 0,47 & 176 & 1,27 & 20 & 16 & 0,0056 & na \\
\hline 69 & 27.06 .2016 & 7,6 & 85,2 & 540 & 244 & 795 & 2765 & 418 & 89 & 0,112 & 2,2 & 0,43 & 155 & 1,24 & 19 & 15 & 0,0042 & na \\
\hline 76 & 04.07.2016 & 7,7 & 84,7 & 606 & 263 & 988 & 3099 & 467 & 94 & 0,111 & 2,3 & 0,44 & 156 & 1,38 & 18 & 15 & 0,0055 & na \\
\hline 83 & 11.07 .2016 & 8,1 & 83,8 & 669 & 271 & 1157 & 3590 & 496 & 100 & 0,100 & 2,2 & 0,41 & 140 & 1,38 & 17 & 14 & 0,0048 & na \\
\hline 90 & 18.07.2016 & 8,1 & 83,1 & 723 & 282 & 1299 & 3880 & 524 & 105 & 0,090 & 2,2 & 0,40 & 130 & 1,41 & 16 & 13 & 0,0041 & na \\
\hline 97 & 25.07 .2016 & 9,7 & 85,3 & 699 & 283 & 1406 & 3564 & 534 & 93 & 0,083 & 2,1 & 0,37 & 120 & 1,40 & 16 & 12 & 0,0037 & na \\
\hline 101 & 29.07 .2016 & 8,3 & 82,2 & 722 & 281 & 1441 & 4139 & 548 & 106 & 0,081 & 2,1 & 0,36 & 113 & 1,41 & 14 & 11 & 0,0037 & na \\
\hline 104 & 01.08 .2016 & 8,6 & 82,4 & 710 & 281 & 1464 & 4081 & 550 & 103 & 0,077 & 2,1 & 0,36 & 111 & 1,41 & 13 & 10,7 & 0,0037 & na \\
\hline 108 & 05.08.2016 & 8,7 & 83,3 & 654 & 281 & 1359 & 4122 & 509 & 105 & 0,073 & 1,99 & 0,39 & 111 & 1,37 & 13 & 10,6 & 0,0055 & 0,065 \\
\hline 111 & 08.08 .2016 & 8,4 & 82,6 & 665 & 280 & 1382 & 4181 & 520 & 105 & 0,072 & 1,98 & 0,37 & 105 & 1,35 & 13 & 10,3 & 0,0052 & 0,064 \\
\hline 115 & 12.08 .2016 & 9,2 & 83,3 & 688 & 285 & 1450 & 4197 & 536 & 105 & 0,070 & 1,97 & 0,37 & 104 & 1,37 & 13 & 10,2 & 0,0055 & 0,066 \\
\hline 118 & 15.08.2016 & 9,1 & 83,2 & 701 & 299 & 1495 & 4321 & 544 & 105 & 0,068 & 2,0 & 0,38 & 102 & 1,37 & 11 & 10,2 & 0,0053 & 0,071 \\
\hline 122 & 19.08.2016 & 8,6 & 82,9 & 613 & 252 & 1373 & 4650 & 517 & 108 & 0,064 & 1,88 & 0,33 & 84 & 1,29 & 11 & 8,1 & 0,0036 & 0,064 \\
\hline 125 & 22.08 .2016 & 9,0 & 83,0 & 673 & 273 & 1527 & 4724 & 559 & 109 & 0,063 & 1,93 & 0,39 & 90 & 1,33 & 11 & 8,7 & 0,0037 & 0,062 \\
\hline 135 & 01.09 .2016 & 8,7 & 82,2 & 687 & 277 & 1585 & 4644 & 563 & 107 & 0,084 & 2,2 & 0,55 & 121 & 1,33 & 15 & 11 & 0,0057 & 0,067 \\
\hline 139 & 05.09 .2016 & 8,9 & 82,5 & 697 & 293 & 1631 & 4786 & 568 & 109 & 0,104 & 2,4 & 0,62 & 144 & 1,37 & 17 & 13 & 0,0071 & 0,073 \\
\hline 146 & 12.09 .2016 & 8,8 & 81,9 & 719 & 295 & 1688 & 4797 & 585 & 109 & 0,113 & 2,5 & 0,66 & 153 & 1,38 & 17 & 14 & 0,0074 & 0,076 \\
\hline 153 & 19.09 .2016 & 8,6 & 81,5 & 689 & 288 & 1695 & 4834 & 580 & 111 & 0,121 & 2,5 & 0,65 & 156 & 1,38 & 19 & 14 & 0,0058 & 0,079 \\
\hline 168 & 04.10 .2016 & 8,1 & 79,9 & 668 & 287 & 1714 & 4991 & 557 & 110 & 0,119 & 2,4 & 0,70 & 162 & 1,35 & 20 & 14 & 0,0057 & 0,073 \\
\hline 174 & 10.10 .2016 & 8,9 & 81,3 & 734 & 302 & 1810 & 4848 & 602 & 109 & 0,129 & 2,4 & 0,74 & 177 & 1,38 & 21 & 15 & 0,0047 & 0,077 \\
\hline 181 & 17.10 .2016 & 8,8 & 81,3 & 693 & 296 & 1748 & 4909 & 571 & 109 & 0,129 & 2,4 & 0,75 & 174 & 1,34 & 21 & 15 & 0,0055 & 0,075 \\
\hline 188 & 24.10 .2016 & 8,6 & 80,8 & 698 & 299 & 1782 & 4919 & 573 & 109 & 0,131 & 2,4 & 0,76 & 178 & 1,32 & 22 & 15 & 0,0050 & 0,074 \\
\hline Reaktor 4.8 & & & & & & & & & & & & & & & & & & \\
\hline & 24.02 .2016 & 7,7 & 86,7 & 475 & 241 & 472 & 2310 & 355 & 10,9 & 0,55 & 7,5 & 1,76 & 774 & 1,29 & 84 & 64 & 0,022 & na \\
\hline & 21.03 .2016 & 8,3 & 88,7 & 484 & 249 & 459 & 2197 & 348 & 9,0 & 0,52 & 6,6 & 1,78 & 755 & 1,35 & 86 & 64 & 0,024 & na \\
\hline & 29.03 .2016 & 8,6 & 89,0 & 484 & 254 & 467 & 2217 & 358 & 8,5 & 0,48 & 6,4 & 1,48 & 622 & 1,30 & 70 & 53 & 0,021 & 0,031 \\
\hline & 04.04 .2016 & 8,5 & 89,1 & 440 & 230 & 452 & 2231 & 338 & 7,3 & 0,40 & 5,2 & 1,76 & 538 & 1,18 & 58 & 44 & 0,0121 & 0,043 \\
\hline & 18.04.2016 & 9,3 & 91,1 & 409 & 216 & 429 & 2033 & 320 & 150 & 0,29 & 4,0 & 0,98 & 388 & 1,20 & 44 & 33 & 0,0129 & 0,044 \\
\hline 2 & 21.04 .2016 & 5,6 & 83,9 & 401 & 206 & 466 & 2309 & 324 & 237 & 0,33 & 4,5 & 1,04 & 451 & 1,09 & 50 & 40 & 0,0099 & na \\
\hline 13 & 02.05 .2016 & 8,1 & 88,0 & 457 & 230 & 477 & 2218 & 356 & 182 & 0,29 & 4,2 & 0,91 & 391 & 1,19 & 44 & 33 & 0,0074 & na \\
\hline 20 & 09.05 .2016 & 7,7 & 88,2 & 440 & 228 & 458 & 2032 & 343 & 149 & 0,24 & 3,6 & 0,77 & 334 & 1,15 & 38 & 29 & 0,0076 & na \\
\hline 28 & 17.05 .2016 & 7,0 & 86,5 & 491 & 240 & 488 & 2207 & 385 & 140 & 0,24 & 3,8 & 0,76 & 314 & 1,23 & 36 & 28 & 0,0071 & na \\
\hline 34 & 23.05 .2016 & 7,6 & 87,5 & 521 & 256 & 523 & 2276 & 402 & 126 & 0,21 & 3,5 & 0,70 & 290 & 1,32 & 34 & 26 & 0,0064 & na \\
\hline 41 & 30.05 .2016 & 7,4 & 87,5 & 483 & 237 & 483 & 2200 & 376 & 106 & 0,176 & 3,0 & 0,58 & 237 & 1,23 & 28 & 22 & 0,0056 & na \\
\hline 48 & 06.06 .2016 & 7,3 & 86,2 & 531 & 254 & 530 & 2345 & 412 & 98 & 0,169 & 3,1 & 0,58 & 229 & 1,35 & 27 & 22 & 0,0049 & na \\
\hline 55 & 13.06.2016 & 7,4 & 86,5 & 485 & 227 & 478 & 2230 & 378 & 83 & 0,157 & 2,7 & 0,51 & 191 & 1,22 & 23 & 18 & 0,0043 & na \\
\hline 62 & 20.06 .2016 & 7,6 & 86,9 & 564 & 258 & 695 & 2598 & 439 & 88 & 0,140 & 3,0 & 0,53 & 197 & 1,38 & 22 & 18 & 0,0056 & na \\
\hline 69 & 27.06 .2016 & 7,4 & 84,9 & 584 & 258 & 840 & 2852 & 449 & 89 & 0,129 & 2,9 & 0,48 & 175 & 1,34 & 21 & 17 & 0,0044 & na \\
\hline 76 & 04.07.2016 & 8,1 & 84,5 & 644 & 274 & 985 & 3251 & 501 & 101 & 0,130 & 3,3 & 0,52 & 177 & 1,45 & 20 & 16 & 0,0056 & na \\
\hline 83 & 11.07 .2016 & 7,9 & 83,7 & 693 & 278 & 1149 & 3487 & 509 & 98 & 0,121 & 3,4 & 0,47 & 156 & 1,45 & 18 & 14 & 0,0057 & na \\
\hline 90 & 18.07.2016 & 8,0 & 83,3 & 719 & 280 & 1261 & 3783 & 519 & 102 & 0,105 & 3,2 & 0,44 & 141 & 1,41 & 16 & 13 & 0,0041 & na \\
\hline 97 & 25.07 .2016 & 8,7 & 84,9 & 645 & 268 & 1264 & 3635 & 498 & 96 & 0,091 & 2,9 & 0,38 & 121 & 1,32 & 15 & 11 & 0,0039 & na \\
\hline 101 & 29.07 .2016 & 8,8 & 83,4 & 735 & 287 & 1438 & 4019 & 551 & 104 & 0,095 & 2,9 & 0,42 & 127 & 1,48 & 14 & 11 & 0,0045 & na \\
\hline 104 & 01.08 .2016 & 8,3 & 83,0 & 682 & 278 & 1405 & 3979 & 534 & 102 & 0,085 & 2,6 & 0,39 & 117 & 1,41 & 13 & 10,8 & 0,0043 & na \\
\hline 108 & 05.08.2016 & 8,3 & 82,7 & 618 & 275 & 1310 & 4007 & 485 & 101 & 0,082 & 2,3 & 0,42 & 112 & 1,35 & 13 & 10,5 & 0,0055 & 0,068 \\
\hline 111 & 08.08.2016 & 8,6 & 83,2 & 659 & 283 & 1361 & 4075 & 516 & 102 & 0,078 & 2,3 & 0,42 & 112 & 1,36 & 13 & 10,5 & 0,0048 & 0,068 \\
\hline 115 & 12.08 .2016 & 8,5 & 82,5 & 663 & 278 & 1381 & 4064 & 518 & 102 & 0,076 & 2,2 & 0,40 & 106 & 1,34 & 13 & 10,1 & 0,0050 & 0,072 \\
\hline 118 & 15.08 .2016 & 9,0 & 83,5 & 663 & 289 & 1417 & 4240 & 524 & 103 & 0,073 & 2,1 & 0,40 & 102 & 1,35 & 10,1 & 10,0 & 0,0055 & 0,071 \\
\hline 122 & 19.08.2016 & 8,7 & 82,5 & 678 & 277 & 1543 & 4650 & 567 & 109 & 0,069 & 2,0 & 0,40 & 98 & 1,33 & 12 & 9,2 & 0,0047 & 0,069 \\
\hline 125 & 22.08 .2016 & 8,7 & 82,8 & 665 & 272 & 1524 & 4595 & 554 & 107 & 0,063 & 1,91 & 0,42 & 92 & 1,34 & 11 & 8,9 & 0,0042 & 0,067 \\
\hline 135 & 01.09 .2016 & 8,4 & 81,5 & 656 & 283 & 1555 & 4678 & 543 & 228 & 0,089 & 2,2 & 0,58 & 124 & 1,32 & 15 & 11 & 0,0057 & 0,070 \\
\hline 139 & 05.09.2016 & 8,7 & 81,8 & 684 & 300 & 1612 & 4700 & 561 & 221 & 0,103 & 2,3 & 0,65 & 145 & 1,35 & 17 & 13 & 0,0064 & 0,075 \\
\hline 146 & 12.09 .2016 & 9,0 & 82,5 & 681 & 303 & 1628 & 4591 & 557 & 203 & 0,112 & 2,4 & 0,66 & 150 & 1,33 & 18 & 14 & 0,0065 & 0,078 \\
\hline 153 & 19.09.2016 & 8,5 & 81,9 & 661 & 293 & 1615 & 4781 & 558 & 198 & 0,118 & 2,5 & 0,69 & 151 & 1,33 & 19 & 14 & 0,0055 & 0,077 \\
\hline 168 & 04.10 .2016 & 7,9 & 80,0 & 646 & 287 & 1636 & 4846 & 540 & 171 & 0,118 & 2,5 & 0,71 & 159 & 1,35 & 19 & 14 & 0,0057 & 0,075 \\
\hline 174 & 10.10 .2016 & 8,5 & 81,0 & 676 & 298 & 1730 & 4793 & 558 & 162 & 0,128 & 2,6 & 0,76 & 173 & 1,32 & 21 & 15 & 0,0051 & 0,082 \\
\hline 181 & 17.10 .2016 & 8,7 & 80,6 & 710 & 305 & 1811 & 4845 & 582 & 155 & 0,135 & 2,8 & 0,79 & 180 & 1,37 & 21 & 16 & 0,0054 & 0,081 \\
\hline 188 & 24.10 .2016 & 8,8 & 81,0 & 737 & 309 & 1824 & 4854 & 604 & 150 & 0,140 & 3,0 & 0,81 & 187 & 1,38 & 23 & 16 & 0,0052 & 0,083 \\
\hline
\end{tabular}


(11) Projektphase 2: Elementgehalte der Makro- und Mikronährstoffe (Quasi)kontinuierliche Laborfermenterversuche am DBFZ Reaktor 4.9: Referenzreaktor (Spurenelementvollversorgung)

\begin{tabular}{|c|c|c|c|c|c|c|c|c|c|c|c|c|c|c|c|c|c|c|}
\hline \multicolumn{4}{|c|}{ Substitutionsversuch 1+2 } & \multicolumn{6}{|c|}{ Makronährstoffe } & \multicolumn{9}{|c|}{ Mikronährstoffe } \\
\hline \multicolumn{2}{|c|}{ Reaktor 4.9} & \multicolumn{2}{|c|}{ Messmethode: } & \multirow{2}{*}{$\begin{array}{l}\text { ICP-OES } \\
\text { P } 178.222\end{array}$} & \multirow{2}{*}{$\begin{array}{c}\text { ICP-OES } \\
\text { S } 181.972\end{array}$} & \multirow{2}{*}{$\begin{array}{l}\text { ICP-OES } \\
\text { Cа 317.933r }\end{array}$} & \multicolumn{2}{|c|}{ ICP-OES ICP-OES } & ICP-OES & \multirow{2}{*}{$\begin{array}{l}\text { ICP-OES } \\
\text { Co } 230.786\end{array}$} & \multirow{3}{*}{$\begin{array}{r}\text { ICP-OES } \\
\text { Ni 231.604 } \\
\mathbf{N i}\end{array}$} & \multirow{3}{*}{$\begin{array}{r}\text { ICP-OES } \\
\text { Mo } 202.032 \\
\text { Mo }\end{array}$} & \multirow{3}{*}{$\begin{array}{r}\text { ICP-OES } \\
\mathrm{Fe} 234.350 \\
\mathbf{F e}\end{array}$} & \multirow{3}{*}{$\begin{array}{r}\text { ICP-OES } \\
\text { CU } 327.395 \\
\text { Cu }\end{array}$} & \multirow{3}{*}{$\begin{array}{r}\text { ICP-OES } \\
\text { Mn } 257.610 \\
\text { Mn }\end{array}$} & \multirow{3}{*}{$\begin{array}{r}\text { ICP-OES } \\
\text { Zn } 206.200 \\
\text { Zn }\end{array}$} & \multirow{3}{*}{$\begin{array}{r}\text { ICP-MS } \\
\text { Se } 77 \text { KED } \\
\text { Se }\end{array}$} & \multirow{3}{*}{$\begin{array}{r}\text { ICP-OES } \\
\text { V 292.401 } \\
\mathbf{V}\end{array}$} \\
\hline $\mathrm{mg} / \mathrm{kg} \mathrm{FN}$ & & & Element: & & & & K $766.491 \mathrm{r}$ & Mg 279.078r & Na 588.995r & & & & & & & & & \\
\hline $\begin{array}{l}\text { Versuchs- } \\
\text { tag }\end{array}$ & $\begin{array}{l}\text { Probe- } \\
\text { nahme }\end{array}$ & $\begin{array}{c}\text { TS } \\
\text { [Gew.-\%] }\end{array}$ & $\begin{array}{c}\text { oTS } \\
\text { [Gew..\%] }\end{array}$ & $\mathbf{P}$ & $\mathbf{s}$ & $\mathrm{Ca}$ & $\mathbf{K}$ & $\mathbf{M g}$ & $\mathrm{Na}$ & Co & & & & & & & & \\
\hline & 24.02 .2016 & 7,9 & 87,0 & 472 & 243 & 472 & 2257 & 354 & 12 & 0,55 & 7,4 & 1,68 & 757 & 1,27 & 83 & 63 & 0,022 & $\overline{\text { na }}$ \\
\hline & 21.03 .2016 & 7,9 & 87,8 & 490 & 255 & 474 & 2367 & 364 & 8,9 & 0,55 & 6,5 & 1,89 & 758 & 1,36 & 87 & 65 & 0,025 & 0,043 \\
\hline & 29.03.2016 & 9,1 & 89,3 & 467 & 244 & 477 & 2347 & 356 & 7,7 & 0,54 & 6,2 & 2,3 & 729 & 1,27 & 80 & 60 & 0,0192 & 0,027 \\
\hline & 04.04.2016 & 8,0 & 86,7 & 513 & 247 & 489 & 2298 & 391 & 10,4 & 0,49 & 6,4 & 1,81 & 760 & 1,32 & 86 & 65 & 0,028 & 0,041 \\
\hline & 18.04.2016 & 7,7 & 88,2 & 429 & 227 & 448 & 2257 & 325 & 157 & 0,49 & 5,4 & 1,70 & 668 & 1,18 & 77 & 57 & 0,022 & 0,030 \\
\hline 2 & 21.04 .2016 & 7,5 & 87,7 & 451 & 233 & 465 & 2266 & 344 & 207 & 0,41 & 5,4 & 1,47 & 537 & 1,23 & 64 & 45 & 0,0108 & na \\
\hline 13 & 02.05 .2016 & 8,6 & 88,4 & 459 & 237 & 484 & 2240 & 361 & 170 & 0,41 & 5,4 & 1,28 & 553 & 1,22 & 62 & 46 & 0,0102 & na \\
\hline 20 & 09.05 .2016 & 7,4 & 86,8 & 482 & 250 & 501 & 2241 & 381 & 152 & 0,44 & 5,7 & 1,37 & 602 & 1,28 & 67 & 50 & 0,0129 & na \\
\hline 28 & 17.05 .2016 & 8,8 & 88,6 & 488 & 249 & 497 & 2225 & 389 & 129 & 0,43 & 5,5 & 1,35 & 582 & 1,26 & 67 & 49 & 0,0121 & na \\
\hline 34 & 23.05 .2016 & 7,3 & 88,9 & 499 & 258 & 519 & 2291 & 397 & 116 & 0,46 & 5,7 & 1,45 & 630 & 1,32 & 71 & 54 & 0,0118 & na \\
\hline 41 & 30.05 .2016 & 7,6 & 86,1 & 502 & 259 & 515 & 2325 & 397 & 101 & 0,46 & 5,5 & 1,44 & 626 & 1,32 & 71 & 54 & 0,0139 & na \\
\hline 48 & 06.06 .2016 & 7,8 & 86,5 & 533 & 269 & 538 & 2384 & 420 & 92 & 0,48 & 5,8 & 1,53 & 656 & 1,41 & 75 & 57 & 0,0149 & na \\
\hline 55 & 13.06.2016 & 7,6 & 86,5 & 521 & 263 & 531 & 2405 & 413 & 80 & 0,47 & 5,7 & 1,51 & 644 & 1,39 & 74 & 56 & 0,0140 & na \\
\hline 62 & 20.06 .2016 & 7,7 & 86,7 & 541 & 266 & 555 & 2400 & 425 & 73 & 0,49 & 5,9 & 1,55 & 682 & 1,45 & 74 & 55 & 0,0164 & na \\
\hline 69 & 27.06 .2016 & 7,5 & 85,7 & 515 & 256 & 533 & 2364 & 395 & 61 & 0,47 & 5,7 & 1,51 & 651 & 1,34 & 74 & 55 & 0,0132 & na \\
\hline 76 & 04.07.2016 & 8,2 & 86,6 & 527 & 257 & 548 & 2403 & 406 & 57 & 0,48 & 5,9 & 1,53 & 656 & 1,40 & 72 & 54 & 0,0167 & na \\
\hline 83 & 11.07.2016 & 7,8 & 85,1 & 552 & 262 & 540 & 2486 & 410 & 51 & 0,50 & 5,8 & 1,66 & 634 & 1,43 & 80 & 55 & 0,0192 & na \\
\hline 90 & 18.07.2016 & 7,8 & 84,6 & 616 & 264 & 592 & 2605 & 431 & 46 & 0,51 & 6,4 & 1,61 & 694 & 1,45 & 76 & 55 & 0,0141 & na \\
\hline 97 & 25.07 .2016 & 7,7 & 85,3 & 530 & 252 & 534 & 2485 & 401 & 40 & 0,49 & 6,3 & 1,57 & 667 & 1,38 & 76 & 56 & 0,0161 & na \\
\hline 101 & 29.07 .2016 & 9,0 & 86,9 & 605 & 276 & 581 & 2603 & 434 & 40 & 0,51 & 6,6 & 1,64 & 709 & 1,49 & 78 & 57 & 0,0192 & na \\
\hline 104 & 01.08 .2016 & 7,9 & 85,6 & 567 & 268 & 571 & 2643 & 436 & 38 & 0,52 & 6,4 & 1,66 & 715 & 1,52 & 77 & 57 & 0,0197 & na \\
\hline 108 & 05.08 .2016 & 7,8 & 85,4 & 537 & 269 & 509 & 2526 & 401 & 35 & 0,52 & 6,2 & 1,82 & 682 & 1,47 & 81 & 59 & 0,030 & 0,082 \\
\hline 111 & 08.08.2016 & 7,6 & 85,0 & 531 & 266 & 506 & 2611 & 400 & 34 & 0,52 & 6,2 & 1,82 & 671 & 1,46 & 80 & 58 & 0,028 & 0,077 \\
\hline 115 & 12.08.2016 & 7,3 & 84,0 & 546 & 279 & 513 & 2757 & 415 & 33 & 0,53 & 6,3 & 1,85 & 682 & 1,46 & 81 & 60 & 0,033 & 0,078 \\
\hline 118 & 15.08.2016 & 9,1 & 87,2 & 538 & 271 & 497 & 2779 & 407 & 30 & 0,54 & 6,6 & 1,88 & 653 & 1,42 & 79 & 56 & 0,035 & 0,077 \\
\hline 122 & 19.08.2016 & 7,4 & 84,3 & 525 & 264 & 524 & 2955 & 420 & 30 & 0,55 & 6,6 & 2,0 & 674 & 1,46 & 78 & 57 & 0,036 & 0,071 \\
\hline 125 & 22.08.2016 & 7,9 & 85,0 & 553 & 272 & 532 & 2858 & 431 & 28 & 0,56 & 6,8 & 2,3 & 705 & 1,51 & 81 & 59 & 0,041 & 0,076 \\
\hline 135 & 01.09 .2016 & 8,0 & 85,1 & 579 & 287 & 542 & 3071 & 445 & 26 & 0,56 & 7,2 & 2,6 & 726 & 1,57 & 83 & 61 & 0,043 & 0,086 \\
\hline 139 & 05.09 .2016 & 8,5 & 86,2 & 573 & 288 & 548 & 3085 & 445 & 24 & 0,57 & 7,4 & 2,6 & 716 & 1,55 & 83 & 60 & 0,039 & 0,080 \\
\hline 146 & 12.09 .2016 & 7,6 & 84,6 & 571 & 282 & 525 & 3004 & 443 & 22 & 0,56 & 7,2 & 2,5 & 710 & 1,53 & 82 & 60 & 0,039 & 0,075 \\
\hline 153 & 19.09.2016 & 7,5 & 84,5 & 571 & 280 & 523 & 3033 & 457 & 22 & 0,56 & 7,2 & 2,4 & 708 & 1,57 & 84 & 62 & 0,035 & 0,098 \\
\hline 168 & 04.10 .2016 & 7,6 & 85,3 & 574 & 276 & 507 & 2915 & 449 & 18 & 0,57 & 7,1 & 2,5 & 718 & 1,53 & 84 & 61 & 0,028 & 0,080 \\
\hline 174 & 10.10 .2016 & 7,5 & 84,9 & 567 & 282 & 500 & 2886 & 438 & 16 & 0,57 & 6,9 & 2,6 & 717 & 1,55 & 84 & 62 & 0,021 & 0,089 \\
\hline 181 & 17.10.2016 & 8,0 & 85,7 & 542 & 267 & 474 & 2842 & 424 & 15 & 0,53 & 6,7 & 2,5 & 684 & 1,46 & 79 & 57 & 0,0175 & 0,090 \\
\hline 188 & 24.10 .2016 & 8,0 & 85,8 & 530 & 266 & 466 & 2820 & 409 & 14 & 0,54 & 6,5 & 2,4 & 686 & 1,44 & 79 & 58 & 0,0180 & 0,083 \\
\hline
\end{tabular}


(12) Projektphase 3: Elementgehalte der Makro- und Mikronährstoffe (Quasi)kontinuierliche Laborfermenterversuche am DBFZ Reaktor 4.5 und 4.6: Substitutionsversuch 3: Deutsches Weidelgras 1 und 2

\begin{tabular}{|c|c|c|c|c|c|c|c|c|c|c|c|c|c|c|c|c|c|c|}
\hline \multicolumn{4}{|c|}{ Substitutionsversuch 3} & \multicolumn{6}{|c|}{ Makronährstoffe } & \multicolumn{9}{|c|}{ Mikronährstoffe } \\
\hline \multirow{2}{*}{\multicolumn{2}{|c|}{$\begin{array}{l}\text { Reaktor } 4.5 \text { und } 4.6 \\
\mathrm{mg} / \mathrm{kg} \text { FM }\end{array}$}} & \multicolumn{2}{|c|}{ Messmethode: } & \multirow{3}{*}{$\begin{array}{r}\text { ICP-OES } \\
\text { P } 178.222 \\
\mathbf{P}\end{array}$} & \multirow{3}{*}{$\begin{array}{r}\text { ICP-OES } \\
\text { S } 181.972 \\
\mathbf{S}\end{array}$} & \multirow{2}{*}{$\begin{array}{l}\text { ICP-OES } \\
\text { Ca } 317.933 r\end{array}$} & \multirow{3}{*}{$\begin{array}{r}\text { ICP-OES } \\
\text { K 766.491r } \\
\mathbf{K}\end{array}$} & \multirow{2}{*}{$\begin{array}{l}\text { ICP-OES } \\
\text { Mg 279.078r }\end{array}$} & ICP-OES & \multirow{3}{*}{$\begin{array}{r}\text { ICP-OES } \\
\text { Co } 230.786 \\
\text { Co }\end{array}$} & \multirow{3}{*}{$\begin{array}{r}\text { ICP-OES } \\
\text { Ni } 231.604 \\
\mathbf{N i}\end{array}$} & \multirow{3}{*}{$\begin{array}{r}\text { ICP-OES } \\
\text { Mo 202.032 } \\
\text { Mo }\end{array}$} & \multirow{3}{*}{$\begin{array}{r}\text { ICP-OES } \\
\mathrm{Fe} 234.350 \\
\mathbf{F e}\end{array}$} & \multirow{3}{*}{$\begin{array}{r}\text { ICP-OES } \\
\text { Сu } 327.395 \\
\text { Cu }\end{array}$} & \multirow{3}{*}{$\begin{array}{r}\text { ICP-OES } \\
\text { Mn } 257.610 \\
\text { Mn }\end{array}$} & ICP-OES & ICP-MS & ICP-OES \\
\hline & & & Element: & & & & & & Na 588.995r & & & & & & & Zn 206.200 & Se 77 KED & v 292.401 \\
\hline $\begin{array}{l}\text { Versuchs- } \\
\text { tag }\end{array}$ & $\begin{array}{l}\text { Probe- } \\
\text { nahme }\end{array}$ & $\underset{\text { [Gew.-\%] }}{\text { TS }}$ & $\begin{array}{c}\text { oTS } \\
\text { [Gew..\%] }\end{array}$ & & & $\mathrm{Ca}$ & & $\mathbf{M g}$ & $\mathrm{Na}$ & & & & & & & $\mathbf{Z n}$ & Se & v \\
\hline Reaktor 4.5 & & & & & & & & & & & & & & & & & & \\
\hline & 21.11 .2016 & 6,2 & 83,1 & 516 & 235 & 433 & 2514 & 369 & 73 & 0,44 & 7,8 & 1,57 & 527 & 1,51 & 66 & 46 & 0,0181 & 0,094 \\
\hline & 12.12.2016 & 6,5 & 84,8 & 475 & 241 & 448 & 2464 & 335 & 51 & 0,32 & 7,3 & 1,13 & 374 & 1,50 & 42 & 32 & 0,0121 & 0,089 \\
\hline & 02.01.2017 & 7,2 & 85,7 & 522 & 256 & 517 & 2517 & 367 & 41 & 0,27 & 7,7 & 0,90 & 325 & 1,67 & 31 & 28 & 0,0102 & 0,111 \\
\hline 51 & 14.06.2017 & 7,8 & 87,0 & 415 & 226 & 529 & 2485 & 300 & 49 & 0,182 & 2,6 & 0,59 & 233 & 2,3 & 31 & 23 & 0,0082 & 0,068 \\
\hline 63 & 26.06.2017 & 7,5 & 86,0 & 426 & 230 & 552 & 2912 & 294 & 70 & 0,167 & 2,2 & 0,56 & 182 & 2,1 & 26 & 19 & 0,0055 & 0,057 \\
\hline 84 & 17.07.2017 & 8,1 & 86,2 & 472 & 250 & 627 & 3011 & 310 & 93 & 0,137 & 2,0 & 0,52 & 164 & 1,85 & 22 & 21 & 0,0061 & 0,065 \\
\hline 105 & 07.08.2017 & 8,3 & 85,7 & 492 & 245 & 627 & 3316 & 326 & 108 & 0,106 & 2,6 & 0,42 & 171 & 1,66 & 17 & 17 & 0,0048 & 0,074 \\
\hline 126 & 28.08.2017 & 8,8 & 85,8 & 531 & 250 & 651 & 3455 & 355 & 118 & 0,101 & 4,2 & 0,41 & 146 & 1,57 & 15 & 13 & 0,0044 & 0,089 \\
\hline 147 & 18.09.2017 & 8,4 & 84,6 & 553 & 259 & 690 & 3617 & 374 & 129 & 0,095 & 4,4 & 0,42 & 120 & 1,56 & 14 & 11 & 0,0035 & 0,086 \\
\hline 168 & 09.10 .2017 & 8,3 & 85,4 & 481 & 248 & 669 & 3388 & 322 & 107 & 0,086 & 3,9 & 0,38 & 96 & 1,43 & 13 & 9,7 & 0,0031 & 0,068 \\
\hline 182 & 23.10.2017 & 8,7 & 85,3 & 492 & 239 & 681 & 3651 & 339 & 106 & 0,068 & 3,8 & 0,36 & 98 & 1,42 & 12 & 8,3 & 0,0033 & 0,073 \\
\hline 203 & 13.11.2017 & 5,5 & 79,8 & 330 & 177 & 513 & 2733 & 225 & 1211 & 0,108 & 4,2 & 0,30 & 79 & 1,05 & 9,0 & 6,0 & 0,0019 & 0,053 \\
\hline 224 & 04.12 .2017 & 3,9 & 77,3 & 309 & 134 & 398 & 2035 & 213 & 781 & 0,138 & 4,2 & 0,25 & 74 & 0,95 & 7,4 & 4,6 & 0,0015 & 0,047 \\
\hline 238 & 18.12.2017 & 4,8 & 81,6 & 349 & 147 & 466 & 2122 & 249 & 632 & 0,165 & 4,0 & 0,27 & 78 & 0,92 & 8,4 & 4,8 & na & 0,063 \\
\hline 259 & 08.01.2018 & 6,0 & 84,2 & 380 & 174 & 591 & 2313 & 270 & 439 & 0,162 & 3,3 & 0,29 & 77 & 0,97 & 10,0 & 5,6 & na & 0,062 \\
\hline 280 & 29.01.2018 & 7,3 & 85,7 & 431 & 213 & 654 & 2626 & 310 & 334 & 0,177 & 2,6 & 0,32 & 63 & 0,98 & 10,5 & 5,6 & na & 0,060 \\
\hline 301 & 19.02 .2018 & 7,3 & 85,4 & 424 & 209 & 643 & 2694 & 288 & 228 & 0,164 & 2,1 & 0,26 & 57 & 1,04 & 9,8 & 5,3 & na & 0,062 \\
\hline 322 & 12.03 .2018 & 7,3 & 83,3 & 436 & 218 & 672 & 2829 & 328 & 214 & 0,174 & 2,0 & 0,27 & 106 & 1,11 & 10,5 & 5,5 & na & 0,172 \\
\hline 345 & 04.04 .2018 & 7,2 & 85,0 & 416 & 215 & 645 & 2925 & 290 & 158 & 0,163 & 1,93 & 0,26 & 50 & 1,09 & 9,6 & 5,3 & na & 0,061 \\
\hline 364 & 23.04 .2018 & 7,3 & 83,5 & 442 & 219 & 669 & 2665 & 318 & 146 & 0,196 & 2,1 & 0,25 & 83 & 1,11 & 10,8 & 5,6 & na & 0,139 \\
\hline Reaktor 4.6 & & & & & & & & & & & & & & & & & & \\
\hline & 21.11 .2016 & 6,2 & 83,2 & 526 & 240 & 439 & 2544 & 375 & 74 & 0,44 & 7,4 & 1,61 & 534 & 1,49 & 67 & 47 & 0,0164 & 0,096 \\
\hline & 12.12.2016 & 6,6 & 85,0 & 495 & 243 & 452 & 2470 & 349 & 52 & 0,30 & 5,3 & 1,13 & 370 & 1,41 & 43 & 33 & 0,0124 & 0,080 \\
\hline & 02.01.2017 & 7,1 & 86,3 & 524 & 254 & 526 & 2490 & 370 & 41 & 0,23 & 4,7 & 0,88 & 305 & 1,57 & 31 & 28 & 0,0092 & 0,085 \\
\hline 51 & 14.06.2017 & 7,6 & 86,1 & 422 & 231 & 550 & 2551 & 310 & 49 & 0,20 & 3,2 & 0,66 & 244 & 2,4 & 32 & 24 & 0,0077 & 0,070 \\
\hline 63 & 26.06.2017 & 8,2 & 86,6 & 480 & 255 & 615 & 2883 & 317 & 69 & 0,197 & 3,2 & 0,67 & 214 & 2,3 & 30 & 22 & 0,0067 & 0,067 \\
\hline 84 & 17.07.2017 & 8,6 & 86,5 & 479 & 243 & 616 & 3179 & 316 & 94 & 0,145 & 3,2 & 0,56 & 179 & 1,90 & 22 & 20 & 0,0056 & 0,067 \\
\hline 105 & 07.08.2017 & 8,4 & 86,0 & 490 & 241 & 639 & 3327 & 330 & 110 & 0,107 & 3,5 & 0,49 & 167 & 1,71 & 18 & 17 & 0,0050 & 0,075 \\
\hline 126 & 28.08.2017 & 9,1 & 86,0 & 541 & 259 & 690 & 3581 & 357 & 122 & 0,090 & 3,2 & 0,47 & 134 & 1,65 & 16 & 14 & 0,0037 & 0,079 \\
\hline 147 & 18.09.2017 & 8,2 & 84,7 & 521 & 241 & 657 & 3582 & 357 & 128 & 0,075 & 3,3 & 0,44 & 109 & 1,46 & 13 & 10,3 & 0,0040 & 0,070 \\
\hline 168 & 09.10 .2017 & 8,7 & 85,2 & 525 & 253 & 690 & 3544 & 354 & 113 & 0,071 & 2,8 & 0,42 & 91 & 1,46 & 13 & 9,9 & 0,0037 & 0,070 \\
\hline 182 & 23.10.2017 & 8,8 & 86,3 & 487 & 247 & 674 & 3455 & 333 & 103 & 0,062 & 2,2 & 0,38 & 73 & 1,34 & 12 & 8,6 & 0,0034 & 0,055 \\
\hline 203 & 13.11.2017 & 6,1 & 80,7 & 369 & 182 & 544 & 2698 & 251 & 1286 & 0,080 & 1,86 & 0,32 & 63 & 1,03 & 9,1 & 6,5 & 0,0023 & 0,042 \\
\hline 224 & 04.12.2017 & 4,8 & 81,0 & 331 & 147 & 441 & 1991 & 234 & 815 & 0,117 & 1,88 & 0,26 & 58 & 0,87 & 8,2 & 5,5 & 0,0017 & 0,041 \\
\hline 238 & 18.12.2017 & 5,1 & 83,2 & 335 & 151 & 462 & 2090 & 240 & 678 & 0,131 & 1,73 & 0,26 & 58 & 0,86 & 8,0 & 4,8 & na & 0,045 \\
\hline 259 & 08.01.2018 & 5,4 & 83,8 & 344 & 164 & 535 & 2315 & 240 & 474 & 0,124 & 1,32 & 0,27 & 50 & 0,84 & 8,9 & 5,3 & na & 0,040 \\
\hline 280 & 29.01 .2018 & 7,0 & 86,5 & 379 & 194 & 589 & 2361 & 253 & 305 & 0,136 & 1,18 & 0,27 & 46 & 0,89 & 9,6 & 5,3 & na & 0,047 \\
\hline 301 & 19.02 .2018 & 6,6 & 87,3 & 403 & 187 & 598 & 2598 & 263 & 151 & 0,132 & 0,96 & 0,24 & 41 & 0,96 & 9,4 & 5,1 & na & 0,050 \\
\hline 322 & 12.03 .2018 & 7,1 & 82,0 & 480 & 215 & 665 & 2836 & 348 & 208 & 0,145 & 1,06 & 0,26 & 70 & 1,12 & 10,4 & 5,4 & na & 0,102 \\
\hline 345 & 04.04.2018 & 7,4 & 83,5 & 492 & 225 & 696 & 2956 & 353 & 168 & 0,147 & 0,97 & 0,27 & 60 & 1,18 & 10,8 & 5,8 & na & 0,090 \\
\hline 364 & 23.04.2018 & 7,4 & 82,3 & 489 & 219 & 698 & 3032 & 359 & 155 & 0,151 & 0,99 & 0,26 & 82 & 1,17 & 11 & 5,9 & na & 0,125 \\
\hline
\end{tabular}


(13) Projektphase 3: Elementgehalte der Makro- und Mikronährstoffe (Quasi)kontinuierliche Laborfermenterversuche am DBFZ Reaktor 4.7 und 4.8: Substitutionsversuch 4: Durchwachsene Silphie 1 und 2

\begin{tabular}{|c|c|c|c|c|c|c|c|c|c|c|c|c|c|c|c|c|c|c|}
\hline \multicolumn{4}{|c|}{ Substitutionsversuch 4} & \multicolumn{6}{|c|}{ Makronährstoffe } & \multicolumn{9}{|c|}{ Mikronährstoffe } \\
\hline \multirow{2}{*}{\multicolumn{2}{|c|}{ Reaktor 4.7 und 4.8}} & \multirow{2}{*}{\multicolumn{2}{|c|}{ Messmethode: }} & \multirow{2}{*}{$\begin{array}{l}\text { ICP-OES } \\
\text { P } 178.222\end{array}$} & \multirow{2}{*}{$\begin{array}{l}\text { ICP-OES } \\
\text { S } 181.972\end{array}$} & \multirow{2}{*}{$\begin{array}{l}\text { ICP-OES } \\
\text { Ca } 317.933 r\end{array}$} & \multirow{2}{*}{\multicolumn{2}{|c|}{ ICP-OES ICP-OES }} & ICP-OES & \multirow{3}{*}{$\begin{array}{r}\text { ICP-OES } \\
\text { Co } 230.786 \\
\text { Co }\end{array}$} & \multirow{3}{*}{ 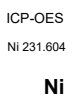 } & \multirow{3}{*}{$\begin{array}{r}\text { ICP-OES } \\
\text { Mo } 202.032 \\
\text { Mo }\end{array}$} & \multirow{3}{*}{$\begin{array}{r}\text { ICP-OES } \\
\mathrm{Fe} 234.350 \\
\mathbf{F e}\end{array}$} & \multirow{3}{*}{$\begin{array}{r}\text { ICP-OES } \\
\text { Сu } 327.395 \\
\mathbf{C u}\end{array}$} & \multirow{3}{*}{$\begin{array}{r}\text { ICP-OES } \\
\text { Mn } 257.610 \\
\text { Mn }\end{array}$} & \multirow{3}{*}{$\begin{array}{r}\text { ICP-OES } \\
\text { Zn } 206.200 \\
\text { Zn }\end{array}$} & ICP-MS & ICP-OES \\
\hline & & & & & & & & & Na 588.995r & & & & & & & & Se 77 KED & $\mathrm{V}_{292.401}$ \\
\hline $\begin{array}{l}\text { Versuchs- } \\
\text { tag }\end{array}$ & $\begin{array}{l}\text { Probe- } \\
\text { nahme }\end{array}$ & $\begin{array}{c}\text { TS } \\
{[\text { Gew.-\%] }}\end{array}$ & $\begin{array}{c}\text { OTS } \\
\text { [Gew.-\%] }\end{array}$ & $\mathbf{P}$ & $\mathbf{s}$ & $\mathrm{Ca}$ & K & Mg & $\mathrm{Na}$ & & & & & & & & Se & v \\
\hline Reaktor 4.7 & & & & & & & & & & & & & & & & & & \\
\hline & 21.11 .2016 & 6,3 & 83,2 & 534 & 249 & 448 & 2632 & 381 & 75 & 0,44 & 7,4 & 1,63 & 538 & 1,53 & 65 & 47 & 0,0179 & 0,099 \\
\hline & 12.12.2016 & 6,5 & 85,4 & 515 & 244 & 465 & 2538 & 365 & 54 & 0,31 & 5,3 & 1,14 & 381 & 1,43 & 46 & 34 & 0,0133 & 0,077 \\
\hline & 02.01.2017 & 7,0 & 85,3 & 528 & 250 & 514 & 2472 & 372 & 43 & 0,22 & 4,6 & 0,84 & 318 & 1,59 & 32 & 27 & 0,0098 & 0,091 \\
\hline 51 & 14.06.2017 & 8,0 & 86,6 & 442 & 234 & 859 & 2632 & 380 & 33 & 0,21 & 2,8 & 0,65 & 250 & 2,5 & 33 & 24 & 0,0068 & 0,071 \\
\hline 63 & 26.06.2017 & 8,3 & 87,0 & 438 & 227 & 1053 & 2774 & 385 & 40 & 0,165 & 2,5 & 0,53 & 192 & 2,2 & 26 & 20 & 0,0065 & 0,056 \\
\hline 84 & 17.07.2017 & 9,2 & 86,8 & 459 & 233 & 1343 & 2945 & 447 & 52 & 0,151 & 3,0 & 0,45 & 177 & 1,97 & 21 & 19 & 0,0056 & 0,068 \\
\hline 105 & 07.08.2017 & 9,5 & 86,4 & 463 & 234 & 1575 & 3264 & 483 & 72 & 0,114 & 3,3 & 0,37 & 201 & 1,78 & 16 & 16 & 0,0053 & 0,084 \\
\hline 126 & 28.08.2017 & 11,5 & 87,4 & 526 & 248 & 1871 & 3688 & 581 & 72 & 0,087 & 2,7 & 0,31 & 158 & 1,80 & 15 & 14 & 0,0051 & 0,080 \\
\hline 147 & 18.09.2017 & 11,3 & 88,4 & 454 & 221 & 1705 & 3212 & 504 & 66 & 0,064 & 2,5 & 0,25 & 116 & 1,42 & 10,8 & 9,6 & 0,0037 & 0,064 \\
\hline 168 & 09.10 .2017 & 9,5 & 78,9 & 375 & 173 & 1372 & 2856 & 424 & 3652 & 0,134 & 3,1 & 0,45 & 193 & 1,17 & 22 & 17 & 0,0049 & 0,057 \\
\hline 182 & 23.10.2017 & 9,3 & 77,3 & 375 & 176 & 1405 & 2842 & 410 & 4037 & 0,147 & 3,2 & 0,48 & 194 & 1,18 & 23 & 17 & 0,0043 & 0,051 \\
\hline 203 & 13.11.2017 & 8,7 & 77,3 & 355 & 174 & 1292 & 2749 & 388 & 3745 & 0,184 & 4,3 & 0,65 & 266 & 1,16 & 29 & 21 & 0,0050 & 0,049 \\
\hline 224 & 04.12.2017 & 4,3 & 59,3 & 253 & 133 & 1061 & 2780 & 324 & 3218 & 0,20 & 3,8 & 0,75 & 259 & 1,06 & 27 & 25 & 0,0062 & 0,037 \\
\hline 238 & 18.12.2017 & 6,6 & 81,3 & 274 & 135 & 1145 & 2108 & 311 & 1844 & 0,22 & 4,5 & 0,64 & 266 & 0,95 & 30 & 20 & na & 0,052 \\
\hline 259 & 08.01.2018 & 7,3 & 83,5 & 312 & 156 & 1359 & 2445 & 357 & 1238 & 0,197 & 4,2 & 0,60 & 244 & 0,98 & 29 & 19 & na & 0,054 \\
\hline 280 & 29.01 .2018 & 8,2 & 84,9 & 336 & 167 & 1499 & 2617 & 386 & 806 & 0,182 & 3,4 & 0,54 & 220 & 0,97 & 27 & 18 & na & 0,056 \\
\hline 301 & 19.02 .2018 & 8,9 & 85,6 & 396 & 187 & 1802 & 2696 & 456 & 534 & 0,179 & 5,0 & 0,55 & 248 & 1,26 & 29 & 18 & na & 0,079 \\
\hline 322 & 12.03 .2018 & 9,3 & 85,0 & 421 & 200 & 1838 & 3090 & 480 & 403 & 0,20 & 6,6 & 0,67 & 280 & 1,41 & 30 & 19 & na & 0,112 \\
\hline 345 & 04.04 .2018 & 10,3 & 86,3 & 469 & 216 & 1933 & 3142 & 524 & 272 & 0,185 & 4,8 & 0,59 & 258 & 1,42 & 31 & 19 & na & 0,090 \\
\hline 364 & 23.04 .2018 & 8,9 & 84,4 & 449 & 213 & 1756 & 3290 & 524 & 209 & 0,174 & 3,4 & 0,54 & 237 & 1,31 & 29 & 19 & na & 0,079 \\
\hline aktor 4.8 & & & & & & & & & & & & & & & & & & \\
\hline & 21.11 .2016 & 6,1 & 83,1 & 515 & 240 & 427 & 2579 & 368 & 74 & 0,44 & 7,4 & 1,59 & 520 & 1,49 & 63 & 46 & 0,0171 & 0,098 \\
\hline & 12.12.2016 & 6,6 & 85,0 & 514 & 248 & 472 & 2539 & 361 & 54 & 0,31 & 5,9 & 1,18 & 392 & 1,48 & 47 & 35 & 0,0141 & 0,078 \\
\hline & 02.01.2017 & 7,3 & 85,1 & 565 & 261 & 534 & 2552 & 400 & 46 & 0,25 & 5,4 & 0,92 & 341 & 1,69 & 35 & 39 & 0,0105 & 0,094 \\
\hline 51 & 14.06.2017 & 7,9 & 86,9 & 411 & 223 & 814 & 2522 & 358 & 36 & 0,198 & 2,9 & 0,62 & 245 & 2,4 & 32 & 23 & 0,0066 & 0,068 \\
\hline 63 & 26.06.2017 & 8,4 & 87,1 & 414 & 217 & 1012 & 2762 & 383 & 39 & 0,153 & 2,6 & 0,53 & 191 & 2,1 & 26 & 19 & 0,0066 & 0,062 \\
\hline 84 & 17.07.2017 & 9,0 & 87,2 & 452 & 230 & 1389 & 2786 & 422 & 48 & 0,142 & 3,0 & 0,44 & 186 & 2,0 & 22 & 20 & 0,0056 & 0,069 \\
\hline 105 & 07.08.2017 & 9,2 & 86,3 & 429 & 216 & 1486 & 3020 & 463 & 57 & 0,110 & 3,3 & 0,37 & 182 & 1,69 & 16 & 17 & 0,0051 & 0,080 \\
\hline 126 & 28.08.2017 & 10,0 & 86,8 & 481 & 230 & 1701 & 3312 & 527 & 65 & 0,096 & 2,8 & 0,33 & 153 & 1,73 & 14 & 13 & 0,0034 & 0,078 \\
\hline 147 & 18.09.2017 & 10,3 & 87,2 & 464 & 223 & 1713 & 3250 & 512 & 67 & 0,066 & 2,5 & 0,27 & 118 & 1,46 & 11 & 9,7 & 0,0038 & 0,067 \\
\hline 168 & 09.10 .2017 & 10,7 & 87,9 & 491 & 234 & 1835 & 2885 & 493 & 60 & 0,136 & 2,7 & 0,43 & 183 & 1,51 & 22 & 17 & 0,0057 & 0,057 \\
\hline 182 & 23.10.2017 & 9,6 & 85,9 & 491 & 234 & 1832 & 3405 & 524 & 68 & 0,141 & 2,5 & 0,45 & 188 & 1,42 & 23 & 18 & 0,0058 & 0,060 \\
\hline 203 & 13.11.2017 & 9,9 & 85,4 & 484 & 229 & 1863 & 3492 & 524 & 71 & 0,125 & 2,3 & 0,46 & 194 & 1,25 & 23 & 16 & 0,0052 & 0,059 \\
\hline 224 & 04.12 .2017 & 10,2 & 86,2 & 455 & 212 & 1765 & 3491 & 522 & 63 & 0,128 & 2,0 & 0,47 & 197 & 1,17 & 26 & 18 & 0,0054 & 0,058 \\
\hline 238 & 18.12.2017 & 9,2 & 86,8 & 446 & 208 & 1941 & 2890 & 487 & 58 & 0,142 & 1,81 & 0,48 & 203 & 1,14 & 27 & 18 & na & 0,055 \\
\hline 259 & 08.01.2018 & 9,5 & 85,5 & 415 & 198 & 1886 & 3344 & 480 & 69 & 0,137 & 1,78 & 0,47 & 187 & 1,03 & 25 & 16 & na & 0,058 \\
\hline 280 & 29.01 .2018 & 9,5 & 86,2 & 432 & 212 & 1830 & 3341 & 499 & 71 & 0,156 & 1,92 & 0,50 & 195 & 1,08 & 27 & 18 & na & 0,057 \\
\hline 301 & 19.02 .2018 & 9,5 & 85,7 & 440 & 211 & 1855 & 3416 & 510 & 75 & 0,151 & 2,8 & 0,51 & 216 & 1,21 & 27 & 17 & na & 0,074 \\
\hline 322 & 12.03 .2018 & 8,7 & 84,3 & 454 & 213 & 1876 & 3257 & 523 & 77 & 0,182 & 5,0 & 0,56 & 245 & 1,40 & 28 & 18 & na & 0,100 \\
\hline 345 & 04.04 .2018 & 9,3 & 84,8 & 458 & 214 & 1894 & 3360 & 532 & 81 & 0,179 & 5,0 & 0,57 & 243 & 1,42 & 27 & 18 & na & 0,103 \\
\hline 364 & 23.04.2018 & 9,2 & 84,6 & 468 & 219 & 1838 & 3324 & 540 & 91 & 0,188 & 5,1 & 0,59 & 256 & 1,44 & 29 & 18 & na & 0,104 \\
\hline
\end{tabular}

(14) Projektphase 3: Elementgehalte der Makro- und Mikronährstoffe (Quasi)kontinuierliche Laborfermenterversuche am DBFZ Reaktor 4.9: Referenzreaktor (Spurenelementvollversorgung)

\begin{tabular}{|c|c|c|c|c|c|c|c|c|c|c|c|c|c|c|c|c|c|c|}
\hline \multicolumn{4}{|c|}{ Substitutionsversuch $3+4$} & \multicolumn{6}{|c|}{ Makronährstoffe } & \multicolumn{9}{|c|}{ Mikronährstoffe } \\
\hline \multirow{2}{*}{\multicolumn{2}{|c|}{$\begin{array}{l}\text { Reaktor } 4.9 \\
\mathrm{mg} / \mathrm{kg} \mathrm{FM}\end{array}$}} & & essmethode: & ICP-OES & ICP-OES & ICP-OES & ICP-OES & ICP-OES & ICP-OES & ICP-OES & ICP-OES & ICP-OES & ICP-OES & ICP-OES & ICP-OES & ICP-OES & ICP-MS & ICP-OES \\
\hline & & & Element: & P 178.222 & S 181.972 & Са $317.933 \mathrm{r}$ & K $766.491 \mathrm{r}$ & Mg 279.078r & Na 588.995r & Co 230.786 & Ni 231.604 & Mo 202.032 & Fe 234.350 & Си 327.395 & Mn 257.610 & Zn 206.200 & Se 77 KED & $\mathrm{V} 292.401$ \\
\hline $\begin{array}{l}\text { Versuchs- } \\
\text { tag }\end{array}$ & $\begin{array}{l}\text { Probe- } \\
\text { nahme }\end{array}$ & $\begin{array}{c}\text { TS } \\
\text { [Gew. } \%]\end{array}$ & $\begin{array}{c}\text { oTS } \\
\text { [Gew.\%] }\end{array}$ & $\mathbf{P}$ & $\mathbf{s}$ & $\mathrm{Ca}$ & $\mathbf{K}$ & Mg & $\mathrm{Na}$ & Co & $\mathrm{Ni}$ & Mo & $\mathrm{Fe}$ & $\mathrm{Cu}$ & $\mathrm{Mn}$ & $\mathrm{Zn}$ & Se & $\mathbf{v}$ \\
\hline & 21.11 .2016 & 6,4 & 83,8 & 522 & 246 & 436 & 2591 & 372 & 74 & 0,47 & 7,6 & 1,72 & 577 & 1,49 & 69 & 50 & 0,0193 & 0,102 \\
\hline & 12.12 .2016 & 6,6 & 84,4 & 514 & 252 & 461 & 2525 & 362 & 53 & 0,49 & 7,2 & 1,77 & 600 & 1,50 & 74 & 53 & 0,0195 & 0,104 \\
\hline & 02.01.2017 & 7,4 & 85,1 & 542 & 264 & 540 & 2582 & 387 & 43 & 0,54 & 7,6 & 1,93 & 679 & 1,68 & 81 & 59 & 0,022 & 0,125 \\
\hline 51 & 14.06.2017 & 7,9 & 86,1 & 455 & 246 & 536 & 2527 & 339 & 25 & 0,66 & 7,9 & 1,74 & 676 & 2,8 & 87 & 61 & 0,0174 & 0,097 \\
\hline 63 & 26.06 .2017 & 8,3 & 87,2 & 443 & 236 & 515 & 2512 & 330 & 21 & 0,62 & 7,8 & 1,65 & 640 & 2,4 & 81 & 58 & 0,0172 & 0,093 \\
\hline 84 & 17.07.2017 & 9,1 & 88,6 & 455 & 237 & 516 & 2306 & 338 & 17 & 0,58 & 8,5 & 1,62 & 707 & 2,1 & 84 & 64 & 0,0157 & 0,079 \\
\hline 105 & 07.08.2017 & 8,4 & 88,0 & 452 & 237 & 553 & 2311 & 355 & 22 & 0,55 & 9,3 & 1,51 & 750 & 1,81 & 79 & 60 & 0,0135 & 0,100 \\
\hline 126 & 28.08.2017 & 9,0 & 88,0 & 515 & 263 & 589 & 2551 & 403 & 16 & 0,55 & 9,3 & 1,61 & 772 & 1,77 & 84 & 62 & 0,0174 & 0,071 \\
\hline 147 & 18.09.2017 & 8,4 & 87,8 & 469 & 243 & 531 & 2422 & 381 & 12 & 0,52 & 7,4 & 1,46 & 682 & 1,51 & 76 & 56 & 0,0144 & 0,053 \\
\hline 168 & 09.10 .2017 & 9,2 & 88,2 & 496 & 242 & 535 & 2624 & 408 & 10,2 & 0,52 & 6,5 & 1,45 & 709 & 1,42 & 84 & 63 & 0,0160 & 0,045 \\
\hline 182 & 23.10.2017 & 9,5 & 86,4 & 476 & 240 & 526 & 2566 & 372 & 1311 & 0,52 & 6,2 & 1,60 & 670 & 1,39 & 80 & 61 & 0,0130 & 0,041 \\
\hline 203 & 13.11.2017 & 8,4 & 85,4 & 508 & 244 & 550 & 2735 & 399 & 857 & 0,51 & 6,2 & 1,54 & 714 & 1,31 & 82 & 57 & 0,0146 & 0,037 \\
\hline 224 & 04.12 .2017 & 8,7 & 86,4 & 500 & 236 & 526 & 2788 & 399 & 563 & 0,53 & 5,6 & 1,50 & 704 & 1,23 & 85 & 62 & 0,0144 & 0,039 \\
\hline 238 & 18.12.2017 & 8,2 & 86,6 & 482 & 230 & 549 & 2708 & 387 & 449 & 0,55 & 5,4 & 1,60 & 729 & 1,19 & 89 & 63 & na & 0,029 \\
\hline
\end{tabular}


(15) Zusatzversuch: Elementgehalte der Makro- und Mikronährstoffe (Quasi)kontinuierliche Laborfermenterversuche am DBFZ -

Reaktor 4.11 und 4.12: Mais-Silage-Monovergärung mit reduzierter Spurenelemente-Zugabe

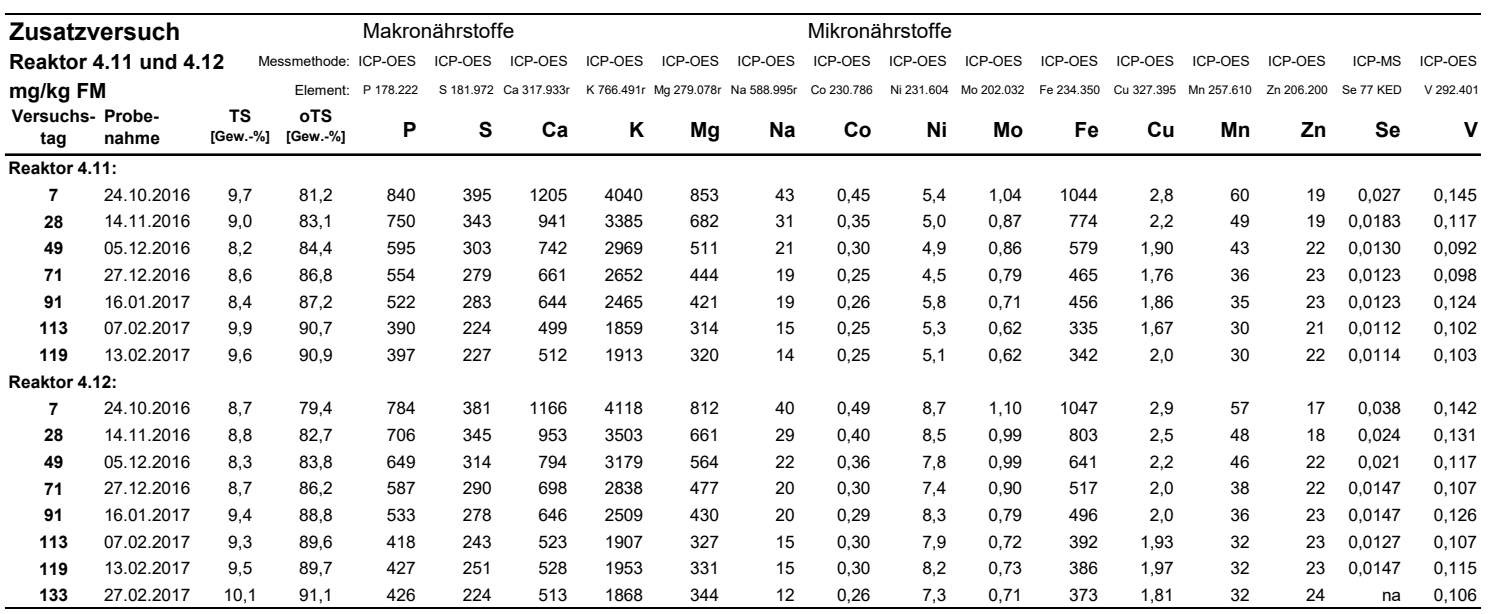




\section{Wissenschaftlicher Werdegang}

2015 - 2020 Promotionsstudium und Wissenschaftlicher Mitarbeiter, Georg-AugustUniversität Göttingen, Interdisziplinäres Zentrum für Nachhaltige Entwicklung (IZNE)

2013 -2014 Wissenschaftlicher Mitarbeiter, Georg-August-Universität Göttingen, Interdisziplinäres Zentrum für Nachhaltige Entwicklung (IZNE)

2010 - 2013 Studium der Geowissenschaften (Master of Science), Georg-AugustUniversität Göttingen, Thema der Masterarbeit: "Bodenbelastung und Elementtransfer vom Boden in Kulturpflanzen im Auenbereich der Oker bei Ohrum (Landkreis Wolfenbüttel, Niedersachsen)"

2009 - 2013 Studentische Hilfskraft, Georg-August-Universität Göttingen, Geowissenschaftliches Zentrum (GZG) und Interdisziplinäres Zentrum für Nachhaltige Entwicklung (IZNE)

2006 - 2010 Studium der Geowissenschaften (Bachelor of Science), Georg-AugustUniversität Göttingen, Thema der Bachelorarbeit: "Elementtransfer von kontaminierten und unkontaminierten Böden in Pappeln und Weiden"

\section{Beteiligung an Forschungsprojekten}

„Spurenelemente durch Energiepflanzen - Stoffströme und Handlungsempfehlungen für eine optimierte Prozessbiologie in Biogasanlagen - Teilvorhaben 1: (Spuren)elemente und Methodenentwicklung, Verfügbarer Anteil' im Fermentermaterial“" (Fachagentur Nachwachsende Rohstoffe e.V., Bundesministerium für Ernährung und Landwirtschaft, Promotionsprojekt)

"Nachhaltige Nutzung von Energie aus Biomasse im Spannungsfeld von Klimaschutz, Landschaft und Gesellschaft - Teilprojekt G2: Schadstoffemissionen bei der Verbrennung von Waldholz und Kurzumtriebshölzern von nicht belasteten und belasteten Standorten“ (Niedersächsisches Ministerium für Wissenschaft und Kultur)

"Screening und Selektion von Amarantsorten und -linien als spurenelementreiches Biogassubstrat" - Projektpartner des Technologie- und Förderzentrums (TFZ) in Straubing (Bayerisches Staatsministerium für Ernährung, Landwirtschaft und Forsten)

\section{Veröffentlichungen}

PASOLD, T. (2020): Charakterisierung minimaler und maximaler Hemmkonzentrationen verschiedener Spurenelemente in Biogasprozessen und Entwicklung eines vereinfachten Bestimmungsverfahrens. Dissertation, Göttingen, 242 S. (vorliegende Arbeit)

Nguyen, T.P., Ruppert, H., Pasold, T., Sauer, B. (2020): Paddy soil geochemistry, uptake of trace elements by rice grains (Oryza sativa) and resulting health risks in the Mekong River Delta, Vietnam. Environmental Geochemistry and Health 42 (8), 2377-2397, Link: https://doi.org/10.1007/s10653-019-00456-7 
Nguyen, T.P., Ruppert, H., Sauer, B., Pasold, T. (2020): Harmful and nutrient elements in paddy soils and their transfer into rice grains (Oryza sativa) along two river systems in northern and central Vietnam. Environmental Geochemistry and Health 42 (1), 191-207. Link: https://doi.org/10.1007/s10653-019-00333-3

Sauer, B., Sträuber, H., Fahlbusch, W., Hey, K., PAsold, T., Pröter, J., Rauber, R., RUPPERT, H., ZECHENDORF, M. (2018): Spurenelemente durch Energiepflanzen - Stoffströme und Handlungsempfehlungen für eine optimierte Prozessbiologie in Biogasanlagen (NiCo). Schlussbericht zum Vorhaben, Fachagentur Nachwachsende Rohstoffe e.V. (Förderkennzeichen: 22014813, 22019014, 22019114, 22019214), 85 S.

Link: https://www.fnr-server.de/ftp/pdf/berichte/22014813.pdf

Sauer, B., Sträuber, H., Hey, K., Fahlbusch, W., Pasold, T., Dittrich-ZechenDORF, M., PRÖTER, J., RUPPERT, H., RAUBER, R. (2018): Spurenelemente durch Energiepflanzen - Stoffströme und Handlungsempfehlungen für eine optimierte Prozessbiologie in Biogasanlagen (NiCo). In: ProFair Consult+Project GmbH (Hrsg.): Tagungsband zum 11. Biogas-Innovationskongress 2018, Mai 2018, Osnabrück, 33-44.

Nguyen, T.P., Ruppert, H., Pasold, T., Fahlbusch, W., Sauer, B. (2018): The transfer of critical elements from soils to rice grains in the Mekong River delta area, Vietnam. $20^{\text {th }}$ EGU General Assembly, EGU2018, Proceedings from the conference held 4-13 April, April 2018, Wien, 4709.

Link: https://meetingorganizer.copernicus.org/EGU2018/EGU2018-4709-2.pdf

Pasold, T., Dittrich-Zechendorf, M., Sträuber, H., SAuer, B., RupPert, H. (2017): Auswirkungen von Cobalt- und Nickelmangel auf quasi-kontinuierliche Biogasprozesse und methanogene Gemeinschaften. KTBL-Schrift 512, FNR/KTBL-Kongress Biogas in der Landwirtschaft - Stand und Perspektiven, September 2017, Bayreuth, 338-339.

Link: https://www.ktbl.de/fileadmin/user_upload/Artikel/Energie/Biogastagung/11512.pdf

Fahlbusch, W., Pospiech, S., Sauer, B., Pasold, T., Ruppert, H. (2017): Trace elements in plants - correction methods for adhering particles to get real plant uptake. Goldschmidt2017 Abstract, Goldschmidt2017, August 2017, Paris, 1094.

Link: https://goldschmidtabstracts.info/2017/1094.pdf

Pospiech, S., Fahlbusch, W., Pasold, T., Sauer, B., Ruppert, H. (2017): Elementanalytik an ICP-OES und ICP-MS - Handbuch für die Probenpräparation, Messung und Auswertung. Interne Wissenssammlung der Arbeitsgruppe Umweltgeochemie (Skript), Göttingen, $75 \mathrm{~S}$.

Pospiech, S., Fahlbusch, W., Sauer, B., Pasold, T., RupPert, H. (2017): Alteration of trace element concentrations in plants by adhering particles - Methods of correction. Chemosphere 182, 501-508. Link: https://doi.org/10.1016/j.chemosphere.2017.05.038

SeIDEl, T., Orasche, J., PAsOld, T., RupPeRT, H. (2017): Schadstoffemissionen bei der Verbrennung von Holz, Stroh und Biogas. In: Ruppert, H., Ibendorf, J. (Hrsg.): Bioenergie im Spannungsfeld - Wege zu einer nachhaltigen Bioenergieversorgung. Universitätsverlag Göttingen, 355-380. Link: https://doi.org/10.17875/gup2017-1042

Pospiech, S., Sauer, B., Fahlbusch, W., Pasold, T., Tolosana-Delgado, R., RUPPERT, H. (2016): Trace element transfer into the plant: What can we learn from element ratios? Goldschmidt Conference Abstracts, Goldschmidt2016, Juni-Juli 2016, Yokohama, 2532. Link: https://goldschmidtabstracts.info/2016/2532.pdf 
SAuer, B., Ruppert, H., RAuber, R., StrÄUber, H., PrÖter, J., Hey, K., FAHLBusch, W., DitTRICH-ZECHENDORF, M., PASOLD, T. (2015): Verbundvorhaben: Spurenelemente durch Energiepflanzen - Stoffströme und Handlungsempfehlungen für eine optimierte Prozessbiologie in Biogasanlagen. KTBL-Schrift 508, FNR/KTBL-Kongress Biogas in der Landwirtschaft - Stand und Perspektiven, September 2015, Potsdam, 354-355.

Link: https://www.ktbl.de/fileadmin/user_upload/Artikel/Energie/Biogastagung/11508.pdf

\section{Vorträge und Posterpräsentationen}

PASOLD, T. (2019): Minimale und maximale Spurenelementkonzentrationsbereiche in Biogasprozessen. Vortrag, Regenerative Energiesysteme (Vorlesung, B.ÖSM.115.3, Wintersemester 2018/2019), Januar 2019, Göttingen.

Nguyen, T.P., Ruppert, H., Pasold, T., Fahlbusch, W., Sauer, B. (2018): The transfer of critical elements from soils to rice grains in the Mekong River delta area, Vietnam. Posterpräsentation, European Geosciences Union (EGU) General Assembly 2018, April 2018, Wien.

PASOLD, T. (2018): Minimale und maximale Spurenelementkonzentrationsbereiche in Biogasprozessen. Vortrag, Fachtagung NiCo: Spurenelemente durch Energiepflanzen Stoffströme und Handlungsempfehlungen für eine optimierte Prozessbiologie in Biogasanlagen, März 2018, Göttingen.

PASOLD, T. (2018): Verarmungs- und Substitutionsversuche in (quasi)kontinuierlichen Laborfermentern. Vortrag, Seminar zur Sedimentologie und Umweltgeologie, Januar 2018, Göttingen.

Pasold, T., Dittrich-Zechendorf, M., SträUber, H., SAuer, B., RupPert, H. (2017): Auswirkungen von Cobalt- und Nickelmangel auf quasi-kontinuierliche Biogasprozesse und methanogene Gemeinschaften. Posterpräsentation, FNR/KTBL-Kongress Biogas in der Landwirtschaft - Stand und Perspektiven, September 2017, Bayreuth.

Fahlbusch, W., Pospiech, S., Sauer, B., Pasold, T., Ruppert, H. (2017): Trace elements in plants - correction methods for adhering particles to get real plant uptake. Posterpräsentation, Goldschmidt2017, August 2017, Paris.

PASOld, T. (2016): Nickel und Cobalt - essenzielle Spurenelemente für die Biogasbildung: Minimale Konzentrationsbereiche und Substitutionsversuche in (quasi)kontinuierlichen Laborfermentern. Vortrag, Seminar zur Sedimentologie und Umweltgeologie, November 2016, Göttingen.

Nguyen, T.P., Ruppert, H., Sauer, B., Pasold, T., Fahlbusch, W (2018): Heavy metal contamination in paddy soils and rice in Vietnam. Posterpräsentation, 9th GeoSymposium of Young Researchers Silesia 2016, August-September 2016, Kroczyce.

SAuer, B., RupPert, H., Rauber, R., SträUber, H., Pröter, J., Hey, K., FAhlbusch, W., DitTRICH-ZEChENDORF, M., PASOlD, T. (2015): Verbundvorhaben: Spurenelemente durch Energiepflanzen - Stoffströme und Handlungsempfehlungen für eine optimierte Prozessbiologie in Biogasanlagen. Posterpräsentation, FNR/KTBL-Kongress Biogas in der Landwirtschaft - Stand und Perspektiven, September 2015, Potsdam. 


\section{Betreute Abschlussarbeiten (Zweitgutachten)}

CAESAR, M. (2017): Wachstum, Elementgehalte und Nutzungspotenziale von Azolla filiculoides in verschiedenen Reinigungsstufen der Göttinger Kläranlage. Bachelorarbeit, Georg-August-Universität Göttingen, $83 \mathrm{~S}$.

PFEIFFER, A. (2016): Auswirkungen des Sojaanbaus auf die Herkunftsregionen in Südamerika und Konsequenzen für Landwirtschaft und Umwelt. Bachelorarbeit, GeorgAugust-Universität Göttingen, $30 \mathrm{~S}$.

STÜRZEBECHER, T. (2016): Versuch der Phosphorbilanzierung zweier Biogasanlagen in Süd-Niedersachsen. Bachelorarbeit, Georg-August-Universität Göttingen, 65 S.

Petering, A. (2015): Schwermetallkontamination in Böden und Weizenkörnern im Raum Harlingerode (Bad Harzburg, Landkreis Goslar, Niedersachsen). Bachelorarbeit, Georg-August-Universität Göttingen, 55 S.

Zwosta, L. (2015): Potenzial- und Nutzungsanalyse von kontaminierten Standorten am Beispiel Harlingerode in Niedersachsen. Bachelorarbeit, Georg-August-Universität Göttingen, $61 \mathrm{~S}$. 